\title{
Mechanisms of Dexamethasone-Induced
}

\section{Hypertension}

Sharon Leng Hong Ong

BSc (Med) MBBS FRACP

A thesis submitted for the degree of Doctor of Philosophy

of the Australian National University

May 2010

High Blood Pressure Research Unit

John Curtin School of Medical Research

Australian National University

Canberra, Australia 
CIIE AUSTRAL

एonal unives

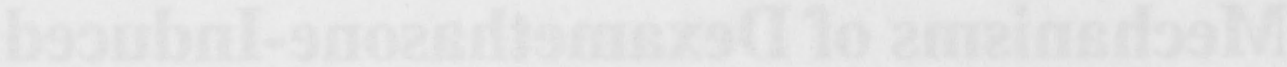




\section{DECLARATION}

The work in this thesis contains no material previously submitted for the award of any degree or diploma in any university or tertiary education institution. The results presented in this thesis are, except where otherwise acknowledged, my own original work.

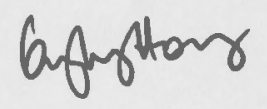

Sharon L H Ong

May 2010 
This thesis is dedicated

to the loving memory of my father

Ong Teng Sim 


\section{ACKNOWLEDGEMENTS}

I am extremely grateful to my supervisor, Professor Judith Whitworth for taking me on and giving me this wonderful opportunity to learn and develop as a researcher. I have learnt very important skills that have helped me significantly in my current roles as a Nephrologist, a lecturer, a researcher and a clinical and research supervisor. I sincerely thank her for her support, encouragement, wisdom and guidance throughout the course of my research studies. I also express my heartfelt gratitude for her continual guidance, beyond her retirement, with finalising this thesis. It was indeed a privilege to be part of her well-organised team.

I am also grateful to my co-supervisor, Dr Yi Zhang, for her assistance, encouragement and support during my $\mathrm{PhD}$ research studies.

I would like to thank the staff at the High Blood Pressure Research Unit for their technical assistance- Matthew Sutton, Janine Vickers, Leanne Langton and Kate Mackenzie. I am particularly indebted to Matthew Sutton for his generous assistance with blood pressure measurements (Chapters 4,6 and 8) and during cull days (Chapters 6 and 8). I am also grateful for the technical assistance provided by Janine Vickers with blood pressure measurements (Chapter 7) and plasma nitrate/nitrite analysis (Chapters 7 and 9). I would also like to thank 
Dr Harpreet Vohra for her expert advice and assistance in flow cytometry, and Dr Cheng Wen for his tips on rat surgery.

I also graciously acknowledge the Research and Education Foundation of the Royal Australasian College of Physicians and the National Heart Foundation for generously awarding me the Jacquot Research Entry Scholarship and the Postgraduate Research Scholarship, respectively. This financial support had allowed me pursue this full-time $\mathrm{PhD}$ research degree. Part of this research project was funded by an NHMRC Project Grant (Grant number 418026). I am also very grateful for the travel grants from the International Society of Hypertension, the Australia and New Zealand Society of Nephrology and the High Blood Pressure Research Council of Australia that enabled me to attend international and national conferences. I acknowledge the financial support of the High Blood Pressure Research Unit, JCSMR in my travel to the International Society of Hypertension Scientific Meeting in Berlin.

I would also like to thank our collaborators from University of Western Australia, Professor Kevin Croft and Dr Trevor Mori, who performed the plasma $F_{2^{-}}$ isoprostane assays.

ACT Pathology, The Canberra Hospital had kindly provided pathology services. 
I would like to thank my husband, Gary, for his support and encouragement during these years. I thank him for enduring the countless nights and weekends spent in the laboratory.

I would also like to acknowledge my mother and brother for their love and support; and finally, my in-laws for all their help during the busy and hectic times. 


\section{ABSTRACT}

Adrenocortical steroids with predominant glucocorticoid activity, both naturallyoccurring and synthetic, have long been recognised as a cause of hypertension. Models of glucocorticoid-induced hypertension (GC-HT) in the rat such as adrenocorticotrophic hormone-induced hypertension (ACTH-HT) and dexamethasone-induced hypertension (DEX-HT) are well-characterised, although the mechanisms contributing to hypertension are less well-understood. Previous studies have shown that ACTH-HT is associated with increased renal vascular resistance, nitric oxide deficiency and oxidative stress. The latter was shown to be linked to the $\mathrm{NAD}(\mathrm{P}) \mathrm{H}$ oxidase but not the xanthine oxidase pathways. As there were proven differences in the pathogenesis of these two models of GC-HT, mechanisms of ACTH-HT cannot be assumed in DEX-HT.

The main aim of this project was to evaluate the mechanisms of DEX-HT, namely the haemodynamic mechanisms and the role of oxidative stress. The role of $\beta$ adrenergic receptor blockade was also evaluated. In some studies, experiments on both dexamethasone (DEX)- and ACTH-HT were conducted for comparison.

DEX (10-20 $\mu \mathrm{g} / \mathrm{rat} /$ day) raised blood pressure in rats without significant metabolic side effects such as precipitous weight loss. The haemodynamic experiments showed that DEX-HT in rats was associated with increased total 
peripheral resistance and decreased total peripheral conductance, although these were not critical features for the production of DEX-HT.

This project showed that the xanthine oxidase pathway and mitochondria were not the major source of superoxide contributing to oxidative stress in DEX-HT. Mitochondrial superoxide, evaluated using a novel mitochondrial specific fluorogenic probe (mito-hydroethidine) via flow cytometry designed for this purpose, was also shown in this project not to play a major role in ACTH-HT in rats.

The arachidonic acid metabolite 20-hydroxyeicosatetraenoic acid, which is increased by nitric oxide deficiency and known to cause oxidative stress and exacerbate nitric oxide deficiency, also did not play a significant role in the pathogenesis of DEX-HT. Propranolol, both a beta adrenergic receptor blocker and lipid peroxidation inhibitor, was not effective in preventing GC-HT.

In conclusion, an increase in total peripheral resistance and nitric oxide-redox imbalance appear to be features of DEX-HT in the rat although the exact mechanism by which DEX raises blood pressure in human remains incompletely understood. Further studies exploring 1) the differences in mechanisms between naturally-occuring and synthetic glucocorticoids and 2) the relevance of oxidative stress and nitric oxide-redox imbalance in human GC-HT are important in advancing our understanding in this form of hypertension. 


\section{CONTENTS}

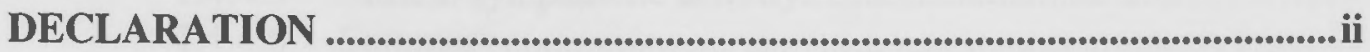

ACKNOWLEDGEMENTS ......................................................................................... iv

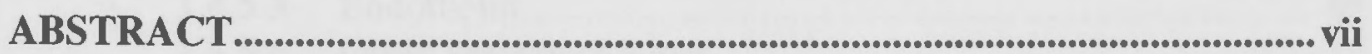

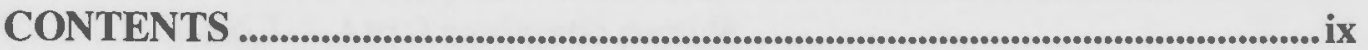

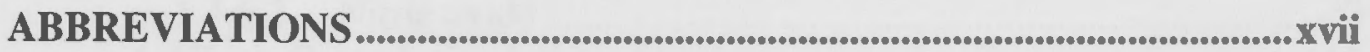

PUBLICATIONS AND PRESENTATIONS ARISING FROM THIS

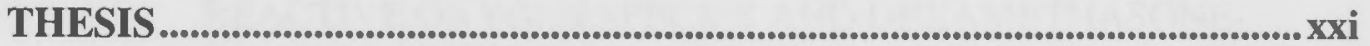

PRESENTATIONS................................................................................................xxiii

\section{CHAPTER 1: MECHANISMS OF DEXAMETHASONE-INDUCED}

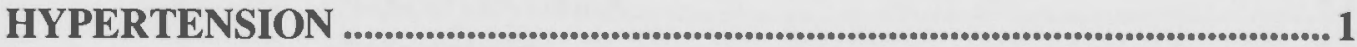

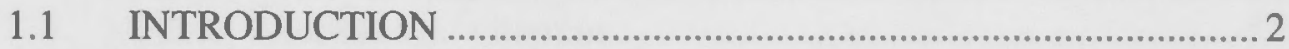

1.2 GLUCOCORTICOID HORMONES .................................................... 3

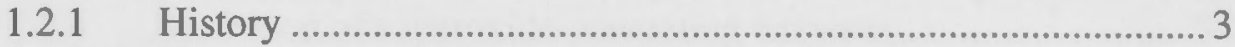

1.2.2 Naturally-occuring glucocorticoids........................................... 5

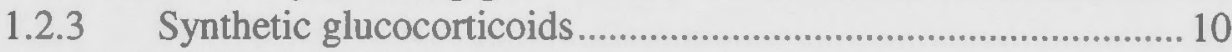

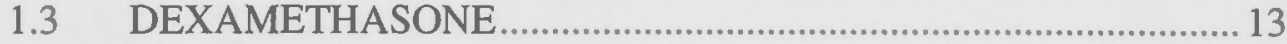

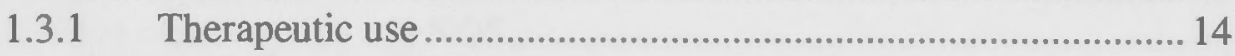

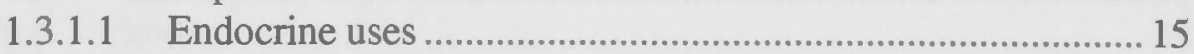

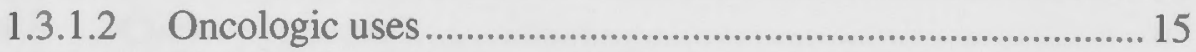

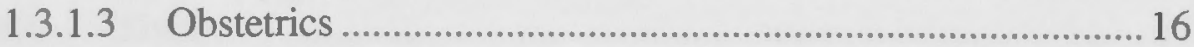

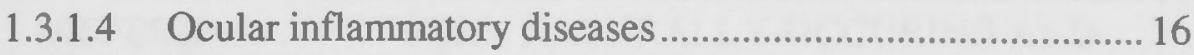

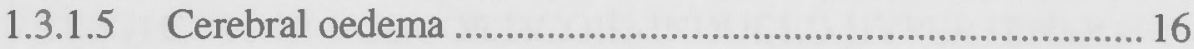

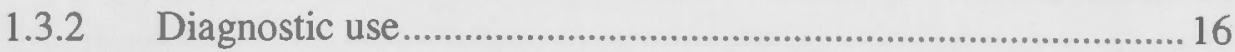

1.3.2.1 Overnight dexamethasone suppression test .......................... 17

1.3.2.2 Formal dexamethasone suppression test ................................ 17

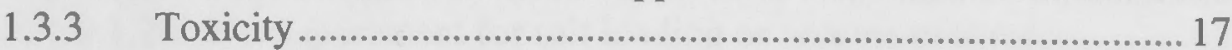

1.3.4 Blood pressure effect of dexamethasone...................................... 18

1.4 MECHANISMS OF DEXAMETHASONE-INDUCED

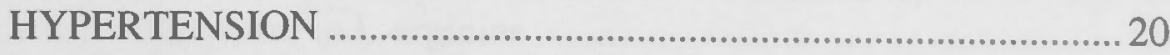

1.4.1 Sodium and water retention ........................................................ 20

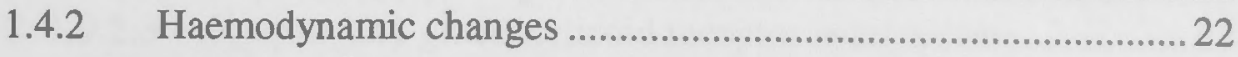


1.4.3 Increased vascular pressor responsiveness...................................23

1.4.4 Increased sympathetic nervous system activity .......................... 26

1.4.4.1 Changes in adrenergic receptors ............................................2 27

1.4.4.2 Changes in catecholamine synthetic pathway .........................22

1.4.4.3 Changes in neuropeptide Y ................................................... 29

1.4.4.4 Changes in plasma noradrenaline and adrenaline levels ........30

1.4.4.5 Muscle sympathetic activity.....................................................32

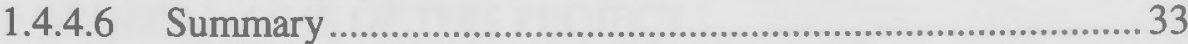

1.4.5 Excess vasopressor hormone ………………................................34

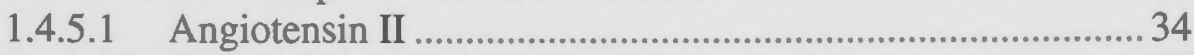

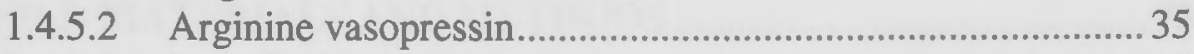

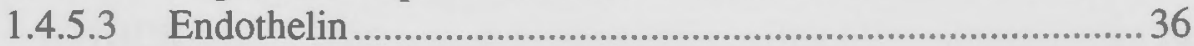

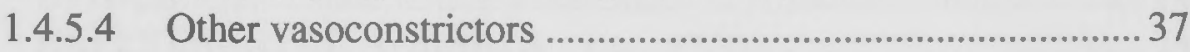

1.4.6 Deficiency in vasodilator hormone ............................................37

1.4.6.1 Atrial natriuretic peptide ...................................................... 37

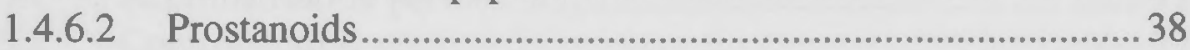

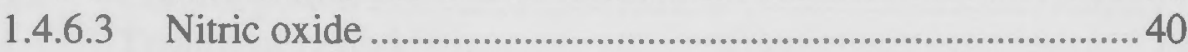

1.4.7 Central stimulation of blood pressure ..........................................45

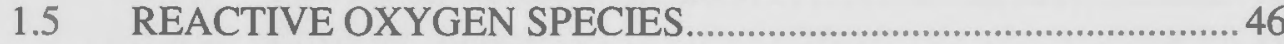

1.6 REACTIVE OXYGEN SPECIES AND HYPERTENSION ..............48

1.7 REACTIVE OXYGEN SPECIES AND DEXAMETHASONE-

INDUCED HYPERTENSION......................................................... 49

1.7.1 Experimental evidence for dexamethasone-induced oxidative stress.

1.7.2 Effect of antioxidants on dexamethasone-induced hypertension51

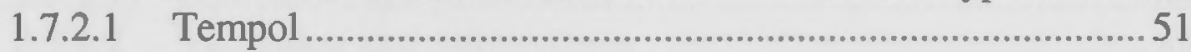

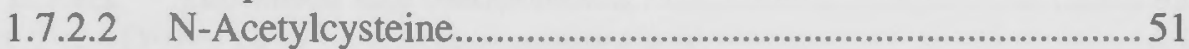

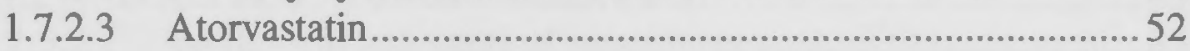

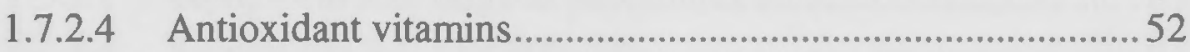

1.7.3 Altered expression and activity of antioxidant enzymes.............53

1.7.4 Inhibition of superoxide-generating enzymes ............................54

1.8 ROLE OF VASCULAR ROS IN GC-HT .......................................5

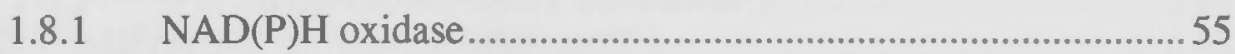

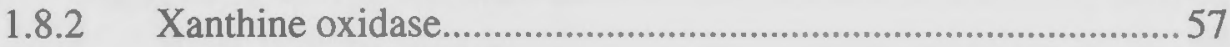

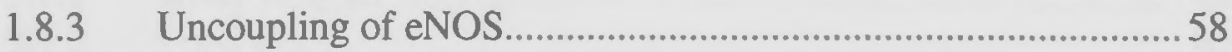

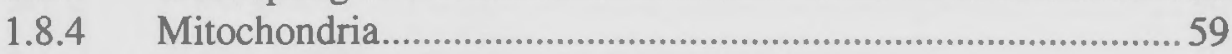

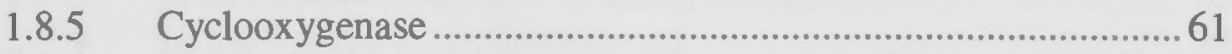

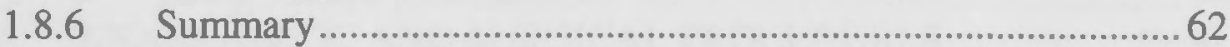

1.9 DIFFERENCES BETWEEN NATURALLY-OCCURING AND SYNTHETIC GLUCOCORTICOID-INDUCED HYPERTENSION

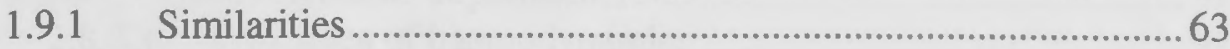

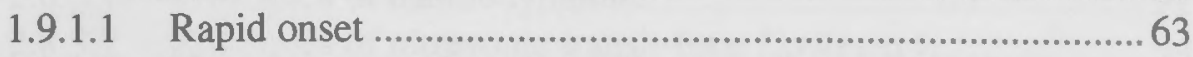

1.9.1.2 No requirement for salt loading or volume expansion...........63

1.9.1.3 Nitric oxide-redox imbalance.................................................64

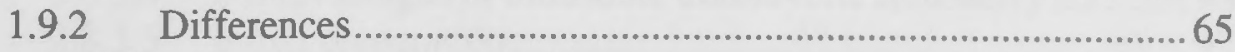

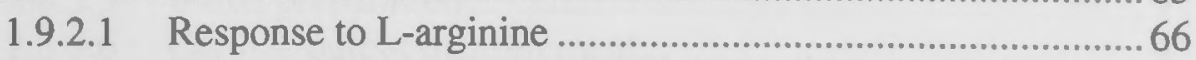

1.9.2.2 Urinary 20-hydroxyeicosatetraenoic acid ..............................66

1.9.2.3 Response to glucocorticoid receptor antagonism...................67 
1.9.2.4 Response to vasopressin antagonism .....................................68

1.9.2.5 Response to aspirin ..............................................................68

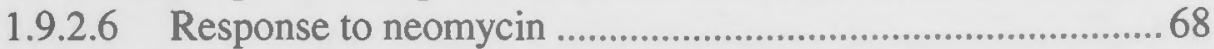

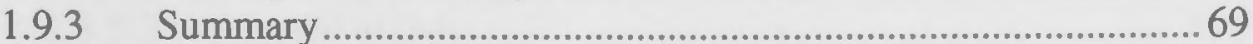

1.10 SUMMARY-MECHANISMS OF DEXAMETHASONE-INDUCED

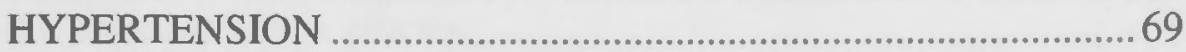

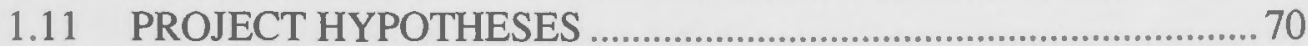

1.12 GENERAL AIMS OF THIS PROJECT ……................................... 70

1.13 SPECIFIC AIMS OF THIS PROJECT ……….................................. 71

CHAPTER 2: MATERIALS AND METHODS ...................................................... 73

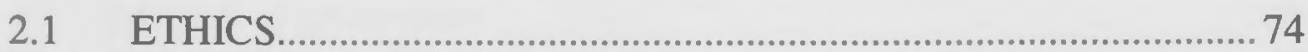

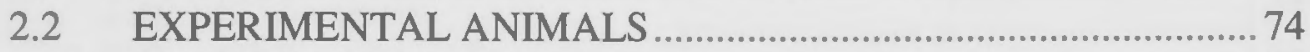

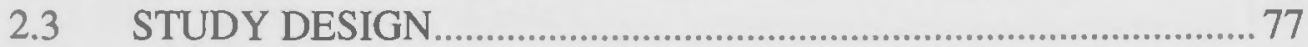

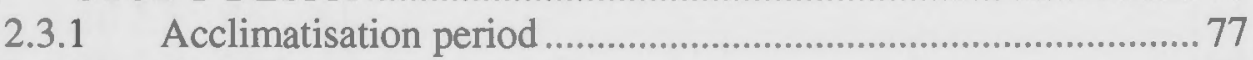

2.3.2 Control period ...........................................................................

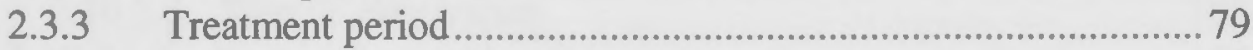

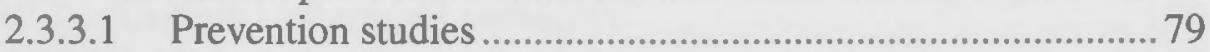

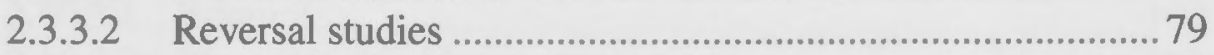

2.4 DRUG ADMINISTRATION PROTOCOL........................................ 81

2.4.1 Dexamethasone preparation and administration .......................... 81

2.4.2 ACTH preparation .................................................................... 82

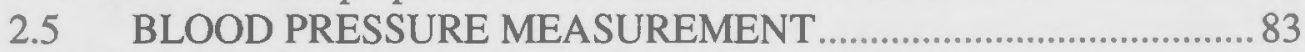

2.5.1 Indirect blood pressure measurement ........................................ 83

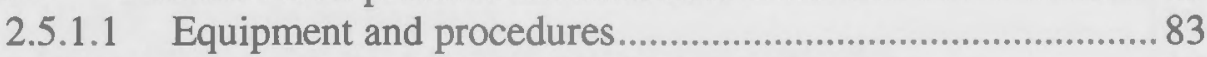

2.5.1.2 Validation and maintenance................................................ 85

2.5.2 Direct blood pressure measurement ............................................... 85

2.5.2.1 Equipment and surgical procedures ...................................... 85

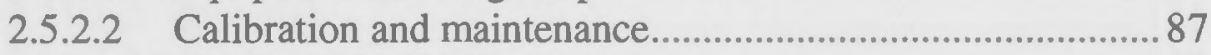

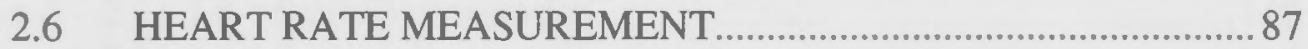

2.6.1 Via tail-cuff sphygmomanometry ............................................... 87

2.6.2 Via Millar carotid artery transducer .......................................... 88

2.7 METABOLIC MEASUREMENTS_.......................................................

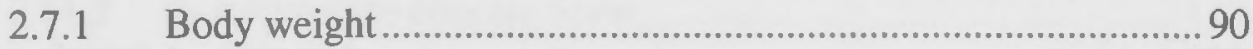

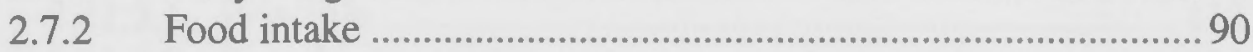

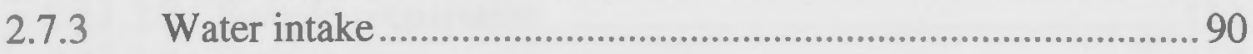

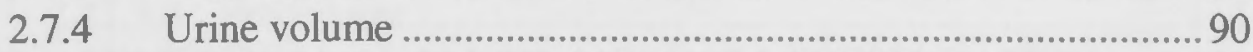

2.8 ANAESTHETIC AND SURGICAL PROCEDURES ........................91

2.8.1 Presurgical treatment ............................................................... 91

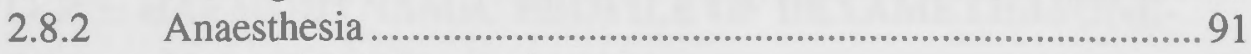

2.8.3 Haemodynamic experiments................................................... 92

2.8.3.1 Principles of haemodynamics ............................................. 92

2.8.3.2 Principles of ultrasonic transit-time flowmetry...................... 94

2.8.3.3 Advantages of ultrasonic transit-time flowmetry ...................96

2.8.3.4 Disadvantages of ultrasonic transit-time flowmetry ............. 96

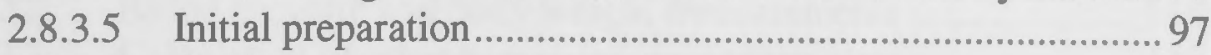

2.8.3.6 Cardiac output measurement.................................................97

2.8.3.7 Regional blood flow measurements .....................................99 
2.8.4 Sacrifice and blood collection procedures ................................. 99

2.8.5 Organ weight determination..................................................... 100

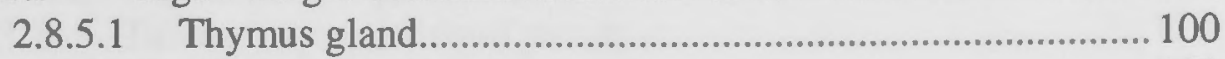

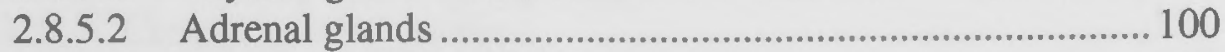

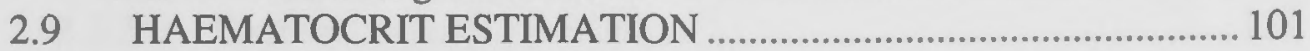

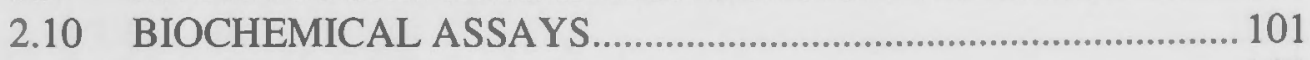

2.10.1 Plasma nitrite and nitrate assay ................................................. 101

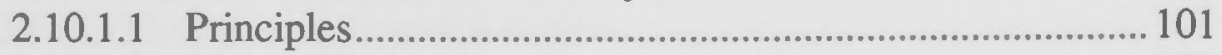

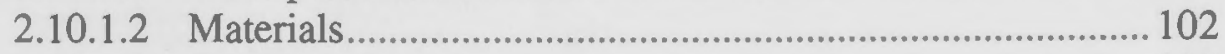

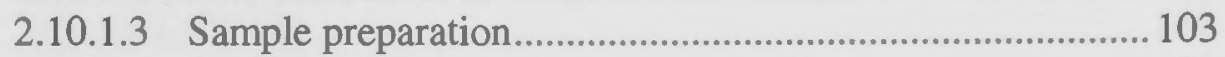

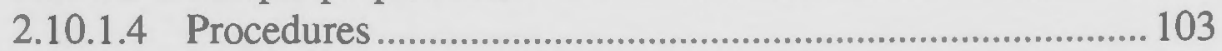

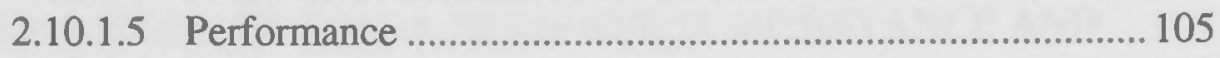

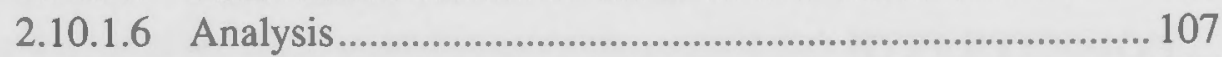

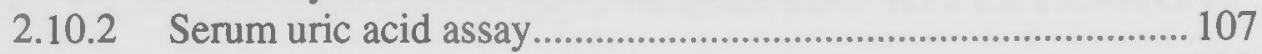

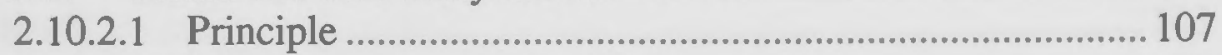

2.10.2.2 Materials and procedures................................................... 108

2.10.2.3 Sample preparation.............................................................. 108

2.10.3 Urinary sodium and potassium .............................................. 109

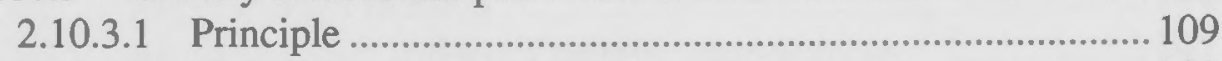

2.10.3.2 Materials and procedures..................................................... 109

2.10.3.3 Sample preparation.......................................................... 109

2.10.4 Urinary creatinine assay ........................................................... 109

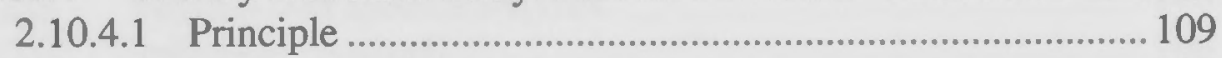

2.10.4.2 Materials and procedures.................................................. 110

2.10.4.3 Sample preparation.............................................................. 110

2.11 DETECTION OF REACTIVE OXYGEN SPECIES …………......... 110

2.11.1 Lucigenin-enhanced chemiluminescence assay ......................... 110

2.11.1.1 Principle ................................................................................ 110

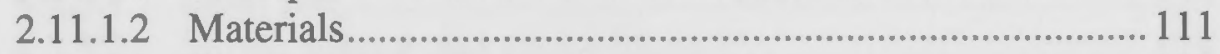

2.11.1.3 Sample preparation............................................................. 112

2.11.1.4 Procedures ................................................................... 112

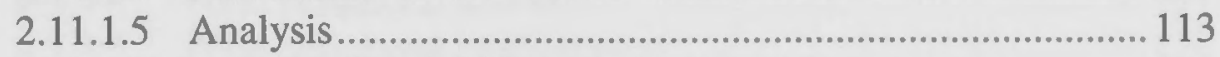

2.11.2 Kidney mitochondrial superoxide analysis .............................. 113

$2.11 .3 \quad F_{2}$-isoprostane concentration..................................................... 113

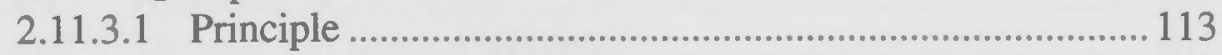

2.11.3.2 Sample preparation............................................................ 113

2.11.3.3 Procedures..................................................................... 114

2.12 STATISTICAL ANALYSIS AND DATA PRESENTATION ....... 114

CHAPTER 3: HAEMODYNAMIC PROFILE OF DEXAMETHASONEINDUCED HYPERTENSION IN THE RAT........................................................ 115

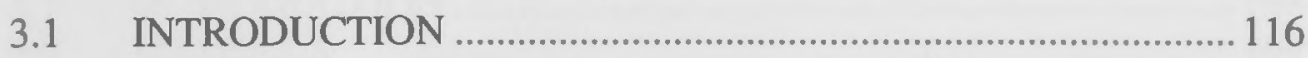

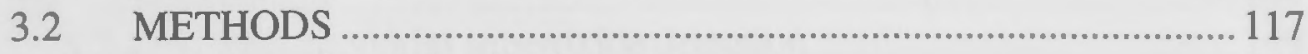

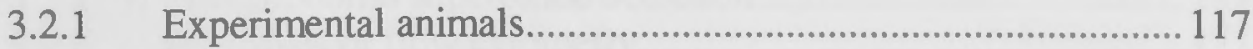

3.2.2 Blood pressure and body weight measurements ....................... 118

3.2.3 Calculation of other haemodynamic parameters ........................ 118

3.2.4 Haematocrit estimation ........................................................... 119

3.2.5 Thymus and adrenal weights................................................. 119 


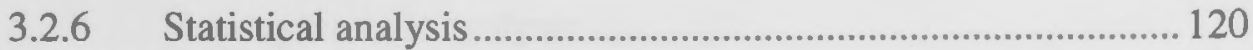

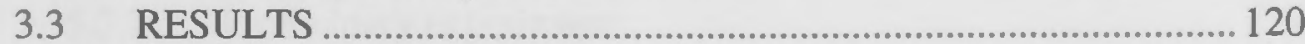

3.3.1 Tail-cuff systolic blood pressure............................................. 120

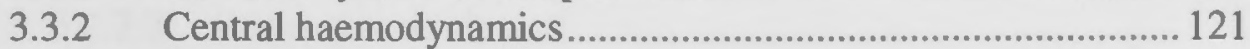

3.3.3 Regional haemodynamics ...................................................... 128

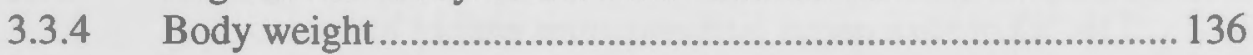

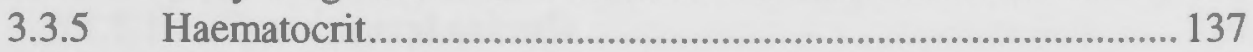

3.3.6 Thymus and adrenal weights.................................................... 137

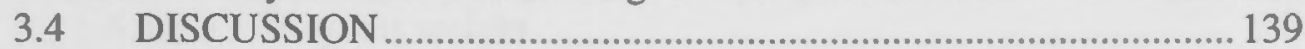

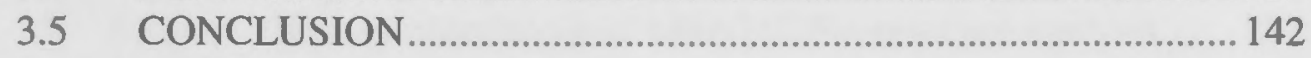

CHAPTER 4: ROLE OF TOTAL PERIPHERAL RESISTANCE AND CONDUCTANCE IN DEXAMETHASONE-INDUCED HYPERTENSION

IN THE RAT ....................................................................................................... 143

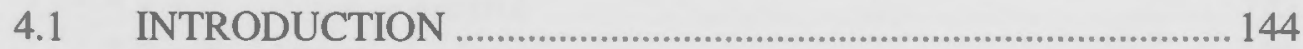

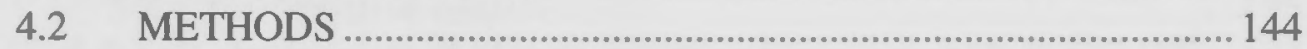

4.2.1 Experimental animals........................................................... 144

4.2.2 Blood pressure and body weight measurement......................... 145

4.2.3 Calculation of other haemodynamic parameters...................... 145

4.2.4 Haematocrit estimation ............................................................ 146

4.2.5 Thymus and adrenal weights................................................ 146

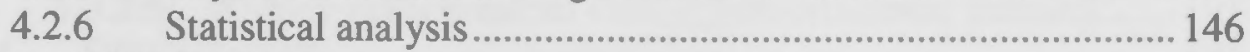

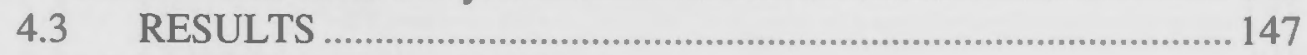

4.3.1 Tail-cuff systolic blood pressure.............................................. 147

4.3.2 Direct blood pressure measurements ........................................ 148

4.3.3 Haemodynamic effects......................................................... 153

4.3.3.1 Heart rate........................................................................ 153

4.3.3.2 Stroke volume and stroke index ......................................... 154

4.3.3.3 Cardiac output and cardiac index ....................................... 155

4.3.3.4 Total peripheral resistance .............................................. 157

4.3.3.5 Total peripheral conductance ............................................ 158

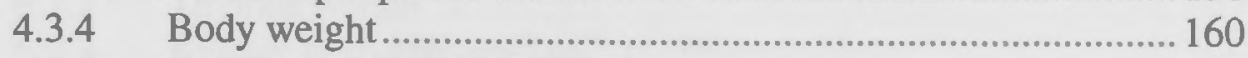

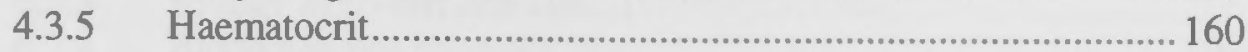

4.3.6 Thymus and adrenal weights.................................................. 161

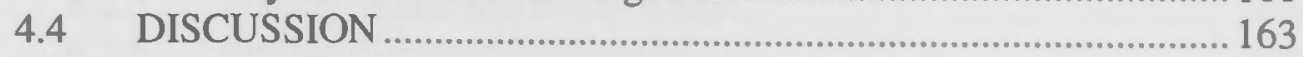

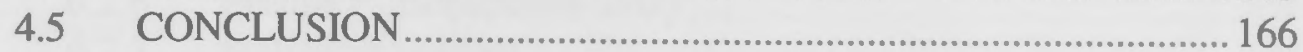

\section{CHAPTER 5: EFFECTS OF GLUCOCORTICOID ON RAT KIDNEY} MITOCHONDRIAL SUPEROXIDE

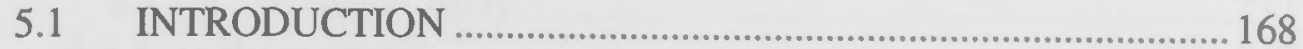

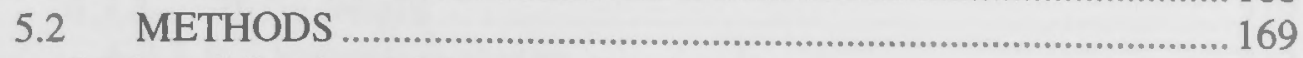

5.2.1 Mitochondrial superoxide detection......................................... 169

5.2.2 Principles of flow cytometry ................................................. 171

5.2.3 Single cell suspension ........................................................... 172

5.2.4 Standardisation of cell number .............................................. 173

5.2.5 Mitochondrial staining ....................................................... 173 


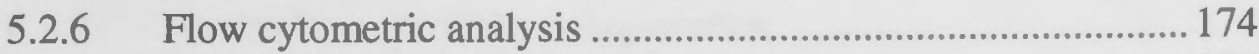

5.2.7 Preliminary experiments ....................................................... 177

5.2.7.1 Optimisation of Mito-HE concentration .............................. 177

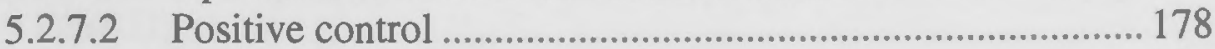

5.2.7.3 Negative control................................................................ 179

5.2.8 Assessment of kidney mitochondrial superoxide in GC-HT ... 179

5.2.8.1 Experimental animals..................................................... 179

5.2.8.2 Systolic blood pressure and body weight measurements .... 180

5.2.8.3 Thymus weight....................................................................... 180

5.2.8.4 Kidney mitochondrial Mito-HE fluorescence analysis ....... 181

5.2.8.5 Kidney mitochondrial $\mathrm{DiIC}_{1}(5)$ fluorescence analysis........ 181

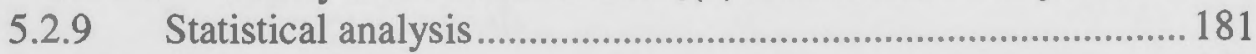

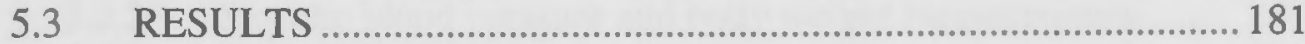

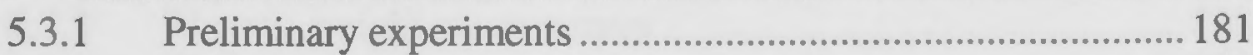

5.3.1.1 Optimisation of Mito-HE concentration .............................. 181

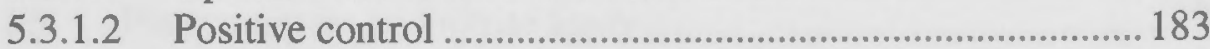

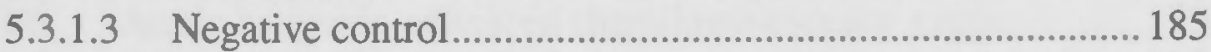

5.3.2 Assessment of kidney mitochondrial superoxide in GC-HT ... 187

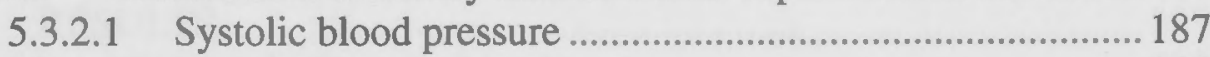

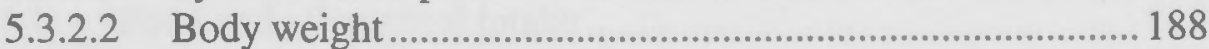

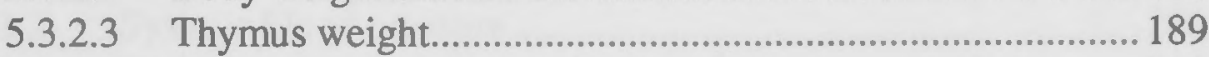

5.3.2.4 Kidney mitochondrial Mito-HE fluorescence analysis ....... 190

5.3.2.5 Kidney mitochondrial $\mathrm{DiIC}_{1}(5)$ fluorescence intensity....... 191

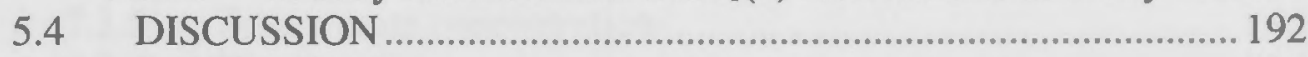

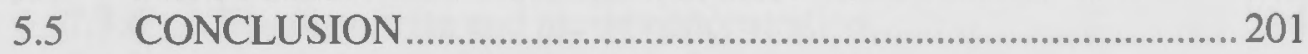

\section{CHAPTER 6: ROLE OF MITOCHONDRIAL SUPEROXIDE IN}

GLUCOCORTICOID-INDUCED HYPERTENSION IN THE RAT ......... 203

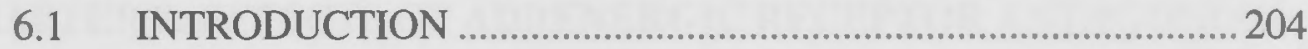

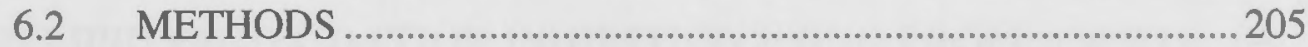

6.2.1 Tail-cuff blood pressure and body weight measurements........207

6.2.2 Thymus weight ....................................................................... 207

6.2.3 Kidney Mito-HE and DilC $_{1}$ (5) fluorescence.............................2208

6.2.4 Blood glucose concentration.....................................................2208

6.2.5 Plasma nitrate and nitrite assay .............................................. 208

6.2.6 Plasma $\mathrm{F}_{2}$-isoprostane assay ............................................... 209

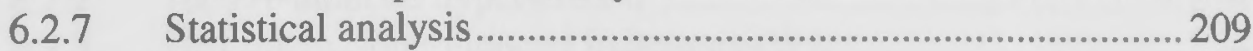

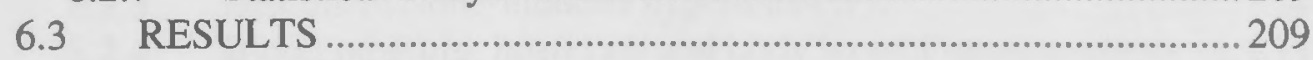

6.3.1 Systolic blood pressure ............................................................209

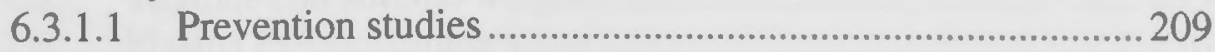

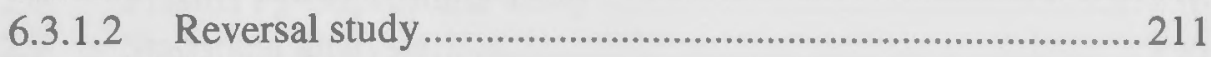

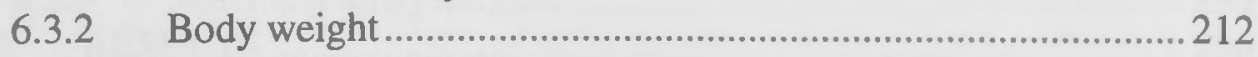

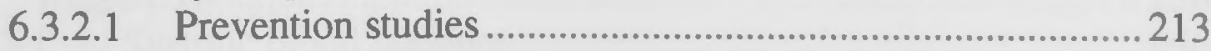

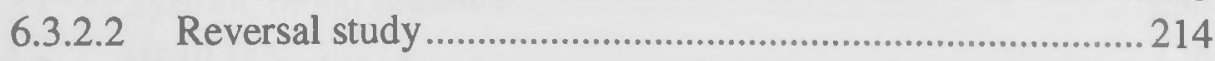

6.3.3 Thymus weight ..................................................................... 216

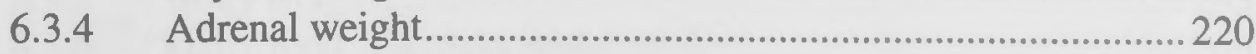

6.3.5 Blood glucose concentration..................................................... 221

6.3.6 Plasma nitrate and nitrite concentration................................... 223 
6.3.7 Plasma $\mathrm{F}_{2}$-isoprostane concentration ............................... 224

6.3.8 Kidney Mito-HE fluorescence ............................................ 225

6.3.9 Kidney $\mathrm{DiIC}_{1}(5)$ fluorescence........................................... 227

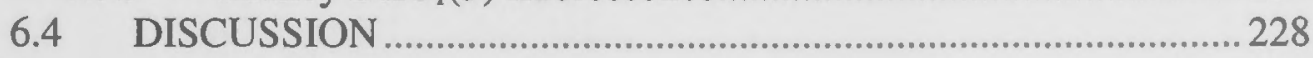

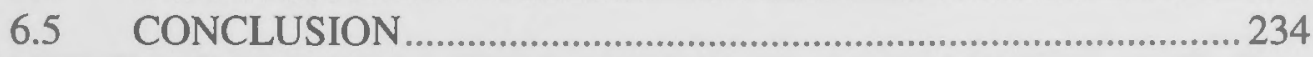

CHAPTER 7: ROLE OF XANTHINE OXIDASE IN DEXAMETHASON-

INDUCED HYPERTENSION IN THE RAT..................................................235

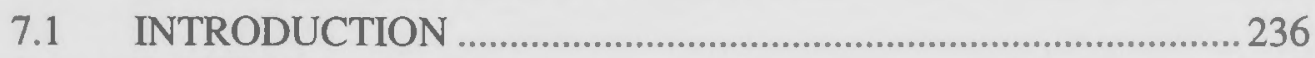

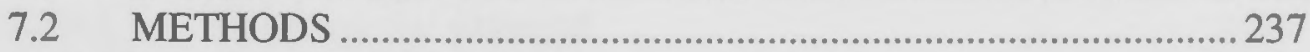

7.2.1 Experimental animals.................................................... 237

7.2.2 Systolic blood pressure and body weight measurements ......... 238

7.2.3 Thymus weight measurement .............................................. 238

7.2.4 Serum urate measurement .................................................... 238

7.2.5 Plasma nitrate and nitrite assay ......................................... 239

7.2.6 Aortic lucigenin-enhanced chemiluminescence assay ............ 239

7.2.7 Data and statistical analysis ............................................. 239

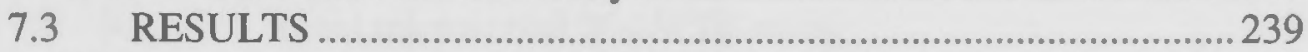

7.3.1 Food and allopurinol intake .............................................. 239

7.3.2 Systolic blood pressure ..................................................... 240

7.3.3 Body weight ................................................................... 241

7.3.4 Thymus weight ......................................................... 242

7.3.5 Serum urate concentration ................................................. 243

7.3.6 Plasma nitrate and nitrite concentration............................... 244

7.3.7 Aortic lucigenin-enhanced chemiluminescence..................... 245

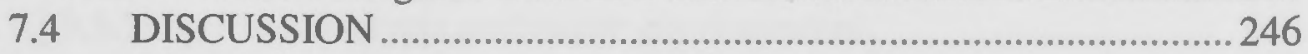

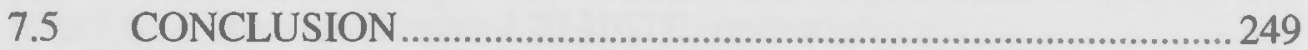

CHAPTER 8: EFFECTS OF ADRENERGIC RECEPTOR ANTAGONISM

AND LIPID PEROXIDATION INHIBITION USING PROPRANOLOL IN

GLUCOCORTICOID-INDUCED HYPERTENSION IN THE RAT ........ 250

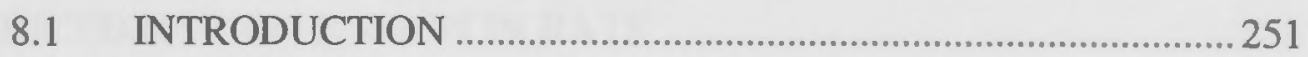

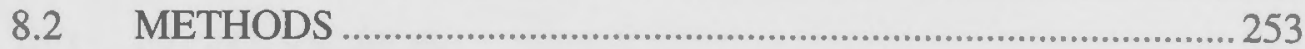

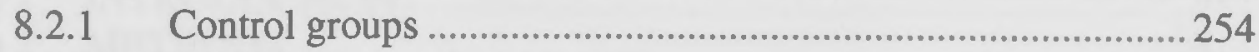

8.2.2 ACTH-induced hypertension ................................................. 254

8.2.3 Dexamethasone-induced hypertension .................................... 254

8.2.4 Blood pressure, heart rate and body weight measurements .... 255

8.2.5 Thymus and adrenal weights.................................................... 255

8.2.6 Plasma $\mathrm{F}_{2}$-isoprostane assay ..............................................2.256

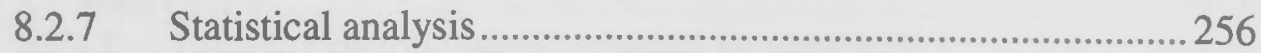

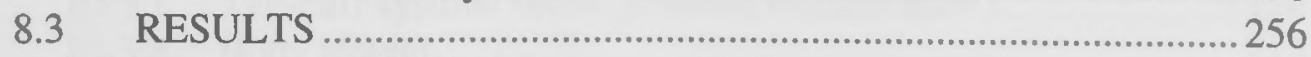

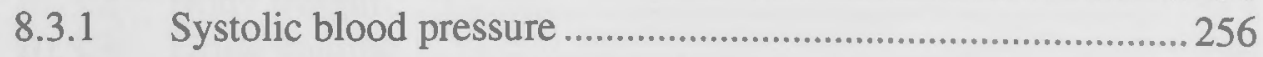

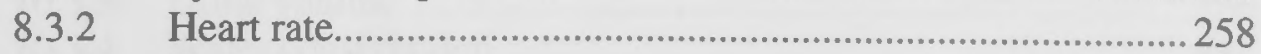

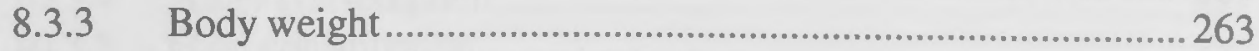

8.3.4 Daily food and propranolol intake ...........................................2.266

8.3.5 Thymus weight .....................................................................2272 


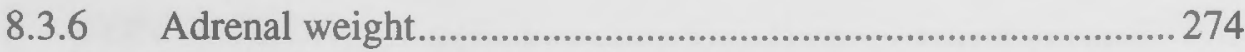

8.3.7 Plasma $\mathrm{F}_{2}$-isoprostane concentration ...................................... 276

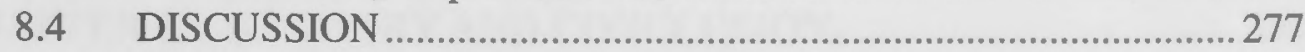

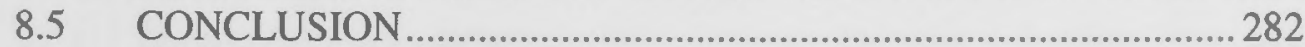

\section{CHAPTER 9: ROLE OF 20-HYDROXYEICOSATETRAENOIC ACID IN} DEXAMETHASONE-INDUCED HYPERTENSION IN THE RAT .

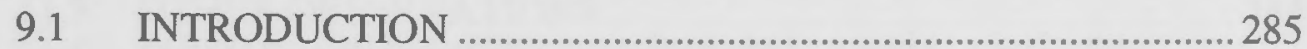

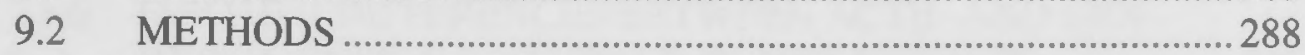

9.2.1 Experimental protocol...........................................................28 288

9.2.2 Tail-cuff systolic blood pressure and body weight measurements

9.2.3 Metabolic measurements .......................................................290

9.2.4 Thymus weight measurement .....................................................2290

9.2.5 Plasma nitrate and nitrite assay ...............................................290

9.2.6 Renal microsomal 20-HETE assay .....................................2. 292

9.2.6.1 Isolation of renal microsomes ........................................... 292

9.2.6.2 Renal microsomal 20-HETE assay ..................................2292

9.2.7 Plasma $F_{2}$-isoprostane assay .............................................2293

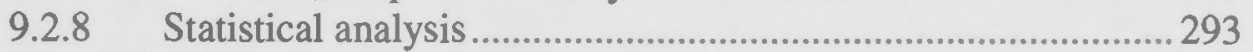

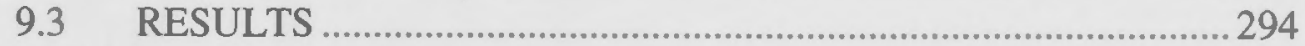

9.3.1 Tail-cuff systolic blood pressure................................................294

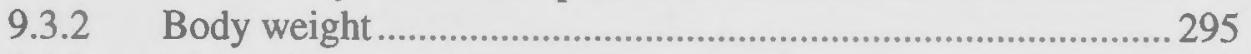

9.3.3 Metabolic parameters _.........................................................298

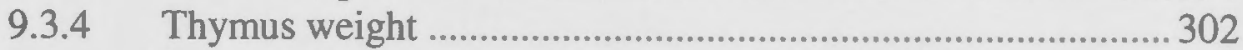

9.3.5 Plasma nitrate and nitrite concentration....................................303

9.3.6 Renal microsomal 20-HETE concentration ..............................304

9.3.7 Plasma $\mathrm{F}_{2}$-isoprostane concentration ....................................... 305

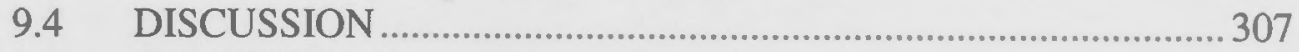

9.5 CONCLUSION …................................................................... 311

CHAPTER 10: METABOLIC PROFILES OF DEXAMETHASONEINDUCED HYPERTENSION IN RATS......................................................... 312

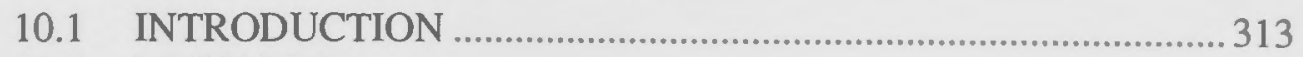

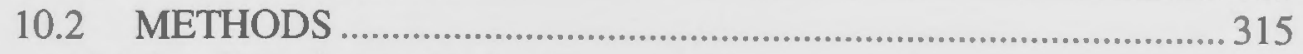

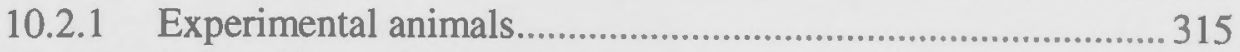

10.2.2 Tail-cuff systolic blood pressure measurements ....................... 315

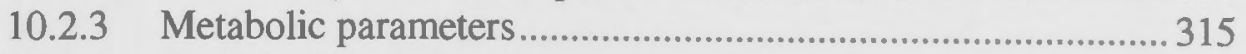

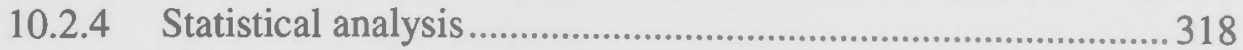

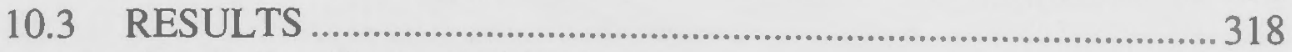

10.3.1 Tail-cuff systolic blood pressure measurements ........................ 318

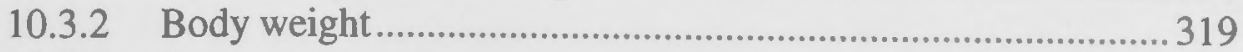

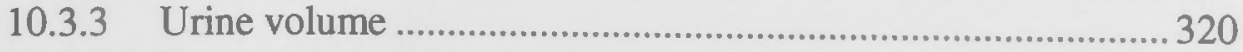

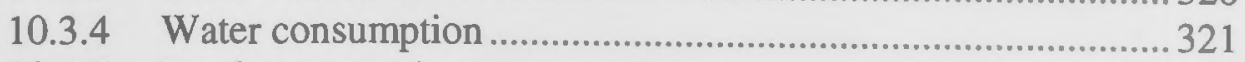

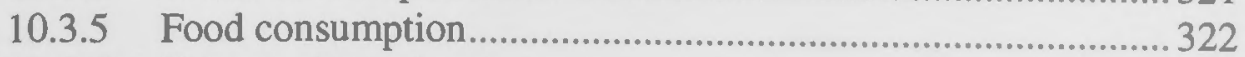

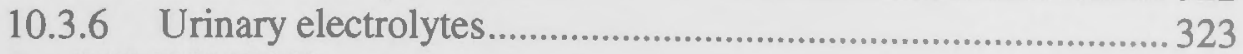

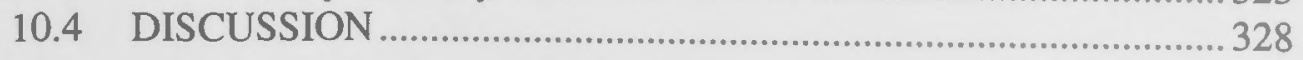




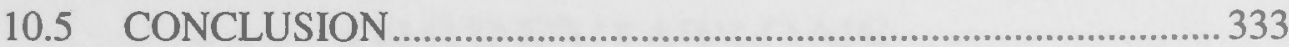

CHAPTER 11: SUMMARY AND CONCLUSION............................................334

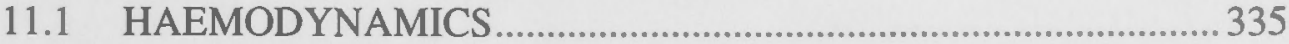

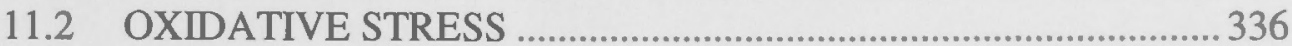

$11.3 \quad$ NITRIC OXIDE DEFICIENCY ........................................................ 338

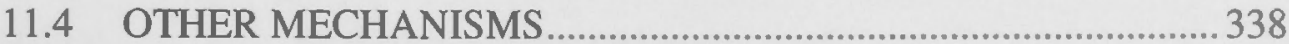

11.5 OTHER OBSERVATIONS ............................................................ 339

11.6 FURTHER DIFFERENCES BETWEEN ACTH- AND DEXHYPERTENSION IDENTIFIED IN THIS PROJECT ................... 339

11.6.1 Effects on body weight .......................................................... 340

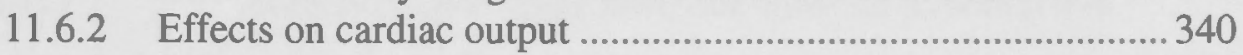

11.6.3 Effects on plasma $F_{2}$-isoprostane concentration ...................... 340

11.6.4 The role of 20-HETE ............................................................ 341

11.7 CONCLUSION AND FUTURE DIRECTIONS .............................. 341

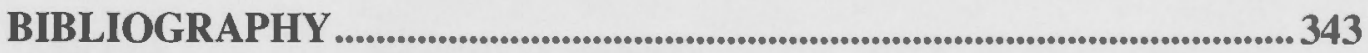




\section{ABBREVIATIONS}

20-HETE

$\mathrm{ACTH}$

ACTH-HT

$\mathrm{ADH}$

Adr

ANOVA

ANP

AVP

$\mathrm{BH}_{4}$

C

cGMP

CI

$\mathrm{CO}$

$\mathrm{CRH}$

CV

DBP

DETC

DEX

DEX-HT

$\operatorname{DiIC}_{1}(5)$

DMSO
20-Hydroxyeicosatetraenoic acid

Adrenocorticotrophic hormone

Adrenocorticotrophic hormone-induced hypertension

Antidiuretic hormone

Adrenaline

Analysis of variance

Atrial natriuretic peptide

Arginine vasopressin

Tetrahydrobiopterin

Control days

Cyclic guanosine monophosphate

Cardiac index

Cardiac output

Corticotrophin releasing hormone

Coefficient of variance

Diastolic blood pressure

Diethylthiocarbamate

Dexamethasone

Dexamethasone-induced hypertension

$1,1^{\prime}, 3,3,3^{\prime}, 3^{\prime}$-hexamethylindodicarbocyanine iodide

Dimethylsulfoxide 


\begin{tabular}{|c|c|}
\hline DNA & Deoxyribonucleic acid \\
\hline DOCA & Deoxycorticosterone acetate \\
\hline EDTA & Ethylenediaminetetraacetic acid \\
\hline eNOS & Endothelial nitric oxide synthase \\
\hline ET-1 & Endothelin 1 \\
\hline $\mathrm{Ex} / \mathrm{Em}$ & Excitation/emission \\
\hline FSC & Forward scatter \\
\hline GABA & Gamma-aminobutyric acid \\
\hline GC-HT & Glucocorticoid-induced hypertension \\
\hline GTP & Guanosine triphosphate \\
\hline HBF & Hindquarter blood flow \\
\hline HCAEC & Human coronary artery endothelial cells \\
\hline HD LA & High dose alpha-lipoic acid \\
\hline HE & Hydroethidine \\
\hline HET0016 & N-hydroxy-N'-(4-butyl-2 methylphenyl)formamidine \\
\hline HO-Etd+ & 2 Hydroxy ethidium \\
\hline HO-Mito-Etd+ & 2 Hydroxy-Mito-ethidium \\
\hline HPMVEC & Human pulmonary micorvascular endothelial cells \\
\hline HR & Heart rate \\
\hline HVR & Hindquarter vascular resistance \\
\hline i.p. & Intraperitoneal \\
\hline i.v. & Intravenous \\
\hline iNOS & Inducible nitric oxide synthase \\
\hline K & Potassium \\
\hline
\end{tabular}




\begin{tabular}{|c|c|}
\hline LA & Alpha-lipoic acid \\
\hline MAP & Mean arterial pressure \\
\hline MBF & Mesenteric blood flow \\
\hline MFI & Mean fluorescence intensity \\
\hline Mito-HE & MitoSOX Red \\
\hline $\mathrm{mnSOD}$ & Manganese superoxide dismutase \\
\hline mRNA & Messenger ribonucleic acid \\
\hline MVR & Mesenteric vascular resistance \\
\hline MW & Molecular weight \\
\hline NAC & $\mathrm{N}$-acetylcysteine \\
\hline $\mathrm{NaCl}$ & Saline/ sodium chloride \\
\hline $\mathrm{NAD}(\mathrm{P}) \mathrm{H}$ & Nicotinamide adenine dinucleotide phosphate \\
\hline NADH & Nicotinamide adenine dinucleotide \\
\hline NAdr & Noradrenaline \\
\hline NO & Nitric oxide \\
\hline NOS & Nitric oxide synthase \\
\hline NOx & Nitrate and nitrite \\
\hline NPY & Neuropeptide Y \\
\hline ns & Not significant \\
\hline$P$ & Pre-treatment days \\
\hline p.o. & Per oral \\
\hline PBS & Phosphate bufferred saline \\
\hline $\mathrm{PGE}_{2}$ & Prostaglandin $\mathrm{E}_{2}$ \\
\hline $\mathrm{PGI}_{2}$ & Prostacyclin \\
\hline
\end{tabular}


PNMT Phenylethanolamine $N$-Methyltransferase

RAS Renin-angiotensin system

RBF Renal blood flow

ROS Reactive oxygen species

RVR Renal vascular resistance

s.c.

Subcutaneous

SBP Systolic blood pressure

SD Standard deviation

SEM Standard error of mean

SHR Spontaneously hypertensive rat

SI Stroke index

SOD Superoxide dismutase

SV Stroke volume

$\mathrm{T} \quad$ Treatment days

TPC Total peripheral conductance

TPR Total peripheral resistance

vs Versus 


\section{PUBLICATIONS AND PRESENTATIONS}

\section{ARISING FROM THIS THESIS}

\section{Publications}

Ong SLH, Zhang Y, Sutton M, Whitworth JA. Haemodynamics of dexamethasone-induced hypertension in the rat. Hypertens Res 2009; 32(10): 889894.

Zhang Y, Wu JHY, Vickers JJ, Ong SLH, Temple SEL, Mori TA, Croft KD, Whitworth JA. The role of 20-hydroxyeicosatetraenoic acid in glucocorticoidinduced hypertension. J Hypertens 2009; 27(8): 1609-1616.

Ong SLH, Zhang Y, Whitworth JA. Mechanisms of dexamethasone-induced hypertension. Curr Hypertens Rev 2009; 5(1): 61-74.

Ong SLH, Zhang Y, Whitworth JA. Reactive oxygen species and glucocorticoidinduced hypertension. Clin Exp Pharmacol Physiol 2008; 35(4):477-482.

Ong SLH, Vickers JJ, Zhang Y, McKenzie KUS, Walsh CE, Whitworth JA. Role of xanthine oxidase in dexamethasone-induced hypertension in rats. Clin Exp Pharmacol Physiol 2007; 34(5-6): 522-524. 


\section{In Press}

Ong SLH, Whitworth JA. How do glucocorticoids cause hypertension: role of nitric oxide deficiency, oxidative stress and eicosanoids. Clin of North Am (in press)

\section{In Preparation}

Ong SLH, Sutton M, Vohra H, Zhang Y, Mori TA, Croft KD, Whitworth JA. Mitochondrial superoxide production in glucocorticoid-hypertensive rats. 


\section{PRESENTATIONS}

\section{International Presentations}

Ong SLH, Sutton M, Vohra H, Zhang Y, Whitworth JA. Alpha lipoic acid prevents glucocorticoid-induced hypertension in the rat. Oral poster presentation at the International Society of Hypertension Scientific Meeting, Berlin, June 2008.

Ong SLH, Vohra H, Sutton M, Zhang Y, Whitworth JA. Kidney mitochondrial superoxide production in glucocorticoid-induced hypertension in rats. Poster presentation at the International Society of Hypertension Scientific Meeting, Berlin, June 2008.

Zhang Y, Croft KD, Vickers JJ, Ong SLH, Mori TA, Whitworth JA. HET0016, a 20-hydroxyeicosatetraenoic acid inhibitor, prevents and reverses adrenocorticotrophic hormone-induced hypertension. Oral poster presentation at the International Society of Hypertension Scientific Meeting, Berlin, June 2008.

\section{National Presentations}

Ong SLH, Sutton M, Zhang Y, Whitworth JA. Haemodynamics of dexamethasone-induced hypertension in the rat. Poster presentation at the Australian and New Zealand Society of Nephrology, Tasmania, September 2009. 
Ong SLH, Zhang Y, Whitworth JA. Haemodyamic profile of dexamethasoneinduced hypertension in the rat. Poster presentation at the High Blood Pressure Research Council of Australia, Melbourne, December 2008.

Ong SLH, Sutton M, Vohra H, Zhang Y, Whitworth JA. Dexamethasoneinduced hypertension in rats is prevented but not reversed by alpha-lipoic acid. Oral poster presentation at the Australian and New Zealand Society of Nephrology, Newcastle, September 2008.

Ong SLH, Sutton M, Vohra H, Zhang Y, Whitworth JA. Alpha-lipoic acid partially prevents adrenocorticotrophin-induced hypertension in the rat. Poster presentation at the High Blood Pressure Research Council Australia Annual Scientific Meeting, Adelaide, December 2007.

Ong SLH, Sutton M, Vohra H, Zhang Y, Whitworth JA. Assessment of mitochondrial superoxide production in kidney cells of glucocorticoid-induced hypertension in the rat. Poster presentation at the High Blood Pressure Research Council of Australia Annual Scientific Meeting, Adelaide, December 2007.

Ong SLH, Vickers JJ, Zhang Y, McKenzie KUS, Whitworth JA. Role of xanthine oxidase in dexamethasone-induced hypertension in the rat. Poster presentation the High Blood Pressure Research Council of Australia Annual Scientific Meeting, Brisbane, December 2006. 


\section{Local Presentations}

Ong SLH, Sutton M, Vohra H, Zhang Y, Whitworth JA. The antioxidant alpha lipoic acid prevents but does not reverse dexamethasone-induced hypertension in the rat. Oral presentation at the Canberra Region Annual Scientific Meeting, June 2008.

Ong SLH, Sutton M, Vohra H, Zhang Y, Whitworth JA. The antioxidant alpha lipoic acid prevents but does not reverse dexamethasone-induced hypertension in the rat. Poster presentation at the Australian Society of Medical Research, Australian Capital Territory Young Investigator Forum, Canberra, June 2008.

Ong SLH, Vohra H, Zhang Y, Whitworth JA. Fluorogenic assessment of the role of mitochondrial superoxide in dexamethasone-induced hypertension in the rat. Poster presentation at the Australian Society of Medical Research, Australian Capital Territory Young Investigator Forum, Canberra, June 2007.

Ong SLH, Vickers JJ, Zhang Y, McKenzie KUS, Whitworth JA. Role of xanthine oxidase in dexamethasone-induced hypertension in the rat. Poster presentation at the Australian Society of Medical Research, Australian Capital Territory Young Investigator Forum, Canberra, June 2006. 


\section{CHAPTER 1}

Mechanisms of Dexamethasone-Induced Hypertension 


\subsection{INTRODUCTION}

The involvement of glucocorticoid hormones in hypertension has long been recognised. Cushing's syndrome due to chronic glucocorticoid excess, either endogenous or exogenous, is associated with hypertension. In addition, local cortisol excess due to impaired glucocorticoid metabolism resulting in hypertension has also been implicated in conditions such as apparent mineralocorticoid excess, licorice abuse, renal failure and essential hypertension (Walker, Best et al. 1996).

Overproduction of cortisol, the major glucocorticoid hormone in humans, can either be due to primary pathology of the adrenal glands (such as hyperplasia, adenoma or carcinoma) or secondary to pituitary-derived (Cushing's Disease) or ectopic adrenocorticotrophic hormone (ACTH) secretion. Approximately $80 \%$ of adults and $47 \%$ of children and adoslescents with Cushing's syndrome due to endogenous glucocorticoid overproduction present with hypertension (Magiakou, Smyrnaki et al. 2006). Hypertension is a more common problem in patients who develop Cushing's syndrome due to administration of glucocorticoid or ACTH. To date, several forms of synthetic glucocorticoids are available for treatment of a variety of clinical conditions including autoimmune diseases, inflammatory diseases, organ transplantation and cancer. Chronic usage of systemic glucocorticoids at supraphysiological doses, sometimes inevitable for certain medical conditions, often leads to complications such as hypertension, a significant cardiovascular risk factor. 
This chapter will review the literature on the proposed pathogenetic mechanisms of hypertension due to a potent synthetic glucocorticoid, dexamethasone (DEX). This chapter also includes a segment featuring the similarities and differences between naturally-occurring and synthetic glucocorticoid hormones.

\subsection{GLUCOCORTICOID HORMONES}

\subsubsection{History}

Both glucocorticoid and mineralocorticoid hormones are forms of corticosteroid hormones produced by the adrenal glands. This gland was first described by Eustachius (Eustachius 1564). The vital function of this gland was only recognised in the nineteenth century by Addison who described the syndrome of adrenal insufficiency in his monograph in 1855 (Addison 1937). This finding was further substantiated by Brown-Sequard who found rapidly fatal outcomes from unilateral or bilateral adrenalectomy in animals (Brown-Sequard 1856). These findings had driven the search for a hormone replacement therapy for adrenal insufficiency.

In contrast to the observations by Addison and Brown-Sequard, Harvey Cushing described the syndrome of hypercortism in 1912 but ascribed it to disorders of the adrenal, thyroid and pineal glands, as well as the ovaries and testes (Medvei 1991). It was then realised by Julius Bauer that this syndrome was explained by "sole hyperfunction of the adrenals" (Bauer 1950). The recognition of 
hypercortism subsequently led to the establishment of the link between the anterior pituitary and the adrenal gland, the identification and purification of $\mathrm{ACTH}$, the steroid hormones and their synthetic analogues.

Cortisone became available following successful extraction from sheep and cattle adrenal cortices by Kendall and Reichstein (Mason, Myers et al. 1936; Reichstein 1962). They both shared the Nobel Prize with Hench, who observed a remarkable remission of rheumatoid arthritis with cortisone injection (Glyn 1998).

The discovery of the therapeutic effects of corticosteroid hormones had revolutionised the treatments of various medical conditions. It also led to the development of many synthetic derivatives of different potencies. 


\subsubsection{Naturally-occuring glucocorticoids}

In humans, cortisol or hydrocortisone is the predominant glucocorticoid produced from cholesterol, the basic precursor of corticosteroid hormones, by the zona fasciculata and reticularis of the adrenal cortex. Its synthesis involves alterations to the cholesterol chemical structure via a series of enzymatic reactions. Due to the lack of adrenal 17-hydroxylase activity in rodents, the predominant glucocorticoid is corticosterone.

The pathway of steroidogenesis in humans is summarised in Figure 1.1. There are 5 important steps in the pathway of cortisol synthesis.

1. Cholesterol is converted to pregnenolone by cholesterol side chain cleavage enzyme (cholesterol desmolase).

2. Pregnenolone is then converted to either progesterone by $3 \beta-$ hydroxysteroid dehydrogenase or to 17-hydroxypregnenolone by $17 \alpha$ hydroxylase.

3. 17-Hydroxyprogesterone is then formed from either 17hydroxypregnenolone by $3 \beta$-hydroxysteroid dehydrogenase or by $17 \alpha-$ hydroxylation of progesterone. 
4. 21ß-Hydroxylation of 17-hydroxyprogesterone results in the formation of 11-deoxycortisol.

5. 11ß-Hydroxylation of 11-deoxycortisol leads to the production of cortisol. 


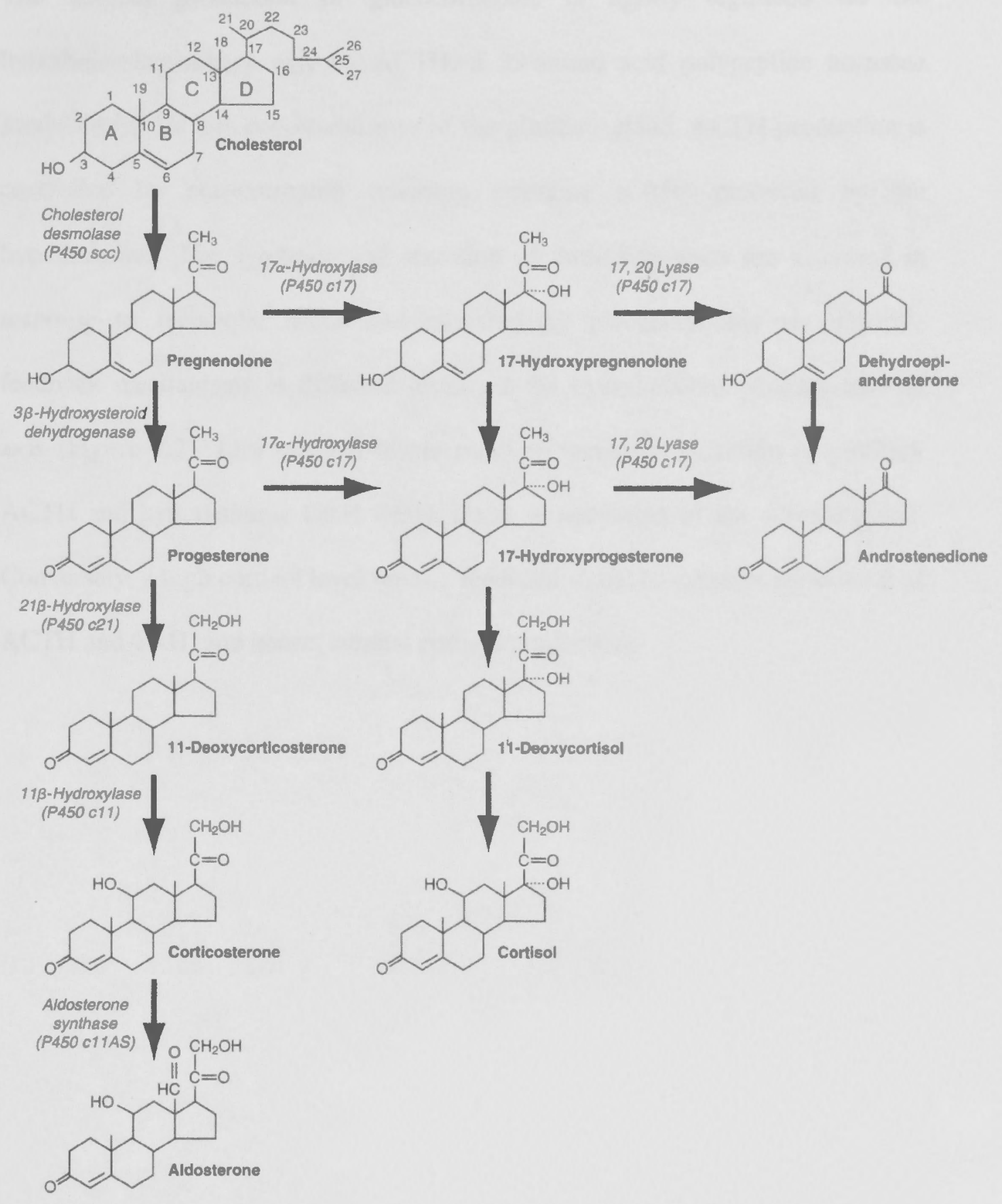

Figure 1.1: Pathways for synthesis of corticosteroids by the adrenal cortex

(Guyton and Hall 2000). 
The adrenal production of glucocorticoids is tightly regulated via the hypothalamic-pituitary axis by ACTH, a 39-amino acid polypeptide hormone produced by the adrenocorticotropes of the pituitary gland. ACTH production is controlled by corticotrophin releasing hormone (CRH) produced by the hypothalamus. The synthesis and secretion of these hormones are activated in response to metabolic needs and inhibited by glucocorticoids via negative feedback mechanisms at different levels of the hypothalamus-pituitary-adrenal axis (Figure 1.2). Low cortisol levels result in increased secretion of pituitary ACTH and hypothalamic CRH which result in activation of the adrenal gland. Conversely, a high cortisol level sends a feedback signal to suppress the release of ACTH and CRH; and hence, adrenal cortisol production. 


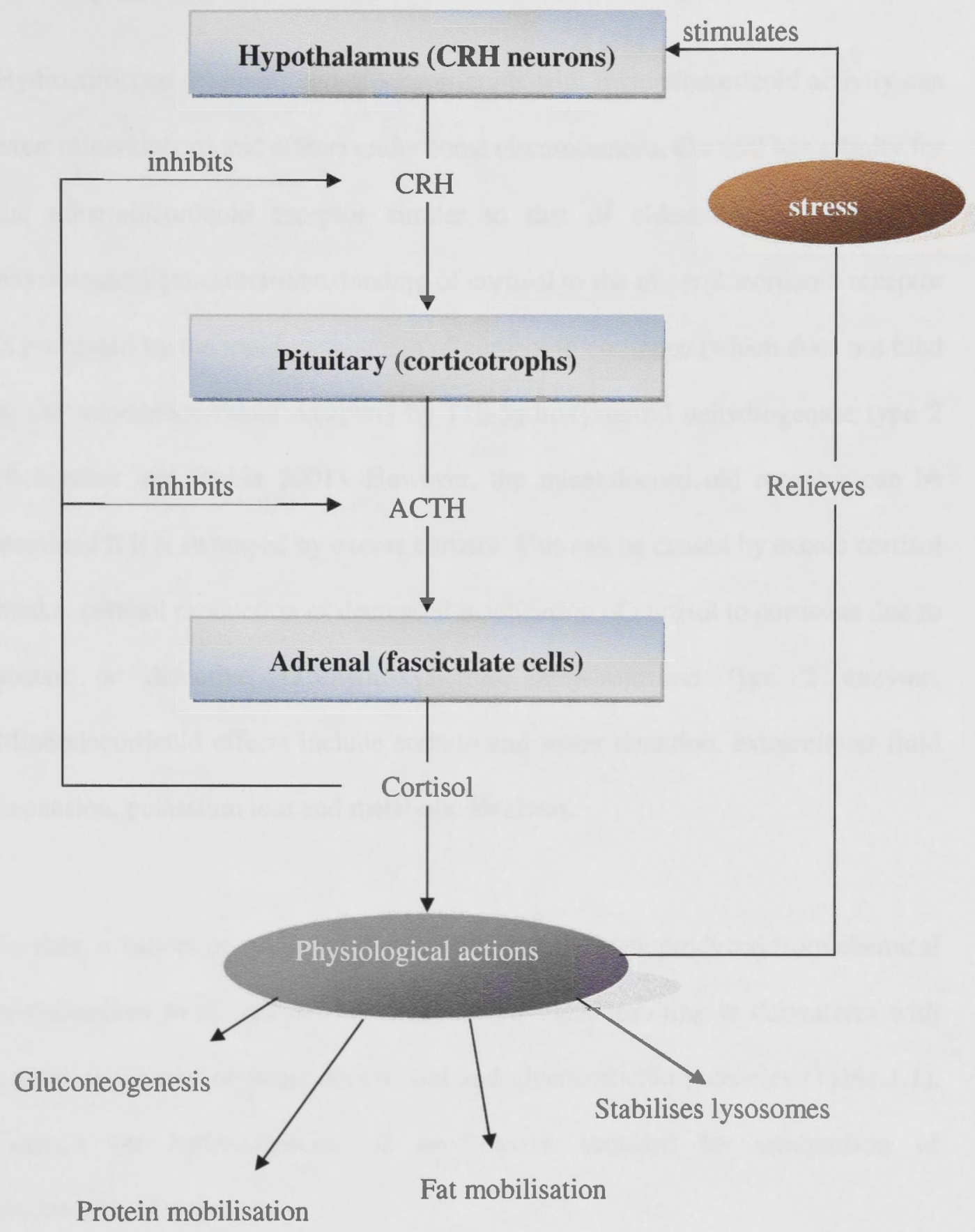

Figure 1.2: Mechanism for regulation of glucocorticoid secretion. ACTH: adrenocorticotrophic hormone, CRH: corticotrophin releasing hormone. 


\subsubsection{Synthetic glucocorticoids}

Hydrocortisone (cortisol) and glucocorticoids with mineralocorticoid activity can exert mineralocorticoid effects under some circumstances. Cortisol has affinity for the mineralocorticoid receptor similar to that of aldosterone. However, at physiological concentrations, binding of cortisol to the minerolocorticoid receptor is prevented by the rapid metabolism of cortisol to cortisone (which does not bind to the mineralocorticoid receptor) by $11 \beta$-hydroxysteroid dehydrogenase type 2 (Schimmer and Parker 2001). However, the mineralocorticoid receptor can be activated if it is swamped by excess cortisol. This can be caused by excess cortisol intake, cortisol production or decreased inactivation of cortisol to cortisone due to absent or defective 11ß-hydroxysteroid dehydrogenase type 2 enzyme. Mineralocorticoid effects include sodium and water retention, extracellular fluid expansion, potassium loss and metabolic alkalosis.

To date, a variety of synthetic glucocorticoids have been produced from chemical modifications to the cortisol molecule (Figure 1.3) resulting in derivatives with greater separation of mineralocorticoid and glucocorticoid potencies (Table 1.1). Cortisol (or hydrocortisone) is used as a standard for comparison of glucocorticoid potency.

Systemic administration of exogenous glucocorticoids also provides inhibitory feedback signals to suppress $\mathrm{CRH}, \mathrm{ACTH}$ and adrenal glucocorticoid production. 


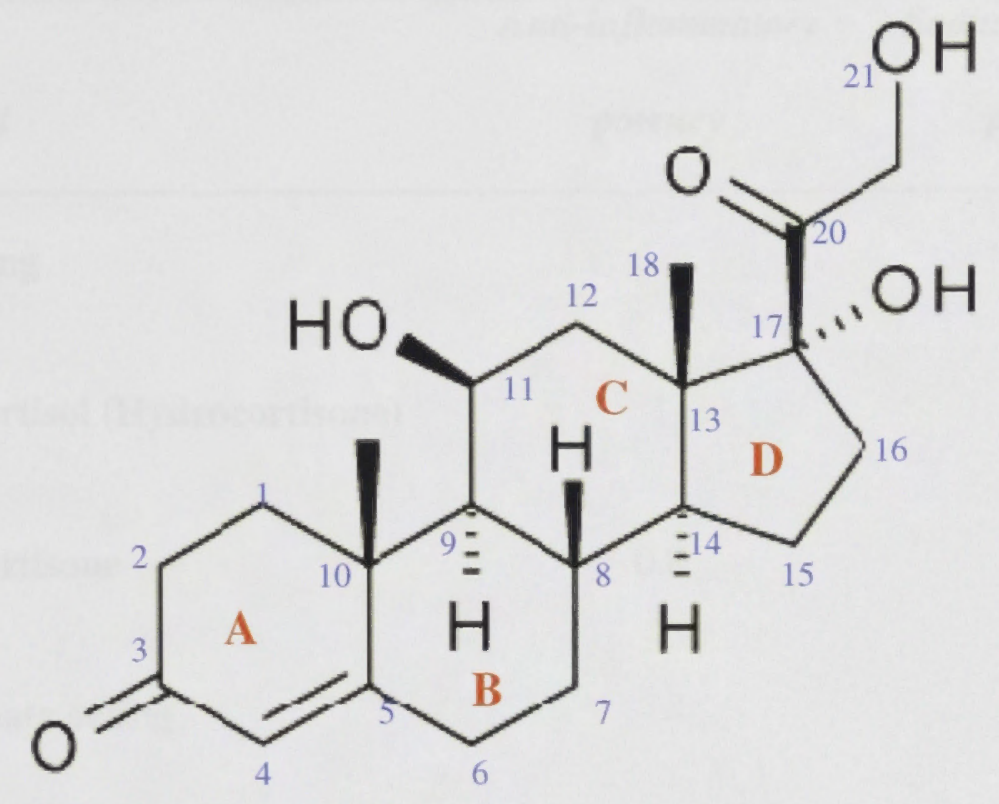

Figure 1.3: Cortisol (Hydrocortisone). The 4,5 double bond and 3-keto group on ring $\mathrm{A}$ are both necessary for glucocorticoid and mineralocorticoid effects whereas the $11 \beta$-hydroxyl group on ring $\mathrm{C}$ is required for glucocorticoid activity only (Schimmer and Parker 2001). The 17a-hydroxyl group on ring D, a substituent for cortisol, is needed for optimal glucocorticoid effect (Schimmer and Parker 2001). The hydroxyl group at C21 on the side chain arising from ring D which is found in all naturally-occuring corticosteroids and most synthetic derivatives is essential for mineralocorticoid function but not glucocorticoid (Schimmer and Parker 2001). 
Table 1.1: Relative potencies of representative glucocorticoids (Rang, Dale et al. 2007).

\section{Anti-inflammatory Sodium retaining}

Compound

potency

potency

Short acting

Cortisol (Hydrocortisone)

1

1

Cortisone

0.8

0.8

Intermediate acting

Prednisone

Prednisolone

6a-methylprednisolone

Triamcinolone

Long acting

Betamethasone

Dexamethasone
4

4

5

5

none

0.8

0.8 minimal

negligible

25
25 minimal 


\subsection{DEXAMETHASONE}

DEX is a synthetic glucocorticoid that is approximately 25 times more potent than cortisol (hydrocortisone). The increased anti-inflammatory and decreased sodiumretaining potencies of DEX relative to cortisol are consequences of chemical modifications that may alter the affinity and intrinsic activity at corticosteroid receptors, absorption, protein binding, rate of metabolic transformation, rate of excretion or membrane permeability (Schimmer and Parker 2001).

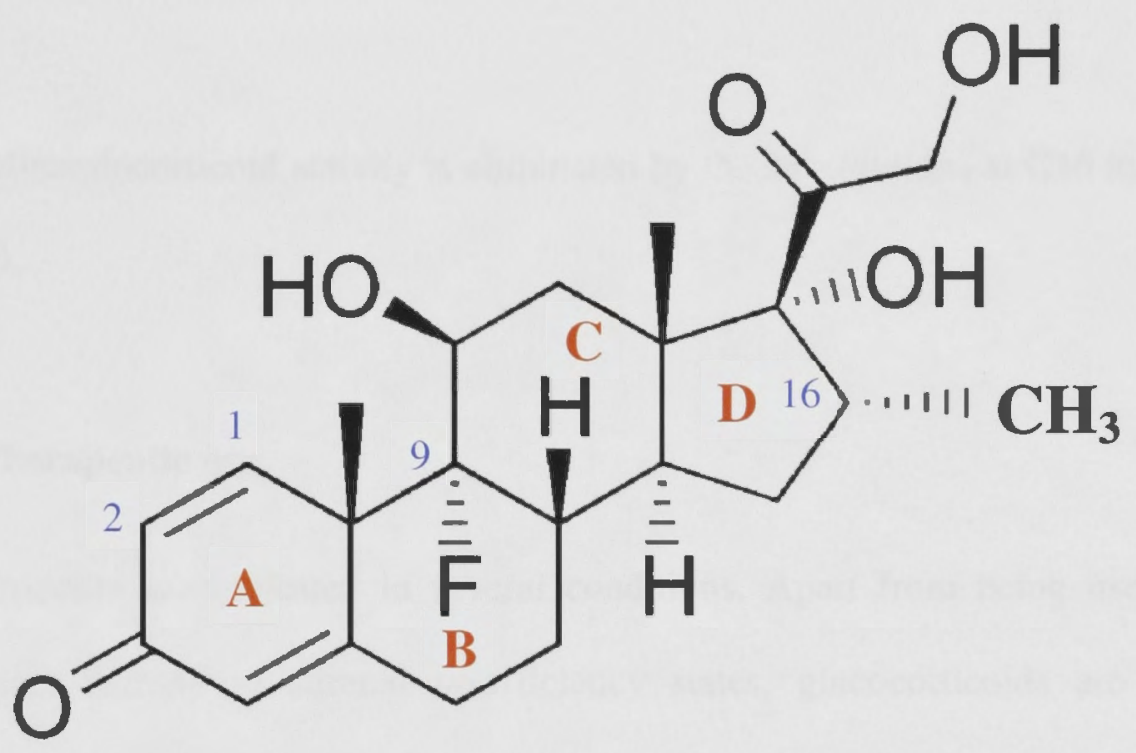

Figure 1.4: Molecular structure of DEX. 
Figure 1.4 features the molecular structure of DEX. DEX is synthesised by 3 structural modifications to the basic cortisol structure.

1. The addition of another double bond in the 1,2 position of ring $A$ selectively results in a four fold increase in the glucocorticoid activity compared to hydrocortisone.

2. Fluorination in ring $\mathrm{B}$ at the $9 a$ position augments glucocorticoid and mineralocorticoid activity.

3. Mineralocorticoid activity is eliminated by the substitutions at $\mathrm{Cl} 16$ in ring D.

\subsubsection{Therapeutic use}

Glucocorticoids are indicated in several conditions. Apart from being used as replacement therapy in adrenal insufficiency states, glucocorticoids are also commonly used for non-endocrine conditions such as allergic disease, inflammatory diseases, autoimmune disorders, organ transplantation and malignancies. For most inflammatory and autoimmune diseases, the shorter acting prednisone and methylprednisolone are preferred over the long-acting DEX to facilitate dose tapering. However, DEX is favoured over other glucocorticoids in certain conditions highlighted below. 


\subsubsection{Endocrine uses}

DEX may be given to patients with either adrenal insufficiency due to structural or functional adrenal abnormalities, or from structural or functional lesions of the pituitary or hypothalamus.

\subsubsection{Oncologic uses}

DEX is used in multiple myeloma where it is either given alone or in combination with thalidomide (thal-dex protocol) or with Adriamycin (doxorubicin) and vincristine (VAD protocol).

DEX is also given to treat nausea in patients with widespread cancer. This works by the augmentation of the antiemetic effects of $5 \mathrm{HT}_{3}$ receptor antagonists, reduction of peritumoral inflammation and prostaglandin production.

DEX can also decrease vasogenic oedema due to central nervous system tumours (intracranial and spinal cord) by stabilising the blood brain barrier. The exact mechanism is poorly elucidated. The upregulation of the blood brain barrierstabilising factor angiopoietin- 1 and downregulation of the blood brain barrierpermeating factor vascular endothelial growth factor due to DEX were thought to be possible mechanisms (Kim, Lee et al. 2008). 


\subsubsection{Obstetrics}

DEX, which crosses the placenta (Wintour, Alcorn et al. 1994) may be given to women at risk of pre-term delivery to promote fetal lung maturity. It can also be given prenatally to mothers of female fetuses with classical congenital adrenal hyperplasia, diagnosed via prenatal detection of 21-hydroxylase deficiency, to prevent virilisation of external genitalia.

\subsubsection{Ocular inflammatory diseases}

Topical ocular preparations (solution or ointment) are available for use in ocular inflammatory diseases. These include anterior uveitis, external inflammatory eye disease secondary to infections and cicatricial pemphigoid, and post-operative inflammation.

\subsubsection{Cerebral oedema}

Apart from oedema due to neoplastic cerebral lesions, DEX is also used for cerebral oedema due to infections, trauma, intracranial haemorrhage and post cerebral irradiation.

\subsubsection{Diagnostic use}

The DEX suppression test can be used to diagnose Cushing's syndrome and Cushing's disease. 


\subsubsection{Overnight dexamethasone suppression test}

The purpose of this test is to determine if patients with clinical signs suggestive of hypercortisolism demonstrate biochemical evidence of increased cortisol biosynthesis. Suppression of plasma cortisol (measured at $8 \mathrm{am}$ ) to less than $<140$ $\mathrm{nmol} / \mathrm{L}(5 \mu \mathrm{g} / \mathrm{dl}$ ) with $1 \mathrm{mg}$ of oral DEX administered the night before (at $11 \mathrm{pm}$ ) excludes Cushing's syndrome (Nieman, Biller et al. 2008).

\subsubsection{Formal dexamethasone suppression test}

This test is used in the differential diagnosis of biochemically-confirmed Cushing's syndrome. Baseline plasma cortisol levels are documented before administering oral DEX (0.5 mg, every 6 hours for 48 hours) (Stewart 2008). This dose significantly suppresses cortisol production in normal subjects and those with cortisol increases due to obesity and stress, but not in individuals with Cushing's syndrome. This is then followed by a high dose phase where patients are given high dose oral DEX (2 mg, every 6 hours for 48 hours) (Stewart 2008). This dose will suppress plasma cortisol in Cushing's syndrome due to pituitary disease (Cushing's disease) but not from primary adrenal cortical lesions or ectopic ACTH production.

\subsubsection{Toxicity}

Continued use of systemic DEX at supraphysiological dosages can result in adverse effects common to any other glucocorticoids. These include adrenal suppression with risk of adrenal insufficiency following acute withdrawal, 
hypertension, immunosuppression with increased risk of infections, glucose intolerance, diabetes mellitus, osteoporosis, osteonecrosis, myopathy, risk of peptic ulcer disease, cataracts and behavioural changes.

\subsubsection{Blood pressure effect of dexamethasone}

Hypertension is a common manifestation of DEX use. In normotensive human subjects, hypertension can be induced with oral DEX ( $1 \mathrm{mg}$ every 8 hours) (Pirpiris, Sudhir et al. 1992; Mangos, Walker et al. 2006). This blood pressure effect is reproducible in laboratory animals including rats (Zhang, Croft et al. 2004; Hu, Zhang et al. 2006; Krug, Zhang et al. 2008), mice (Wallerath, Witte et al. 1999; Wallerath, Godecke et al. 2004), sheep (Whitworth, Coghlan et al. 1979) and dogs (Nakamoto, Suzuki et al. 1991). In rats, DEX dose of as a low as $1 \mu \mathrm{g}$ (administered via subcutaneous infusion over 4 weeks) (Tonolo, Fraser et al. 1988), has been shown to result in a significant increase in SBP.

The exact mechanism of DEX-induced hypertension (DEX-HT) is unknown. Perturbations in the various pathophysiological systems affecting blood pressure such as plasma volume, renin-angiotensin-aldosterone system, sympathetic activity, vasopressor and vasodepressor systems have been proposed as contributing to DEX-HT. Mechanisms of DEX-HT including the role of these factors and the interactions between them will be examined below (Table 1.2). 
Table 1.2: Postulated mechanisms of dexamethasone-induced hypertension

1. Sodium retention and volume expansion

2. Hemodynamic changes

3. Increased vascular pressor responsiveness

4. Increased sympathetic nervous system activity

5. Vasopressor hormone excess

i. renin-angiotensin system

ii. arginine vasopressin

iii. endothelin

iv. cathecholamine

v. neuropeptide $Y$

6. Vasodilator hormone deficiency

i. Atrial natriuretic peptide

ii. Prostanoids

iii. Nitric oxide

7. Central stimulation of blood pressure

8. Oxidative stress

i. $\mathrm{NAD}(\mathrm{P}) \mathrm{H}$

ii. oxidase

iii. Xanthine oxidase

iv. Uncoupling of eNOS

v. Mitochondria

vi. Cyclooxygenase 


\subsection{MECHANISMS}

\section{HYPERTENSION}

\subsubsection{Sodium and water retention}

The notion that glucocorticoids induce hypertension through activation of renal mineralocorticoid receptors arises from observations that cortisol produces renal sodium retention and hypertension (Stewart, Walker et al. 1995). However, evidence for a causal relationship between sodium retention and glucocorticoidinduced hypertension (GC-HT) is lacking. It is now clear that sodium retention is not the cause of either synthetic or naturally-occuring GC-HT, in humans or rats (Whitworth, Gordon et al. 1989; Montrella-Waybill, Clore et al. 1991; Williamson, Kelly et al. 1996; Li, Wen et al. 1999). Experimental cortisolinduced hypertension in humans (Montrella-Waybill, Clore et al. 1991; Williamson, Kelly et al. 1996) and adrenocorticotrophic hormone-induced hypertension (ACTH-HT) in rats $(\mathrm{Li}$, Wen et al. 1999) are not prevented by mineralocorticoid receptor blockade with spironolactone. Synthetic glucocorticoids elevate blood pressure in humans without any sodium retention or plasma volume expansion (Whitworth, Gordon et al. 1989). Grunfeld and colleagues have also demonstrated in rats that the mineralocorticoid receptor antagonist RU28318, at a dose that lowered blood pressure in mineralocorticoid hypertension (Moura and Worcel 1981), failed to modify hypertension induced by a glucocorticoid receptor agonist RU26988 (Grunfeld, Eloy et al. 1985). They have also found that the glucocorticoid receptor antagonist RU38486 prevented 
and improved glucocorticoid receptor agonist RU26988-induced hypertension without altering body weight, urinary water and sodium excretion (Grunfeld, Eloy et al. 1985).

DEX has high affinity for glucocorticoid receptors and low affinity for mineralocorticoid receptors. It effectively induces nuclear translocation of both glucocorticoid and mineralocorticoid receptors but stimulates mineralocorticoid receptor-mediated transactivation at a much lower capacity than aldosterone. 11Ketodexamethasone, the oxidation product of $11 \beta$ hydroxysteroid dehydrogenase 2, also demonstrated a weak binding affinity for mineralocorticoid receptors and requires a high concentration to achieve nuclear translocation without activating mineralocorticoid receptor-mediated transcription of a reporter gene (Rebuffat, Tam et al. 2004) Even though DEX can bind to mineralocorticoid receptors, DEX raises blood pressure in man without mineralocorticoid effects as evidenced by the absence of urinary sodium retention and increase in body weight (Whitworth, Gordon et al. 1989). It produces diuresis in preterm infants (Bos, van Asselt et al. 2000) and promotes excretion of a water load in adrenal insufficiency in rats (Bengele, McNamara et al. 1977; Hayamizu, Kanda et al. 1994). DEX-HT in humans was accompanied by natriuresis without any changes in body weight or plasma volume (Whitworth, Gordon et al. 1989). In the rat, DEX decreases body weight (Zhang, Croft et al. 2004; Hu, Zhang et al. 2006) and increases haematocrit by $5 \%(\mathrm{Hu}$, Zhang et al. 2006). 
The increase in blood pressure due to DEX is independent of sodium loading or retention. Okuno et al. confirmed that the hypertensive effect of DEX $(2.5 \mathrm{mg} / \mathrm{L}$ drinking fluids or approximately $30-60 \mu \mathrm{g} /$ day, given orally) in rats was not influenced by sodium loading with oral $1 \% \mathrm{NaCl}$ (Okuno, Suzuki et al. 1981).

\subsubsection{Haemodynamic changes}

Haemodynamic studies provide insights into the effects of DEX in the different vascular beds. Hypertension due to a large dose of DEX $(0.5 \mathrm{mg} / \mathrm{kg} /$ day, given orally) in dogs was accompanied by a reduction in cardiac output and an increase in calculated total peripheral resistance (Nakamoto, Suzuki et al. 1991; Nakamoto, Suzuki et al. 1992). In humans, DEX (1 mg orally three times daily for 7 days) increased mean arterial pressure from $82 \pm 3$ to $91 \pm 3 \mathrm{mmHg}(p<$ 0.001) and calculated total peripheral vascular resistance from 21.9 to $24.3 \mathrm{mmHg} / \mathrm{L}$ per minute $( \pm 0.4, p<0.01)$ without affecting the cardiac output (Pirpiris, Sudhir et al. 1992).

Studies of the effect of DEX on the regional haemodynamics are limited. In a study evaluating the effects of DEX $(125 \mu \mathrm{g} / \mathrm{kg} /$ hour, intravenous infusion over 24 hours) on the regional haemodynamic responses to lipopolysaccharide in rats, DEX treatment alone (before administration of lipopolysaccharide) increased the mean arterial pressure, hindquarter blood flow and conductance; and decreased renal and mesenteric blood flow and conductance (Gardiner, Kemp et al. 1996). 
Currently available data indicate that DEX-HT, in both humans and dogs, is characterised by increases in calculated total peripheral resistance. It has been unclear whether this represents a coexisting feature or a pathogenic mechanism of DEX-HT. This issue was addressed in this project and is discussed in Chapters 3 and 4.

\subsubsection{Increased vascular pressor responsiveness}

Hypertension is associated with structural, mechanical (compliance and distensibility) and functional abnormalities of blood vessels that result in decreased lumen diameter of small arteries and arterioles (Intengan and Schiffrin 2000). Structural impairment of the blood vessel wall is unlikely to play a major role in experimental DEX-HT where the hypertensive action of DEX occurs rapidly within 1-2 days (Nakamoto, Suzuki et al. 1991; Pirpiris, Sudhir et al. 1992; Zhang, Croft et al. 2004; Hu, Zhang et al. 2006; Krug, Zhang et al. 2008). Furthermore, there are no reports confirming the presence of vascular hypertrophy histologically in DEX-induced hypertensive subjects or animals.

Functional aberrations of the vascular smooth muscle cells, manifesting as increased sensitivity and reactivity to vasoconstrictors (i.e. increased vascular pressor responsiveness) have been described in DEX-HT in humans, rats and dogs (Handa, Kondo et al. 1984; Russo, Fraser et al. 1989; Russo, Fraser et al. 1990; Nakamoto, Suzuki et al. 1991; Pirpiris, Sudhir et al. 1992). Greater rises in forearm vascular resistance in response to infusions of angiotensin II and 
noradrenaline were observed in human subjects with DEX-induced $(3 \mathrm{mg} /$ day, given orally) hypertension versus controls, consistent with DEX increasing sensitivity to these vasoconstrictors. (Pirpiris, Sudhir et al. 1992). Pretreatment with oral DEX $(0.3 \mathrm{mg} / \mathrm{dL}$ drinking water, approximately $0.1 \mathrm{mg} / \mathrm{day}$ or $0.5 \mathrm{mg} / \mathrm{kg} / \mathrm{day}$ ) in rats enhanced blood pressure elevation due to noradrenaline (Handa, Kondo et al. 1984). A similarly enhanced pressor response to noradrenaline was observed in DEX-HT $(0.5 \mathrm{mg} / \mathrm{kg} /$ day, given orally $)$ in dogs but in contrast to the human study (Pirpiris, Sudhir et al. 1992), pressor response to angiotensin II was unaltered (Nakamoto, Suzuki et al. 1991). In vitro perfusion of isolated rat mesenteric vasculatures resulted in a reduced threshold and increased maximal response to noradrenaline in DEX-induced hypertensive rats $(2 \mu \mathrm{g} / \mathrm{rat} /$ day, approximately $7-9.5 \mu \mathrm{g} / \mathrm{kg} /$ day, given orally for 28 days) but not in controls (Russo, Fraser et al. 1989; Russo, Fraser et al. 1990). However, these effects were not reproducible with AVP and potassium chloride infusions (Russo, Fraser et al. 1989; Russo, Fraser et al. 1990). Ijima and Malik demonstrated that DEX-HT $(1.8 \mathrm{mg} / \mathrm{kg} /$ week or average of approximately $257 \mu \mathrm{g} / \mathrm{kg} / \mathrm{day}$, for 2 weeks) in rats was associated with increased pressor response to arginine vasopressin but not to noradrenaline or angiotensin II (Iijima and Malik 1988).

These studies, which were undertaken in different species under different experimental conditions, showed some discrepancies in the responses (or lack of response) to vasoconstrictors including angiotensin II, noradrenaline and arginine vasopressin (Handa, Kondo et al. 1984; Russo, Fraser et al. 1989; Russo, Fraser et al. 1990; Nakamoto, Suzuki et al. 1991; Pirpiris, Sudhir et al. 1992). 
Nevertheless, these results suggested a role for DEX in increasing the sensitivity of vascular smooth muscle cells to vasoconstrictors, and vasoconstriction and potentiation of the effects of vasoconstrictors may contribute to DEX-HT.

The mechanisms underlying this heightened response to vasoconstrictors induced by DEX have not been fully elucidated. Sato et al. demonstrated a DEX-induced decrease in threshold for the production of inositol triphosphate by cultured vascular smooth muscle cells in response to angiotensin II and arginine vasopressin. This effect was thought to be mediated by the glucocorticoid receptor as it was completely blocked by a specific glucocorticoid antagonist RU38486 (Sato, Suzuki et al. 1992). This group later identified that DEX (30 mg/L drinking water, approximately $0.1 \mathrm{mg} / \mathrm{rat} /$ day or $0.5 \mathrm{mg} / \mathrm{kg} /$ day) in rats stimulated vascular angiotensin II type 1A receptor mRNA even prior to the onset of hypertension (Sato, Suzuki et al. 1994).

Another possible mechanism for DEX-induced increased vascular pressor reactivity is alteration in sodium/potassium pump activity (Pamnani, Clough et al. 1978; Stern, Palant et al. 1994). In an in vitro study, direct stimulation of sodium/potassium-pump-mediated cation transport in cultured rat aortic smooth mucle cells was demonstrated after 18-24 hour incubation with $10^{-9} \mathrm{M}$ DEX (Stern, Palant et al. 1994). Sodium/potassium pump activity in the tail artery of DEX-induced hypertensive (initial DEX dose of $12.5 \mathrm{mg}$ followed by $6.5 \mathrm{mg}$ weekly, subcutaneous injections) uninephrectomised rats was significantly increased (Pamnani, Clough et al. 1978). 
Whilst increased vascular sensitivity or reactivity to vasoconstrictors may be a feature of DEX administration, it remains unclear whether these changes are sufficient to account, if at all, for the hypertension. Despite the evidence presented above, there are reports that have not found any pressor effects of either acute or chronic DEX treatments (acute: $20 \mathrm{mg}$ intravenously to human subjects (Sambhi, Weil et al. 1962); and chronic: $0.5 \mathrm{mg} /$ day for 14 days intramuscularly to mongrel dogs (Lefer, Manwaring et al. 1966)) on arterial responses to noradrenaline and angiotensin II.

Other factors contributing to increased pressor responsiveness in DEX-HT include decreased synthesis of vasodilator prostanoids and increased expression of angiotensin 1A and AVP receptors, discussed in Sections 1.4.6.2, 1.4.5.1 and 1.4 .5 .2 , respectively.

\subsubsection{Increased sympathetic nervous system activity}

Several studies have evaluated the effect of DEX on sympathetic nerve activity and the role of sympathetic nervous system as a mediator of DEX-HT, but with conflicting results. This is, in part, due to the methods used to measure sympathetic nerve function. There are no published reports evaluating the role of sympathetic nervous system in DEX-HT by means of ganglionic blockade. However, there are several other pieces of evidence from both direct (muscle sympathetic activity) and indirect (expression of adrenergic receptors, catecholamine synthesis, plasma catecholamine and plasma and tissue 
neuropeptide Y) assessments of sympathetic nerve activity in DEX-HT which are presented below.

\subsubsection{Changes in adrenergic receptors}

DEX can alter the balance of $\alpha 1$-adrenergic receptor availability in vascular smooth muscles (Haigh and Jones 1990). In DDT1 MF2 hamster smooth muscle cell culture, DEX $\left(10^{-6} \mathrm{M}\right.$ ) was shown to result in $2.8 \pm 0.7$ (mean $\pm \mathrm{SEM}$ ) fold increase in the expression of $\alpha 1 \mathrm{~B}$ adrenergic receptor genes and 1.8 \pm 0.2 -fold increase in the expression of $\alpha 1-\beta$ adrenergic receptors (Sakaue and Hoffman 1991). As discussed in Section 1.5.3, these alterations may contribute to the increase in vascular reactivity and pressor responsiveness seen in DEX-treated humans and experimental animals (Handa, Kondo et al. 1984; Russo, Fraser et al. 1989; Russo, Fraser et al. 1990; Nakamoto, Suzuki et al. 1991; Pirpiris, Sudhir et al. 1992).

\subsubsection{Changes in catecholamine synthetic pathway}

Tyrosine hydroxylase: Kumai et al. showed that hypertension in rats induced by subcutaneous DEX injections at $1 \mathrm{mg} / \mathrm{kg} /$ day for 2 days was associated with elevated adrenaline and noradrenaline levels in plasma and adrenal medulla; and increased expression and activity of tyrosine hydroxylase, a rate-limiting enzyme that converts L-tyrosine to dihydrophenylalanine (precursor for dopamine) (Kumai, Asoh et al. 2000). They have also demonstrated that inhibition of tyrosine hydroxylase with $\alpha$-methyl-p-tyrosine reversed DEX-HT (Kumai, Asoh 
et al. 2000). Intravenous DEX (2 $\mathrm{mg}$, single dose) in normotensive human subjects resulted in elevated plasma dopamine and adrenaline levels that could be blocked by the tyrosine hydroxylase inhibitor alpha-methyl-p-tyrosine (Watanabe, Noshiro et al. 1995).

Phenylethanolamine $N$-methyltransferase: The activity of phenylethanolamine $N$ methyltransferase (PNMT), an enzyme that converts noradrenaline to adrenaline, in peripheral tissues (atria, ventricle and skeletal muscle) was significantly increased with chronic subcutaneous DEX treatment at $1 \mathrm{mg} / \mathrm{kg} /$ day for $12-14$ days in both intact and adrenalectomised rats (Kennedy and Ziegler 1991; Kennedy, Elayan et al. 1993). The increase in activity, which was secondary to DEX-induced increase in PNMT level rather than activation of existing enzyme, was associated with restoration of adrenalectomy-induced decrease in adrenaline production in the atria (Kennedy and Ziegler 1991). In another experiment, these authors demonstrated that administration of a highly selective peripheral PNMT inhibitor in adrenalectomised DEX-induced hypertensive rats successfully normalised blood pressure (Kennedy, Elayan et al. 1993).

Whilst these studies suggest a role for tyrosine hydroxylase and non-adrenal PNMT in the pathogenesis of DEX-HT, it is not possible to draw any definitive conclusions about the role of increased sympathetic activity in DEX-HT as both tyrosine hydroxylase and PNMT activities are very indirect measures of sympathetic function. 


\subsubsection{Changes in neuropeptide $Y$}

Neuropeptide Y (NPY) is a 36 amino acid peptide that is found in abundance within neurons both in the central (Adrian, Allen et al. 1983; Allen, Adrian et al. 1983) and peripheral (Gu, Polak et al. 1983; Lundberg, Terenius et al. 1983) nervous system. In the periphery, it coexists with noradrenaline in sympathetic neurons that innervate the blood pressure-controlling structures: blood vessels, heart and kidneys (Sundler, Bottcher et al. 1993). It is released upon sympathetic stimulation and thus, is used as an index of sympathetic activity. NPY can modulate vascular tone by inhibiting the release of noradrenaline and NPY itself at the presynaptic NPY receptors (Haass, Cheng et al. 1989; Pernow and Lundberg 1989). At the postsynaptic receptor, NPY causes direct vasoconstriction (especially coronary, cerebral, mesenteric and renal vascular beds) (Edvinsson, Ekblad et al. 1984; Echtenkamp and Dandridge 1989; Tanaka, Mori et al. 1997) and enhances the vasoconstricting effect of vasopressors including noradrenaline and histamine (Edvinsson, Ekblad et al. 1984).

A role for NPY in GC-HT was proposed following observations that DEX can influence NPY gene expression and tissue content in neuroendocrine tissue and cell lines (Corder, Pralong et al. 1988; Wilding, Gilbey et al. 1993; Myrsen, Ahren et al. 1996; Myrsen-Axcrona, Karlsson et al. 1997). DEX-induced NPY expression in islet cells (in vivo DEX dose $2 \mathrm{mg} / \mathrm{kg}$ /day, intraperitoneal injections, 12 days) (Myrsen, Ahren et al. 1996) and insulin-producing cell line RINm5F (in vitro DEX concentration 100 nM, 5 days) (Myrsen-Axcrona, Karlsson et al. 1997) has been demonstrated. Increases in rat hypothalamic NPY mRNA (Wilding, 
Gilbey et al. 1993) and content (Corder, Pralong et al. 1988; Wilding, Gilbey et al. 1993) have also been shown following DEX treatment $(0.4 \mathrm{mg} / \mathrm{kg} / \mathrm{day}$, subcutaneously (Wilding, Gilbey et al. 1993) - $0.5 \mathrm{mg} / \mathrm{kg} / \mathrm{day}$, intraperitoneally (Corder, Pralong et al. 1988)). The only evaluation of plasma NPY levels in glucocorticoid hypertension was conducted by Tabarin et al. who found that plasma NPY was not elevated in either hypertensive $(n=15)$ or normotensive patients ( $\mathrm{n}=11)$ with Cushing's syndrome (Tabarin, Minot et al. 1992). There is insufficient evidence to conclude that changes in plasma or tissue NPY content play a role in DEX-HT as direct measurement of NPY tissue content and plasma level, consequent on alterations in its formation, release and clearance, in DEXHT has not been reported.

\subsubsection{Changes in plasma noradrenaline and adrenaline levels}

DEX effects on plasma noradrenaline and adrenaline concentrations are inconsistent in the literature (Table 1.4). Acute $\operatorname{DEX}(2.5,25$ and $250 \mu \mathrm{g}$ or approximately $0.01,0.1$ and $1 \mathrm{mg} / \mathrm{kg}$, respectively, given subcutaneously) did not alter plasma adrenaline and noradrenaline levels in normal rats (Brown and Fisher 1986). On the other hand, rats made hypertensive with 2-day DEX injections ( $1 \mathrm{mg} / \mathrm{kg} / \mathrm{day}$, given subcutaneously) had significantly raised plasma adrenaline and noradrenaline (Kumai, Asoh et al. 2000). In another study in rats, $0.1 \mathrm{mg} / \mathrm{kg}$ subcutaneous DEX raised plasma adrenaline but did not alter plasma noradrenaline concentration (Silvan, Martinez-Mateos et al. 2007). 
Similar variations in plasma adrenaline and noradrenaline concentrations were documented in normotensive human subjects following DEX treatment (Rothschild, Langlais et al. 1984; Watanabe, Noshiro et al. 1995) (Table 1.3).

Whilst plasma adrenaline and noradrenaline concentrations have been used as an index of sympathetic activity, they have limitations. Static measurements are influenced by changes in renal function and hence, plasma catecholamine clearance. Discrepancies probably also reflect differences in species studied, dose and duration of DEX treatments. 
Table 1.3: Changes in plasma noradrenaline and adrenaline concentrations induced by DEX treatment in normotensive humans and rats

\begin{tabular}{ccccc} 
Species & DEX dose & $\begin{array}{c}\text { Plasma } \\
\text { NAdr }\end{array}$ & $\begin{array}{c}\text { Plasma } \\
\text { Adr }\end{array}$ & Reference \\
\hline Human & $2 \mathrm{mg}$, i.v. stat. & $\uparrow$ & $\downarrow$ & $\begin{array}{c}\text { (Watanabe, Noshiro } \text { et } \\
\text { al. 1995) }\end{array}$ \\
Human & $1 \mathrm{mg}$, p.o. stat. & $\leftrightarrow$ & $\leftrightarrow$ & $\begin{array}{c}\text { (Rothschild, Langlais } \\
\text { et al. 1984) }\end{array}$ \\
& & & & \\
Rat & $0.01,0.1,1 \mathrm{mg} / \mathrm{kg}$, & & & (Brown and Fisher \\
& s.c. stat. & $\downarrow$ & & 1986) \\
Rat & $1 \mathrm{mg} / \mathrm{kg}$, s.c. 2 days & $\uparrow$ & $\uparrow$ & (Kumai, Asoh et al. \\
& & & & 2000) \\
Rat & $0.1 \mathrm{mg} / \mathrm{kg}$, s.c. stat. & $\leftrightarrow$ & $\uparrow$ & (Silvan, Martinez- \\
& & & & Mateos et al. 2007)
\end{tabular}

Adr: adrenaline, NAdr: noradrenaline.

\subsubsection{Muscle sympathetic activity}

Direct assessment of sympathetic vasomotor drive to skeletal muscle has been evaluated in six healthy male subjects, treated with oral DEX ( $3 \mathrm{mg} /$ day) for five days. DEX significantly increased systolic blood pressure from a pre-DEX pressure of $115 \pm 1$ to $125 \pm 2 \mathrm{mmHg}$ (mean \pm SEM, $P<0.01$ ) but completely suppressed resting spontaneous sympathetic activity in five subjects and markedly 
reduced it in the other (Macefield, Williamson et al. 1998). Stimulated sympathetic activity induced by cold pressor stimulus, end-inspiratory and endexpiratory apnoea were also attenuated by DEX (Macefield, Williamson et al. 1998).

\subsubsection{Summary}

Although indirect methods have provided some useful information about the effects of DEX on sympathetic nerve activity, direct recording of sympathetic nerve traffic remains a more reliable technique (Grassi and Esler 1999). Based on the data of Macefield et al. using the latter technique, it is evident that DEX-HT, at least in humans, is not due to increased sympathetic drive (Macefield, Williamson et al. 1998). 


\subsubsection{Excess vasopressor hormone}

\subsubsection{Angiotensin II}

DEX (4 mg/kg, intraperitoneally) given to rats 16 and 24 hours before sacrifice significantly increased plasma and liver renin substrate (angiotensinogen) without significant alteration in plasma renin activity (Eggena and Barrett 1988). In DEXHT in rats $(2.5 \mathrm{mg}$ DEX/L drinking water, approximately $30-60 \mu \mathrm{g} /$ day or 0.17 $0.27 \mathrm{mg} / \mathrm{kg} /$ day), plasma renin substrate was increased (Okuno, Suzuki et al. 1981) but plasma renin activity was within normal range (Okuno, Suzuki et al. 1981; Suzuki, Handa et al. 1982). The absence of raised plasma renin activity in these studies suggests that glucocorticoid-induced increase in plasma renin substrate does not necessarily indicate activation of the renin-angiotensin system.

Another way of evaluating the role of angiotensin II in the development of DEXHT is by utilising angiotensin II receptor blockers or angiotensin converting enzyme inhibitors in in vivo models. Suzuki et al. found that DEX $(2.5 \mathrm{mg} / \mathrm{L}$ drinking water, approximately $30-60 \mu \mathrm{g} /$ day or $0.17-0.27 \mathrm{mg} / \mathrm{kg} / \mathrm{day}$ ) raised blood pressure, daily urine volume, urinary sodium excretion and fluid intake without altering plasma renin activity (Suzuki, Handa et al. 1982). There was partial prevention of DEX-HT with the angiotensin antagonist saralasin and partial reversal with both saralasin and angiotensin converting enzyme inhibitor SQ14225 without significant differences in volume status, as indicated by body weight, daily urine volume and fluid intake, between the groups. The blood pressure lowering effects of saralasin and SQ14225 were abolished by bilateral 
nephrectomy (Suzuki, Handa et al. 1982). These results highlight a partial contribution of vasoconstrictor angiotensin II in the development of DEX-HT but do not differentiate whether this is due to angiotensin II excess or glucocorticoidinduced increase in angiotensin II sensitivity.

Apart from its vasoconstrictive capacity, angiotensin II stimulates angiogenesis, vascular smooth muscle hypertrophy and hyperplasia, myocardial hypertrophy and vascular extracellular matrix synthesis (Ferrario and Chappell 2008). It is also a powerful stimulus of superoxide production by the mitochondria, via the activation of mitochondrial ATP-sensitive potassium channel openers, and NAD(P)H oxidase pathway (Griendling, Minieri et al. 1994; Kimura, Zhang et al. 2005) (Figure 1.2). The role of oxidative stress including NAD(P)H oxidasemediated superoxide in DEX-HT is discussed in Section 1.5.7.

\subsubsection{Arginine vasopressin}

Administration of arginine vasopressin (AVP) to normotensive rats resulted in dose dependent increases in mean arterial pressure (Iijima and Malik 1988). This increase was accentuated by DEX administration $(1.8 \mathrm{mg} \cdot \mathrm{kg} / \mathrm{week}$, given as weekly subcutaneous injections for 2 weeks). Administration of $\mathrm{d}\left(\mathrm{CH}_{2}\right)_{5} \mathrm{Tyr}(\mathrm{Me}) \mathrm{AVP}$, an AVP $\mathrm{V}_{1}$ receptor antagonist, reduced mean arterial pressure in DEX-treated rats but not in control rats (Iijima and Malik 1988). The contribution of AVP to the pathogenesis of DEX-HT appears to be mediated by increased expression of the $V_{1 a} A V P$ receptor due to increased mRNA stability 
(Murasawa, Matsubara et al. 1995) and not increased plasma AVP concentration (Nakamoto, Suzuki et al. 1991).

\subsubsection{Endothelin}

DEX has been shown to induce endothelin release from cultured human umbilical vein endothelial cells (Huang, Whitworth et al. 1995) and cultured rat and rabbit vascular smooth muscle cells (Kanse, Takahashi et al. 1991; Roubert, Viossat et al. 1993). Plasma endothelin level was reported to be increased in rats receiving DEX (2 mg/kg/day for 12 days) (Takahashi, Suda et al. 1991). A $50 \%$ increase in circulating endothelin concentration was also reported in human subjects treated with another synthetic glucocorticoid, prednisolone (50 mg on day $1,25 \mathrm{mg}$ on days 2 and 3, and 10mg on days 4 and 5) (Borcsok, Schairer et al. 1998). It is unclear whether this association represents the pathological process responsible for DEX-HT. Further in vivo studies using an endothelin antagonist in DEX-HT models is necessary to confirm this hypothesis. 


\subsubsection{Other vasoconstrictors}

The influences of other vasoconstrictors such as catecholamines and NPY have been discussed in Sections 1.5.4.2 and 1.5.4.3, respectively.

\subsubsection{Deficiency in vasodilator hormone}

Of the naturally-occurring vasodilators, atrial natriuretic peptide, prostanoids and nitric oxide have been examined in DEX-HT models. There are no data on the role of other vasodilators (eg endothelium-derived hyperpolarizing factor) in the pathogenesis of DEX-HT.

\subsubsection{Atrial natriuretic peptide}

The role of atrial natriuretic peptide (ANP) in DEX-HT is controversial. Currently available evidence from both in vivo and in vitro studies indicates conflicting results on the effect of DEX on tissue and plasma ANP concentrations. On one hand, plasma atrial natriuretic peptide (ANP) concentrations were significantly lower in rats made hypertensive with subcutaneous DEX infusions at low dosages $(1,2,5$ and $10 \mu \mathrm{g} /$ day) (Tonolo, Fraser et al. 1988). In these rats, the ANP values were negatively correlated with blood pressure (Tonolo, Fraser et al. 1988). On the other hand, DEX treatment $(1 \mathrm{mg} /$ day or $4 \mathrm{mg} / \mathrm{kg} /$ day, subcutaneously for 2 days) increased expression of atrial, ventricular and pulmonary ANP gene, and plasma ANP levels in both intact and adrenalectomised rats (Gardner, Hane et al. 1986). The DEX-induced increases in plasma ANP and atrial ANP mRNA levels were independent of volume status as they were not suppressed by water 
deprivation for 96 hours prior to sacrifice and sample collection (Gardner, Hane $e t$ al. 1986). Garcia et al. demonstrated that the synthesis and release of ANP by the atria of adrenalectomised rats are regulated by $\operatorname{DEX}(0.1 \mathrm{mg} / \mathrm{kg} / \mathrm{day}$ via miniosmotic pump subcutaneous infusion for 5 days) and can only occur when co-administered with a mineralocorticoid which exerts a permissive effect on the regulatory role of DEX on ANP production (Garcia, Debinski et al. 1985). In another study, ANP levels from atrial slices and extract obtained from DEX $(0.1 \mathrm{mg} / \mathrm{kg} / \mathrm{day}$, intraperitoneal injection, 4 days)-treated rats were significantly higher compared to control rats but were not reproducible by in vitro incubation of atrial slices with varying concentrations of DEX $\left(10^{-7}-10^{-4} \mathrm{M}\right.$, for 1 and 4 hours) (Lachance, Garcia et al. 1986). The latter result was likely due to inadequate incubation time.

It is unclear whether the contrasting effects of DEX on tissue and plasma ANP concentrations are related to the dose and administration of $\mathrm{DEX}$ and/or variations in experimental conditions, rat strain or model. Nevertheless, the role of ANP availability and activity in DEX-HT merits further investigation.

\subsubsection{Prostanoids}

The notion that a reduction in vasodilator prostanoids plays a role in the development of DEX-HT arose from observations in in vitro studies that DEX can inhibit the biosynthesis of prostaglandins via the inhibition of phospholipase $A_{2}$ activity, mediated by a transferable phopholipase $A_{2}$ inhibitory protein (Flower 
and Blackwell 1979; Hirata, Schiffmann et al. 1980; Russo-Marie and Duval 1982), and subsequent reduction in arachidonic acid.

The roles of prostaglandins and prostacyclins in the development of DEX-HT have been examined, but with conflicting results. The discrepancies may be ascribed to variability in species and experimental protocols. There have been reports indicating that hypertension in rats due to DEX ( $3 \mathrm{mg} / \mathrm{L}$, drinking water, approximately $146-160 \mu \mathrm{g} / \mathrm{day}, 7$ days) was associated with decreased urinary excretion of PGI-M, a marker of prostacyclin $\left(\mathrm{PGI}_{2}\right.$ ) biosynthesis (Falardeau and Martineau 1989). Handa et al. concluded that DEX-HT in the rat (DEX dose: $0.1 \mathrm{mg} /$ day or $0.5 \mathrm{mg} / \mathrm{kg} /$ day, orally) was associated with sustained inhibition of prostaglandin synthesis based on their finding of reduced urinary prostaglandin $E_{2}$ $\left(\mathrm{PGE}_{2}\right)$ excretion prior to the onset of hypertension (Handa, Kondo et al. 1984). There are no in vivo studies in humans evaluating the role of prostanoids in DEXHT but studies using cultured human umbilical vein endothelial cells showed that thrombin- and histamine-stimulated prostacyclin $\left(\mathrm{PGI}_{2}\right)$ (Lewis, Campbell et al. 1986; Huang, Whitworth et al. 1995) and histamine-stimulated $\mathrm{PGE}_{2}$ (Lewis, Campbell et al. 1986) release from this human cell line were inhibited by DEX $\left(10^{-7} \mathrm{M}\right.$ or approximately $0.04 \mu \mathrm{g} / \mathrm{mL}$ (Lewis, Campbell et al. 1986) and $1 \mu \mathrm{g} / \mathrm{mL}($ Huang, Whitworth et al. 1995)).

On the other hand, Nasjletti et al. demonstrated that DEX-HT in rats (DEX dose: $2.5 \mathrm{mg} / \mathrm{kg} /$ week, given subcutaneously) was associated with an increase in circulating and urinary excretion of $\mathrm{PGE}_{2}$ in the setting of reduced renomedullary 
production (Nasjletti, Erman et al. 1984). This was attributed to decreased degradation of this vasodilator prostaglandin in the kidney and other extrapulmonary tissues (Nasjletti, Erman et al. 1984). This conclusion was further supported by another study which found increased renal and urinary $\mathrm{PGE}_{2}$ and 6oxo-PGF 1 , a stable derivative of $\mathrm{PGI}_{2}$, in DEX-induced hypertensive rats (DEX dose: $2.5 \mathrm{mg} / \mathrm{L}$ drinking water or approximately $0.3 \mathrm{mg} / \mathrm{kg} /$ day) (Codde and Beilin 1985). Furthermore, they have shown that cod liver oil diet prevented DEX-HT despite causing a fall in vasodilator prostaglandins (Codde and Beilin 1985). In a recent study from our own laboratory, low dose DEX (1 $\mu \mathrm{g} / \mathrm{rat} / \mathrm{day}$, subcutaneous injection) resulted in hypertension in rats without altering urinary $\mathrm{PGI}_{2}$ (Zhang, Hu et al. 2008).

Whilst there are data in keeping with the hypothesis that DEX-HT is linked to a reduction in vasodilator prostanoids, it remains unclear if this is a significant cause of DEX-HT.

\subsubsection{Nitric oxide}

Nitric oxide (NO) deficiency due to administration of nitric oxide synthase inhibitors (Rees, Palmer et al. 1989; Wen, Li et al. 2000a) or endothelial nitric oxide synthase (eNOS) gene inactivation (Huang, Huang et al. 1995) is associated with hypertension. Although there are no published data on the role of NO based on acute NO synthase blockade or sequential blockade of vasoactive systems in $\mathrm{DEX}-\mathrm{HT}$, there is accumulating evidence suggesting a role for NO deficiency in 
the pathogenesis of DEX-HT. Plasma nitrate/nitrite, a marker of total body NO synthesis, are reduced in rats (Mondo, Yang et al. 2006) and mice (Wallerath, Witte et al. 1999; Wallerath, Godecke et al. 2004) made hypertensive by DEX treatment. The reduced availability of NO might result from a range of influences on the NO biosynthetic pathways: i) alteration in the activity and expression of NOS ii) decreased availability of tetrahydrobiopterin $\left(\mathrm{BH}_{4}\right)$, a NOS cofactor, iii) decreased NO precursor L-arginine and iv) increased $\mathrm{NO}$ removal via its interaction with superoxide to form peroxynitrite (Figure 1.5).

Altered nitric oxide synthase expression: DEX substantially suppressed the expression of eNOS mRNA and protein in cultured human and bovine endothelial cells, as well as in aorta, kidney and liver of DEX-induced hypertensive rats (Wallerath, Witte et al. 1999). Furthermore, the hypertensive effect of DEX seen in wild type mice was not evident in their eNOS knockout counterparts (Wallerath, Godecke et al. 2004). This is compatible with the proposal that DEXHT involves downregulation of eNOS gene expression and resultant decrease in vasodilator NO.

Decreased tetrahydrobiopterin availability: Tetrahydrobiopterin $\left(\mathrm{BH}_{4}\right)$ is an essential cofactor for all the nitric oxide synthase isoforms (Schmidt, Werner et al. 1992) (Figure 1.5). Simmons et al. demonstrated that DEX-induced decrease in intracellular $\mathrm{BH}_{4}$ content in cultured rat cardiac microvascular endothelial cells, mediated by suppression of guanosine triphosphate (GTP) cyclohydrolase gene expression, was associated with suppressed nitrogen oxide production (Simmons, 
Ungureanu-Longrois et al. 1996). In another study, ex vivo analysis of aortic segments of DEX $(5 \mathrm{mg} / \mathrm{kg}$, subcutaneous implant)-induced hypertensive rats revealed decreased GTP cyclohydrolase gene expression, dampened eNOS activity, increased vasoconstrictor response to phenylephrine and absent vascular contraction to NOS inhibitor $N^{\omega}$-nitro-L-arginine (Johns, Dorrance et al. 2001). Based on these results, they proposed that $\mathrm{BH}_{4}$ deficiency consequent on downregulation of GTP cyclohydrolase results in eNOS-dependent regulation of vascular contractility and may play a role in the development of GC-HT.

In contrast, $\mathrm{BH}_{4}$ administration in vivo in DEX-hypertensive rats did not alter systolic blood pressure (Miao, Zhang et al. 2007).

Decreased L-arginine availability: Nitric oxide is synthesized from L-arginine via the catalytic action of NOS with co-production of L-citrulline (Figure 1.5). Induced depletion of L-arginine in rats resulted in hypertension and decreased NO synthesis, as evidenced by reduced urinary nitrate and cyclic GMP (Wakabayashi, Yamada et al. 1994), illustrating the importance of L-arginine in the maintenance of nitric oxide homeostasis. However, L-arginine supplementation did not prevent or reverse DEX-HT (Li, Fraser et al. 1997; Hu, Zhang et al. 2006). This could mean that either L-arginine deficiency is not a predominant feature of DEX-HT or there is a defective L-arginine delivery system that results in a state of relative intracellular L-arginine deficiency. DEX-induced abnormalities in L-arginine transport have been demonstrated in cardiac microvascular endothelial cells 
(Simmons, Ungureanu-Longrois et al. 1996). The state of L-arginine transporter in the DEX-induced hypertensive model has not been examined.

Increased nitric oxide inactivation: NO has a very short half life. Under normal circumstances, it diffuses rapidly across cell membrane into red blood cells where it is removed by its interaction with haemoglobin (Azarov, Huang et al. 2005). It can also be inactivated within the vessel wall by its interaction with superoxide to form a powerful oxidant, peroxynitrite (Pacher, Beckman et al. 2007). This reaction occurs faster that the removal of superoxide by superoxide dismutase. In the presence of vascular oxidative stress, endothelial NO can be rapidly quenched by excess superoxide resulting in a state of NO deficit. The role of oxidative stress in DEX-HT will be reviewed in Section 1.6. 


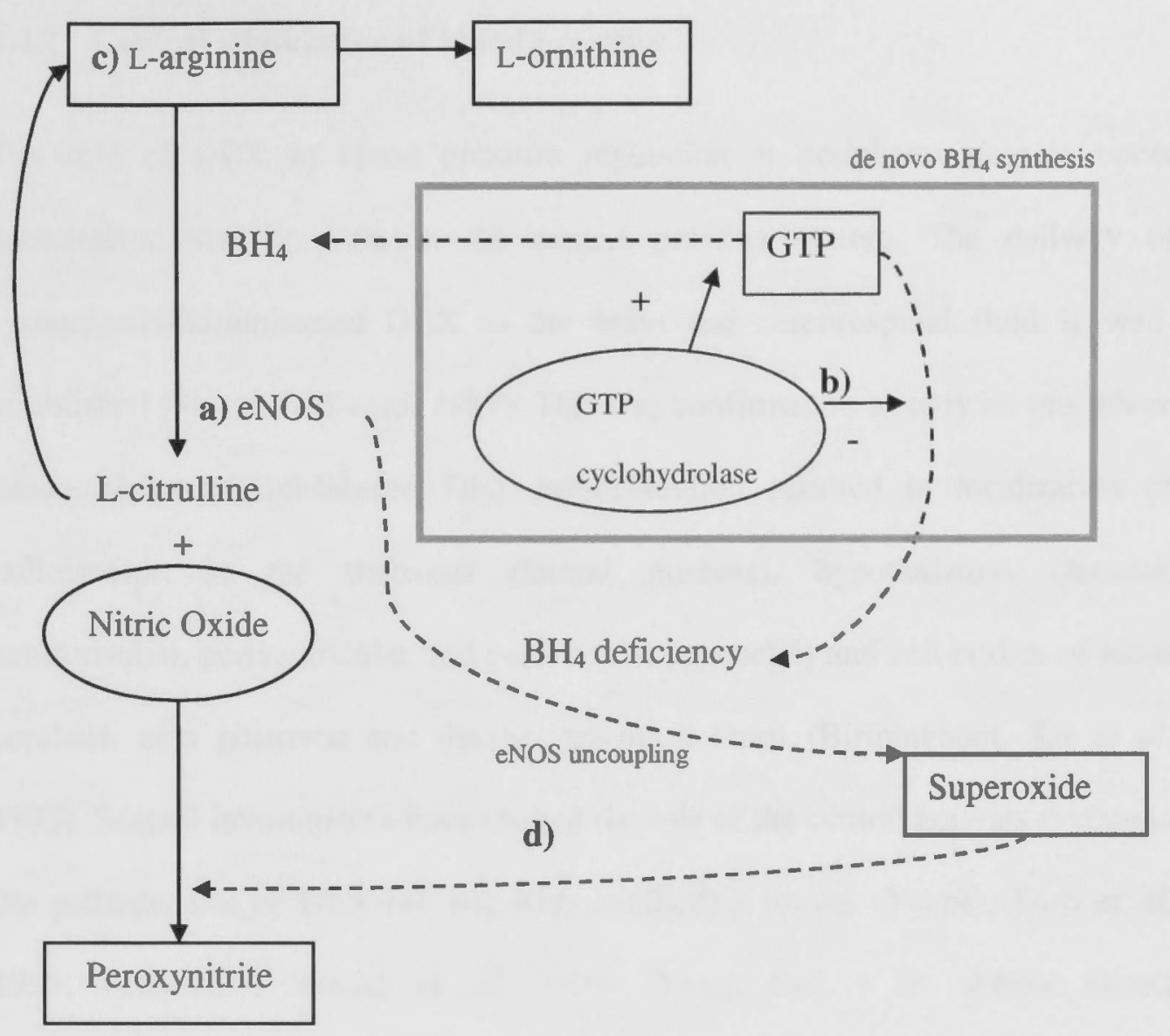

Figure 1.5: Biochemical pathways controlling NO bioavailability. DEX treatment can decrease NO by altering four main pathways marked in this diagram. a) Downregulation of eNOS or decrease in eNOS activity by DEX can decrease NO synthesis; b) Downregulation of GTP cyclohydrolase by DEX results in decreased de novo $\mathrm{BH}_{4}$ biosynthesis and $\mathrm{BH}_{4}$ deficiency; c) Decrease in L-arginine availability due to alterations to its transporter by DEX; d) DEX-induced oxidative stress increases NO inactivation through peroxynitration, a reaction that is significantly faster than dismutation of superoxide by superoxide dismutase. $\mathrm{BH}_{4}$ : tetrahydrobiopterin, eNOS: endothelial nitric oxide synthase, GTP: guanosine triphosphate. 


\subsubsection{Central stimulation of blood pressure}

The role of DEX in blood pressure regulation at peripheral sites is better documented than its role in the central nervous system. The delivery of systemically-administered DEX to the brain and cerebrospinal fluid is wellestablished (Siegal, Soti et al. 1997). This was confirmed in a study on rats where subcutaneous tritium-labeled DEX administration resulted in localization of radioisotope in the thalamus (lateral nucleus), hypothalamus (arcuate, ventromedial, periventricular and paraventricular nuclei) and cell bodies of locus ceruleus, area postrema and nucleus tractus solitarii (Birmingham, Sar et al. 1993). Several investigators have studied the role of the central nervous system in the pathogenesis of DEX-HT but with conflicting results (Tonolo, Soro et al. 1993; Nakamoto, Suzuki et al. 1995; Wang, Ou et al. 2005). Direct microinjections of DEX into bilateral rat nucleus tractus solitarii resulted in a transient hypertensive response acutely at lower doses (12.5 and 25 pmol) and a longer hypertensive response at higher doses (50 and 100 pmol) (Wang, Ou et al. 2005). The increase in blood pressure was found to be mediated by glucocorticoid receptor-independent interaction with $\mathrm{GABA}_{\mathrm{A}}$ and $\mathrm{GABA}_{\mathrm{B}}$ receptors and glucocorticoid-receptor dependent non-transcriptional activation of phosphtidylinositol 3-kinase/protein kinase Akt pathway. However, the possibility of systemic absorption and DEX interactions at peripheral sites was not accounted for in this study. In contrast, intracerebroventricular DEX ( 1 and $10 \mu \mathrm{g} / \mathrm{kg} / \mathrm{day}$ for 7 days in dogs (Nakamoto, Suzuki et al. 1995), and approximately $1 \mu \mathrm{g} / \mathrm{kg} / \mathrm{day}$ for 24 days in rats (Tonolo, Soro et al. 1993)) lowered blood pressure. In the 
former study, co-administration of intracerebroventricular glucocorticoid receptor antagonist, RU38486, abolished the antihypertensive effects (Nakamoto, Suzuki et al. 1995). Differences in dosages, treatment duration and central sites of DEX administration may account for these discrepancies. Nevertheless, the role of the central nervous system in the pathogenesis of DEX-HT remains unclear.

\subsection{REACTIVE OXYGEN SPECIES}

Reactive oxygen species (ROS) at low levels under normal physiological conditions play a pivotal role in innate immunity, cell signalling and regulation of vascular integrity. They consist of biologically active oxygen radicals (superoxide, hydroxyl, carbonate, peroxyl and alkoxyl radicals) and highly reactive non-radicals (hydrogen peroxide, hypochlorus acid, fatty acid hydroperoxides, reactive aldehydes and singlet oxygen) which are generated in a constant fashion as by-products of normal cellular metabolism (Bayir 2005) (Table 1.4). Superoxide and hydrogen peroxide are generated from one and two electron reduction of molecular oxygen respectively. These two ROS molecules serve as precursors of other ROS through a cascade of reactions catalysed by a host of antioxidant enzymes. Superoxide is unstable in aqueous environment and is rapidly dismutated by superoxide dismutase (SOD) to hydrogen peroxide. This in turn is detoxified by catalase and glutathione peroxidase into water and oxygen. 
Table 1.4: Reactive oxygen species

\section{Oxygen radicals Highly reactive non radicals}

$\begin{array}{ll}\text { Superoxide } & \text { Hydrogen peroxide } \\ \text { Hydroxyl } & \text { Hypochlorus acid } \\ \text { Carbonate } & \text { Fatty acid hydroperoxides } \\ \text { Peroxyl } & \text { Reactive aldehydes } \\ \text { Alkoxyl } & \text { Singlet oxygen }\end{array}$

The antioxidant enzymes and low molecular weight antioxidant molecules such as glutathione and ascorbate are responsible for maintaining ROS at normal physiological levels (Crawford and Davies 1994). Imbalance between ROS production and antioxidant defence leads to oxidative stress. This phenomenon is linked to various pathologies including cardiovascular diseases, hypertension, neurodegenerative diseases, cancer, inflammatory conditions and diabetes mellitus (Dhalla, Temsah et al. 2000; Sayre, Smith et al. 2001; Valko, Leibfritz et al. 2007). 


\subsection{REACTIVE OXYGEN SPECIES AND HYPERTENSION}

Patients with essential hypertension have higher levels of free radical production, lower NO availability, lower levels of vitamin E and SOD, and higher concentrations of lipid peroxidation product malondialdehyde than normotensive controls (Kumar and Das 1993; Armas-Padilla, Armas-Hernandez et al. 2007). Hypertension due to unilateral renal artery stenosis in pigs (Lerman, Nath et al. 2001) and chronic angiotensin II infusion (10 ng/ $/ \mathrm{kg} / \mathrm{min}$ ) in rats (Reckelhoff, Zhang et al. 2000) and pigs (Haas, Krier et al. 1999) was accompanied by increased $\mathrm{F}_{2}$-isoprostane, a product of arachidonic acid peroxidation. There was overproduction of aortic superoxide in spontaneously hypertensive rats (Wu, Millette et al. 2001) which could be prevented by tempol, an antioxidant, at the pre-hypertensive stage (Nabha, Garbern et al. 2005). Hypertensive Dahl saltsensitive rats displayed elevated urinary excretions of $F_{2}$-isoprostane and 8 hydroxy $2^{\prime}$ deoxyguanosine (another biomarker of oxidative stress) (Kushiro, Fujita et al. 2005), raised superoxide production in the mesenteric microvasculature detected by tetranitroblue tetrazolium dye and increased plasma hydrogen peroxide levels (Swei, Lacy et al. 1997). Additionally, experimental induction of oxidative stress in normotensive rats, by reduction of tissue glutathione synthase content with an inhibitor buthionine sulfoximine $(30 \mathrm{mmol} / \mathrm{L}$ drinking water for 2 weeks), led to hypertension (Vaziri, Wang et al. 2000).

ROS can result in hypertension through the loss of NO-mediated vasodilatation; sodium and water retention consequent on increased sodium reabsorption, 
glomerular damage and nephron loss; central stimulation of sympathetic outflow; and impaired carotid baroreceptor responses (Harrison, Gongora et al. 2007).

\subsection{REACTIVE OXYGEN SPECIES AND DEXAMETHASONE- INDUCED HYPERTENSION}

The concept that oxidative stress is important in the pathogenesis of DEX-HT emerged from a range of experimental evidence.

\subsubsection{Experimental evidence for dexamethasone-induced oxidative stress}

Iuchi et al. demonstrated that pre-treatment of human umbilical vein endothelial cells with DEX enhanced ROS generation in a time and dose-dependent manner (Iuchi, Akaike et al. 2003). They have also shown that patients with glucocorticoid excess have increased immunostaining of nitrotryrosine residue, a stable biomarker of NO radical-derived oxidants, in the skeletal muscle vasculature. However, immunohistochemical staining for nitrotyrosine in adrenal, thymus, kidney and liver tissues from DEX-induced hypertensive rats did not demonstrate any changes compared to controls (Tan, Schyvens et al. 2005).

In another study, bilateral adrenalectomy and treatment with the glucocorticoid antagonist RU486 resulted in attenuation of elevated hydroethidine staining while DEX injection in adrenalectomised rats restored the oxidative reaction of hydroethidine (Suzuki, Swei et al. 1995). 
Lucigenin-enhanced chemiluminescence is widely used for the study of oxidative stress in various animal models of cardiovascular diseases (Rajagopalan, Kurz et al. 1996; Dobrian, Schriver et al. 2001; Wu, Millette et al. 2001; Paravicini, Gulluyan et al. 2002). Ex vivo analysis of aorta from DEX-induced hypertensive rats showed increased $\mathrm{NAD}(\mathrm{P}) \mathrm{H}$-activated lucigenin-enhanced chemiluminescence (Mondo, Yang et al. 2006a). These authors have also demonstrated increased plasma superoxide concentration measured using the Fenton reaction method in DEX-induced hypertensive rats (Mondo, Yang et al. 2006).

Because of the limitations associated with direct detection of reactive oxygen and nitrogen species in clinical settings, biomarkers of oxidative modifications, which are more stable, have been used to assess physiological and pathological redox states (Dalle-Donne, Rossi et al. 2006). Among the biomarkers, F2-isoprostanes, prostaglandin $\mathrm{F}_{2 \alpha}$-like compounds, are reliable markers of oxidative stress and free radical-catalysed lipid peroxidation of esterified arachidonic acid (DalleDonne, Rossi et al. 2006). Plasma $F_{2}$-isoprostane was increased in DEX-HT in rats (Zhang, Croft et al. 2004; Miao, Zhang et al. 2007).

To date, there is no convincing evidence for increased ROS production in DEXHT in humans. There was, however, some evidence implicating oxidative stress in endogenous GC-HT in humans. Iuchi et. al. demonstrated increased vascular production of peroxynitrite (through immunostaining of nitrotyrosine residues in vascular tissues) in a patient with HT associated with Cushing's syndrome (Iuchi, 
Akaike et al. 2003), but cortisol treatment in healthy humans did not result in a significant increase in urinary $\mathrm{F}_{2}$-isoprostane excretion. (Kelly JJ, Mangos $\mathrm{GJ}$ and Whitworth JA, unpublished data)

\subsubsection{Effect of antioxidants on dexamethasone-induced hypertension}

In rats, hypertension induced by DEX was reversed and prevented by tempol, a superoxide scavenger (Zhang, Croft et al. 2004). Additionally, drugs with antioxidant properties such as $\mathrm{N}$-acetylcysteine (Krug, Zhang et al. 2008), atorvastatin (Mondo, Yang et al. 2006; Mondo, Yang et al. 2006a) and folic acid (Miao, Zhang et al. 2007) ameliorated DEX-HT in rats suggesting a role for oxidative stress (see below).

\subsubsection{Tempol}

Improvement in DEX-HT following tempol treatment was not accompanied by reduction in $\mathrm{F}_{2}$-isoprostane levels (Zhang, Croft et al. 2004). Tempol, a membrane permeable antioxidant, may modify intracellular ROS which are not detectable by plasma $\mathrm{F}_{2}$-isoprostane concentrations.

\subsubsection{N-Acetylcysteine}

The hypotensive effect of N-acetylcysteine may be mediated by increase in NOmediated vasodilatation (Girouard, Chulak et al. 2003), antioxidant properties via replenishing intracellular cysteine required for the synthesis of tissue glutathione (Ruffmann and Wendel 1991), and down-regulation of NAD(P)H oxidase 
activities and expression (Zhang, Fujii et al. 2004). In DEX-treated rats, Nacetylcysteine partially prevented the development but had no effect on established hypertension (Krug, Zhang et al. 2008).

\subsubsection{Atorvastatin}

Atorvastatin given orally at $50 \mathrm{mg} / \mathrm{kg} /$ day for 14 days prevented DEX-HT in rats (Mondo, Yang et al. 2006). Accompanying this blood pressure response, DEXinduced hypertensive rats on atorvastatin also showed suppressed aortic superoxide production, measured using the lucigenin-enhanced chemiluminescence assay; upregulation of eNOS mRNA and improved acetylcholine-induced vasodilatation (Mondo, Yang et al. 2006). This study showed that hypertension due to DEX is in part mediated by oxidative stress.

\subsubsection{Antioxidant vitamins}

Folic acid supplementation prevented and partially reversed DEX-HT in the rat (Miao, Zhang et al. 2007). This antihypertensive effect of folic acid was not accompanied a significant decrease in plasma homocysteine, increase in tetrahydrobiopterin $\left(\mathrm{BH}_{4}\right)$ or decreased $\mathrm{F}_{2}$-isoprostane concentrations (Miao, Zhang et al. 2007). However, the possibility of a decrease in intracellular ROS by folic acid has not been excluded. There are no published data on the effects of antioxidant vitamins such as vitamins $\mathrm{C}$ and $\mathrm{E}$ in DEX-HT. 


\subsubsection{Altered expression and activity of antioxidant enzymes}

Changes in the availability and activity of endogenous antioxidant enzymes, such as SODs, catalase, thioredoxin reductase and GSH-related enzymes, are seen in response to oxidative stress (Wassmann, Wassmann et al. 2004). Rajashree and Puvanakrishnan found that hypertensive DEX-treated rats had increased tissue markers of lipid peroxidation (thiobarbituric acid reactants), decreased catalase and SOD levels in the heart but not in the kidney (Rajashree and Puvanakrishnan 1999). They also demonstrated a decrease followed by a subsequent increase in glutathione S-transferase and glutathione peroxidase activity with DEX treatment (Rajashree and Puvanakrishnan 1999). Furthermore, oxidative stress induced by DEX in veal calves was associated with activation of glutathione peroxidase (Carletti, Cantiello et al. 2007). It is likely that there is an element of a compensatory induction, at transcriptional level, of antioxidant protein and enzymes in response to exposure to ROS (Crawford and Davies 1994). 


\subsubsection{Inhibition of superoxide-generating enzymes}

ROS reduction by inhibition of the different superoxide synthetic pathways has provided further evidence for a role for ROS in DEX-HT in the rat. (See below.)

\subsection{ROLE OF VASCULAR ROS IN GC-HT}

There is increasing evidence implicating vascular ROS in the pathogenesis of hypertension (Touyz and Schiffrin 2004; Harrison, Gongora et al. 2007). Superoxide is produced first and through a cascade of catalytic processes, other ROS are generated. Although a variety of enzymes contribute to production of superoxide, the predominant sources in the vasculature are $\mathrm{NAD}(\mathrm{P}) \mathrm{H}$ oxidase, xanthine oxidase, uncoupled eNOS, and mitochondria (Cai and Harrison 2000). (Figure 1.6) 


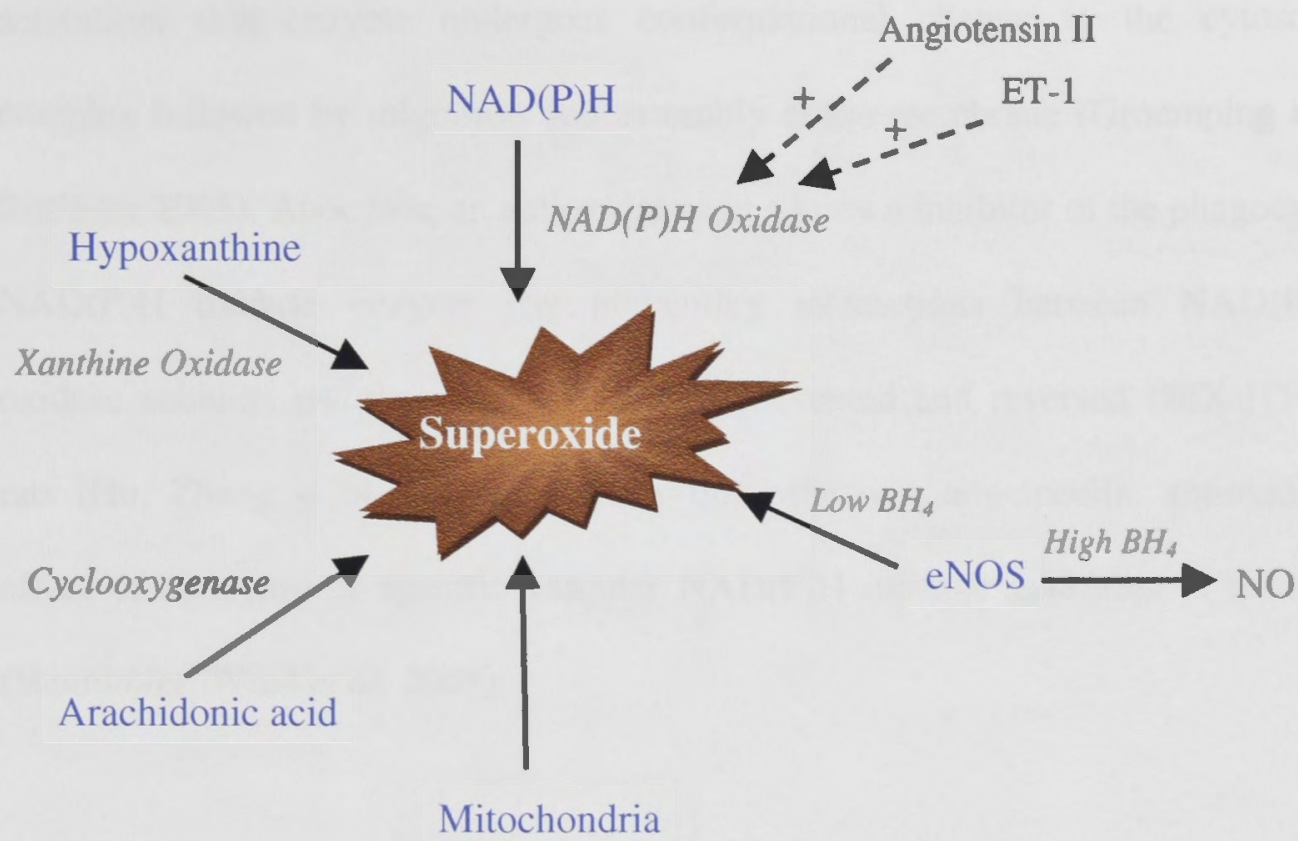

Figure 1.6: Major sources of superoxide in the vasculature. Ang II: angiotensin II, $\mathrm{BH}_{4}$ : tetrahydrobiopterin, ET-1: Endothelin-1, NAD(P)H: reduced nicotinamine adenine dinucleotide phosphate, NO: nitric oxide.

\subsubsection{NAD(P)H oxidase}

$\mathrm{NAD}(\mathrm{P}) \mathrm{H}$ oxidase is one of the major producers of vascular superoxide, expressed in all layers of the blood vessel wall (Pagano, Ito et al. 1995; Pagano, Clark et al. 1997; Wang, Pagano et al. 1998; Sorescu, Weiss et al. 2002). It is a multimeric enzyme composed of a membrane-bound catalytic domain (gp91phox/Nox2, Nox1 or Nox4), regulatory cytosolic subunits (p22phox, p47phox, p67phox, p40phox) and low molecular weight G-protein (Rac 1 or Rac

2) (Bengtsson, Gulluyan et al. 2003; Lassegue and Clempus 2003). Upon 
activation, this enzyme undergoes conformational change in the cytosolic complex followed by migration and assembly at the membrane (Groemping and Rittinger 2005). Apocynin, an antioxidant and a known inhibitor of the phagocytic $\mathrm{NAD}(\mathrm{P}) \mathrm{H}$ oxidase enzyme (by preventing interactions between $\mathrm{NAD}(\mathrm{P}) \mathrm{H}$ oxidase subunits $\mathrm{p} 47 \mathrm{phox}$ and gp9lphox), prevented and reversed DEX-HT in rats (Hu, Zhang et al. 2006). Whether this reflects a non-specific antioxidant effect of apocynin or specific vascular $\mathrm{NAD}(\mathrm{P}) \mathrm{H}$ oxidase inhibition is unclear (Heumuller, Wind et al. 2008).

Superoxide production by $\mathrm{NAD}(\mathrm{P}) \mathrm{H}$ oxidase can be stimulated by angiotensin II (Figure 1.6). In an in vitro study, incubation of vascular smooth muscle cells with angiotensin II resulted in increased superoxide production, largely mediated by activation of both $\mathrm{NADH}$ and $\mathrm{NAD}(\mathrm{P}) \mathrm{H}$ oxidases (Griendling, Minieri et al. 1994). The angiotensin II ATl receptor antagonist losartan $(25 \mathrm{mg} / \mathrm{kg} / \mathrm{day}$, in drinking water for 2 days) significantly decreased vascular superoxide production, as assessed by lucigenin-enhanced chemiluminescence, in angiotensin II-induced hypertension in rats. This demonstrated a role for the angiotensin type 1 receptor in $\mathrm{NAD}(\mathrm{P}) \mathrm{H}$ oxidase stimulated superoxide production. In our laboratory, treatment with losartan at a dose known to fully prevent ACTH-HT, partially prevented and reversed DEX-HT in rats (He 2008).

Further examination of the role of $\mathrm{NAD}(\mathrm{P}) \mathrm{H}$ oxidase enzyme using other strategies such as $\mathrm{NAD}(\mathrm{P}) \mathrm{H}$ oxidase subunit knockout mice, will be necessary to confirm its role in DEX-HT. 


\subsubsection{Xanthine oxidase}

Xanthine oxidase catalyses the oxidation of hypoxanthine and xanthine to uric acid, with superoxide formed as by-products. Whilst this superoxide-generating pathway is implicated in the development of hypertension in spontaneously hypertensive (Nakazono, Watanabe et al. 1991; Suzuki, DeLano et al. 1998) and Dahl salt-sensitive rats (Swei, Lacy et al. 1999), it has been shown not to be the major source of superoxide in ACTH-HT in the rat as the xanthine oxidase inhibitor allopurinol (200 mg/kg/day, given orally) failed to prevent and reverse ACTH-HT despite lowering plasma uric acid (Zhang, Chan et al. 2005). In contrast, allopurinol $(0.4 \mathrm{mg} / \mathrm{mL}$ drinking water, approximately $45 \mathrm{mg} / \mathrm{kg} /$ day $)$ was shown to be effective in lowering mean blood pressure in high dose DEXinduced hypertensive rats ( $0.5 \mathrm{mg} / \mathrm{kg} /$ day) (Wallwork, Parks et al. 2003). One of the limitations of this study was the use of a high dose DEX-HT model. High DEX dose is known to result in significant weight loss due to plasma volume contraction and skeletal muscle catabolism (Tonolo, Fraser et al. 1988); and consequently activate the renin-angiotensin-aldosterone system, increase endogenous glucocorticoid levels (Stricker, Vagnucci et al. 1979) and suppress ANP production (Sebaai, Lesage et al. 2002). Further clarification of the role of xanthine oxidase inhibition in the prevention of DEX-HT, using a much lower DEX dose so as to minimise these confounding effects is necessary. 


\subsubsection{Uncoupling of eNOS}

Fully-reduced $\mathrm{BH}_{4}$ serves as an important cofactor for endothelial nitric oxide synthase (eNOS) which catalyses the conversion of L-arginine to L-citrulline and NO (Figure 1.5). Decreased $\mathrm{BH}_{4}$ availability, which promotes eNOS uncoupling, leads to the formation of superoxide instead of NO (Vasquez-Vivar, Kalyanaraman et al. 1998).

DEX-HT (5 mg subcutaneous DEX pellet delivering approximately $0.79 \mathrm{mg} / \mathrm{kg} /$ day) in the rat is associated with downregulation of vascular GTP cyclohydrolase I, the rate-limiting enzyme for de novo biosynthesis of $\mathrm{BH}_{4}$ (Mitchell, Dorrance et al. 2003). Abnormal endothelium-dependent vasorelaxation in the aortic rings of the DEX-induced hypertensive rats was corrected by sepiapterin, a $\mathrm{BH}_{4}$ donor (Mitchell, Dorrance et al. 2003). However, in vivo administration of $\mathrm{BH}_{4}$ failed to prevent $\mathrm{DEX}-\mathrm{HT}(10 \mu \mathrm{g} / \mathrm{rat} /$ day, given subcutaneously) in the rat despite appropriate increases in plasma biopterin levels (Miao, Zhang et al. 2007).

In a $\mathrm{BH}_{4}$-free environment, L-arginine did not inhibit superoxide production but rather, augmented superoxide generation that could only be reversed by the addition of reduced $\mathrm{BH}_{4}$ (Vasquez-Vivar, Martasek et al. 2002). L-Arginine supplementation did not prevent, reverse or exacerbate DEX-HT (10 $\mu \mathrm{g} / \mathrm{rat} / \mathrm{day}$, given subcutaneously) in the rat ( $\mathrm{Li}$, Fraser et al. 1997). As eNOS is downregulated by DEX treatment (Wallerath, Witte et al. 1999; Schafer, 
Wallerath et al. 2005), its involvement in superoxide production via its uncoupled state is unlikely to be significant. Results from recent studies using in vivo sepiapterin (which increases $\mathrm{BH}_{4}$ production via a salvage pathway involving the conversion of sepiapterin to dihydrobiopterin and then, to $\mathrm{BH}_{4}$ ) and the NOS inhibitor N-nitro-L-arginine (which minimises eNOS acitivity and thus, eNOS uncoupling) in DEX-induced hypertensive rats were consistent with this proposal (Thida, Earl et al. 2010). Sepiapterin did not prevent or reverse DEX-HT in rats despite significantly increased total plasma biopterin concentrations (Thida, Earl et al. 2010). N-Nitro-L-arginine did not relieve DEX-HT but exacerbated it (Thida, Earl et al. 2010).

These findings showed that oxidative stress due to eNOS uncoupling does not play a significant role in the pathogenesis of DEX-HT.

\subsubsection{Mitochondria}

Whilst the main function of mitochondria is to produce adenosine triphosphate via electron transfer in the electron transport chain resulting in reduction of oxygen to water, it also results in superoxide formation in the event of electron leakage within this chain. Complex I and III are the primary source of superoxide within the mitochondria. Approximately $0.2-2 \%$ of oxygen used by the mitochondria is reduced to superoxide in this electron transport chain (Madamanchi and Runge 2007). As a charged radical, superoxide produced within the mitochondria does not diffuse across the mitochondrial membrane into the cytosol readily (Han, 
Antunes et al. 2003). It exits the organelle through voltage dependent anion channels and more commonly, as membrane-permeable hydrogen peroxide following its dismutation by intermembrane $\mathrm{Cu}, \mathrm{Zn}$-superoxide dismutase (Han, Antunes et al. 2003).

Apart from being an important source of superoxide, the mitochondrion is also a target of oxidative injury (Madamanchi and Runge 2007). Oxidative damage to the mitochondria is known to damage mitochondrial DNA, compromise oxidative phosphorylation potentials, decrease energy production and lead to additional production of mitochondrial reactive oxygen species (Madamanchi and Runge 2007). Mitochondrial DNA damage is increasingly recognised as an important etiological factor in cardiovascular disease including hypertension (Ramachandran, Levonen et al. 2002; Ballinger 2005).

Mitochondrial superoxide overproduction has also been implicated in the pathogenesis of hypertension in some models. El Midaoui et al. demonstrated that hypertension in rats treated chronically with glucose is associated with increased mitochondrial superoxide production (El Midaoui, Elimadi et al. 2003). In these rats, oral supplementation of alpha-lipoic acid $(500 \mathrm{mg} / \mathrm{kg}$ rat chow) blunted the increase in cardiac mitochondrial superoxide and prevented hypertension. Despite this observation, the sequential relationship between these factors remains unclear. It was also uncertain whether the blood pressure lowering effect was due to the antioxidant properties of alpha-lipoic acid or the direct result of a lowered mitochondrial superoxide level. The role of mitochondrial-derived superoxide 
and the effect of alpha-lipoic acid on DEX-HT have not been examined previously.

\subsubsection{Cyclooxygenase}

Endothelial cyclooxygenase is involved in regulation of vascular tone, endothelial function and pressor reactivity to agonists. Arachidonic acid metabolism by endothelial cyclooxygenase is a source of superoxide anions as demonstrated in studies on cerebral arteries in cats and dogs (Kontos, Wei et al. 1985; Cosentino, Sill et al. 1994).

The role of cyclooxygenase-related superoxide overproduction in DEX-HT has not been fully evaluated. Aspirin, a non-selective cyclooxygenase inhibitor with antioxidant properties, tended to prevent DEX-HT $(P<0.07)$ in rats but failed to reverse it (Zhang, Miao et al. 2007). This modest effect could be due to its nonspecific antioxidant properties or aspirin-induced stimulation of NO production via eNOS acetylation (Taubert, Berkels et al. 2004) and increased cyclic GMP (Grosser and Schroder 2003) rather than inhibition of cyclooxygenase-related oxidative stress. Furthermore, indomethacin treatment potentiated the hypertensive effect of low dose DEX $(0.1 \mathrm{mg} / \mathrm{kg} /$ day, given orally $)$ in dogs (Nakamoto, Suzuki et al. 1992). However, the extent of oxidative stress was not evaluated in this study. The hypertensive response could be a consequence of relative imbalance of vasodilator and vasoconstrictor prostaglandins from the DEX and indomethacin doses used. 
Whether oxidative stress due to cyclooxygenase pathway has a role in DEX-HT remains to be determined.

\subsubsection{Summary}

There is a body of evidence implicating oxidative stress in the pathogenesis of DEX-HT. DEX-HT is associated with raised plasma $\mathrm{F}_{2}$-isoprostane and lucigeninenhanced chemiluminescence. In rats, DEX-HT is prevented and reversed by antioxidants tempol, apocynin, folic acid, $\mathrm{N}$-acetylcysteine and aspirin. Oxidative stress in DEX-HT has been shown to involve the NAD(P)H oxidase pathway but not uncoupled eNOS. The role of xanthine oxidase-stimulated superoxide production in DEX-HT needs further evaluation. The role superoxide produced by the mitochondria and cyclooxygenase in DEX-HT has not been evaluated.

\subsection{DIFFERENCES BETWEEN NATURALLY-OCCURING AND SYNTHETIC GLUCOCORTICOID-INDUCED HYPERTENSION}

DEX, the most potent synthetic glucocorticoid, is commonly used for study of the generic effects of glucocorticoid in different disease states and in various biological pathways. DEX however exhibits some features that are different from those of naturally-occurring glucocorticoids. The similarities and differences between DEX- and ACTH-HT are summarised below. ACTH-HT is explicable in terms of ACTH-stimulated cortisol (Whitworth, Saines et al. 1984; Connell, 
Whitworth et al. 1987) and corticosterone (Mangos, Turner et al. 2000) secretions in humans and rats, respectively.

\subsubsection{Similarities}

\subsubsection{Rapid onset}

Development of GC-HT, due to both naturally-occurring and synthetic glucocorticoid hormones, is rapid. Hypertension due to oral cortisol at supraphysiological doses ( 80 and $200 \mathrm{mg} /$ day) is evident within 24 hours with the peak blood pressure occurring at day 4 or 5 of treatment in humans (Connell, Whitworth et al. 1987; Kelly, Tam et al. 1998). ACTH administration produces rapid onset of hypertension in sheep (Scoggins, Allen et al. 1979), rats (Turner, Wen et al. 1998) and humans (Connell, Whitworth et al. 1987; Whitworth 1992). Similarly, DEX raises blood pressure in rats $(10 \mu \mathrm{g} / \mathrm{rat} /$ day, subcutaneously) (Zhang, Croft et al. 2004; Hu, Zhang et al. 2006; Krug, Zhang et al. 2008), dogs $(0.5 \mathrm{mg} / \mathrm{kg} / \mathrm{day}$, orally) (Nakamoto, Suzuki et al. 1991; Nakamoto, Suzuki et al. 1992) and humans (3 mg/day, orally) (Pirpiris, Sudhir et al. 1992) within 1-2 days.

\subsubsection{No requirement for salt loading or volume expansion}

In both forms of GC-HT, salt loading and volume expansion are not prerequisites for their hypertensinogenic effects. Excess sodium is not required for the development of GC-HT. DEX raises blood pressure independent of sodium and intravascular volume expansion in man (Whitworth, Gordon et al. 1989). 
Likewise, ACTH-HT is not entirely dependent on this mechanism even though sodium excess can magnify its hypertensive response in both man and sheep (Humphrey, Fan et al. 1983; Whitworth, Saines et al. 1985). Furthermore, dietary sodium restriction $(15 \mathrm{mmol} /$ day $)$ did not prevent ACTH-HT $(1 \mathrm{mg} /$ day, given intramuscularly) in man despite limiting the mineralocorticoid effects (Connell, Whitworth et al. 1988). Cortisol-induced hypertension developed in sheep following sodium depletion (urinary sodium loss of $603 \pm 49 \mathrm{mmol}$ ) although the extent of the increase was less than that in control sheep (Mills, Coghlan et al. 1986).

Cortisol, at maximal physiological doses, exhibits mineralocorticoid activity. However, spironolactone blocked the mineralocorticoid effects of cortisol without preventing the blood pressure rise (Williamson, Kelly et al. 1996). In addition, the administration of intravenous deoxycorticosterone (1 mg/day or $40 \mu \mathrm{g} / \mathrm{hour})$ in man for 5 days reproduced the antinatriuretic properties of cortisol without the hypertensive effects (Whitworth, Saines et al. 1984).

\subsubsection{Nitric oxide-redox imbalance}

As discussed in the earlier sections, both DEX- and ACTH-HT are associated with increased superoxide production and decreased NO bioavailability.

Plasma biomarkers of oxidative stress and NO availability: Plasma $\mathrm{F}_{2^{-}}$ isoprostanes, reliable markers of lipid peroxidation and systemic oxidative stress, 
were elevated in both ACTH- (Zhang, Jang et al. 2003; Zhang, Chan et al. 2005; Miao, Zhang et al. 2007) and DEX-HT in rats (Zhang, Croft et al. 2004; Miao, Zhang et al. 2007). Similarly, plasma reactive nitrogen intermediates (nitrate/nitrite), a marker of NO availability, are reduced in cortisol- (Kelly, Tam et al. 1998), corticosterone-(Mangos, Turner et al. 2000), ACTH- (Wen, Li et al. 2000; Turner, Mangos et al. 2001) and DEX-HT (Wallerath, Godecke et al. 2004; Mondo, Yang et al. 2006; Mondo, Yang et al. 2006a; Miao, Zhang et al. 2007).

Responses to antioxidants and $\mathrm{BH}_{4}$ supplementation: Both ACTH- and DEX-HT were prevented and reversed by antioxidants: folic acid (Miao, Zhang et al. 2007), N-acetylcysteine (Mondo, Zhang et al. 2006; Krug, Zhang et al. 2008) and superoxide scavenger tempol (Zhang, Jang et al. 2003; Zhang, Croft et al. 2004). Hypertension due to both ACTH and DEX was prevented and reversed by apocynin (Zhang, Chan et al. 2005; Hu, Zhang et al. 2006) implicating a role for superoxide-generating $\mathrm{NAD}(\mathrm{P}) \mathrm{H}$ oxidase in this condition. Oxidative stress in these models of hypertension was not due to uncoupling of eNOS as hypertension was not prevented by administration of $\mathrm{BH}_{4}$ (Zhang, Pang et al. 2004; Miao, Zhang et al. 2007).

\subsubsection{Differences}

Despite the evidence implicating oxidative stress and NO-superoxide imbalance, blood pressure responses to treatments known to modify the synthesis of NO and superoxide were variable. 


\subsubsection{Response to $L$-arginine}

As discussed earlier, L-arginine deficiency is not a characteristic of DEX-HT. LArginine treatment $(500 \mathrm{mg} / \mathrm{kg} /$ day) increased plasma nitrate/nitrite (NOx) concentrations but failed to prevent hypertension in DEX-treated rats ( $\mathrm{Li}$, Fraser et al. 1997; Hu, Zhang et al. 2006). In contrast, ACTH-HT is associated with decreased plasma L-arginine, L-citrulline and NOx concentrations (Wen, Li et al. 2000). Established ACTH-HT was prevented and partially reversed by L-arginine, but not D-arginine supplementation (Turner, Wen et al. 1996; Wen, Li et al. 2000).

The blood pressure lowering effect of L-arginine in corticosterone- and ACTHHT is largely due to restoration of NO by increased availability of NOS substrate as increases in blood pressure with both corticosterone- and ACTH treatments were prevented by L-arginine but not D-arginine (Turner, Wen et al. 1996; Mangos, Turner et al. 2000); and co-administration of L-arginine and the competitive NOS inhibitor, N-nitro L-arginine negated the blood pressure lowering effect of L-arginine in ACTH-HT (Wen, Li et al. 2000a).

\subsubsection{Urinary 20-hydroxyeicosatetraenoic acid}

ACTH-HT, but not DEX-HT, is associated with increased urinary 20hydroxyeicosatetraenoic acid excretion (Zhang, Hu et al. 2008). 
20-Hydroxyeicosatetraenoic acid (20-HETE) is a cytochrome P450-derived arachidonic acid metabolite that has potent vasoconstrictive properties. Synthesis of 20-HETE can be inhibited by NO (Roman 2002). Overproduction of 20-HETE in ACTH-HT might be due to NO deficiency although this does not explain the findings in DEX-HT.

\subsubsection{Response to glucocorticoid receptor antagonism}

Dihydroepiandrosterone, an endogenous steroid with anti-glucocorticoid activity, prevented DEX-HT (Shafagoj, Opoku et al. 1992) but not ACTH-HT (Li, Wen et al. 1996).

DEX-HT was prevented by the glucocorticoid antagonist RU486 at a dose (50 mg subcutaneous pellet) that did not reverse weight loss (Kalimi 1989). With ACTHHT, a dose of RU486 (70 mg/kg every third day) that reversed weight loss did not modify blood pressure ( $\mathrm{Li}$, Wen et al. 1999). Partial prevention of the blood pressure rise was seen with high dose RU486 (70 mg/kg/day) ( $\mathrm{Li}$, Wen et al. 1999).

These differences suggest glucocorticoid receptor activation is more predominant in DEX-HT. 


\subsubsection{Response to vasopressin antagonism}

Vasopressin antagonism with the AVP $V_{1}$ receptor antagonist [1-( $\beta$-mercapto- $\beta, \beta$ cyclopentamethylene propionic acid) 2-(O-methyl)-Tyrosine]-AVP significantly decreased mean arterial pressure in DEX-induced hypertensive rats but not in saline-treated control rats (Iijima and Malik 1988). Conversely, ACTH-HT was not modified by either acute or chronic inhibition of the AVP $V_{\text {la }}$ receptor using OPC 21268 (Fraser, Turner et al. 2000) at a dose proven to be effective in blocking $V_{1}$ receptors (Burrell, Phillips et al. 1994).

\subsubsection{Response to aspirin}

Aspirin, a non-selective cyclooxygenase inhibitor and an antioxidant, prevented and partially reversed ACTH-HT but not DEX-HT in rats (Zhang, Miao et al. 2007). The postulated antihypertensive mechanisms of aspirin in the ACTH-HT model include inhibition of cyclooxygensase- and NAD(P)H oxidase-mediated superoxide production, decrease in superoxide-mediated NO inactivation, increase in NO production and increase in cyclic GMP availability. The reason for the differences between DEX- and ACTH-HT is unclear.

\subsubsection{Response to neomycin}

Neomycin attenuates the blood pressure rise in rats following $\mathrm{ACTH}$, corticosterone or prednisolone but not DEX (Fraillon, Wynne et al. 1984). It remains unclear whether the blood pressure changes were due to altered patterns of enterohepatic handling and metabolism or the intrinsic properties of neomycin. 


\subsubsection{Summary}

The observed differences between naturally-occuring GC-HT and DEX-HT suggest that hypertension due to these glucocorticoids involves different pathophysiological perturbations of NO-redox imbalance.

\subsection{SUMMARY- MECHANISMS OF DEXAMETHASONE-INDUCED HYPERTENSION}

There is convincing evidence indicating that sodium retention with a resultant volume expansion is not a mechanism of DEX-HT. Whilst the major cause for DEX-HT remains unclear, there is a body of evidence implicating nitric oxideredox imbalance, in part, due to increased vascular superoxide production. The likely source of superoxide is the $\mathrm{NAD}(\mathrm{P}) \mathrm{H}$ oxidase pathway. DEX-HT is also associated with increased total peripheral resistance and heightened pressor response to vasocontrictors but not raised sympathetic activity. The roles of endogenous vasoconstrictors and vasodilators in the pathogenesis of DEX-HT are variable, with possible links to NO, prostanoids, angiotensin II, arginine vasopressin, endothelins, catecholamines, neuropeptide $\mathrm{Y}$ and atrial natriuretic peptide. 


\subsection{PROJECT HYPOTHESES}

The hypotheses of this project were that:

1. increase in TPR will be essential to raise blood pressure with DEX.

2. there is ROS overproduction from mitochondria in GC-HT and hypertension can be prevented and reversed by inhibiting mitochondrial superoxide production using alpha-lipoic acid.

3. allopurinol, a xanthine oxidase inhibitor, can prevent and reverse DEX-HT by means of ROS reduction.

4. propranolol, a $\beta$-adrenergic receptor blocker and lipid peroxidase inhibitor, can prevent GC-HT.

5. DEX-HT is associated with increased production of 20-HETE and specific inhibition of 20-HETE production will reverse DEX-HT.

\subsection{GENERAL AIMS OF THIS PROJECT}

The main aim of this project was to evaluate the mechanisms of DEX-HT, namely the haemodynamic mechanisms, the role of oxidative stress and NO deficiency. 
The role of $\beta$-adrenergic receptor blockade in DEX-HT and metabolic effects of DEX in rats were also evaluated. In some experiments, evaluations of both DEXand ACTH-HT were conducted for comparison purposes.

\subsection{SPECIFIC AIMS OF THIS PROJECT}

This project comprised 7 studies (Table 1.5).

Two studies examined the haemodynamic mechanisms of DEX-HT. The first evaluated the haemodynamic characteristics of DEX-HT in the rat (Chapter 3). This was followed by evaluation of the role of TPR and conductance in DEX-HT (Chapter 4). The roles of mitochondrial superoxide (Chapters 5 and 6) and lipid peroxidation (Chapter 8) were evaluated in both DEX- and ACTH-HT. In Chapter 7, the role of xanthine oxidase, another source of reactive oxygen species, was examined in DEX-HT. The role of $\beta$-adrenergic activity (Chapter 8) and 20HETE (Chapter 9) in the pathogenesis of DEX-HT were also evaluated. Metabolic profiles of DEX-induced hypertensive rats were documented in Chapter 10. 
Table 1.5: Studies in this project

\section{HAEMODYNAMICS}

Chapter 3: Haemodynamic profile of DEX-HT in the rat

Chapter 4: The role of total peripheral resistance and

conductance in DEX-HT in the rat

\section{OXIDATIVE STRESS}

Chapters 5 \& 6: Role of mitochondrial superoxide in GC-HT in the rat

Chapter 7: Role of xanthine oxidase in DEX-HT in the rat

Chapter 8: The effects of adrenergic receptor antagonism and lipid peroxidation inhibition using propranolol in GC-HT in the rat

\section{NITRIC OXIDE DEFICIENCY}

Chapter 9: Role of 20-hydroxyeicosatetraenoic acid in DEX-HT in the rat

\section{OTHER MECHANISMS/ OBSERVATIONS}

The role of adrenergic receptors- as in Chapter 8 above

Chapter 10: Metabolic profiles of DEX-HT in rats 


\section{CHAPTER 2}

Materials and Methods 


\section{$2.1 \quad$ ETHICS}

This project was approved by the Animal Experimentation Ethics Committee (Approval numbers: J.HB.17.03, J.HB.20.05, J.HB.22.06 and J.HB.24.06).

\subsection{EXPERIMENTAL ANIMALS}

Rats are used in the study of GC-HT because of the relevance of this model to human GC-HT (Whitworth, Schyvens et al. 2001; Whitworth, Zhang et al. 2006).

Male Sprague-Dawley rats with an initial body weight of $180-280 \mathrm{~g}$ were used in this project (Figure 2.1). This outbred stain is one of the most widely used animals in experimental hypertension. Only male rats are used to minimise gender-related variability to the results. These rats were purchased from Animal Resource Centre, Perth. This certified facility produces and sells laboratory animals that are free from pathogens. (http://www.arc.wa.gov.au/overview.php) 


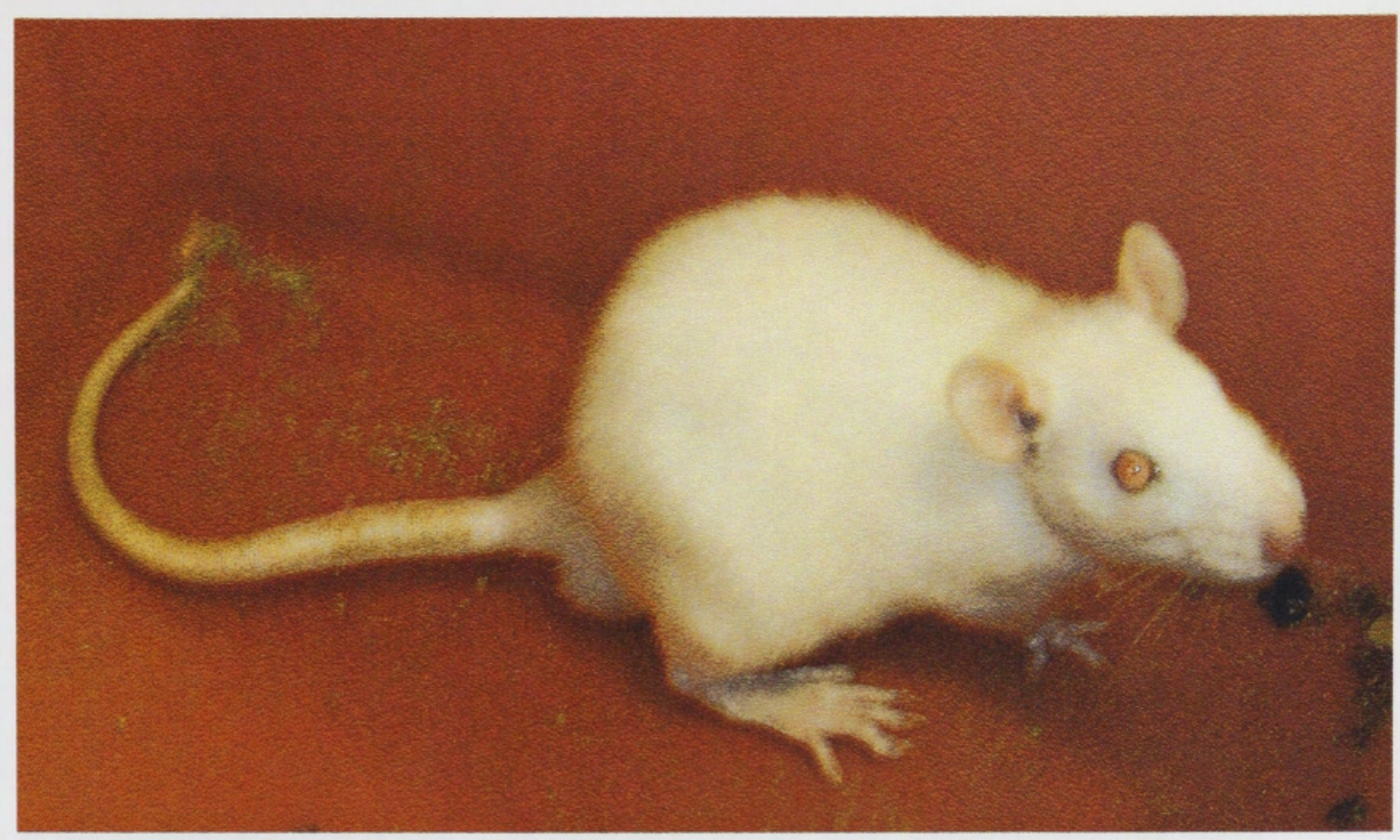

Figure 2.1: Male Sprague-Dawley albino rat

The rats were housed in a purpose-built laboratory in John Curtin School of Medical Research, Australian National University. The laboratory has ducted air conditioning $\left(21 \pm 1{ }^{\circ} \mathrm{C}\right)$ and operates 12-hour light/dark cycles. The rats were housed communally in groups of 2-3 per cage. An individually-ventilated caging system was used (Techniplast, Buguggiate, Italy) (Figure 2.2). These cages were made of transparent plastic and thus, allowed interaction and socialisation. The cages were lined with aspen wood chip bedding (Tapvei, Finland). The animals had free access to standard rat chow (Gordon's Specialty Stockfeeds Pty. Ltd., Yanderra, Australia) and tap water. 


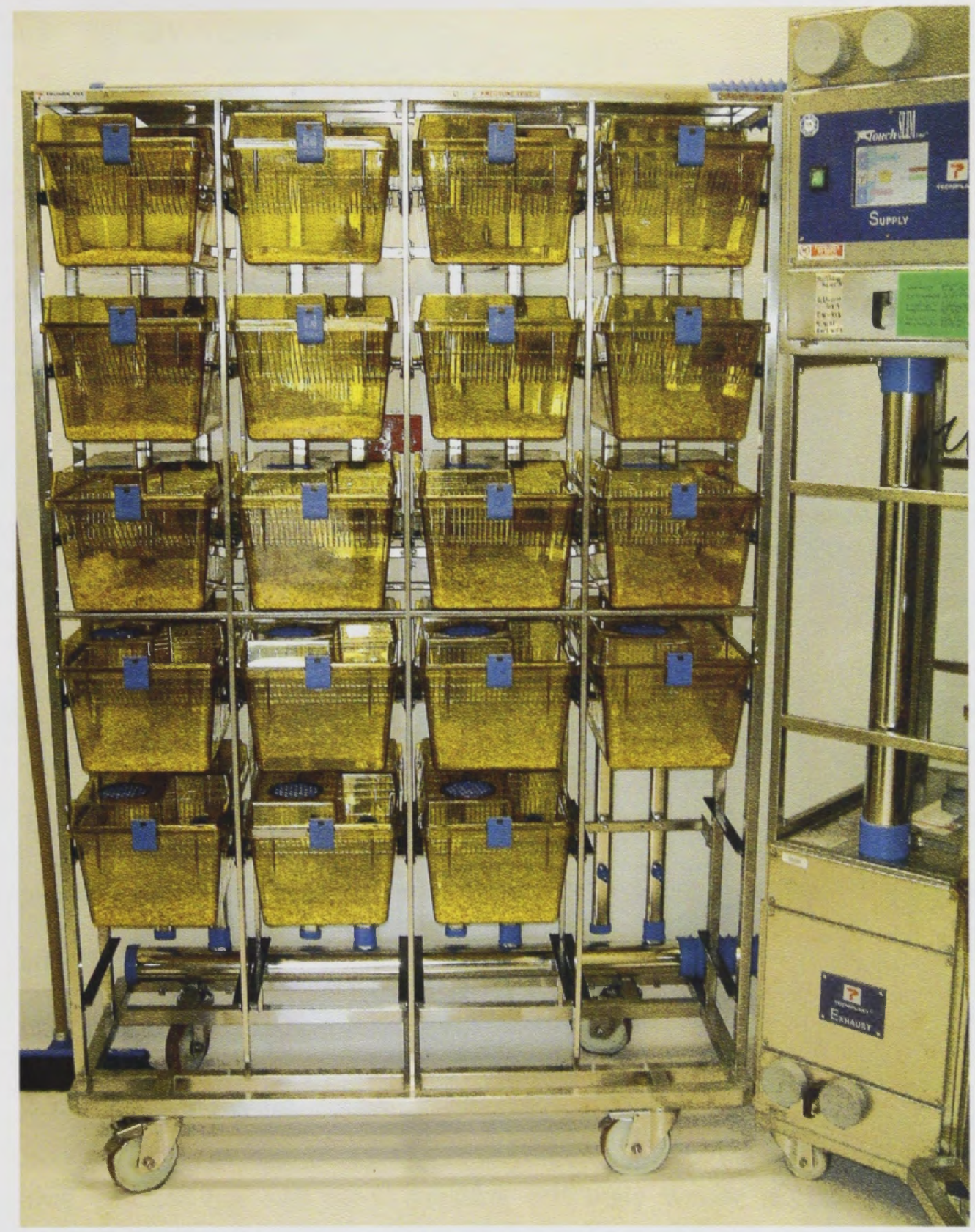

Figure 2.2: Individually-ventilated caging system 


\subsection{STUDY DESIGN}

This project consisted of 7 studies which followed a general experimental protocol for animal handling, treatment and tail cuff blood pressure measurement. There was inter-study variability with regard to the treatments, surgical procedures and assays.

The main experimental protocol consisted of 3 main phases- acclimatisation, control and treatment periods (Figure 2.3).

\subsubsection{Acclimatisation period}

The rats were allowed to familiarise with the environment, handling and blood pressure measuring equipment prior to any experimental procedure for 1-2 weeks after their arrival.

\subsubsection{Control period}

Two baseline tail cuff blood pressure and body weight measurements were obtained prior to drug administration on day C4 (4 days prior to treatment) and C2 ( 2 days prior to treatment). The prefix " $\mathrm{C}$ " represents control days. 


\section{Prevention Study}

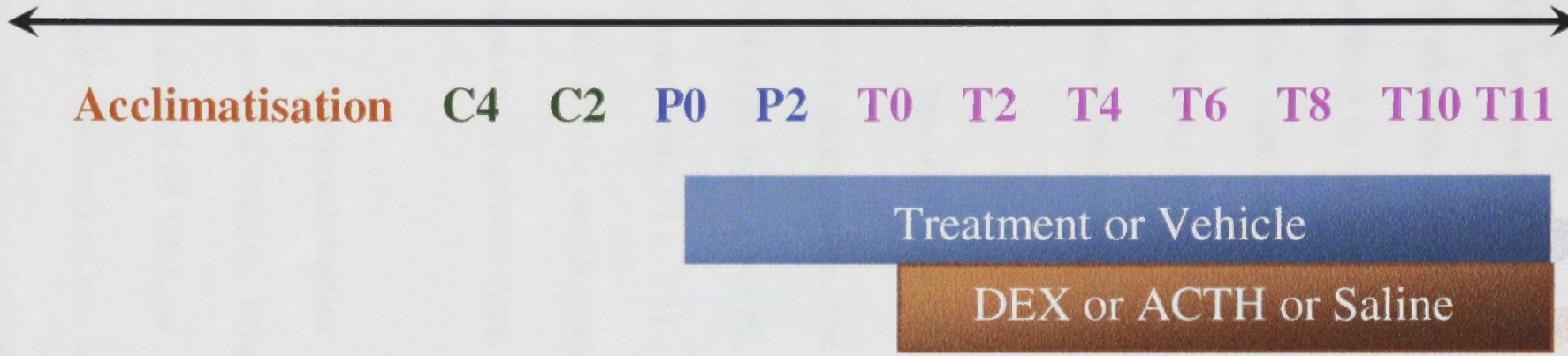

\section{Reversal Study}

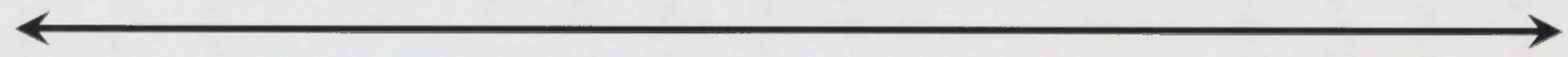

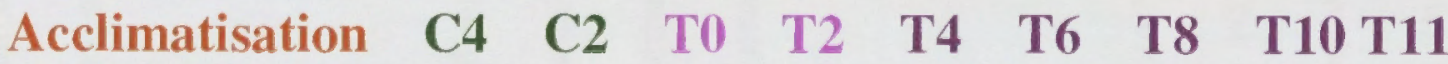

\section{DEX or ACTH or Saline}

Treatment or Vehicle 


\subsubsection{Treatment period}

GC-HT was produced by the administration of either DEX or ACTH (Section 2.4). In addition, several other treatments known to inhibit the pathways proposed to contribute to GC-HT were used to evaluate the mechanisms of GC-HT. These treatments were study-specific and are listed in Table 2.1. The timing and duration of drug administration were variable, and determined by whether it was a prevention or reversal study.

\subsubsection{Prevention studies}

In the prevention studies, the rats were pre-treated with the drugs listed on Table 2.1 for 4 days, from $\mathrm{P} 0$ to $\mathrm{P} 2$, prior to the administration of $\mathrm{ACTH}$ or DEX which were given from $\mathrm{T} 0$ to $\mathrm{T} 11$. The aim was to evaluate if the treatment of interest could prevent the development of GC-HT. The prefixes "P" and "T" denote pretreatment and treatment days, respectively.

\subsubsection{Reversal studies}

In the reversal studies, drug treatment was commenced four days after the commencement of DEX or ACTH. The aim was to evaluate the effect of the treatment of interest in rats with established hypertension due to glucocorticoid. Reversal studies were performed for a particular treatment only if a complete prevention was demonstrated. 
Table 2.1: Summary of studies and drug treatments

\begin{tabular}{|c|c|c|c|}
\hline Chapter & Study Title & $\begin{array}{l}\text { Saline/DEX/ } \\
\text { ACTH (s.c) }\end{array}$ & Other treatments \\
\hline 3 & $\begin{array}{l}\text { Haemodynamic profile of DEX-HT in } \\
\text { the rat }\end{array}$ & DEX/Saline & $\begin{array}{l}\text { Vehicle (Saline), } \\
\text { gavage }\end{array}$ \\
\hline 4 & $\begin{array}{l}\text { Role of total peripheral resistance and } \\
\text { conductance in DEX-HT in the rat }\end{array}$ & DEX/Saline & $\begin{array}{l}\text { Minoxidil or Vehicle } \\
\text { (Saline), gavage }\end{array}$ \\
\hline 5 & $\begin{array}{l}\text { Effects of GC on rat kidney } \\
\text { mitochondrial superoxide }\end{array}$ & $\begin{array}{l}\text { DEX/ACTH/ } \\
\text { Saline }\end{array}$ & Nil \\
\hline 6 & $\begin{array}{l}\text { Role of mitochondrial superoxide in } \\
\qquad \text { GC-HT in the rat }\end{array}$ & $\begin{array}{l}\text { DEX/ACTH/ } \\
\text { Saline }\end{array}$ & $\begin{array}{l}\text { Alpha lipoic acid or } \\
\text { Vehicle (Ground } \\
\text { food), p.o. }\end{array}$ \\
\hline 7 & $\begin{array}{l}\text { Role of xanthine oxidase in DEX-HT } \\
\text { in the rat }\end{array}$ & DEX/Saline & $\begin{array}{l}\text { Allopurinol or } \\
\text { Vehicle (Ground } \\
\text { food), p.o. }\end{array}$ \\
\hline 8 & $\begin{array}{c}\text { Effects of adrenergic receptor } \\
\text { antagonism and lipid peroxidation } \\
\text { inhibition using propranolol in GC-HT } \\
\text { in the rat }\end{array}$ & $\begin{array}{l}\text { DEX/ACTH/ } \\
\text { Saline }\end{array}$ & $\begin{array}{l}\text { Propranolol or } \\
\text { Vehicle (Ground } \\
\text { food), p.o. }\end{array}$ \\
\hline 9 & $\begin{array}{l}\text { Role of } 20 \text {-hydroxyeicosatetraenoic } \\
\text { acid in DEX-HT in the rat }\end{array}$ & DEX/Saline & $\begin{array}{l}\text { HET } 0016 \text { or Vehicle } \\
\text { (Lecithin), i.p. }\end{array}$ \\
\hline 10 & Metabolic profiles of DEX-HT in rats & DEX/Saline & $\begin{array}{l}\text { Vehicle (Saline), } \\
\text { gavage }\end{array}$ \\
\hline
\end{tabular}


During the control and treatment phases, indirect SBP readings were measured on alternate days using tail-cuff plethysmography (See Section 2.5.1).

\subsection{DRUG ADMINISTRATION PROTOCOL}

The preparation and administration of DEX and ACTH are discussed here but that of other drugs in the relevant chapters.

\subsubsection{Dexamethasone preparation and administration}

DEX is a synthetic glucocorticoid which has very active glucocorticoid activity and minimal mineralocorticoid activity (Rang, Dale et al. 2007).

DEX solution was prepared by diluting $1 \mathrm{~mL}$ of $4 \mathrm{mg} / \mathrm{mL}$ stock solution (DBL dexamethasone sodium phosphate, David Bull Laboratories, Mulgrave, Australia) in $29 \mathrm{~mL}$ sterile saline $(0.9 \% \mathrm{NaCl})$. The final concentration of the DEX solution was $131.57 \mu \mathrm{g} / \mathrm{mL}$ DEX sodium phosphate which contains $100 \mu \mathrm{g} / \mathrm{mL}$ DEX.

DEX was injected subcutaneously once daily at a dose of $20 \mu \mathrm{g} / \mathrm{rat}(0.2 \mathrm{~mL} / \mathrm{rat}$, approximately $0.06 \mathrm{mg} / \mathrm{kg}$ body weight) in the haemodynamic and metabolic studies (Chapter 3, 4 and 10) and at a dose of $10 \mu \mathrm{g} / \mathrm{rat}(0.1 \mathrm{~mL} / \mathrm{rat}$, approximately $0.03 \mathrm{mg} / \mathrm{kg}$ body weight) for the other studies. Equivalent volumes of normal saline were used in control rats for these studies. The timing and duration of DEX or vehicle treatments is discussed separately in each study. 
The solution was drawn into a $1 \mathrm{~mL}$ syringe and injected subcutaneously using a 25 gauge needle (Terumo Medical Corporation, Elkton, USA). The rat was restrained gently but firmly under a towel to prevent movements during the subcutaneous DEX injection after the tail-cuff SBP and body weight measurements.

\subsubsection{ACTH preparation}

The ACTH preparation used (tetracosactrin $1 \mathrm{mg} / \mathrm{mL}$, Synacthen Depot, Novartis Pharmaceuticals, Sydney, Australia) is a synthetic polypeptide that consists of the first 24 of 39 amino acids found in the endogenous ACTH molecule. ACTH suspension was prepared by diluting $1 \mathrm{~mL}$ of $1 \mathrm{mg} / \mathrm{mL}$ stock suspension in $4 \mathrm{~mL}$ sterile saline $(0.9 \% \mathrm{NaCl})$. The final concentration of the preparation was $0.2 \mathrm{mg} / \mathrm{mL}$. The dose administered was $0.2 \mathrm{mg} / \mathrm{kg} / \mathrm{day}(0.1 \mathrm{~mL} / 100 \mathrm{~g} / \mathrm{day}$, subcutaneous injections). Equivalent volume of normal saline was used in control rats.

As with DEX treatment, ACTH was administered subcutaneously using the technique described in Section 2.4.1. 


\subsection{BLOOD PRESSURE MEASUREMENT}

\subsubsection{Indirect blood pressure measurement}

\subsubsection{Equipment and procedures}

Blood pressure was measured in conscious rats using the indirect tail-cuff sphygmomanometer (Figure 2.4). Experiments were performed in the morning on alternate days, in a separate quiet room within the laboratory. The rats were placed in plastic restrainers on a heating plate $\left(39-40^{\circ} \mathrm{C}\right.$, SDR Clinical Technology, Sydney, Australia) for approximately 12 minutes before recording the SBP readings. This caused tail artery vasodilation which was necessary for the detection of the oscillatory pulse wave by the pneumatic sensor bulbs and pulse transducers (Narco Biosystems, Houston, USA). The bulbs, which were securely taped to the tail using adhesive tapes (Leukofix, Smith \& Nephew Pty Ltd., Mount Waverley, Australia) were connected to the pneumatic transducers. Analogue signals were amplified by an amplifier (PA100, SDR Clinical Technology, Sydney, Australia) and converted to digital signals by a Biopac MP100 processor (Biopac Systems Inc., Goleta, USA). The blood pressure data were then acquired using the HyperRat software (SDR Clinical Technology, Sydney, Australia) on a Macintosh computer.

Systolic blood pressure readings were acquired by inflating a pneumatic cuff that was applied to the base of the rat tail (proximal to the pneumatic bulb sensor). The cuff, which was powered by an electrosphygmomanometer (Narco Biosystems, 
Houston, USA) was inflated to $225 \mathrm{mmHg}$ to occlude the tail artery and then deflated. The return of the pulse signal during deflation marked the SBP. The SBP readings were recorded on the computer.

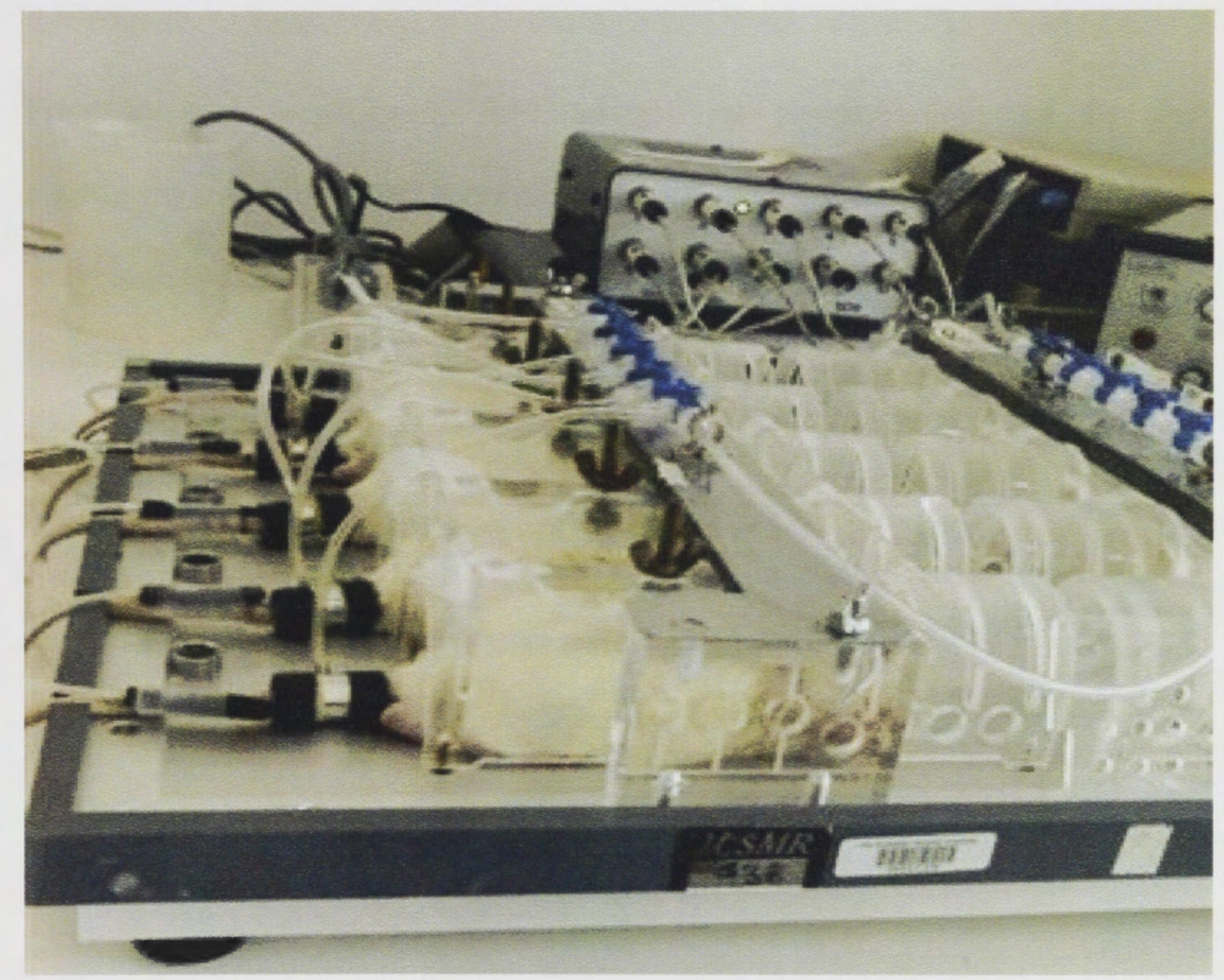

Figure 2.4: Tail-cuff sphygmomanometer

Up to 30 readings were recorded from each rat and the mean of the four median recordings, among which the difference was less than $10 \mathrm{mmHg}$, were accepted as the SBP. 


\subsubsection{Validation and maintenance}

This method has been previously compared with the 'gold standard' of a direct blood pressure measurement technique using a biotelemetry system with both giving similar blood pressure results (Fraser, Turner et al. 2001).

Prior to each experiment, the maximal air pressure delivered by the electrosphygmomanometer for cuff inflation was checked using a mercury sphygmomanometer. The tubing and occlusion cuffs were also checked for air leaks prior to any actual blood pressure recordings.

\subsubsection{Direct blood pressure measurement}

Direct blood pressure measurements were carried out only in the haemodynamic experiments (Chapters 3 and 4).

\subsubsection{Equipment and surgical procedures}

Following the induction of general anaesthesia using pentobarbitone (see Section 2.8.2 below), the rats were placed on a heating pad (surface temperature $38-40^{\circ} \mathrm{C}$, D.L.C Australia Pty. Ltd., Hoppers Crossing, Australia) in a dorsally recumbent position. The ventral aspect of the neck was shaved using an electric clipper (Thrive, Daito Electric Machine Ind Co, Ltd, Osaka, Japan). A midline ventral skin incision was made to expose the strap muscles of the neck. Subsequent surgical procedures were performed using a stereomicroscope (Leica MZ75, Leica Microscopy Systems Ltd, Heerbrugg, Switzerland). Haemostasis was achieved 
using a battery operated cautery. Blunt dissection along the direction of the muscle fibres exposed the trachea and thyroid gland. Further blunt dissection laterally towards the left of the animal exposed the left jugular vein and left common carotid artery. Approximately $2 \mathrm{~cm}$ of the left common carotid artery was gently isolated from the adherent connective tissues, vagus and cervical sympathetic nerves. Once the artery was isolated, three $5 / 0$ silk sutures (B.Braun Surgical, Melsungen, Germany) were passed around the artery and placed approximately $1 \mathrm{~cm}$ apart- one at the cranial end, the other one at the caudal end and the third in the middle between the cranial and caudal sutures. The cranial suture was tied to occlude the carotid artery. The middle and caudal sutures were tied loosely around the artery. A micro vascular clamp (HD-S, S\&T, Neuhausen, Switzerland) was placed over the caudal suture to prevent bleeding during the insertion of the catheter tip transducer (Mikro-Tip SPR-320; Millar Instruments Inc., Houston, USA). To insert the Millar catheter, an incision was made to the artery between the cranial and middle sutures using a 25 gauge needle with its sharp tip bent at $90^{\circ}$. Using the same needle, the Millar catheter was introduced into the artery in the direction of the heart. The middle suture was tightened to prevent bleeding when the artery clip was removed. The Millar catheter was advanced to the level of the aortic arch. Both the middle and caudal sutures were tightened to secure the catheter to the carotid artery. The Millar catheter was then connected to a bridge amplifier and the PowerLab ${ }^{\circledR}$ data acquisition system (AD Instruments, Sydney, Australia). The latter was connected to a computer running the Chart ${ }^{\mathrm{TM}} 5$ for Windows software (AD Instruments, Sydney, Australia). The 
mean arterial pressure, systolic and diastolic blood pressures were recorded on the computer.

\subsubsection{Calibration and maintenance}

The Millar catheter was calibrated with a pressure gauge calibration kit consisting of an aneroid sphygmomanometer prior to each use.

The catheter was soaked in enzyme cleaner (Tergazyme, Alconox Inc., White Plains, USA) after each use to remove any blood clot or protein build-up that might affect the accuracy of the pressure transducer.

\subsection{HEART RATE MEASUREMENT}

Heart rate was measured using the tail-cuff sphygmomanometry, non-invasively and Millar catheter, invasively.

\subsubsection{Via tail-cuff sphygmomanometry}

Heart rate measurements were obtained via this technique as reported in Chapter 10. During the acquisition of SBP readings, heart rate data were also acquired and recorded. The arterial pulsations were detected via the pneumatic pressure transducers and amplified. The signals were digitalised and recorded. Each sine wave was counted as a beat. 
Up to 30 readings were recorded. Readings that were $<200$ and $>500$ beats $/ \mathrm{min}$ were excluded as these were not within the physiological ranges for rats and were likely to be artefactual. All the other heart rate readings were averaged and accepted as the heart rate.

\subsubsection{Via Millar carotid artery transducer}

Heart rate measurements were also measured invasively using the Millar catheter in the haemodynamic studies (Chapters 3 and 4). The arterial pulsations were detected by the transducer, acquired by the PowerLab ${ }^{\circledR}$, amplified by the bridge amp and recorded by the computer running the Chart ${ }^{\mathrm{TM} 5}$ for Windows software. The number of sine waves per minute was used as heart rate.

\subsection{METABOLIC MEASUREMENTS}

Metabolic assessments were performed using stainless steel metabolic cages (Mascot Wire Works Pty Ltd., Sydney, Australia) where the rats were housed singly for 24 hours on alternate days through 12-hour light/dark cycles (Figure 2.5). The construct of this caging system enables measurements of 24hour food and water intakes and urine volume. Under each cage is a removable stainless steel funnel that collects urine and faeces. The lower part of the funnel is a specially-designed pipe that can separate urine and faeces. A graduated plastic container is attached to the end of the funnel for urine collection. Attached to the cages are food canisters that also support water bottles. 


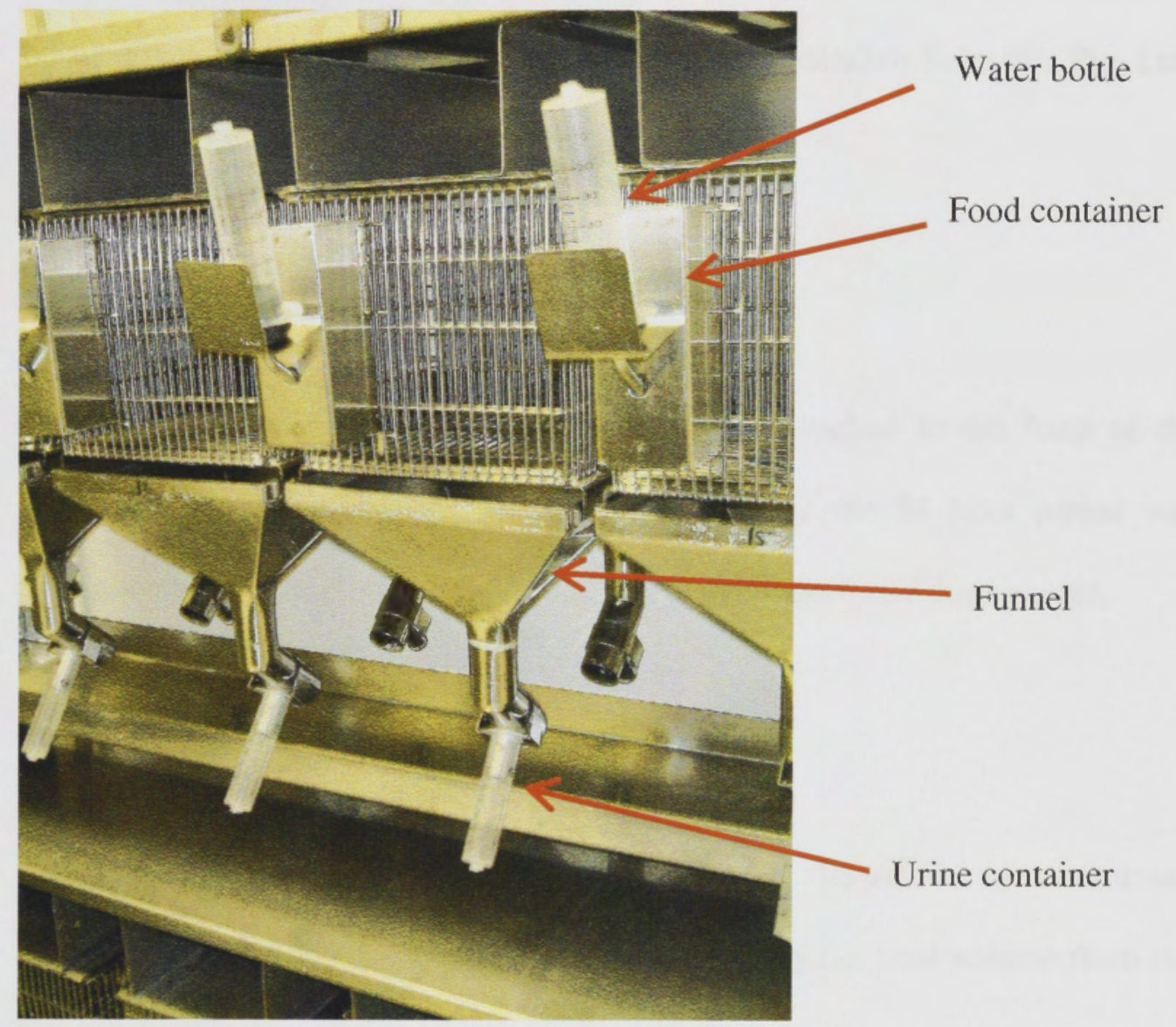

Figure 2.5: Metabolic cages 


\subsubsection{Body weight}

Body weight was measured after tail cuff experiments using a multi-function balance that allows weighing of moving animals. (Australian Scientific Pty. Ltd., Kotara, Australia).

\subsubsection{Food intake}

Pellet rat food was placed in the food container attached to the front of the metabolic cage. The amount of food ingested over the 24 hour period was determined by subtracting the final food weight from the initial food weight.

\subsubsection{Water intake}

The graduated water bottle was filled with tap water. The amount of water drunk over the 24 hour period was calculated by subtracting the final volume from the initial volume.

\subsubsection{Urine volume}

Urine was collected into the graduated container at the bottom of the funnel (Figure 2.5). Urine volume was measured and documented. Two milliliters of urine was collected into Eppendorf tubes and stored in a $-70^{\circ} \mathrm{C}$ freezer. Urinary sodium, potassium and creatinine were analysed at the ACT Pathology, The Canberra Hospital. 


\subsection{ANAESTHETIC AND SURGICAL PROCEDURES}

\subsubsection{Presurgical treatment}

The animals were given the final doses of all treatments prior to surgery.

\subsubsection{Anaesthesia}

All surgical procedures were carried out under general anaesthesia, achieved by either using intraperitoneal pentobarbitone sodium (Nembutal $60 \mathrm{mg} / \mathrm{mL}$, Merial Australia Pty. Ltd., Sydney, Australia) or isoflurane (Laser Animal Health, Pharmachem, Eagle Farm, Australia) inhalational anaesthesia.

Pentobarbitone was used to anaesthetise animals in the haemodynamic (Chapters 3 and 4), metabolic (Chapter 10) and the 20-HETE studies (Chapter 9). Pentobarbitone $(60 \mathrm{mg} / \mathrm{kg}$ or $0.1 \mathrm{~mL} / 100 \mathrm{~g}$ body weight) was drawn into a $1 \mathrm{~mL}$ syringe with a 25 gauge needle. The rat was restrained gently but firmly under a towel to prevent movements during the intraperitoneal injection. The right hind leg was abducted at the hip joint and at the same time, the hindquarter was lifted up. This allowed the abdominal viscera to fall anteriorly with gravity and minimise inadvertent puncture of the abdominal viscera. The injection was made at a site between the liver and the bladder, approximately $1 \mathrm{~cm}$ to the right of the midline. Before the injection, the syringe was withdrawn to ensure that the needle was not in the bladder or a blood vessel. Following the injection, the rat was returned to the cage until anaesthesia was achieved. During longer surgical 
procedures, such as in the haemodynamic studies (Chapters 3 and 4), additional bolus doses of pentobarbitone were required $(6 \mathrm{mg} /$ hour or $0.1 \mathrm{~mL}$ hour) to maintain satisfactory anaesthesia.

Isoflurane inhalational anaesthesia was used in the remainder of the studies (Chapters 5, 6, 7 and 8). It was administered with oxygen ( $2 \mathrm{~L} / \mathrm{min})$ via a vaporiser (Tec 5 Vaporizer, Datex-Ohmeda, Helsingborg, Sweden). Induction of anaesthesia was attained in an anaesthetic chamber using $4 \%$ isoflurane concentration. Isoflurane administration via a nose cone was continued at $2 \%$ to maintain anaesthesia throughout the entire surgical procedure.

The level of anaesthesia was checked by stretching the hind foot outward and pinching the paw pad firmly. If the rat did not retract the foot or move from this manoeuvre, satisfactory anaesthesia had been achieved.

\subsubsection{Haemodynamic experiments}

\subsubsection{Principles of haemodynamics}

Haemodynamics is the study of physics that governs the blood flow within the cardiovascular system. The fundamental principle of haemodynamics in the cardiovascular system is based on the Poiseuille's Law which states that the flow of fluid through a series of tubes is proportional to the pressure difference between the 2 ends of a tube and conductance, but inversely proportional to resistance (Figure 2.6). 


\section{$\mathrm{Q}=\underline{\Delta \operatorname{Pr}^{4} \pi}$ $\eta \mathrm{L} 8$}

$$
\begin{aligned}
& =\Delta \mathrm{P}\left[\frac{\mathrm{r}^{4}}{\eta \mathrm{L}}\right]\left[\frac{\pi}{8}\right) \\
& =\Delta \mathrm{P}\left[\frac{1}{\mathrm{R}}\right]\left[\begin{array}{l}
\frac{\pi}{8} \\
8
\end{array}\right]
\end{aligned}
$$

Figure 2.6: Poiseuille's Law. $\mathrm{Q}=$ flow rate, $\Delta \mathrm{P}=$ difference in pressure between the ends of a tube, $r=$ radius of the tube, $\eta=$ viscocity, $L=$ length of the tube, $\pi / 8=$ proportionality constant and $\mathrm{r}^{4} / \eta \mathrm{L}=$ conductance.

When applied to the cardiovascular system, this equation gives the formula:

$$
\text { Mean Arterial Pressure }=\text { Cardiac Output } \mathbf{x} \text { Total Peripheral Resistance }
$$

Cardiac output $(\mathrm{CO})$ and total peripheral resistance (TPR) are the fundamental parameters that determine arterial pressure. The blood pressure and $\mathrm{CO}$ are measured, whilst TPR is calculated by dividing mean arterial pressure by $\mathrm{CO} . \mathrm{CO}$, 
which is the minute output of blood from the heart, is dependent on the heart rate and stroke volume. TPR is the sum of the resistance of all peripheral vasculature in the systemic circulation. Total peripheral conductance (TPC), which is reciprocal of TPR, is the blood flow normalised to MAP in all peripheral vasculature. Determination of $\mathrm{CO}$ and TPR in a hypertensive model allows characterisation of the haemodynamic profile of the increase in blood pressure and thus, contributes to better understanding of the pathogenesis of hypertension in that model.

In Chapters 3 and 4 , rats underwent surgical procedures that enabled measurements of haemodynamic parameters. In the central haemodynamic experiments, the mean arterial pressure (described in Section 2.5.2), heart rate (described in Section 2.6.2) and cardiac output were measured. In the regional haemodynamic experiments, the mean arterial pressure, and blood flow measurement of the left renal, left common iliac and anterior mesenteric arteries were measured.

\subsubsection{Principles of ultrasonic transit-time flowmetry}

Haemodynamic parameters were measured using an electronic flow detection unit or flowmeter (Transonic T106 Small Animal Blood Flowmeter, Transonic System Inc., Ithaca, USA) with enhanced frequency resolution and volume flow-sensing probes (Transonic 3SS and 1RB perivascular probes, Transonic System Inc., Ithaca, USA). This system uses an ultrasonic transit-time principle to sense blood 
flow in vessels positioned within the flow probe acoustic-sensing window, independent of flow velocity profile, turbulence and haematocrit.

The flow probe consists of 2 ultrasonic transducers, which act as both transmitters and receivers for the ultrasonic beam, within the flow-sensor body and a fixed acoustic reflector (Figure 2.7). An ultrasonic wave transmitted from transducer 1 intersects the vessel, is reflected off the acoustic probe reflector, again intersects the vessel in the reverse direction and is received by transducer 2 (Figure 2.7). The flowmeter accurately measures the transit time which is the time taken for the ultrasonic signals to travel from one transducer to the other.

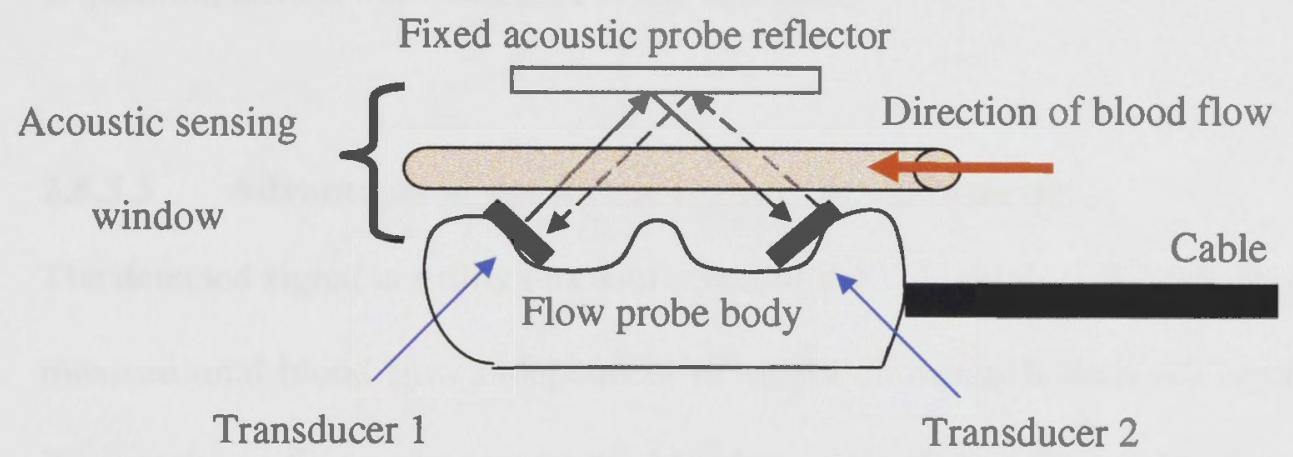

Figure 2.7: Basic structural components of aTransonic perivascular ultrasonic volume flow-sensing probe. upstream transit-time cyele,-- $\rightarrow$ downstream transit-time cycle.

Ultrasonic signals travelling back and forth alternately traverse the flowing blood in the upstream and downstream directions. During the upstream transit-time 
measurement cycle, the ultrasonic signal travels against blood flow. This results in an increase in transit time by a factor dependent on blood flow. The downstream transit-time measurement cycle, where the ultrasonic signal travels with blood flow, is associated with a reduction in transit time by the same magnitude as during the upstream cycle. The difference in integrated transit times between the upstream and downstream cycles is a measure of volume flow rather than velocity (Drost 1978).

The flowmeter automatically detects the scaling and individual calibration factors of flow probe attached to it. It is also attached to an external data acquisition device (PowerLab ${ }^{\circledR}$ data acquisition system). Prior to each recording, the data acquisition device was calibrated to the flowmeter.

\subsubsection{Advantages of ultrasonic transit-time flowmetry}

The detected signal is a direct measurement of net volume flow. Ultrasonic probes measure total blood flow independent of vessel diameter. It does not require the flow probe to fit snugly around the blood vessel and thus, is suitable for vessels smaller than the acoustic window. This is because ultrasonic beams that do not intersect the vessels do not contribute to the volume flow integral. This technique does not require zero calibration and it is, therefore, not subjected to offset and zero adjustment inaccuracies. It is also not affected by vessel-probe alignment, haematocrit and flow turbulence. 
Acoustic couplant or gel is required to fill the area within the acoustic window completely in acute study. Presence of gas bubbles within this space will affect the transmitted signals. Another disadvantage is the need to perform surgical implantation of flow-sensing probe.

\subsubsection{Initial preparation}

Once anaesthesia had been achieved, the rat was placed on a heating pad (surface temperature $38-40^{\circ} \mathrm{C}$ ) in a dorsally recumbent position. The rat was given maintenance intravenous fluid replacement $(0.9 \% \mathrm{NaCl})$ via a tail vein intravenous cannula (24 G Optiva IV catheter, Medex Medical Ltd, Haslingden, Great Britain) using a micro-processor controlled syringe pump (Model 22; Harvard Apparatus Ltd., South Natick, USA) at a rate of $1 \mathrm{~mL} /$ hour. All surgical procedures were performed using a stereomicroscope (Leica MZ7s, Leica Microscopy Systems Ltd, Heerbrugg, Switzerland).

The ventral aspect of the neck was shaved with an electric clipper. The left common carotid artery was cannulated for direct blood pressure and heart rate measurements, as described in Sections 2.5.2 and 2.6.2, respectively.

\subsubsection{Cardiac output measurement}

Cardiac output (CO) was measured using the Transonic 3SS perivascular probe. This probe was connected to the flowmeter that had been connected to the PowerLab $^{\circledR}$ data acquisition system. Cardiac measurements were recorded by the computer using the Chart ${ }^{\mathrm{TM}} 5$ software. 
Mechanical ventilation: With measurement of cardiac haemodynamics, the rat was mechanically-ventilated using a small animal ventilator (Harvard Apparatus Ltd., South Natick, USA) via a tracheal cannula. The trachea was visible through the ventral midline neck incision made for left common carotid cannulation procedure. The adherent connective tissue was dissected away using a blunt curved forceps. The ventral aspect of the $3^{\text {rd }}$ intercartilage space was incised. Haemostasis was achieved using a cautery. A tracheal cannula with Luer adapter (Harvard Apparatus, Holliston, USA) was inserted into the trachea, secured and connected to the ventilator. The respiratory rate was set to 80 breaths per minute. Supplemental oxygen $(2 \mathrm{~mL} / \mathrm{min})$ was administered to the rat via the ventilator.

Cardiac output measurement: Bilateral thoracotomy was performed to expose the heart and aorta. The pericardium was opened and ascending aorta was isolated by blunt dissection. The 3SS perivascular probe was positioned around the ascending aorta. Acoustic gel (SurgiLube; W. Fougera \& Co, Melville, USA) was deposited into the acoustic window of the probe adjacent to the artery to replace the air space.

After at least 20 minutes stabilisation, cardiac output measurements were recorded at $1 \mathrm{~min}$ intervals for $10 \mathrm{~min}$. These values were then averaged. 


\subsubsection{Regional blood flow measurements}

Regional blood flow was measured using the Transonic 1RB perivascular probe (Transonic System Inc., Ithaca, USA) connected to the same blood flow meter. Data acquisition was as described in cardiac output measurement.

Midline laparotomy was performed in anaethesised rats. The intestines were gently deflected to expose the left kidney. The left renal artery, found posterior to the larger renal vein, was carefully located and dissected. The 1RB perivascular probe was then placed around the artery. The left common iliac artery blood flow was used as a marker of hindquarter blood flow. The iliac artery, immediately below the bifurcation of the terminal abdominal aorta, was located and gently dissected before placing the 1RB perivascular probe around it. For mesenteric blood flow measurement, the anterior mesenteric artery was gently dissected to allow the placement of the IRB perivascular probe. Acoustic gel was deposited into the acoustic window of the probe next to the artery, replacing the air space. All the blood flow measurements were recorded after at least 20 minutes stabilisation.

\subsubsection{Sacrifice and blood collection procedures}

In the haemodynamic studies (Chapters 3 and 4), rats were sacrificed after the haemodynamic experiments. In the other studies, (Chapters 5-9), the rats were sacrificed once general anaesthesia had been attained. Bilateral thoracotomy was performed (if not already done for other experiments e.g. cardiac output 
measurements) to expose the heart. The pericardial sac was dissected away. All the rats were sacrificed via exsanguination. This was performed by right ventricular puncture using an 18 gauge needle and a $10 \mathrm{~mL}$ syringe. Blood was aspirated slowly to prevent haemolysis. Approximately $6-8 \mathrm{~mL}$ of blood could be collected from each rat.

Blood was collected into different tubes, depending on the assays required for the different studies. This is discussed in Section 2.9.

\subsubsection{Organ weight determination}

\subsubsection{Thymus gland}

The thymus gland was gently dissected away from the adjacent structures using blunt forceps. It was placed on an absorbent paper to move excess blood and moisture before being weighed using an electronic balance (Sartorius Analytical, Goettingen, Germany). The weight was expressed as $\mathrm{mg} / 100 \mathrm{~g}$ body weight.

\subsubsection{Adrenal glands}

The adrenal glands were located and removed via a median laparotomy incision. Using a pair of blunt curved forceps, the adherent connective tissue was dissected away. Blood and excess moisture were removed using an absorbent paper. The weights of these glands, obtained using an electronic balance, were averaged and expressed as $\mathrm{mg} / 100 \mathrm{~g}$ body weight. 


\subsection{HAEMATOCRIT ESTIMATION}

Blood was collected into heparinised capillary tubes and centrifuged. Haematocrit was measured using a micro-haematocrit reader (Hawksley and Sons Ltd., Sussex, England). Data were expressed as percentage of red blood cells per total volume of blood.

\subsection{BIOCHEMICAL ASSAYS}

\subsubsection{Plasma nitrite and nitrate assay}

\subsubsection{Principles}

Determination of NO is difficult due to its short half life and its highly reactive nature (Knowles and Moncada 1994). Therefore, measurement of nitrite and nitrate (NOx), which are stable end products of NO, in plasma is often used as an indicator of total body NO production. Quantitating nitrite alone is inadequate as nitrite in whole blood is rapidly oxidised to nitrate by haemoglobin prior to plasma preparation. Hence, both nitrite and nitrate have to be measured.

Nitrate is measured as nitrite after enzymatic reduction by nitrate reductase. Nitrite is measured by the Griess colorimetric reaction. The optical density is measured using a microplate reader at a wavelength of $540 \mathrm{~nm}$. 


\subsubsection{Materials}

This assay was performed using reagents provided in a commercially available kit (Endogen, Pierce, Rockford, IL, USA). The kit consisted of the following reagents:

10x Reagent diluent. This is a Hepes based buffer containing detergent and preservatives. One in 10 dilution was performed using deionised water to give $1 \mathrm{x}$ reagent diluent.

Nitrate reductase diluent. This is a phosphate based buffer containing preservatives.

Nitrate reductase. This lyophilised enzyme was reconstituted by adding $1 \mathrm{~mL}$ nitrate reductase diluent into the given vial. One in 2.5 dilution was performed on the reconstituted nitrate reductase concentrate. The diluted enzyme was used directly in the assay.

Reduced $\beta$-nicotinamide adenine dinucleotide (NADH). This lyophilised reagent was reconstituted by adding $1 \mathrm{~mL}$ deionised water into the given vial. Prior to use, 1 in 2 dilution was performed using deionised water.

Nitrite standard. This is a solution of sodium nitrite at $2000 \mu \mathrm{M}$ in water with preservatives. 
Nitrate standard. This is a solution of sodium nitrate at $1000 \mu \mathrm{M}$ in water with preservatives.

Griess reagent I. This is a solution of sulphanilamide in $2 \mathrm{M}$ hydrochloric acid.

Griess reagent II. This is a solution of $\mathrm{N}$-(1-Naphthyl) ethylenediamine in $2 \mathrm{M}$ hydrochloric acid.

\subsubsection{Sample preparation}

Briefly, blood was collected into tubes (BD Vacutainer, Franklin Lakes, USA) containing $3.2 \%$ buffered sodium citrate $(0.109 \mathrm{M})$. Plasma was prepared by centrifuging the blood tubes at $3500 \mathrm{rpm}$ (relative centrifugal force $2465 \mathrm{G}$ ) for 15 min at $4{ }^{\circ} \mathrm{C}$. Plasma was stored at $-70^{\circ} \mathrm{C}$ until assayed.

Plasma samples were thawed immediately prior to use. These were spun for 5 minutes at $13000 \mathrm{rpm}$ at $4{ }^{\circ} \mathrm{C}$. Approximately $300 \mu \mathrm{L}$ of the sample's plasma was aliquoted into ultrafiltration tubes (Ultraforce-MC 30000 NMWL filter unit, Millipore Corp., Bedford, USA) and spun for 25 minutes at $13000 \mathrm{rpm}$ at $4{ }^{\circ} \mathrm{C}$. The filtrate was used directly in the assay.

\subsubsection{Procedures}

Standard curves for both nitrite and nitrate were constructed. Nitrite standards were prepared by sequential dilutions of $2000 \mu \mathrm{M}$ stock nitrite solution with a 
Hepes-based buffer containing detergent and preservatives (1x reagent diluent) to give the following concentrations: $200,100,50,25,12.5,6.25$ and $3.125 \mu \mathrm{M}$. Nitrate standards were prepared by sequential dilutions of $1000 \mu \mathrm{M}$ stock nitrate with $1 \mathrm{x}$ reagent diluent to give the following concentrtions: $100,50,25,12.5,6.25$ and 3.125 $\mu$ M. Plain 1x reagent diluent was used as a zero standard. $200 \mu \mathrm{L}$ of $1 \mathrm{x}$ reagent diluent was pipetted into duplicate blank wells.

Nitrate Assay Procedures: The nitrate standards $(50 \mu \mathrm{L})$, controls ie. unspiked

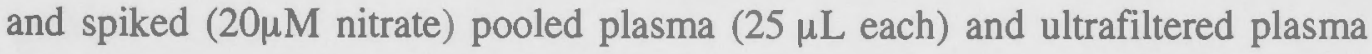
samples $(25 \mu \mathrm{L})$ were pipetted into the wells of a 96-well plate (NUNC Brand Products, Roskilde, Denmark). The controls and plasma samples were further diluted with $25 \mu \mathrm{L} 1 \mathrm{x}$ reagent diluent.

Diluted NADH $(25 \mu \mathrm{L})$ was added to the standard and sample wells. Following this, $25 \mu \mathrm{L}$ of diluted nitrate reductase was added to the standard and sample wells (except the blank wells).

The plate was then incubated at $37^{\circ} \mathrm{C}$ for 30 minutes before Griess Reagents I and II ( $50 \mu \mathrm{L}$ each) were added to the standard and sample wells (except the blank wells).

Nitrite Assay Procedure: Similarly, the nitrite standards $(50 \mu \mathrm{L})$, controls ie. unspiked and spiked ( $20 \mu \mathrm{M}$ nitrate) pooled plasma ( $25 \mu \mathrm{L}$ each) and ultrafiltered 
plasma samples $(25 \mu \mathrm{L}$ ) were pipetted into the wells of a 96-well plate. The controls and plasma samples were further diluted with $25 \mu \mathrm{L} 1 \mathrm{x}$ reagent diluent.

Instead of NADH and nitrate reductase enzyme, $50 \mu \mathrm{L} 1 \mathrm{x}$ reagent diluent was added to the standard and sample wells (except the blank wells).

The plate was also incubated at $37{ }^{\circ} \mathrm{C}$ for 30 minutes before Griess Reagents I and II (50 $\mu \mathrm{L}$ each) were added to the standard and sample wells (except the blank wells).

Both nitrate and nitrite assay plates were incubated for another 10 minutes at room temperature $\left(22-25^{\circ} \mathrm{C}\right)$ to allow the colour to develop. Absorbances for each well were read at $540 \pm 20 \mathrm{~nm}$ after blanking against the blank wells using a microplate reader (THERMOmax Microplate Reader, Molecular Devices, California, USA).

\subsubsection{Performance}

Linearity: The standard curves for nitrate and nitrite constructed as per the method described in Section 2.9.1.4 had a correlation coefficient of 1.00 each.

Precision: Spiked (with $20 \mu \mathrm{M}$ nitrate) and unspiked pooled rat plasma samples were run multiple times in the same assay to determine the intra-assay precision. The mean, standard deviation (SD) and the precision numbers which represent the 
percent coefficient of variance (\% CV) for the concentrations of nitrate determined in the assay are summarised in Table 2.2 below. 
Table 2.2: Assay precision

Quality Controls

Unspiked plasma

16.9

0.09

5.2

Plasma $+20 \mu \mathrm{M}$ nitrate

38.03

CV: coefficient of variance; SD: Standard deviation.

\subsubsection{Analysis}

The net optical density for the standards and samples were calculated by subtracting the average zero standard optical density from the optical density for each standard and sample. The average optical densities for each standard versus nitrate and nitrite concentrations were plotted. The optical densities for each sample were then plotted on the standard curves. Total nitrate and nitrite concentrations can be extrapolated from these graphs.

\subsubsection{Serum uric acid assay}

\subsubsection{Principle}

The uric acid assay was based on the method described by Trivedi (Trivedi, Rebar et al. 1978). Oxidation of uric acid to allantoin by uricase leads to the production of hydrogen peroxide. In the presence of peroxidase, the reaction of hydrogen 
peroxide with 4-aminoantipyrine and 2, 4, 6-tribromo-3-hydroxybenzoic acid forms a quinoneimine dye. The change in absorbance at $548 \mathrm{~nm}$ is proportional to the uric acid concentration in the sample.

\subsubsection{Materials and procedures}

The serum uric acid assay was performed by ACT Pathology at The Canberra Hospital. This laboratory is accredited by NATA.

This assay was performed on the ARCHITECT ${ }^{\circledR}$ c8000 (Abbot Laboratories, Abbott Park, USA) automated assay system using reagents provided in a commercially available kit (Abbott Clinical Chemistry, Abbott Park, USA) consisting of a single reagent solution. The active ingredients of the solution are 4-aminoantipyrine $\quad(0.5 \mathrm{mmol} / \mathrm{L}), 2, \quad 4, \quad 6$-tribromo-3-hydroxybenzoic acid $(1.75 \mathrm{mmol} / \mathrm{L})$, uricase $(>120 \mathrm{U} / \mathrm{L})$, peroxidase $(>500 \mathrm{U} / \mathrm{L})$ and TRIS buffer $50 \mathrm{mmol} / \mathrm{L}$.

\subsubsection{Sample preparation}

Blood was collected into serum tubes (BD Vacutainer, Franklin Lakes, USA). Blood was allowed to clot for at least 1 hour and serum was prepared by centrifuging the blood tubes at $3500 \mathrm{rpm}$ (relative centrifugal force $2465 \mathrm{G}$ ) for 15 min at $4{ }^{\circ} \mathrm{C}$. Serum was stored at $-70{ }^{\circ} \mathrm{C}$ until assayed. 


\subsubsection{Urinary sodium and potassium}

\subsubsection{Principle}

Ion-selective electrodes for sodium and potassium use membranes selective to each of these ions. Voltage or electrical potential that is generated across the membranes between the reference and measuring electrodes (in accordance with the Nernst equation) is compared to previously determined voltages and thus, converted into ion concentration.

\subsubsection{Materials and procedures}

The urinary sodium and potassium assays were performed by ACT Pathology at The Canberra Hospital using the ICT $\mathrm{Na}^{+}, \mathrm{K}^{+}, \mathrm{Cl}^{-}$reagent kit on the ARCHITECT $^{\circledR}$ c8000 (Abbot Laboratories, Abbott Park, USA) automated assay system.

\subsubsection{Sample preparation}

Urine was collected using the metabolic cages as described in Section 2.7.4 and kept at $-70^{\circ} \mathrm{C}$ until assayed.

\subsubsection{Urinary creatinine assay}

\subsubsection{Principle}

Creatinine in the urine reacts with picrate to form a creatinine-picrate complex at an alkaline $\mathrm{pH}$. The rate of increase in absorbance at $500 \mathrm{~nm}$ due to the formation 
of creatinine-picrate complex is directly proportional to the concentration of creatinine in the urine sample.

\subsubsection{Materials and procedures}

This assay was performed by ACT Pathology at The Canberra Hospital using a two-reagent kit consisting of sodium hydroxide $(0.25 \mathrm{~mol} / \mathrm{L})$ and picric acid $\left(20.5 \mathrm{mmol} / \mathrm{L}\right.$ ) on an automated assay system (ARCHITECT $^{\circledR}$ c8000, Abbot Laboratories, Abbott Park, USA).

\subsubsection{Sample preparation}

Urine was collected using the metabolic cages as described in Section 2.7.4 and kept at $-70^{\circ} \mathrm{C}$ until assayed.

\subsection{DETECTION OF REACTIVE OXYGEN SPECIES}

\subsubsection{Lucigenin-enhanced chemiluminescence assay}

\subsubsection{Principle}

Lucigenin-enhanced chemiluminescence was used to determine superoxide production in rat aortic segments resected following sacrifice. The reaction of lucigenin (bis- $\mathrm{N}$-methylacridinium nitrate) with superoxide in its cationic state through a series of reactions resulted in the formation of an unstable dioxetane compound that emits a photon as it decomposes. This can be detected in the scintillation/luminescence counter. 


\subsubsection{Materials}

All solutions were prepared fresh prior to each assay. All chemicals were purchased from Sigma-Aldrich (St Louis, MO, USA).

Hepes-buffered Krebs solution. This solution consists of $100 \mathrm{mM} \mathrm{NaCl}, 5 \mathrm{mM}$ $\mathrm{KCl}, 1 \mathrm{mM} \mathrm{MgSO}$, $2 \mathrm{mM} \mathrm{K}_{2} \mathrm{HPO}_{4}, 25 \mathrm{mM} \mathrm{NaHCO}$, $11 \mathrm{mM}$ glucose, $20 \mathrm{mM}$ Na-Hepes and $2 \mathrm{mM} \mathrm{CaCl}_{2}, \mathrm{pH} 7.4$.

NAD(P)H solution. This is a solution of $\mathrm{NAD}(\mathrm{P}) \mathrm{H}$ at $1 \mathrm{mM}$ in Hepes-buffered Krebs solution. It is stored away from light at $4{ }^{\circ} \mathrm{C}$.

Sodium diethyldithiocarbamate (DETC). This is a solution of DETC at $1 \mathrm{M}$ in warm $\left(37^{\circ} \mathrm{C}\right)$ Hepes-buffered Krebs solution.

Lucigenin solution. This is a solution of lucigenin at $500 \mu \mathrm{M}$ in warm $\left(37^{\circ} \mathrm{C}\right)$ Hepes-buffered Krebs solution. It is kept away from light.

Incubation solution. This is a solution of DETC at $13.5 \mathrm{mM}$ and $\mathrm{NAD}(\mathrm{P}) \mathrm{H}$ at 0.1 $\mathrm{mM}$ in Hepes-buffered Krebs solution.

Assay solution. This is a solution of $\mathrm{NAD}(\mathrm{P}) \mathrm{H}$ at $0.1 \mathrm{mM}$ in Hepes-buffered Krebs solution. 


\subsubsection{Sample preparation}

After sacrificing the rat, a 1-2 cm segment of the thoracic aorta was resected. The aortic segment was washed in saline solution and the adherent connective tissue was carefully dissected. It was bisected and kept on ice in Hepes-buffered Krebs solution until it was ready to be incubated.

\subsubsection{Procedures}

All the aortic segments were incubated for 30 minutes at $37^{\circ} \mathrm{C}$ in incubation solution. $\mathrm{NAD}(\mathrm{P}) \mathrm{H}$ was added to activate $\mathrm{NAD}(\mathrm{P}) \mathrm{H}$ oxidase, the main source of superoxide In the vasculature. The superoxide dismutase inhibitor diethylthiocarbamate (DETC) was used to prevent dismutation of existing superoxide. After incubation, the aortic segments were placed in individual wells of a black 96-well plate containing the assay solution. Lucigenin was added to the wells prior to analysis to give a final concentration of $5 \mu \mathrm{M}$.

The plates were read in a scintillation detector (TopCount NXT Microplate

Scintillation \& Luminescence Counter, PerkinElmer, Woodbridge, Canada). Twelve successive readings were recorded.

The aortic segments were dried at $60{ }^{\circ} \mathrm{C}$ for 1-2 days and the dry weight of each segment was recorded. 


\subsubsection{Analysis}

The first 2 and the last 2 readings from the blank and sample wells were excluded and the mean luminescence, expressed as counts per second (counts/s), was calculated. The mean values from the blank wells were subtracted from the sample wells. The net results were then adjusted to aortic weight by dividing the net luminescence by dry weight of the corresponding aortic tissue and expressed as counts per second per mg of dry wt (counts/s/mg).

\subsubsection{Kidney mitochondrial superoxide analysis}

The method for this assay is described in Chapter 5 .

\subsection{3 $\mathrm{F}_{2}$-isoprostane concentration}

\subsubsection{Principle}

The $F_{2}$-isoprostane family is a group of chemically stable prostaglandin $F_{2}$-like compounds generated during peroxidation of unsaturated fatty acids in membrane phospholipids, namely arachidonic acid. They have been shown to be reliable markers of lipid peroxidation and oxidative stress in vivo. (Morrow, Hill et al. 1990; Morrow and Roberts 1996; Patrono and FitzGerald 1997)

\subsubsection{Sample preparation}

Blood was collected into blood tubes (BD Vacutainer, Franklin Lakes, USA) containing $5.4 \mathrm{mg} \mathrm{K}_{2}$ EDTA and $2 \mathrm{mg}$ glutathione. Plasma was prepared by centrifuging the blood tubes at $3500 \mathrm{rpm}$ (relative centrifugal force $2465 \mathrm{G}$ ) for 15 
min at $4{ }^{\circ} \mathrm{C}$. Butylated hydroxyltoluene was added to plasma samples (final concentration $0.2 \mathrm{mg} / \mathrm{mL}$ ) before being stored at $-70^{\circ} \mathrm{C}$ until assayed.

\subsubsection{Procedures}

Plasma $\mathrm{F}_{2}$-isprostane was assayed by Associate Professor Kevin Croft, University of Western Australia using stable isotope dilution capillary gas/ electron capture negative ionization mass spectrometry (Mori, Croft et al. 1999).

\subsection{STATISTICAL ANALYSIS AND DATA PRESENTATION}

Results were expressed as mean \pm SEM. SBP, body weight, heart rate (Chapter 8), urine volume (Chapter 10) and food and water consumption (Chapter 10) measurements between and within groups were analysed by repeated-measure analysis of variance, with Greenhouse-Geisser adjustment for multisample asphericity. Comparisons between groups for thymus weight and adrenal weight, haemodynamic parameters, biochemical assay results (plasma NOx, serum urate, plasma $F_{2}$-isoprostane), blood glucose level, haematocrit, aortic lucigeninenhanced chemiluminescence, flow cytometric results and food and water consumption were carried out using unpaired t-tests. To minimise the family-wise type 1 error, the Ryan-Holm stepdown Bonferroni procedure was applied to the raw $P$ values to give $P$ : 


\section{CHAPTER 3}

Haemodynamic Profile of Dexamethasone-Induced

Hypertension in the Rat 


\subsection{INTRODUCTION}

The haemodynamics of DEX-HT have been evaluated in both humans (Pirpiris, Sudhir et al. 1992) and dogs (Nakamoto, Suzuki et al. 1991). DEX-HT in humans ( $3 \mathrm{mg} / \mathrm{day}$ ) was associated with increased TPR without any change in CO (Pirpiris, Sudhir et al. 1992). Whilst these are useful data depicting the haemodynamic patterns of DEX-HT, little is known about the regional flow patterns contributing to the raised TPR. In dogs, DEX-HT $(0.5 \mathrm{mg} / \mathrm{kg} /$ day $)$ was found to be associated with increased TPR and decreased CO (Nakamoto, Suzuki et al. 1991). Whether these changes represent features necessary for the development of DEX-HT has not been previously evaluated. In that study, only CO, TPR and renal blood flow were evaluated leaving other haemodynamic parameters unexamined. Furthermore, the relevance of the dog model of DEX-HT to that in humans remains unclear. For the purpose of examining the mechanisms of GC-HT, the rat is generally more applicable to the human situation (Whitworth, Schyvens et al. 2001; Whitworth, Zhang et al. 2006).

Haemodynamic profiles of cortisol- and ACTH-HT have been evaluated. Cortisol-induced hypertension (50 mg orally, every 6 hours for 5 days) in man was associated with a rise in $\mathrm{CO}$ and renal vascular resistance but no change in calculated TPR (Connell, Whitworth et al. 1987). The beta-adrenergic receptor blocker atenolol prevented the increase in $\mathrm{CO}$ but not the blood pressure increase in cortisol-hypertensive human subjects (Pirpiris, Yeung et al. 1993). ACTH-HT in rats has been shown to be associated with increased $\mathrm{CO}$ and renal vascular 
resistance (Wen, Fraser et al. 1998) In another series of experiments, Wen et al. demonstrated that ramipril successfully prevented both ACTH-HT and rise in renal vascular resistance, whereas neither preventing rise in $\mathrm{CO}$ nor decreasing TPR altered ACTH-HT in the rat (Wen, Fraser et al. 1999). These studies showed that increase in renal vascular resistance is necessary for the development of ACTH-HT. In sheep, ACTH-HT was also associated with increased CO but prevention of $\mathrm{CO}$ increase by $\beta$-adrenoreceptor blockade did not modify the rise in blood pressure (Graham, Allen et al. 1980). Because of the differences between ACTH-HT and DEX-HT described in Chapter 1, inferences cannot be drawn about DEX-HT based on these results in ACTH-HT.

In this study, we evaluated the central and regional haemodynamic profiles of DEX-HT using ultrasonic transit-time flowmetry. This method has been validated for measurement of cardiac output and regional blood flow in rats (Wen, Li et al. 1996).

\subsection{METHODS}

\subsubsection{Experimental animals}

This study was approved by the Animal Experimentation Ethics Committee of the Australian National University (Protocol No. J.HB.22.06). Forty one male Sprague-Dawley rats (initial body weight of 200-250 g) were housed and acclimatised as described in Sections 2.2 and 2.3 of Chapter 2. Nineteen rats 
(sham treatment, $\mathrm{n}=9$ and $\mathrm{DEX}, \mathrm{n}=10$ ) were used in the central haemodynamic study and 22 rats (sham treatment, $\mathrm{n}=11$ and $\mathrm{DEX}, \mathrm{n}=11$ ) in the regional haemodynamic study. These rats received saline by gavage $(0.1 \mathrm{~mL} / 100 \mathrm{~g}$ body weight/day, from day $\mathrm{P} 0)$ and subcutaneous saline $(0.2 \mathrm{~mL} / \mathrm{rat} /$ day $)$ or $\mathrm{DEX}$ injections (20 $\mu \mathrm{g} / \mathrm{rat} / \mathrm{day}, \mathrm{s.c.})$ from day T0-T11.

\subsubsection{Blood pressure and body weight measurements}

Non-invasive systolic blood pressure (SBP) was measured in conscious rats between 9-11 am on alternate days using the tail-cuff method described in Section 2.5.1. Body weights were measured on alternate days after the tail-cuff SBP measurements.

Direct blood pressure measurements were recorded under pentobarbitone anaesthesia $(60 \mathrm{mg} / \mathrm{kg}$, i.p.) on the last day of experiment (T11) where the mean arterial pressure (MAP), systolic and diastolic blood pressures (DBP) and heart rate (HR) were measured using a catheter tip pressure transducer (Mikro-Tip SPR320; Millar Instruments Inc., Houston, USA) that was inserted into the left carotid artery and advanced to the level of the aortic arch. Surgical approaches for cardiac output and regional flow measurements are described in Section 2.8.3.

\subsubsection{Calculation of other haemodynamic parameters}

The following formulae were used to calculate the other haemodynamic parameters. 
- Cardiac Index $(\mathrm{CI})=\mathrm{CO} / 100 \mathrm{~g}$ body weight

- Stroke Volume $(\mathrm{SV})=\mathrm{CO} / \mathrm{HR}$

- $\quad$ Stroke Index $(\mathrm{SI})=\mathrm{CI} / \mathrm{HR}$

- Total peripheral resistance $(\mathrm{TPR})=\mathrm{MAP} / \mathrm{CO}$

- Total peripheral conductance $(\mathrm{TPC})=1 / \mathrm{TPR}$

- Regional vascular resistance $=$ MAP/Regional blood flow

- Regional vascular conductance $=1 /$ Regional vascular resistance

\subsubsection{Haematocrit estimation}

After the haemodynamic experiments, the animals were sacrificed via exsanguination. Blood was collected for haematocrit estimation as described in Section 2.9 of Chapter 2.

\subsubsection{Thymus and adrenal weights}

Thymus wet weight, expressed relative to body weight (grams thymus weight per $100 \mathrm{~g}$ body weight), was used as a marker of glucocorticoid activity. The surgical technique is described in Section 2.8.5.1 of Chapter 2.

Adrenal wet weight, expressed relative to body weight (grams adrenal weight per $100 \mathrm{~g}$ body weight), was used to assess the efficacy of DEX administration. The surgical technique is described in Section 2.8.5.2 of Chapter 2. 


\subsubsection{Statistical analysis}

Results were expressed as mean \pm SEM. Statistical analysis were as described in Section 2.12 of Chapter 2.

\subsection{RESULTS}

\subsubsection{Tail-cuff systolic blood pressure}

Sham injections with sterile saline did not alter SBP (T0: 126 \pm 3 , T10: $124 \pm 2 \mathrm{mmHg}, \mathrm{n}=21$ ). DEX significantly increased SBP from $123 \pm 2$ on day T0 to $142 \pm 2 \mathrm{mmHg}$ on day $\mathrm{T} 10(\mathrm{n}=20, P<0.0005)$. In comparison with salinetreated rats $(n=20)$, SBP in the DEX-treated group $(n=21)$ was significantly higher $(P<0.0005)$ (Figure 3.1$)$. 


\section{Tail-Cuff Systolic Blood Pressure}

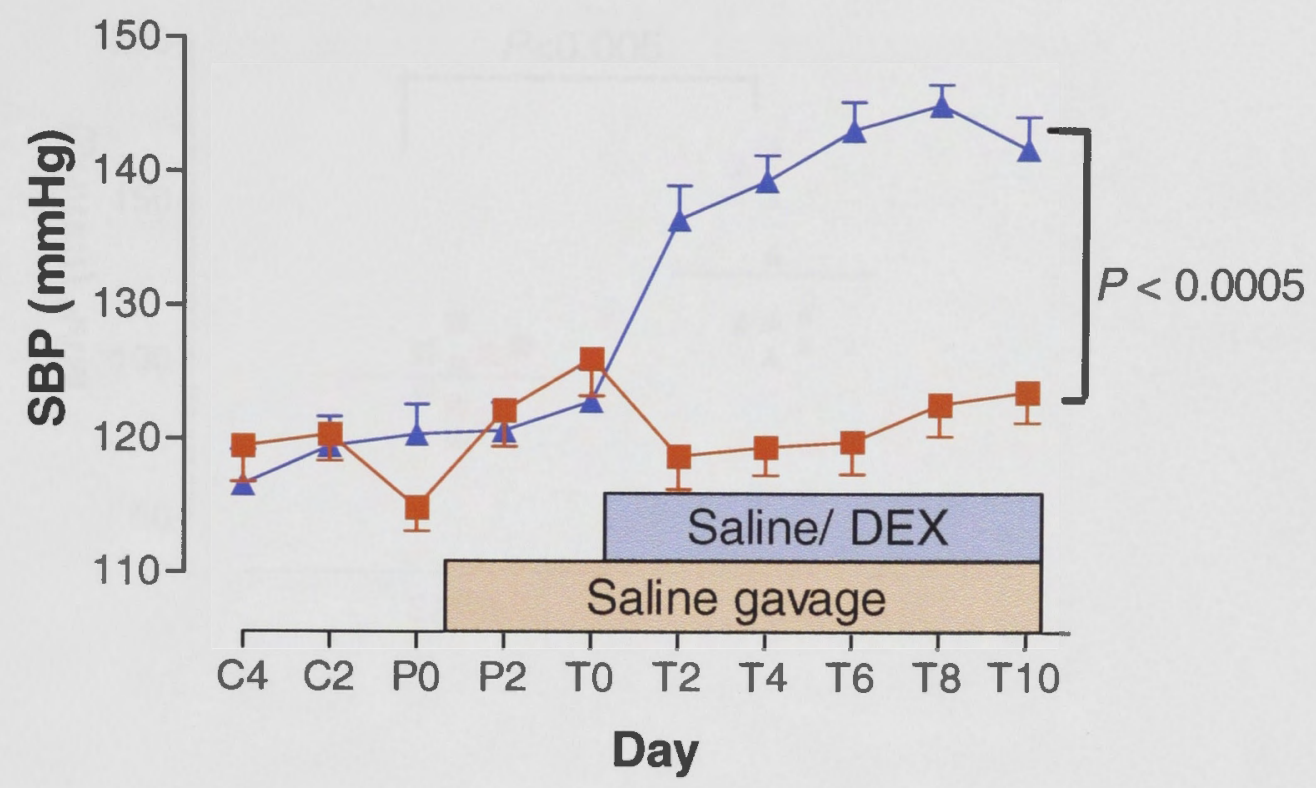

Figure 3.1: Tail-cuff systolic blood pressure. - Saline, $n=20 ; \Delta \quad D E X, n=21$.

\subsubsection{Central haemodynamics}

DEX-treated rats $(\mathrm{n}=10)$ had significantly higher MAP $(P<0.005)$, systolic and diastolic blood pressure $(P<0.005)$ (Figure 3.2), TPR $(P<0.05)$ and lower TPC $(P<0.005)$ (Figure 3.3$)$ in comparison to the sham treatment group $(n=9)$. There was no significant difference in HR, CO, CI, SV and SI between DEX $(n=9)$ and sham $(\mathrm{n}=10)$ treatment groups (Figures 3.4-3.6, Table 3.1). 


\section{Mean Arterial Pressure}

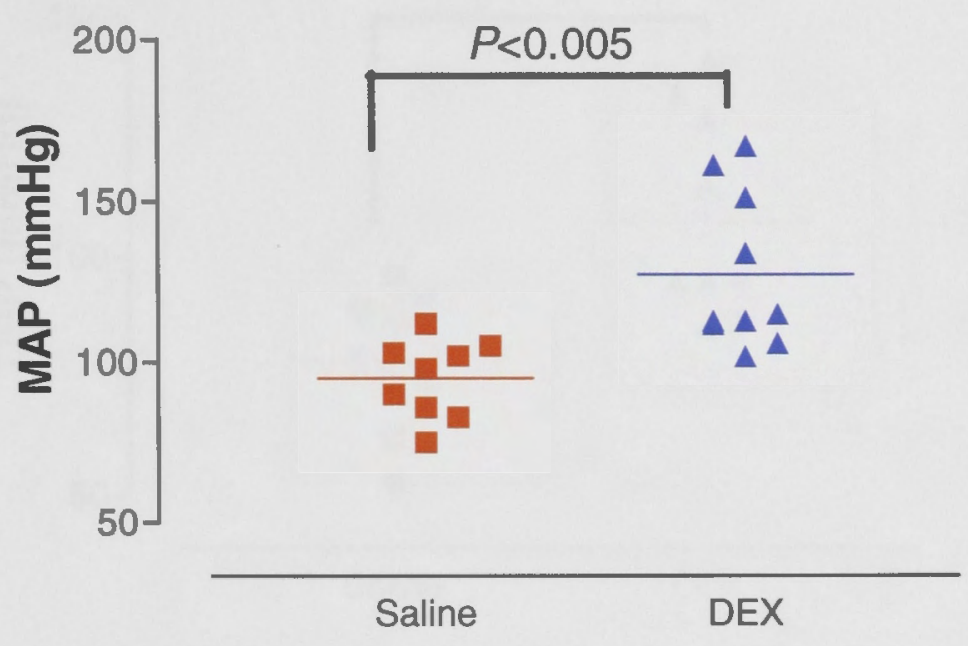

Direct Systolic Blood Pressure

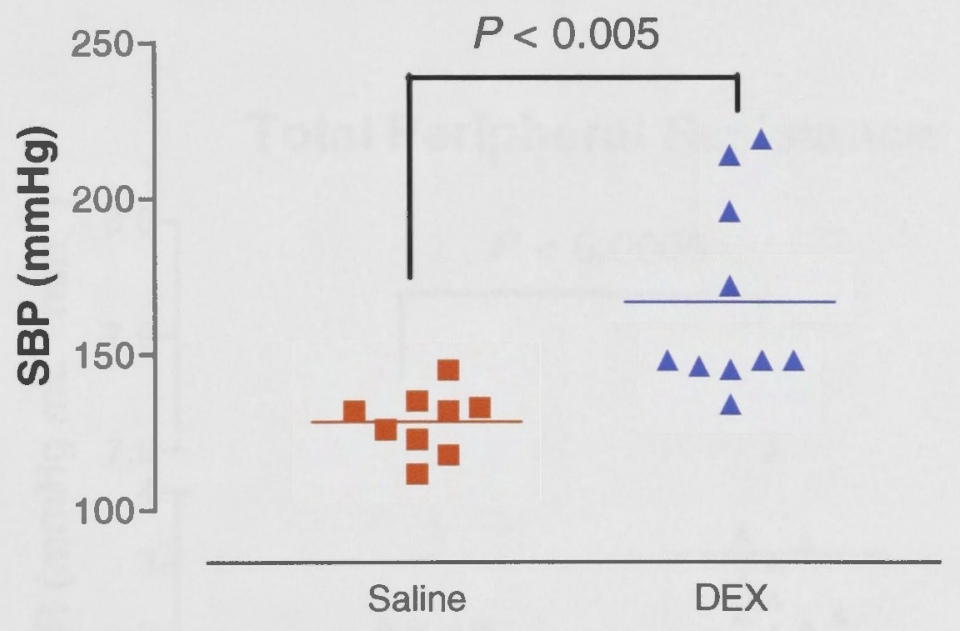




\section{Direct Diastolic Blood Pressure}

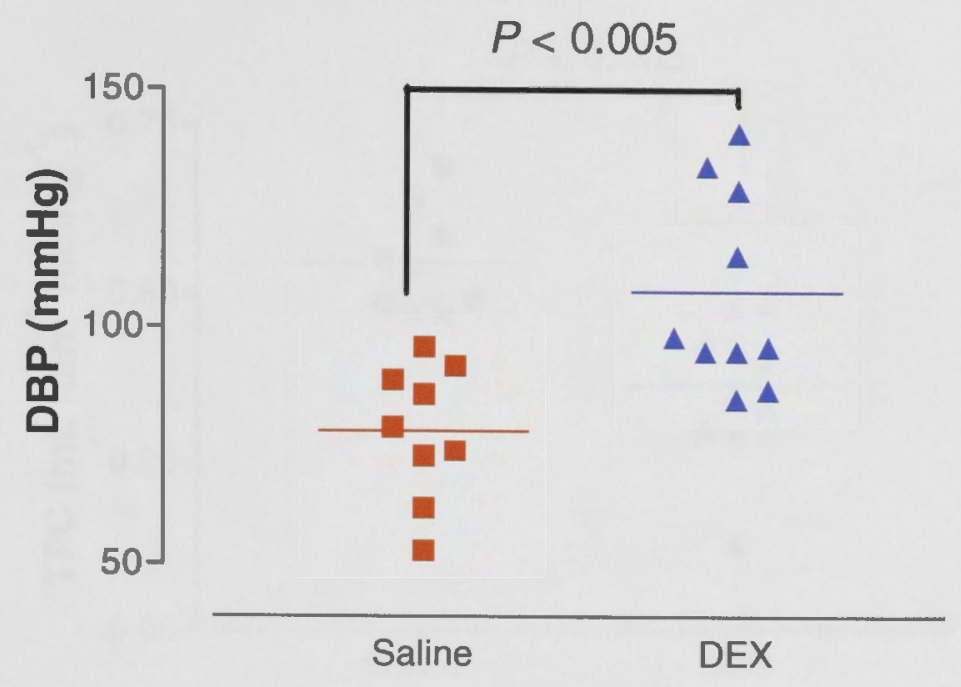

Figure 3.2: Comparisons of blood pressure readings between sham and DEX treatments. $\square$ Saline, $\mathrm{n}=9 ; \Delta$ DEX, $\mathrm{n}=10$.

\section{Total Peripheral Resistance}

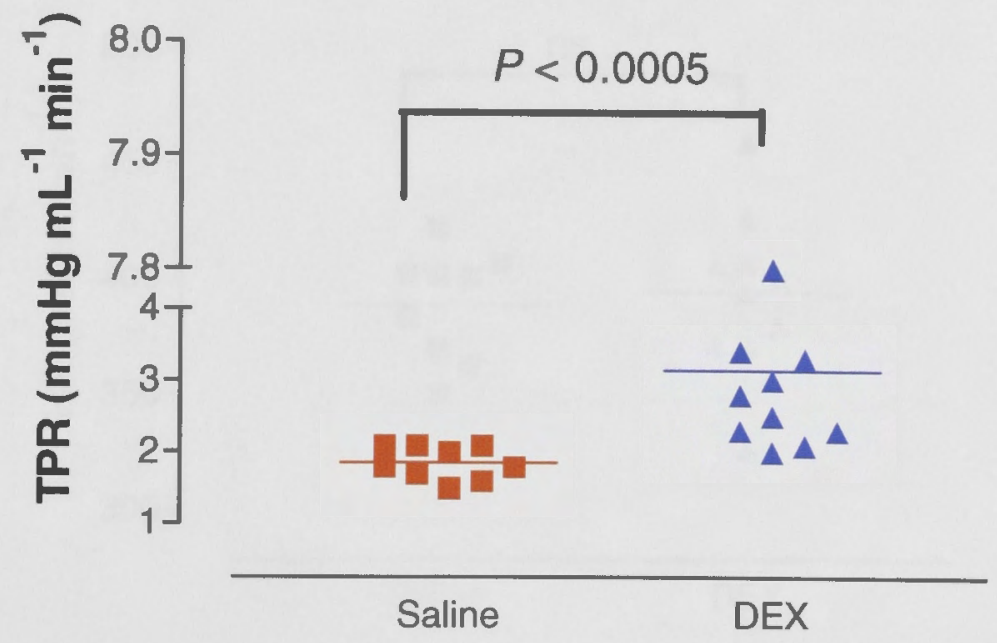




\section{Total Peripheral Conductance}

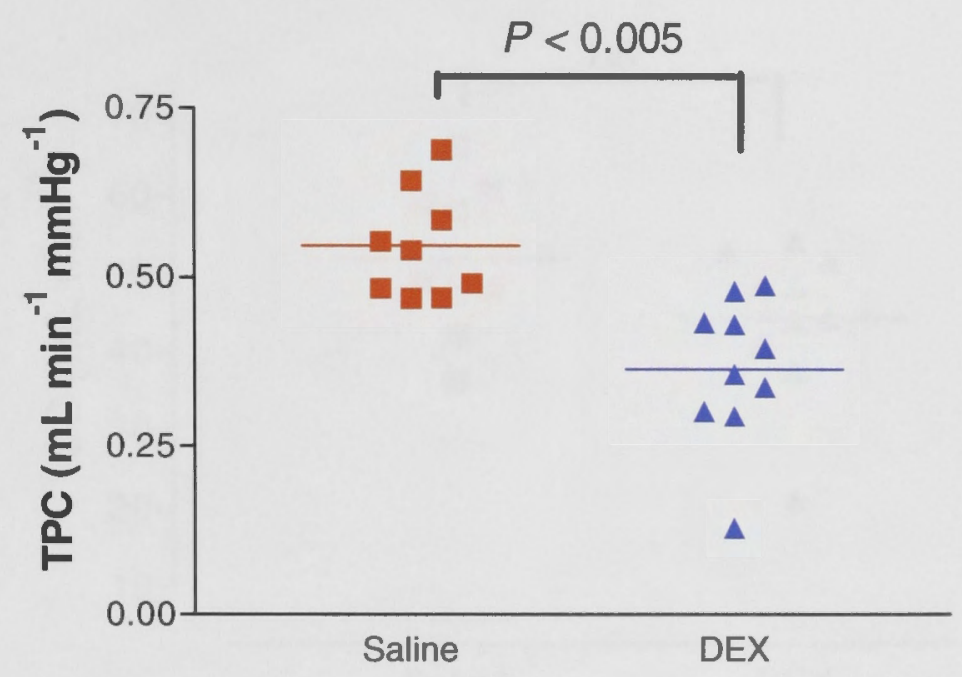

Figure 3.3: Comparisons of total peripheral resistance and total peripheral conductance between sham and DEX treatments. Saline, $\mathrm{n}=9 ; \boldsymbol{\Delta}$ DEX, $\mathrm{n}=10$.

\section{Heart Rate}

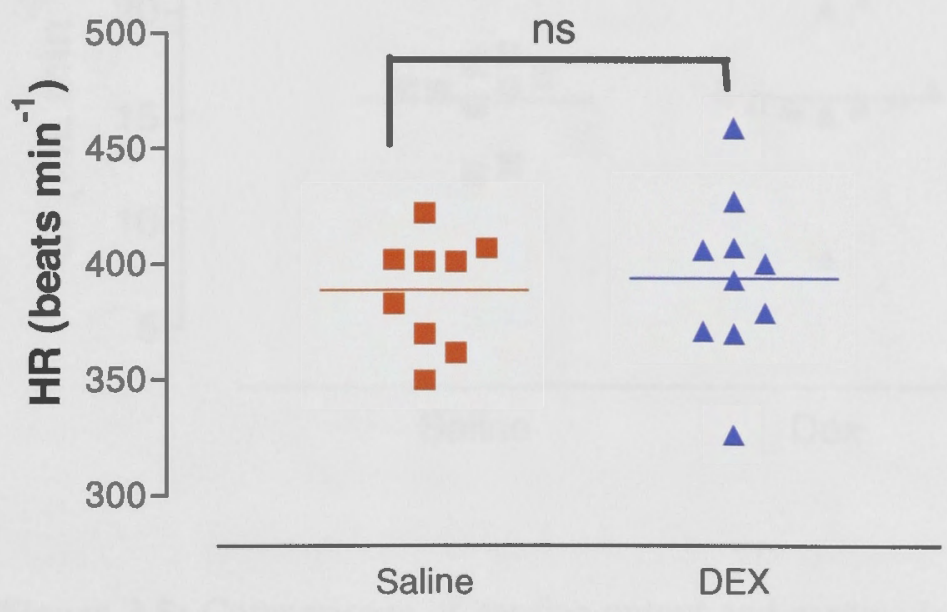

Figure 3.4: Comparisons of heart rate between sham and DEX treatments. Saline, $\mathrm{n}=9 ; \boldsymbol{\Delta}$ DEX, $\mathrm{n}=10$. 


\section{Cardiac Output}

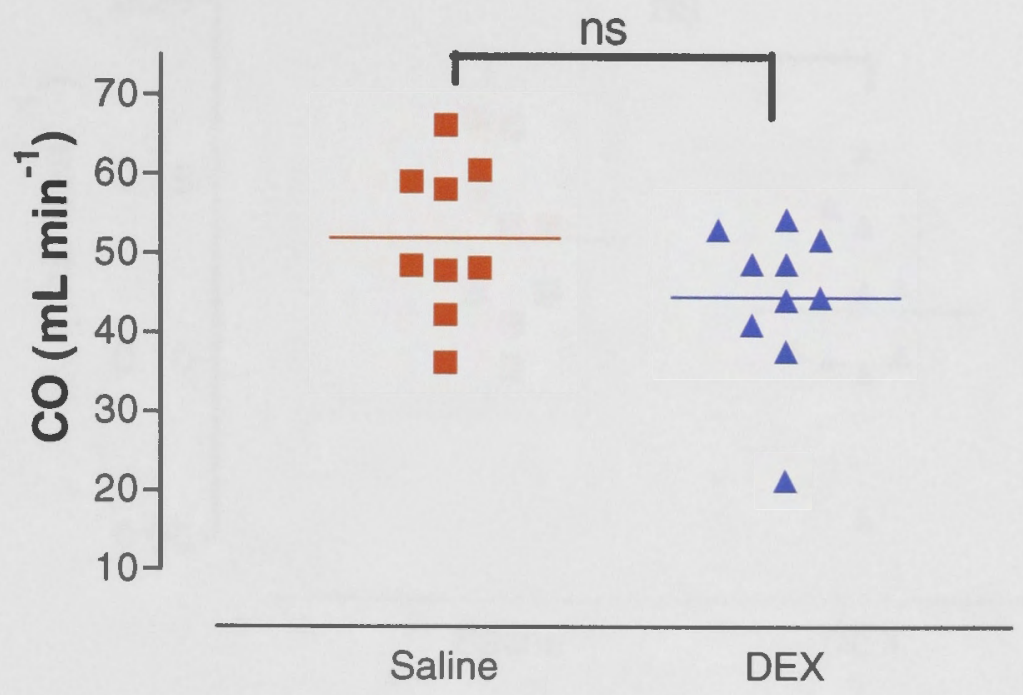

\section{Cardiac Index}

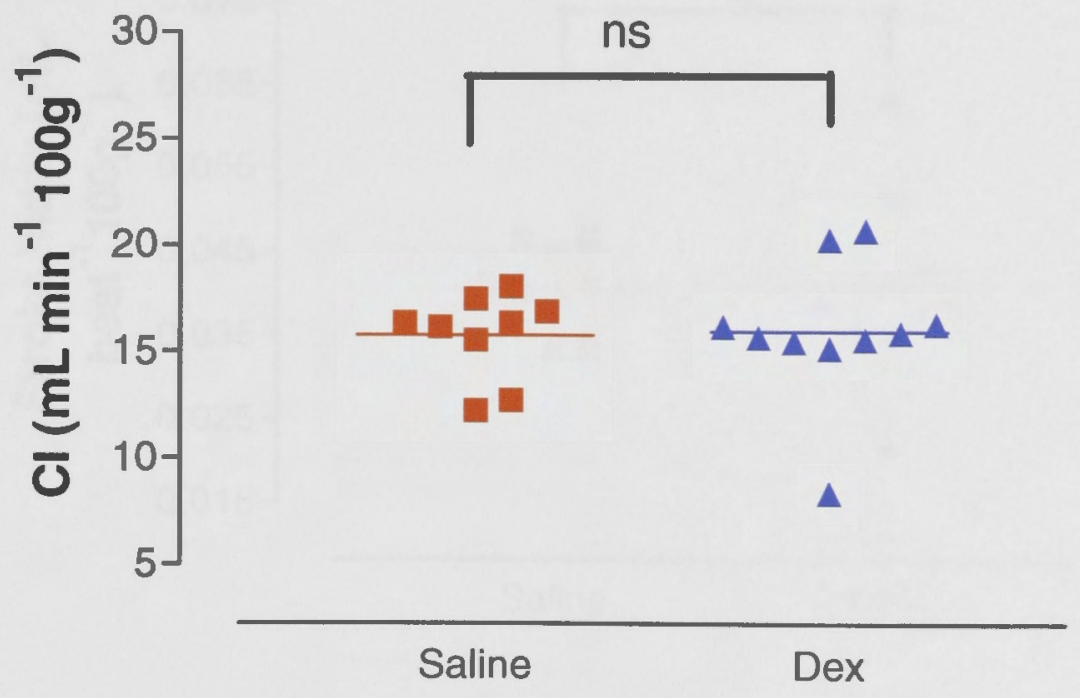

Figure 3.5: Comparisons of cardiac output and cardiac index between sham and DEX treatments. - Saline, $\mathrm{n}=9 ; \Delta \mathrm{DEX}, \mathrm{n}=10$. 


\section{Stroke Volume}

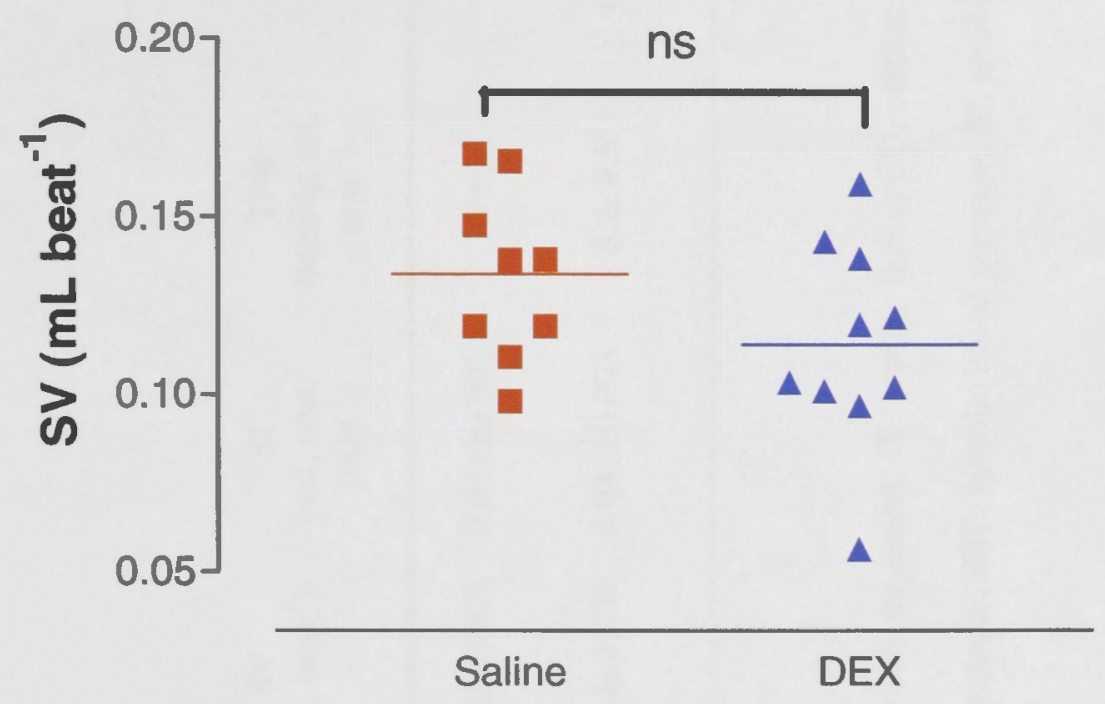

\section{Stroke Index}

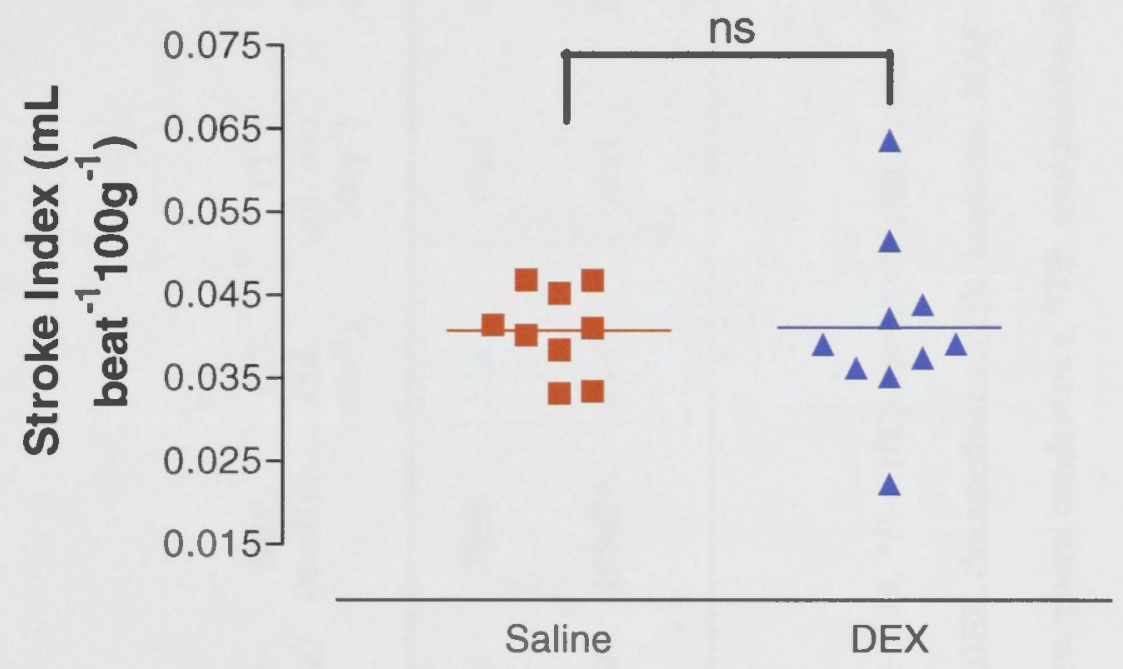

Figure 3.6: Comparisons of stroke volume and stroke index between sham and DEX treatments. $\approx$ Saline, $\mathrm{n}=9 ; \quad \Delta \mathrm{DEX}, \mathrm{n}=10$. 
Table 3.1: Central haemodynamic effects of sham and DEX treatments

\begin{tabular}{|c|c|c|c|c|c|c|c|c|c|c|}
\hline $\begin{array}{l}\text { Treatment } \\
\text { Groups }\end{array}$ & $\begin{array}{c}\text { MAP } \\
(\mathrm{mmHg})\end{array}$ & $\underset{(\mathrm{mmHg})}{\mathrm{SBP}}$ & $\begin{array}{c}\text { DBP } \\
(\mathrm{mmHg})\end{array}$ & $\begin{array}{c}\mathrm{CO} \\
(\mathrm{mL} \\
\left.\mathrm{min}^{-1}\right)\end{array}$ & $\begin{array}{c}\mathrm{CI} \\
\left(\mathrm{mL} \min ^{-1}\right. \\
\left.100 \mathrm{~g}^{-1}\right)\end{array}$ & $\begin{array}{l}\mathrm{HR} \\
\text { (beats } \\
\min ^{-1} \text { ) }\end{array}$ & $\begin{array}{c}\mathrm{SV} \\
\left(\mathrm{mL} \text { beat }^{-1}\right)\end{array}$ & $\begin{array}{c}\text { SI } \\
\left(\mathrm{mL} \mathrm{beat}^{-1}\right. \\
\left.100 \mathrm{~g}^{-1}\right)\end{array}$ & $\begin{array}{c}\text { TPR } \\
\left(\begin{array}{c}\mathrm{mmHg} \mathrm{mL}^{-1} \\
\left.\mathrm{~min}^{-1}\right)\end{array}\right.\end{array}$ & 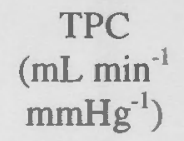 \\
\hline $\begin{array}{l}\text { Sham } \\
\mathbf{n}=9\end{array}$ & $95 \pm 4$ & $128 \pm 3$ & $78 \pm 5$ & $52 \pm 3$ & $16 \pm 1$ & $389 \pm 8$ & $0.134 \pm 0.008$ & $0.041 \pm 0.002$ & $1.9 \pm 0.1$ & $0.55 \pm 0.03$ \\
\hline $\begin{array}{c}\text { DEX } \\
n=10\end{array}$ & $127 \pm 8^{*}$ & $167 \pm 10^{*}$ & $108 \pm 7 *$ & $45 \pm 3$ & $16 \pm 1$ & $394 \pm 11$ & $0.114 \pm 0.009$ & $0.041 \pm 0.003$ & $3.2 \pm 0.5^{\dagger}$ & $0.36 \pm 0.03 *$ \\
\hline
\end{tabular}

Data were expressed as mean \pm SEM. ${ }^{*} P<0.005$ versus sham treatment, ${ }^{\dagger} P<0.05$ versus sham treatment. CI, cardiac index; CO, cardiac output; DBP, diastolic blood pressure; DEX, dexamethasone; HR, heart rate; MAP, mean arterial pressure; SBP, systolic blood pressure; SI, stroke index; SV, stroke volume; TPC, total peripheral conductance; TPR, total peripheral resistance. 


\subsubsection{Regional haemodynamics}

In comparison with sham treatment $(n=11)$, DEX treatment $(n=11)$ increased MAP $(P<0.05)$, SBP $(P<0.005)$ and DBP $(P<0.05)$ (Figure 3.7$)$ but did not significantly change renal, mesenteric or common iliac blood flow, vascular resistance or vascular conductance measurements (Figures 3.8-3.10, Table 3.2).

a) Mean Arterial Pressure

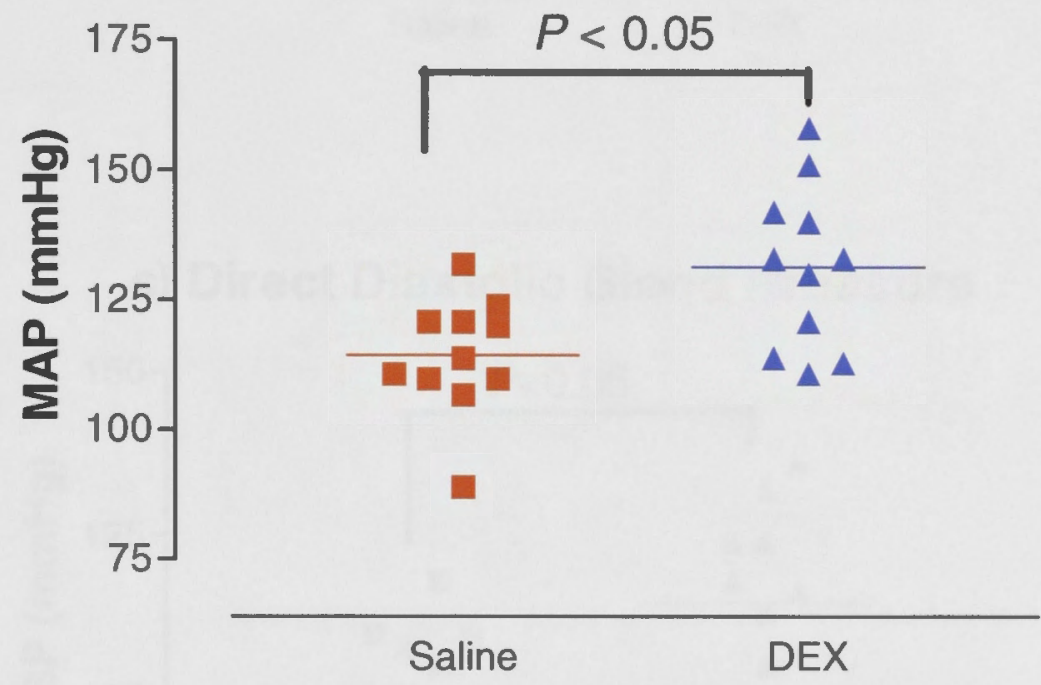


b) Direct Systolic Blood Pressure

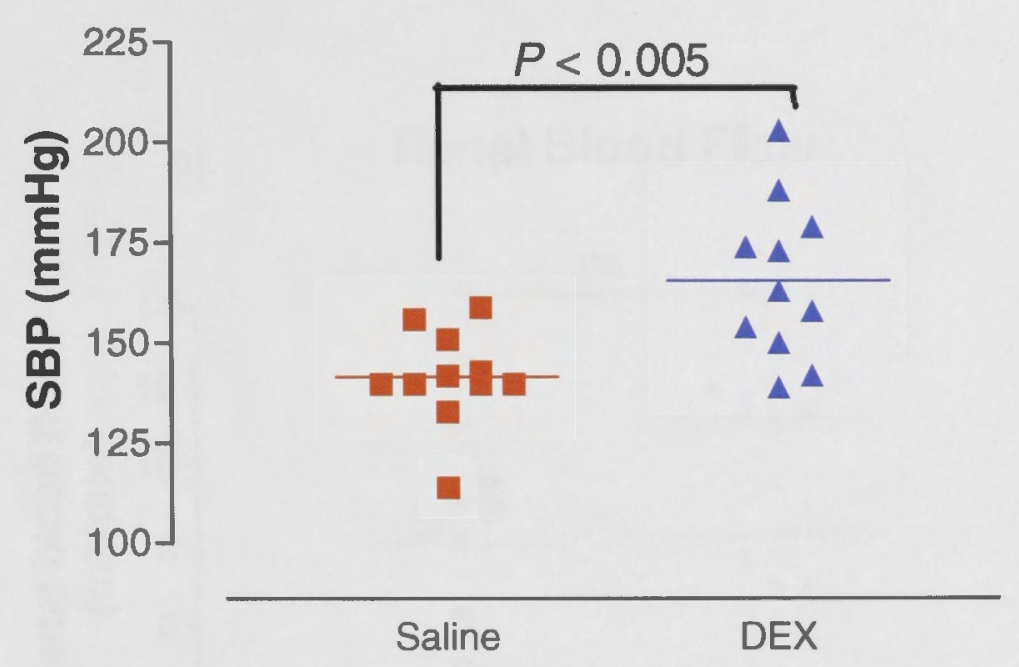

c) Direct Diastolic Blood Pressure

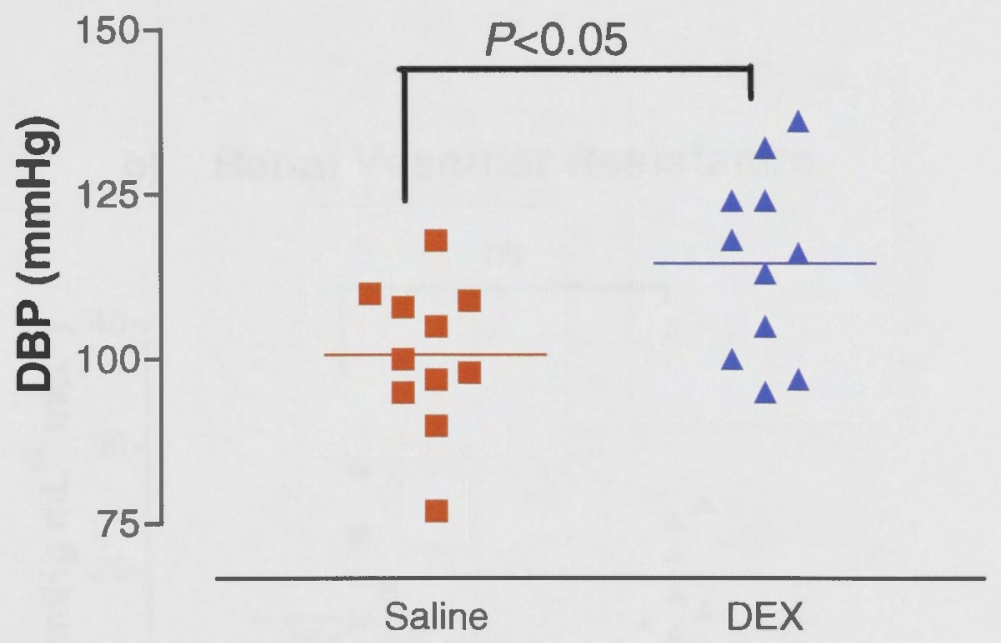

Figure 3.7: Comparisons of blood pressure readings between sham and DEX treatments. $\square$ Saline, $\mathrm{n}=11 ; \Delta \mathrm{DEX}, \mathrm{n}=11$. 
a) Renal Blood Flow

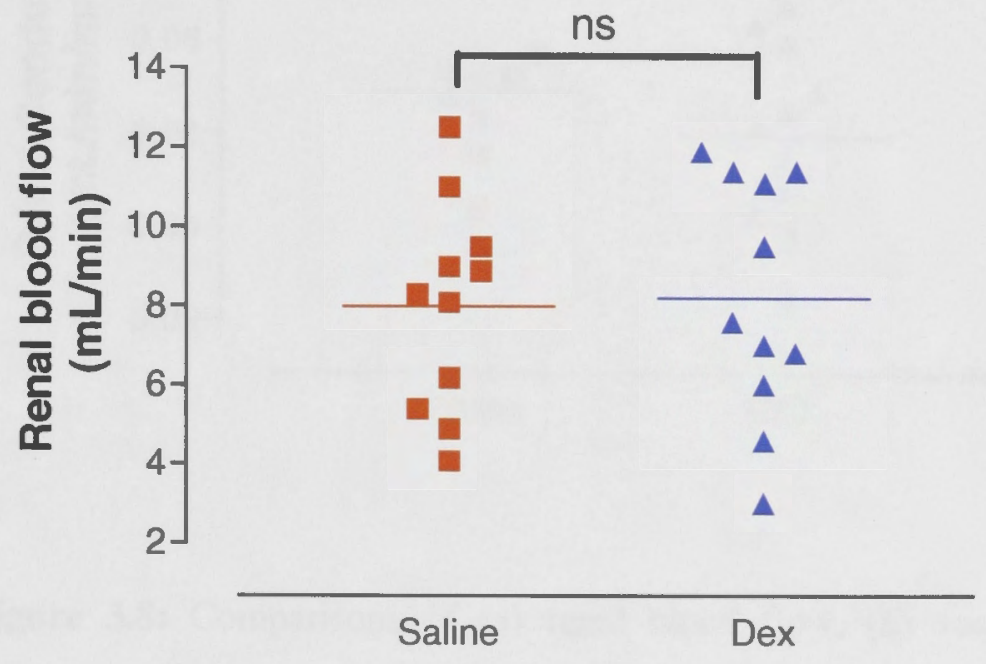

b) Renal Vascular Resistance

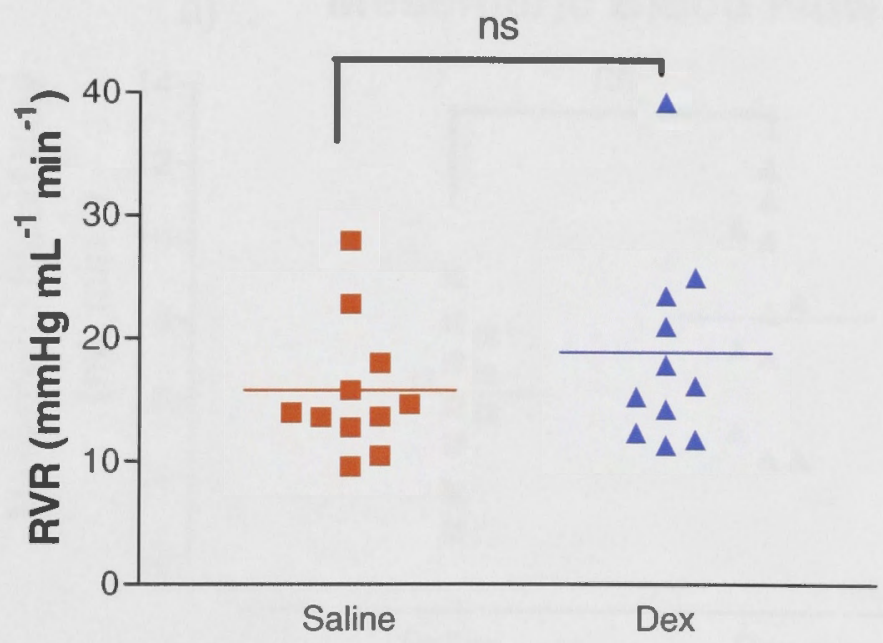




\section{c) Renal Vascular Conductance}

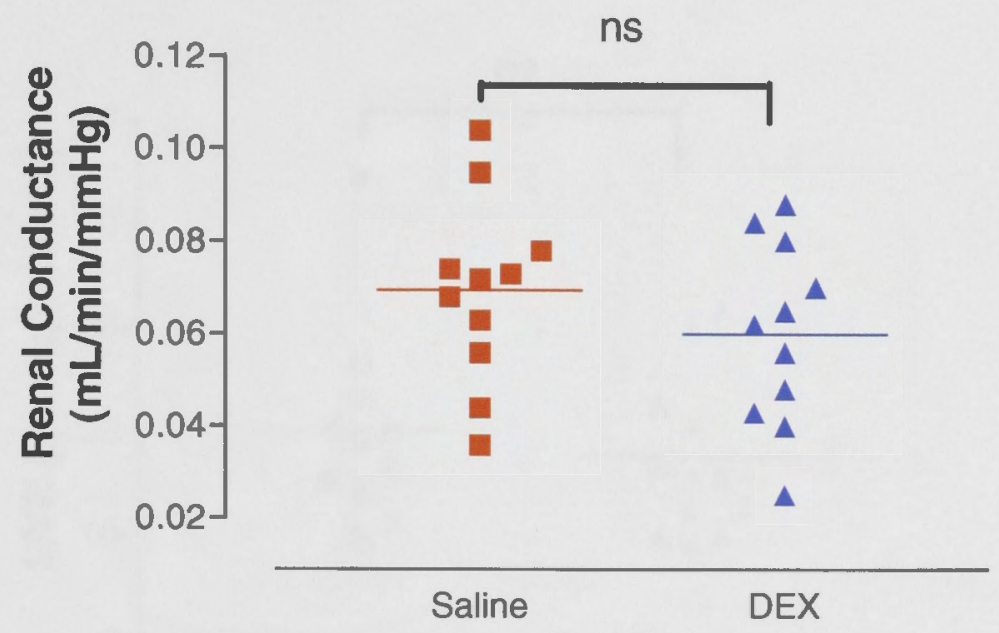

Figure 3.8: Comparisons of (a) renal blood flow, (b) vascular resistance and (c) vascular conductance in saline and DEX treatments. Saline, $\mathrm{n}=11 ; \Delta \mathrm{DEX}, \mathrm{n}=11$.

a) Mesenteric Blood Flow

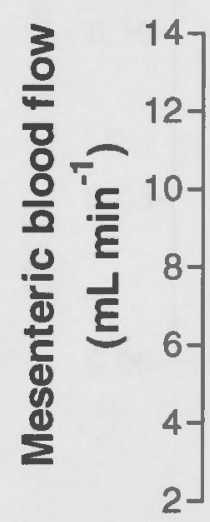

ns

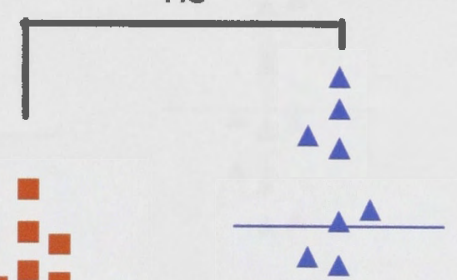

$\Delta$

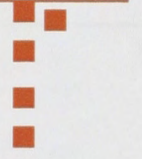

$\Delta \mathbf{\Delta}$

Saline

Dex 


\section{b) Mesenteric Vascular \\ Resistance}

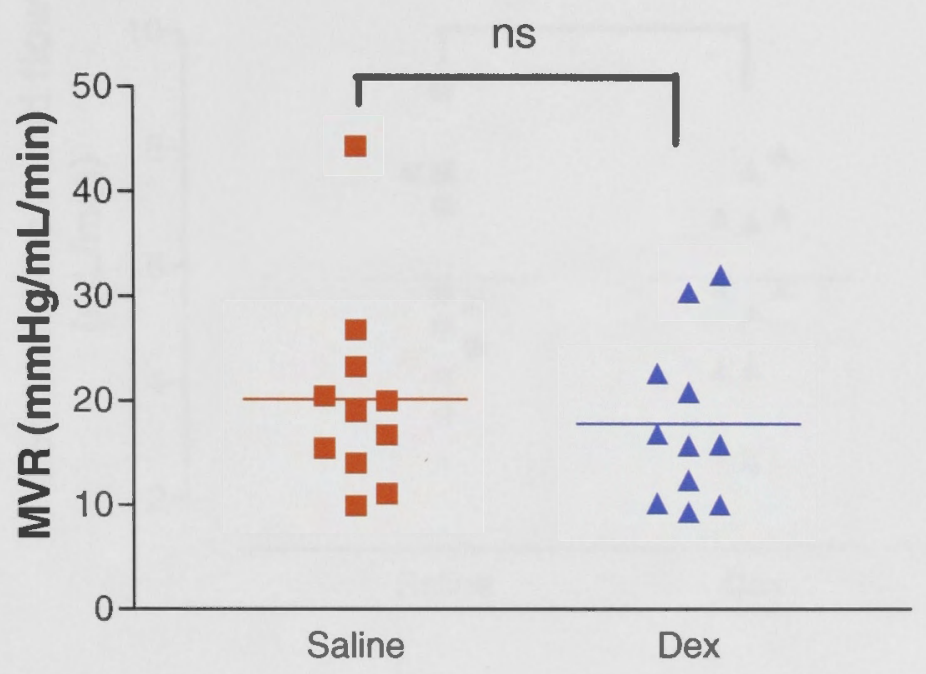

c) Mesenteric Vascular

Conductance

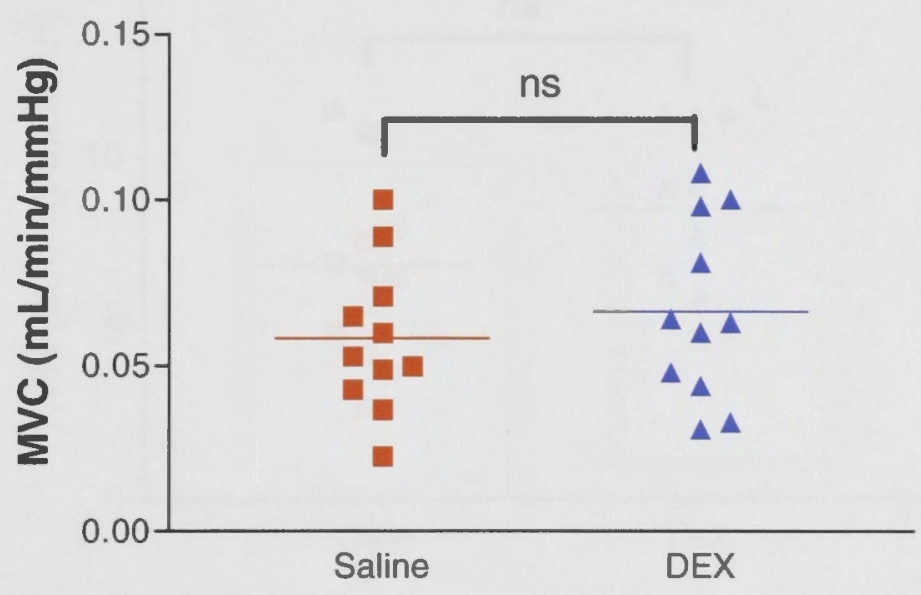

Figure 3.9: Comparisons of (a) mesenteric blood flow, (b) vascular resistance and (c) vascular conductance in saline and DEX treatments. - Saline, $\mathrm{n}=11$; $\Delta$ DEX, $\mathrm{n}=11$. 
a) Hindquarter Blood Flow

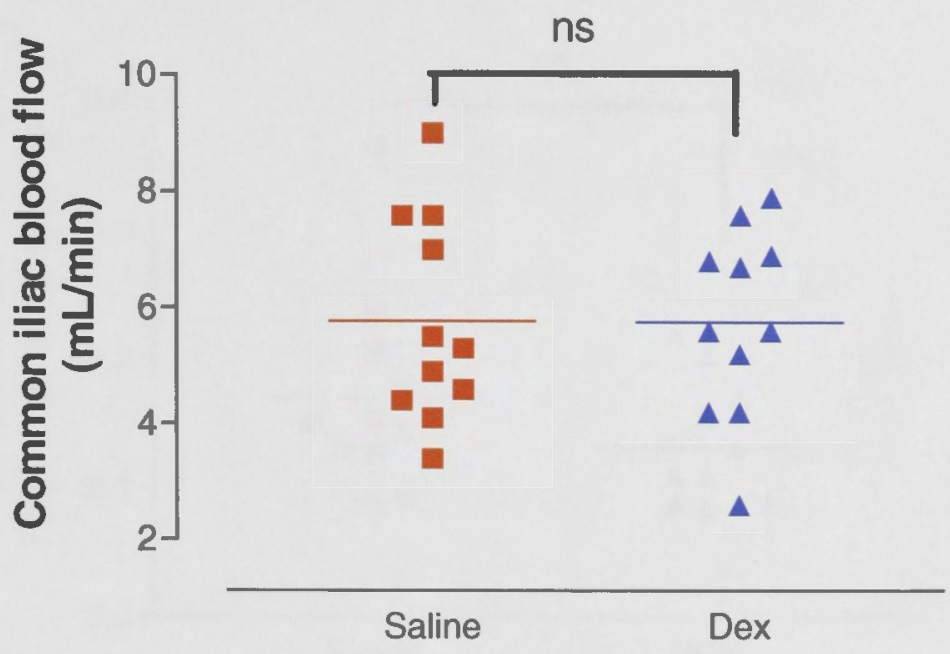

b) Hindquarter Vascular Resistance

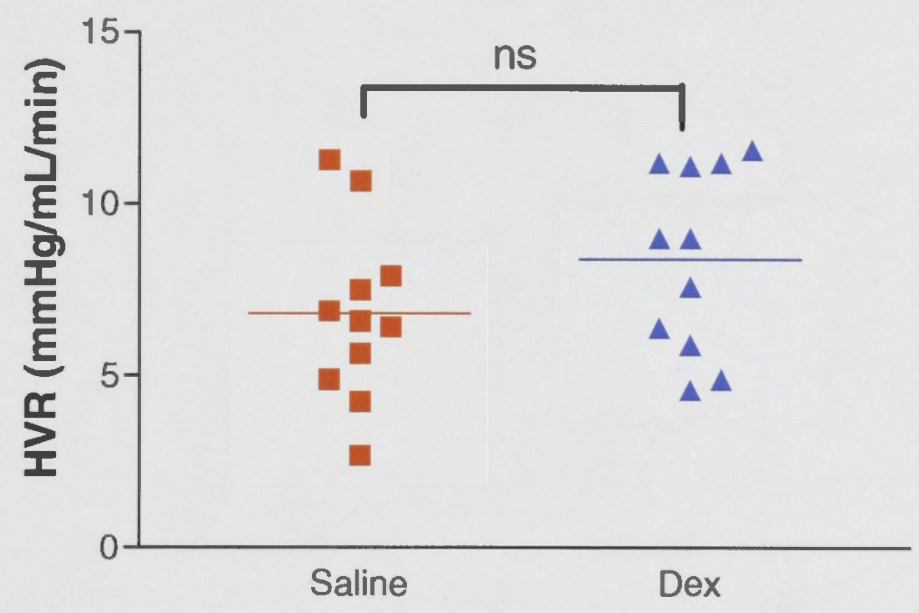




\section{c) Hindquarter Vascular}

Conductance

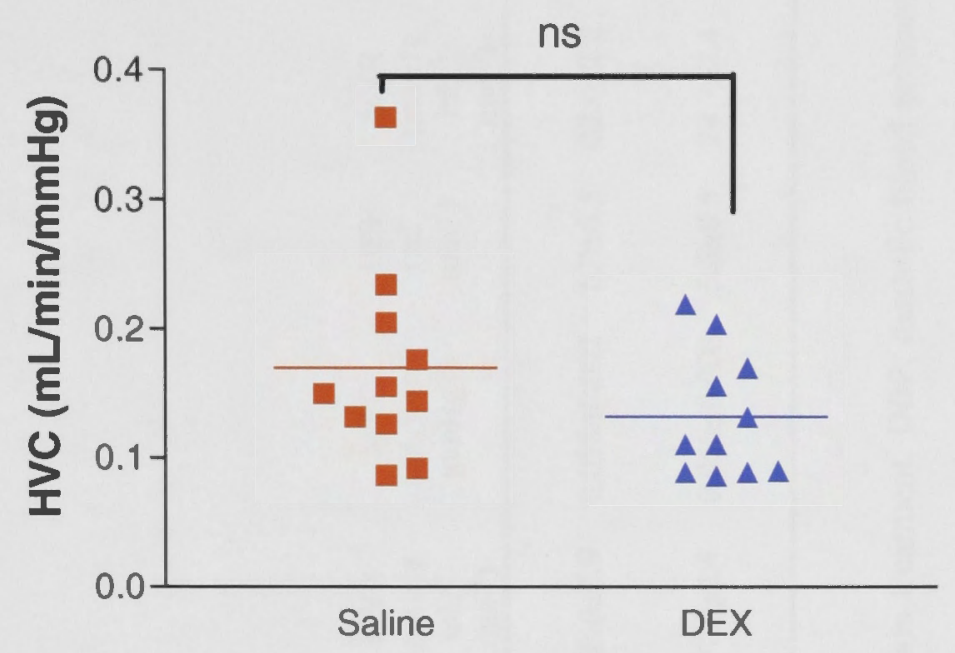

Figure 3.10: Comparisons of hindquarter blood flow, vascular resistance and vascular conductance in saline and DEX treatments. - Saline, $\mathrm{n}=11 ; \Delta \mathrm{DEX}, \mathrm{n}=11$. 
Table 3.2: Regional haemodynamic effects of sham and DEX treatments

\begin{tabular}{|c|c|c|c|c|c|c|c|c|c|c|c|c|}
\hline $\begin{array}{l}\text { Treatment } \\
\text { Groups }\end{array}$ & $\begin{array}{c}\text { MAP } \\
(\mathrm{mmHg})\end{array}$ & $\begin{array}{c}\text { SBP } \\
(\mathrm{mmHg})\end{array}$ & $\begin{array}{c}\text { DBP } \\
(\mathrm{mmHg})\end{array}$ & $\begin{array}{c}\text { RBF } \\
\left(\mathrm{mL} \min ^{-1}\right)\end{array}$ & $\begin{array}{c}\text { RVR } \\
\left(\mathrm{mmHg} \mathrm{mL}^{-1}\right. \\
\left.\min ^{-1}\right)\end{array}$ & $\begin{array}{c}\text { RVC } \\
\left(\mathrm{mL} \mathrm{min}^{-1}\right. \\
\left.\mathrm{mmHg}^{-1}\right)\end{array}$ & $\begin{array}{l}\text { MBF } \\
(\mathrm{mL} \\
\left.\mathrm{min}^{-1}\right)\end{array}$ & $\begin{array}{c}\text { MVR } \\
\left(\mathrm{mmHg}^{-1} \mathrm{~g}\right. \\
\mathrm{mL}^{-1} \\
\left.\mathrm{~min}^{-1}\right)\end{array}$ & $\begin{array}{c}\text { MVC } \\
\left(\mathrm{mL} \mathrm{min}^{-1}\right. \\
\left.\mathrm{mmHg}^{-1}\right)\end{array}$ & $\begin{array}{l}\text { HBF } \\
(\mathrm{mL} \\
\left.\mathrm{min}^{-1}\right)\end{array}$ & $\begin{array}{c}\text { HVR } \\
(\mathrm{mmHg} \\
\mathrm{mL}^{-1} \\
\left.\mathrm{~min}^{-1}\right) \\
\end{array}$ & $\begin{array}{c}\text { HVC } \\
\left(\mathrm{mL} \mathrm{min}^{-1}\right. \\
\left.\mathrm{mmHg}^{-1}\right)\end{array}$ \\
\hline $\begin{array}{c}\text { Sham } \\
n=11\end{array}$ & $114 \pm 3$ & $142 \pm 4$ & $101 \pm 4$ & $8.0 \pm 0.8$ & $16.0 \pm 1.6$ & $0.069 \pm 0.006$ & $6.0 \pm 0.6$ & $20.0 \pm 2.9$ & $0.058 \pm 0.007$ & $6.0 \pm 0.5$ & $22.0 \pm 2.2$ & $0.05 \pm 0.01$ \\
\hline $\begin{array}{l}\text { DEX } \\
n=11\end{array}$ & $132 \pm 5^{\dagger}$ & $166 \pm 6^{*}$ & $115 \pm 4^{\dagger}$ & $8.2 \pm 0.9$ & $18.9 \pm 2.5$ & $0.060 \pm 0.006$ & $8.2 \pm 0.8$ & $17.8 \pm 2.4$ & $0.066 \pm 0.008$ & $5.8 \pm 0.5$ & $24.7 \pm 2.4$ & $0.04 \pm 0.04$ \\
\hline
\end{tabular}

Data were expressed as mean \pm SEM. ${ }^{*} P<0.005$ versus sham treatment, ${ }^{\dagger} P<0.05$ versus sham treatment. DBP, diastolic blood pressure; DEX, dexamethasone; HBF, hindquarter blood flow; HVC, hindquarter vascular conductance; HVR, hindquarter vascular resistance; MAP, mean arterial pressure; MBF, mesenteric blood flow; MVC, mesenteric vascular conductance; MVR, mesenteric vascular resistance; RBF, renal blood flow; RVC, renal vascular conductance; RVR, renal vascular resistance; SBP, systolic blood pressure. 


\subsubsection{Body weight}

There was an initial decrease in body weight from $285 \pm 4 \mathrm{~g}$ on day $\mathrm{T} 0$, following the initiation of DEX treatment, to $277 \pm 4 \mathrm{~g}$ on day T2 $(P<0.0005)$ (Figure 3.11). Subsequently, body weight remained stable at that level throughout the entire treatment period $(n=21)$. On the other hand, rats on sham treatment gained weight progressively over the entire experimental period from $280 \pm 7 \mathrm{~g}$ on day T0 to $328 \pm 7 \mathrm{~g}$ on day $\mathrm{T} 11(\mathrm{n}=20, P<0.0005)$.

\section{Body Weight}

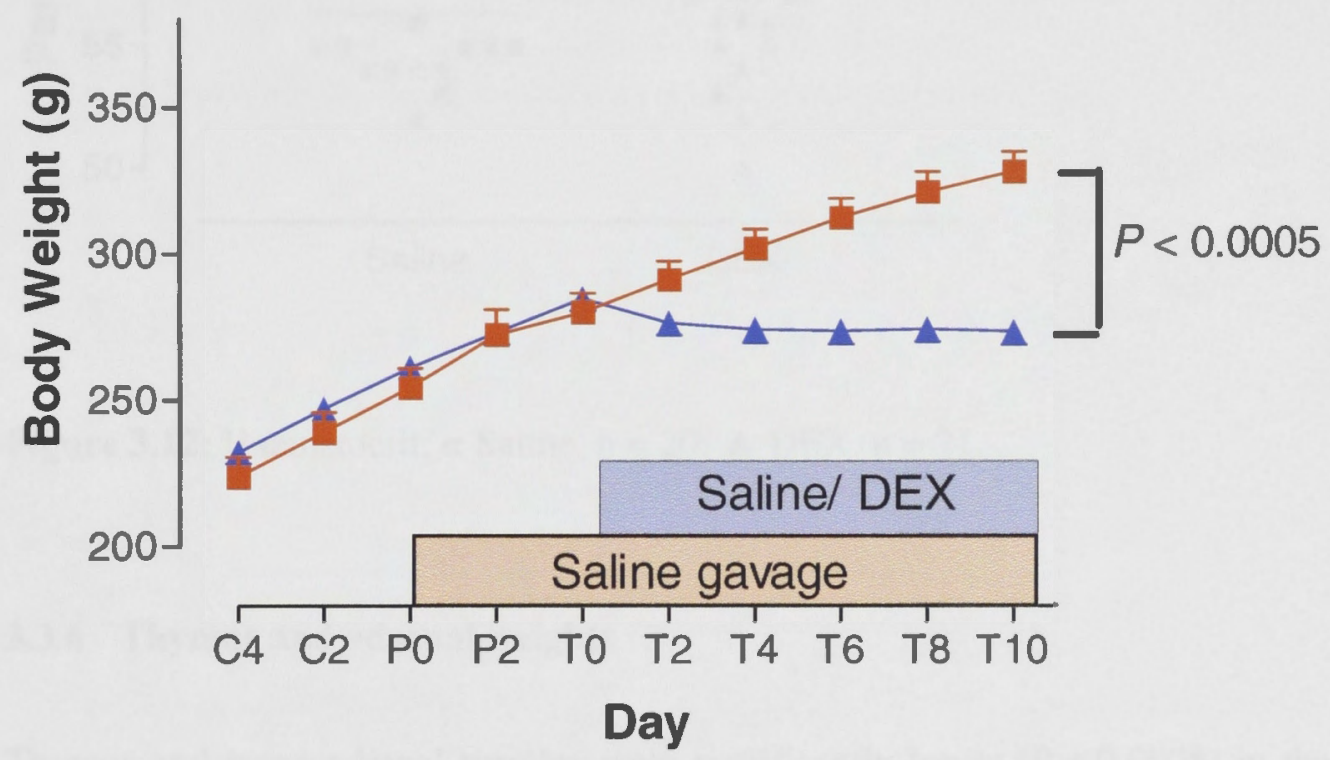

Figure 3.11: Body weight. - Saline, $n=20 ; \Delta$ DEX, $n=21$. 


\subsubsection{Haematocrit}

There was no significant difference in haematocrit between saline $(56 \pm 1 \%$, $\mathrm{n}=20)$ and DEX-treated rats $(58 \pm 1 \%, \mathrm{n}=21)$ (Figure 3.12).

\section{Haematocrit}

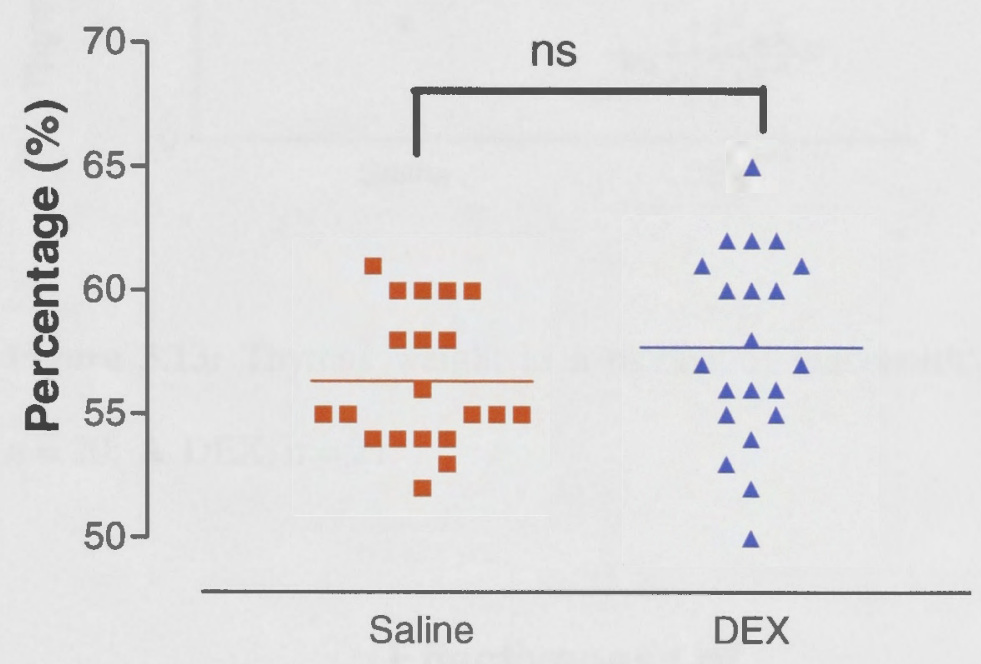

Figure 3.12: Haematocrit. $=$ Saline, $n=20 ; \Delta D E X, n=21$.

\subsubsection{Thymus and adrenal weights}

Thymus and mean adrenal weights were significantly lower $(P<0.0005)$ in the DEX-treated group (thymus: $53 \pm 5$; adrenal: $3.9 \pm 0.2 \mathrm{mg} / 100 \mathrm{~g}$ body weight, $\mathrm{n}=21$ ) compared with sham treatment group (thymus: $163 \pm 9$; adrenal: $6 \pm 0.2$ $\mathrm{mg} / 100 \mathrm{~g}$ body weight, $\mathrm{n}=20$ ) (Figures 3.13 and 3.14 ). 


\section{Glucocorticoid Activity}

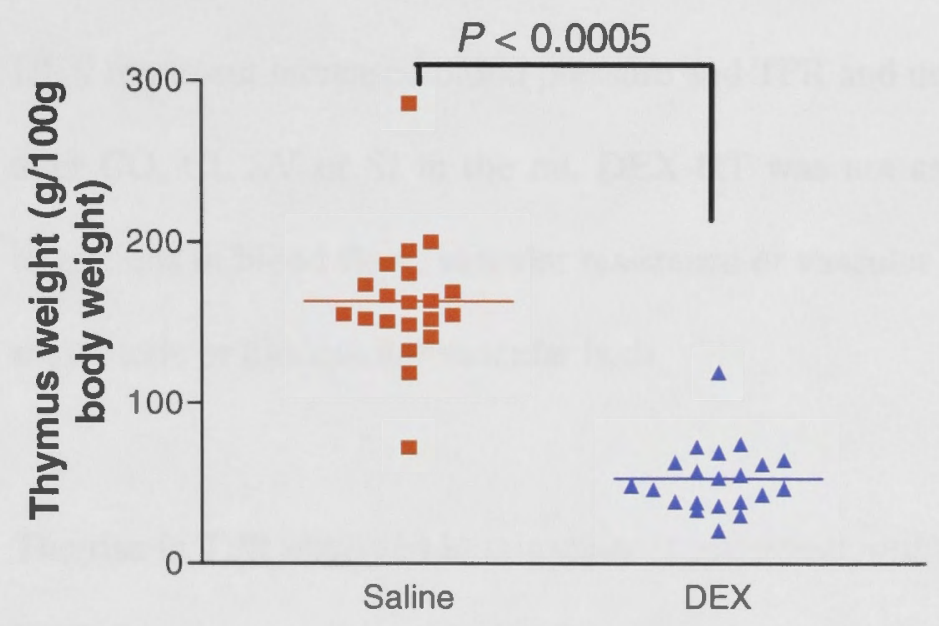

Figure 3.13: Thymus weight as a marker of glucocorticoid activity. - Saline, $\mathrm{n}=20 ; \Delta$ DEX, $\mathrm{n}=21$.

\section{Effectiveness of Dexamethasone Administration}

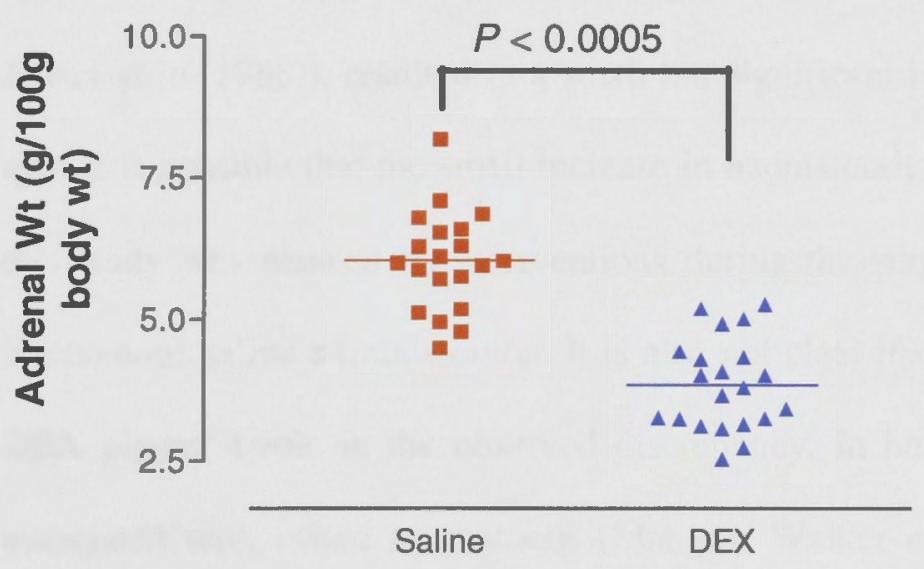

Figure 3.14: Adrenal weight as a marker of effective DEX administration. Saline, $\mathrm{n}=20 ; \boldsymbol{\Delta}$ DEX, $\mathrm{n}=21$. 


\subsection{DISCUSSION}

DEX treatment increased blood pressure and TPR and decreased TPC; but did not alter $\mathrm{CO}, \mathrm{CI}, \mathrm{SV}$ or SI in the rat. DEX-HT was not associated with significant alterations in blood flow, vascular resistance or vascular conductance in the renal, mesenteric or hindquarter vascular beds.

The rise in TPR observed in this study is consistent with the results obtained from DEX-hypertensive humans (Pirpiris, Sudhir et al. 1992) and dogs (Nakamoto, Suzuki et al. 1995). It is unlikely that alterations in blood viscosity, which could in turn alter vascular resistance and conductance, contribute to the increase in TPR in DEX-HT. In this study, DEX-HT was not associated with any change in haematocrit. This finding was different from those reported by $\mathrm{Hu}$ et. al. and Tonolo et. al. showing that DEX, at lower doses $(20 \mu \mathrm{g} / \mathrm{kg} /$ day which is approximately 5-6 $\mu \mathrm{g} / \mathrm{rat} / \mathrm{day}$ (Hu, Zhang et al. 2006) and $2 \mu \mathrm{g} / \mathrm{rat} /$ day (Tonolo, Fraser et al. 1988)), resulted in a small but significant increase in haematocrit in rats. It is possible that the small increase in haematocrit due to DEX treatment in this study was masked by interventions during the surgical procedures such as intravenous saline administration. It is also not clear if differences in the dose of DEX played a role in the observed discrepancy. In humans, DEX-HT was not associated with raised haematocrit (Mangos, Walker et al. 2006). DEX-HT in dogs was not accompanied by a significant alteration in plasma osmolality (Nakamoto, Suzuki et al. 1991). 
Another explanation for the increase in TPR is DEX-induced changes to vascular tone. There is increasing evidence linking deficiency in the vasorelaxant nitric oxide to the pathogenesis of DEX-HT. Plasma NOx, a marker of NO synthesis, was significantly reduced in DEX-hypertensive humans (Mangos, Walker et al. 2006) and rodents (Wallerath, Witte et al. 1999; Wallerath, Godecke et al. 2004). There are also reports implicating DEX in potentiating vascular pressor responsiveness to noradrenaline and/or angiotensin II in humans (Pirpiris, Sudhir et al. 1992), rats (Handa, Kondo et al. 1984) and dogs (Nakamoto, Suzuki et al. 1991).

The observed increase in TPR and decrease in TPC may reflect cumulative changes from a number of peripheral vascular beds including those evaluated in this study. In addition, these changes could also be a consequence of alterations in a site or sites other than the renal, mesenteric or hindquarter vascular system.

This study did not show any significant differences in the blood flow, resistance or conductance in the renal, mesenteric or hindquarter vascular beds. However, acute DEX administration at a significantly higher dose $(3 \mathrm{mg} / \mathrm{kg}$ or approximately $900 \mu \mathrm{g} / \mathrm{rat} / \mathrm{day}$, continuous infusion over 24 hours) in conscious rats resulted in increased MAP, and decreased renal and mesenteric blood flows and vascular conductance (Gardiner, Kemp et al. 1996). These differences could be due to a variety of factors such as DEX dose differences, duration of DEX treatment and the use of anaesthesia. Data obtained from studies using high DEX dose need to be interpreted with caution as volume depletion through diuresis 
(Chabria and Gaitonde 1966) due to high dose DEX may confound haemodynamic assessments of DEX-HT.

In this study, haemodynamic parameters were measured under pentobarbitone general anaesthesia $(60 \mathrm{mg} / \mathrm{kg}$, intraperitoneal injection). There are reports documenting the impact of anaesthetic agents on cardiovascular measurements (Koeppen, Katz et al. 1979; Janssen, De Celle et al. 2004; Saha, Saha et al. 2007). Pentobarbitone ( $50 \mathrm{mg} / \mathrm{kg}$, i.p.) has been shown to decrease $\mathrm{CI}$ and $\mathrm{HR}$ in mice (Janssen, De Celle et al. 2004). Koeppen et al. have also demonstrated that pentobarbitone significantly reduced renal blood flow. Mechanical ventilation can also affect haemodynamic parameters. Sellden et al. have shown that changing ventilatory pattern in rats under choralose general anaesthesia from spontaneous breathing to artificial ventilation resulted in a significant decrease in CO and SV; and a significant increase in MAP, HR and TPR (Sellden, Sjovall $e t$ al. 1986). In view of these limitations, appropriately-matched controls that also underwent similar experimental and surgical conditions were used to minimise the confounding effects associated with acute haemodynamic experiments.

Thymus and adrenal weights were used as surrogate markers of the effectiveness of DEX administration as effective DEX delivery will decrease thymus and adrenal weights. DEX induces apoptosis in thymocytes and thus, results in thymic atrophy (Sun, Dinsdale et al. 1992; Zavitsanou, Nguyen et al. 2007). Adrenal atrophy was due to suppression of endogenous glucorticoid production by exogenous DEX administration. 


\subsection{CONCLUSION}

DEX-HT is associated with a decrease in TPC and increase in TPR. These changes cannot be ascribed simply to individual alterations in conductance or resistance in the renal, mesenteric or hindquarter circulations.

The haemodynamics of DEX-HT in rats are different from those in ACTH-HT in rats. ACTH-HT is associated with raised $\mathrm{CO}$ and renal vascular resistance, and DEX-HT with increase in TPR and decrease in TPC. These findings support the notion that the mechanisms of DEX-HT (synthetic glucocorticoid) are different from those of ACTH-HT (ACTH-stimulated endogenous glucocorticoid). 


\section{CHAPTER 4}

Role of Total Peripheral Resistance and Conductance in Dexamethasone-Induced Hypertension in the Rat 


\subsection{INTRODUCTION}

As shown in Chapter 3, DEX-HT in rats is associated with an increase in TPR and decrease in TPC. In this study, using ultrasonic transit-time flowmetry as described in Chapter 2, we further evaluated the role of TPR and TPC in DEX-HT in rats by the administration of the vasodilator minoxidil at a dose known to reduce TPR in rats (Wen, Fraser et al. 1999).

\subsection{METHODS}

\subsubsection{Experimental animals}

This study was approved by the Animal Experimentation Ethics Committee of the Australian National University (Protocol No. J.HB.22.06). The general methodology for this study was as described in Chapters 2 and 3 (Section 3.2).

Twenty rats weighing 200-250 g were randomly divided into 2 groups, with 10 rats in each group.

1. Minoxidil $(15 \mathrm{mg} / \mathrm{kg} /$ day, gavage $)+$ saline $(0.2 \mathrm{~mL} / \mathrm{rat} /$ day, $\mathrm{sc})$

2. Minoxidil $(15 \mathrm{mg} / \mathrm{kg} /$ day, gavage $)+\operatorname{DEX}(20 \mu \mathrm{L} / \mathrm{rat} /$ day, sc $)$ 
Minoxidil suspension (15 $\mathrm{mg} / \mathrm{mL})$ was prepared daily by mixing minoxidil powder (Sigma-Aldrich, St. Louis, USA) in $0.9 \% \mathrm{NaCl}$ solution. All rats received minoxidil suspension ( $15 \mathrm{mg} / \mathrm{kg} /$ day or $0.1 \mathrm{~mL} / 100 \mathrm{~g}$ body weight) by gavage daily between 3-4 pm for 16 days from P0 to T11. From day T0-T11, one group was co-administered DEX $(n=10)$, and the other group, saline $(n=10)$ via subcutaneous injections daily between $3-4 \mathrm{pm}$.

\subsubsection{Blood pressure and body weight measurement}

Tail-cuff SBP measurement was performed on alternate days at 9-11 am using the method as described in Sections 2.5.1 (Chapter 2) and 3.2.2 (Chapter 3). Body weights were measured on alternate days after the tail-cuff SBP measurements.

Direct blood pressure and heart rate (HR) measurements were recorded as per the method described in Sections 2.5.2 and 2.6.2 of Chapter 2; and Section 3.2.2 of Chapter 3. In this study, only central haemodynamic profiles were evaluated. Surgical approaches for CO measurements are described in Section 2.8.3.

\subsubsection{Calculation of other haemodynamic parameters}

The following haemodynamic parameters were calculated.

- Cardiac Index $(\mathrm{CI})=\mathrm{CO} / 100 \mathrm{~g}$ body weight

- $\quad$ Stroke Volume (SV) = CO/HR 
- $\quad$ Stroke Index $(\mathrm{SI})=\mathrm{CI} / \mathrm{HR}$

- $\quad \mathrm{TPR}=\mathrm{MAP} / \mathrm{CO}$

- $\quad \mathrm{TPC}=1 / \mathrm{TPR}$

\subsubsection{Haematocrit estimation}

Haematocrit estimation was performed as described in Sections 2.9 and 3.2.4.

\subsubsection{Thymus and adrenal weights}

Thymus wet weight, expressed relative to body weight (grams thymus weight per $100 \mathrm{~g}$ body weight), was used as a marker of glucocorticoid activity. Adrenal wet weight, expressed relative to body weight (grams adrenal weight per $100 \mathrm{~g}$ body weight), was used to assess DEX treatment efficacy. The surgical methods were as described in Section 2.8.5.1 of Chapter 2.

\subsubsection{Statistical analysis}

Data from this study was compared with central haemodynamic data from rats described in Chapter 3, which were used as controls.

Controls:

1. Vehicle (saline, $0.1 \mathrm{~mL} / 100 \mathrm{~g}$, gavage $)+$ Saline $(0.2 \mathrm{~mL} / \mathrm{rat}, \mathrm{s.c}),. \mathrm{n}=9$ 
2. Vehicle (saline, $0.1 \mathrm{~mL} / 100 \mathrm{~g}$, gavage $)+\mathrm{DEX}(20 \mu \mathrm{L}$ rat, s.c. $), \mathrm{n}=10$

Results were expressed as mean \pm SEM. Statistical analysis were as described in Section 2.12 of Chapter 2.

\subsection{RESULTS}

\subsubsection{Tail-cuff systolic blood pressure}

DEX treatment increased tail-cuff SBP from $125 \pm 4$ to $140 \pm 3 \mathrm{mmHg}$ (T0-T10, $P<0.005, \mathrm{n}=9$ ) whilst sham injections with saline did not alter SBP (T0: $128 \pm$ 4 to $\mathrm{T} 10: 120 \pm 4 \mathrm{mmHg}, \mathrm{n}=10$ ). Tail-cuff SBP in the DEX-treated group $(\mathrm{n}=10)$ was significantly higher than in the sham treatment group $(P<0.005$, $\mathrm{n}=9$ ). Tail-cuff SBP in the minoxidil + DEX group tended to rise from day T0 to day T10 but was not statistically significant $(P=0.086, \mathrm{n}=10)$. Minoxidil treatment did not alter SBP in saline-treated rats (Figure 4.1). 


\section{Tail-cuff Systolic Blood \\ Pressure}

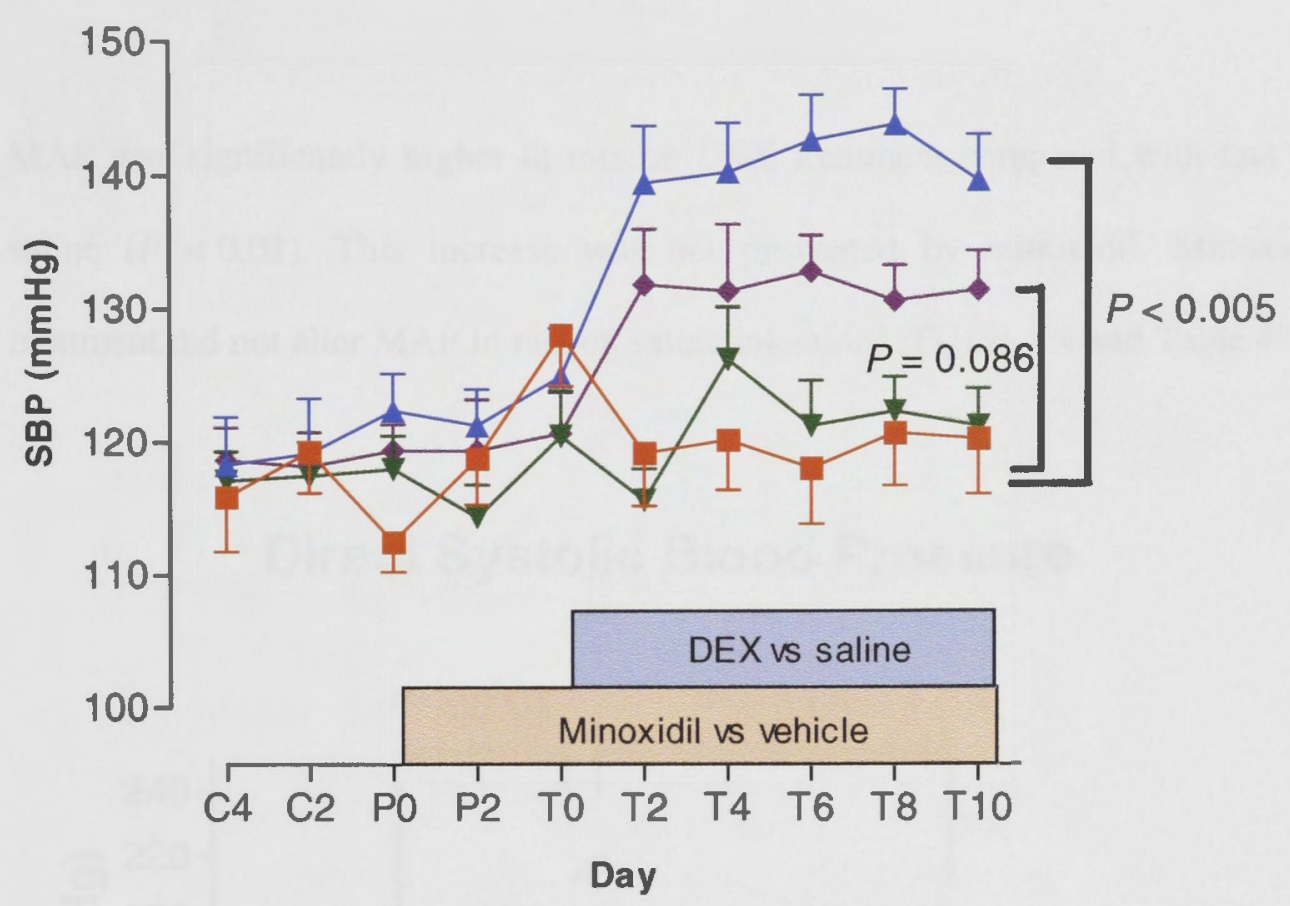

Figure 4.1: Tail-cuff SBP. $\approx$ Vehicle + Saline, $n=9 ; \Delta$ Vehicle + DEX, $n=10$;

$\nabla$ Minoxidil + Saline, $\mathrm{n}=10 ;-$ Minoxidil + DEX, $\mathrm{n}=10$.

\subsubsection{Direct blood pressure measurements}

Both direct systolic and diastolic blood pressure (DBP) measurements obtained on the last day of experiment (T11) were significantly higher in the DEX-treated rats compared with saline-treated control rats. Rats on minoxidil + DEX treatments had significantly lower SBP than those on DEX treatment alone $\left(P^{\prime}<0.05\right)$. However, there was no significant difference in direct DBP between DEX- and minoxidil + DEX-treated rats. Interestingly, minoxidil treatment significantly 
increased direct DBP $\left(P^{\prime}<0.05\right)$ but not direct SBP or MAP in rats receiving saline injections (Figures 4.2 and 4.3; and Table 4.1).

MAP was significantly higher in rats on DEX treatment compared with rats on saline $\left(P^{\prime}<0.01\right)$. This increase was not prevented by minoxidil. Minoxidil treatment did not alter MAP in rats on saline injections (Figure 4.4 and Table 4.1).

\section{Direct Systolic Blood Pressure}

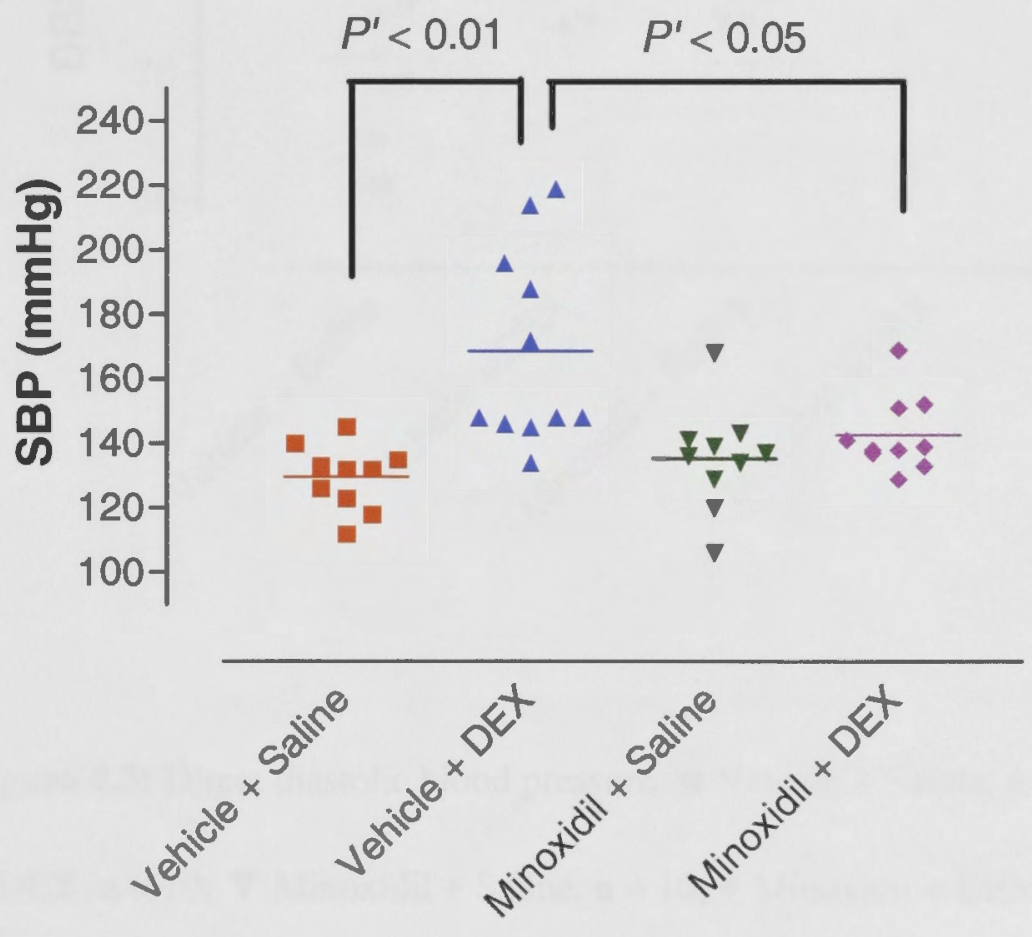

Figure 4.2: Direct systolic blood pressure. Vehicle + Saline, $n=9 ; \boldsymbol{\Delta}$ Vehicle + DEX, $\mathrm{n}=10 ; \boldsymbol{\nabla}$ Minoxidil + Saline, $\mathrm{n}=10 ;$ Minoxidil + DEX, $\mathrm{n}=10$. 


\section{Direct Diastolic Blood Pressure}

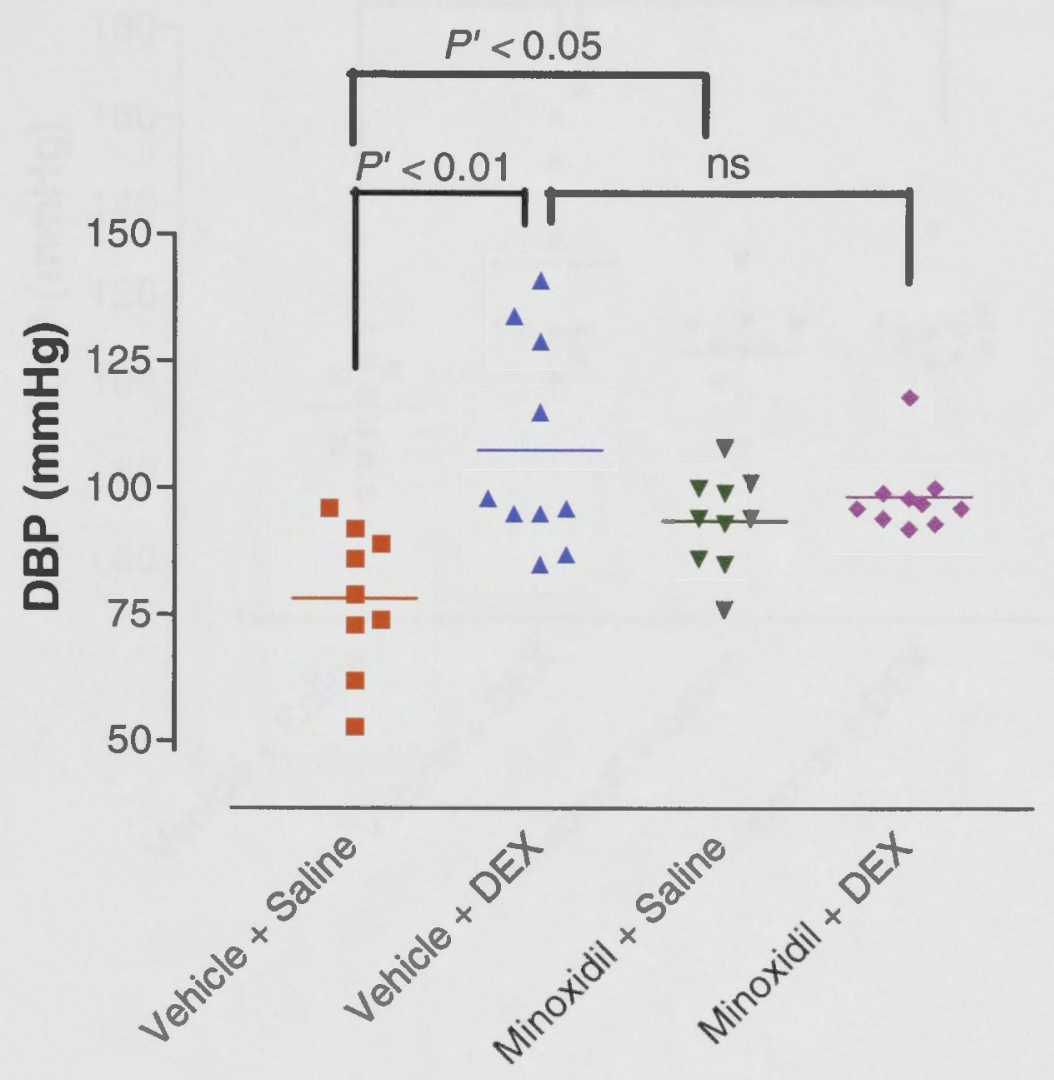

Figure 4.3: Direct diastolic blood pressure. $n$ Vehicle + Saline, $n=9 ; \boldsymbol{\Delta}$ Vehicle + DEX, $\mathrm{n}=10 ; \boldsymbol{\nabla}$ Minoxidil + Saline, $\mathrm{n}=10 ;-$ Minoxidil + DEX, $\mathrm{n}=10$. 


\section{Mean Arterial Pressure}

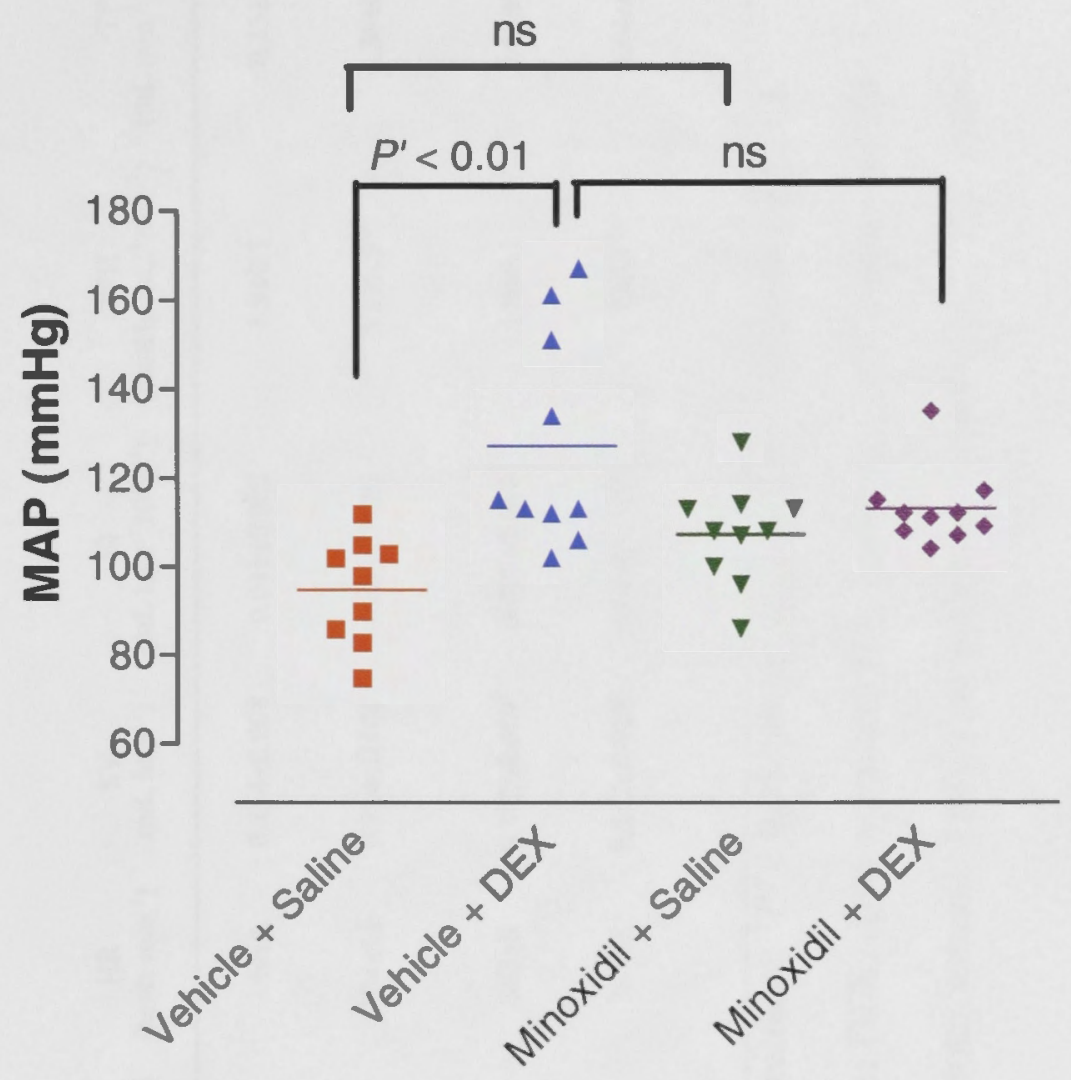

Figure 4.4: Mean arterial pressure. $\square$ Vehicle + Saline, $n=9 ; \Delta$ Vehicle + DEX, $\mathrm{n}=10 ; \boldsymbol{\nabla}$ Minoxidil + Saline, $\mathrm{n}=10 ;$ Minoxidil + DEX, $\mathrm{n}=10$ 
Table 4.1: $\quad$ Central haemodynamic effects of sham and dexamethasone treatments with and without minoxidil

\begin{tabular}{|c|c|c|c|c|c|c|c|c|c|c|}
\hline $\begin{array}{l}\text { Treatment } \\
\text { group }\end{array}$ & $\begin{array}{l}\text { MAP } \\
(\mathrm{mmHg})\end{array}$ & $\underset{(\mathrm{mmHg})}{\mathrm{SBP}}$ & $\begin{array}{c}\mathrm{DBP} \\
(\mathrm{mmHg})\end{array}$ & $\begin{array}{c}\mathrm{CO} \\
\left(\mathrm{mL} \min ^{-1}\right)\end{array}$ & $\underset{\left(\mathrm{mL} \min ^{-1} 100 \mathrm{~g}^{-1}\right)}{\mathrm{Cl}}$ & $\begin{array}{c}\text { HR } \\
\text { (beats } \min ^{-1} \text { ) }\end{array}$ & $\begin{array}{c}\text { SV } \\
\left(\mathrm{mL} \text { beat }^{-1}\right)\end{array}$ & $\begin{array}{c}\mathrm{SI} \\
(\mathrm{mL} \text { beat } \\
\left.{ }^{-1} 100 \mathrm{~g}^{-1}\right)\end{array}$ & $\begin{array}{c}\text { TPR } \\
\left(\mathrm{mmHg} \mathrm{mL} \mathrm{min}^{-1}\right)\end{array}$ & $\begin{array}{c}\text { TPC } \\
\left(\mathrm{mL} \mathrm{min}^{-1} \mathrm{mmHg}^{-1}\right)\end{array}$ \\
\hline $\begin{array}{l}\text { Sham } \\
n=9\end{array}$ & $95 \pm 4$ & $128 \pm 3$ & $78 \pm 5$ & $52 \pm 3$ & $16 \pm 1$ & $389 \pm 8$ & $0.134 \pm 0.008$ & $0.041 \pm 0.002$ & $1.9 \pm 0.1$ & $0.55 \pm 0.03$ \\
\hline $\begin{array}{c}\text { DEX } \\
\mathrm{n}=10\end{array}$ & $127 \pm 8^{*}$ & $167 \pm 10^{*}$ & $108 \pm 7 *$ & $45 \pm 3$ & $16 \pm 1$ & $394 \pm 11$ & $0.114 \pm 0.009$ & $0.041 \pm 0.003$ & $3.2 \pm 0.5 *$ & $0.36 \pm 0.03^{*}$ \\
\hline $\begin{array}{l}\text { Minoxidil + sham } \\
n=10\end{array}$ & $107 \pm 4$ & $135 \pm 5$ & $94 \pm 3^{+}$ & $70 \pm 4^{\dagger}$ & $22 \pm 2^{+}$ & $383 \pm 9$ & $0.182 \pm 0.009^{\dagger}$ & $0.057 \pm 0.005^{\dagger}$ & $1.6 \pm 0.1$ & $0.65 \pm 0.04^{\dagger}$ \\
\hline $\begin{array}{c}\text { Minoxidil + DEX } \\
\mathbf{n}=10\end{array}$ & $113 \pm 3 *$ & $143 \pm 4^{\ddagger}$ & $98 \pm 2$ & $72 \pm 4^{\ddagger}$ & $25 \pm 1^{*}$ & $395 \pm 8$ & $0.182 \pm 0.010^{\ddagger}$ & $0.064 \pm 0.003^{\ddagger}$ & $1.6 \pm 0.1^{\ddagger}$ & $0.64 \pm 0.04^{\ddagger}$ \\
\hline
\end{tabular}

Data were expressed as mean \pm SEM. ${ }^{*} P^{\prime}<0.01$ versus sham treatment; ${ }^{\dagger} P^{\prime}<0.05$ versus sham; ${ }^{\ddagger} P^{\prime}<0.05$ versus DEX. CI, cardiac index; CO, cardiac output; DBP, diastolic blood pressure; DEX, dexamethasone; HR, heart rate; MAP, mean arterial pressure; SBP, systolic blood pressure; SI, stroke index; SV, stroke volume; TPC, total peripheral conductance; TPR, total peripheral resistance. 


\subsubsection{Haemodynamic effects}

\subsubsection{Heart rate}

Heart rate was not significantly altered by DEX or minoxidil treatments (Figure 4.5 and Table 4.1).

\section{Heart Rate}

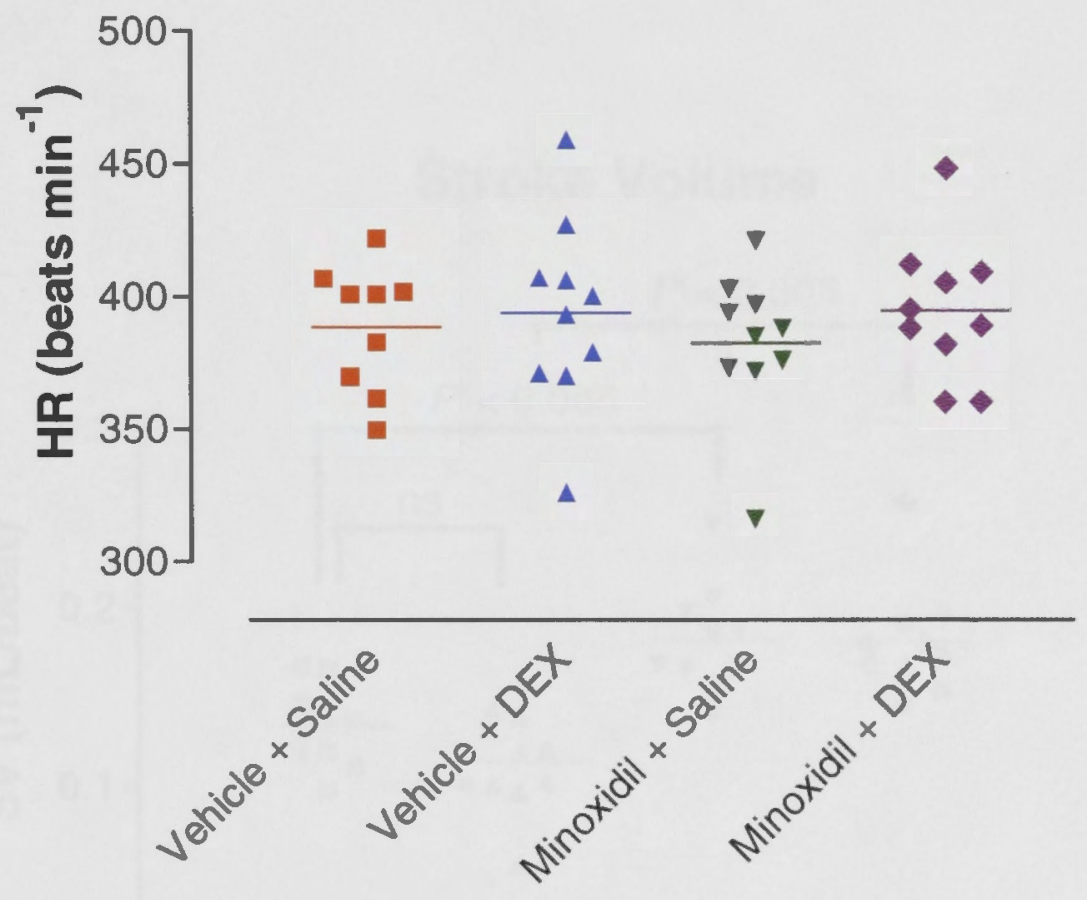

Figure 4.5: Heart rate. Vehicle + Saline, $n=9 ; \Delta$ Vehicle + DEX, $n=10 ; \nabla$ Minoxidil + Saline, $\mathrm{n}=10 ;$ Minoxidil + DEX, $\mathrm{n}=10$. 


\subsubsection{Stroke volume and stroke index}

There was no significant difference in SV and SI between the DEX-treated and sham groups. In comparison with the sham group, rats on minoxidil + sham treatments had significantly higher SV $\left(P^{\prime}<0.005\right)$ and SI $\left(P^{\prime}<0.05\right)$. Similarly, minoxidil + DEX treatments resulted in significantly higher SV $\left(P^{\prime}<0.005\right)$ and SI $\left(P^{\prime}<0.005\right)$ compared with DEX treatment alone (Figures 4.6 and 4.7 ; and Table 4.1).

\section{Stroke Volume}

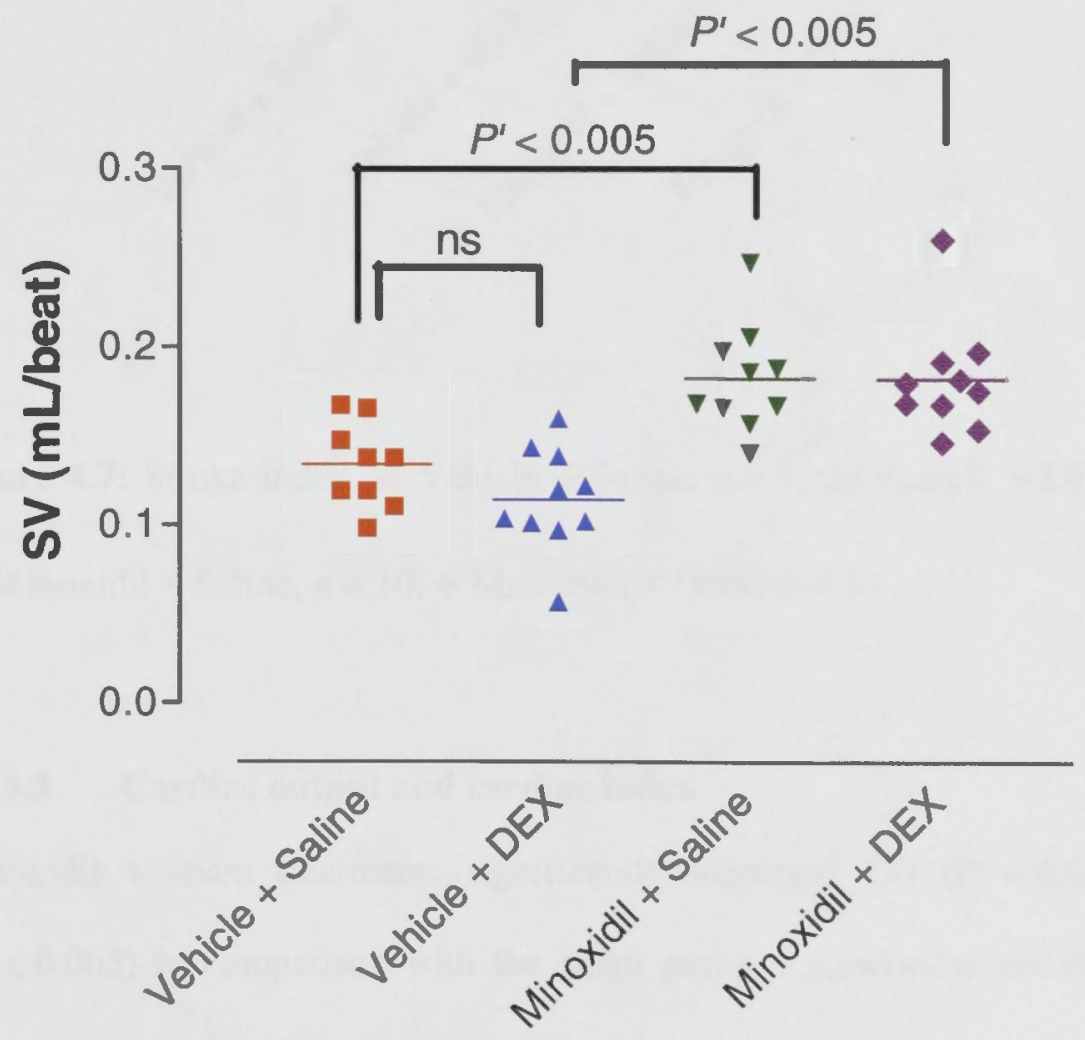

Figure 4.6: Stroke volume. - Vehicle + Saline, $n=9 ; \Delta$ Vehicle + DEX, $n=10$;

$\nabla$ Minoxidil + Saline, $\mathrm{n}=10 ;$ Minoxidil $+\mathrm{DEX}, \mathrm{n}=10$. 


\section{Stroke Index}

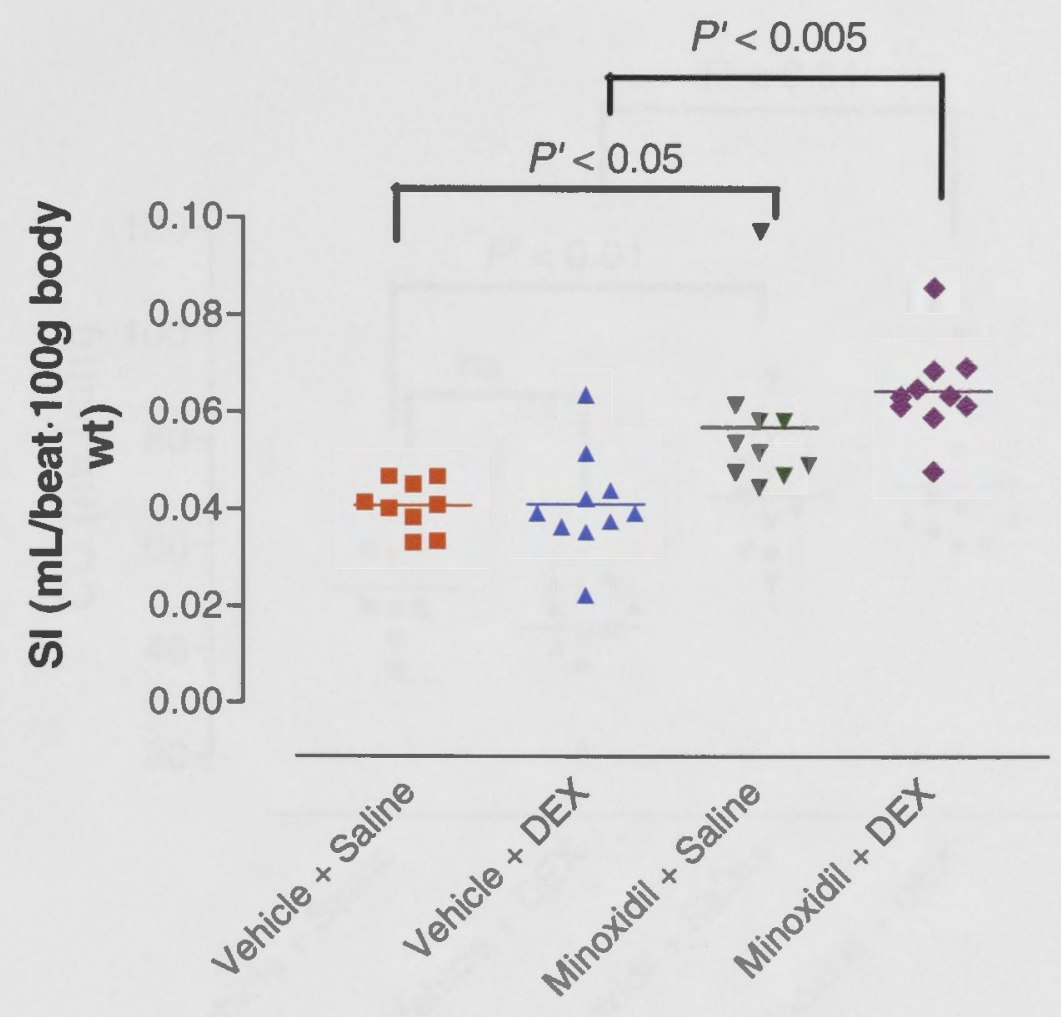

Figure 4.7: Stroke index. - Vehicle + Saline, $n=9 ; \Delta$ Vehicle + DEX, $n=10$; $\boldsymbol{\nabla}$ Minoxidil + Saline, $\mathrm{n}=10 ;$ Minoxidil + DEX, $\mathrm{n}=10$.

\subsubsection{Cardiac output and cardiac index}

Minoxidil + sham treatments significantly increased $\mathrm{CO}\left(P^{\prime}<0.01\right)$ and $\mathrm{CI}$ $\left(P^{\prime}<0.005\right)$ in comparison with the sham group. Likewise, minoxidil + DEXtreated rats had significantly higher $\mathrm{CO}\left(P^{\prime}<0.01\right)$ and $\mathrm{CI}\left(P^{\prime}<0.005\right)$ compared with DEX-treated rats (Figures 4.8 and 4.9; and Table 4.1). 


\section{Cardiac Output}

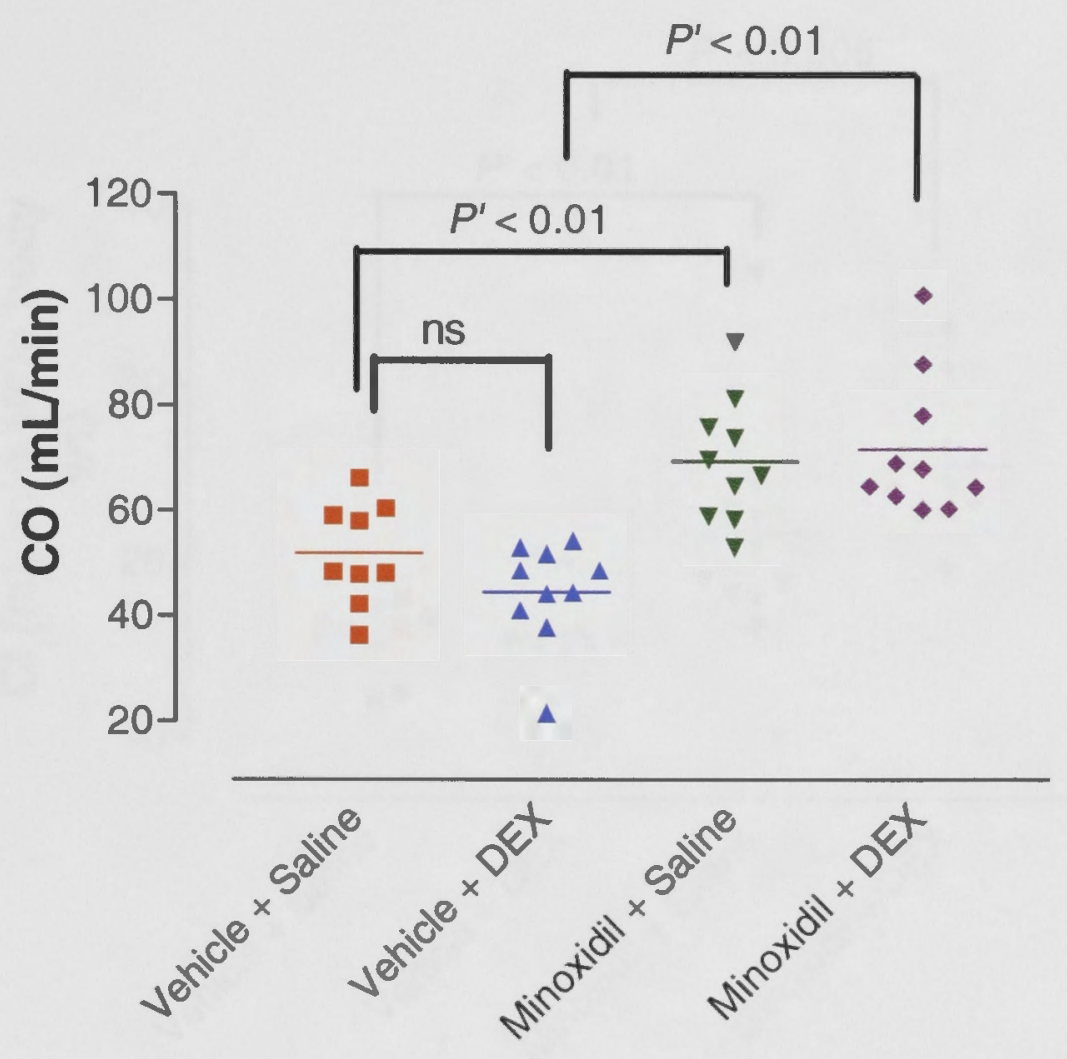

Figure 4.8: Cardiac output. a Vehicle + Saline, $n=9 ; \Delta$ Vehicle + DEX, $n=10$;

$\boldsymbol{\nabla}$ Minoxidil + Saline, $\mathrm{n}=10 ;$ Minoxidil + DEX, $\mathrm{n}=10$. 


\section{Cardiac Index}

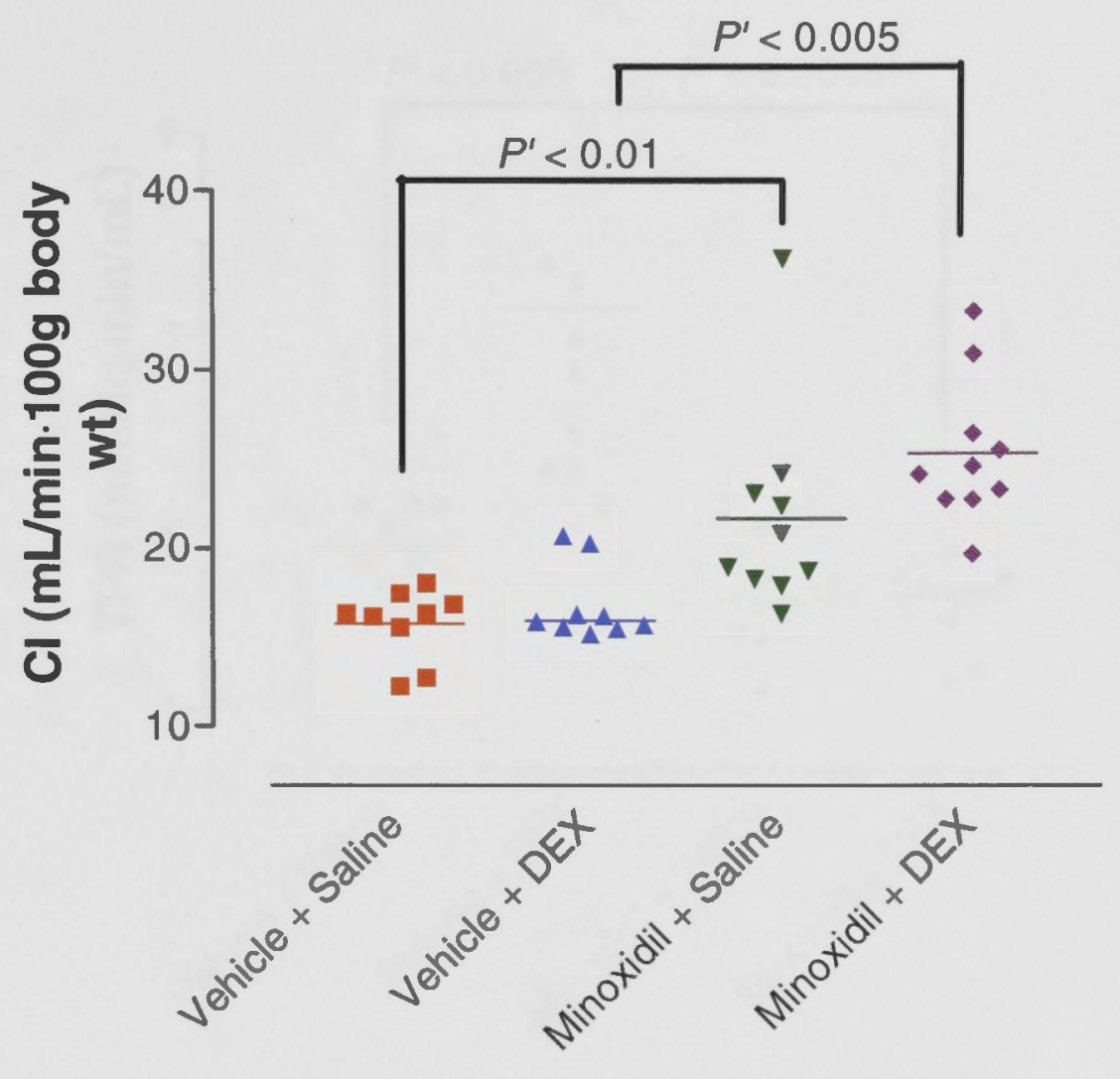

Figure 4.9: Cardiac index. $\square$ Vehicle + Saline, $n=9 ; \Delta$ Vehicle + DEX, $n=10$;

Minoxidil + Saline, $\mathrm{n}=10 ;$ Minoxidil + DEX, $\mathrm{n}=10$.

\subsubsection{Total peripheral resistance}

TPR was significantly increased in the DEX-treated group compared with the sham treatment group $\left(P^{\prime}<0.05\right)$ and was significantly lower in the minoxidil + DEX-treated group in comparison to DEX-treated group $\left(P^{\prime}<0.05\right)$ (Figure 4.10 and Table 4.1). 


\section{Total Peripheral Resistance}

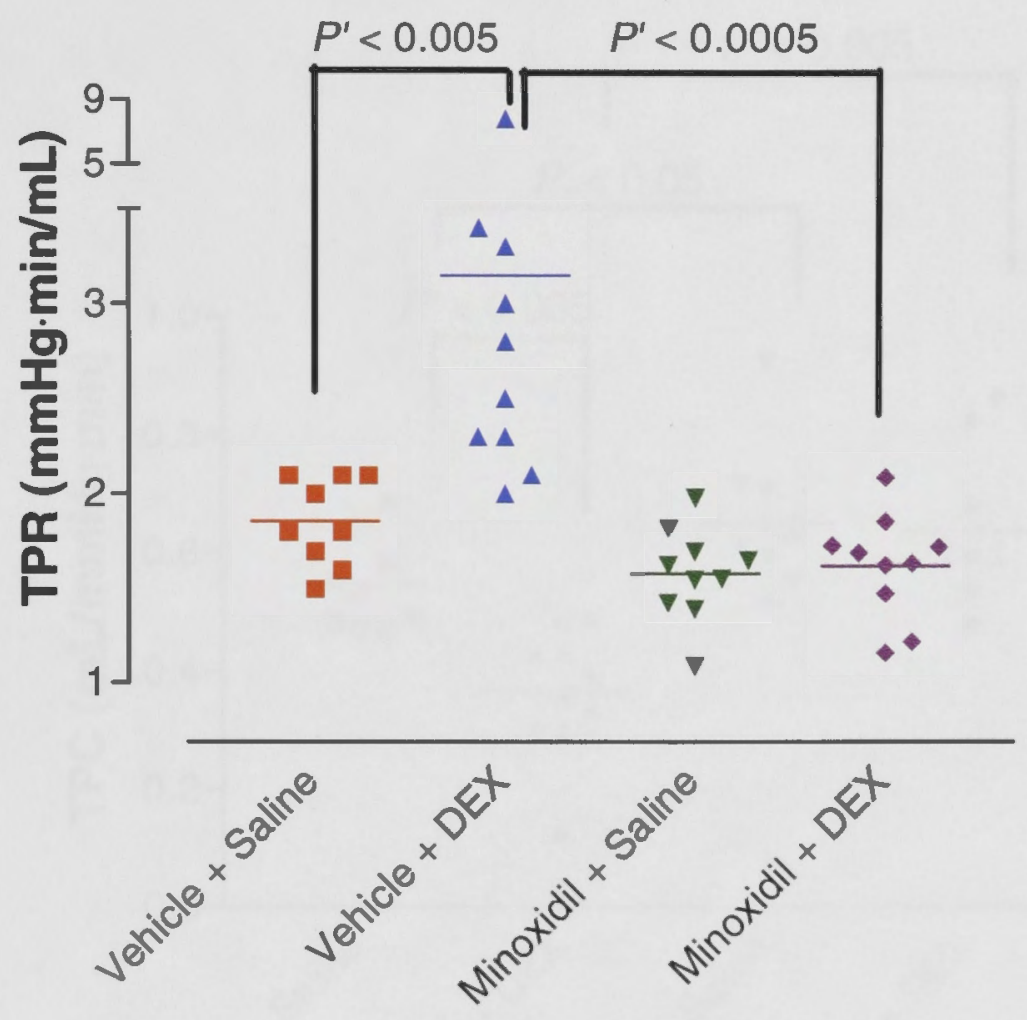

Figure 4.10: Total peripheral resistance. $n$ Vehicle + Saline, $n=9 ; \Delta$ Vehicle + DEX, $\mathrm{n}=10 ; \boldsymbol{\nabla}$ Minoxidil + Saline, $\mathrm{n}=10 ;-$ Minoxidil + DEX, $\mathrm{n}=10$.

\subsubsection{Total peripheral conductance}

TPC in the DEX-treated group was significantly lower than in the sham treatment group $\left(P^{\prime}<0.005\right)$. Rats on minoxidil + DEX had significantly higher TPC than those on DEX without minoxidil $\left(P^{\prime}<0.005\right)$. Minoxidil + sham treatments significantly increased TPC in comparison to sham treatment $\left(P^{\prime}<0.05\right)$ (Figures 4.11 and Table 4.1). 


\section{Total Peripheral Conductance}

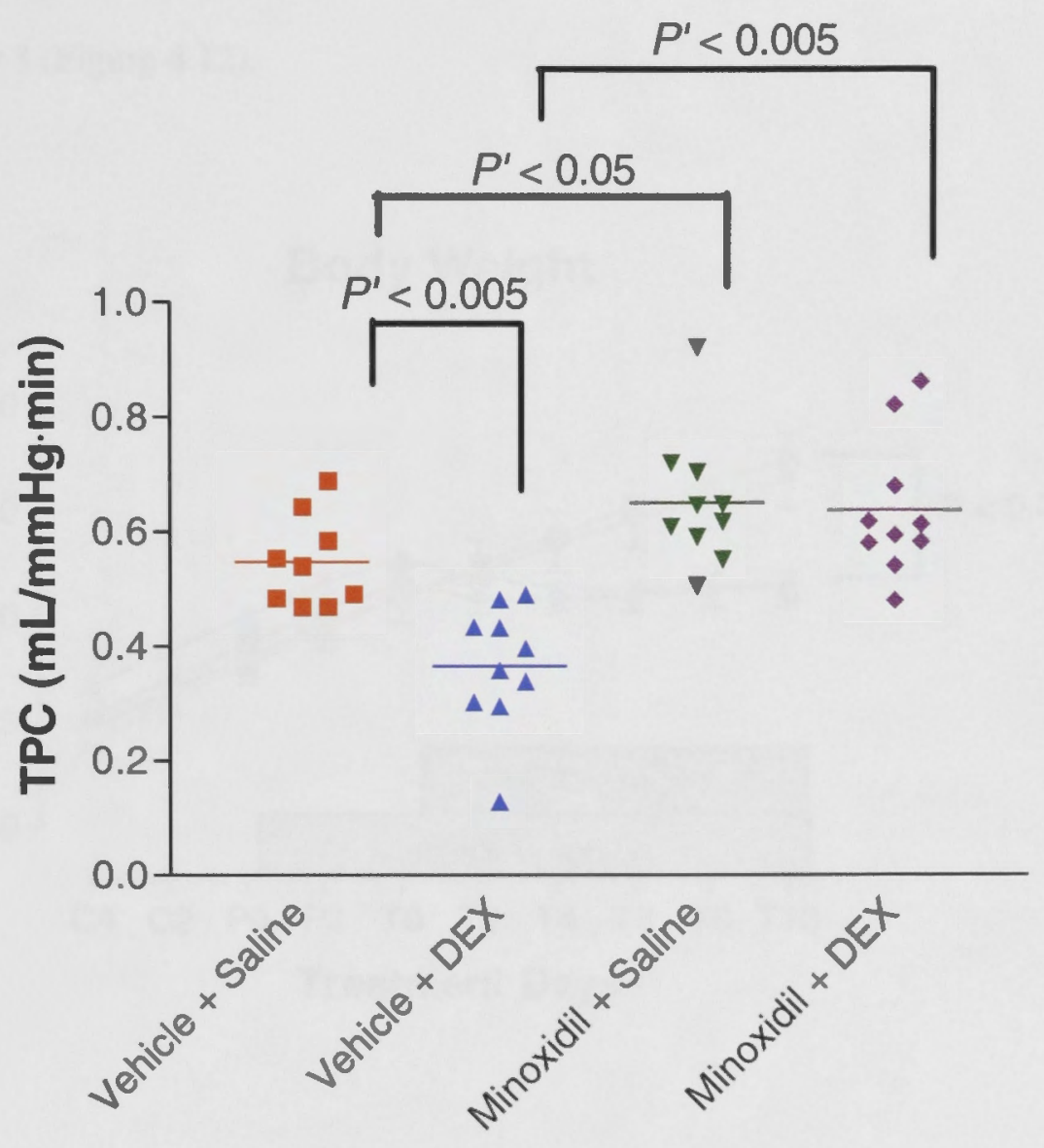

Figure 4.11: Total peripheral conductance. Vehicle + Saline, $n=9 ; \Delta$ Vehicle + DEX, $\mathrm{n}=10 ; \nabla$ Minoxidil + Saline, $\mathrm{n}=10 ;$ Minoxidil + DEX, $\mathrm{n}=10$. 


\subsubsection{Body weight}

Minoxidil treatment did not alter the DEX-induced body weight changes seen in Chapter 3 (Figure 4.12).

\section{Body Weight}

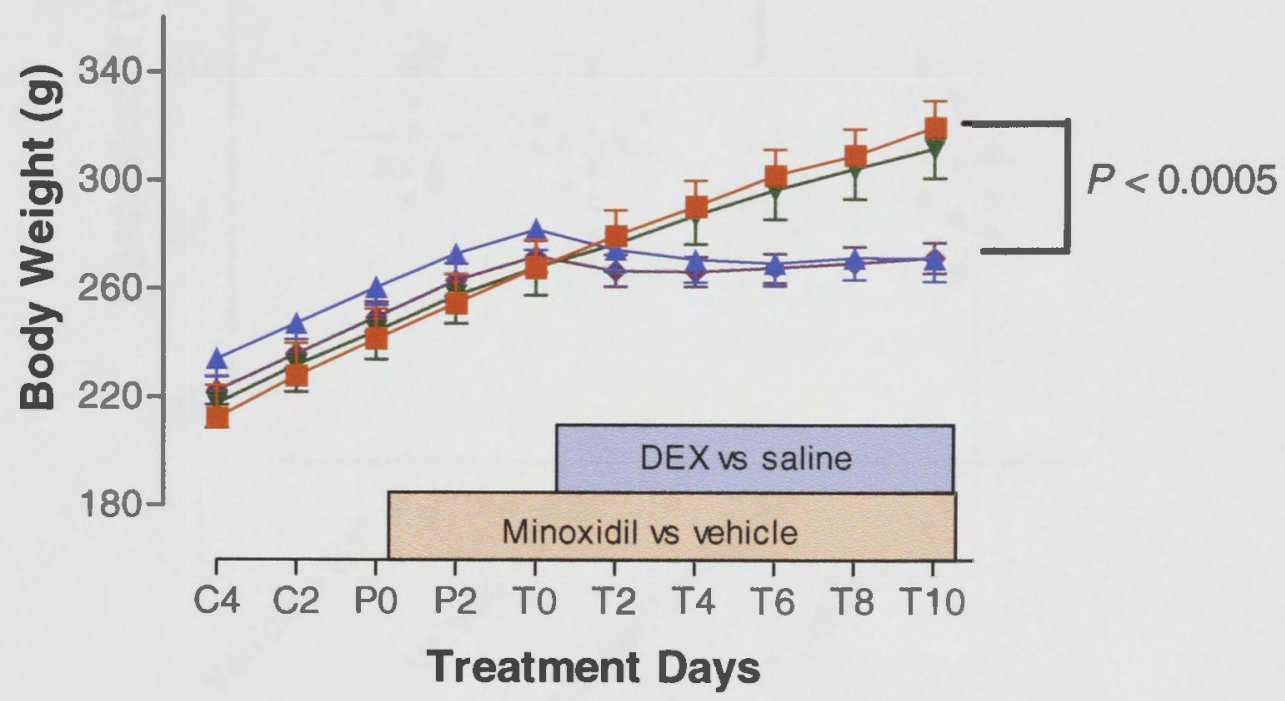

Figure 4.12: Body weight. $\|$ Vehicle + Saline, $n=9 ; \Delta$ Vehicle + DEX, $n=10$;

$\boldsymbol{\nabla}$ Minoxidil + Saline, $\mathrm{n}=10 ;$ Minoxidil + DEX, $\mathrm{n}=10$.

\subsubsection{Haematocrit}

There was no significant difference in haematocrit between DEX-treated and sham rats (sham: $56 \pm 1.1, \mathrm{n}=9$; DEX: $55 \pm 0.9 \%, \mathrm{n}=10$ ). Minoxidil + sham treatments produced a significant decrease in haematocrit compared with sham treatment alone (sham: $56 \pm 1.1, \mathrm{n}=9$; minoxidil + sham: $52 \pm 0.6 \%, \mathrm{n}=10$, 
$P<0.005$ ). This decrease was not seen in minoxidil + DEX-treated rats (DEX: $55 \pm 0.9$; minoxidil + DEX: $55 \pm 1.6 \%, n=10$ each) (Figure 4.13)

\section{Haematocrit}

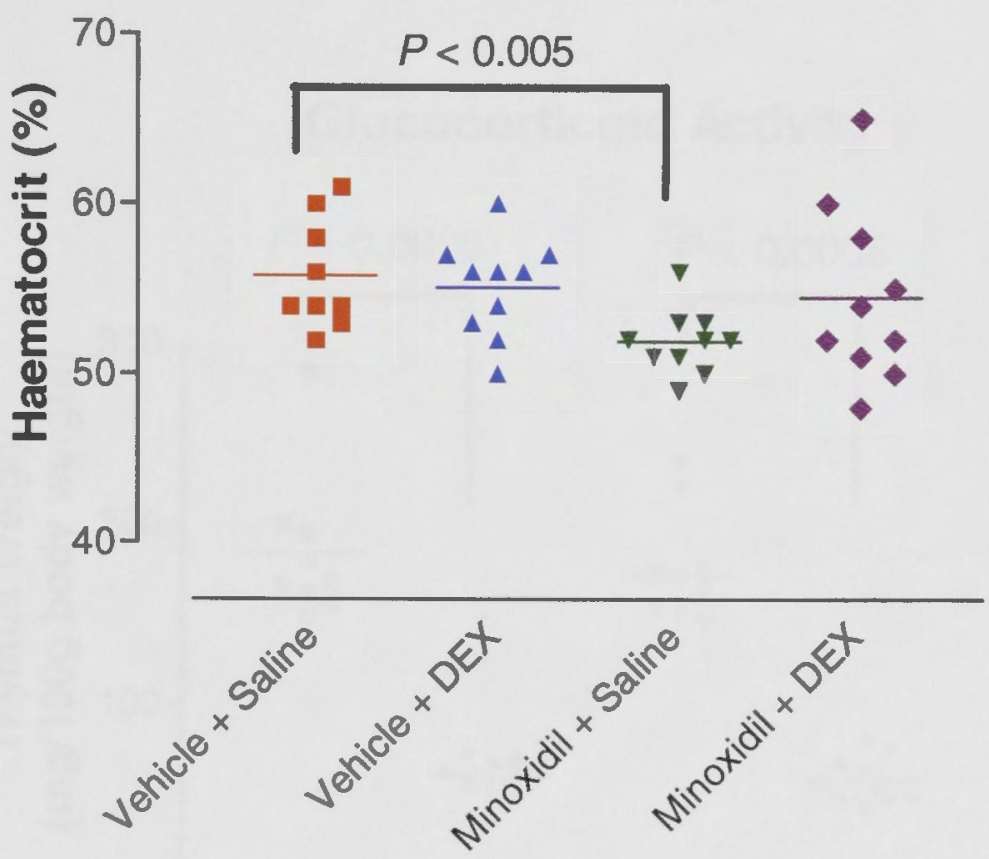

Figure 4.13: Haematocrit. Vehicle + Saline, $n=9 ; \Delta$ Vehicle + DEX, $n=10$;

$\nabla$ Minoxidil + Saline, $\mathrm{n}=10 ;$ Minoxidil + DEX, $\mathrm{n}=10$.

\subsubsection{Thymus and adrenal weights}

DEX treatment, regardless of the presence of minoxidil treatment, resulted in significant decreases in thymus (saline: $185 \pm 14, n=9$ versus DEX: $60 \pm 3$, $\mathrm{n}=10$ and minoxidil + saline: $172 \pm 10, \mathrm{n}=10$ versus minoxidil + DEX: $60 \pm 4$ 
$\mathrm{mg} / 100 \mathrm{~g}, \mathrm{n}=10$; both $P<0.0005$ ) (Figure 4.14) and mean adrenal weights (saline: $5.7 \pm 0.2, \mathrm{n}=9$ versus $\mathrm{DEX}: 3.7 \pm 0.3, \mathrm{n}=10$ and minoxidil + saline: $5.6 \pm 0.4, \mathrm{n}=10$ versus minoxidil + DEX: $3.6 \pm 0.1 \mathrm{mg} / 100 \mathrm{~g}, \mathrm{n}=10$; both $P<0.0005$ ) (Figure 4.15).

\section{Glucocorticoid Activity}

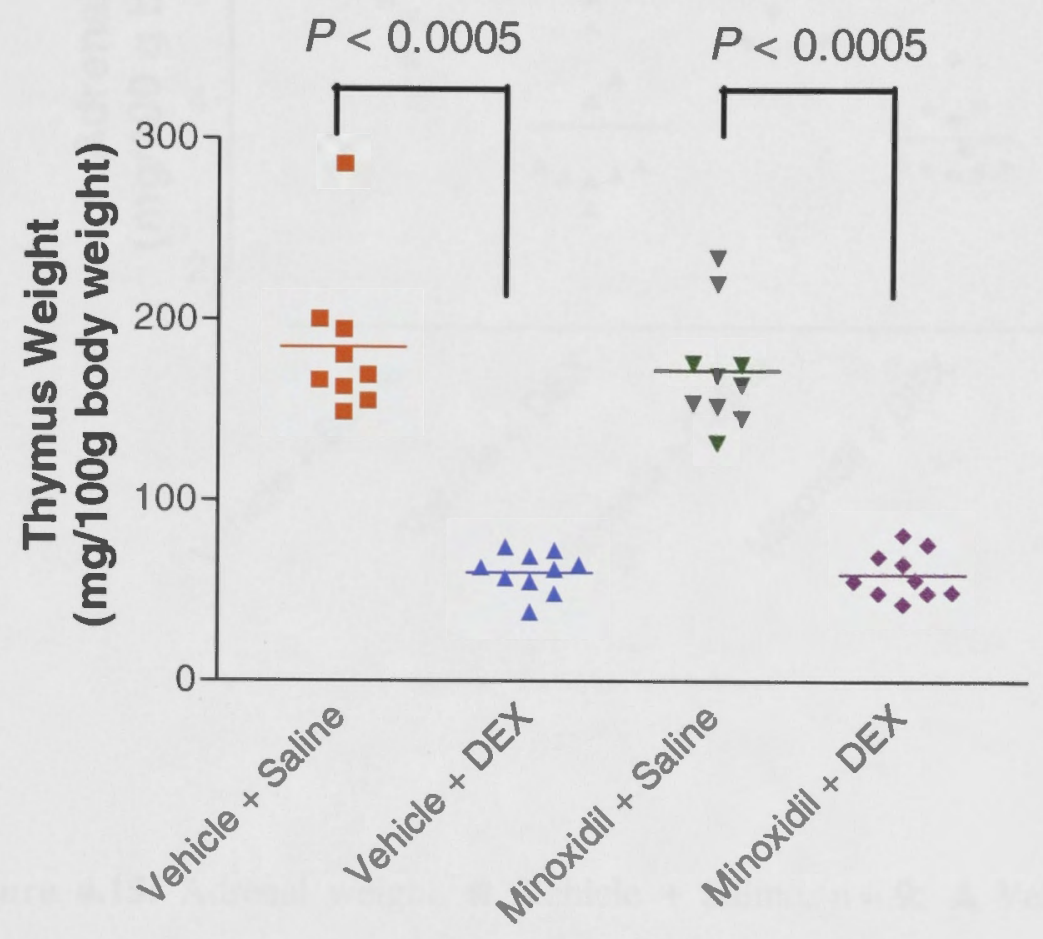

Figure 4.14: Thymus weight. $n$ Vehicle + Saline, $n=9 ; \Delta$ Vehicle + DEX, $\mathrm{n}=10 ; \boldsymbol{\nabla}$ Minoxidil + Saline, $\mathrm{n}=10 ;$ Minoxidil + DEX, $\mathrm{n}=10$ 


\section{Efficacy of Dexamethasone Administration}

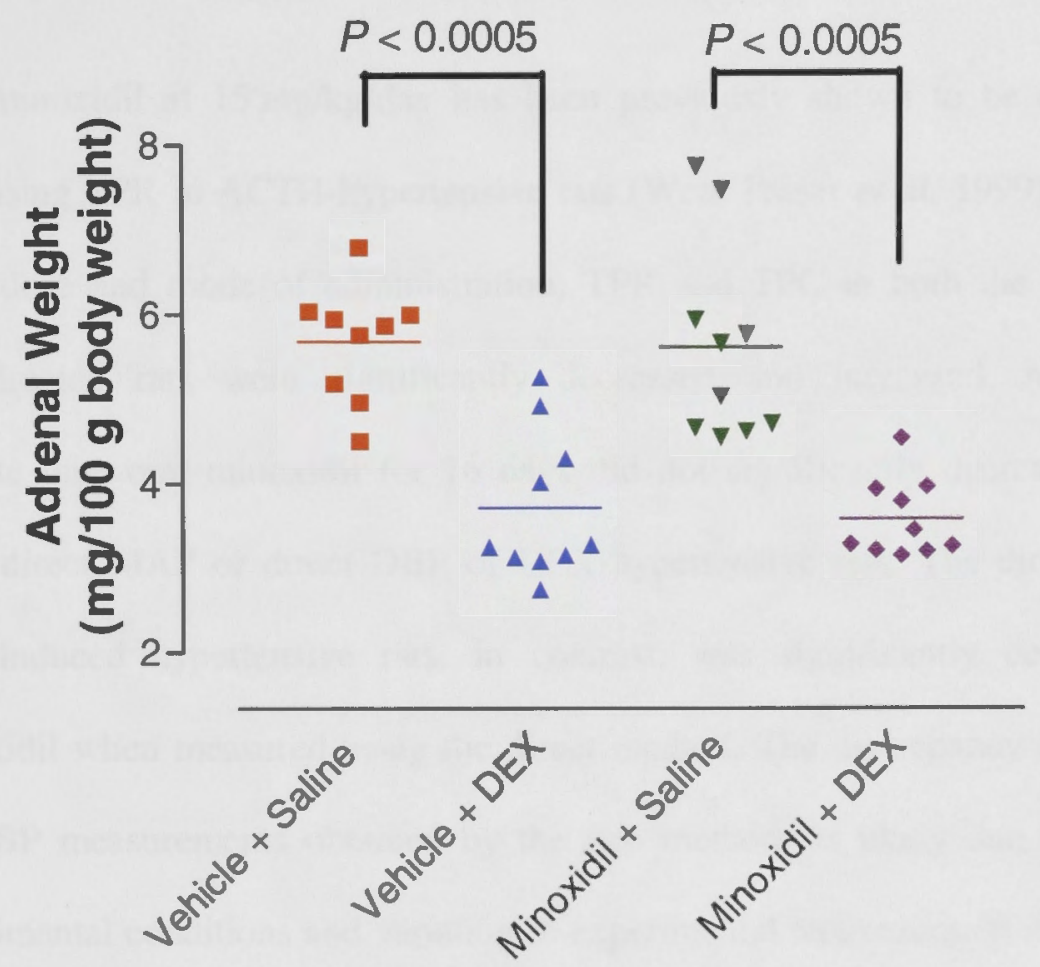

Figure 4.15: Adrenal weight. Vehicle + Saline, $n=9 ; \Delta$ Vehicle + DEX, $\mathrm{n}=10 ; \boldsymbol{\nabla}$ Minoxidil + Saline, $\mathrm{n}=10 ;$ Minoxidil + DEX, $\mathrm{n}=10$.

\subsection{DISCUSSION}

As shown in Chapter 3, DEX-HT in the rat is associated with raised TPR and reduced TPC. Minoxidil treatment for 16 days significantly decreased TPR and 
increased CO and TPC in DEX-hypertensive rats but did not prevent the hypertension.

Oral minoxidil at $15 \mathrm{mg} / \mathrm{kg} /$ day has been previously shown to be effective in decreasing TPR in ACTH-hypertensive rats (Wen, Fraser et al. 1999). Using the same dose and mode of administration, TPR and TPC in both the saline- and DEX-treated rats were significantly decreased and increased, respectively. Despite this, oral minoxidil for 16 days did not significantly decrease tail-cuff SBP, direct MAP or direct DBP of DEX-hypertensive rats. The direct SBP of DEX-induced hypertensive rats, in contrast, was significantly decreased by minoxidil when measured using the direct method. The discrepancy observed in the SBP measurements obtained by the two methods is likely due to different experimental conditions and variation in experimental techniques. With the direct method, the rats were anaesthetised and mechanically-ventilated. Limitations associated with these manoeuvres have been discussed in Chapter 3. Tail-cuff SBP, on the other hand, was obtained in restrained conscious animals without the undesirable side effects of general anaesthesia and mechanical ventilation. The tail-cuff system is a reliable method for assessing SBP in rats. It was previously shown, in our hands, to produce results that are comparable to telemetry (Fraser, Turner et al. 2001).

Although direct SBP of DEX-hypertensive rats was significantly reduced by minoxidil, increases in direct MAP and DBP due to DEX were not significantly lowered. Furthermore, DBP in the minoxidil + saline group was significantly 
increased compared with the saline-only group. There are 2 possible explanations for this absence of blood pressure lowering effect of minoxidil. Firstly, changes in TPR and TPC are not critical to the development of DEX-HT, though they might contribute to the maintenance of the hypertensive state. Secondly, the blood pressure lowering effect of minoxidil may have been offset by volume factors. $\mathrm{CO}$ and $\mathrm{CI}$, in both the minoxidil + saline- and minoxidil + DEX-treated rats were increased. As there was no change in HR between the treatment groups, minoxidil-induced elevations in $\mathrm{CO}$ and $\mathrm{Cl}$ were also associated with increases in SV and SI in the saline and DEX-treated rats. It is likely that these increases counteracted all the vasodilator effect of minoxidil. The mechanism responsible for the increases in $\mathrm{CO}$ and $\mathrm{CI}$ and the loss of blood pressure lowering effect of minoxidil, is mainly related to plasma volume expansion. In this study, we observed a decrease in haematocrit due to minoxidil in the saline-treated group. Several studies have also suggested that minoxidil treatment resulted in plasma and blood volume expansion in rats (Leenen and Prowse 1987; Sanz, Lopez Novoa et al. 1990; Tsoporis, Fields et al. 1991). In the present study, minoxidil did not alter haematocrit in the DEX-HT rats despite increasing $\mathrm{CO}$ and $\mathrm{CI}$. It remains unclear if DEX can inhibit the fall in haematocrit due to minoxidil.

The reduction of both thymus and adrenal weights were signs of effective DEX delivery to these animals. 


\subsection{CONCLUSION}

Concomitant treatment with the vasodilator minoxidil prevented the rise in TPR and decrease in TPC but did not completely prevent the rise in blood pressure due to DEX, which in this case was associated with increase in $\mathrm{CO}$ and $\mathrm{CI}$. Thus, raised TPR and decrease in TPC are features of DEX-HT that may not be critical for production of DEX-HT and may be overcome by increase in CO. 


\section{CHAPTER 5}

Effects of Glucocorticoid on Rat Kidney

Mitochondrial Superoxide 


\subsection{INTRODUCTION}

The mitochondrion is an important source of ROS, primarily the superoxide radical and consequently its dismutation product, hydrogen peroxide, which can form highly reactive hydroxyl radicals. It consumes approximately $90 \%$ of the cell's oxygen and $1-4 \%$ of oxygen reacting with the mitochondrial respiratory chain is incompletely reduced to ROS (Richter 1988; Lenaz 1998). The electron transport chain is generally accepted as the main source of ROS in the mitochondria. Based on studies in isolated mitochondria utilising different substrates or inhibitors to support or inhibit cell respirations, it is concluded that the main sites of superoxide generation within the electron transport chain are complex I (NADH dehydrogenase) and III (cytochrome $b c_{l}$ complex) with the release of superoxide towards the mitochondrial matrix (Muller, Liu et al. 2004). The electron transport chain is, however, not the only major supply of ROS within the mitochondria. Recent studies on neural tissues have revealed that alphaketoglutarate dehydrogenase, an important Kreb cycle enzyme, also contributes to ROS generation in the mitochondria (Starkov, Fiskum et al. 2004; Adam-Vizi 2005; Tretter and Adam-Vizi 2005).

Besides being the producer of ROS, the mitochondrion is also susceptible to oxidative injury. Overproduction and the resultant accumulation of mitochondrialderived ROS can damage mitochondrial components such as mitochondrial DNA and membrane, leading to mitochondrial dysfunction which can further exacerbate mitochondrial ROS production. 
As there are a number of different pathways contributing to the production of superoxide in cells apart from the mitochondria such as the $\mathrm{NAD}(\mathrm{P}) \mathrm{H}$ oxidase, xanthine oxidase and eNOS in its uncoupled form (Chapter 1), proper assessment of mitochondrial superoxide production requires analysis at this subcellular level. Furthermore, superoxide generated by the mitochondria does not permeate the inner mitochondrial membrane and is compartmentalised within this organelle away from the cytosol (Han, Antunes et al. 2003).

The aim of this study was to establish and validate a simple quantitative assay to detect superoxide within the mitochondria and to determine the effect of glucocorticoids in kidney mitochondrial superoxide levels.

\subsection{METHODS}

This study was approved by the Animal Experimentation Ethics Committee of the Australian National University (Protocol No. J. HB. 20.05).

\subsubsection{Mitochondrial superoxide detection}

One of the methods includes analysing superoxide oxidation products in isolated mitochondrial particles. First, mitochondria from rat kidney were isolated using differential ultra-centrifugation of cell suspension prepared from homogenised kidney. This process was based on a method described previously (Pallotti and Lenaz 2001). The mitochondrial content was standardised by quantitation of the 
protein content using the Bradford method (Bradford 1976) (BioRad, Gladesville, Australia) using bovine serum albumin as standard. After staining the isolated mitochondria with a number of fluorogenic probes- nonylacridine orange to identify mitochondrial particles, $1,1^{\prime}, 3,3,3^{\prime}, 3^{\prime}$-hexamethylindodicarbocyanine iodide $\left(\mathrm{DilC}_{1}(5)\right)$ to assess mitochondrial membrane potential and dihydroethidine to assess superoxide generation (all fluorogenic probes were purchased from Molecular Probes, Invitrogen, USA), the stained isolated mitochondria were assessed via flow cytometry (Mattiasson 2004; Mattiasson 2004a). This technique was abandoned as this multi-step process involved in this method was timeconsuming and not suitable for analysis of a large number of fresh specimens on the days the animals were sacrificed. Instead, I utilised another fluorescent method which involved analysing mitochondrial superoxide in intact live cells. Analysis of mitochondria in live cells bypasses this tedious process of mitochondrial isolation. It also allows for separation of artefacts of isolated mitochondrial preparations from actual signals generated by mitochondrial superoxide.

In this latter technique, a novel fluorogenic probe, triphenylphosphoniumhydroethidine (Mito-HE) (MitoSOX ${ }^{\mathrm{TM}}$ Red, Molecular Probes, Invitrogen, USA), which is a derivative of hydroethidine (HE) was used. HE is widely used to detect intracellular superoxide anion. It reacts with superoxide to form ethidium $\left(\mathrm{Etd}^{+}\right)$ and its hydroxylated product $\left(\mathrm{HO}-\mathrm{Etd}^{+}\right.$) both of which become highly fluorescent when intercalated with cellular nucleic acids (Robinson, Janes et al. 2006). Mito-HE is derived by linking a triphenylphosphonium cation to the HE 
moiety. The positive charge in the phosphonium group allows Mito-HE to selectively accumulate in the mitochondria of live cells with $100-1000$ fold affinity, as described in other compounds with the phosphonium side-chain (Ross, Kelso et al. 2005). Mito-HE is oxidised in the similar manner to HE to result in the formation of Mito- $\mathrm{Etd}^{+}$which fluoresces following excitation.

As Mito-HE accumulates in mitochondria as a function of mitochondrial membrane potential, cells were also stained with $\operatorname{DiIC}_{1}(5)$, a mitochondrial potentiometric probe to ensure that cell treatment with Mito-HE did not depolarise mitochondrial membrane. This phenomenon can lead to extra-mitochondrial oxidation of Mito-HE causing false positive results. $\mathrm{DiIC}_{1}(5)$ is a cationic cyanine dye which penetrates the cytosol of eukaryotic cells and accumulates in cells in response to membrane potential (Shapiro, Natale et al. 1979; Shapiro 2000). It however, accumulates mainly in the mitochondria with active membrane potentials at concentrations below $100 \mathrm{nM}$.

\subsubsection{Principles of flow cytometry}

Flow cytometry is a powerful tool that allows multi-parametric analysis of cells within a heterogenous cell population. It uses the principles of light excitation and scattering to generate specific data of cells such as size, granularity or complexity, phenotype and health. 
The cells, as they pass through the laser beam, scatter the light in different angles. Forward scatter (FSC) is the amount of light that scatters in the forward direction. Its magnitude is proportional to cell size. Side scatter (SSC), on the other hand, is a measure of granularity or complexity within the cell. It is a large angle scatter caused by structures within the cell. The intensity of the light scatter captured by the detectors is converted to voltage pulse which is then presented graphically.

Fluorescent molecules are commonly used in flow cytometry to study cell characteristics. When cells treated with fluorochromes are excited by light with a correct wavelength, they emit fluorescent signals which follow the same paths as the FSC and SSC signals. These fluorescent signals travel through a series of filters and mirrors to reach the detector for that particular wavelength. Fluorescence data are collected via the same method as FSC and SSC data. The intensity of the fluorescence is converted into voltage signals proportional to the emissions emitted. All of the voltage pulses are collected and presented graphically as in Figure 5.2.

\subsubsection{Single cell suspension}

Rats were sacrificed under anaesthesia using the method described in Section 2.8 of Chapter 2. The left kidney was located and removed via a median laparotomy incision. The left rat kidney was harvested and immediately placed in chilled phosphate buffered saline (PBS). Perinephric fat and serosal membrane were trimmed, before cutting the kidney into smaller pieces. The kidney was then 
homogenised manually using a cell sieve and a $5 \mathrm{~mL}$ syringe plunger in a petri dish containing $10 \mathrm{~mL}$ of chilled PBS. The cells were then filtered using a $2000 \mu \mathrm{m}$ cell filter.

\subsubsection{Standardisation of cell number}

Trypan blue (Fluka, Seelze, Germany) was used to stain dead cells. The cells were counted using a Neubauer haemocytometer (Brightline ${ }^{\circledR}$, American Optical Corporation, Buffalo, USA) counting chamber and a microscope at $100 x$ magnification. In a viable cell preparation, the proportion of dead cells should be less than $20 \%$. A total cell count of $2.0 \times 10^{6}$ per sample was required for flow cytometry analysis.

\subsubsection{Mitochondrial staining}

The cells were incubated with Mito-HE and $\mathrm{DiIC}_{1}(5)$. The approximate excitation and emission (Ex/Em) spectral peaks for Mito-HE are 510/580 and for $\mathrm{DiIC}_{1}(5)$ are $638 / 658 \mathrm{~nm}$. These two dyes are compatible as the fluorescent products for Mito-HE and DiIC $_{1}(5)$ are efficiently excited by the 488 and $633 \mathrm{~nm}$ lasers, respectively without overlap in their emission peaks.

Mito-HE and $\mathrm{DiIC}_{1}(5)$ were reconstituted with dimethylsulfoxide (DMSO) to $5 \mathrm{mM}$ and $3 \mathrm{mM}$ respectively. These stock solutions were further diluted using PBS to the required concentrations. The DMSO and PBS solutions used on MitoHE were percolated with nitrogen. Mito-HE (final concentration $2 \mu \mathrm{M}$, see 
validation study below) and $\mathrm{DiIC}_{1}(5)$ (final concentration $10 \mathrm{nM}$ ) solutions were added to the cells and incubated for 10 minutes at $37^{\circ} \mathrm{C}$. All samples were prepared in duplicate.

\subsubsection{Flow cytometric analysis}

The cells were analysed immediately after incubation with Mito-HE and $\operatorname{DiIC}_{1}(5)$. The cells were protected from light at all times to prevent quenching of fluorescence signals.

Flow cytometric analysis was performed using a FACSort BD (Becton Dickinson, San Jose, USA) flow cytometer equipped with $488 \mathrm{~nm}$ Argon and $633 \mathrm{~nm}$ diode lasers. Excitation of the Mito-HE oxidation products was performed using the $488 \mathrm{~nm}$ laser. The emissions generated by Mito-HE oxidation products were detected by the FL2 channel. This channel can detect emissions in the wavelength range of $585 \pm 30 \mathrm{~nm}$. DiIC $(5)$ was excited by the $633 \mathrm{~nm}$ laser and the emissions were detected by the FL 4 channel. This channel can detect emissions in the wavelength range of $660 \pm 20 \mathrm{~nm}$.

Data acquisition and analysis were performed using the CellQuest software (Becton Dickinson Immunocytometry Systems, San Jose, USA).

Based on light scattering properties in the FSC and the SSC modes, the cells were gated to exclude cell debris (Figure 5.1). Dead cells are recognised flow 
cytometrically by very low fluorescence and relatively low FSC and SSC. This gate was drawn to cover a large SSC area to ensure all cell lines from the kidneys were covered, whilst excluding cell debris.

Using the "medium" sampling rate, 15000 gated events per sample were analysed.

The geometric mean fluorescence intensity (MFI) was used. The geometric MFI values of the samples were obtained by subtracting fluorescence of the stained specimen, obtained in the region marked $\mathrm{M} 2$ in Figure $5.2 \mathrm{~b}$, with autofluorescence of unstained specimen obtained in the region marked $\mathrm{Ml}$ in Figure 5.2a.

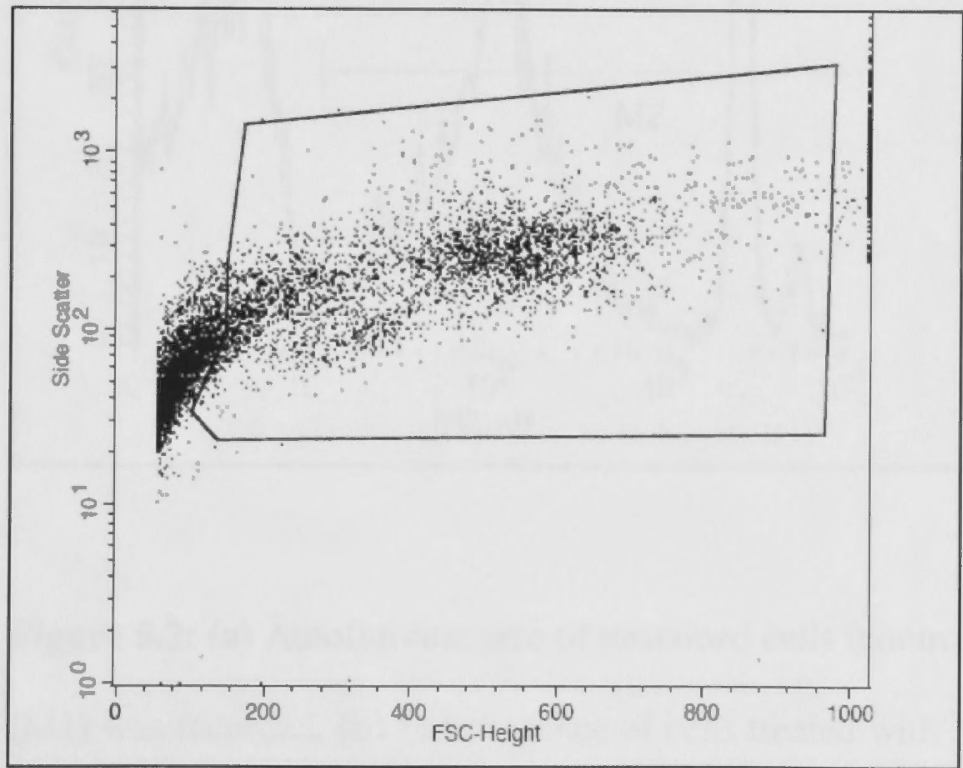

Figure 5.1: Cells were gated to exclude debris. FSC = forward scatter. 
a

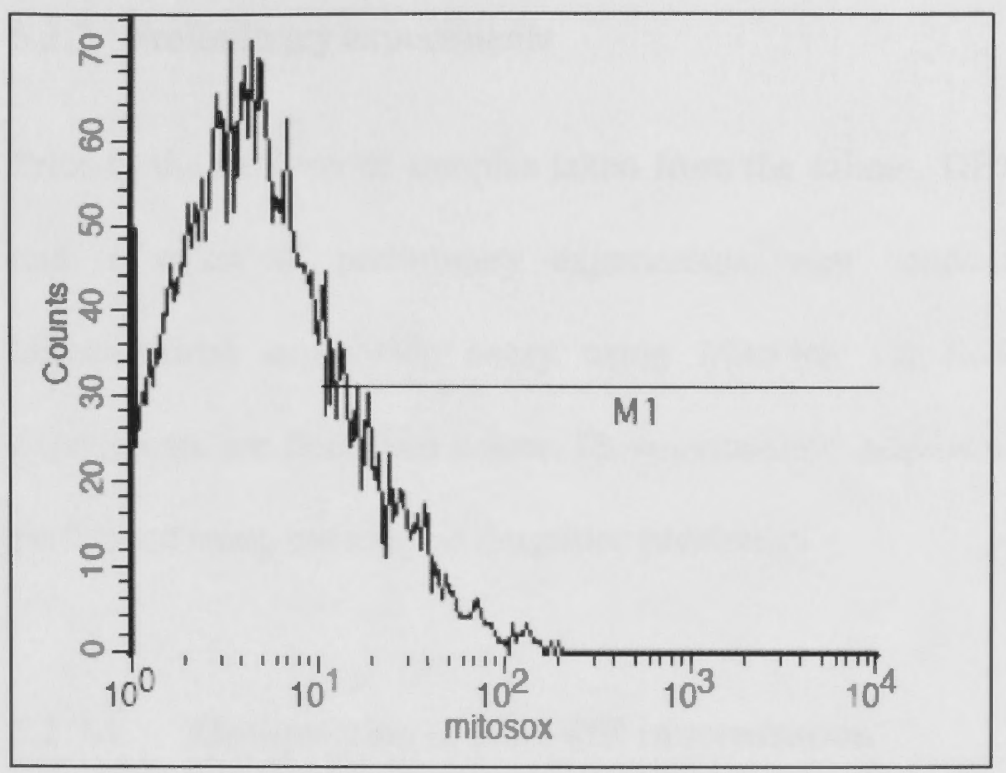

b

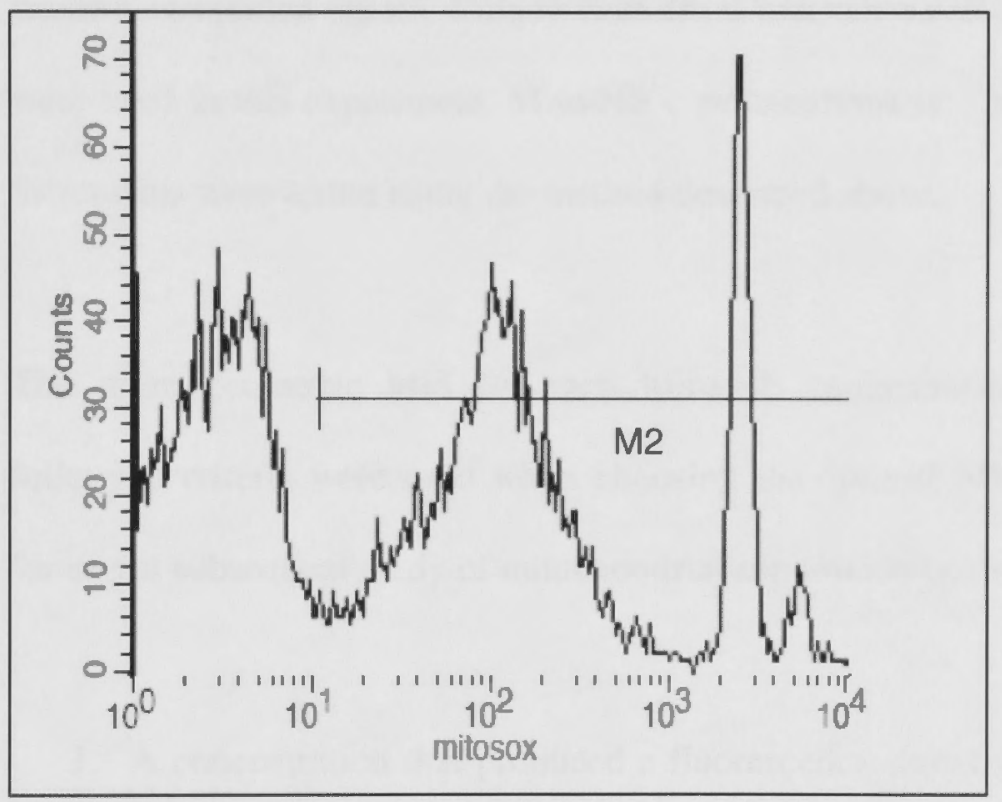

Figure 5.2: (a) Autofluorescence of unstained cells (control) in the marked region (M1) was recorded. (b) Fluorescence of cells treated with Mito-HE in the marked region (M2) was recorded. Net geometric MFI was calculated by subtracting the geometric MFI obtained in M2 region from M1 region. 


\subsubsection{Preliminary experiments}

Prior to the analysis of samples taken from the saline-, DEX and ACTH-treated rats, a series of preliminary experiments were conducted to validate the mitochondrial superoxide assay using Mito-HE via flow cytometry. These experiments are described below. Flow cytometric acquisition and analysis were performed using the method described previously.

\subsubsection{Optimisation of Mito-HE concentration}

This experiment was conducted to evaluate the concentration of Mito-HE which resulted in optimal signal. Kidney cells from four untreated Sprague-Dawley rats were used in this experiment. Mito-HE concentrations of 1 to $10 \mu \mathrm{M}$ with $1 \mu \mathrm{M}$ increments were tested using the method described above.

The mean geometric MFI for each Mito-HE concentration was plotted. The following criteria were used when choosing the optimal Mito-HE concentration for use in subsequent study of mitochondrial superoxide generation:

1. A concentration that produced a fluorescence signal that was not affected by background autofluorescence.

2. A concentration that was not cytotoxic. Changes in cell morphology due to cell damage can alter the scatter properties of the cells. 
3. A concentration that produced maximal separation of negative and positive population at the above flow cytometric settings.

\subsubsection{Positive control}

Antimycin A (Sigma-Aldrich, St Louis, USA) is a mixture product originating from Streptomycin kitazawensis which consists of Antimycin Al and A3a (Nakayama, Okamoto et al. 1956). It blocks the mitochondrial electron transport chain at the Complex III and results in the breakdown of the proton gradient across the inner mitochondrial membrane and thus, the mitochondrial membrane potential (Campo, Kinnally et al. 1992; Pham, Robinson et al. 2000). Antimycin A, at concentrations ranging from 2 to $20 \mu \mathrm{M}$, can also increase mitochondrial superoxide production in both isolated mitochondrial preparations and live cells (Du, Daniels et al. 2006; Meany, Poe et al. 2006; Robinson, Janes et al. 2006; Mukhopadhyay, Rajesh et al. 2007). I used $5 \mu \mathrm{M}$ Antimycin A to establish a positive control to validate mitochondrial superoxide generation with Mito-HE. To ensure that this concentration did not collapse the mitochondrial membrane potential, a phenomenon that could lead to a false positive Mito-HE signal, cells were counterstained with $\operatorname{DilC}_{1}(5)$.

Antimycin A (MW 548.7) was reconstituted in $95 \%$ ethanol to give a $400 \mu \mathrm{M}$ solution. It was further dissolved in PBS before being used to incubate the cells at a final concentration of $5 \mu \mathrm{M}$. Cells were incubated with Antimycin A for 15 minutes prior to staining with Mito-HE and $\mathrm{DiIC}_{1}(5)$. The geometric MFI for both dyes were evaluated using the flow cytometric analysis method described above. 


\subsubsection{Negative control}

Tiron or sodium dihydroxybenzene disulfonate (Fluka, Steinheim, Germany), a membrane permeable superoxide mimetic agent, was used to lower superoxide in the mitochondria. It has been widely used in concentrations of $10-30 \mathrm{mM}$ to evaluate the biological responses of superoxide in vitro (Hein and Kuo 1998; MacKenzie and Martin 1998; Arimura, Egashira et al. 2001). (Kondo-Nakamura, Shintani-Ishida et al.). Tiron ( $5 \mathrm{mM}$ ) has also been successfully used to inhibit carbon monoxide-induced superoxide production in cardioblastic $\mathrm{H} 9 \mathrm{c} 2$ cells in vitro. It was reported that brief exposure (approximately $15 \mathrm{~min}$ ) of cells to concentrations of 50 and $100 \mathrm{mM}$ resulted in no cytotoxicity (Krishna, Liebmann et al. 1992). In the present study, I used $5 \mathrm{mM}$ Tiron to establish a negative control to validate mitochondrial superoxide generation with Mito-HE.

Tiron (MW 314.20), which was prepared in PBS, was added to the cells to give a final concentration of $50 \mathrm{mM}$ before the addition of Mito-HE and $\mathrm{DilC}_{1}(5)$ dyes.

\subsubsection{Assessment of kidney mitochondrial superoxide in GC-HT}

In this part of the study, mitochondrial superoxide in kidney cells of rats treated with saline, DEX or ACTH were evaluated.

\subsubsection{Experimental animals}

Male Sprague-Dawley rats (initial body weight of $250-270 \mathrm{~g}$ ) were housed and acclimatised as described in Sections 2.2 and 2.3 of Chapter 2. 
The rats were treated with $\mathrm{ACTH}(0.2 \mathrm{mg} / \mathrm{kg} / \mathrm{day}), \mathrm{DEX}(0.03 \mathrm{mg} / \mathrm{kg} /$ day $)$ or sterile saline (vehicle for $\mathrm{ACTH}$ and $\mathrm{DEX}, 0.9 \% \mathrm{NaCl}, 0.1 \mathrm{~mL} / \mathrm{rat} /$ day) for 12 days (T0-T11) after 4 control days (C4-C1).

Group 1. Sterile saline $(n=16$, of which 10 rats were pooled from the studies in Chapter 6)

Group 2. $\mathrm{ACTH}(\mathrm{n}=15$, of which 10 rats were pooled from the studies in Chapter 6)

Group 3. DEX ( $n=15$, of which 10 rats were pooled from the studies in Chapter 6)

\subsubsection{Systolic blood pressure and body weight measurements}

The animals underwent second-daily tail cuff experiments from day $\mathrm{T} 0$ until day T10 at 9-11 am using the method as described in Section 2.5.1 of Chapter 2. Body weight measurements were recorded on alternate days after the tail-cuff SBP measurements.

\subsubsection{Thymus weight}

At the end of the experiment, these rats were sacrificed under anaesthesia. Thymus resection and blood sampling procedures were as described in Sections 2.8.5.1 and 2.8.4 of Chapter 2, respectively. 
Thymus wet weight, expressed relative to body weight (grams thymus weight per $100 \mathrm{~g}$ body weight), was used as a marker of glucocorticoid activity.

\subsubsection{Kidney mitochondrial Mito-HE fluorescence analysis}

Mito-HE fluorescence intensity was used as a marker of mitochondrial superoxide availability. The kidneys were harvested, prepared for Mito-HE staining and analysed using flow cytometry as described in Sections 5.2.3 to 5.2.6 (above).

\subsubsection{Kidney mitochondrial $\operatorname{DilC}_{1}(5)$ fluorescence analysis}

DilC $_{1}(5)$ fluorescence intensity was used to determine the mitochondrial membrane potential. The method for this analysis was described in Sections 5.2.5 and 5.2.6 (above).

\subsubsection{Statistical analysis}

Results were expressed as mean \pm SEM. Statistical analysis were as described in Section 2.12 of Chapter 2.

\subsection{RESULTS}

\subsubsection{Preliminary experiments}

\subsubsection{Optimisation of Mito-HE concentration}

Concentrations up to $3 \mu \mathrm{M}$ resulted in a steady increase in Mito-HE geometric MFI. This signal plateaued at concentrations between 3-6 $\mu \mathrm{M}$ and declined at 
concentrations $>6 \mu \mathrm{M}$ (Figure 5.3 and Table 5.1). To minimise risk of cytotoxicity initiated by Mito-HE, which might in turn result in cell and mitochondrial damage, a concentration $1 \mu \mathrm{M}$ below the concentration that produced the maximal geometric MFI (ie $2 \mu \mathrm{M}$ ) was chosen.

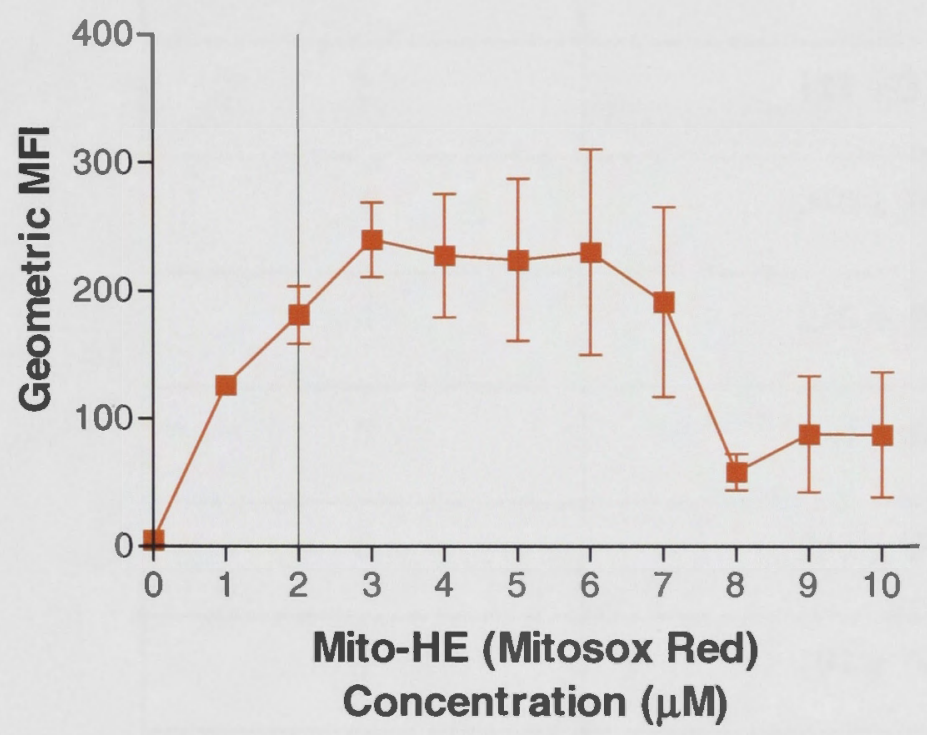

Figure 5.3: Mito-HE titration curve, $n=4$. 
Table 5.1: Mito-HE geometric mean fluorescence intensity generated by the different Mito-HE concentrations

\begin{tabular}{|c|c|}
\hline Mito-HE concentration & Geometric MFI (mean \pm SEM) \\
\hline 0 & $5 \pm 1$ \\
\hline 1 & $126 \pm 7$ \\
\hline 2 & $181 \pm 23$ \\
\hline 3 & $240 \pm 29$ \\
\hline 4 & $228 \pm 48$ \\
\hline 5 & $224 \pm 63$ \\
\hline 6 & $230 \pm 80$ \\
\hline 7 & $191 \pm 74$ \\
\hline 8 & $59 \pm 14$ \\
\hline 9 & $88 \pm 45$ \\
\hline 10 & $88 \pm 49$ \\
\hline
\end{tabular}

\subsubsection{Positive control}

Cells pre-treated with $5 \mu \mathrm{M}$ Antimycin A had significantly higher Mito-HE fluorescence ( $694 \pm 105, \mathrm{n}=6$ ) compared with untreated cells (control, $213 \pm 27$, $\mathrm{n}=6, P<0.005$ ) (Figure 5.4). Antimycin $\mathrm{A}$ at $5 \mu \mathrm{M}$ did not significantly reduce 
mitochondrial membrane potential as indicated by $\mathrm{DiIC}_{1}(5)$ geometric MFI (control: $136 \pm 49$ and Antimycin A: $72 \pm 12, \mathrm{n}=6$ each, $n s$ ) (Figure 5.5).

\section{Positive Control: Mitochondrial Superoxide}

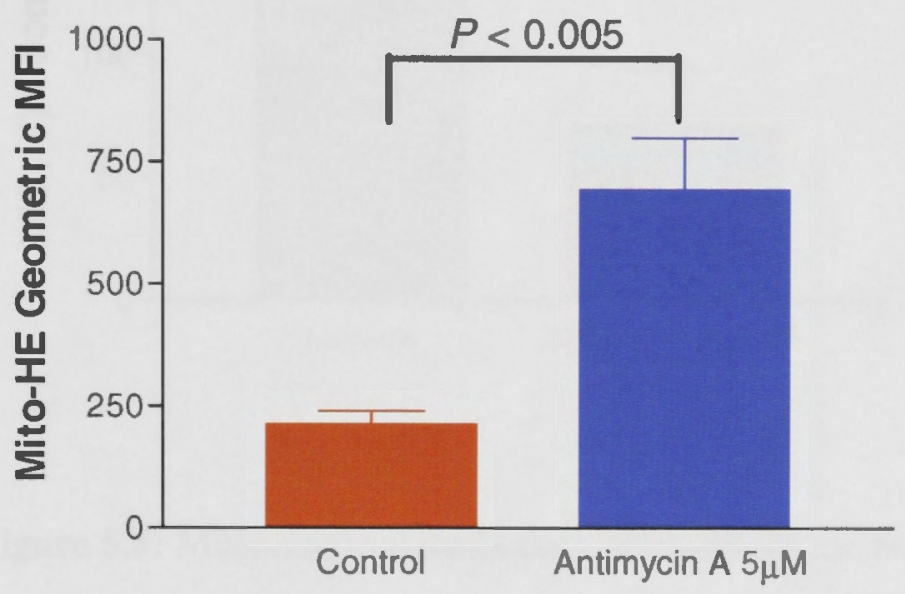

Figure 5.4: Mitochondrial superoxide availability for the positive control established using Antimycin A. Control, $n=6$, Antimycin A, $n=6$. 


\section{Positive Control: Mitochondrial Membrane Potential}

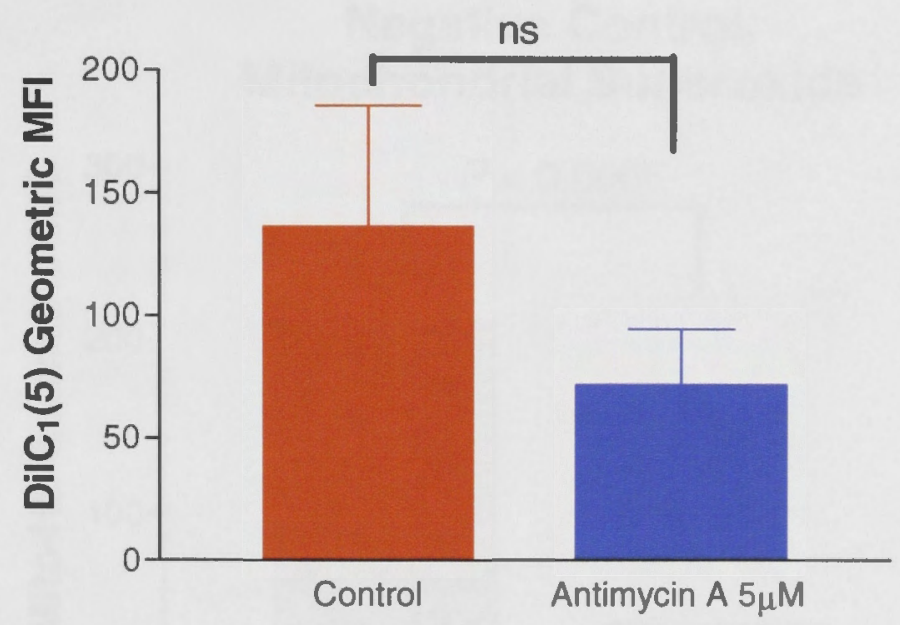

Figure 5.5: Mitochondrial membrane potential for the positive control established using Antimycin A. Control, $\mathrm{n}=6$, Antimycin $\mathrm{A}, \mathrm{n}=6$, $\mathrm{ns}=$ not significant.

\subsubsection{Negative control}

Tiron at $50 \mathrm{mM}$ resulted in significant reduction in Mito-HE geometric MFI ( $37 \pm 6, \mathrm{n}=6)$ compared to controls $(213 \pm 27, \mathrm{n}=6, P<0.0005)$ (Figure 5.6). At this concentration, Tiron did not significantly alter mitochondrial membrane potential as represented by DilC $_{1}(5)$ geometric MFI (control: $136 \pm 49$ and Tiron: $115 \pm 34, \mathrm{n}=6$ each, $n s$ ) (Figure 5.7). 


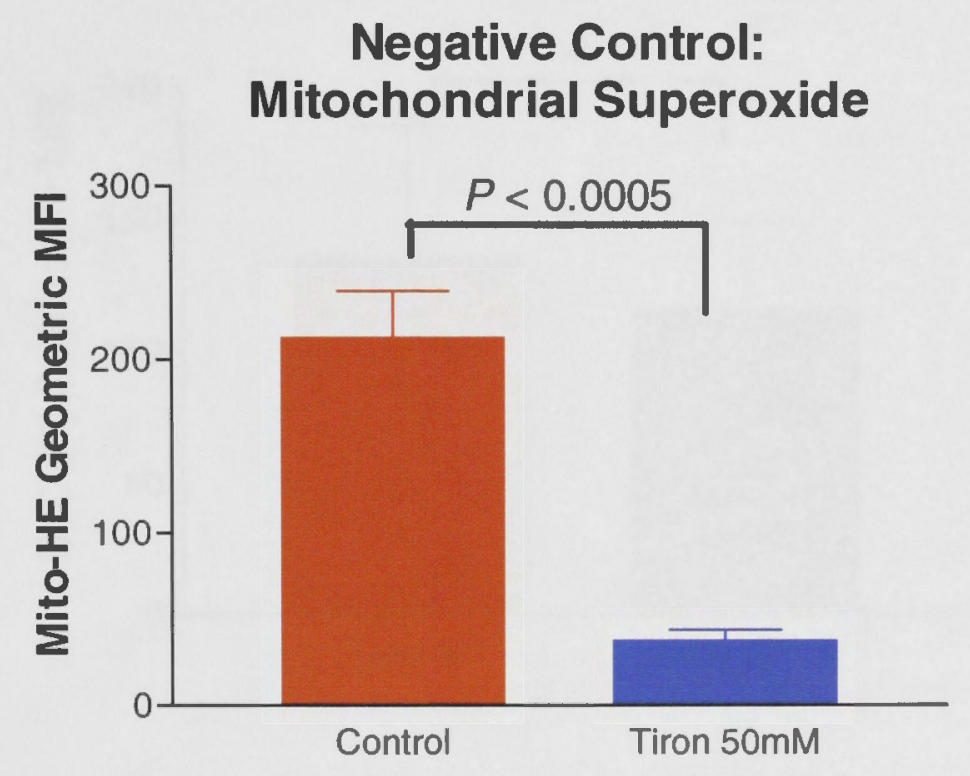

Figure 5.6: Mitochondrial superoxide availability for the negative control established using Antimycin A. Control, $\mathrm{n}=6$, Tiron, $\mathrm{n}=6$. 


\section{Negative Control: Mitochondrial Membrane Potential}

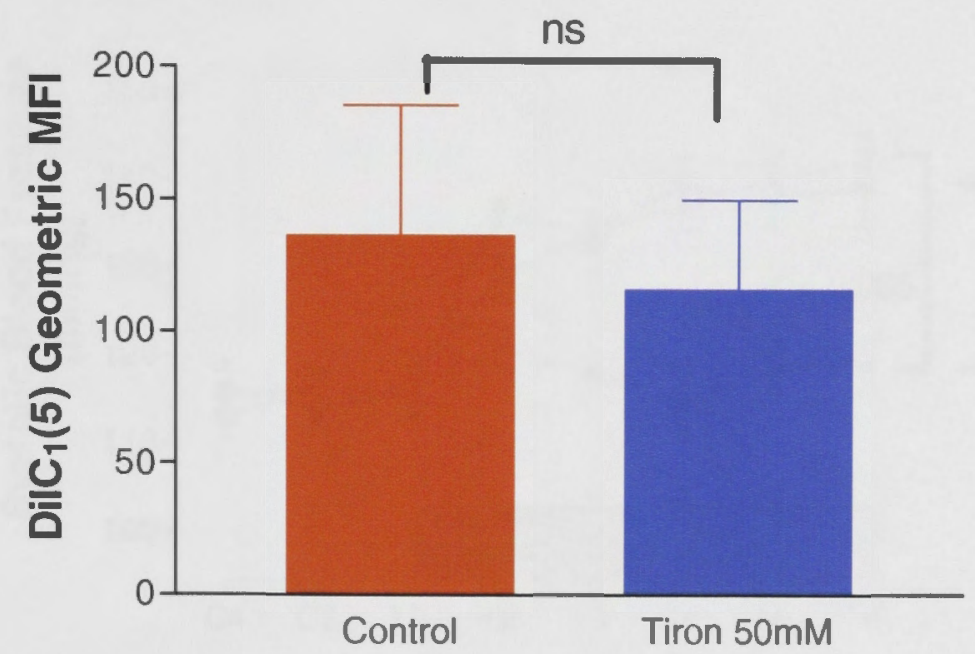

Figure 5.7: Mitochondrial membrane potential for the negative control established using Tiron. Control, $\mathrm{n}=6$, Tiron, $\mathrm{n}=6$, $\mathrm{ns}=$ not significant.

\subsubsection{Assessment of kidney mitochondrial superoxide in GC-HT}

\subsubsection{Systolic blood pressure}

SBP was significantly elevated in DEX-treated rats from $116 \pm 2 \mathrm{mmHg}$ on day T0 to $142 \pm 3 \mathrm{mmHg}$ on day T10 $(\mathrm{n}=15, P<0.0005)$. Similarly, SBP in ACTHtreated rats was significantly raised from $116 \pm 3 \mathrm{mmHg}$ on day $\mathrm{T0}$ to $139 \pm 5 \mathrm{mmHg}$ on day T10 $(\mathrm{n}=15, P<0.0005)$. Saline did not alter SBP (T0: $116 \pm 2 \mathrm{mmHg}$, and T10: $119 \pm 3 \mathrm{mmHg}, \mathrm{n}=16, n s$ ) (Figure 5.8). 
SBP readings were significantly higher in the DEX and ACTH-treated groups compared with the saline-treated groups $\left(P^{\prime}<0.001\right.$ each) (Figure 5.8).

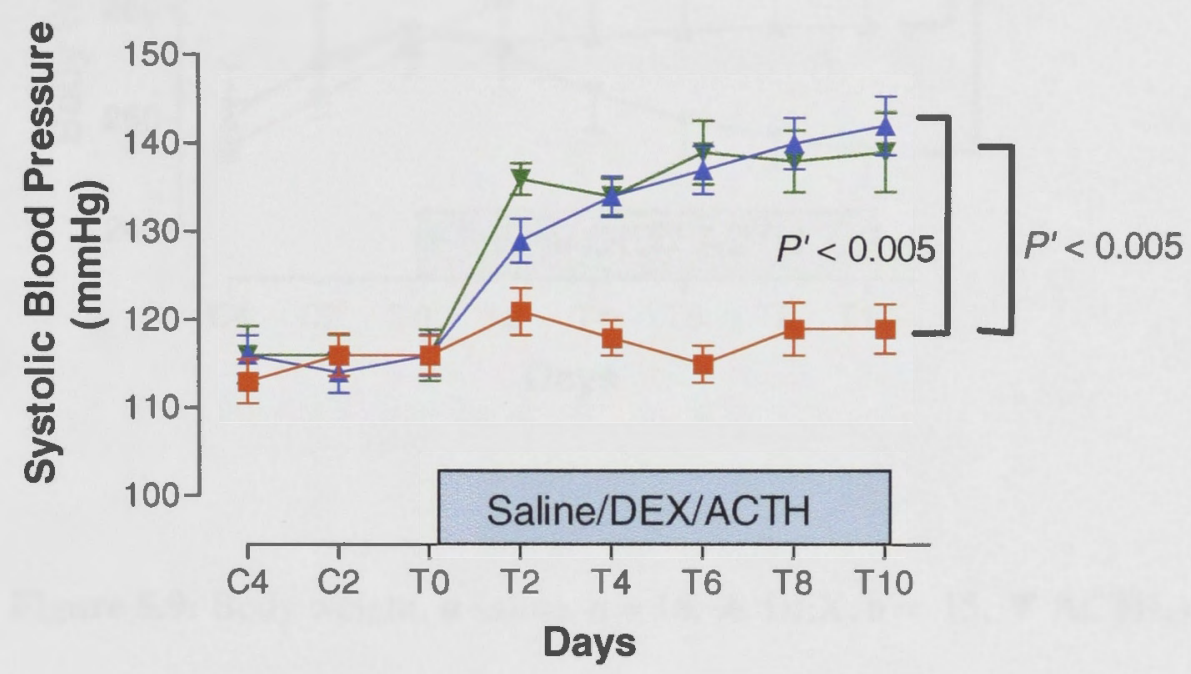

Figure 5.8: Systolic blood pressure. saline, $n=16, \Delta$ DEX, $n=15, \nabla A C T H$, $\mathrm{n}=15$.

\subsubsection{Body weight}

There was a significant weight increase in rats treated with saline from day T0 $(273 \pm 5 \mathrm{~g})$ to day T10 $(310 \pm 5 \mathrm{~g}, \mathrm{n}=15, P<0.0005)$. DEX-treated rats had no significant body weight change between day $\mathrm{T} 0(277 \pm 9 \mathrm{~g})$ to day $\mathrm{T} 10$ $(277 \pm 8 \mathrm{~g}, \mathrm{n}=15, n s)$. Rats on ACTH treatment had progressive weight loss from $276 \pm 8 \mathrm{~g}$ on day T0 to $254 \pm 6 \mathrm{~g}(\mathrm{n}=16, P<0.0005)$ (Figure 5.9$)$.

Overall, rats treated with DEX and ACTH had significantly lower body weight than those on saline treatment $\left(P^{\prime}<0.001\right.$ each) (Figure 5.9). 


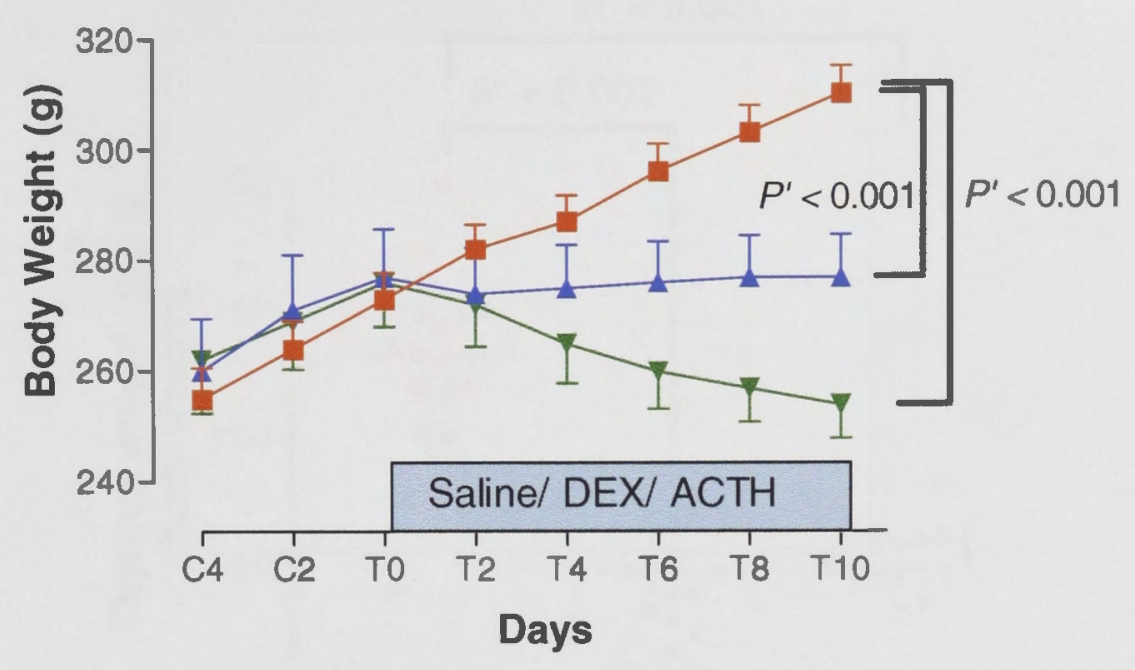

Figure 5.9: Body weight. $n$ saline, $\mathrm{n}=16, \Delta \mathrm{DEX}, \mathrm{n}=15, \boldsymbol{\mathrm { ACTH }}, \mathrm{n}=15$.

\subsubsection{Thymus weight}

Thymus weight in DEX- $(47 \pm 5 \mathrm{mg} / \mathrm{g}$ body weight, $\mathrm{n}=15)$ and ACTH-treated rats $(59 \pm 10 \mathrm{mg} / \mathrm{g}$ body weight, $\mathrm{n}=15)$ was significantly lower compared to saline-treated rats $\left(132 \pm 7 \mathrm{mg} / \mathrm{g}\right.$ body weight, $\mathrm{n}=16, P^{\prime}<0.001$ each) (Figure 5.10). 


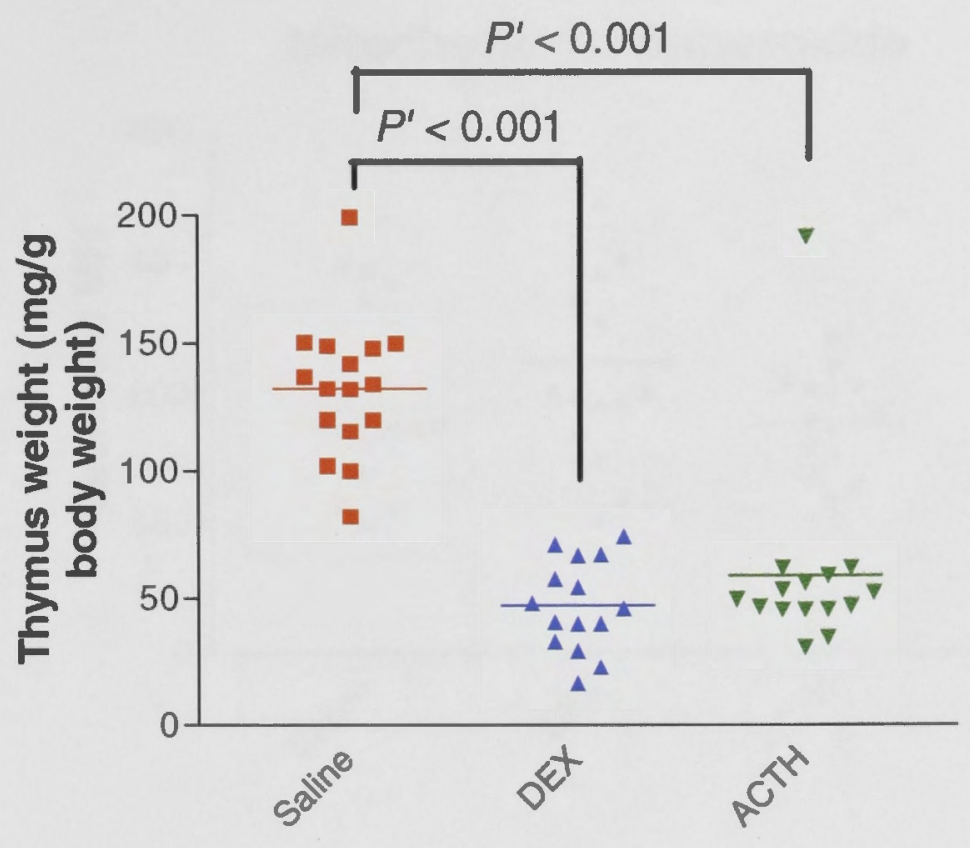

Figure 5.10: Thymus weight. - saline, $\mathrm{n}=16, \boldsymbol{\Delta}$ DEX, $\mathrm{n}=15, \nabla$ ACTH, $\mathrm{n}=15$.

\subsubsection{Kidney mitochondrial Mito-HE fluorescence analysis}

There was no difference in kidney mitochondrial Mito-HE fluorescence intensity between the (DEX- $223 \pm 20, n=15)$ and ACTH-treated groups $(176 \pm 9, n=15)$ compared with saline-treated group ( $188 \pm 16, n=16, n s)$ (Figure 5.11). 


\section{Mitochondrial Superoxide}

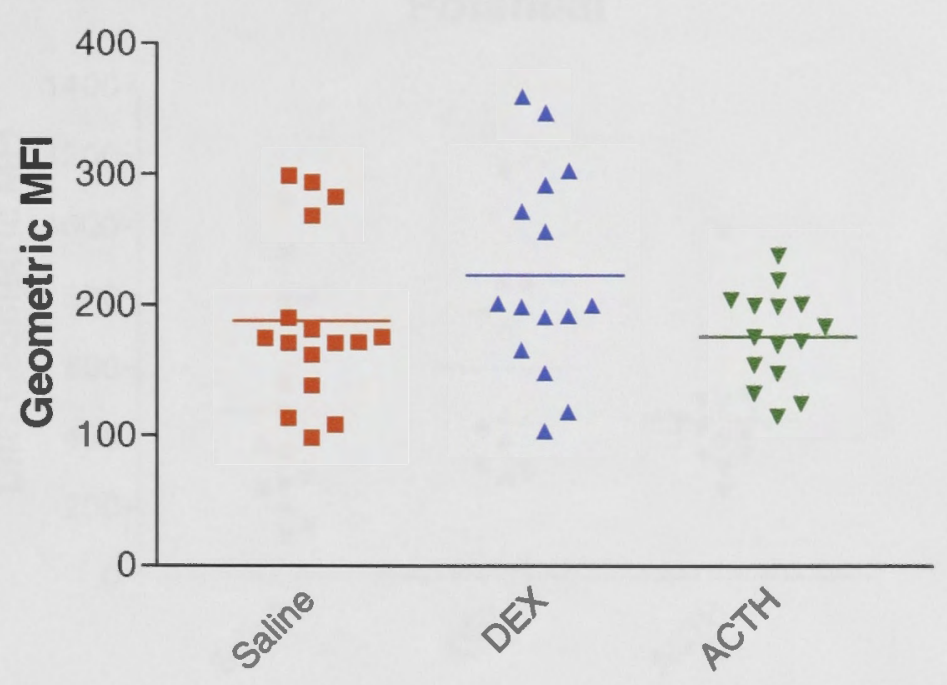

Figure 5.11: Kidney mitochondrial Mito-HE geometric mean fluorescence intensity as marker of mitochondrial superoxide availability. a saline, $\mathrm{n}=16$, DEX, $\mathrm{n}=15, \nabla$ ACTH, $\mathrm{n}=15$.

\subsubsection{Kidney mitochondrial $\operatorname{DiIC}_{1}(5)$ fluorescence intensity}

There was no difference in kidney mitochondrial $\operatorname{DilC}_{1}(5)$ fluorescence intensity between DEX- $(599 \pm 75, \mathrm{n}=13)$ and ACTH- $(451 \pm 49, \mathrm{n}=13)$ treated groups compared with saline-treated group $(476 \pm 82, \mathrm{n}=14, n s)$. 


\section{Mitochondrial Membrane \\ Potential}

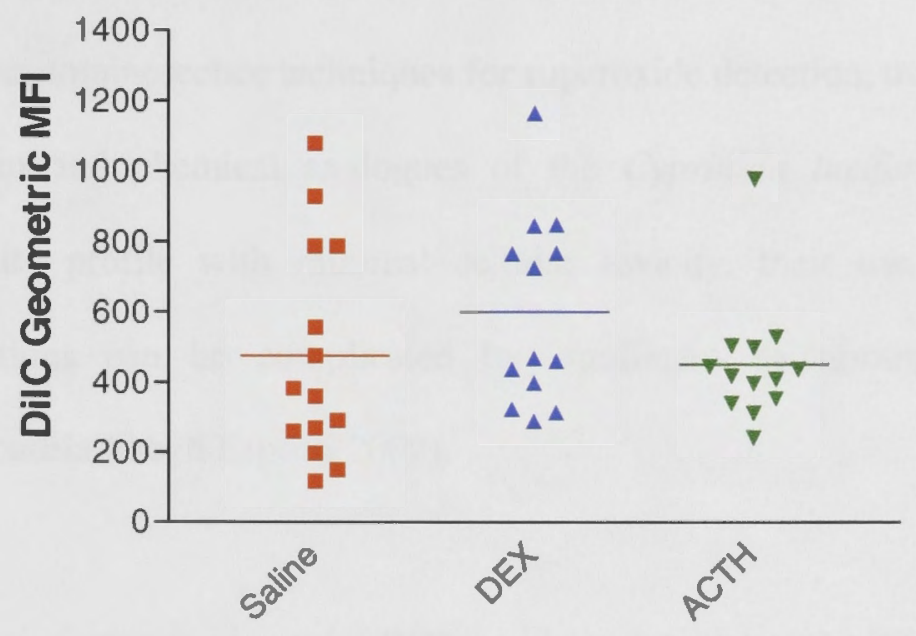

Figure 5.12: Kidney mitochondrial $\operatorname{DilC}_{1}(5)$ mean fluorescence intensity as marker of mitochondrial membrane potential. saline, $\mathrm{n}=14, \mathbf{\Delta E X}, \mathrm{n}=13$, $\mathrm{ACTH}, \mathrm{n}=13$.

\subsection{DISCUSSION}

Laboratory assessment of reactive oxygen species remains very challenging. Superoxide, the first ROS to be generated, has a very short half life. Its rapid dismutation by MnSOD and interaction with other compounds make its detection rather difficult and arduous. Earlier techniques which employed spectrophotometry such as cytochrome c reduction, adenochrome formation and nitroblue tetrazolium are associated with limited specificity and sensitivity 
(Tarpey and Fridovich 2001). Luminescent and fluorescent probes are increasingly used for measurement of ROS in cells and tissues. Despite claims that chemiluminescence techniques for superoxide detection, using probes such as lucigenin and chemical analogues of the Cyprinida luciferin, offer a better sensitivity profile with minimal cellular toxicity, their use in mitochondrial preparations can be complicated by significant background reactions with mitochondria (Degli Esposti 2002).

Information on mitochondrial ROS has been based largely on studies performed on mechanically-isolated mitochondrial preparations or submitochondrial particles. Mechanical isolation of mitochondria from their natural cellular environment through differential centrifugation can affect the bioenergetics of the electron transport chain. Nohl et al. argued that previous claims that mitochondrial ROS production are byproducts of cellular respirations may simply reflect bioenergetic artefacts consequent on mechanical mitochondrial isolation (Nohl, Gille et al. 2005). Some workers examined mitochondrial ROS in intact live cells to avoid this artefact though this remains challenging experimentally (Dawson, Gores et al. 1993; Vanden Hoek, Shao et al. 1997; Duranteau, Chandel et al. 1998). Whilst this is possible for detection of hydrogen peroxide which is membrane permeable and stable, the detection of mitochondrial superoxide in live cells becomes more problematic as it is heavily affected by mitochondrial superoxide dismutase, a tightly regulated superoxide scavenger within the mitochondria. Furthermore, their activity varies with the different cell lines. One 
of the techniques that is increasingly used is in situ evaluation of mitochondrial superoxide using a novel fluorescence dye, Mito-HE.

In this experiment, Mito-HE probe was used in flow cytometry to detect mitochondrial superoxide in live cells. The use of flow cytometry allows a large number of cells to be analysed rapidly. Simultaneous quantitative measurements of a number of parameters such as mitochondrial superoxide and membrane potential, or other markers are also possible with this technique. In addition, cell debris can also be excluded from analysis. With this method, which only detects fluorescence associated with particles, lower Mito-HE concentrations can be used. Whilst $5 \mu \mathrm{M}$ is recommended for other fluorometric techniques, lower Mito-HE concentrations can be used in flow cytometry. In this study, we have shown that Mito-HE concentration of more than $5 \mu \mathrm{M}$ can result in reductions in mitochondrial membrane potentials and decrease uptake and fluorescence of Mito-HE in this cell preparation. It is also important to do parallel assessment of mitochondrial membrane potential to ensure that cell treatments, including MitoHE dye loading itself, do not depolarise the mitochondria in the cells as Mito-HE requires a membrane potential across the inner mitochondrial membrane to accumulate there. Otherwise, Mito-HE can be oxidised elsewhere in the cell and result in falsely positive signals for mitochondrial superoxide.

There are also a number of limitations associated with the use of the HE-based assay. Superoxide reacts with Mito-HE to form two highly fluorescent compounds, HO-Mito-Etd ${ }^{+}$and Mito-Etd ${ }^{+}$that can be detected optimally by 
excitation wavelengths of 396 and $510 \mathrm{~nm}$ respectively. HO-Mito-Etd ${ }^{+}$is more specific for superoxide whilst Mito-Etd ${ }^{+}$can be generated by other non-specific oxidation products (Robinson, Janes et al. 2006). Standard lasers available on flow cytometers can excite fluorophores at 488 and $405 \mathrm{~nm}$. Whilst excitation wavelength at $488 \mathrm{~nm}$ fluoresces Mito-HE oxidation product very well, its specificity can be enhanced by using lower excitation wavelengths (eg $405 \mathrm{~nm}$ when used for flow cytometry) (Robinson, Janes et al. 2006). However, at this near ultraviolet wavelength, the autofluorescence can be intense and may be difficult to exclude fully from flow data analysis. In this study, a flow cytometer with an excitation wavelength of $488 \mathrm{~nm}$ was used as the issue associated with decreased specificity was accounted for by having a saline-treated control group in the study. Any increase in fluorescent signal generated by the glucocorticoidinduced hypertensive group in comparison with the control group could therefore be presumed to correlate with mitochondrial superoxide. Another limitation with the use of a fluorescent dye is that Mito-HE is prone to photo-oxidation and its oxidation product susceptible to photo-bleaching. Thus light exposure needs to be kept to a minimum during the experiment as was done in this study.

Antimycin A and Tiron were used as positive and negative controls respectively to validate mitochondrial superoxide generation with Mito-HE. The validation studies described in Sections 5.2.6 and 5.2.7 showed that Mito-HE can detect an increase in kidney mitochondrial superoxide induced biochemically using Antimycin A and a decrease due to the scavenging effect of Tiron. Although Antimycin A can result in loss of mitochondrial membrane potential, a 
phenomenon known to result in the uptake of Mito-HE in sites other than the mitochondria, the concentration used in this study $(5 \mu \mathrm{M})$ did not affect the membrane potential. Tiron at $50 \mathrm{mM}$ also did not significantly reduce mitochondrial membrane potential, and thus is unlikely to affect the specificity of the results. The main limitation of these controls was that the stimulation and inhibition of mitochondrial superoxide were done in in vitro rather than in in vivo models. However, the latter was not feasible given the toxicity of these agents.

In this study, cells from the kidney were used for several reasons. Firstly, the kidney is a highly-vascularised organ, rich in resistance blood vessels. Secondly, the kidney is relevant to GC-HT. We have previously shown that ACTH-HT, a model mainly mediated by cortisol, is associated with increased renal vascular resistance (Wen, Fraser et al. 1998; Wen, Fraser et al. 1999). Furthermore, the rise in $\mathrm{BP}$ due to $\mathrm{ACTH}$ in rats was accompanied by reductions in iNOS and eNOS gene expression in the kidney (Lou, Wen et al. 2001).

Although the Mito-HE probe has mainly been used in high performance liquid chromatography and fluorescent microscopy, I have independently developed a method for its use in flow cytometry for our laboratory with technical assistance from Dr Harpreet Vohra (Flow Cytometry Resource Facility, John Curtin School of Medical Research). Since the development of this technique for use in the studies of the role of mitochondrial superoxide in GC-HT (current chapter and Chapter 6), there have been a number of other researchers who have published work using Mito-HE in flow cytometry. Table 5.1 summarises the studies to date 
using Mito-HE in flow cytometry. Whilst there were similarities with the flow technique used in these studies, such as the use of $488 \mathrm{~nm}$ excitation wavelength, there were also several important differences that need to be highlighted.

Most of these authors used $5 \mu \mathrm{M}$ Mito-HE, as per the manufacturer's protocol (Product information via http://probes.invitrogen.com/media/pis/mp36008.pdf) which was developed using live bovine pulmonary epithelial cells, MRC5 human lung fibroblasts and mouse 3T3 fibroblasts for used in fluorescence microscopy. Thus, the concentration for optimal fluorescence without causing cytotoxicity for other cell lines cannot be assumed. The optimal Mito-HE concentration needs to be individually optimised and assessed based on the different cell lines and experimental conditions.

Another fundamental difference between the present study and some of those listed on Table 5.1 was the concurrent determination of the mitochondrial membrane potential. This is a crucial step as uptake of Mito-HE into mitochondria is a function of mitochondrial membrane potential (Robinson, Janes et al. 2008). False positive results can be a consequence of collapsed mitochondrial membrane potential rather than excess mitochondrial superoxide production. Only a few studies performed this crucial analysis (Zhang, Soboloff et al. 2006; Dvorak, Payne et al. 2007; Payne, Weber et al. 2007; Cordero, De Miguel et al. 2010). These investigators assessed the mitochondrial membrane potential to assess mitochondrial integrity but failed to recognise the confounding effect of collapsed mitochondrial membrane potential on fluorescence analysis using Mito-HE. 
Furthermore, validations with positive and negative controls were not performed in some of the studies listed (Table 5.2).

Some of these authors have validated the flow cytometric technique with confocal microscopy and confirmed that the fluorescence signal due to the oxidation products of Mito-HE was indeed a consequence of mitochondrial superoxide production. Using confocal microscopy, Mukhopadhyay et al also showed that Mito-HE staining is enhanced in dead cells (Mukhopadhyay, Rajesh et al. 2007). Nevertheless, dead and damaged cells have already being excluded from the present study and further analysis using confocal microscopy is unlikely to provide any extra information. 
Table 5.2: Use of Mito-HE in flow cytometry

\begin{tabular}{|c|c|c|c|c|c|c|}
\hline Cell Type & Method & $\begin{array}{c}\text { Mito-HE } \\
\text { concentration } \\
(\mu \mathrm{M})\end{array}$ & $\begin{array}{l}\text { Positive } \\
\text { Control }\end{array}$ & $\begin{array}{l}\text { Negative } \\
\text { Control }\end{array}$ & $\begin{array}{c}\text { Membrane } \\
\text { Potential } \\
\text { Assessment }\end{array}$ & References \\
\hline $\begin{array}{c}\text { Oesophageal } \\
\text { adenocarcinoma (Seg 1) } \\
\text { cells }\end{array}$ & $\begin{array}{l}\text { Flow } \\
\text { cytometry }\end{array}$ & 5 & - & - & Mitotracker Red & (Dvorak, Payne et al. 2007) \\
\hline $\begin{array}{c}\text { Colon carcinoma (HCT } \\
116) \text { cells }\end{array}$ & $\begin{array}{l}\text { Flow } \\
\text { cytometry }\end{array}$ & 5 & Deoxycholate & - & Mitotracker Red & (Payne, Weber et al. 2007) \\
\hline $\begin{array}{c}\text { Human diffuse large cell } \\
\text { lymphoma (OCI-Ly 19), } \\
\text { Human Burkitts } \\
\text { Lymphoma (Ramos), } \\
\text { normal human B cells }\end{array}$ & $\begin{array}{c}\text { Flow } \\
\text { cytometry }\end{array}$ & 5 & - & - & - & (Brookes, Morse et al. 2007) \\
\hline HCAEC & $\begin{array}{l}\text { Flow } \\
\text { cytometry }\end{array}$ & 5 & - & - & - & $\begin{array}{l}\text { (Rajesh, Mukhopadhyay et al. } \\
\text { 2007) }\end{array}$ \\
\hline
\end{tabular}




\begin{tabular}{|c|c|c|c|c|c|c|}
\hline Cell Type & Method & $\begin{array}{c}\text { Mito-HE } \\
\text { concentration } \\
(\boldsymbol{\mu} \mathbf{M})\end{array}$ & $\begin{array}{c}\text { Positive } \\
\text { Control }\end{array}$ & $\begin{array}{c}\text { Negative } \\
\text { Control }\end{array}$ & $\begin{array}{c}\text { Membrane } \\
\text { Potential } \\
\text { Assessment }\end{array}$ & References \\
\hline $\begin{array}{c}\text { Rat embryonic ventricular } \\
\text { myocardial (H9c2) cells } \\
\text { and HCAEC }\end{array}$ & $\begin{array}{c}\text { Flow } \\
\text { cytometry, } \\
\text { confocal } \\
\text { microscopy }\end{array}$ & 5 & $\begin{array}{c}\text { Antimycin A, } \\
\text { Paraquat } \\
\text { permeable } \\
\text { SOD }\end{array}$ & $\begin{array}{c}\text { (Mukhopadhyay, Rajesh et al. } \\
\text { 2007a) }\end{array}$ \\
\hline HCAEC, PMVEC & $\begin{array}{c}\text { Flow } \\
\text { cytometry }\end{array}$ & 5 & $\begin{array}{c}\text { Antimycin A, } \\
\text { doxorubicin }\end{array}$ & $\begin{array}{c}\text { Cell- } \\
\text { permeable } \\
\text { SOD }\end{array}$ & (Mukhopadhyay, Rajesh et al. \\
\hline Human spermatozoa & $\begin{array}{c}\text { Flow } \\
\text { cytometry, } \\
\text { confocal } \\
\text { microscopy }\end{array}$ & 2 & Rotenone & - & - & (Koppers, De Iuliis et al. 2008) \\
\hline Blood mononuclear cells & $\begin{array}{c}\text { Flow } \\
\text { cytometry }\end{array}$ & 1 & - & Mitotracker Red & $\begin{array}{l}\text { (Cordero, De Miguel et al. } \\
\text { 2010) }\end{array}$ \\
\hline
\end{tabular}

HCAEC = human coronary artery endothelial cells , HPMVEC $=$ Human pulmonary micorvascular endothelial cells, SOD = superoxide dismutase. 
The in vivo physiological studies verified previous findings that both ACTH and DEX increased SBP in rats (Zhang, Jang et al. 2003; Hu, Zhang et al. 2006). This increase in blood pressure was accompanied by a decrease in thymus weight implicating increased glucocorticoid activity induced by ACTH and DEX injections; and oxidative stress as indicated by an increase in plasma $F_{2}$ isoprostane concentration (Ong, Zhang et al. 2008) (Sections 6.3.7, 8.3.7, 11.2). In this study, however, ACTH and DEX did not result in a significant increase in Mito-HE geometric MFI despite producing blood pressure elevation. Possibly the increase in mitochondrial superoxide generation in GC-hypertensive rats was not large enough for a change to be detected. Another possible explanation is that Mito-HE oxidation by superoxide (rate constant of $\sim 10^{6} \mathrm{M}^{-1} \mathrm{~s}^{-1}$ ) produced in the impermeable compartment could not surpass the efficacy of endogenous mitochondrial superoxide dismutase (rate constant $>10^{9} \mathrm{M}^{-1} \mathrm{~s}^{-1}$ ). Nevertheless, this study did not find any evidence that mitochondrial superoxide plays a major role in the pathogenesis of GC-HT.

\subsection{CONCLUSION}

In this study, kidney mitochondrial superoxide was not increased in glucocorticoid-induced hypertensive rats, suggesting that mitochondrial superoxide does not play a significant role in the pathogenesis of glucocorticoid hypertension. 
The role of mitochondrial superoxide in GC-HT is further evaluated and discussed in Chapter 6. 


\section{CHAPTER 6}

Role of Mitochondrial Superoxide in Glucocorticoid-

Induced Hypertension in the Rat 


\subsection{INTRODUCTION}

Mitochondrial ROS have been implicated in the pathogenesis of hypertension. Treatment with alpha-lipoic acid, an antioxidant that can improve mitochondrial function (Hagen, Ingersoll et al. 1999), prevented both hypertension and mitochondrial ROS overproduction due to hyperglycemia in rats (El Midaoui, Elimadi et al. 2003) and thus suggested a role for mitochondrial ROS in this form of experimental hypertension. Mitochondrial superoxide dismutase deficiency has been shown to be linked with susceptibility to hypertension with aging and high salt intake in mouse (Rodriguez-Iturbe, Sepassi et al. 2007). The role of mitochondrial ROS overproduction in the development of glucocorticoid-induced hypertension is unclear.

Alpha-lipoic acid is a naturally-occuring short chain fatty acid that serves as an important cofactor for many enzyme complexes including mitochondrial respiratory enzymes. Exogenously-administered alpha-lipoic acid has been shown to be an effective antioxidant. Urinary $\mathrm{F}_{2}$-isprostane concentration, a biomarker of lipid peroxidation, was significantly reduced after treatment with $600 \mathrm{mg} / \mathrm{day}$ alpha-lipoic acid orally for 2 months in humans (Marangon, Devaraj et al. 1999). Apart from this, alpha-lipoic acid has also been shown to improve mitochondrial function. A single dose of alpha-lipoic acid (100 mg/kg i.p.) resulted in improvement in mitochondrial function, determined by mitochondrial oxygen consumption and complex I, II and IV activities, in endotoxemic rats (Vanasco, Cimolai et al. 2008). Furthermore, alpha-lipoic acid supplementation $(0.5 \% \mathrm{w} / \mathrm{w})$ 
improved the average mitochondrial membrane potential in old rats hepatocytes to that of young rats (Hagen, Ingersoll et al. 1999). Mitochondrial membrane potential is another indicator of mitochondrial health as it reflects the metabolic activity and integrity of mitochondrial membrane (Distelmaier, Koopman et al. 2008).

\subsection{METHODS}

This study was approved by the Animal Experimentation Ethics Committee of the Australian National University (Protocol No. J. HB. 20.05). Male SpragueDawley rats were housed and acclimatised as described in Sections 2.2 and 2.3 of Chapter 2. The general methodology for this study was as described in Chapter 2.

Alpha-lipoic acid powder (Sigma, St. Louis, USA) was administered by mixing in ground food and given to rats overnight (16-18 hours). Normal saline $(0.9 \% \mathrm{NaCl}$, $0.1 \mathrm{~mL} / \mathrm{rat} /$ day $), \operatorname{DEX}(10 \mu \mathrm{g} / \mathrm{rat} /$ day $)$ and ACTH $(0.2 \mathrm{mg} / \mathrm{kg} /$ day $)$ were administered from day T0 to T11 using the technique described in Section 2.3 of Chapter 2.

The rats were randomly divided into 9 treatment groups. The alpha-lipoic acid prevention study was performed in both DEX-and ACTH-HT. The reversal study was only performed in DEX-HT as alpha-lipoic acid completely prevented DEXHT. 


\section{Control groups}

Control rats were allocated $30 \mathrm{~g}$ of plain ground food per rat per night (16-18 hours) from P0. Pelleted food was given during the control days and daytime (from approximately 0900 to 1700 hours) from P0.

Group 1: Saline $(\mathrm{n}=10)$

Group 2: DEX $(\mathrm{n}=10)$

Group 3: $\mathrm{ACTH}(\mathrm{n}=10)$

\section{Alpha lipoic acid prevention studies}

In the prevention studies, rats were pre-treated with either alpha-lipoic acid (500 mg/kg in food) (El Midaoui, Elimadi et al. 2003) in ground food overnight (16-18 hours) from P0, 4 days before commencement of saline, DEX or ACTH injections. In another group of 4 rats, high dose alpha-lipoic acid $(5 \mathrm{~g} / \mathrm{kg}$ of ground food, starting at P0) was given prior to the commencement of ACTH injections from T0-T11.

Group 4. Alpha-lipoic acid + saline $(n=10)$

Group 5. Alpha-lipoic acid + DEX $(n=10)$ 
Group 6. Alpha-lipoic acid + ACTH $(n=10)$

Group 7. Alpha-lipoic acid (high dose) + ACTH ( $n=4)$

\section{Alpha lipoic acid reversal study}

In the reversal studies, oral alpha-lipoic acid-laced food was given 4 days after the subcutaneous injections (T4-T11).

Group 8. Saline + alpha-lipoic acid $(\mathrm{n}=10)$

Group 9. DEX + alpha-lipoic acid $(n=10)$

\subsubsection{Tail-cuff blood pressure and body weight measurements}

The animals underwent second-daily tail cuff experiments from day C4 until day T10 at 9-11 am using the method described in Section 2.5.1 of Chapter 2. Body weight measurements were recorded on alternate days after the tail-cuff SBP measurements.

\subsubsection{Thymus weight}

On day T11, rats were sacrificed under isoflurane anaesthesia. Thymus resection were as described in Section 2.8.5.1 of Chapter 2. 
Thymus wet weight, expressed relative to body weight (grams thymus wet weight per $100 \mathrm{~g}$ body weight), was used as a marker of glucocorticoid activity.

\subsubsection{Kidney Mito-HE and DiIC $_{1}(5)$ fluorescence}

The kidneys were resected and prepared for mitochondrial superoxide determination using Mito-HE fluorescence analysis via flow cytometry technique as described in Section 5.2.3 of Chapter 5. This was used as marker of mitochondrial superoxide availability. Double staining with $\operatorname{DiIC}_{1}(5)$ was performed using the technique described in Section 5.2 .5 of Chapter 5 to assess mitochondrial membrane potential.

\subsubsection{Blood glucose concentration}

Blood was drawn via cardiac puncture as described in Section 2.8.4 of Chapter 2. A drop of blood was placed on the glucometer strip (Precision Plus Blood Glucose Electrodes, Abbott Laboratories, MA, USA) and the blood glucose concentration was read on a glucometer (MediSense 2, Abbott Laboratories, Bedford, MA, USA).

\subsubsection{Plasma nitrate and nitrite assay}

Plasma nitrate and nitrite (NOx) concentrations, measured using the Griess colorimetric reaction described in Section 2.10 .1 of Chapter 2, were used as a marker of endogenous $\mathrm{NO}$ availability. 


\subsubsection{Plasma $F_{2}$-isoprostane assay}

Plasma $\mathrm{F}_{2}$-isoprostane concentration was used as a marker of systemic lipid peroxidation. The assay technique was described in Section 2.11 .3 of Chapter 2 .

\subsubsection{Statistical analysis}

Results were expressed as mean \pm SEM. Statistical analysis were as described in Section 2.12 of Chapter 2.

\subsection{RESULTS}

\subsubsection{Systolic blood pressure}

DEX increased SBP from $115 \pm 3$ to $139 \pm 4 \mathrm{mmHg}(\mathrm{T} 0-\mathrm{T} 10, P<0.005)$ and ACTH from $110 \pm 3$ to $133 \pm 4 \mathrm{mmHg}$ (T0-T10, $P<0.0005$ ). Sham injection with sterile saline did not modify SBP (T0: $113 \pm 2, \mathrm{~T} 10$ : $119 \pm 3 \mathrm{mmHg}$ ). Between group comparisons showed that SBP in the groups receiving DEX and ACTH were significantly higher than in the saline-treated group $(n=10$ each, $\left.P^{\prime}<0.001\right)$.

\subsubsection{Prevention studies}

There was no significant change in SBP in the alpha-lipoic acid + saline group (T0: $111 \pm 2, \mathrm{~T} 10: 118 \pm 3 \mathrm{mmHg}, n s$ ). With alpha-lipoic acid pre-treatment, DEX did not significantly increase SBP (T0: $117 \pm 4$, T10: $126 \pm 5 \mathrm{mmHg}, n s$ ). However, there was a significant increase in SBP in ACTH-treated rats receiving 
alpha-lipoic acid pretreatment (T0: $115 \pm 2$ to T10: $129 \pm 5 \mathrm{mmHg}, P<0.05$ ). Despite this, there was a significant decrease in SBP in the alpha-lipoic acid + ACTH group $\left(P^{\prime}<0.001\right)$ when compared with the ACTH-only group, indicating partial prevention by alpha-lipoic acid. A tenfold increase in alpha-lipoic acid dose $(100 \mathrm{mg} / \mathrm{rat} / \mathrm{day})$ did not decrease the SBP further. There was no difference in SBP between the alpha lipoic acid + saline-treated group and the saline-only group. SBP of the alpha-lipoic acid + DEX-treated group was significantly lower than that of the DEX-only group $\left(P^{\prime}<0.001\right)$ (Figures 6.1a and b).

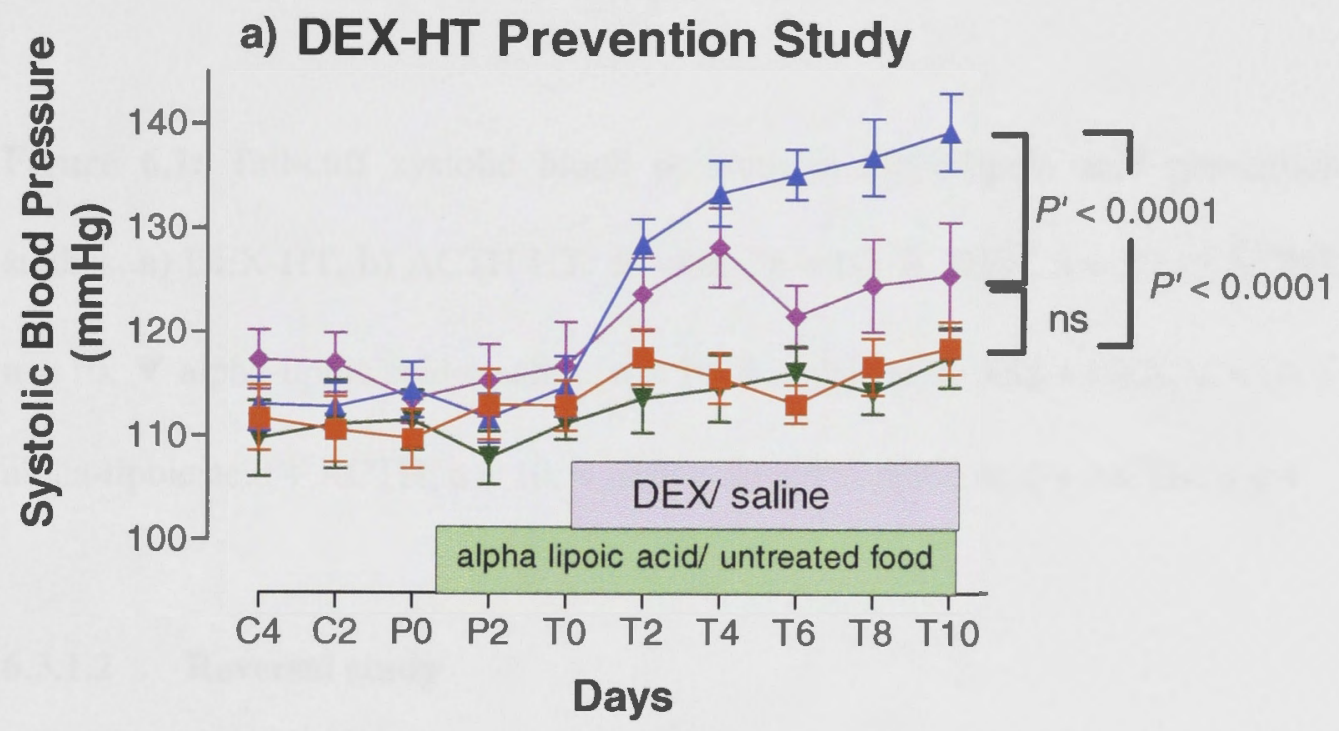




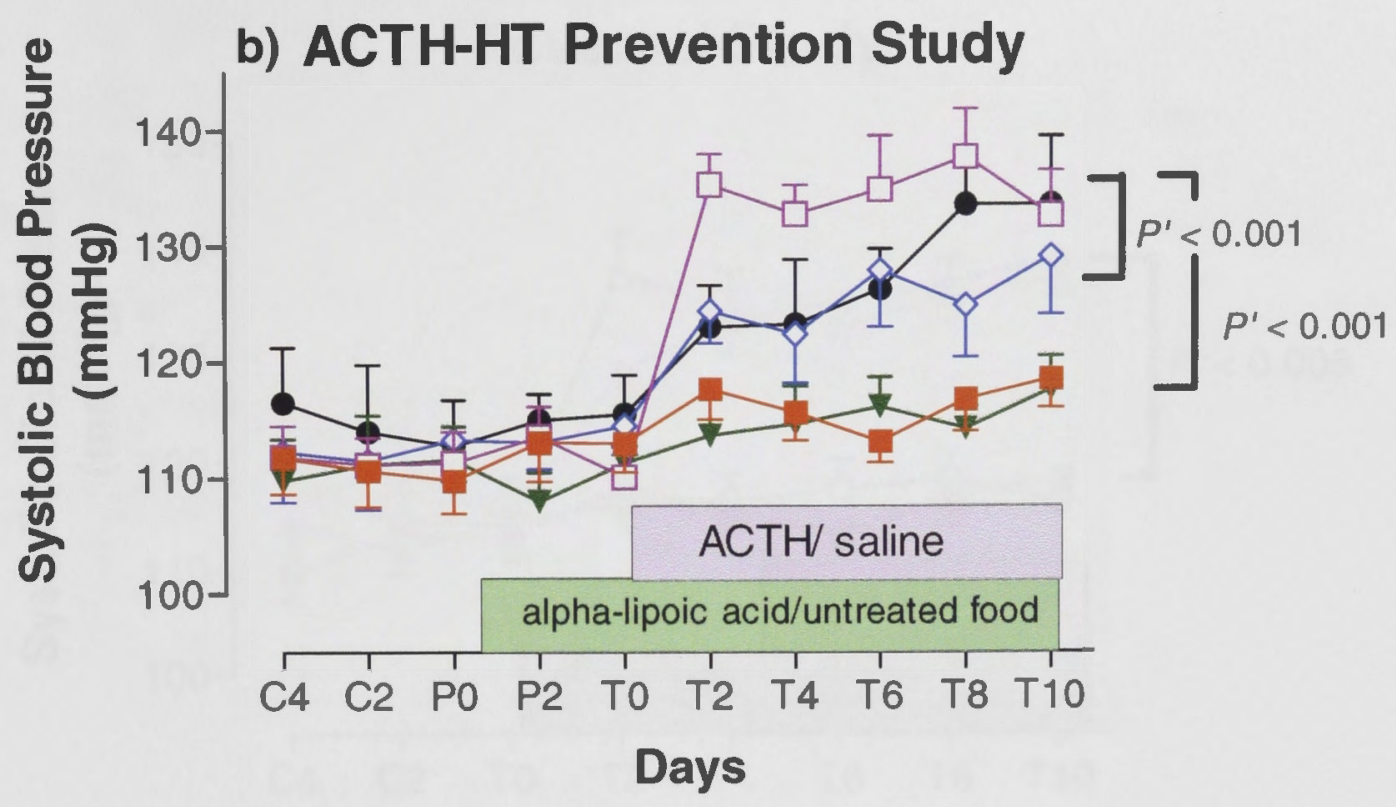

Figure 6.1: Tail-cuff systolic blood pressure in alpha-lipoic acid prevention studies. a) DEX-HT, b) ACTH-HT. a saline, $\mathrm{n}=10$; $\triangle \mathrm{DEX}, \mathrm{n}=10$; $\square \mathrm{ACTH}$, $\mathrm{n}=10 ; \boldsymbol{\nabla}$ alpha-lipoic acid + saline, $\mathrm{n}=10 ;$ alpha-lipoic acid $+\mathrm{DEX}, \mathrm{n}=10, \diamond$ alpha-lipoic acid + ACTH, $\mathrm{n}=10$, $\bullet$ high dose alpha-lipoic acid $+\mathrm{ACTH}, \mathrm{n}=4$.

\subsubsection{Reversal study}

The increase in SBP due to DEX was not reversed by alpha-lipoic acid (10 mg/rat/day) treatment. Systolic BP on day T4, day 1 of alpha-lipoic acid treatment, was $134 \pm 4 \mathrm{mmHg}$ and on $\mathrm{T} 10$ was $137 \pm 2 \mathrm{mmHg}(\mathrm{n}=10, n s)$. As in the prevention study, alpha-lipoic acid did not alter SBP in the saline treated group (T4: $133 \pm 3 \mathrm{mmHg}$; T10: $139 \pm 4 \mathrm{mmHg}, \mathrm{n}=10, n s$ ) (Figure 6.2). 


\section{Reversal Study}

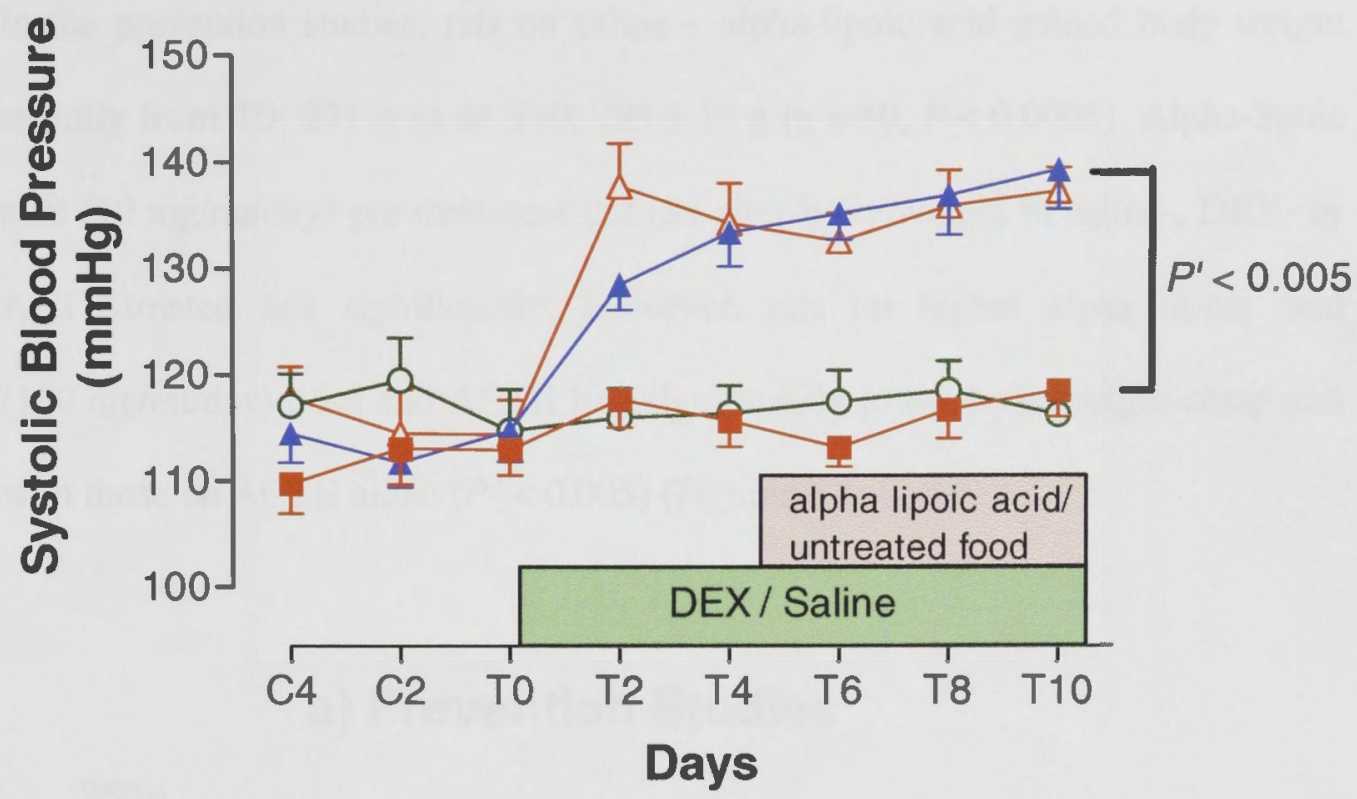

Figure 6.2: Tail-cuff systolic blood pressure in alpha-lipoic acid/ DEX-HT reversal study. $=$ saline, $\mathrm{n}=10 ; \Delta \mathrm{DEX}, \mathrm{n}=10 ; \circ$ saline + alpha-lipoic acid, $\mathrm{n}=$ 10; $\triangle \mathrm{DEX}+$ alpha-lipoic acid, $\mathrm{n}=10$.

\subsubsection{Body weight}

Body weight of saline-treated rats increased steadily from $270 \pm 6$ to $306 \pm 7 \mathrm{~g}$ (T0-T10, $P<0.0005$ ). DEX-treated rats did not gain significant body weight (T0: $272 \pm 9, \mathrm{~T} 10: 277 \pm 7 \mathrm{~g}, \mathrm{~T} 0-\mathrm{T} 10, n s)$ whilst $\mathrm{ACTH}$ treatment resulted in significant weight loss (from T0: $283 \pm 11$ to T10: $258 \pm 8 \mathrm{~g}, P<0.001$, $P<0.0005)$. In these studies, the DEX- and ACTH-treated groups showed significantly lower body weights than the saline-treated group $\left(P^{\prime}<0.005\right)$. 


\subsubsection{Prevention studies}

In the prevention studies, rats on saline + alpha-lipoic acid gained body weight steadily from T0: $271 \pm 11$ to T10: $295 \pm 12 \mathrm{~g}$ ( $\mathrm{n}=10, P<0.0005)$. Alpha-lipoic acid (10 mg/rat/day) pre-treatment did not alter body weight in saline-, DEX- or ACTH-treated rats significantly. However, rats on higher alpha lipoic acid (100 mg/rat/day) dose and ACTH had significantly lower body weight compared with those on ACTH alone $\left(P^{\prime}<0.005\right)$ (Figures 6.3a and b).

\section{a) Prevention Studies}

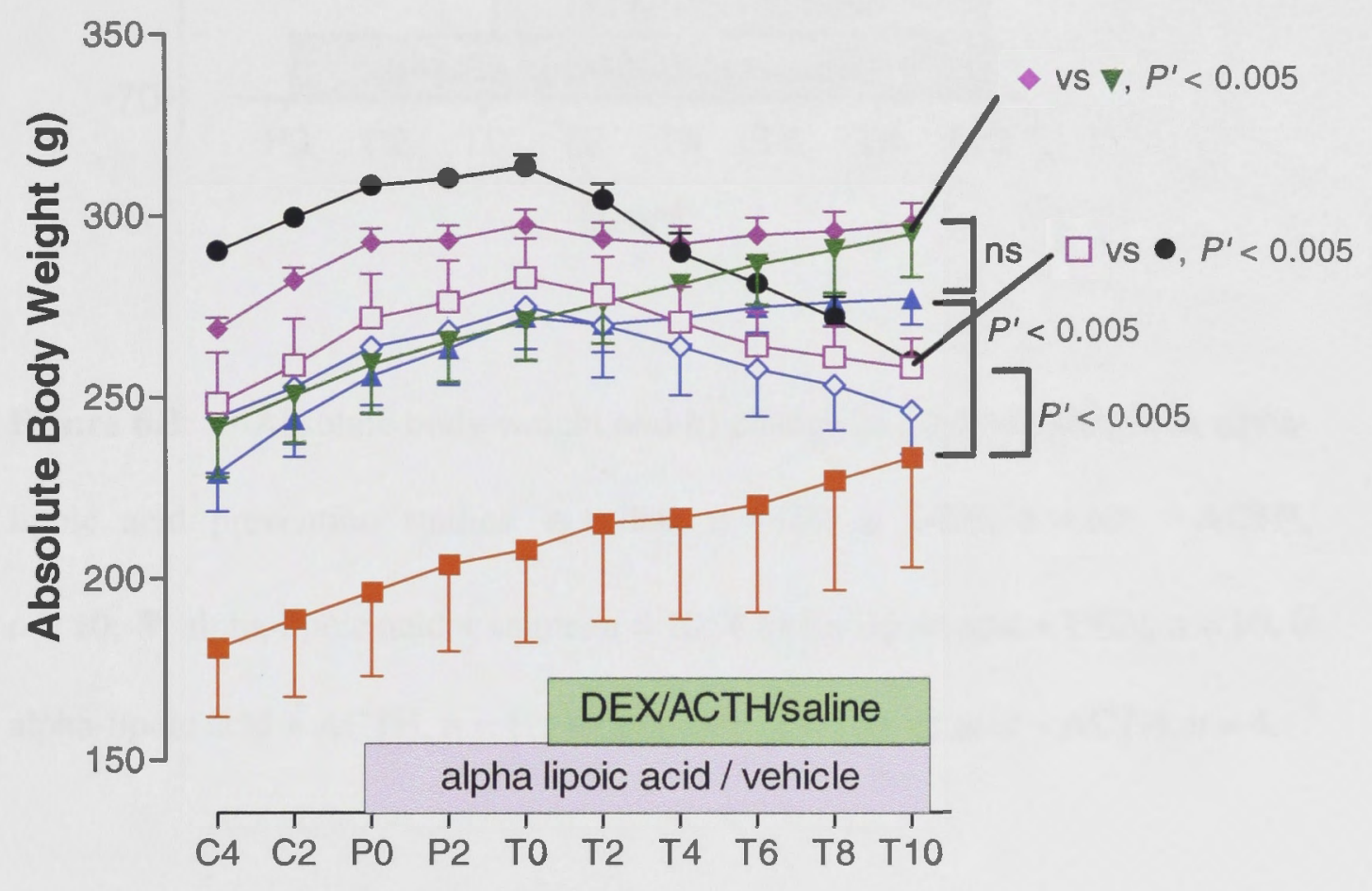

Days 


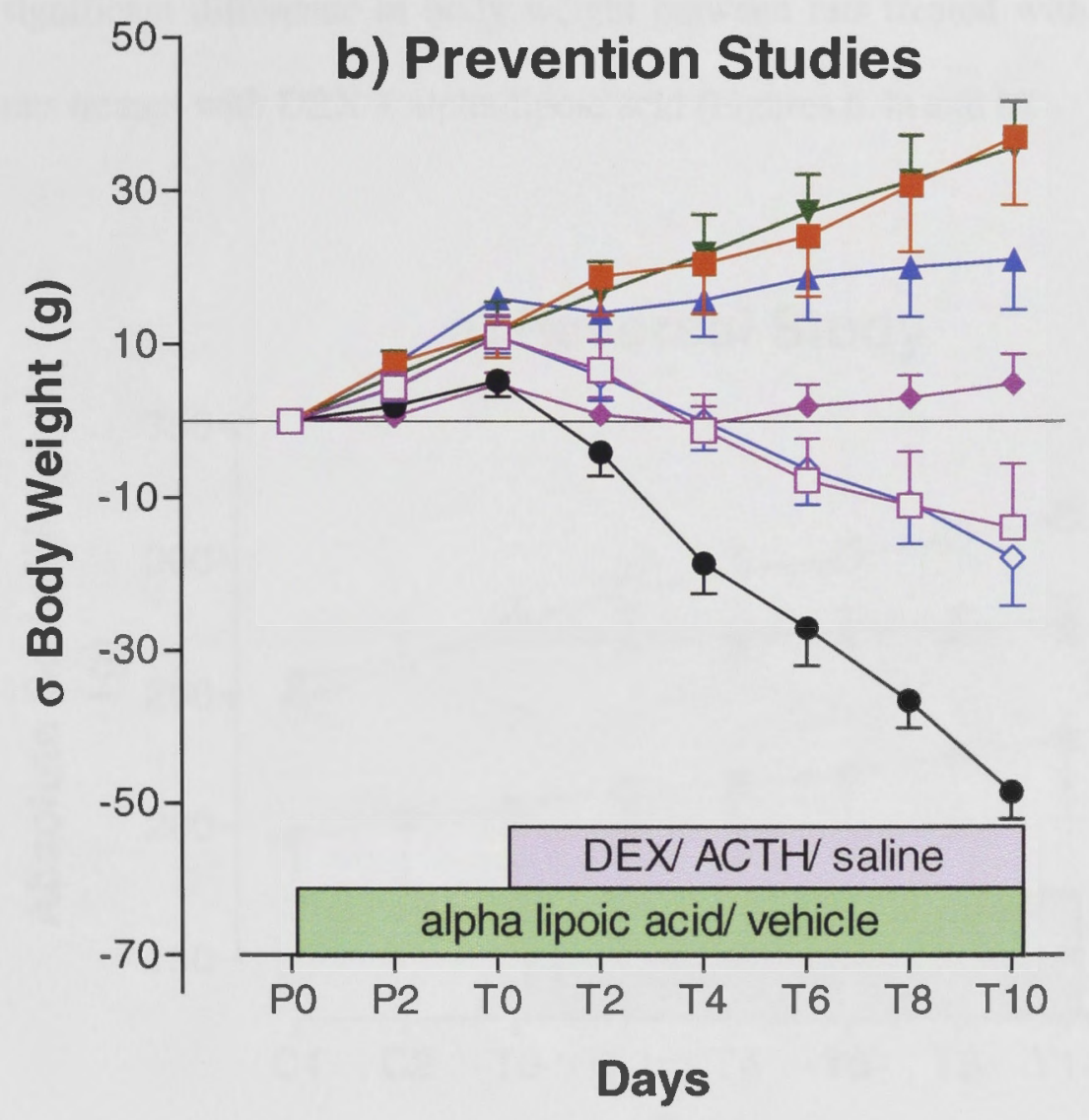

Figure 6.3: a) Absolute body weight and b) change $(\Delta)$ in body weight in alphalipoic acid prevention studies. a saline, $\mathrm{n}=10$; $\Delta$ DEX, $\mathrm{n}=10$; $\square \mathrm{ACTH}$, $\mathrm{n}=10 ; \boldsymbol{\nabla}$ alpha-lipoic acid + saline, $\mathrm{n}=10 ;$ alpha-lipoic acid + DEX, $\mathrm{n}=10, \diamond$ alpha-lipoic acid + ACTH, $n=10, \bullet$ high dose alpha lipoic acid + ACTH, $n=4$.

\subsubsection{Reversal study}

In the reversal study, rats on DEX + alpha-lipoic acid failed to gain weight during the course of the study (from T4: $277 \pm 10$ to T10: $278 \pm 8 \mathrm{~g}, \mathrm{n}=10, n s$ ) whilst those on saline + alpha-lipoic acid gained weight progressively from day $276 \pm$ $10 \mathrm{~g}$ on day T4 to $296 \pm 10 \mathrm{~g}$ on day T10 $\left(\mathrm{n}=10, P^{\prime}<0.0005\right)$. There was no 
significant difference in body weight between rats treated with DEX alone and rats treated with DEX + alpha-lipoic acid (Figures 6.4a and b).

\section{a) Reversal Study}

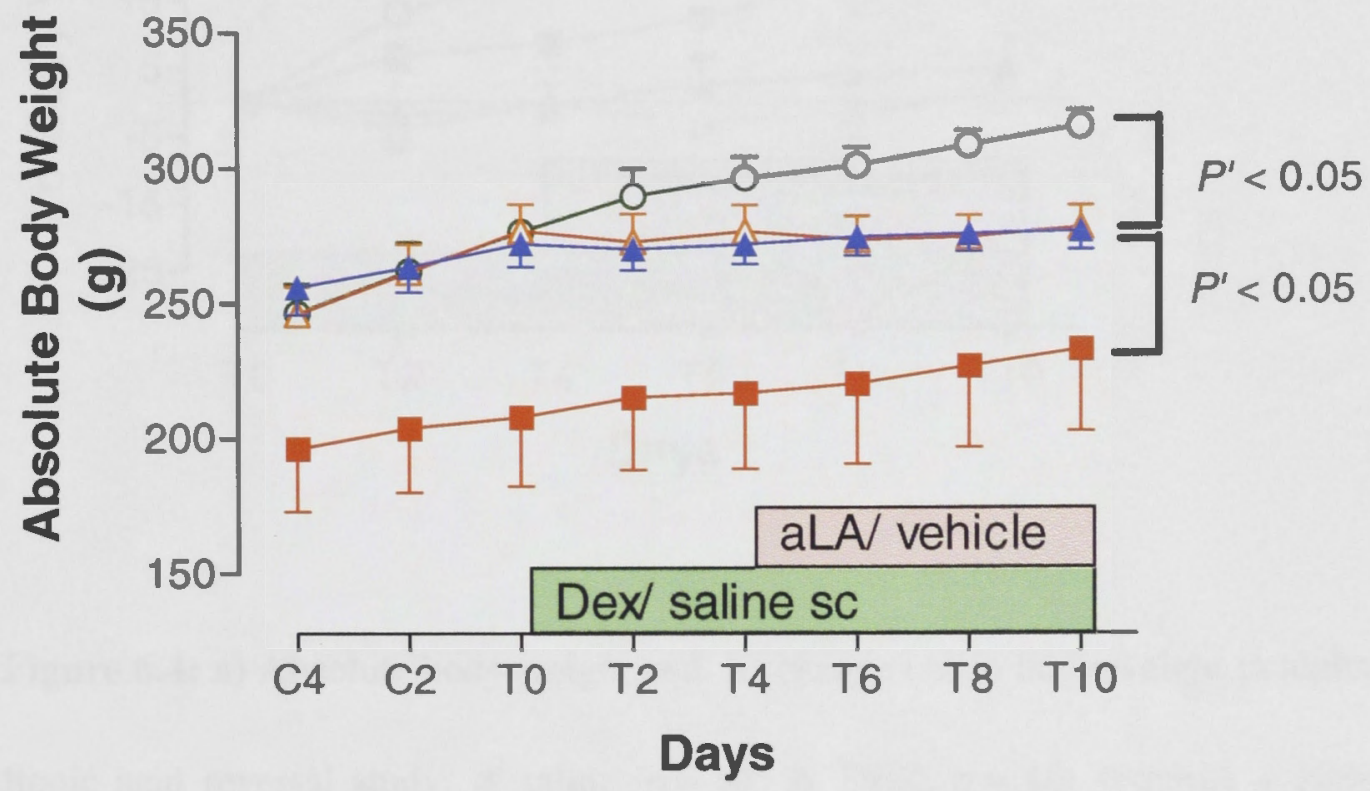




\section{b) Reversal Study}

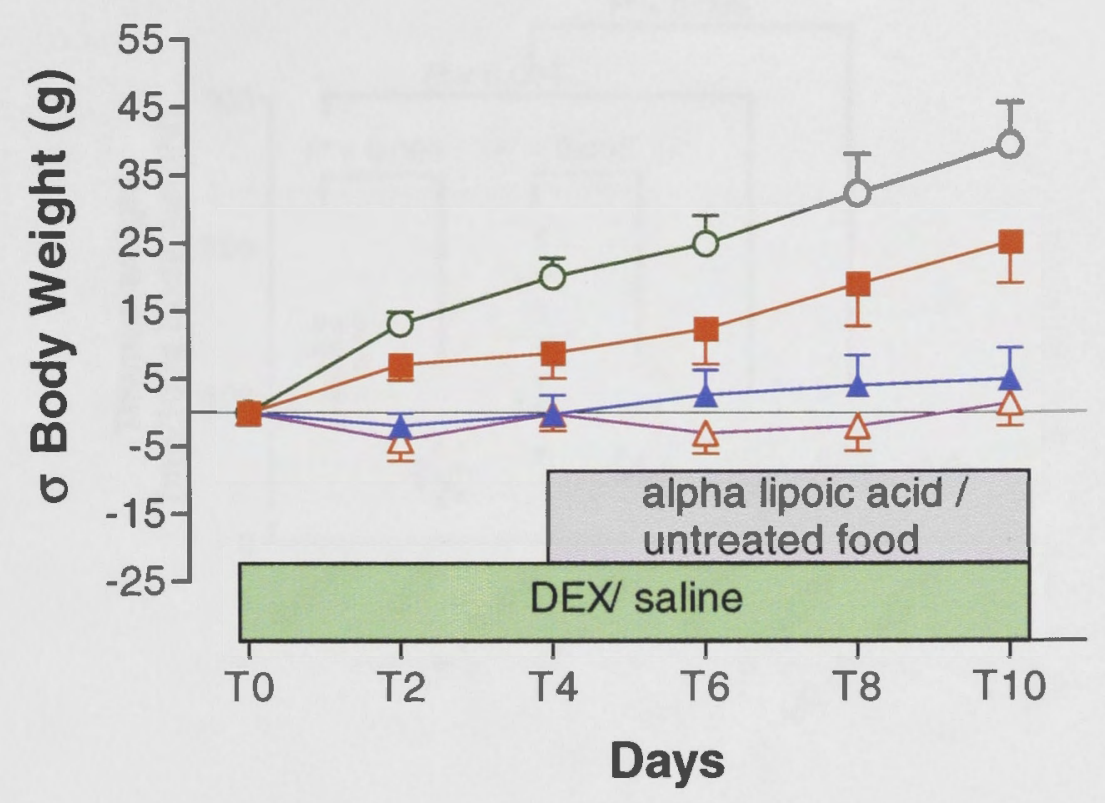

Figure 6.4: a) Absolute body weight and, b) change $(\Delta)$ in body weight in alphalipoic acid reversal study. a saline, $\mathrm{n}=10 ; \boldsymbol{\Delta}$ DEX, $\mathrm{n}=10 ; \circ$ saline + alpha lipoic acid, $\mathrm{n}=10 ; \triangle \mathrm{DEX}+$ alpha lipoic acid, $\mathrm{n}=10$.

\subsubsection{Thymus weight}

Thymus wet weight was significantly lower with DEX and ACTH treatments compared with saline $\left(P^{\prime}<0.005\right)$ regardless of the presence of alpha-lipoic acid treatment $\left(P^{\prime}<0.005\right)$ (Figure 6.5 and Table 6.1). 


\section{a) Prevention Study}

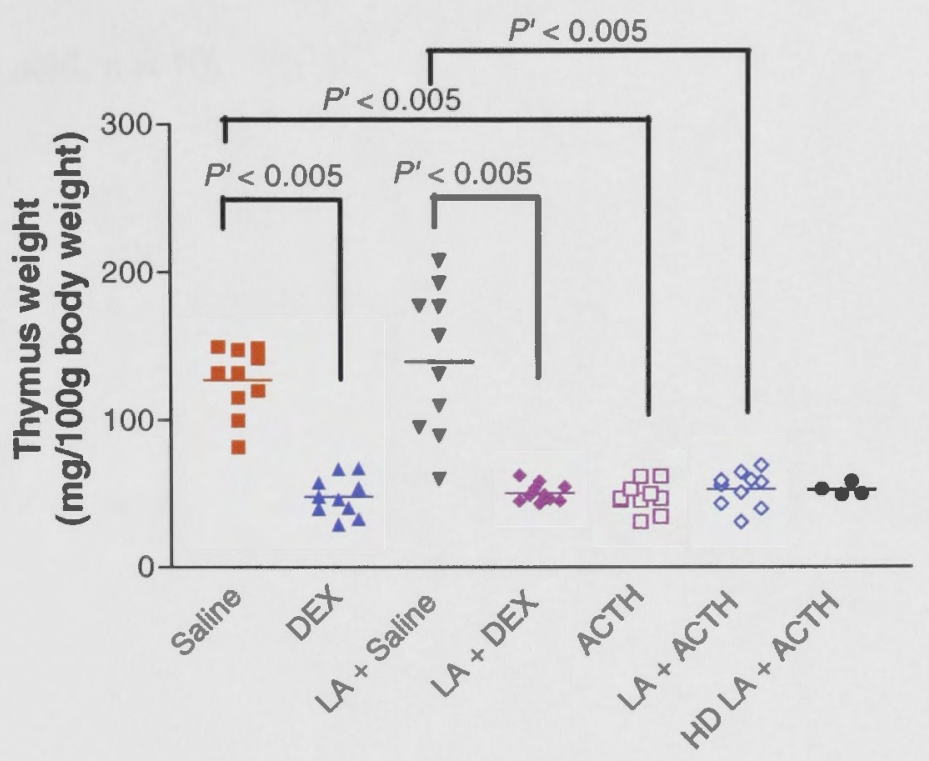

b) Reversal Study

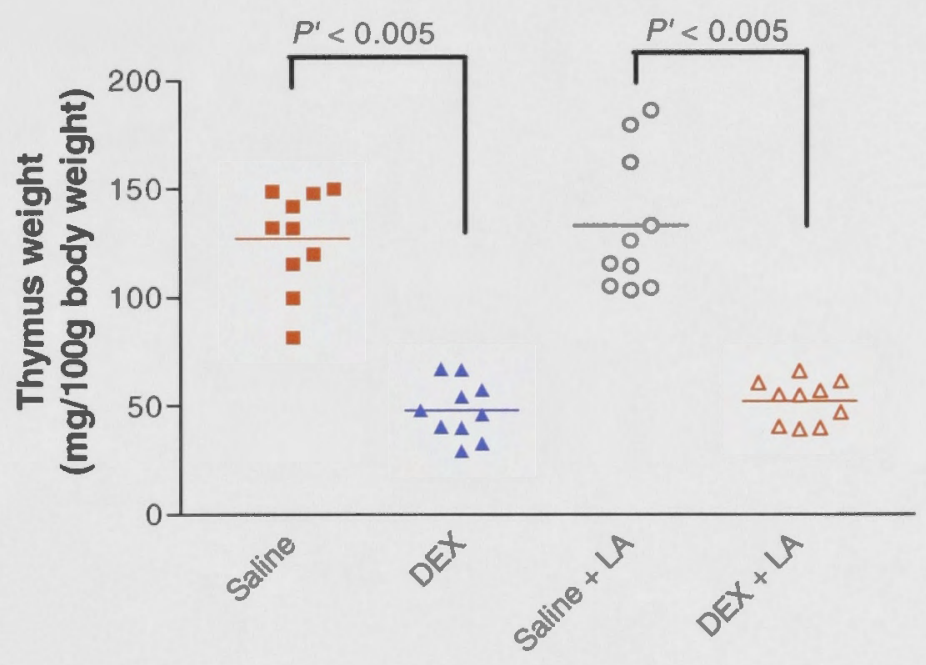

Figure 6.5: a) Thymus weight in the prevention study: $=$ saline, $n=10 ; \Delta D E X$, $\mathrm{n}=10 ; \square \mathrm{ACTH}, \mathrm{n}=10 ; \mathbf{v}$ alpha-lipoic acid (LA) + saline, $\mathrm{n}=10 ;$ alpha-lipoic acid + DEX, $n=10, \diamond$ alpha-lipoic acid + ACTH, $n=10, \bullet$ high dose alpha-lipoic acid (HD LA) + ACTH, $\mathrm{n}=4$, b) Thymus weight in the reversal study: " saline, 
$\mathrm{n}=10 ; \Delta \mathrm{DEX}, \mathrm{n}=10 ;$ o saline + alpha-lipoic acid, $\mathrm{n}=10 ; \Delta \mathrm{DEX}+$ alphalipoic acid, $\mathrm{n}=10$. 
Table 6.1: Biological measurements for alpha lipoic acid studies.

\begin{tabular}{|c|c|c|c|c|c|c|c|}
\hline Groups & $\begin{array}{l}\text { Thymus } \\
\text { (mg/100g body } \\
\text { weight) }\end{array}$ & $\begin{array}{l}\text { Adrenal (mg/100g } \\
\text { body weight) }\end{array}$ & $\begin{array}{l}\text { Blood Glucose } \\
(\mathrm{mmol} / \mathrm{L})\end{array}$ & $\begin{array}{c}\text { Plasma NOx } \\
(\mu M)\end{array}$ & $\begin{array}{l}\text { Plasma } \mathrm{F}_{2-} \\
\text { Isoprostane } \\
(\mathrm{nmol} / \mathrm{L})\end{array}$ & $\begin{array}{l}\text { Mito-HE } \\
\text { (geometric } \\
\text { MFI) }\end{array}$ & $\begin{array}{c}\mathrm{DiIC}_{1}(5) \\
\text { (geometric } \\
\text { MFI) }\end{array}$ \\
\hline Saline & $127 \pm 7.2$ & $8 \pm 0.5$ & $11 \pm 0.3$ & $9.3 \pm 1.2$ & $4.7 \pm 0.3$ & $198 \pm 15$ & $73 \pm 8$ \\
\hline DEX & $48 \pm 4.1^{*}$ & $5 \pm 0.2 *$ & $9 \pm 0.4^{*}$ & $12.3 \pm 0.9$ & $7.1 \pm 0.6^{*}$ & $223 \pm 21$ & $107 \pm 18$ \\
\hline ACTH & $48 \pm 3.2 *$ & $41 \pm 4.4^{*}$ & $11 \pm 0.6$ & $8.2 \pm 0.8$ & $5.4 \pm 0.4$ & $196 \pm 7$ & $110 \pm 13$ \\
\hline LA + saline $p$ & $140 \pm 15.8$ & $8 \pm 0.2$ & $11 \pm 0.6$ & $13.0 \pm 1.5$ & $4.9 \pm 0.3$ & $201 \pm 11$ & $184 \pm 12 *$ \\
\hline $\mathrm{LA}+\mathrm{DEX} p$ & $50 \pm 2.0^{\S}$ & $6 \pm 0.2^{\S \dagger}$ & $10 \pm 0.3$ & $11.2 \pm 1.0$ & $5.7 \pm 0.2^{\dagger}$ & $220 \pm 18$ & $131 \pm 8$ \\
\hline $\mathrm{LA}+\mathrm{ACTH} p$ & $53 \pm 3.7^{\S}$ & $43 \pm 3.5^{8}$ & $10 \pm 0.5$ & $13.1 \pm 3.4$ & $5.4 \pm 0.3$ & $217 \pm 13$ & $152 \pm 20$ \\
\hline HD LA + ACTH p & $53 \pm 2.1$ & $47 \pm 2.1$ & $13 \pm 1.1$ & $8.1 \pm 1.0$ & $4.6 \pm 0.6$ & $273 \pm 10^{a}$ & $125 \pm 6$ \\
\hline Saline + LA r & $133 \pm 10.0$ & $7 \pm 0.5$ & $12 \pm 0.5^{*}$ & $12.7 \pm 2.3$ & $5.4 \pm 0.2$ & $166 \pm 18$ & $161 \pm 10^{*}$ \\
\hline $\mathrm{DEX}+\mathrm{LA} \mathrm{r}$ & $52 \pm 3.1^{\#}$ & $5 \pm 0.4^{\# \prime}$ & $12 \pm 0.8^{\dagger}$ & $10.9 \pm 1.5$ & $5.6 \pm 0.5$ & $178 \pm 20$ & $134 \pm 12$ \\
\hline
\end{tabular}

LA: alpha-lipoic acid, HD LA: high dose alpha-lipoic acid, p: prevention, r: reversal. $*^{*} P^{\prime}<0.05$ vs saline, ${ }^{\S} P^{\prime}<0.005$ vs LA + saline prevention, " $P^{\prime}<0.01$ vs saline + LA reversal, ${ }^{\dagger} P^{\prime}<0.05$ vs DEX, ${ }^{x} P^{\prime}<0.0005$ vs ACTH. 


\subsubsection{Adrenal weight}

The adrenal weight for rats on DEX treatment was significantly lower than in those on saline injections $\left(\boldsymbol{P}^{\prime}<0.0005\right)$ (Figure 6.6a and Table 6.1). ACTH treated rats had significantly higher adrenal weight than saline treated rats (Figure 6.6b and Table 6.1). Alpha-lipoic acid partially prevented the fall in adrenal weight due to DEX in the prevention study $\left(P^{\prime}<0.01\right)$ (Figure 6.6a). This effect was not observed in the DEX-HT reversal or ACTH-HT prevention studies (Figures $6.6 \mathrm{a}$ and $\mathrm{b}$ ).

a)

\section{DEX Efficacy}

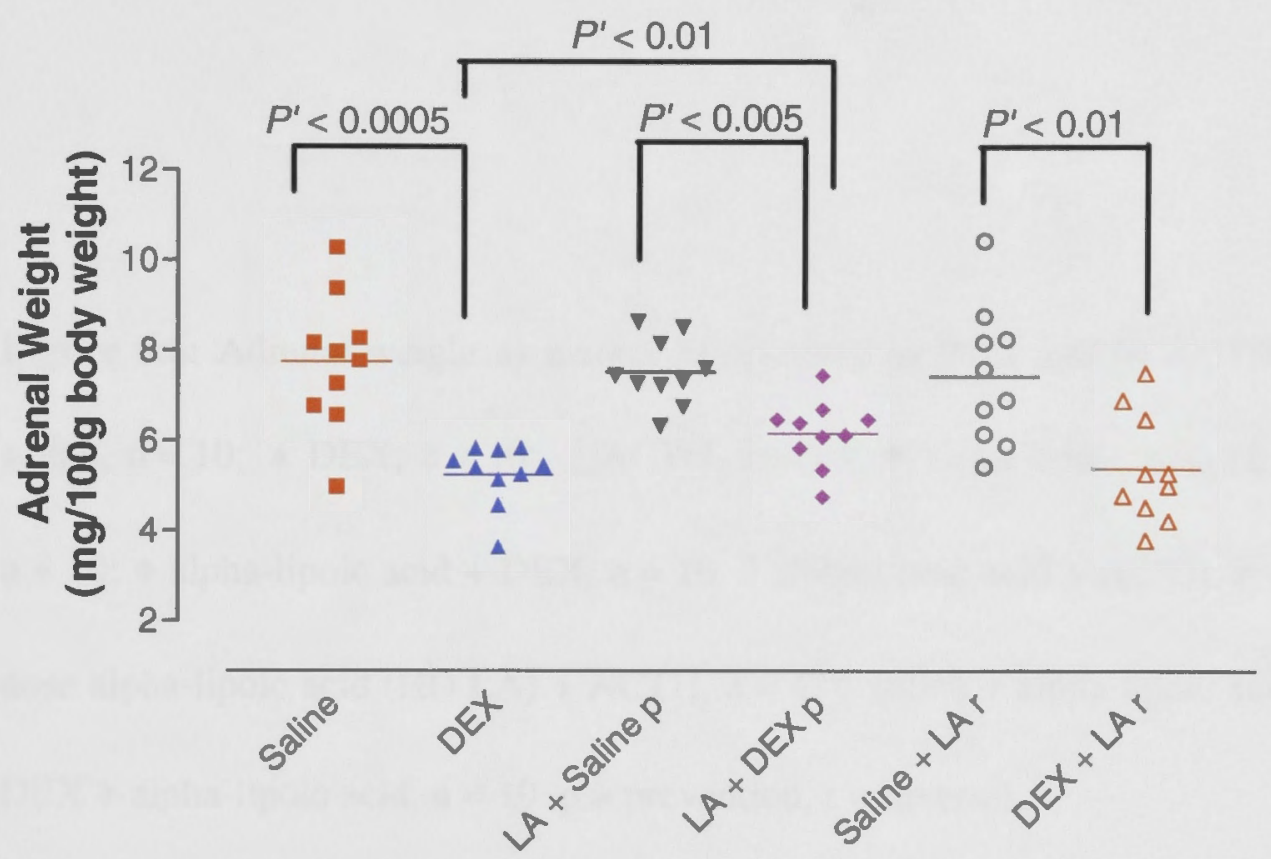


b) ACTH Efficacy

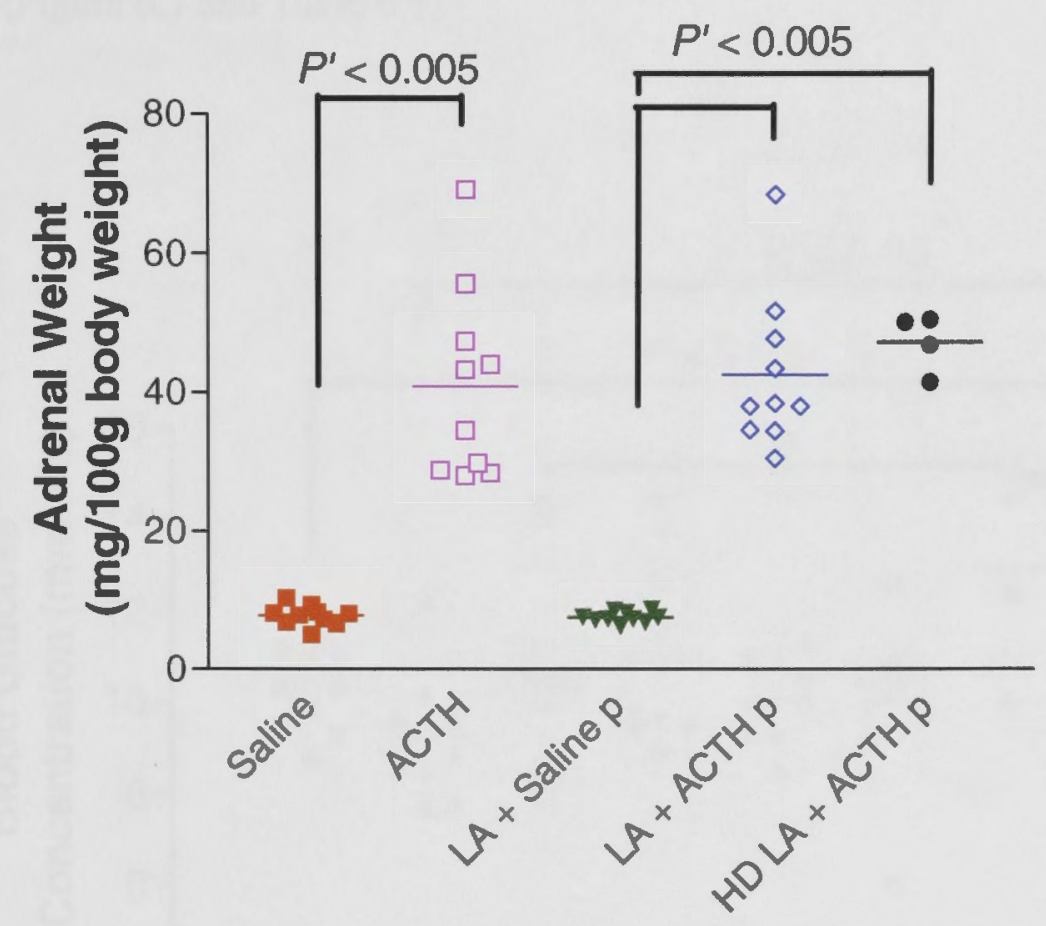

Figure 6.6: Adrenal weight as marker of effective a) DEX and b) ACTH delivery. saline, $\mathrm{n}=10 ; \Delta \mathrm{DEX}, \mathrm{n}=10 ; \square \mathrm{ACTH}, \mathrm{n}=10 ; \nabla$ alpha-lipoic acid (LA) + saline, $\mathrm{n}=10 ; \diamond$ alpha-lipoic acid + DEX, $\mathrm{n}=10, \diamond$ alpha-lipoic acid $+\mathrm{ACTH}, \mathrm{n}=10, \bullet$ high dose alpha-lipoic acid (HD LA) + ACTH, $n=4$, ○ saline + alpha-lipoic acid, $n=10 ; \Delta$ $\mathrm{DEX}+$ alpha-lipoic acid, $\mathrm{n}=10 . \mathrm{p}=$ prevention, $\mathrm{r}=$ reversal.

\subsubsection{Blood glucose concentration}

Neither DEX nor ACTH altered the blood glucose concentrations in rats. Alpha lipoic acid increased blood glucose concentration in saline and DEX-treated rats in the 
reversal study. This effect was not observed in the DEX-HT and ACTH-HT prevention studies (Figure 6.7 and Table 6.1).

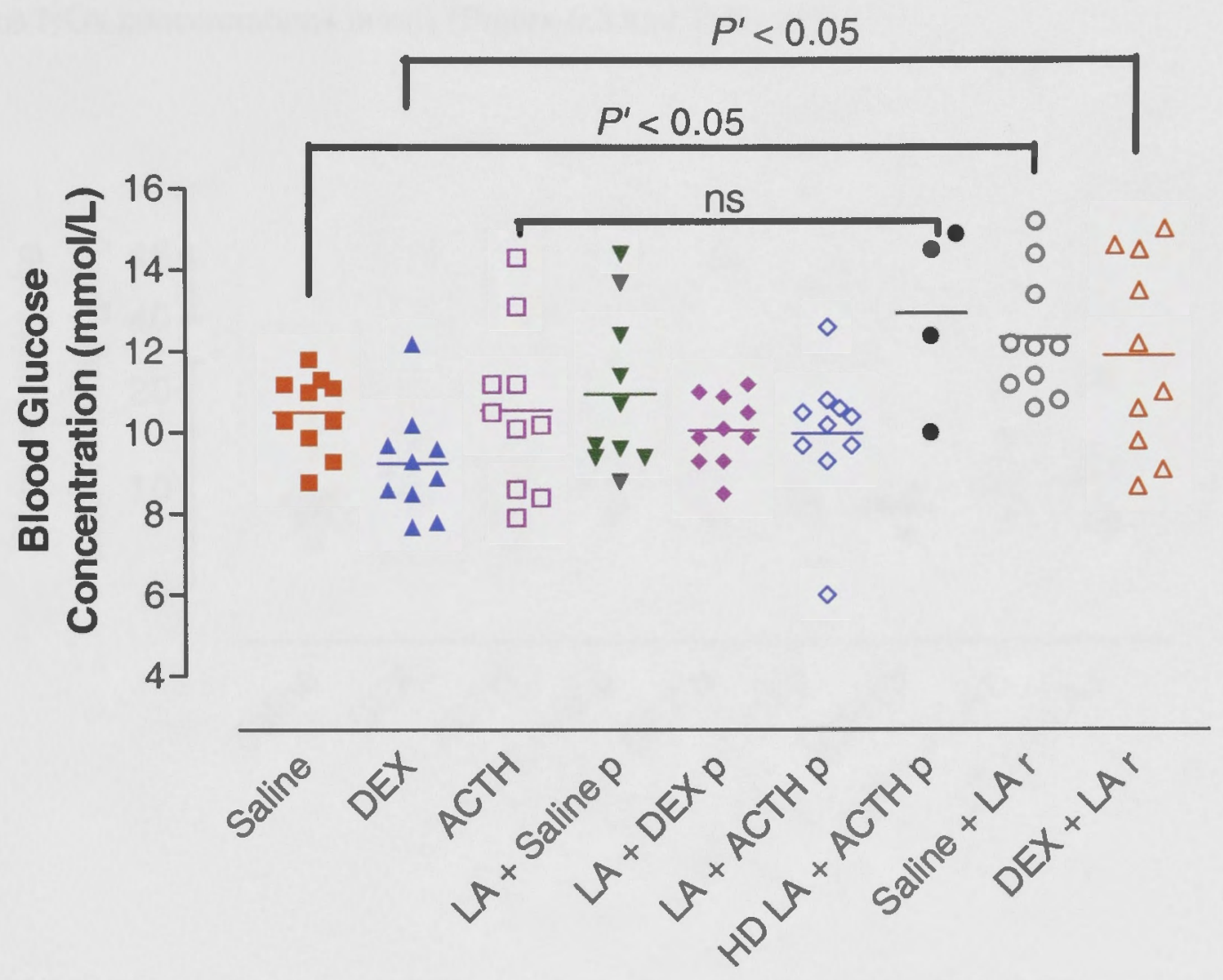

Figure 6.7: Blood glucose concentration. $=$ saline, $\mathrm{n}=10 ; \Delta \mathrm{DEX}, \mathrm{n}=10 ; \square \mathrm{ACTH}$, $\mathrm{n}=10 ; \boldsymbol{\nabla}$ alpha-lipoic acid (LA) + saline, $\mathrm{n}=10 ;$ alpha-lipoic acid + DEX, $\mathrm{n}=10$, $\checkmark$ alpha-lipoic acid + ACTH, $\mathrm{n}=10, \bullet$ high dose alpha lipoic acid (HD LA) + ACTH, $\mathrm{n}=4$, o saline + alpha-lipoic acid, $\mathrm{n}=10 ; \Delta \mathrm{DEX}+$ alpha-lipoic acid, $\mathrm{n}=10$. $\mathrm{p}=$ prevention, $\mathrm{r}=$ reversal . 


\subsubsection{Plasma nitrate and nitrite concentration}

There was no significant difference in plasma NOx concentration in DEX- and ACTHtreated rats compared with saline-treated rats. Alpha-lipoic acid also did not alter the plasma NOx concentrations in rats (Figure 6.8 and Table 6.1).

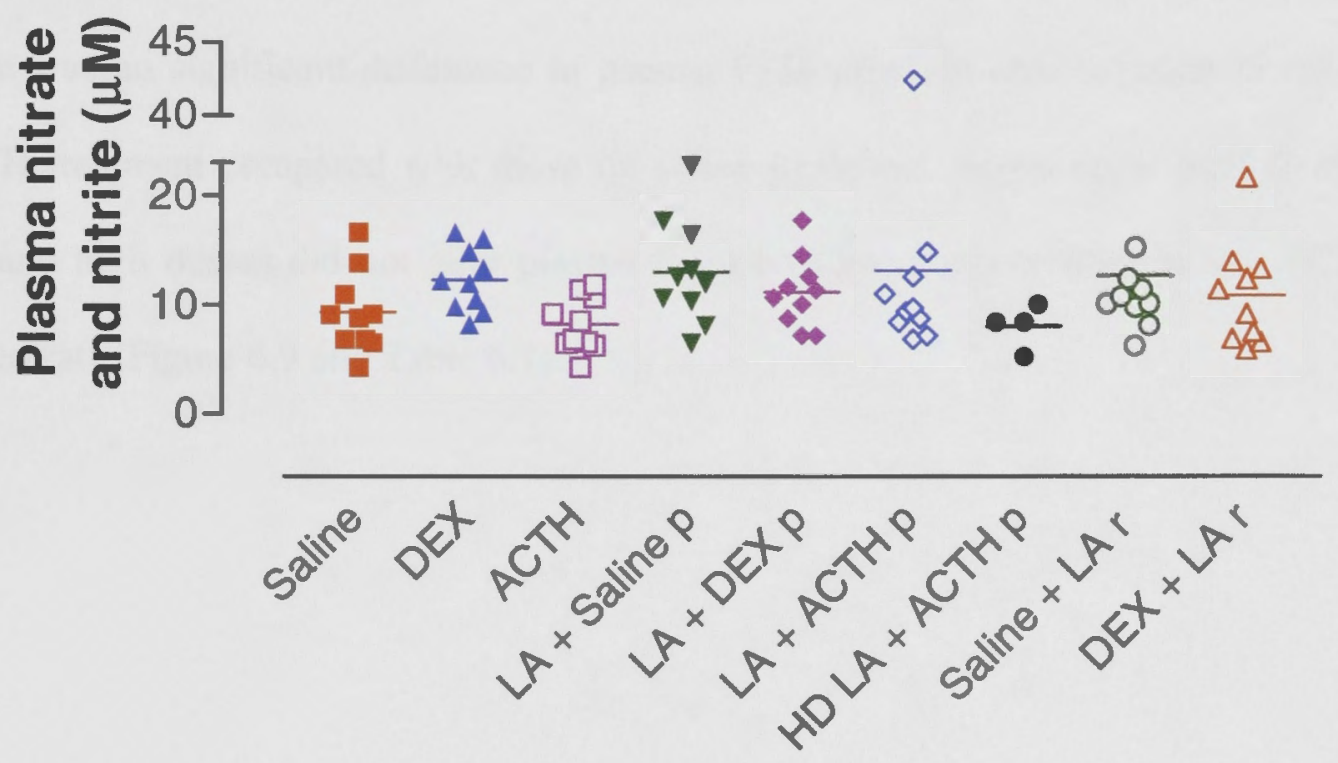

Figure 6.8: Plasma nitrate and nitrite concentration. $=$ saline, $\mathrm{n}=10 ; \Delta \mathrm{DEX}, \mathrm{n}=10$ ACTH, $n=10 ; \nabla$ alpha-lipoic acid $(\mathrm{LA})+$ saline, $\mathrm{n}=10 ;$ alpha lipoic acid + DEX, $\mathrm{n}=10, \diamond$ alpha-lipoic acid + ACTH, $\mathrm{n}=10, \bullet$ high dose alpha-lipoic acid (HD LA) + ACTH, $n=4$, o saline + alpha-lipoic acid, $n=10 ; \Delta \mathrm{DEX}+$ alpha-lipoic acid, $\mathrm{n}=10$. $\mathrm{p}=$ prevention, $\mathrm{r}=$ reversal . 


\subsubsection{Plasma $\mathrm{F}_{2}$-isoprostane concentration}

The DEX-treated group had significantly higher plasma $\mathrm{F}_{2}$-isoprostane concentration compared with the saline-treated group $\left(P^{\prime}<0.01\right)$. Rats pre-treated with alpha-lipoic acid before DEX treatment had significantly lower plasma $F_{2}$-isoprostane concentration than those on DEX alone $\left(P^{\prime}<0.05\right)$. However, no significant difference was observed in the DEX-HT reversal study between the DEX and DEX + alpha-lipoic acid groups. There was no significant difference in plasma $F_{2}$-isoprostane concentration in rats on ACTH treatment compared with those on saline treatment. Alpha-lipoic acid (both at low and high doses) did not alter plasma $\mathrm{F}_{2}$-isoprostane concentration in the $\mathrm{ACTH}$ treated rats (Figure 6.9 and Table 6.1). 


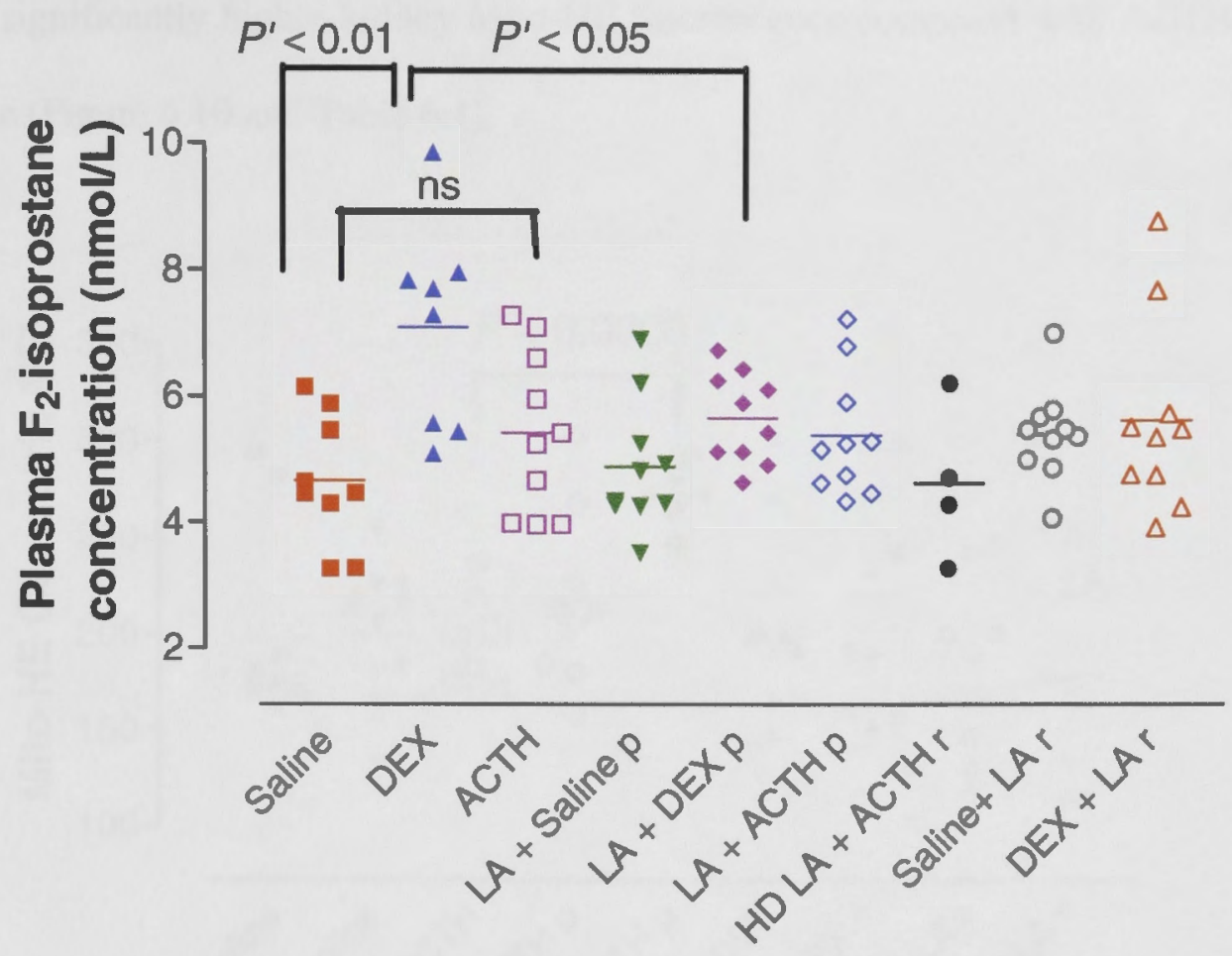

Figure 6.9: Plasma $F_{2}$-isoprostane concentration. - saline, $n=9 ; \Delta D E X, n=8$;

ACTH, $n=10 ; \nabla$ alpha-lipoic acid (LA) + saline, $n=10 ;$ alpha-lipoic acid + DEX, $\mathrm{n}=10, \diamond$ alpha-lipoic acid $+\mathrm{ACTH}, \mathrm{n}=10, \bullet$ high dose alpha-lipoic acid (HD LA) + ACTH, $n=4$, ○ saline + alpha-lipoic acid, $n=10 ; \triangle D E X+$ alpha-lipoic acid, $n=10$. $\mathrm{p}=$ prevention, $\mathrm{r}=$ reversal

\subsubsection{Kidney Mito-HE fluorescence}

There was no significant difference in kidney Mito-HE fluorescence between DEX- or ACTH- and saline-treated rats. Rats treated with high dose alpha-lipoic acid and ACTH 
had significantly higher kidney Mito-HE fluorescence compared with ACTH treatment alone (Figure 6.10 and Table 6.1).

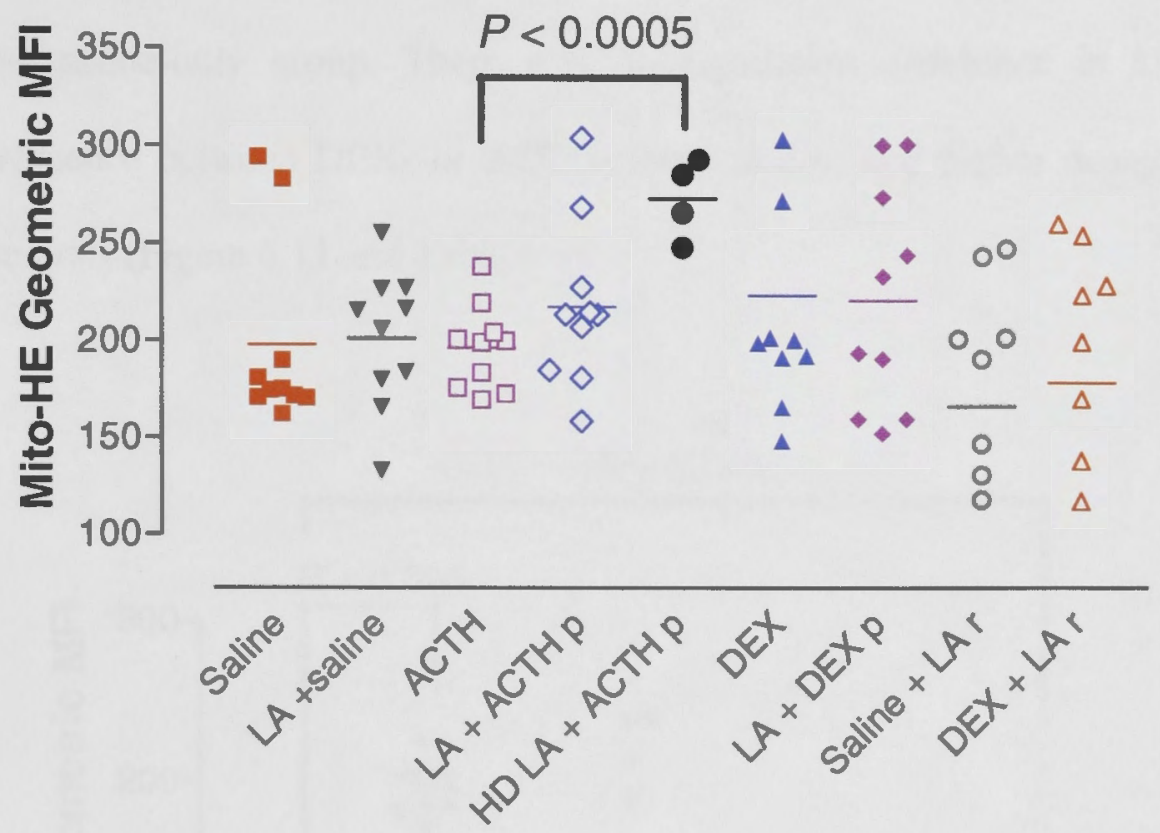

Figure 6.10: Mito-HE geometric MFI. - saline, $\mathrm{n}=10 ; \Delta \mathrm{DEX}, \mathrm{n}=10 ; \square \mathrm{ACTH}$, $\mathrm{n}=10 ; \boldsymbol{\nabla}$ alpha-lipoic acid $(\mathrm{LA})+$ saline, $\mathrm{n}=10 ;$ alpha-lipoic acid $+\mathrm{DEX}, \mathrm{n}=10$, $\diamond$ alpha-lipoic acid + ACTH, $n=10, \bullet$ high dose alpha-lipoic acid (HD LA) + ACTH, $\mathrm{n}=4$, $\circ$ saline + alpha-lipoic acid, $\mathrm{n}=10 ; \Delta$ DEX + alpha-lipoic acid, $\mathrm{n}=10$. $\mathrm{p}=$ prevention, $\mathrm{r}=$ reversal . 


\subsubsection{Kidney $\operatorname{DiIC}_{1}(5)$ fluorescence}

$\operatorname{DilC}_{1}(5)$ mean geometric fluorescence was significantly higher in the groups which received alpha-lipoic acid (in the prevention and reversal studies) and saline compared to the saline-only group. There was no significant difference in kidney $\operatorname{DiIC}_{1}(5)$ fluorescence between DEX- or ACTH- (both at low and higher dosages) and salinetreated rats (Figure 6.11 and Table 6.1).

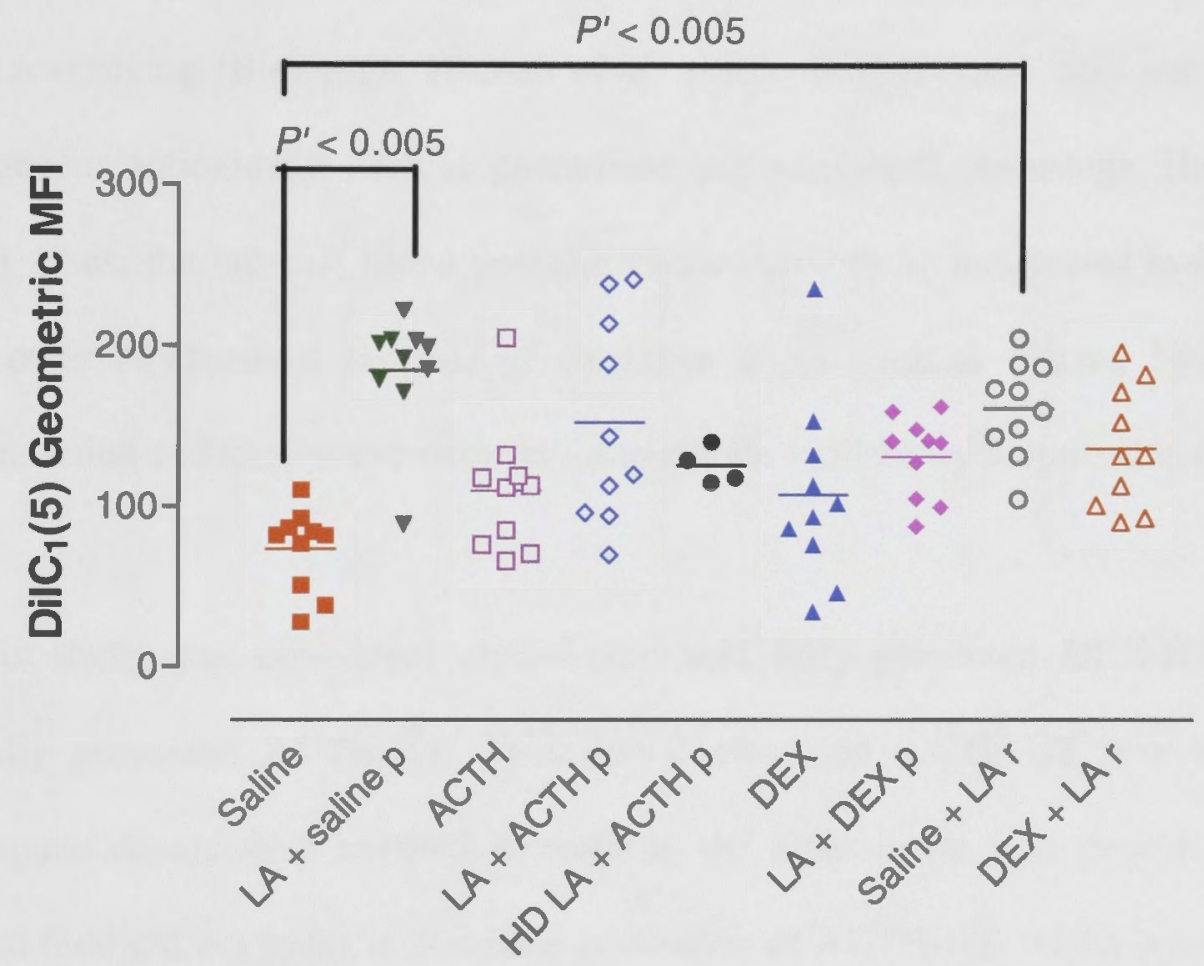

Figure 6.11: $\operatorname{DiIC}_{1}(5)$ geometric MFI. — saline, $\mathrm{n}=10 ; \triangle \mathrm{DEX}, \mathrm{n}=10 ; \square \mathrm{ACTH}$, $\mathrm{n}=10 ; \boldsymbol{\nabla}$ alpha-lipoic acid (LA) + saline, $\mathrm{n}=10 ;-$ alpha-lipoic acid + DEX, $\mathrm{n}=10$ $\diamond$ alpha-lipoic acid $+\mathrm{ACTH}, \mathrm{n}=10, \bullet$ high dose alpha-lipoic acid (HD LA) + ACTH, $\mathrm{n}=4$, $\circ$ saline + alpha-lipoic acid, $\mathrm{n}=10 ; \Delta$ DEX + alpha-lipoic acid, $\mathrm{n}=10$. $\mathrm{p}=$ prevention, $\mathrm{r}=$ reversal . 


\subsection{DISCUSSION}

Even though alpha-lipoic acid has been shown to be effective in improving mitochondrial function and decreasing mitochondrial superoxide in rats (Hagen, Ingersoll et al. 1999; El Midaoui, Elimadi et al. 2003), its effect is not entirely specific to mitochondria. The antioxidant effect of alpha-lipoic acid which is mediated by both the original alpha-lipoic acid compound and its metabolite dihydrolipoic acid, a more potent antioxidant, acts via a number of mechanisms such as metal chelation and direct ROS scavenging (Biewenga, Haenen et al. 1997). Dihydrolipoic acid can regenerate endogenous antioxidants such as glutathione and vitamin C (Biewenga, Haenen et al. 1997). Thus, the tail-cuff blood pressure results need to be interpreted in conjunction with other biochemical analysis of oxidative stress such as plasma $F_{2}$-isoprostane concentration and kidney mitochondrial superoxide availability described in Chapter 6 .

In this study, the antioxidant alpha-lipoic acid fully prevented DEX-HT but only partially prevented ACTH-HT. This partial effect on ACTH-HT was not due to inadequate dosing as a ten-fold increase in the alpha-lipoic acid dose to $5 \mathrm{~g} / \mathrm{kg}$ of ground food did not result in complete prevention of ACTH-HT. Alpha-lipoic acid was less effective in reversing established DEX-HT. Using the same dosage used in the prevention study, alpha-lipoic acid only partially reversed DEX-HT. The blood pressure lower effects of alpha-lipoic acid did not correlate directly with mitochondrial superoxide availability as demonstrated by the kidney Mito-HE assay. 
The Mito-HE mean fluorescence intensity on kidney cells was not altered by DEX, ACTH or alpha-lipoic acid (500 mg/kg of ground food). However, alpha lipoic acid at $5 \mathrm{~g} / \mathrm{kg}$ of ground food resulted in significantly higher Mito-HE mean fluorescence intensity. This was not associated with a decrease in mitochondrial membrane potential, a phenomenon known to result in a false increase in Mito-HE signals. It is possible that alpha-lipoic acid at a higher dose results in oxidative stress, most likely due to increased mitochondrial superoxide generation. The role of alpha-lipoic acid and its active metabolite, dihydrolipoic acid, as pro-oxidants has been reported in the literature (Biewenga, Haenen et al. 1997; Moini, Packer et al. 2002; Çakatay 2006). In this study, the increase in mitochondrial superoxide production is unlikely to translate to systemic oxidative stress given the lack of change in the plasma $\mathrm{F}_{2}$-isoprostane concentration observed in this group, although the sample size for this group is very small and there may not have been significant power to detect a change. Another postulation is that superoxide generated within the mitochondria is not readily available to extramitochondrial structures. Superoxide, being a charged radical, does not permeate the mitochondrial membrane into the cytoplasm easily (Han, Antunes et al. 2003). It leaves the mitochondria via voltage-dependent anion channels and more commonly, as membrane-permeable hydrogen peroxide following its dismutation by intermembrane $\mathrm{Cu}, \mathrm{Zn}$-superoxide dismutase (Han, Antunes et al. 2003).

Plasma $F_{2}$-isoprostane concentration was significantly increased in DEX-but not ACTH-hypertensive rats. The latter finding was in contrast to previous findings that ACTH-HT was associated with raised plasma $F_{2}$-isoprostane concentration, suggesting insufficient power to detect a difference (Zhang, Wu et al. 2009). Pooled $F_{2}$-isoprostane 
concentration data from our laboratory showed that ACTH-hypertensive rats $(n=46)$ had a significant higher plasma $F_{2}$-isoprostane concentration than the normotensive saline-treated rats $(\mathrm{n}=48)\left(\mathrm{Ong}\right.$, Zhang et al. 2008). The presence of a raised $\mathrm{F}_{2^{-}}$ isoprostane concentration in DEX-treated rats again confirmed the role of oxidative stress in its pathogenesis. Pre-treatment with alpha-lipoic acid successfully prevented the increase of plasma $\mathrm{F}_{2}$-isoprostane concentration due to DEX. However, alpha lipoic acid had no effect on the plasma $\mathrm{F}_{2}$-isoprostane in established DEX-HT.

The lower blood pressure readings observed in the alpha-lipoic acid + DEX and alphalipoic acid + ACTH groups in the prevention studies; and DEX + alpha-lipoic acid in the reversal study were not due to inadequate dosing of DEX and ACTH. Similarly, the lower plasma $\mathrm{F}_{2}$-isoprostane in the alpha-lipoic acid + DEX-treated group compared to DEX-only group was not a reflection of insufficient DEX administration. The effectiveness of DEX and ACTH delivery was confirmed by significant lowering of thymus weight in all groups receiving DEX or ACTH. As described in Chapter 3, excess gluococorticoid hormones results in thymocyte apoptosis and thymic involution (Sun, Dinsdale et al. 1992; Zavitsanou, Nguyen et al. 2007). Alpha lipoic acid has no effect on the GC-induced thymic involution.

Adrenal weight was also used as a surrogate marker of effective DEX and ACTH administration. DEX administration showed a reduction in adrenal weight consistent with adrenal suppression associated with exogenous glucocorticoid administration, whilst ACTH stimulation of the adrenal glands resulted in a significant increase in adrenal weight. In this study, alpha-lipoic acid did not alter the adrenal weight in either 
saline- and ACTH-treated rats. It, however, partially inhibited DEX-induced reduction in adrenal weight. The reason for this remains unclear. Whether this has any correlation with DEX activity or the blood pressure reducing effect of alpha-lipoic acid effect remains to be determined. Cremer et al. demonstrated that oral alpha-lipoic acid at 20 , 60 and $180 \mathrm{mg} / \mathrm{kg}$ body weight/day did not alter adrenal weight relative to body weight or the histology of the adrenal glands of Sprague-Dawley rats (Cremer, Rabeler et al. 2006). These authors, however, showed a dose-dependent decrease in body weight in rats treated with alpha-lipoic acid. Without adjusting to body weight changes, these authors found reduction in left adrenal weight only in male rats treated with $60 \mathrm{mg} / \mathrm{kg}$ body weight alpha-lipoic acid and no significant change in right adrenal weight in male rats and both adrenals in female rats. As there were no associated histopathological correlations, they concluded that this change was due to the reduction in body weight gain (Cremer, Rabeler et al. 2006).

In this study, treatment of alpha-lipoic acid ( $500 \mathrm{mg} / \mathrm{kg}$ ground food/day) did not change the body weight profile observed with saline, DEX and ACTH treatments. However, with a higher alpha-lipoic acid dose ( $5 \mathrm{~g} / \mathrm{kg}$ ground food/day) body weight of the ACTH-treated rats fell precipitously. The rate of fall was greater in the group receiving the higher alpha-lipoic acid dose compared with the ACTH-only and alphalipoic acid $(500 \mathrm{mg} / \mathrm{kg}$ ground food/day) + ACTH groups. A similar result was observed by Cremer et al. Alpha-lipoic acid has been shown to exhibit a dose dependent anti-obesity effect in Sprague-Dawley rats, independent of systemic toxicity, taste aversion or associated illness (Kim, Park et al. 2004). Rather, it works via the 
suppression of hypothalamic AMP activated protein kinase, a fuel sensor in cells, which is activated during cellular energy depletion (Kim, Park et al. 2004).

In this study, blood glucose concentration was evaluated because hyperglycaemia has been shown to result in mitochondrial dysfunction (Munusamy and MacMillan-Crow 2009) and is a common feature in humans exposed to excess glucocorticoid hormones. Fasting blood glucose was significantly higher in patients with active Cushing's syndrome than those in remission (Terzolo, Allasino et al. 2004); and age- and sexmatched controls (Faggiano, Pivonello et al. 2003). In this study, however, ACTH and DEX did not alter blood glucose concentration in rats, consistent with previous findings in rats (Zhang, Pang et al. 2004). Interestingly, alpha-lipoic acid significantly increased glucose concentration in the reversal study involving DEX-HT and its saline control. The fact that alpha-lipoic acid increased blood glucose concentration in DEX- and saline-treated rats in the reversal study but not in the prevention study suggests that duration of alpha-lipoic acid treatment is likely to play a role. However, there is no evidence in the literature implicating alpha-lipoic acid as a cause of hyperglycemia. Rather, alpha-lipoic acid at a same dose prevented hyperglycemia in glucose-fed rats (El Midaoui, Wu et al. 2002).

In other studies (Chapters 7, 9), plasma NOx was decreased in DEX-HT. This decrease was not observed in the present studies on DEX- and ACTH-HT, with and without alpha-lipoic acid treatment. This may be influenced by decreased sensitivity of this assay in detecting very low plasma NOx levels seen in all group including the control and DEX-treated groups. In this study, alpha-lipoic acid did not alter the plasma NOx 
concentrations in rats treated with saline, DEX or ACTH. The effect of alpha-lipoic acid on the saline-treated rats was consistent with the results seen in other studies where alpha-lipoic acid given to rats orally ( $20 \mathrm{mg} / \mathrm{kg}$ body weight (Amudha, Josephine et al. 2007) and $1 \mathrm{~g} / \mathrm{L}$ drinking water (Marley, Holt et al. 1999) did not alter plasma NOx in normal controls. In these studies, alpha-lipoic acid prevented the rise in plasma NOx induced by cyclosporine treatment (Amudha, Josephine et al. 2007) and biliary cirrhosis (Marley, Holt et al. 1999) in rats by the inhibition of renal iNOS and total hepatic NOS respectively . In the present study, however, alpha lipoic acid did not alter plasma NOx in DEX or ACTH-treated rats. This is probably because, unlike those studies, DEX and ACTH suppresses eNOS and iNOS in rats (Wallerath, Witte et al. 1999; Lou, Wen et al. 2001).

In summary, alpha-lipoic acid fully prevented DEX-HT, partially prevented ACTH-HT and partially reversed DEX-HT without altering the kidney mitochondrial superoxide availability. This suggests that GC-HT is prevented (and DEX-HT, partially reversed) by alpha-lipoic acid through a mechanism other than mitochondrial superoxide reduction.

The differences observed between DEX- and ACTH-HT, once again, depict the different pathophysiological mechanisms contributing to the two hypertensive models. 


\subsection{CONCLUSION}

This study suggested that mitochondrial superoxide does not play a major role in the pathogenesis of GC-HT in the rat. 


\section{CHAPTER 7}

Role of Xanthine Oxidase in Dexamethasone-Induced

Hypertension in the Rat 


\subsection{INTRODUCTION}

Xanthine oxidase, best known for its role in purine degradation, has broad biological roles (Berry and Hare 2004), and is one of several sources of reactive oxygen species (ROS). In the process of converting hypoxanthine to xanthine and xanthine to uric acid, oxygen is reduced to form superoxide anions. The role of xanthine oxidase has been studied in various forms of hypertension. Inhibition of xanthine oxidase using oxpurinol lowered blood pressure in spontaneously hypertensive rats (Nakazono, Watanabe et al. 1991). Tungsten supplementation aimed at inhibiting xanthine oxidase also resulted in decrease in oxygen radicals and blood pressure in spontaneously hypertensive rats (Suzuki, DeLano et al. 1998).

The effect of xanthine oxidase inhibition on GC-HT has been examined previously. Wallwork and co-workers observed a decrease in mean blood pressure with allopurinol treatment in hypertension produced by high dose DEX $(0.5$ $\mathrm{mg} / \mathrm{kg} /$ day). However, in our hands, allopurinol failed to prevent and reverse ACTH-HT (Zhang, Chan et al. 2005). This study aimed to clarify the role of xanthine oxidase inhibition in the prevention of GC-HT, using a much lower dose of DEX to minimise potential confounding effects of massive dosage. 


\subsection{METHODS}

\subsubsection{Experimental animals}

This study was approved by the Animal Experimentation Ethics Committee of the Australian National University (J.HB.17.03). General methods used in this study have been described in Chapter 2. In this experiment, the rats were given either normal food or allopurinol-laced food for 18 days (from day P0 to T12) and either saline or DEX injection for 14 days (from day T0 to T13).

Thirty male Sprague-Dawley rats weighing 240-260 g were randomly divided into 4 treatment groups.

Group 1 Vehicle and saline $(\mathrm{n}=8)$

Group 2 Allopurinol and saline $(n=7)$

Group 3 Vehicle and DEX $(n=8)$

Group 4 Allopurinol and DEX ( $n=7)$

DEX $(10 \mu \mathrm{g} / \mathrm{rat} / \mathrm{day}, \quad s . c)$ and saline $(0.9 \% \mathrm{NaCl}, 0.1 \mathrm{~mL} / \mathrm{rat} / \mathrm{day})$ were administered via subcutaneous injection at 1200 hours daily, as described in Section 2.4.1. Allopurinol ( $200 \mathrm{mg} / \mathrm{kg} /$ day, Sigma-Aldrich, St Louis, USA) was 
given orally as allopurinol-laced ground food. The total allopurinol dose per cage was adjusted daily based on the weight of the animals. To ensure complete allopurinol intake, the designated rats were given drug-laced food ( $20 \mathrm{~g}$ per rat), which accounted for $90 \%$ of their food intake, at night over 15 hours. Each morning, the remaining ground food was collected and weighed. All animals had access to the normal pellet food during the day time.

\subsubsection{Systolic blood pressure and body weight measurements}

SBP measurements were recorded on alternate days between 10 am to 12 noon, using the tail-cuff method described in Section 2.5.1 of Chapter 2. Body weights were recorded daily after blood pressure measurements.

\subsubsection{Thymus weight measurement}

The rats were sacrificed via exsanguination under isoflurane surgical anaesthesia (Section 2.8.2 of Chapter 2) and thymus wet weight, expressed relative to body weight (grams thymus weight per $100 \mathrm{~g}$ body weight) was used as a marker of glucocorticoid activity as described in Section 2.8.5.1.

\subsubsection{Serum urate measurement}

The efficacy of allopurinol treatment was evaluated by reduction in serum urate concentration, measured using the method described in Section 2.10 .2 by ACT Pathology, The Canberra Hospital, Australia. 


\subsubsection{Plasma nitrate and nitrite assay}

Plasma NOx, measured using the Griess colorimetric reaction described in Section 2.10.1, were used as a marker of endogenous NO availability.

\subsubsection{Aortic lucigenin-enhanced chemiluminescence assay}

This assay, which was described in Section 2.11.1, was performed as a marker of vascular superoxide production.

\subsubsection{Data and statistical analysis}

Results were expressed as mean \pm SEM. Statistical analysis were as described in Section 2.12 (Chapter 2).

\subsection{RESULTS}

\subsubsection{Food and allopurinol intake}

After accounting for the inevitable nightly wastage of ground food, the mean food intake for allopurinol-treated rats was $20 \pm 1 \mathrm{~g} / \mathrm{rat} / 15$-hour night (around $98 \%$ completion) and the mean allopurinol dose consumed was $196 \pm 0.5 \mathrm{mg} / \mathrm{kg} /$ day which was around $98 \%$ of the given dose. 


\subsubsection{Systolic blood pressure}

SBP was raised by DEX but not altered by allopurinol treatment (Figure 7.1). In the DEX-treated group, SBP increased from $110 \pm 2$ to $126 \pm 3 \mathrm{mmHg}$ $(P<0.001)$. SBP was increased in the DEX compared with the saline group $\left(\boldsymbol{P}^{\prime}<0.01\right)$. Allopurinol did not prevent HT in DEX-treated rats and did not alter SBP in saline or DEX-treated rats.

\section{Tail-cuff Systolic Blood Pressure}

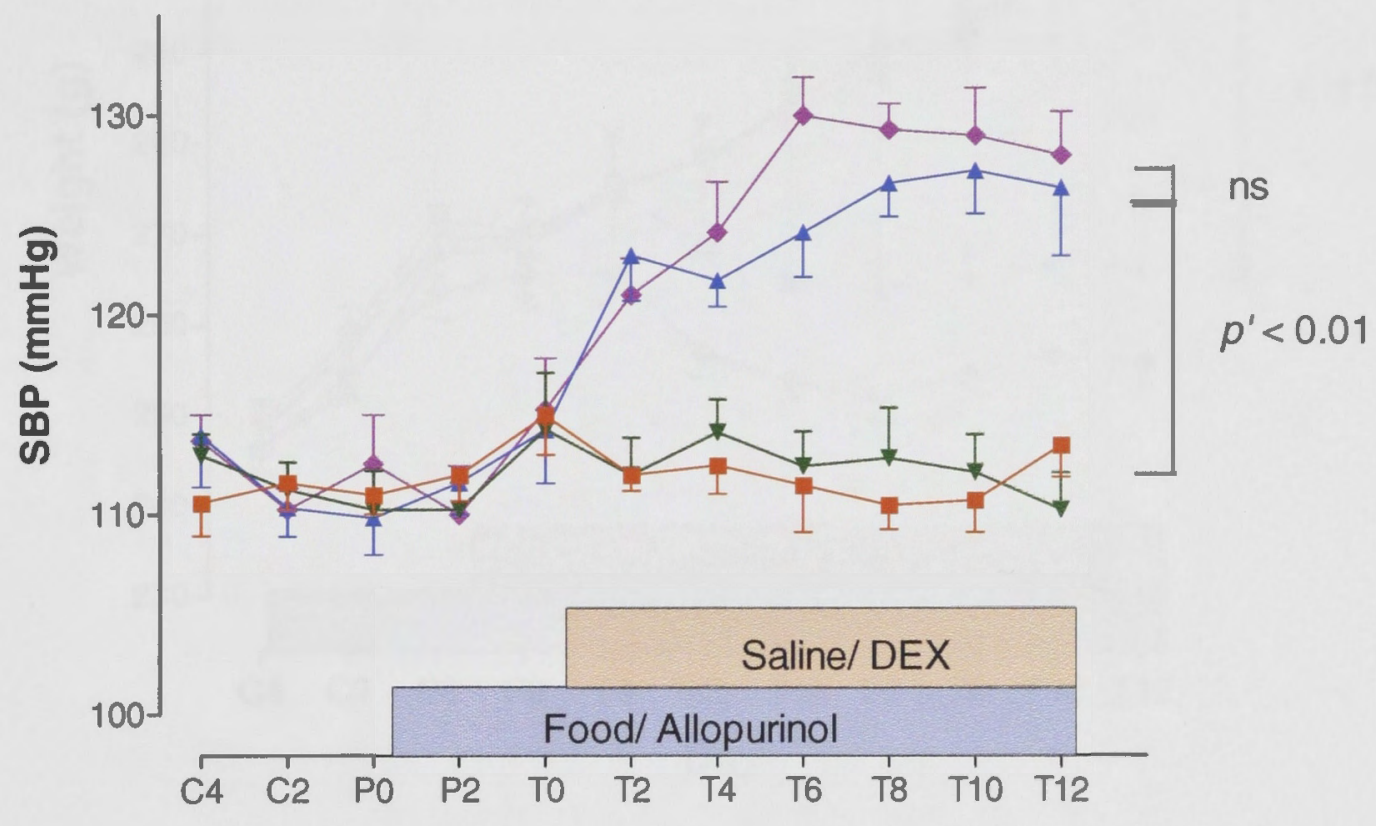

Day

Figure 7.1: Tail-cuff SBP readings from day C4 to day T12. saline only, $\mathrm{n}=8$;

$\triangle$ DEX only, $\mathrm{n}=8 ; \boldsymbol{\nabla}$ allopurinol + saline, $\mathrm{n}=7$; allopurinol $+\mathrm{DEX}, \mathrm{n}=7$. C: control days, P: pre-treatment days, T: treatment days. 


\subsubsection{Body weight}

Body weight fell initially in DEX-treated rats, then stabilised. These changes were not modified by allopurinol. When compared with saline, DEX-treated rats had lower mean body weight (saline: $301 \pm 14 \mathrm{~g}$; DEX: $267 \pm 8 \mathrm{~g}, P^{\prime}<0.01$ ) (Figure 7.2).

\section{Body Weight}

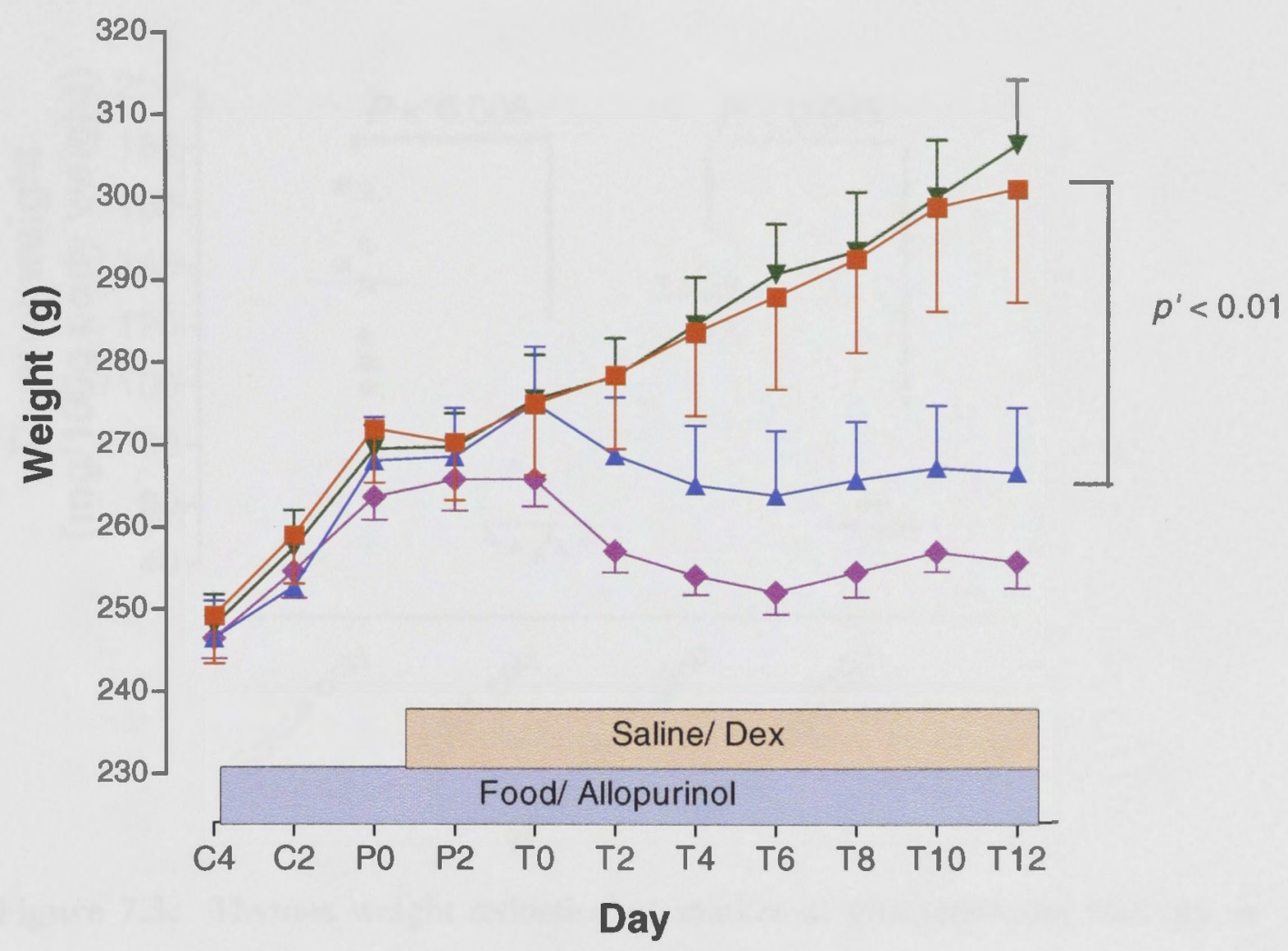

Figure 7.2: Body weight. a saline only, $\mathrm{n}=8 ; \Delta$ DEX only, $\mathrm{n}=8$; $\nabla$ allopurinol + saline, $\mathrm{n}=7$; allopurinol $+\mathrm{DEX}, \mathrm{n}=7$. C: control days, $\mathrm{P}$ : pretreatment days, T: treatment days. 


\subsubsection{Thymus weight}

DEX significantly decreased thymus weight (saline: $134 \pm 9$; DEX: $53 \pm$ $4 \mathrm{mg} / 100 \mathrm{~g}$ body weight, $P<0.001)$ and this significant decrease due to DEX was not altered by allopurinol (allopurinol + saline: $130 \pm 3$; allopurinol + DEX: $53 \pm 3 \mathrm{mg} / 100 \mathrm{~g}$ body weight, $\mathrm{n}=7$ each) (Figure 7.3).

\section{Glucocorticoid Activity}

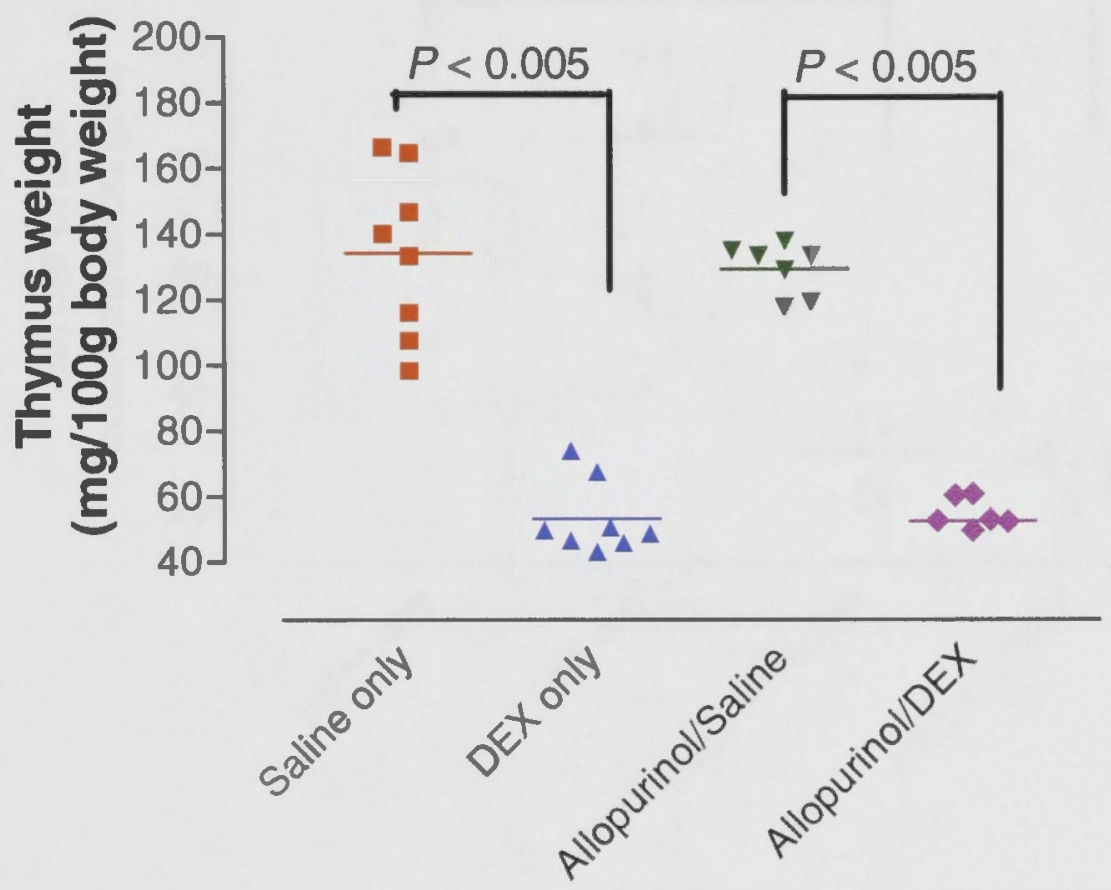

Figure 7.3: Thymus weight reduction as marker of glucocorticoid acitivity.

saline only, $\mathrm{n}=8 ; \boldsymbol{\Delta}$ DEX only, $\mathrm{n}=8 ; \boldsymbol{\nabla}$ allopurinol + saline, $\mathrm{n}=7 ; \diamond$ allopurinol $+\mathrm{DEX}, \mathrm{n}=7$. 


\subsubsection{Serum urate concentration}

Serum urate concentrations were $76 \pm 5 \mu \mathrm{mol} / \mathrm{L}$ in the saline- and $84 \pm 13 \mu \mathrm{mol} / \mathrm{L}$ in the DEX-treated rats, and lower following allopurinol treatment, $30 \pm 3 \mu \mathrm{mol} / \mathrm{L}$ saline and $28 \pm 2 \mu \mathrm{mol} / \mathrm{L} \mathrm{DEX}(P<0.001)$ (Figure 7.4).

\section{Serum Urate}

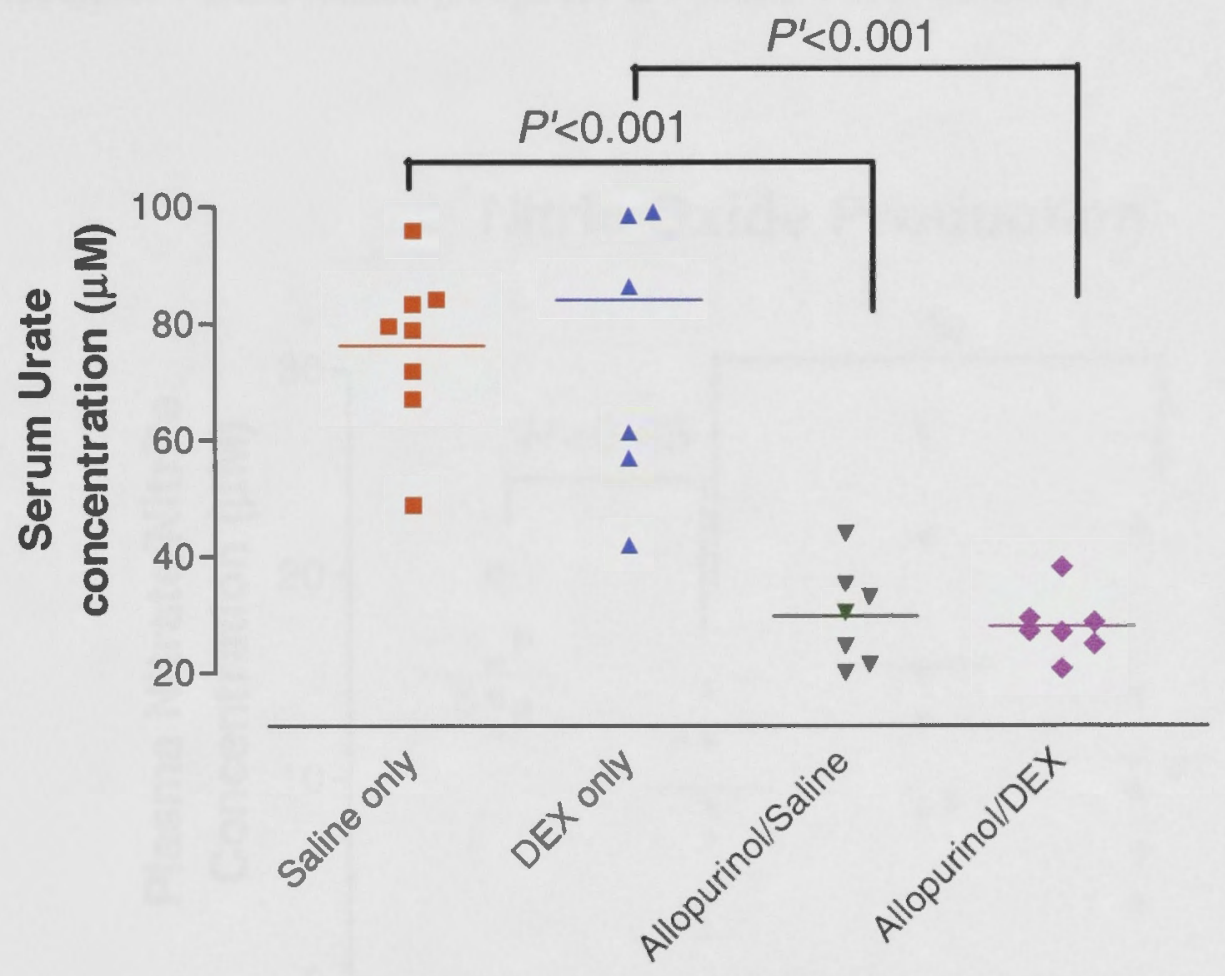

Figure 7.4: Serum urate reduction as a marker of effective allopurinol absorption.

- saline only, $\mathrm{n}=8 ; \boldsymbol{\Delta}$ DEX only, $\mathrm{n}=8 ; \boldsymbol{\nabla}$ allopurinol + saline, $\mathrm{n}=7$; allopurinol $+\mathrm{DEX}, \mathrm{n}=7$. 


\subsubsection{Plasma nitrate and nitrite concentration}

There was a significant decrease in plasma NOx concentrations in DEX-treated rats in comparison with saline-treated rats (saline: $15 \pm 1 \mu \mathrm{M}, \mathrm{n}=8$; $\mathrm{DEX} 10 \pm 2$ $\mu \mathrm{M}, \mathrm{n}=6, P^{\prime}<0.05$ ), but this was not prevented by allopurinol (allopurinol + DEX: $11 \pm 3 \mu \mathrm{M}, \mathrm{n}=6$ ). There was no significant difference in plasma NOx concentrations between the allopurinol + saline- $(16 \pm 3 \mu M, n=6)$ and allopurinol + DEX-treated groups $(11 \pm 3 \mu \mathrm{M}, \mathrm{n}=6)$ (Figure 7.5).

\section{Nitric Oxide Production}

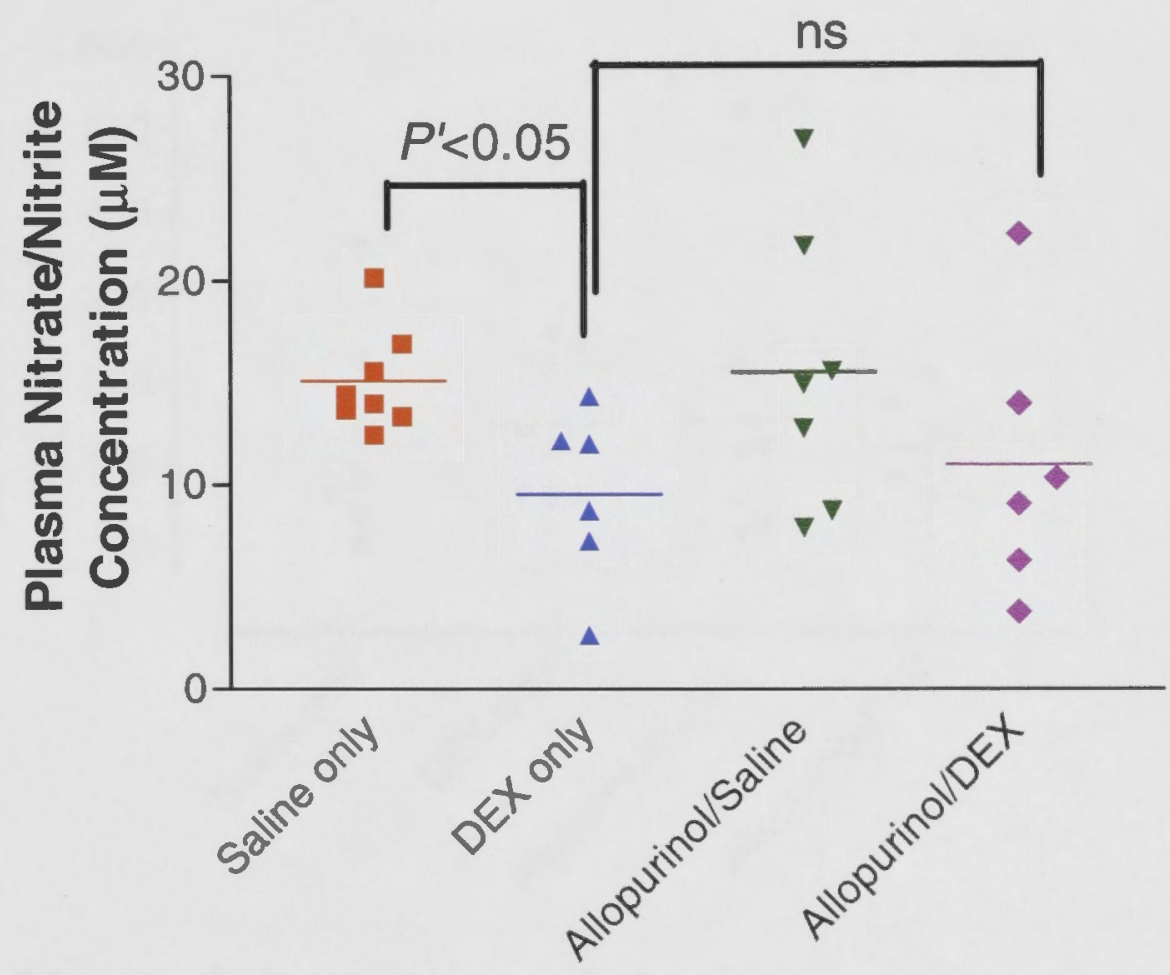

Figure 7.5: Plasma nitrate/nitrite as a marker of total body nitric oxide production. = saline, $\mathrm{n}=8 ; \boldsymbol{\Delta} \mathrm{DEX}, \mathrm{n}=6 ; \boldsymbol{\nabla}$ allopurinol + saline, $\mathrm{n}=7$; allopurinol $+\mathrm{DEX}, \mathrm{n}=6$. 


\subsubsection{Aortic lucigenin-enhanced chemiluminescence}

There was no significant difference in aortic lucigenin-enhanced chemiluminescence in saline- $(182 \pm 47$ count $/ \mathrm{s} / \mathrm{mg}, \mathrm{n}=7)$ and DEX-treated rats $(200 \pm 29$ count/s/mg, $n=8)$. Allopurinol treatment did not significantly alter the aortic lucigenin-enchanced chemiluminescence of the saline and DEX-treated groups (allopurinol + saline: $203 \pm 67$ count/s/mg, $n=7$; allopurinol + DEX: $169 \pm 22$ count/s/mg, $\mathrm{n}=7$ ) (Figure 7.6).

\section{Aortic Superoxide Production}

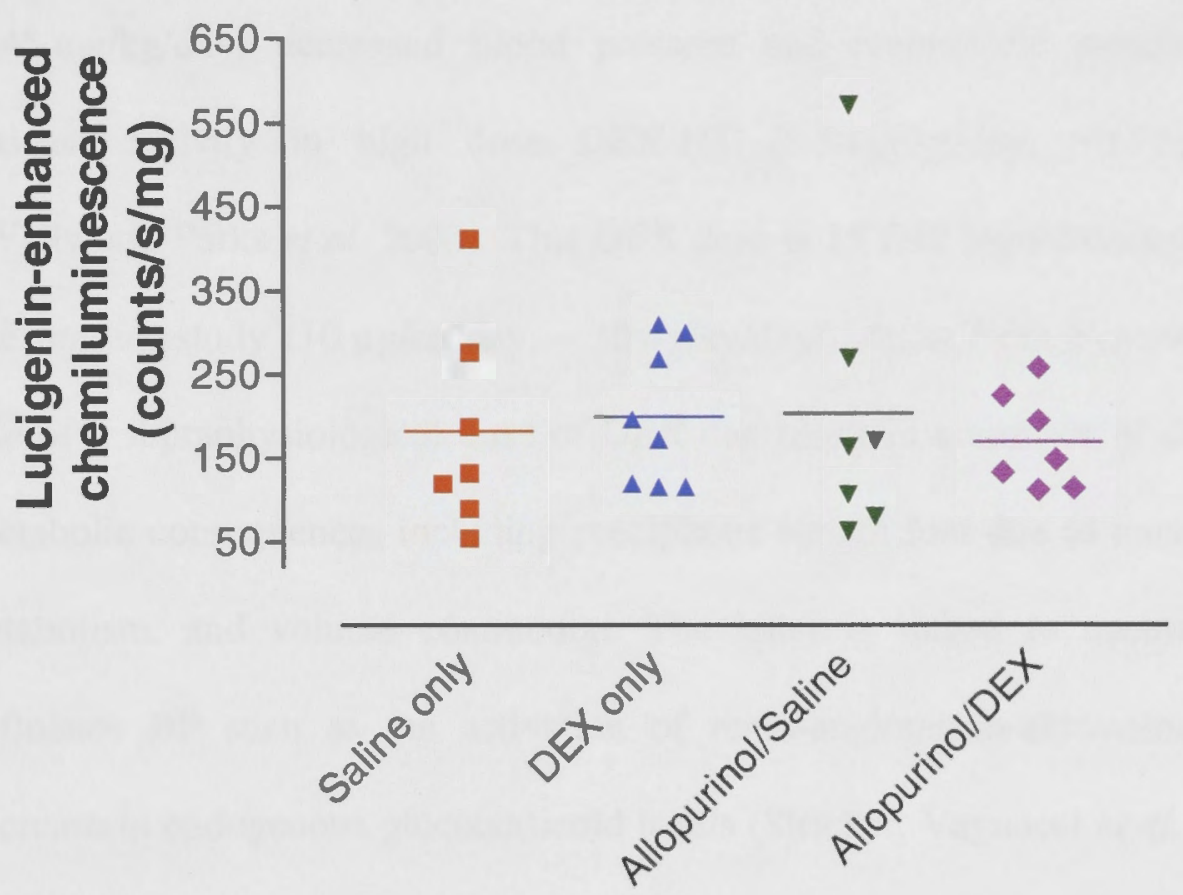

Figure 7.6: Aortic lucigenin-enhanced chemiluminescence as a marker of vascular superoxide production. a saline only, $\mathrm{n}=7 ; \Delta$ DEX only, $\mathrm{n}=8$; $\boldsymbol{\nabla}$ allopurinol + saline, $\mathrm{n}=7 ;$ allopurinol $+\mathrm{DEX}, \mathrm{n}=7$. 


\subsection{DISCUSSION}

As discussed in Chapter 1, ROS are generated mainly generated by the NAD(P)H oxidase and the xanthine oxidase pathways, eNOS in its uncoupled form and mitochondria. The $\mathrm{NAD}(\mathrm{P}) \mathrm{H}$ oxidase pathway (Hu, Zhang et al. 2006) but not uncoupling of eNOS (Li, Fraser et al. 1997; Hu, Zhang et al. 2006; Thida, Earl et al. 2010) plays a role in the pathogenesis of DEX-HT. The present study suggests that xanthine oxidase does not have a major role in DEX-HT.

Wallwork et al. have shown that allopurinol treatment $(0.4 \mathrm{~g} / \mathrm{L}$ drinking water, $\sim 45 \mathrm{mg} / \mathrm{kg} /$ day) decreased blood pressure and cremasteric muscle xanthine oxidase activity in high dose DEX-HT $(0.5 \mathrm{mg} / \mathrm{kg} /$ day, $\sim 150 \mu \mathrm{g} / \mathrm{rat} /$ day $)$ (Wallwork, Parks et al. 2003). This DEX dose is 15 fold higher than that used in the present study (10 $\mu \mathrm{g} / \mathrm{rat} / \mathrm{day}, ~ 30 \mu \mathrm{g} / \mathrm{kg} / \mathrm{day})$. Apart from hypertension, the use of a supraphysiological dose of DEX can result in a number of dose-related metabolic consequences including precipitous weight loss due to muscle and fat catabolism, and volume contraction. The latter is linked to events likely to influence BP such as the activation of renin-angiotensin-aldosterone system, increase in endogenous glucocorticoid levels (Stricker, Vagnucci et al. 1979) and suppression of atrial natriuretic peptide (Sebaai, Lesage et al. 2002). These confounding factors due to excessive DEX dose make such a model unsuitable for examining mechanisms of GC-HT. Consequently, a dose that was sufficient to induce hypertension without significant weight loss was employed in this study 
(Tonolo, Fraser et al. 1988; Li, Fraser et al. 1997; Zhang, Croft et al. 2004; Hu, Zhang et al. 2006; Miao, Zhang et al. 2007).

Wallwork and colleagues did not report serum urate concentration. This allopurinol dose, however, did not result in any significant change in serum urate levels or SBP compared to controls (Zhang Y, unpublished data). As in a previous study (Zhang, Chan et al. 2005), the dose used in this study (196 $\pm 0.5 \mathrm{mg} / \mathrm{kg} / \mathrm{day}$ after correcting for nightly allopurinol-laced food wastage) effectively lowered serum urate levels indicating inhibition of the xanthine oxidase pathway.

Plasma NOx concentrations were significantly decreased by DEX treatment, consistent with other published findings in rats (Mondo, Yang et al. 2006) and mice (Wallerath, Witte et al. 1999; Wallerath, Godecke et al. 2004). This decrease was not prevented by pretreatment with allopurinol, suggesting that DEX-induced NO deficiency was not inhibited by xanthine oxidase blockade.

Aortic lucigenin-enhanced chemiluminescence, a marker of vascular oxidative stress, was not altered by DEX and/or allopurinol. A possible explanation for this negative result is that nitric oxide-redox imbalance in the DEX-HT model is likely to be more marked in resistance vessels compared to larger vessel like the aorta. As shown in Chapter 3, DEX-HT is associated with increased in TPR, a phenomenon contributed mostly by arterioles (resistance vessels). Some may also argue that the lack of a significant difference might be a due to small sample size as similar observations were made in the ACTH-HT model (Zhang, Chan et al. 
2005; Zhang, Miao et al. 2007). Using pooled data from our laboratory, ACTHHT was associated with increased aortic lucigenin-enhanced chemiluminescence (Zhang, Chan et al. 2005). However, pooled data from our laboratory did not shown any significant change in luminescence in DEX-hypertensive rats compared with control rats (Figure 7.7).

\section{Lucigenin-enhanced chemiluminescence: Aortic Superoxide Production}

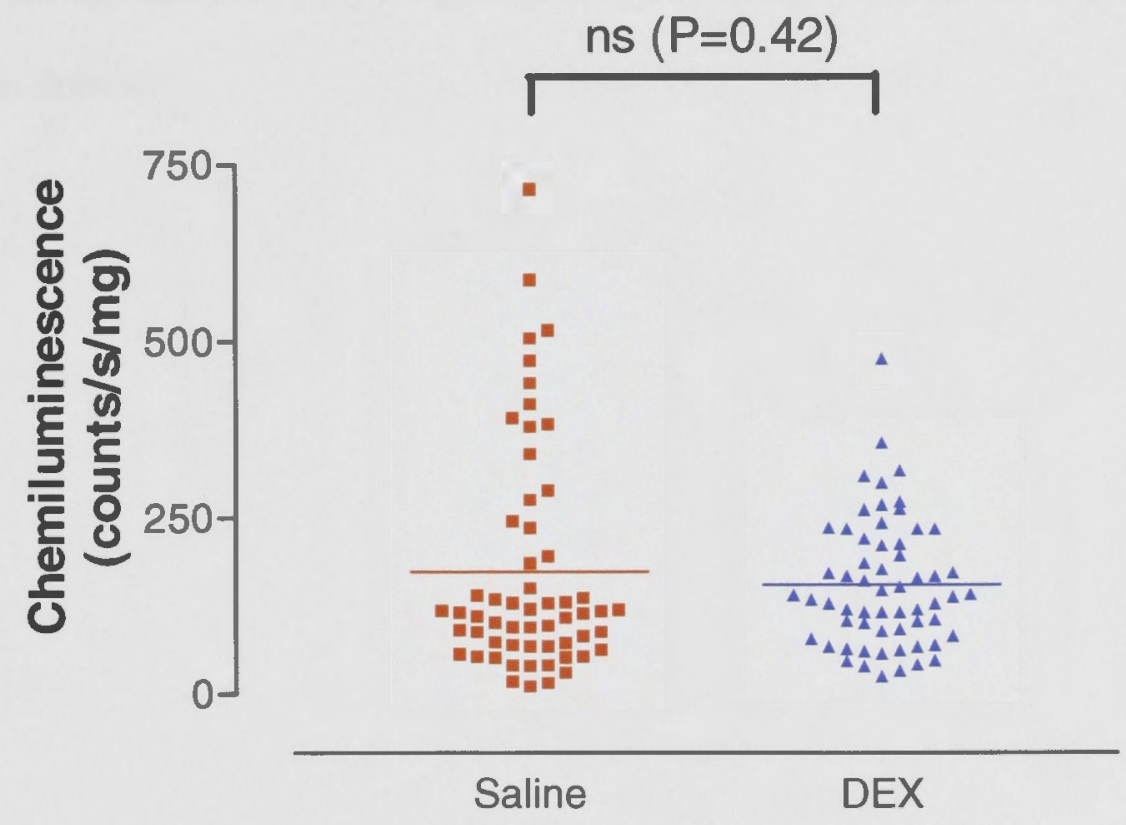

Figure 7.7: Pooled aortic lucigenin-enhanced chemiluminescence data from the High Blood Pressure Research Unit (2006-2008). $=$ Saline, $\mathrm{n}=59 ; \mathbf{\Delta}$ DEX, $\mathrm{n}=$ 60. 


\subsection{CONCLUSION}

This study shows that allopurinol is ineffective in preventing DEX-HT in the rats despite adequate inhibition of the xanthine oxidase enzyme. Thus, the xanthine oxidase pathway does not play a significant role in the pathogenesis of DEX-HT in the rat.

Together with our previous finding showing lack of effect of allopurinol on ACTH-HT (Zhang, Chan et al. 2005), a conclusion that the xanthine oxidase pathway does not play a significant role in the pathogenesis of GC-HT in rats can be drawn. 


\section{CHAPTER 8}

Effects of Adrenergic Receptor Antagonism and Lipid Peroxidation Inhibition Using Propranolol in

Glucocorticoid-Induced Hypertension in the Rat 


\subsection{INTRODUCTION}

Stimulation of the sympathetic nerves in the adrenal medulla results in the release of catecholamines, adrenaline and noradrenaline, into the systemic circulation (Guyton and Hall 2000). Activation of adrenergic receptors by these catecholamines results in slightly prolonged but similar effects to direct sympathetic nerve stimulation. The production of noradrenaline from adrenaline in the adrenal medulla is catalysed by phenylethanolamine $\mathrm{N}$-methyltransferase (PNMT), an enzyme regulated by adrenal glucocorticoid at transcriptional, translational and proteolytic levels (Wong, Siddall et al. 1995). Regulations by glucocorticoid hormones at these different levels occur independently and are different depending on the chronicity of glucocorticoid treatment (Wong, Siddall et al. 1995). Acute glucocorticoid replacement was shown to be associated with increased PNMT mRNA expression without a concomitant increase in PNMT enzyme activity. Chronic replacement on the other hand restored PNMT enzyme activity but decreased mRNA expression (Wong, Siddall et al. 1995).

Increased pressor responsiveness to noradrenaline has been observed in GC-HT in humans (Kurland and Freedberg 1951; Mendlowitz, Naftchi et al. 1961; Pirpiris, Sudhir et al. 1992), rats (Russo, Fraser et al. 1989; Russo, Fraser et al. 1990) and dogs (Nakamoto, Suzuki et al. 1991). In some studies, glucocorticoid treatment was associated with increases in plasma adrenaline (Kumai, Asoh et al. 2000; Silvan, Martinez-Mateos et al. 2007) and noradrenaline concentrations (Watanabe, Noshiro et al. 1995; Kumai, Asoh et al. 2000), but this is not a 
universal finding (Rothschild, Langlais et al. 1984; Brown and Fisher 1986). Rothschild et al showed that DEX administration in human subjects resulted in raised levels of dopamine but not other catecholamines (Rothschild, Langlais et al. 1984). Brown and Fisher, on the other hand, were able to suppress etherinduced increases in plasma adrenaline and noradrenaline (Brown and Fisher 1986).

Whilst it has been shown previously that atenolol, a selective $\beta 1$-adrenoreceptor blocker, did not prevent ACTH-HT (Wen, Fraser et al. 1999) in the rat, it remains unclear if complete blockade of both $\beta 1$ - and $\beta 2$-receptors (and therefore, inhibition of myocardial and smooth muscle contractility, respectively) would be able to produce an effect.

Activation of both $\beta 1$ - and $\beta 2$-adrenoreceptors may play a role in the pathogenesis of ACTH and DEX-HT in the rat. In this study, the role of the beta adrenergic receptors was tested using a non-selective $\beta$-adrenoreceptor blocker propranolol in vivo, a commonly used antihypertensive drug. Beta-adrenergic receptor blockers are useful drugs commonly used in the treatment of hypertension, cardiac failure and ischaemic heart disease. This study will also help identify the utility of $\beta$ adrenoreceptor blockers in GC-HT.

Apart from $\beta$-adrenergic antagonism, the beneficial cardiovascular effects demonstrated by $\beta$-propranolol have been related to its antioxidant effect. Propranolol has been to shown to have hydroxyl radical, hypochlorous acid, 
peroxyl radical, nitric oxide radical and peroxynitrite but not superoxide scavenging activity (Gomes, Costa et al. 2006). This antioxidant property was associated with its intrinsic chemical properties rather than membrane stablising effect which requires a concentration significantly higher than reported serum clinical levels (Mak and Weglicki 1988). These authors subsequently found evidence that a metabolic product of propranolol ring oxidation, 4-hydroxyl propranolol, is a potent antioxidant which can provide anti-LDL oxidation and endothelial cell protection in in vitro experiments (Mak and Weglicki 2004). There have also been studies showing propranolol is a potent inhibitor of lipid peroxidation. These in vitro studies demonstrated effective inhibition of lipid peroxidation in canine sarcolemnal membranes (Mak and Weglicki 1988) and rat liver microsomes (Aruoma, Smith et al. 1991). Since oxidative stress, as discussed in Chapter 1, plays a significant role in glucocorticoid hypertension, the role of propranolol as a lipid peroxidation inhibitor was also examined in both ACTH and DEX-HT.

\subsection{METHODS}

This study was approved by the Animal Experimentation Ethics Committee of the Australian National University (Protocol No. J.HB.20.05). The general methodology for this study was as described in Chapter 2 .

There were 2 sets of experiments in this study. The first evaluated the effects of propranolol on ACTH-HT and the second, on DEX-HT. Data from each of these 
studies were compared with the control groups (groups 1 and 2). Seventy male Sprague-Dawley rats were randomly divided into 6 treatment groups.

\subsubsection{Control groups}

Group 1: Untreated ground food + saline $(0.9 \% \mathrm{NaCl}, 0.1 \mathrm{~mL} / 100 \mathrm{~g} / \mathrm{day}, \mathrm{sc})$, $\mathrm{n}=14$

Group 2: Propranolol (100 mg/kg/day)-laced ground food + saline (1 mL/kg/day, $\mathrm{sc}), \mathrm{n}=10$.

\subsubsection{ACTH-induced hypertension}

Group 3: Untreated ground food + ACTH $(0.2 \mathrm{mg} / \mathrm{kg} / \mathrm{day}, \mathrm{sc}), \mathrm{n}=13$

Group 4: Propranolol-laced ground food + ACTH, $n=10$

\subsubsection{Dexamethasone-induced hypertension}

Group 5: Untreated ground food + DEX (10 $\mu \mathrm{g} / \mathrm{rat} / \mathrm{day}), \mathrm{n}=13$.

Group 6: Propranolol-laced food + DEX, $\mathrm{n}=10$

Propranolol (Inderal, AztraZeneca, North Ryde, Australia) was used in this study. The propranolol tablets were ground and mixed in ground rat food. Propranolol- 
laced food was freshly prepared daily. The total propranolol dose per cage was adjusted daily based on weight of the animals. To ensure complete propranolol ingestion, the animals were given $20 \mathrm{~g}$ of ground food per rat per night over 15 hours. This was approximately $90 \%$ of their food intake at night. Each morning the remaining ground food was collected and weighed. The amount given on subsequent nights was adjusted according to the amount left over on the previous night. During the day, all animals had unlimited access to pellet food.

\subsubsection{Blood pressure, heart rate and body weight measurements}

Tail-cuff SBP and HR measurements were performed on alternate days at 9-11 am using the method as described in Sections 2.5.1 and 2.6.1, respectively (Chapter 2). Body weights were measured on alternate days after the tail-cuff SBP and HR measurements.

\subsubsection{Thymus and adrenal weights}

Thymus wet weight, expressed relative to body weight (grams thymus weight per $100 \mathrm{~g}$ body weight), was used as a marker of glucocorticoid activity. Adrenal wet weight, expressed relative to body weight (grams adrenal weight per $100 \mathrm{~g}$ body weight), was used to assess ACHT and DEX treatment efficacy. The surgical techniques were as described in Section 2.8.5.1 of Chapter 2. 


\subsubsection{Plasma $\mathrm{F}_{2}$-isoprostane assay}

Plasma $\mathrm{F}_{2}$-isoprostane was used as a marker of systemic lipid peroxidation. The assay technique was described in Section 2.11.3 of Chapter 2.

\subsubsection{Statistical analysis}

Results were expressed as mean \pm SEM. Statistical analysis were as described in Section 2.12 of Chapter 2.

\subsection{RESULTS}

\subsubsection{Systolic blood pressure}

SBP was raised by ACTH (from $110 \pm 2$ on day T0 to $134 \pm 3$ on day T12, $P<$ 0.0005 ) and DEX (from $114 \pm 2$ on day T0 to $139 \pm 3 \mathrm{mmHg}$ on day T12, $P<$ 0.0005 ) but not changed by propranolol treatment (from $111 \pm 2$ on day T0 to 112 \pm 2 on day T12, ns). Propranolol treatment did not prevent ACTH- and DEXinduced hypertension in the rat. Systolic blood pressures in the propranolol + ACTH- and propranolol + DEX-treated groups were significantly higher than in the propranolol-only group $\left(P^{\prime}<0.001\right.$ and $P^{\prime}<0.005$, respectively) (Figures 8.1 and 8.2). 


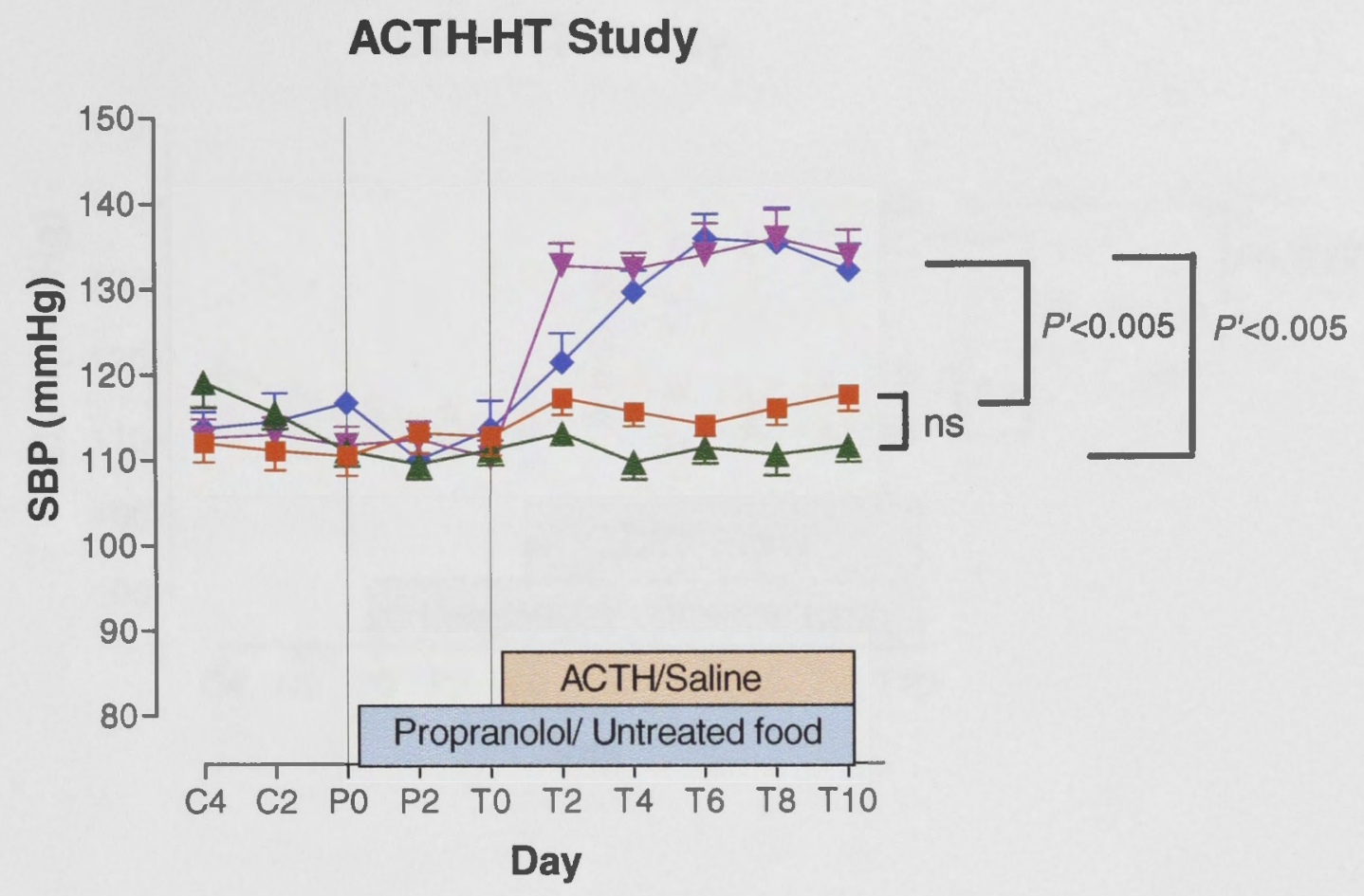

Figure 8.1: Tail-cuff SBP readings from day $\mathrm{C} 4$ to day $\mathrm{T} 10$ in the ACTH-HT prevention study. = Untreated food + saline, $\mathrm{n}=14 ; \boldsymbol{\Delta}$ Propranolol + saline, $\mathrm{n}=10 ; \nabla$ untreated food $+\mathrm{ACTH}, \mathrm{n}=13 ; \bullet$ Propranolol $+\mathrm{ACTH}, \mathrm{n}=10$. 


\section{DEX-HT Study}

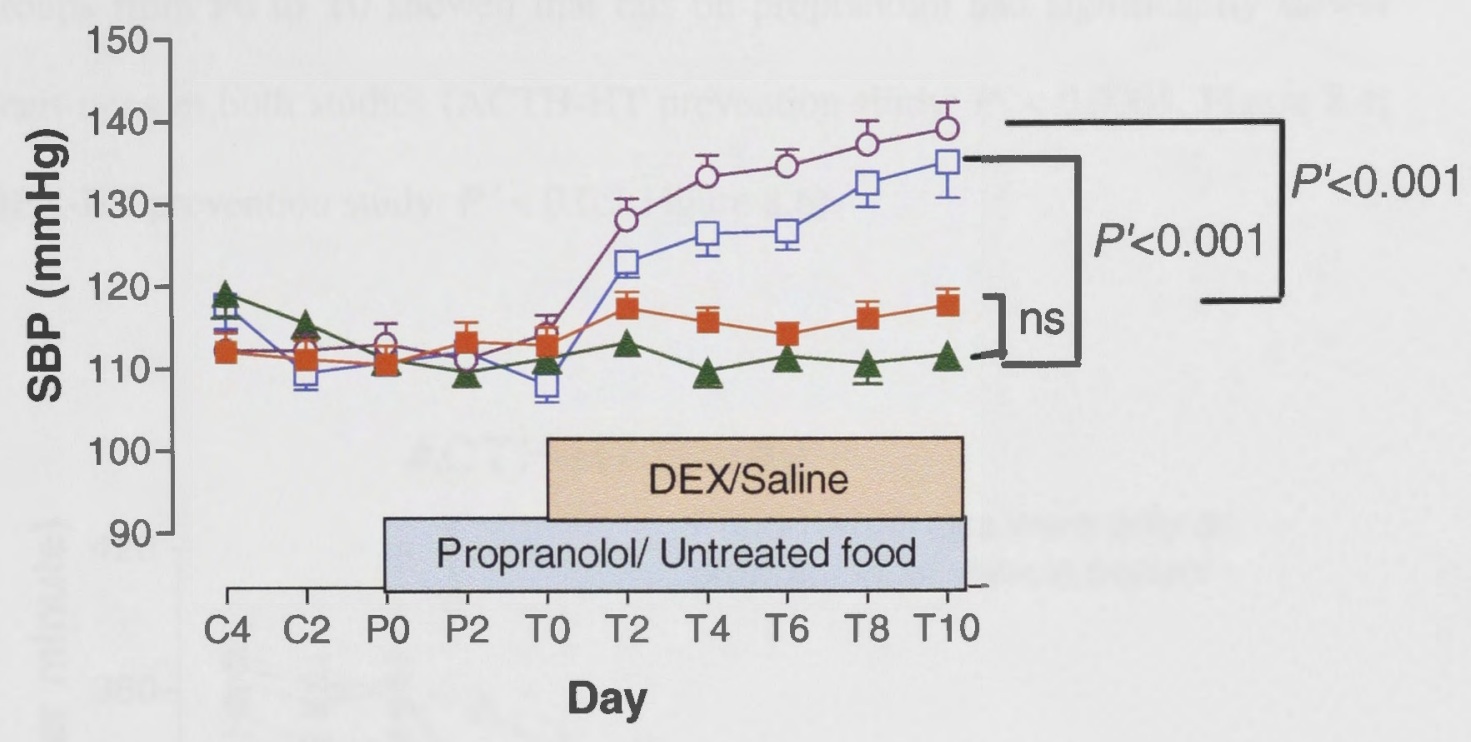

Figure 8.2: Tail-cuff SBP readings from day C4 to day $\mathrm{T} 10$ in the DEX-HT prevention study. = Untreated food + saline, $n=14 ; \boldsymbol{\Delta}$ Propranolol + saline, $\mathrm{n}=10 ; \circ$ Untreated food + DEX, $\mathrm{n}=13 ; \square$ Propranolol + DEX, $\mathrm{n}=10$.

\subsubsection{Heart rate}

There was a progressive fall in the HR throughout the entire duration of the experiment in all groups (Figures 8.3 and 8.5 ). Therefore, in order to evaluate the effectiveness of $\beta$-adrenergic receptor blockade by propranolol, we looked at the HR profile during the initial period of propranolol treatment from day P0 to T0 (Figures 8.3 and 8.5). As the animals were only on either untreated food or propranolol between day P0 to day T0 (HR measurements were obtained prior to treatment on day $\mathrm{T} 0$ ), they were re-grouped to either propranolol or untreated 
food groups for this analysis (Figures 8.4 and 8.6). Comparison between these groups from P0 to T0 showed that rats on propranolol had significantly slower heart rates in both studies (ACTH-HT prevention study: $P^{\prime}<0.0005$, Figure 8.4; DEX-HT prevention study: $P^{\prime}<0.05$, Figure 8.6).

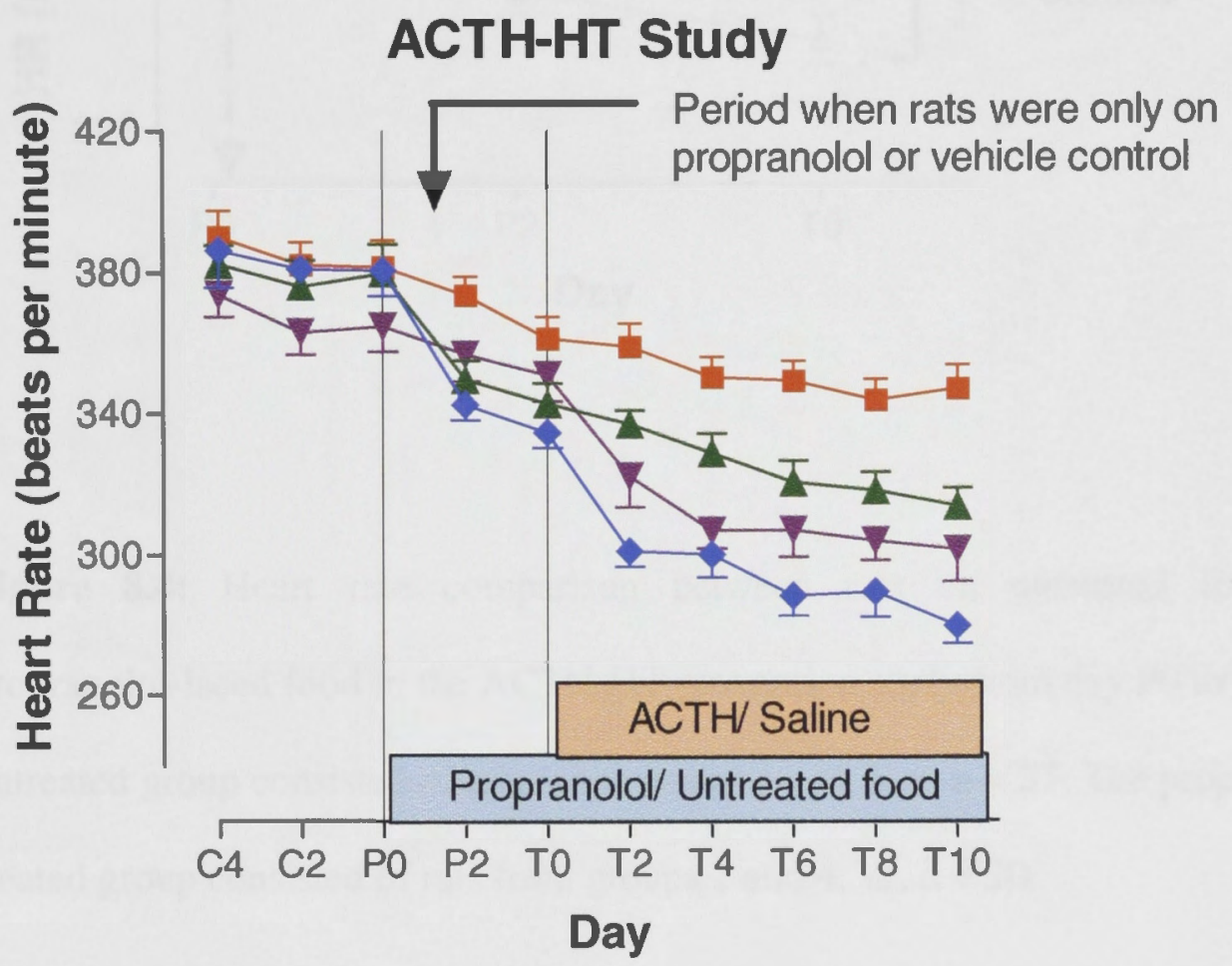

Figure 8.3: Heart rate measurements from day $\mathrm{C} 4$ to $\mathrm{T} 10$. $=$ Untreated food + saline, $\mathrm{n}=14 ; \boldsymbol{\Delta}$ Propranolol + saline, $\mathrm{n}=10 ; \boldsymbol{\nabla}$ Untreated food $+\mathrm{ACTH}$, $\mathrm{n}=13 ; \bullet$ Propranolol + ACTH, $\mathrm{n}=10$. 


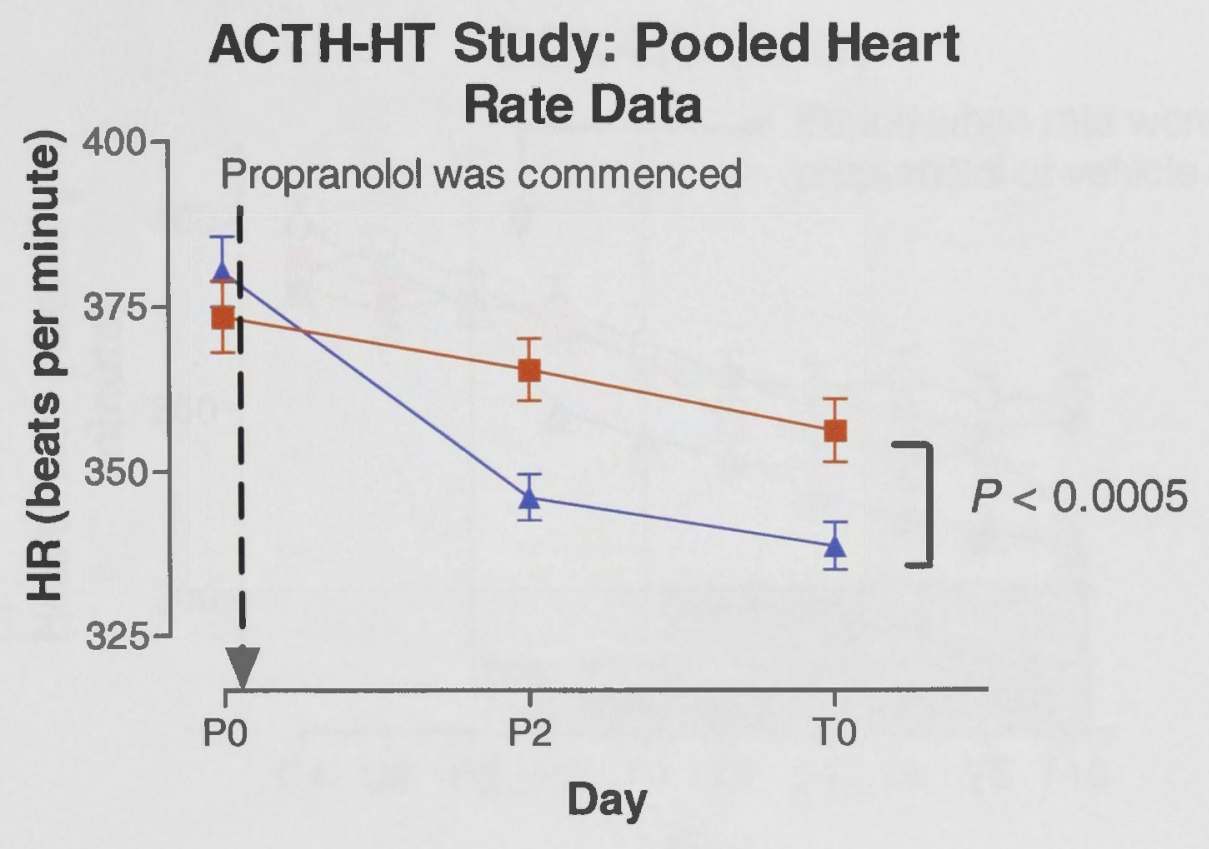

Figure 8.4: Heart rate comparison between rats on untreated food and propranolol-laced food in the ACTH-HT prevention study from day P0 to TO. The untreated group consisted of rats from groups 1 and $3, m, n=27$. The propranololtreated group consisted of rats from groups 2 and $4, \boldsymbol{\Delta}, \mathrm{n}=20$. 


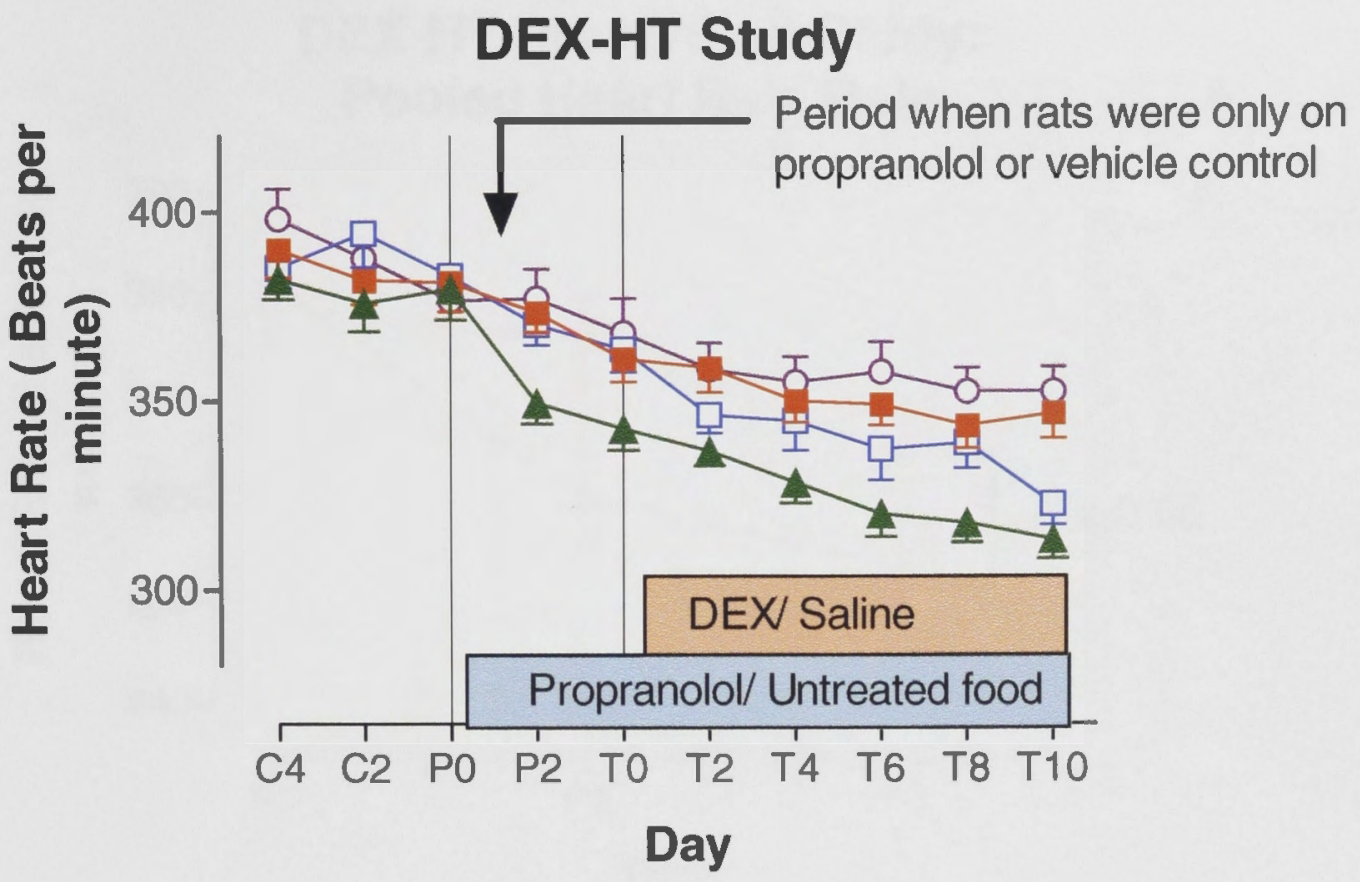

Figure 8.5: Heart rate measurements from day $\mathrm{C} 4$ to $\mathrm{T} 0$. $=$ Untreated food + saline, $\mathrm{n}=14 ; \boldsymbol{\Delta}$ Propranolol + saline, $\mathrm{n}=10 ;$; Untreated food $+\mathrm{DEX}, \mathrm{n}=13$ $\square$ Propranolol + DEX, $\mathrm{n}=10$. 


\section{DEX-HT Prevention Study: Pooled Heart Rate Data}

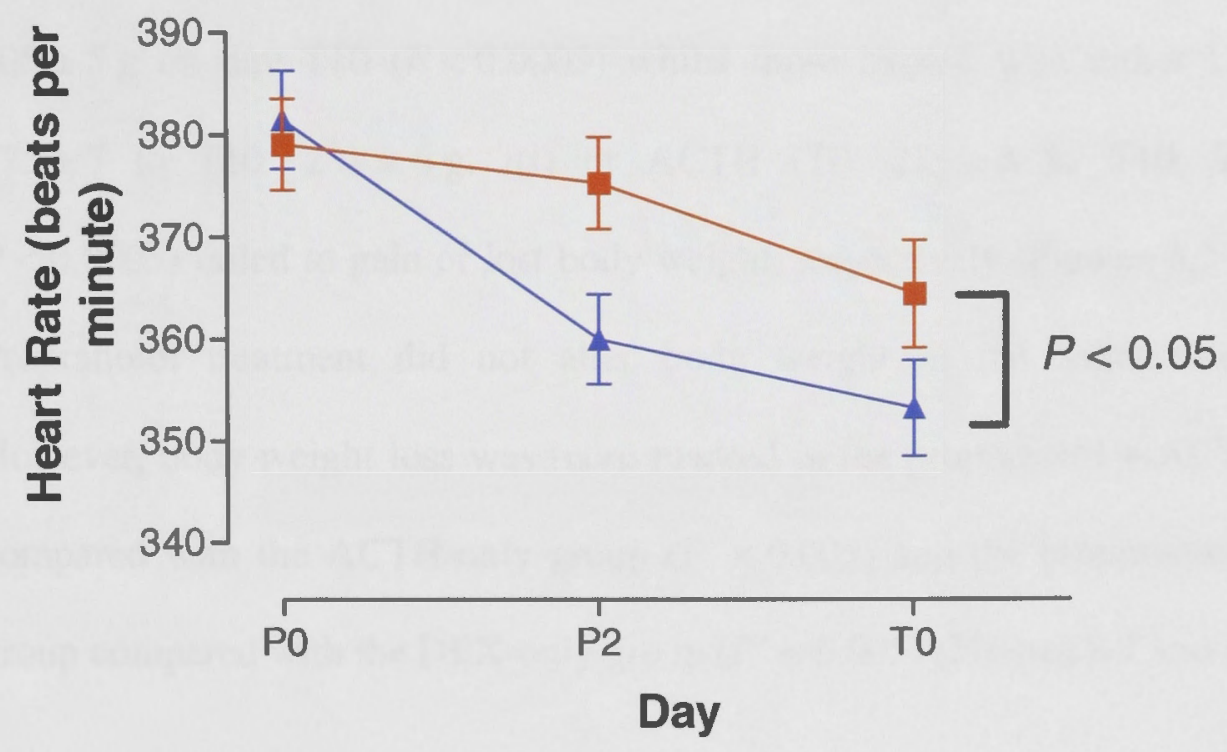

Figure 8.6: Comparison between the rats on untreated food and propranolol-laced food in the DEX-HT prevention study from day P0 to T0. The untreated group consisted of rats from groups 1 and $5, \mathrm{~m}, \mathrm{n}=27$. The propranolol-treated group consisted of rats from groups 2 and $6, \boldsymbol{\Delta}, \mathrm{n}=20$. 


\subsubsection{Body weight}

Rats treated with saline gained weight steadily from $273 \pm 5 \mathrm{~g}$ on day T0 to $305 \pm 5 \mathrm{~g}$ on day T10 $(P<0.0005)$ whilst those treated with either DEX (T0: $275 \pm 7$ to $\mathrm{T} 10: 281 \pm 6 \mathrm{~g}, n s)$ or $\mathrm{ACTH}(\mathrm{T0}: 285 \pm 8$ to $\mathrm{T} 10: 259 \pm 6 \mathrm{~g}$, $P<0.0005$ ) failed to gain or lost body weight, respectively (Figures 8.7 and 8.8 ). Propranolol treatment did not alter body weight in the saline-treated rats. However, body weight loss was more marked in the propranolol + ACTH group compared with the ACTH-only group $\left(P^{\prime}<0.005\right)$ and the propranolol + DEX group compared with the DEX-only group $\left(P^{\prime}<0.005\right)$ (Figures 8.7 and 8.8 ).

a) ACTH-HT Study

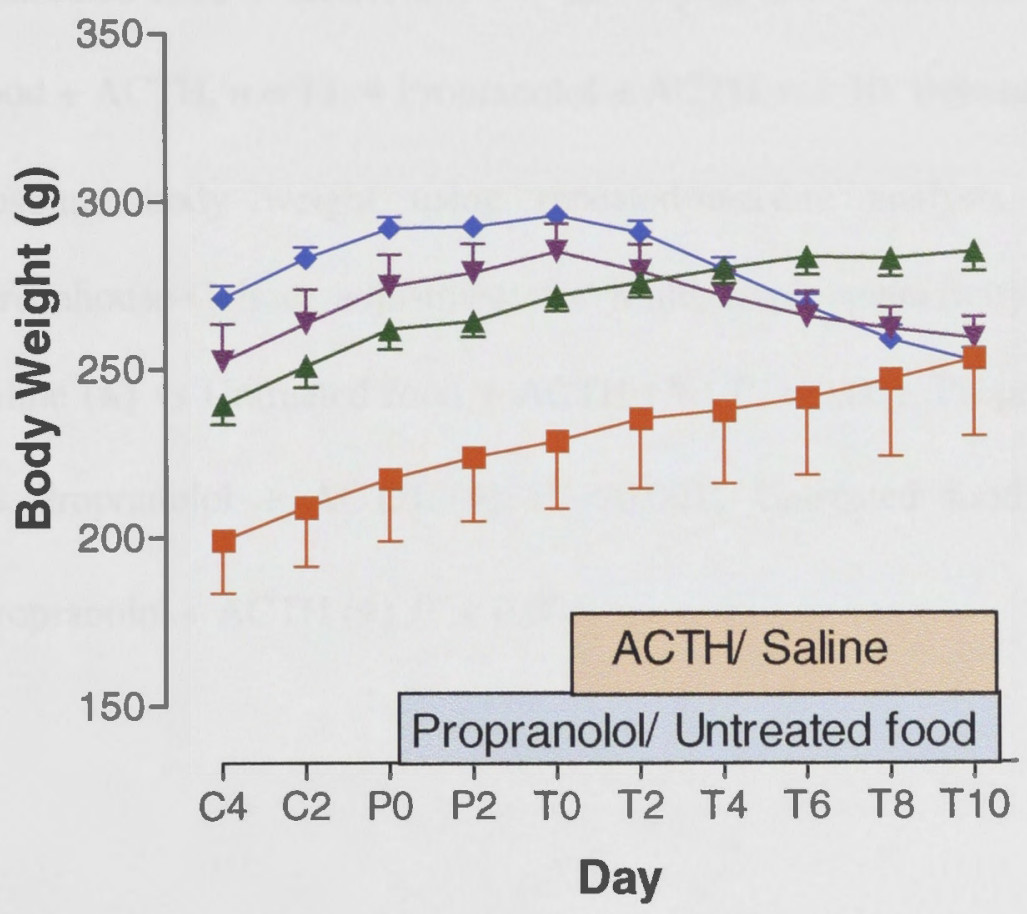




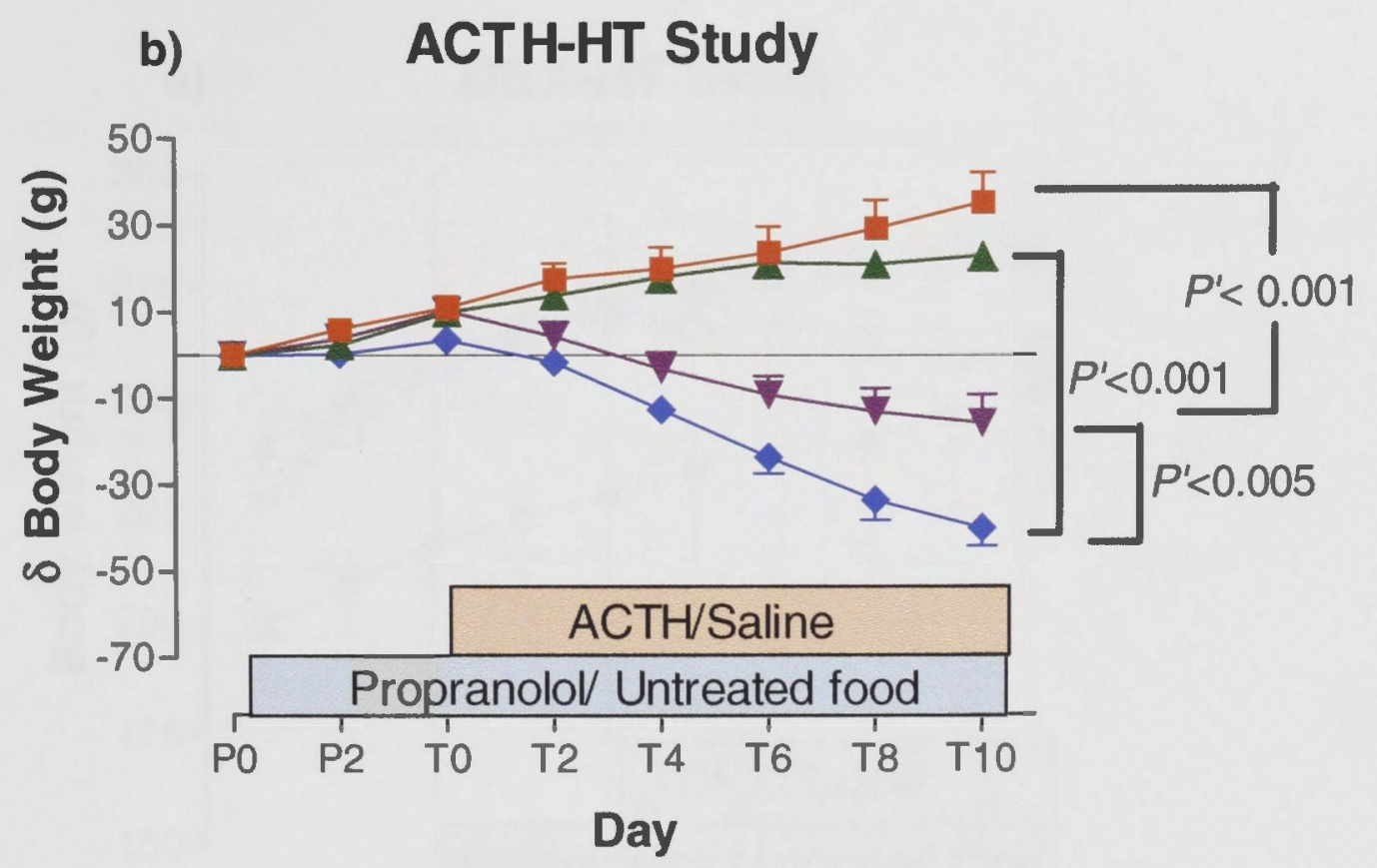

Figure 8.7: a) Absolute body weight and b) change $(\delta)$ in body weight.

Untreated food + saline, $\mathrm{n}=14 ; \boldsymbol{\Delta}$ Propranolol + saline, $\mathrm{n}=10 ; \nabla$ Untreated food $+\mathrm{ACTH}, \mathrm{n}=13 ; \bullet$ Propranolol $+\mathrm{ACTH}, \mathrm{n}=10$. Between group analysis on absolute body weight using repeated-measure analysis of variance, with Greenhouse-Geisser adjustment for multisample asphericity: Untreated food + saline ( $₫$ ) vs Untreated food + ACTH $(\nabla) P^{\prime}<0.001$; Propranolol + saline $(\mathbf{\Delta})$ vs Propranolol $+\mathrm{ACTH}(\diamond) P^{\prime}<0.001$; Untreated food $+\mathrm{ACTH}(\nabla)$ vs Propranolol + ACTH $(\diamond) P^{\prime}<0.005$ 
a) DEX-HT Study

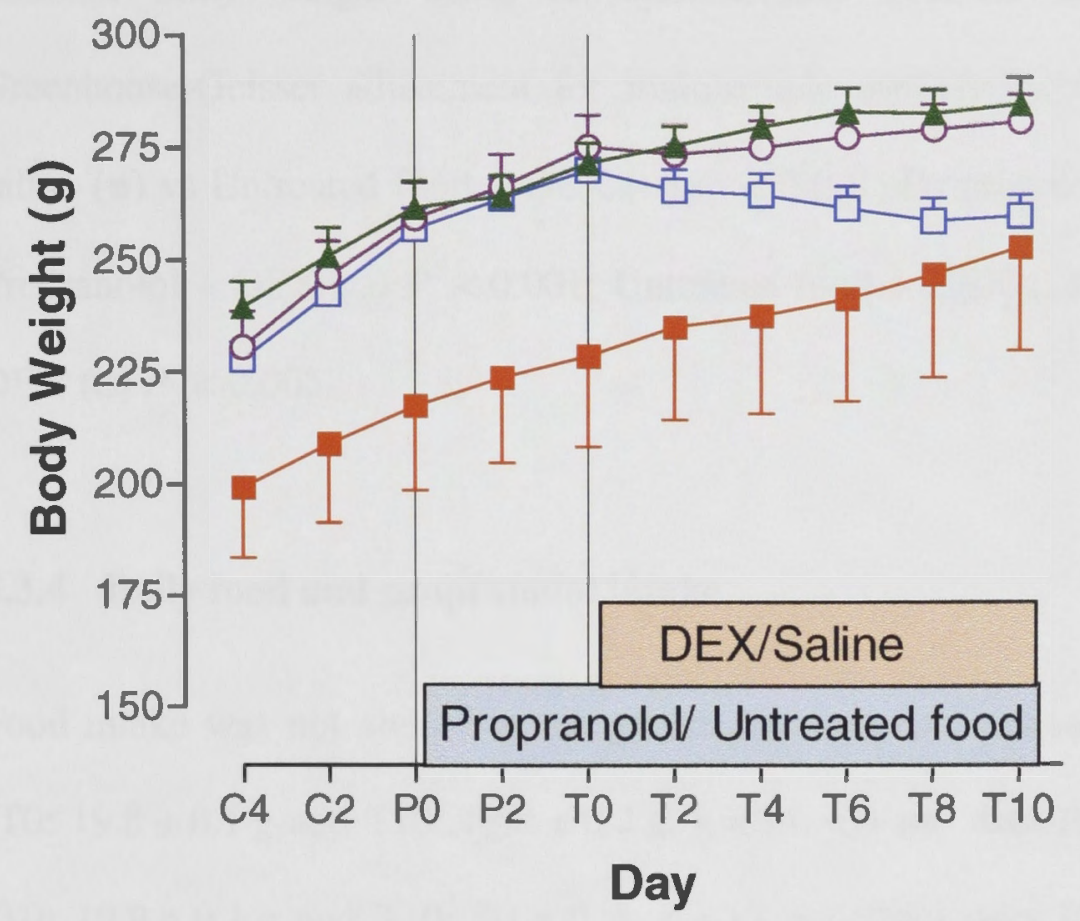

b)

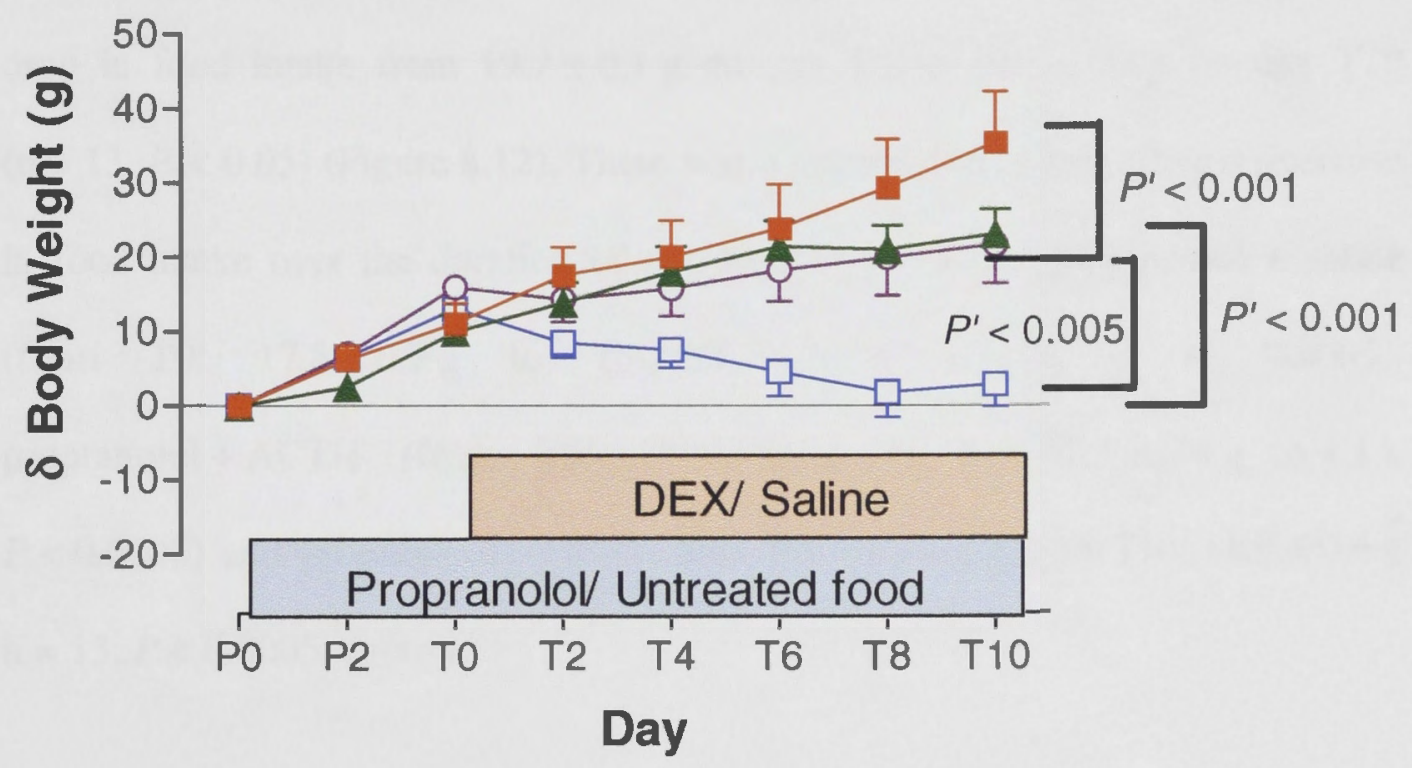

Figure 8.8: a) Absolute body weight and b) change ( $\delta$ ) in body weight. Untreated food + saline, $\mathrm{n}=14 ; \boldsymbol{\Delta}$ Propranolol + saline, $\mathrm{n}=10 ; 0$ Untreated food 
+ DEX, $n=13 ; \square$ Propranolol + DEX, $n=10$. Between group analysis on absolute body weight using repeated-measure analysis of variance, with Greenhouse-Geisser adjustment for multisample asphericity: Untreated food + saline ( $(\boldsymbol{a})$ vs Untreated food $+\operatorname{DEX}(0) P^{\prime}<0.001$; Propranolol + saline $(\boldsymbol{\Delta})$ vs Propranolol + DEX (口) $P^{\prime}<0.001$; Untreated food + DEX (०) vs Propranolol + $\operatorname{DEX}(\square) P^{\prime}<0.005$

\subsubsection{Daily food and propranolol intake}

Food intake was not altered in the groups that received untreated food + saline (T0: $19.8 \pm 0.1 \mathrm{~g}$ and T10: $19.8 \pm 0.2 \mathrm{~g}, \mathrm{n}=14, n s$ ) and untreated food $+\mathrm{ACTH}$ (T0: $19.9 \pm 0.1 \mathrm{~g}$ and T10: $20 \pm 0 \mathrm{~g}, \mathrm{n}=13, n s$ ) from days P0 to T10 (Figure 8.11). Within the untreated food + DEX group, there was a small but significant drop in food intake from $19.7 \pm 0.1 \mathrm{~g}$ on day $\mathrm{T} 0$ to $19.1 \pm 0.3 \mathrm{~g}$ on day $\mathrm{T} 10$ $(\mathrm{n}=13, P<0.05)$ (Figure 8.12$)$. There was a progressive and significant decrease in food intake over the duration of this experiment in the propranolol + saline (from T0: $17.5 \pm 0.2 \mathrm{~g}$ to $\mathrm{T} 10: 13.1 \pm 0.3 \mathrm{~g}, \mathrm{n}=14, \quad P<0.0005$ ), propranolol $+\mathrm{ACTH} \quad$ (from $\mathrm{T} 0: \quad 17.4 \pm 0.4 \mathrm{~g}$ to $\mathrm{T} 10: 12.5 \pm 0.4 \mathrm{~g} \quad \mathrm{n}=13$, $P \cdot<0.0005$ ) and propranolol + DEX (from T0: $17.2 \pm 0.5 \mathrm{~g}$ to T10: $11.9 \pm 0.6 \mathrm{~g}$ $\mathrm{n}=13, P<0.0005)$ groups.

Overall, the mean food intake for rats on saline injection and untreated ground food was $19.8 \pm 0 \mathrm{~g} / \mathrm{rat} / 15$-hour night. This was not significantly altered by ACTH $19.9 \pm 0 \mathrm{~g} / \mathrm{rat} / 15$-hour night (Figure 8.9) or DEX $19.7 \pm 0 \mathrm{~g} / \mathrm{rat} / 15$-hour 
night (Figure 8.10). However, propranolol significantly decreased food intake in saline-, ACTH- (Figure 8.11) and DEX-treated rats (Figure 8.12) $\left(P^{\prime}<0.005\right.$, each). To ensure complete propranolol intake, the amount of food provided was adjusted based on intake of the previous few days. Accounting for the inevitable nightly wastage of ground food, the mean propranolol dose consumed was 95.9 , 93.5 and $95 \mathrm{mg} / \mathrm{kg} /$ day (full dose $100 \mathrm{mg} / \mathrm{kg} /$ day) (Figure 8.13).

Rats treated with both ACTH and propranolol had greater reduction in food intake compare to those on ACTH alone $\left(P^{\prime}<0.005\right.$, Figure 8.11). However, there was no significant change in food intake between the DEX only group and propranolol + DEX group (Figure 8.12). 


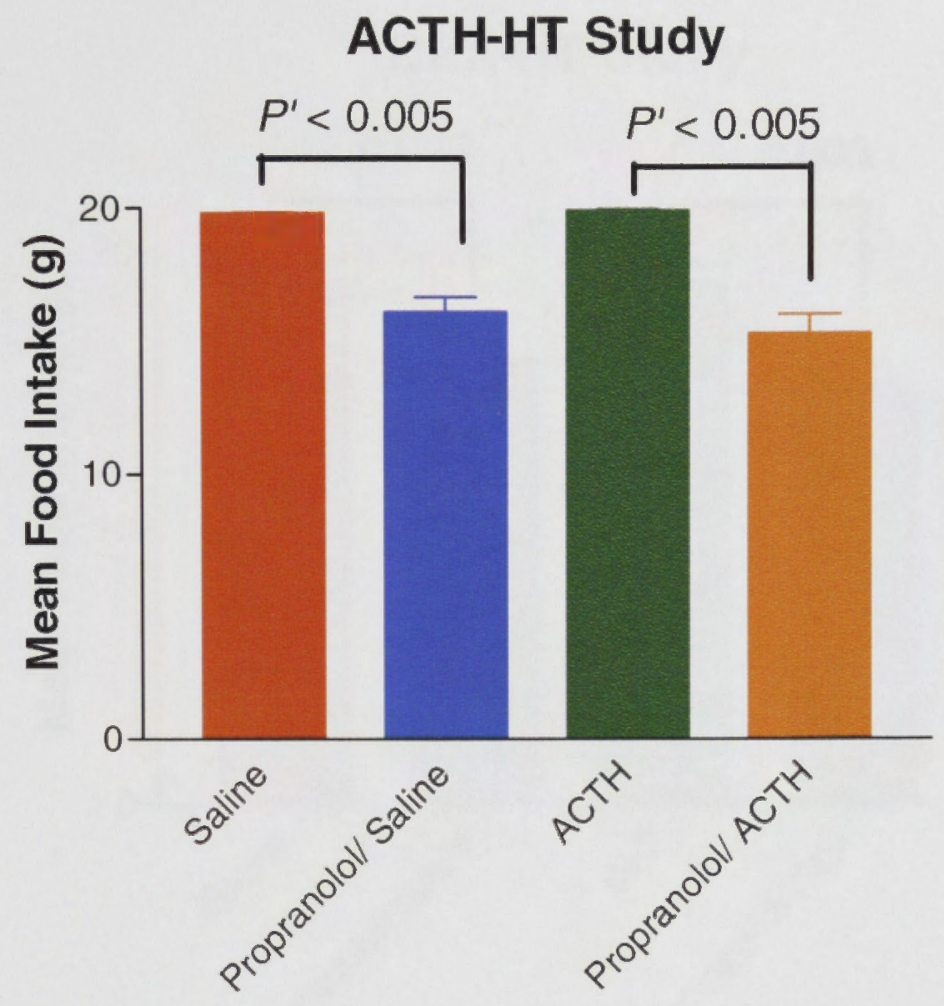

Figure 8.9: Overall mean food intake per rat per 15 hour night. Untreated food + saline, $\mathrm{n}=14 ;$ Propranolol + saline, $\mathrm{n}=10$; Untreated food + ACTH, $\mathrm{n}=13 ;$ Propranolol + ACTH, $\mathrm{n}=10$. 


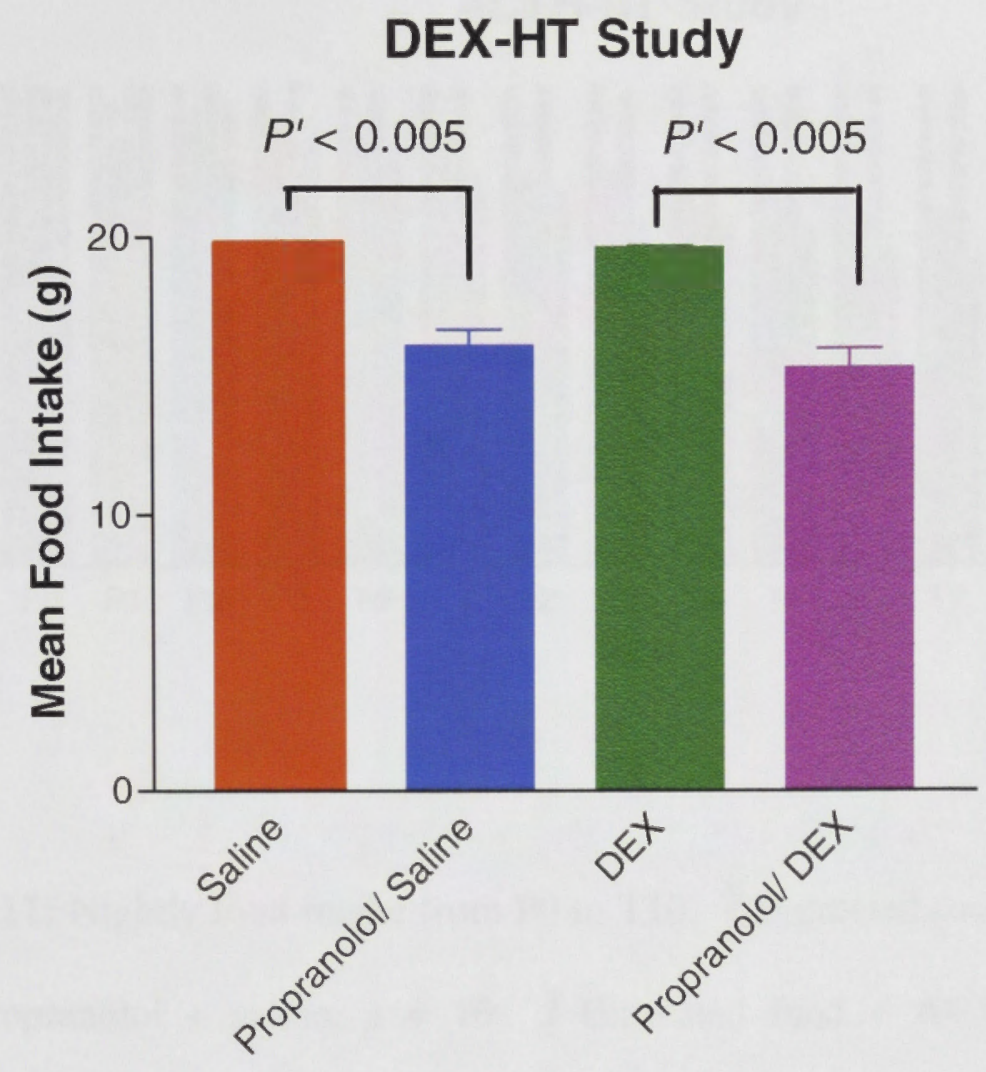

Figure 8.10: Overall mean food intake per rat per 15 hour night. Untreated food + saline, $n=14 ; \quad$ Propranolol + saline, $n=10$; Untreated food + DEX, $\mathrm{n}=13 ;$ Propranolol + DEX, $\mathrm{n}=10$. 
ACTH-HT Study

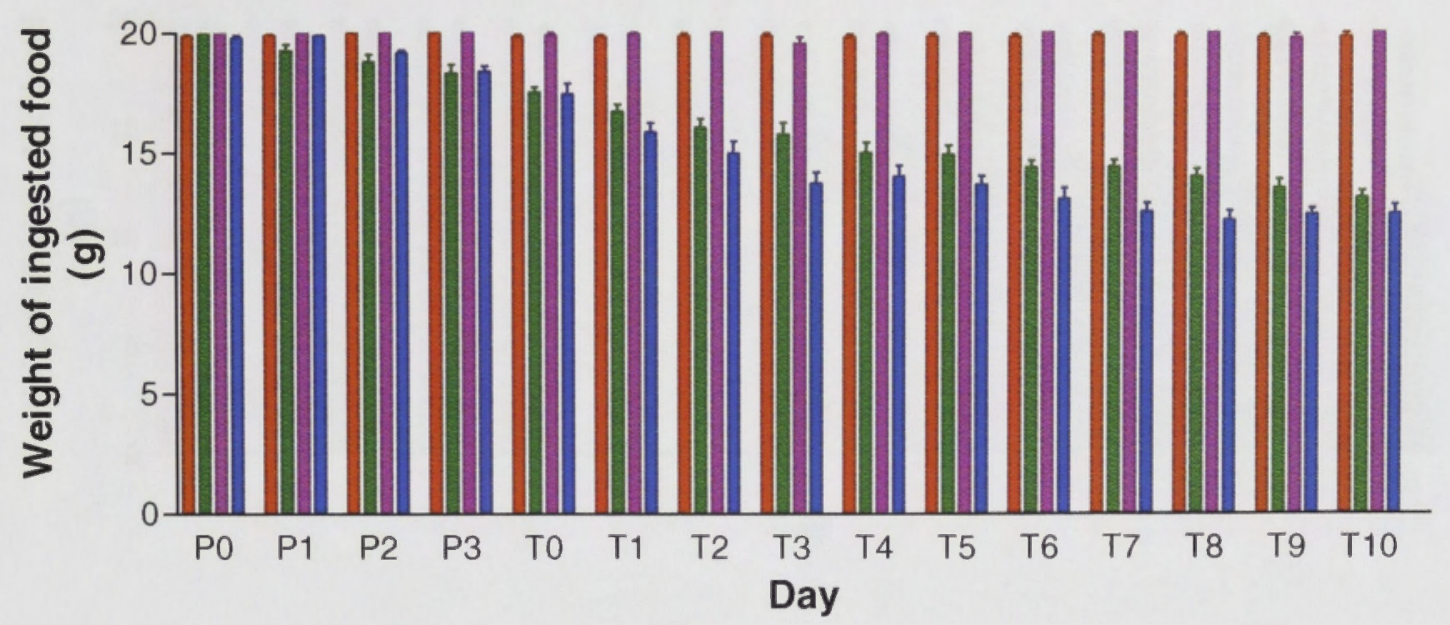

Figure 8.11: Nightly food intake from $\mathrm{P} 0$ to $\mathrm{T} 10$. Untreated food + saline, $\mathrm{n}=$ 14; Propranolol + saline, $\mathrm{n}=10 ;$ Untreated food $+\mathrm{ACTH}, \mathrm{n}=13 ;$ Propranolol + ACTH, $\mathrm{n}=10 . P^{\prime}<0.005$ for comparisons between the untreated food + saline group vs propranolol + saline group; untreated food + ACTH group vs propranolol + ACTH group; and propranolol + saline group vs propranolol + ACTH group using repeated measures analysis of variance. 


\section{DEX-HT Study}

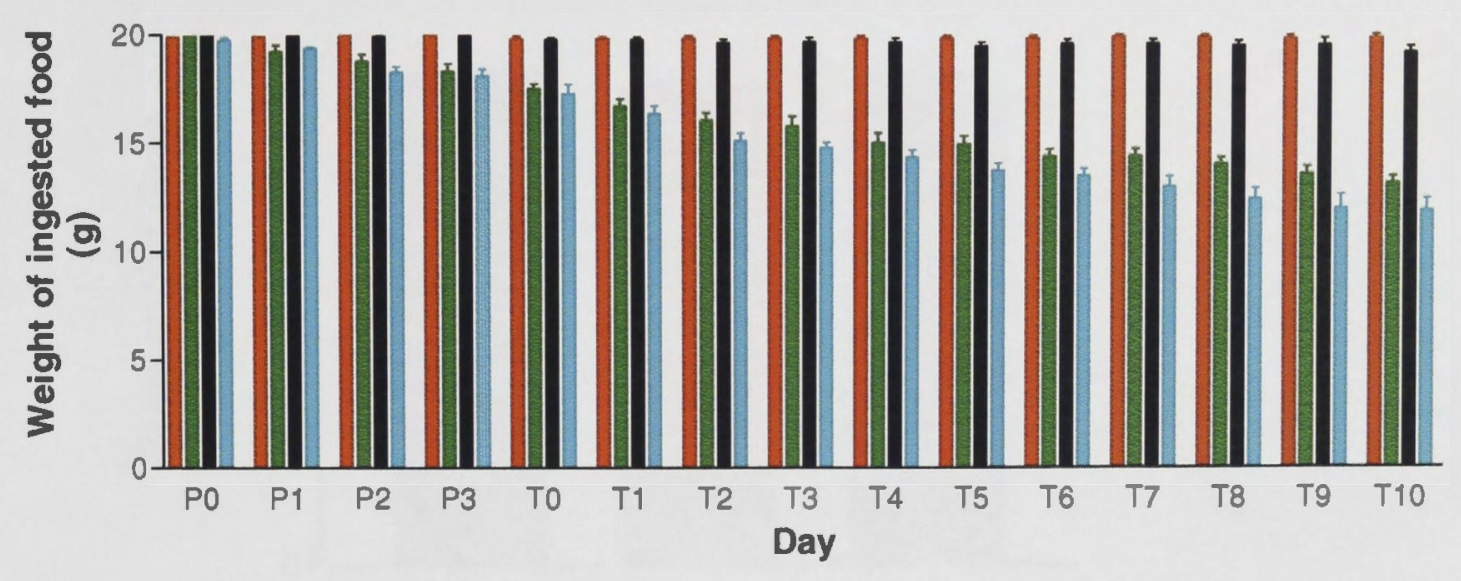

Figure 8.12: Nightly food intake from P0 to T10. Untreated food + saline, $n=$ 14; Propranolol + saline, $\mathrm{n}=10 ;$ Untreated food + DEX, $\mathrm{n}=13$;

Propranolol + DEX, $\mathrm{n}=10 . P^{\prime}<0.005$ for comparisons between the untreated food + saline group vs propranolol + saline group; and untreated food + DEX group vs propranolol + DEX group using repeated measures analysis of variance. 


\section{Propranolol Dose Consumed}

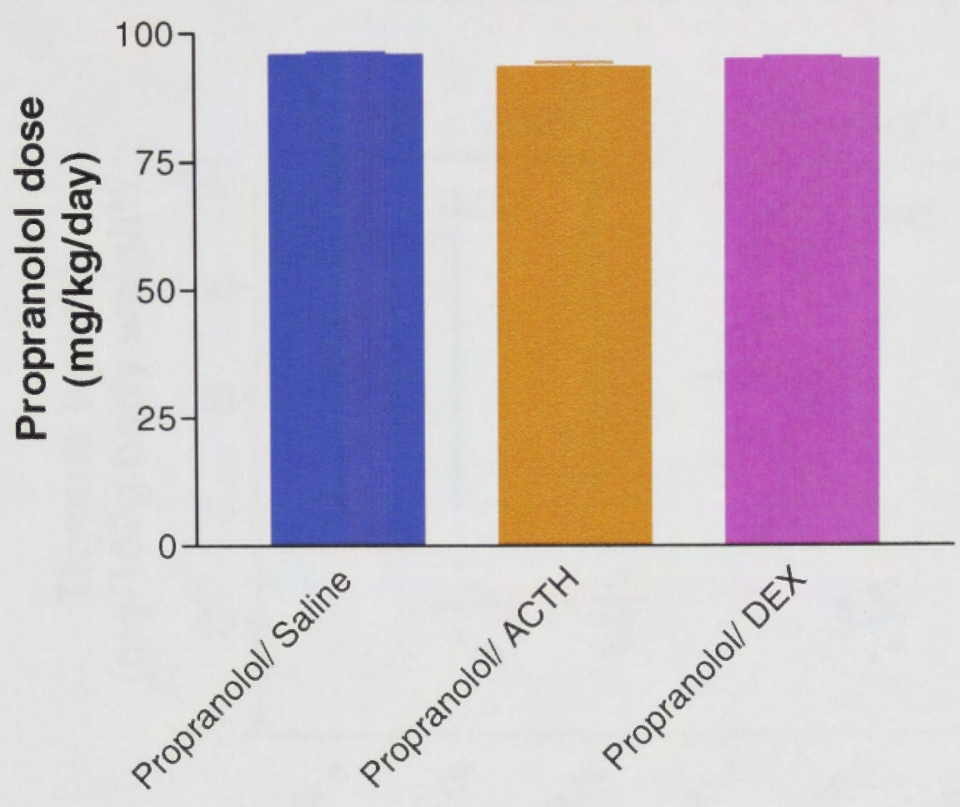

Figure 8.13: $\quad$ Propranolol + saline, $n=10 ; \quad$ Propranolol + ACTH, $n=10$ Propranolol + DEX, $\mathrm{n}=10$.

\subsubsection{Thymus weight}

Thymus weight of rats treated with ACTH and DEX was significantly lower than in those on saline injection $\left(P^{\prime}<0.001\right)$. These changes were not altered by propranolol treatment. Propranolol alone did not modify thymus weight (Figure 8.14 and Table 8.1). 


\section{Glucocorticoid Activity}

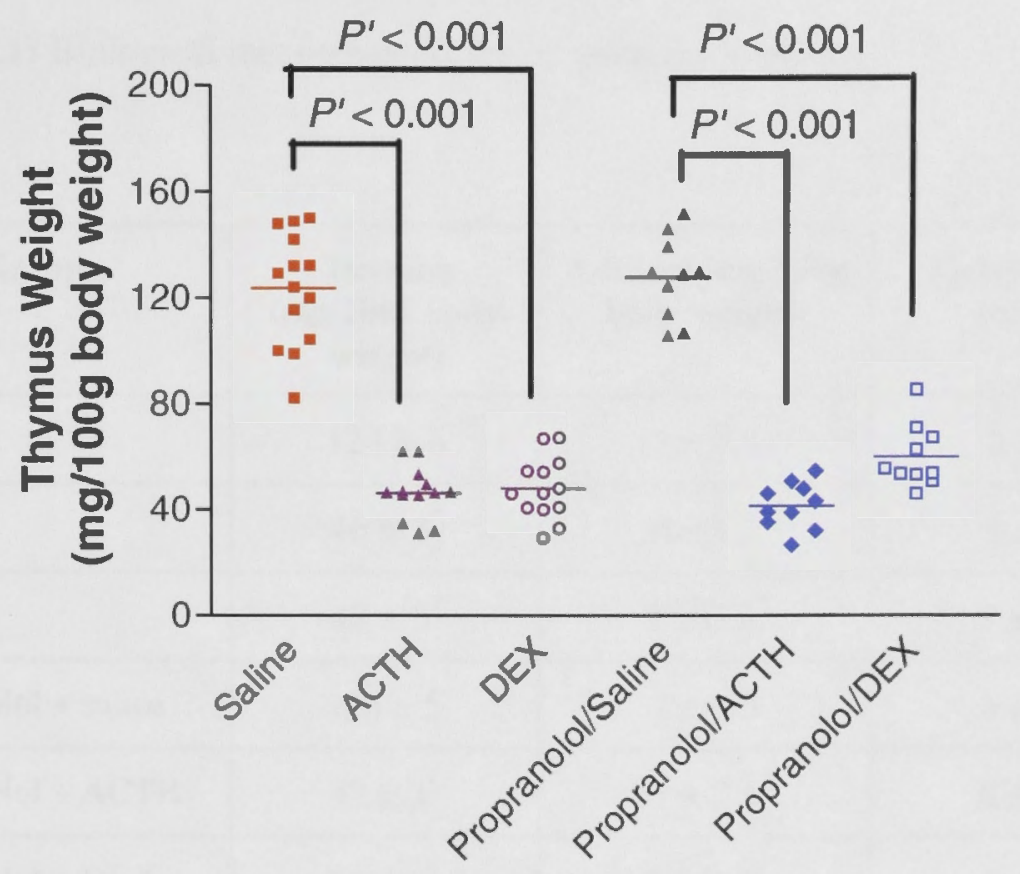

Figure 8.14: Thymus weight as an indicator of glucocorticoid activity.

Untreated food + saline, $\mathrm{n}=14 ; \boldsymbol{\Delta}$ Propranolol + saline, $\mathrm{n}=10 ; \boldsymbol{\nabla}$ Untreated food + ACTH, $n=13 ;$ Propranolol + ACTH, $n=10 ; \circ$ Untreated food + DEX, $\mathrm{n}=13 ; \square$ Propranolol + DEX, $\mathrm{n}=10$. 
Table 8.1: Biological measurements for propranolol studies

\begin{tabular}{|l|c|c|c|}
\hline \multicolumn{1}{|c|}{ Groups } & $\begin{array}{c}\text { Thymus } \\
\text { (mg/100g body } \\
\text { weight) }\end{array}$ & $\begin{array}{c}\text { Adrenal (mg/100g } \\
\text { body weight) }\end{array}$ & $\begin{array}{c}\text { F }_{2} \text {-Isoprostane } \\
\text { (nmol/L) }\end{array}$ \\
\hline Saline & $124 \pm 6$ & $7 \pm 0.4$ & $5 \pm 0.3$ \\
\hline ACTH & $46 \pm 3^{*}$ & $37 \pm 4.2^{*}$ & $5 \pm 0.4$ \\
\hline DEX & $48 \pm 3^{*}$ & $5 \pm 0.2^{*}$ & $7 \pm 0.6^{\dagger}$ \\
\hline Propranolol + saline & $128 \pm 5$ & $7 \pm 0.3$ & $6 \pm 0.2^{\dagger}$ \\
\hline Propranolol + ACTH & $42 \pm 3^{\S}$ & $31 \pm 2.6^{\S}$ & $8 \pm 0.7^{*}$ \\
\hline Propranolol + DEX & $60 \pm 4^{\S}$ & $5 \pm 0.2^{\S}$ & $7 \pm 0.4$ \\
\hline
\end{tabular}

${ }^{*} P^{\prime}<0.001$ vs saline, ${ }^{8} P^{\prime}<0.001$ vs propranolol \pm saline, $\dagger P^{\prime}<0.05$ vs saline, ${ }^{\#} P^{\prime}<0.05$ vs ACTH.

\subsubsection{Adrenal weight}

Adrenal weight was significantly increased by ACTH treatment $\left(P^{\prime}<0.001\right)$ but not by propranolol or saline. The increase in adrenal weight by ACTH was not modified by propranolol treatment (Figure $8.15 \mathrm{a}$ and Table 8.1 ).

DEX treatment significantly decreased adrenal weight $\left(P^{\prime}<0.001\right)$. This reduction was not affected by propranolol treatment (Figure $8.15 \mathrm{~b}$ and Table 8.1). 
a)

ACTH-HT Study

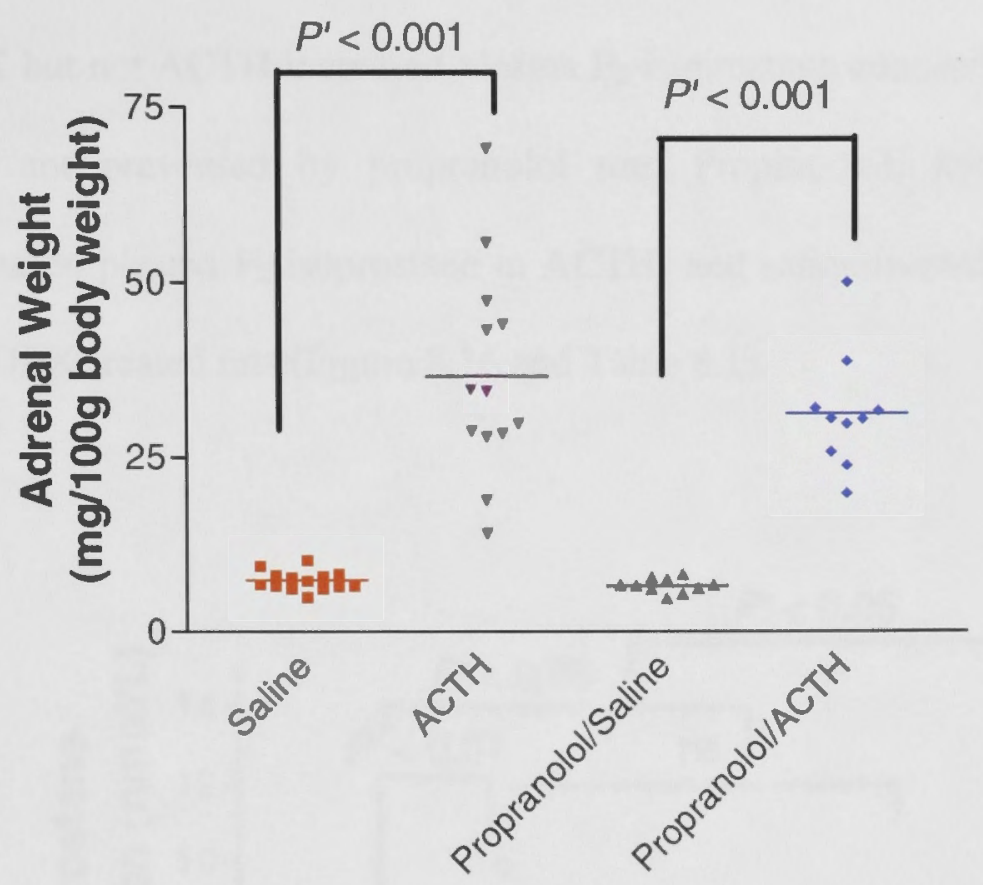

b) DEX-HT Study

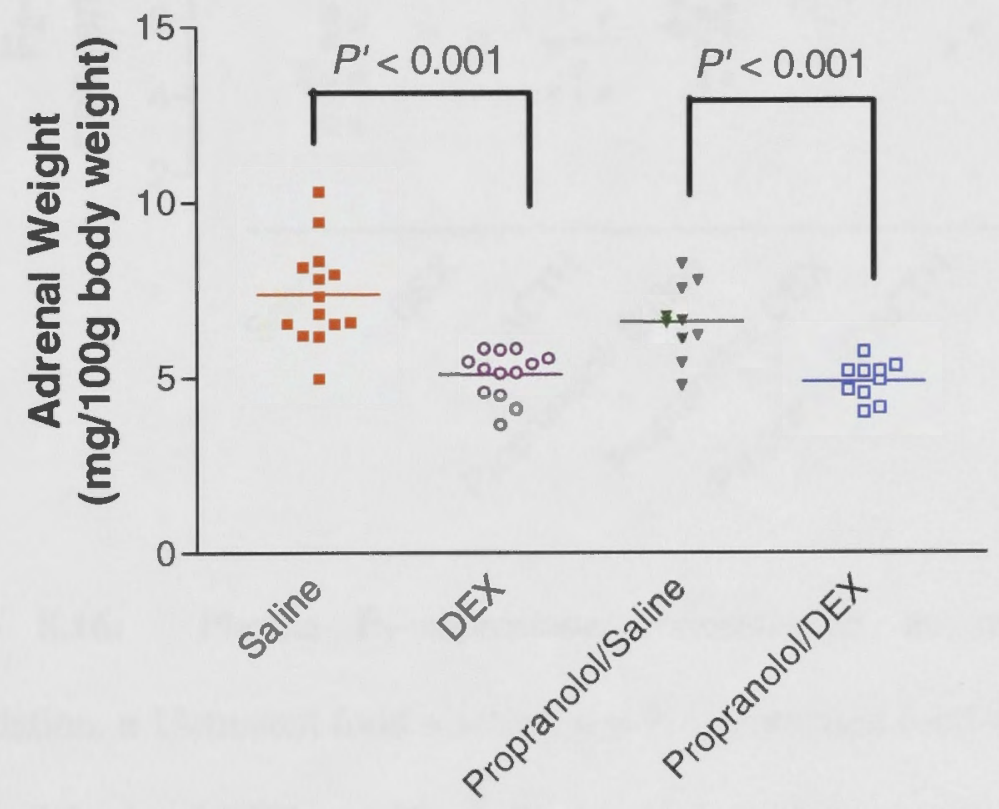

Figure 8.15: Adrenal weight as a marker of effective ACTH (a) and DEX (b) administration. $=$ Untreated food + saline, $\mathrm{n}=14 ; \boldsymbol{\Delta}$ Propranolol + saline, $\mathrm{n}=10$; $\nabla$ Untreated food $+\mathrm{ACTH}, \mathrm{n}=13 ; \bullet$ Propranolol $+\mathrm{ACTH}, \mathrm{n}=10 ; \circ$ Untreated food + DEX, $n=13 ; \square$ Propranolol + DEX, $n=10$. 


\subsubsection{Plasma $\mathbf{F}_{2}$-isoprostane concentration}

DEX but not $\mathrm{ACTH}$ increased plasma $\mathrm{F}_{2}$-isoprostane concentration. This increase was not prevented by propranolol use. Propranolol, however, significantly increased plasma $\mathrm{F}_{2}$-isoprostane in ACTH- and saline-treated rats $\left(P^{\prime}<0.05\right)$ but not DEX-treated rats (Figure 8.16 and Table 8.1).

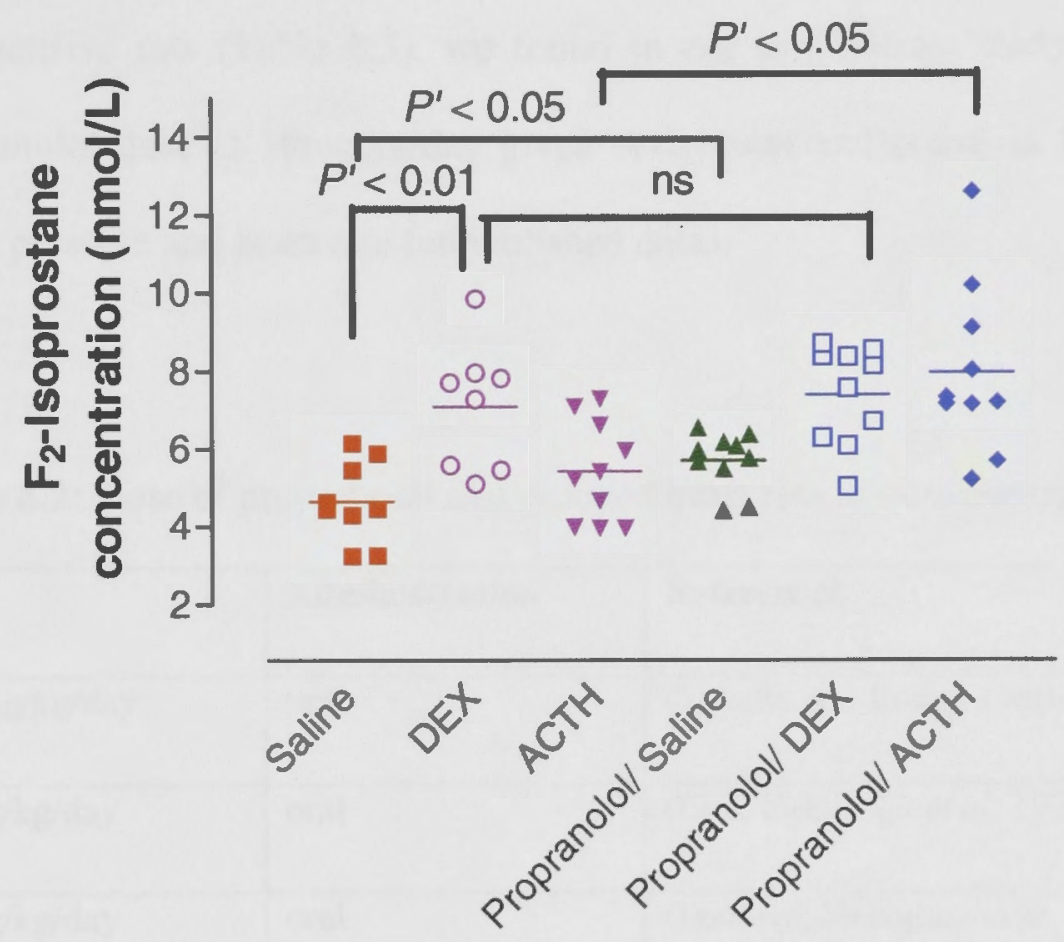

Figure 8.16: Plasma $F_{2}$-isoprostane concentration as marker of lipid peroxidation. = Untreated food + saline, $\mathrm{n}=9$; $\circ$ Untreated food $+\mathrm{DEX}, \mathrm{n}=8$;

Untreated food + ACTH, $n=10 ; \boldsymbol{\Delta}$ Propranolol + saline, $n=10 ; \square$ Propranolol + DEX, $\mathrm{n}=10 ;$ Propranolol + ACTH, $\mathrm{n}=10$. 


\subsection{DISCUSSION}

In this study, propranolol did not prevent GC-HT despite effectively inhibiting $\beta$ adrenergic receptors. The dose used $(100 \mathrm{mg} / \mathrm{kg} /$ day $)$ significantly reduced heart rate in all rats. Thus, the negative results seen in this study were not due to inadequate dosing. Although a range of propranolol dosages and regimens were effective in lowering heart rates in normal rats (Table 8.2) and blood pressure in hypertensive rats (Table 8.3), we found in our preliminary study that a lower propranolol dose at $10 \mathrm{mg} / \mathrm{kg} /$ day given orally was ineffective in lowering both blood pressure and heart rate (unpublished data).

Table 8.2: Dose of propranolol that reduced heart rate in normotensive rats

\begin{tabular}{|l|l|l|}
\hline Dose & Administration & References \\
\hline $100 \mathrm{mg} / \mathrm{kg} /$ day & oral & (Takeda and Bunag 1980) \\
\hline $30 \mathrm{mg} / \mathrm{kg} /$ day & oral & (Ebii, Fukunaga et al. 1991) \\
\hline $30 \mathrm{mg} / \mathrm{kg} /$ day & oral & (Igarashi, Nakajima et al. 1977) \\
\hline $30 \mathrm{mg} / \mathrm{kg} /$ day & oral & (Tsunoda, Takezawa et al. 2000) \\
\hline $5 \mathrm{mg} / \mathrm{kg}$ b.d. & intraperitoneal & (Chakrabarti and Sharma 1993) \\
\hline $2 \mathrm{mg} / \mathrm{kg} /$ day & subcutaneous & (Bunag 1977) \\
\hline
\end{tabular}


Table 8.3: Dose of propranolol that decreased heart rate in hypertensive rats

\begin{tabular}{|l|l|l|l|}
\hline Dose & Administration & Strain & References \\
\hline $100 \mathrm{mg} / \mathrm{kg}$ & oral & SHR & $\begin{array}{l}\text { (Kishi, Kawashima } \text { et al. } \\
1985)\end{array}$ \\
\hline $64 \mathrm{mg} / \mathrm{kg}$ & & SHR & $\begin{array}{l}\text { (Kubo, Esumi } \text { et al. } \\
1977)\end{array}$ \\
\hline $30 \mathrm{mg} / \mathrm{kg}$ & oral & SHR & $\begin{array}{l}\text { (Antonaccio, High } \text { et al. } \\
1986)\end{array}$ \\
\hline $15 \mathrm{mg} / \mathrm{kg}$ & oral & SHR & $\begin{array}{l}\text { (Ishihara, Chin } \text { et al. } \\
1989)\end{array}$ \\
\hline $5 \mathrm{mg} / \mathrm{kg}$ & intraperitoneal & $\begin{array}{l}\text { Stressed-induced } \\
\text { hypertensive rats }\end{array}$ & $\begin{array}{l}\text { (Bennett and Gardiner } \\
1979)\end{array}$ \\
\hline $5 \mathrm{mg} / \mathrm{kg}$ & subcutaneous & $\begin{array}{l}\text { Methylprednisolone and } \\
\text { DOCA-hypertensive }\end{array}$ & $\begin{array}{l}\text { (Burris, Waeber } \text { et al. } \\
1984)\end{array}$ \\
\hline $\begin{array}{l}1.5 \mathrm{mg} / \mathrm{L} \\
\text { water }\end{array}$ & oral & SHR & \begin{tabular}{l} 
(Owens 1987) \\
\hline
\end{tabular}
\end{tabular}

DOCA: deoxycorticosterone acetate, SHR: spontaneously hypertensive rat

Heart rate was used in this study as a surrogate marker of propranolol treatment efficacy as this is a parameter commonly used in the clinical setting to determine the beta blocking effect of this class of medication in humans. Propranolol level is not readily available. Furthermore, identification of a drug in the serum does not necessarily correlate with the desired clinical response. In this experiment, heart rate was assessed non-invasively using the tail cuff equipment at the time blood pressure was measured. This data was readily available from the tail cuff experiment performed to obtain the SBP. Although telemetry may be a better method to measure HR, it was not feasible in this experiment which involved only non-invasive $\mathrm{BP}$ monitoring. 
In this study, we observed a progressive decline in heart rate in both glucocorticoid-treated and saline control rats over time throughout the entire experiment. This was expected as the rats became accustomed to the experimental procedure. As glucocorticoid (DEX, $35 \mu \mathrm{g} / 100 \mathrm{~g}$ body weight, orally) has been shown to reduce heart rate in male Sprague Dawley rats (Roy, De et al. 2009), we assessed the blood pressure changes in these groups during the period after administration of propranolol but before glucocorticoid treatment.

In this study, propranolol was added to food and was given rats overnight, when the rats were most active and awake. The amount of food provided was adjusted daily based on the weight of the animals. To ensure complete propanolol intake, the designated rats were given drug-laced food, which accounted for $90 \%$ of their food intake, at night. Each morning, the remaining food was collected and weighed. Untreated pellet food was subsequently given during the day to make up for the daily deficit.

In this study rats treated with both propranolol and either ACTH or DEX had significant body weight loss compared to control rats. This effect was not seen in the propranolol + saline treated rats. It is unclear why combination treatment with propranolol and glucocorticoid resulted in this. There is no evidence in the literature to suggest any possible interactions between propranolol and ACTH or DEX to exacerbate the catabolic effects of glucocorticoids. The reduction in food intake observed in this study could contribute to the weight loss although 
reduction in food consumption was seen in all groups (ie saline, ACTH and DEXtreated groups).

The role of propranolol in reducing food intake in this study remains unclear. It is possible that propranolol affected the palatability of the ground rat food although this is unlikely to be the reason for the weight changes described above as unlimited normal pellet rat food was provided during the day to ensure that weight loss was not due to food restriction. Propranolol alone has been shown not to alter food intake in rats (Racotta and Soto-Mora 1993). However, it was shown to inhibit the hypophagic effects of the $\beta$ adrenoreceptor agonists salbutamol ( $\beta 1$ ) and isoproterenol ( $\beta 2$ ) (Racotta and Soto-Mora 1993). Whether it can exert an anorexigenic effect when used in conjunction with ACTH or glucocorticoids remain unclear.

The antihypertensive effect of propranolol is likely to be related to the blockade of vasoconstriction induced by sympathetic stimulation. It has been shown that the fall in blood pressure due to propranolol was also associated with reductions in plasma renin and aldosterone concentrations (van den Meiracker, Man in't Veld et al. 1989). Changes in cardiac output and circulating blood volume, however, were not shown to be critical factors (van den Meiracker, Man in't Veld et al. 1989). Propranolol has also been reported to potentiate the effect of atrial natriuretic peptide (Yoshimoto, Naruse et al. 1998). The renin-angiotensin-aldosterone and atrial natriuretic peptide pathways were not tested in this study. 
This study confirmed that activation of the $\beta$-adrenergic pathways is not a main mechanism of GC-HT. This is also true in other aspects of the sympathetic pathways. In one study which directly assessed the sympathetic vasomotor drive to skeletal muscle in human subjects, oral cortisol ( $200 \mathrm{mg} / \mathrm{day}$, for 5 days $)$ and DEX ( $3 \mathrm{mg} /$ day, for 5 days) but not placebo individually increased blood pressure, and suppressed resting and stimulated muscle sympathetic activity (Macefield, Williamson et al. 1998). Noradrenaline spillover rate and noradrenaline uptake studies did not show any increase in sympathetic nervous activity in cortisol-induced hypertension in humans (Sudhir, Jennings et al. 1989). Chemical sympathectomy using 6-hydroxy dopamine did not inhibit or delay the development of ACTH-HT in rats ( $\mathrm{Li}$ and Whitworth 1991) and sympathetic nervous activity was not increased in ACTH-HT in sheep (Spence, Mathai et al. 1989).

Selective inhibition of $\beta 1$-adrenergic receptor with atenolol ( $50 \mathrm{mg} / \mathrm{kg} / \mathrm{day}$, orally) failed to prevent ACTH-HT in rats despite a demonstrable decrease in cardiac output (Wen, Fraser et al. 1999). The outcome was similar with blockade of both the $\beta 1$ - and $\beta 2$-adrenergic receptors using propranolol, suggesting that nonselective activation of $\beta$-adrenergic receptors does not play a significant role in the development of GC-HT. Thus, the utility of $\beta$-adrenergic receptor blockers in GC-HT is limited in this form of hypertension.

As indicated earlier, propranolol is also an antioxidant via its anti-lipid peroxidation effect. Contrary to previous reports demonstrating its effectiveness 
in preventing lipid peroxidation in canine sarcolemnal membranes (Mak and Weglicki 1988) and rat liver microsomes (Aruoma, Smith et al. 1991), propranolol was found to promote an increase in plasma $F_{2}$-isoprostane concentration in saline and ACTH-treated rats in this study (Figure 8.16). The reason for the observed discrepancy is unclear but could be due to differences in the mode of propranolol treatment (in vitro vs in vivo), target tissue of examination (heart and liver vs plasma) and assay used to evaluate lipid peroxidation (malondialdehyde and thiobarbituric vs $\mathbf{F}_{2}$-isoprostane assays). Furthermore, there is no evidence suggesting that high dose propranolol is prooxidant. In the present study, there was no significant difference in the plasma $\mathrm{F}_{2^{-}}$ isoprostane concentration in the DEX + propranolol group compared with DEXonly group (Figure 8.16 ). This is most likely because DEX had already resulted in raised $F_{2}$-isoprostane concentration and further increase was not apparent. With these results, it is also likely that the blood pressure lowering effect of $\beta$ adrenergic blockade was counteracted by an increase in oxidative stress in this study. Therefore, despite being known as an antioxidant, propranolol failed to prevent GC-HT.

\subsection{CONCLUSION}

Propranolol at $100 \mathrm{mg} / \mathrm{kg} /$ day was effective in lowering heart rate in rats. However, despite adequate treatment, propranolol was unsuccessful in preventing ACTH and DEX-HT in rats. Despite previous reports that propranolol is an antioxidant, it served as a pro-oxidant in this study. 
This study, together with previous evidence that atenolol is ineffective in ACTHHT, suggests that $\beta$-adrenoreceptor activation is not a major contributor to GC-HT in the rat and that $\beta$-adrenoreceptor blocker has a limited therapeutic role in GCHT,

This study also showed that lipid peroxidation does not play a major role in the pathogenesis of GC-HT in rats. 


\section{CHAPTER 9}

Role of 20-Hydroxyeicosatetraenoic Acid in

Dexamethasone-Induced Hypertension in the Rat 


\subsection{INTRODUCTION}

Arachidonic acid has long been known to be metabolised by cyclooxygenase and lipoxygenase to prostaglandins, prostacyclines, thromboxanes and leucotrienes. Arachidonic acid was subsequently found to be also metabolised, by cytochrome P450 enzymes to form 19- and 20-hydroxyeicosatetraenoic acids, epoxyeicosatrienoic acids and dihydroeicosatrienoic acids.

20-Hydroxyeicosatetraenoic acid (20-HETE) participates in the regulation of vascular tone in the renal, cerebral, coronary and skeletal muscle arterioles (Miyata and Roman 2005). It is a potent vasoconstrictor which can also potentiate vascular smooth muscle response to vasoconstrictor and myogenic stimuli (Miyata and Roman 2005). Furthermore, 20-HETE plays an important role in regulating renal tubular sodium reabsorption resulting in natriuretic and diurectic properties (Miyata and Roman 2005).

The interactions of NO and 20-HETE have been recognised. The catalytic activity of cytochrome P450 enzymes can be inhibited by NO or NO donors (Morgan, Ullrich et al. 2001). 20-HETE can be inhibited directly and functionally by NO (Alonso-Galicia, Drummond et al. 1997; Sun, Alonso-Galicia et al. 1998; Oyekan, Youseff et al. 1999). In addition, 20-HETE has the ability to reduce eNOS activation (Cheng, Ou et al. 2008) and accelerate degradation of NO by increasing NAD(P)H oxidase-related superoxide overproduction (Wang, Singh et al. 2006), which may further decrease the availability of NO and exacerbate 
hypertension. Therefore, based on these data, 20-HETE production is likely to be increased in the presence of NO deficiency (Figure 9.1).

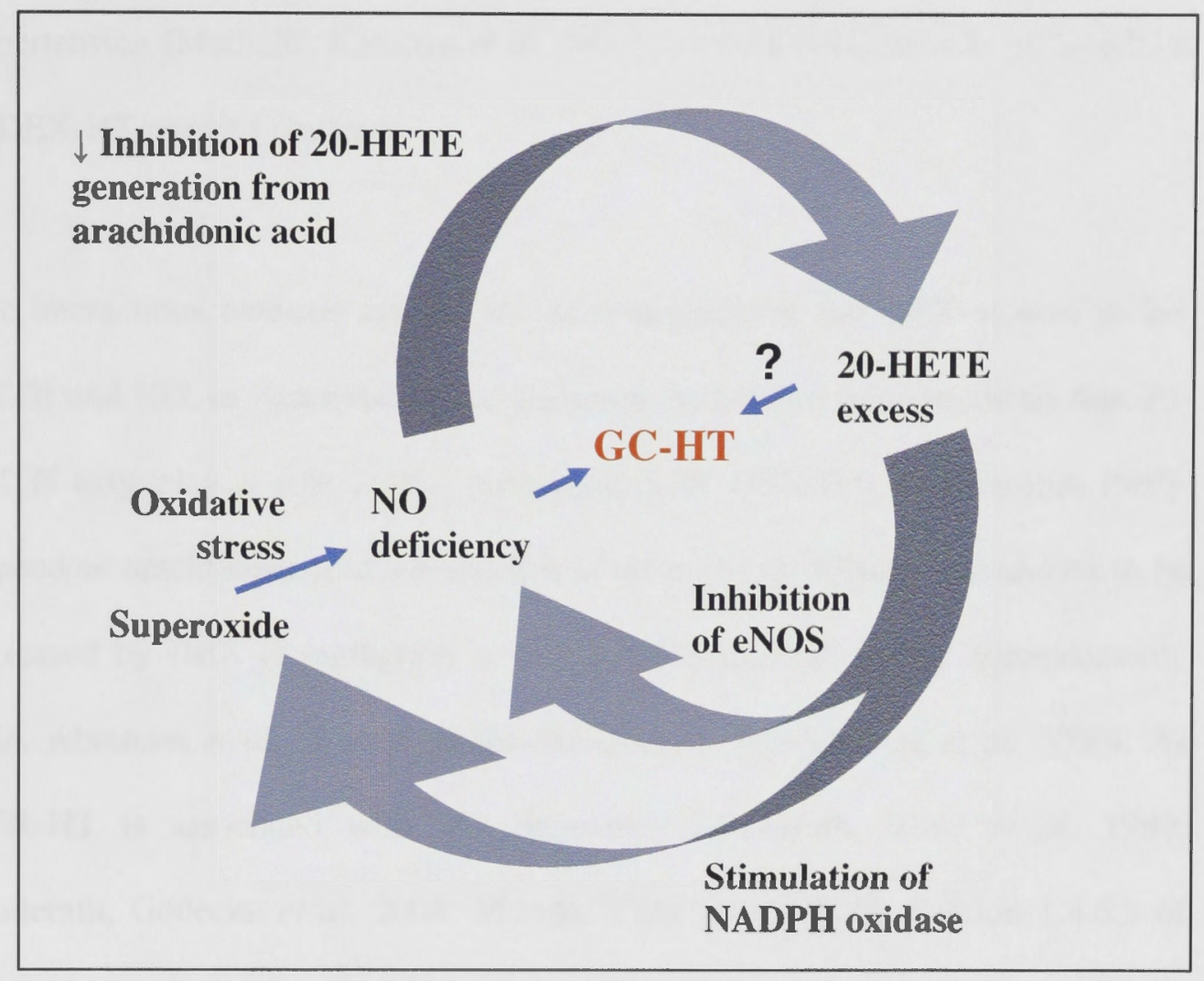

Figure 9.1: Proposed mechanism of GC-HT involving 20-HETE.

There is considerable evidence suggesting that 20-HETE plays a role in the pathogenesis of a number of experimental hypertensive models. Liu et. al. demonstrated that CYP4F2 transgenic mice with increased 20-HETE production have significantly higher blood pressure readings compared with wild-type controls (Liu, Zhao et al. 2009). They have also shown a positive correlation between blood pressure and urinary 20-HETE excretion (Liu, Zhao et al. 2009). Production of 20-HETE is increased in kidneys of androgen-induced hypertensive 
rats (Singh, Cheng et al. 2007). Furthermore, chronic inhibition of 20-HETE production decreased blood pressure in rats treated with angiotensin II suggesting that 20-HETE contributes to the development of angiotensin II-induced hypertension (Muthalif, Karzoun et al. 2000). Nevertheless, the role of 20-HETE in DEX-HT remains unclear.

The interactions between arachidonic acid metabolism and DEX as well as 20HETE and NO, as described in the literature, had led to our hypothesis that 20 HETE may play a role in the pathogenesis of DEX-HT. Cytochrome P450dependent arachidonic acid metabolism in rat proximal tubules was shown to be increased by $\operatorname{DEX}(1 \mathrm{mg} / \mathrm{kg} /$ day sc for 7 days) and reduced by adrenalectomy (Lin, Abraham et al. 1994; Sanchez-Mendoza, Lopez-Sanchez et al. 2000). As DEX-HT is associated with NO deficiency (Wallerath, Witte et al. 1999; Wallerath, Godecke et al. 2004; Mondo, Yang et al. 2006) (Section 1.4.6.3 of Chapter 1 and Section 7.3.6 of Chapter 7), it was also important to evaluate whether excess 20-HETE availability, which is linked with NO deficiency, is a cause of DEX-HT. Despite these results, a recent study from our group showed that DEX-hypertensive rats (DEX $20 \mu \mathrm{g} / \mathrm{kg} /$ day, subcutaneously, for 12 days) did not have increased 20-HETE excretion (Zhang, Hu et al. 2008). Whilst this finding suggests the absence of 20-HETE excess in DEX-hypertensive rats, an increase in plasma and tissue 20-HETE concentrations cannot be assumed. Furthermore, the absence of an increased 20-HETE does not exclude an increase in sensitivity of the vasculature to 20-HETE. The role of 20-HETE in the 
development of DEX-HT can only be assessed by evaluating the blood pressure response of DEX-hypertensive rats using a 20-HETE inhibitor.

Therefore, the aim of this study was to investigate whether renal parenchymal 20HETE is increased by low dose DEX (10 $\mu \mathrm{g} / \mathrm{rat} /$ day); and to investigate if the specific 20-HETE inhibitor $N$-hydroxy- $N^{\prime}$-(4-butyl-2-methylphenyl) formamidine (HET0016) can reverse DEX-HT. The kidney tissue was used because of its relevance in ACTH-HT. We have previously demonstrated that urinary excretion of 20-HETE in ACTH-hypertensive rats was elevated (Zhang, Hu et al. 2008). We have also shown that ACTH increased renal vascular resistance (Wen, Fraser et al. 1998; Wen, Fraser et al. 1999). Furthermore, the rise in BP due to ACTH in rats was accompanied by reductions in iNOS and eNOS gene expression in the kidney (Lou, Wen et al. 2001).

\subsection{METHODS}

\subsubsection{Experimental protocol}

This study was approved by the Animal Experimentation Ethics Committee (J.HB. 23.06). General methods used in this study have been described in Chapter

2. After 8 days treatment with DEX, the rats were injected with HET0016 $(10 \mathrm{mg} / \mathrm{kg} / \mathrm{day}$, in a volume of $2 \mathrm{~mL} / \mathrm{kg})$ or vehicle $(2 \mathrm{~mL} / \mathrm{kg}, 10 \%$ lecithin in saline) intraperitoneally. 
HET0016 was generously donated by Taisho Pharmaceutical Co. Ltd, Saitama, Japan. HET0016, a lipid soluble powder, was prepared in a lecithin-based vehicle (10\% lecithin in normal saline).

Thirty-two male Sprague Dawley rats weighing 250-300 g were divided into 4 treatment groups.

Group 1: Saline $(0.1 \mathrm{~mL} / \mathrm{rat} /$ day subcutaneous injection $)+$ Vehicle $(2 \mathrm{~mL} / \mathrm{kg}$, $10 \%$ lecithin in saline, intraperitoneal injection), $\mathrm{n}=8$

Group 2: Saline + HET 0016 (10 mg/kg/day, intraperitoneal injection), $\mathrm{n}=8$

Group 3: DEX (10 $\mu \mathrm{g} / \mathrm{rat} /$ day subcutaneous injection $)+$ Vehicle, $\mathrm{n}=8$

Group 4: DEX + HET0016, $n=8$

\subsubsection{Tail-cuff systolic blood pressure and body weight measurements}

The animals underwent second-daily tail-cuff experiments until day T8 when SBP readings will be obtained daily. The tail-cuff experimental protocol was unlike the one described in Chapter 2. Because of the limited amount of HET0016 available to our laboratory, the duration of treatment had to be shortened. Therefore, the tail-cuff experimental protocol had to be modified to maximise the number of tailcuff experimental days over the shortened experimental duration. This 
modification to the original tail-cuff experiment highlighted in Chapter 2 is described in Figure 9.2.

Body weight was measured immediately after the tail-cuff experiments.

\subsubsection{Metabolic measurements}

Twenty-four hour food and water consumption and urine volume were measured on the day before the last tail-cuff blood pressure measurement (day T11) using individual metabolic cages as described in Section 2.7 of Chapter 2.

\subsubsection{Thymus weight measurement}

At the end of the experiment, rats were sacrificed under anaesthesia. Thymus resection and blood sampling procedures were as described in Section 2.8 of Chapter 2.

Thymus wet weight, expressed relative to body weight (grams thymus weight per $100 \mathrm{~g}$ body weight), was used as a marker of glucocorticoid activity.

\subsubsection{Plasma nitrate and nitrite assay}

Plasma NOx concentrations, measured using the Griess colorimetric reaction described in Section 2.10.1 of Chapter 2, were used as a marker of endogenous NO availability. 


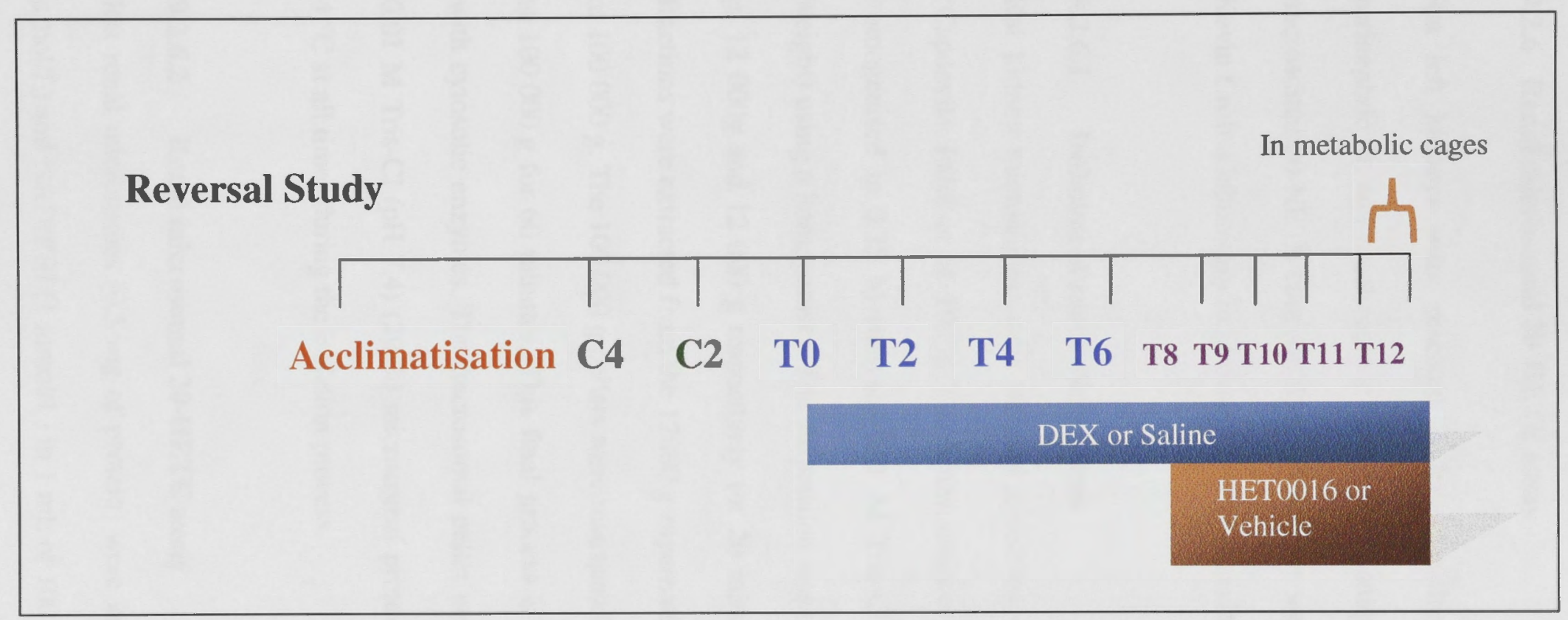

Figure 9.2: Modified tail-cuff experimental protocol. The prefix " $\mathrm{C}$ " denotes control days and " $\mathrm{T}$ " denotes treatment days. 


\subsubsection{Renal microsomal 20-HETE assay}

Rat left kidneys were resected under anaesthesia. They were dissected free of perinephric fat and renal capsule before being snap frozen at $-70{ }^{\circ} \mathrm{C}$ for assay of renal microsomal 20-HETE concentration. This assay was performed by Associate Professor Kevin Croft's laboratory in University of Western Australia.

\subsubsection{Isolation of renal microsomes}

Rat kidney microsomes were isolated according to previously published techniques (Capdevila, Falck et al. 1990). The frozen kidneys were thawed, weighed, minced and homogenised in $0.25 \mathrm{M}$ sucrose, $0.01 \mathrm{M}$ Tris- $\mathrm{Cl}(\mathrm{pH} 7.4)(15 \mathrm{~mL} / \mathrm{g}$ of wet kidney weight) using a homogeniser. The suspension was centrifuged thrice successive at 3000 $\mathrm{g}, 12000 \mathrm{~g}$ and $12000 \mathrm{~g}$ respectively for 20 minutes each time. Finally, microsomal fractions were extracted from the $12000 \mathrm{~g}$ supernatants by centrifugation for 60 minutes at $100000 \mathrm{~g}$. The $100000 \mathrm{~g}$ pellets were resuspended in $0.15 \mathrm{M} \mathrm{KCl}$ and re-centrifuged at $100000 \mathrm{~g}$ for 60 minutes. This final process is necessary to reduce contaminations with cytosolic enzymes. The microsomal pellet was suspended in $0.25 \mathrm{M}$ sucrose and $0.01 \mathrm{M}$ Tris- $\mathrm{Cl}(\mathrm{pH}$ 7.4) (20-30 microsomal protein $/ \mathrm{mL})$. The specimens were kept at $4{ }^{\circ} \mathrm{C}$ at all times during the isolation process.

\subsubsection{Renal microsomal 20-HETE assay}

Rat renal microsomes $(0.5 \mathrm{mg}$ of protein) were incubated with arachidonic acid (100 $\mu \mathrm{mol} / \mathrm{L})$ and $\mathrm{NAD}(\mathrm{P}) \mathrm{H}(1 \mathrm{mmol} / \mathrm{L})$ in $1 \mathrm{~mL}$ of $100 \mathrm{mmol} / \mathrm{L}$ potassium phosphate buffer (pH 7.4). Reactions were started with the addition of $\mathrm{NAD}(\mathrm{P}) \mathrm{H}$, incubated for 15 
minutes at $37^{\circ} \mathrm{C}$, and then stopped with the addition of $20 \mu \mathrm{L} 2 \mathrm{~N} \mathrm{HCl}$. Extraction of the mixture was performed using $2 \mathrm{~mL}$ ethyl acetate. The extract was then evaporated under a nitrogen stream and resuspended in $\mathrm{H}_{2} \mathrm{O}$ :acetonitrile (6:4). The reaction products were separated on an Agilent LiChrosphere C18 reversed phase column using a HewlettPackard series 1100 instrument (Hewlett-Packard, Waldbronn, Germany). The rate of product formation was analysed with ultraviolet detection at $200 \mathrm{~nm}$. The initial mobile phase consisted of acetonitrile: water: acetic acid (40:60:0.1\%) and a flow rate of 1 $\mathrm{mL} / \mathrm{min}$. Compounds were eluted with a linear gradient starting at $40 \%$ acetonitrile and eventually increased to $100 \%$ acetonitrile over 20 minutes. The formation of 20 -HETE, expressed as $\mathrm{nmol} / \mathrm{min} / \mathrm{mg}$ protein, was determined by comparing the peak areas of individual samples with a reference 20-HETE standard curve that was run on the same day as the sample analysis (Cayman Chemicals, Ann Arbor. Michigan. USA).

\subsubsection{Plasma $\mathrm{F}_{2}$-isoprostane assay}

Plasma $F_{2}$-isoprostane concentration was used as a marker of systemic lipid peroxidation. The assay technique was described in Section 2.11.3 of Chapter 2 .

\subsubsection{Statistical analysis}

Results were expressed as mean \pm SEM. Statistical analysis were as described in Section 2.12 of Chapter 2 .

The plasma NOx, renal microsomal 20-HETE, plasma $F_{2}$-isoprostane results for the saline + vehicle and saline + HET0016 groups consist of pooled data from the 20- 
HETE/ACTH-HT reversal and prevention studies performed by other investigators in our laboratory to increase the sample size and hence, statistical power.

\subsection{RESULTS}

\subsubsection{Tail-cuff systolic blood pressure}

SBP in the DEX + vehicle group increased significantly from $121 \pm 3$ on day T0 to $138 \pm 2 \mathrm{mmHg}$ on day $\mathrm{T} 6(P<0.01)$. There was no significant change in SBP following treatment with the vehicle lecithin from day $\mathrm{T} 8(140 \pm 3 \mathrm{mmHg})$ to $\mathrm{T} 12$ $(140 \pm 5 \mathrm{mmHg})$ in this group. Similarly, SBP in the DEX + HET0016 group increased significantly from $120 \pm 4 \mathrm{mmHg}$ on day $\mathrm{T} 0$ to $133 \pm 3 \mathrm{mmHg}$ on day $\mathrm{T} 6(P<0.0005)$ but did not change significantly with the commencement of HET0016 from T8 $(140 \pm 3 \mathrm{mmHg})$ to $\mathrm{T} 12(138 \pm 6 \mathrm{mmHg})$. There was no significant change in SBP in the saline + vehicle group from day $\mathrm{T} 0(118 \pm 2 \mathrm{mmHg})$ to day $\mathrm{T} 6(120 \pm 2 \mathrm{mmHg})$ and from day $\mathrm{T} 8(119 \pm 1 \mathrm{mmHg})$ to day $\mathrm{T} 12(112 \pm 2 \mathrm{mmHg})$. Rat receiving saline and HET0016 treatments also did not show any significant blood pressure change from day T0 $(119 \pm 3 \mathrm{mmHg})$ to $\mathrm{T} 6(118 \pm 2 \mathrm{mmHg})$ and from day $\mathrm{T} 8(121 \pm 2 \mathrm{mmHg})$ to $\mathrm{T} 12$ (119 $\pm 3 \mathrm{mmHg}$ ) (Figure 9.3).

Between-group comparisons showed that SBP was significantly higher in the DEXtreated groups (DEX + vehicle and DEX + HET0016) compared to the saline-treated group from day T0 to day T6 $\left(\mathrm{n}=8, P^{\prime}<0.05\right.$ and $P^{\prime}<0.005$ respectively). Neither 
HET0016 nor vehicle altered the blood pressure profiles from day T8 to T12 in all groups (Figure 9.3).

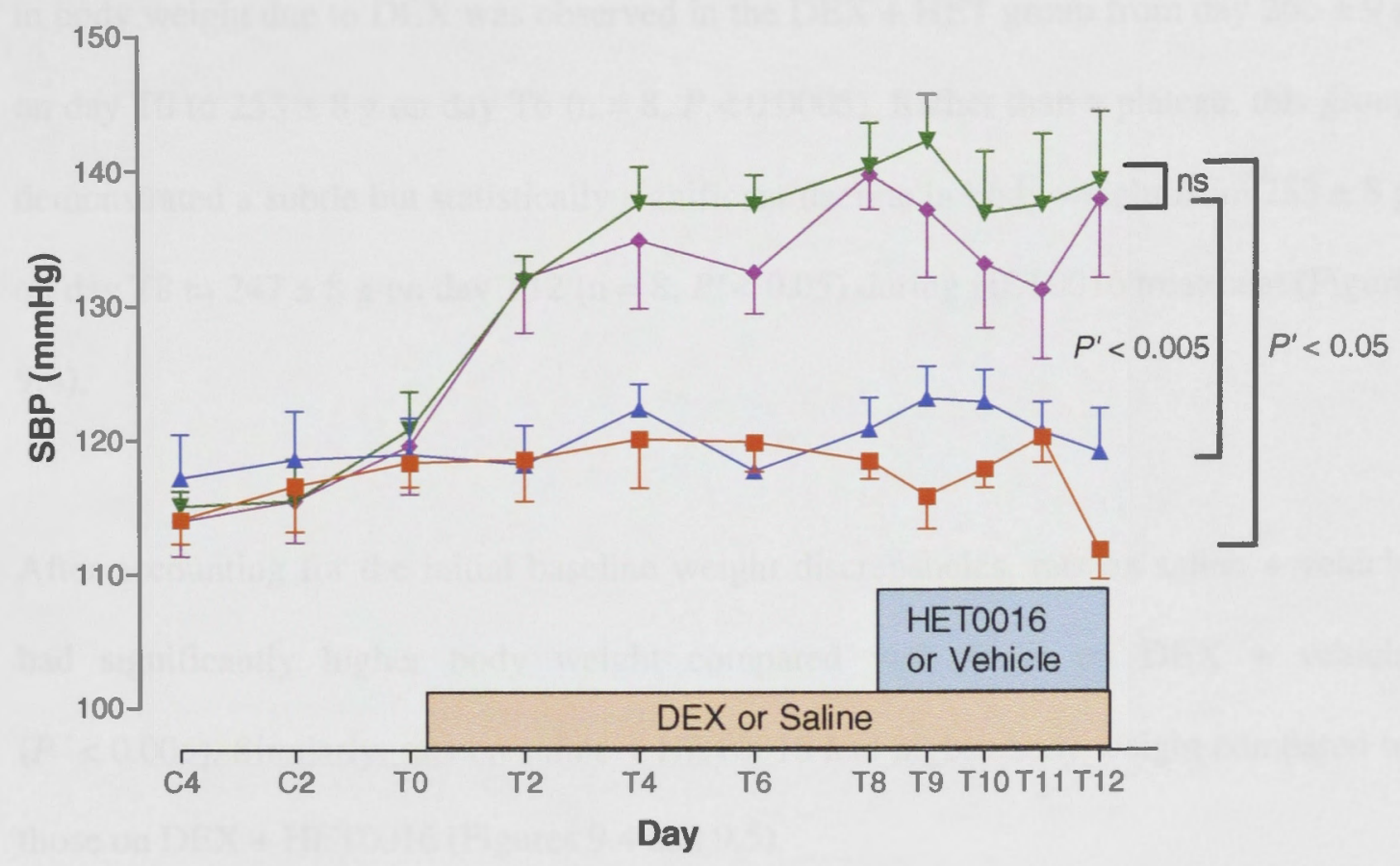

Figure 9.3: Tail-cuff systolic blood pressure. = Saline + Vehicle, $n=8 ; \Delta$ Saline + HET0016, $\mathrm{n}=8 ; \boldsymbol{\nabla}$ DEX + Vehicle, $\mathrm{n}=8 ;-\mathrm{DEX}+$ HET0016, $\mathrm{n}=8$.

\subsubsection{Body weight}

There was an increase in body weight in the saline + vehicle-treated group from 299 $\pm 15 \mathrm{~g}$ on day T0 to $321 \pm 17 \mathrm{~g}$ on day $\mathrm{T} 6(P<0.0005)$. This statistically significant increase in body weight was not observed from day $\mathrm{T} 8$ to $\mathrm{T} 12$ following the commencement of lecithin (T8: $328 \pm 17, \mathrm{~T} 12: 335 \pm 17 \mathrm{~g}, \mathrm{n}=6, n s$ ). However, in the 
DEX + vehicle group, DEX treatment resulted in a slight but significant weight loss from $278 \pm 10 \mathrm{~g}$ on day T0 to $266 \pm 9 \mathrm{~g}$ on day T6, $\mathrm{n}=8, P<0.0005$ ) which plateaued from day T8 $(267 \pm 10 \mathrm{~g})$ to $\mathrm{T} 12(264 \pm 9 \mathrm{~g}, n s)$ whilst on vehicle treatment. Similar fall in body weight due to DEX was observed in the DEX + HET group from day $266 \pm 9 \mathrm{~g}$ on day T0 to $253 \pm 8 \mathrm{~g}$ on day T6 ( $\mathrm{n}=8, P<0.0005)$. Rather than a plateau, this group demonstrated a subtle but statistically significant decline in body weight from $253 \pm 8 \mathrm{~g}$ on day T8 to $247 \pm 8 \mathrm{~g}$ on day T12 $\left(\mathrm{n}=8, P^{\prime}<0.05\right)$ during HET0016 treatment (Figure 9.4).

After accounting for the initial baseline weight discrepancies, rats on saline + vehicle had significantly higher body weight compared with those on DEX + vehicle $\left(P^{\prime}<0.005\right)$. Similarly, rats on saline + HET0016 had higher body weight compared to those on DEX + HET0016 (Figures 9.4 and 9.5). 


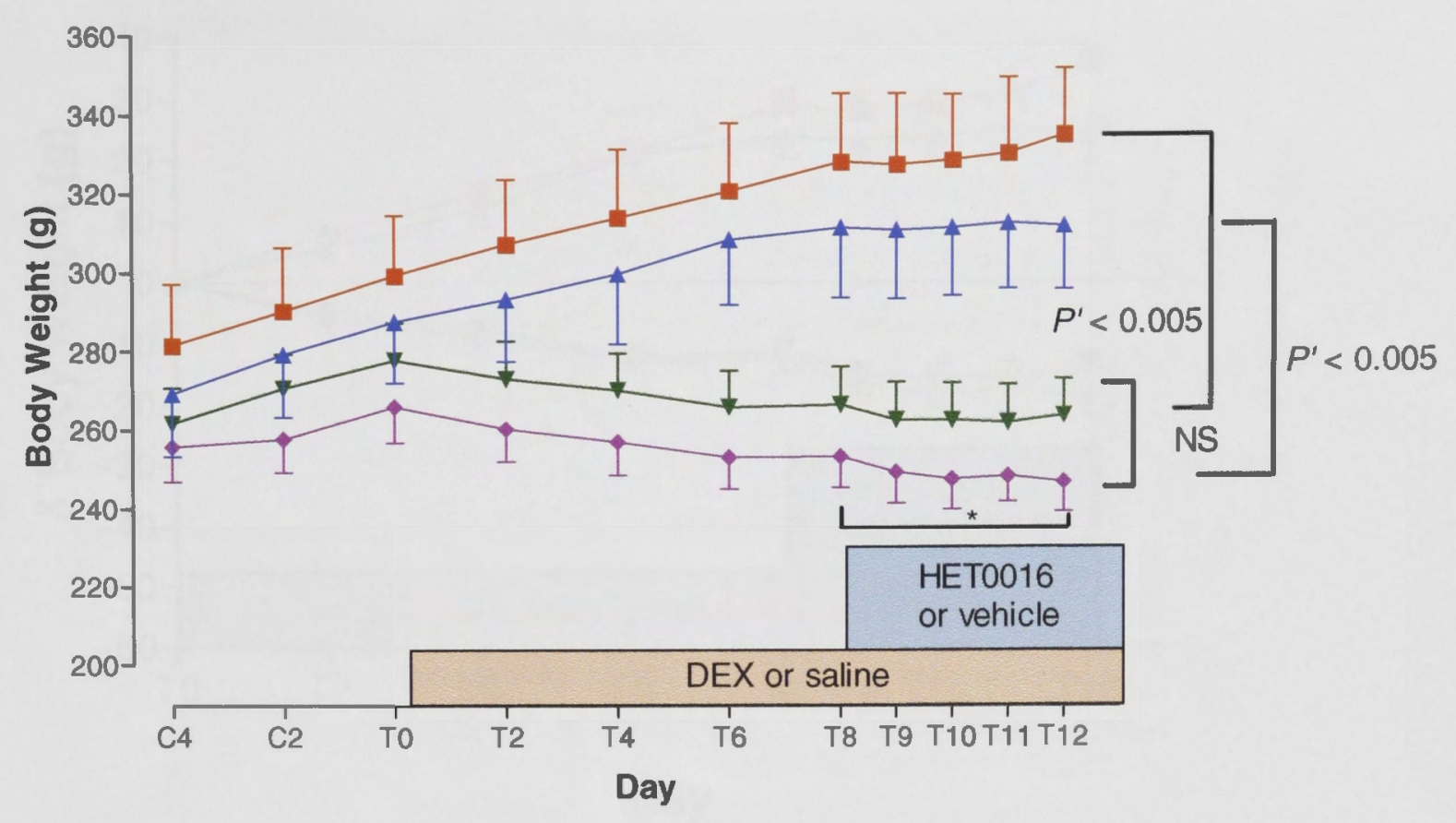

Figure 9.4: Body weight measurements. - Saline + Vehicle, $n=8 ; \Delta$ Saline + HET0016, $\mathrm{n}=8 ; \boldsymbol{\nabla}$ DEX + Vehicle, $\mathrm{n}=8 ;-\mathrm{DEX}+$ HET0016, $\mathrm{n}=8$. * There was a statistically significant decline in body weight from T8 to T12 in the DEX + HET0016 group $\left(P^{\prime}<0.005\right.$, one way ANOVA). 


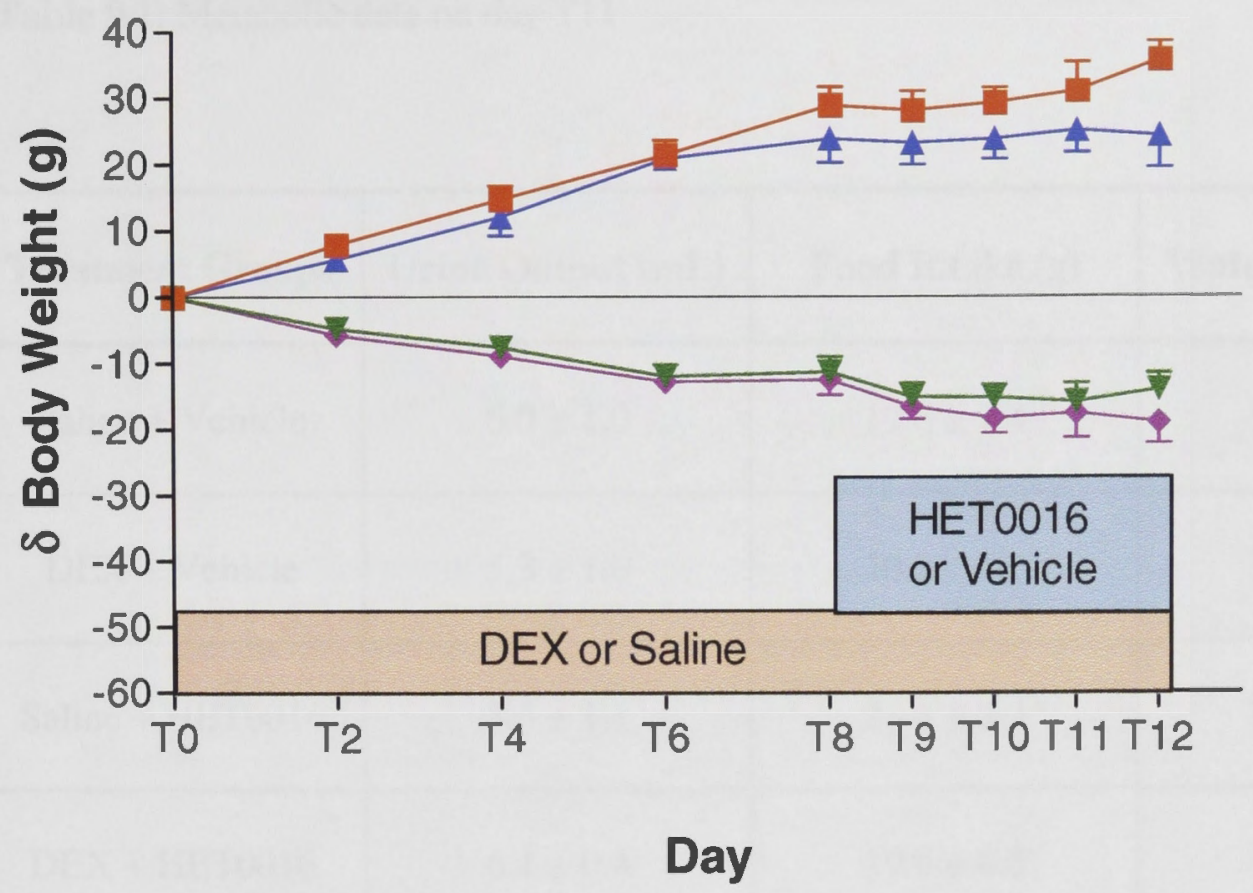

Figure 9.5: Change in body weight. $\approx$ Saline + Vehicle, $n=8 ; \Delta$ Saline + HET0016, $n$ $=8 ; \boldsymbol{\nabla}$ DEX + Vehicle, $\mathrm{n}=8 ;$ DEX + HET0016, $\mathrm{n}=8$.

\subsubsection{Metabolic parameters}

On day $\mathrm{T} 11$, there was no significant difference in urine output, food and water consumption in any treatment group (Table 9.1, Figures 9.6-9.8). 
Table 9.1: Metabolic data on day T11.

\begin{tabular}{|c|c|c|c|}
\hline Treatment Groups & Urine Output (mL) & Food Intake (g) & Water Intake (mL) \\
\hline Saline + Vehicle & $6.0 \pm 1.0$ & $19.6 \pm 1.4$ & $36.1 \pm 2.2$ \\
\hline DEX + Vehicle & $5.3 \pm 1.0$ & $20.8 \pm 1.1$ & $30.1 \pm 2.2$ \\
\hline Saline + HET0016 & $4.3 \pm 1.1$ & $21.4 \pm 2.3$ & $33.1 \pm 2.6$ \\
\hline DEX + HET0016 & $6.1 \pm 0.4$ & $19.9 \pm 0.5$ & $34.8 \pm 4.3$ \\
\hline
\end{tabular}

Results were expressed as mean \pm SEM. 


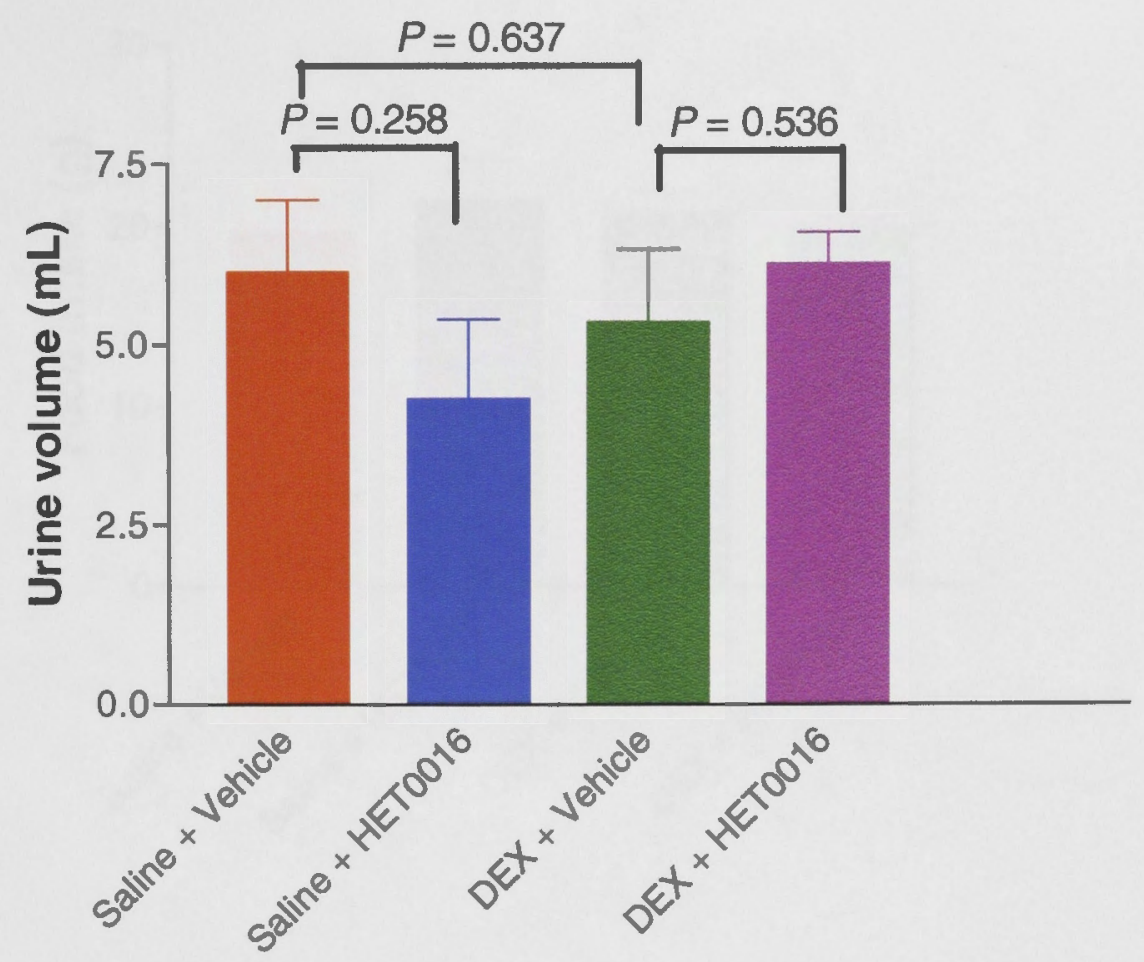

Figure 9.6: 24 hour urine volume on day T11. Saline + Vehicle, $n=8$; Saline + HET0016, $\mathrm{n}=8$; DEX + Vehicle, $\mathrm{n}=8$; DEX + HET0016, $\mathrm{n}=8$. 


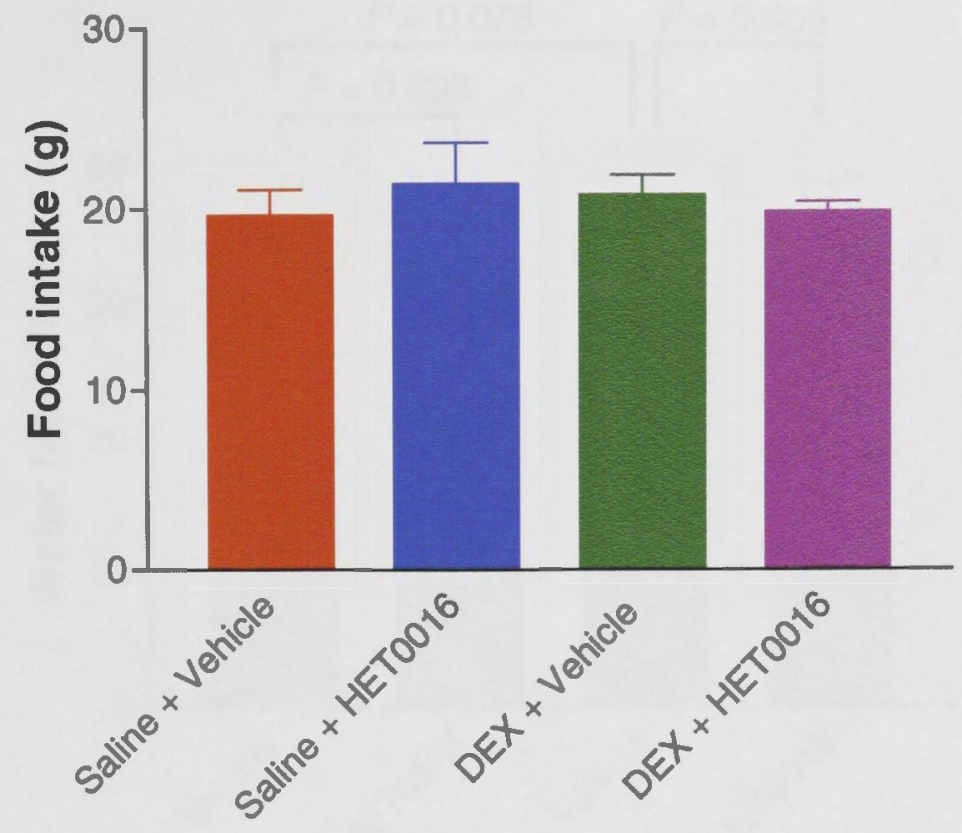

Figure 9.7: 24-hour food consumption. Saline + Vehicle, $\mathrm{n}=8$; Saline + HET0016, $\mathrm{n}=8$; DEX + Vehicle, $\mathrm{n}=8$; DEX + HET0016, $\mathrm{n}=8$. 


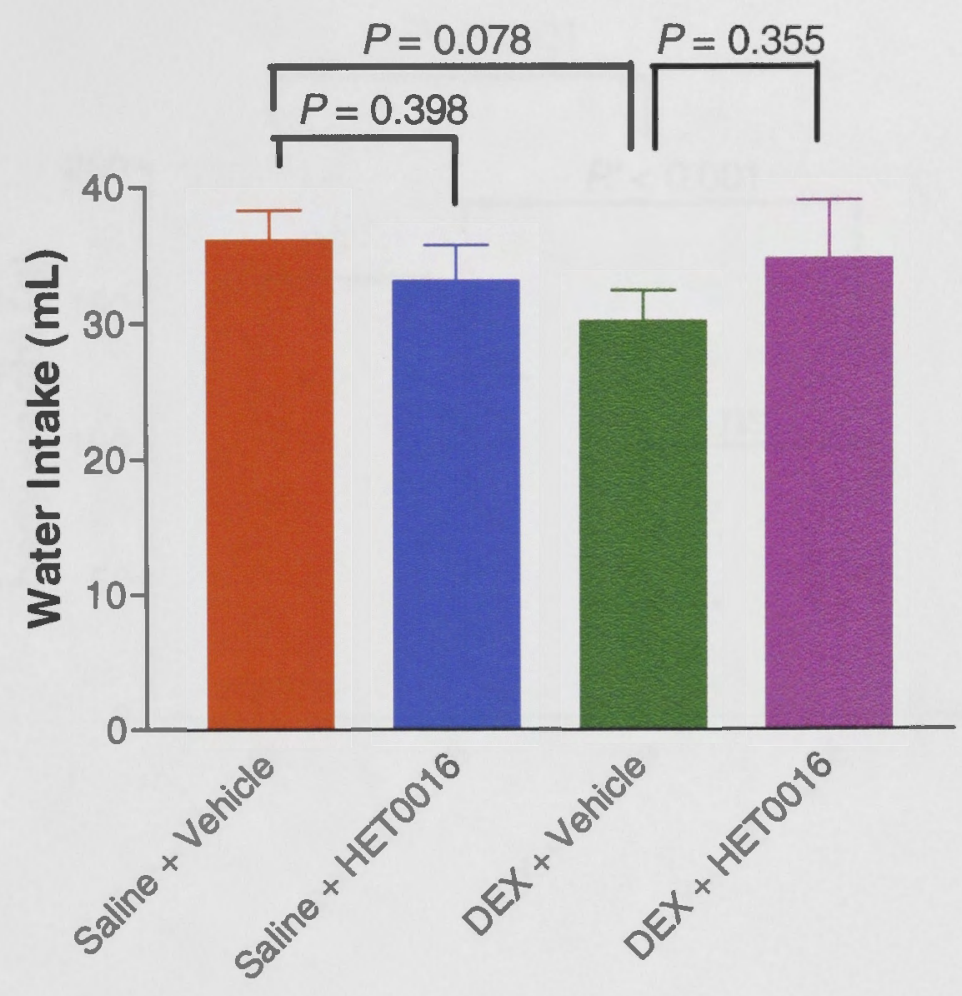

Figure 9.8: 24-hour water consumption. Saline + Vehicle, $\mathrm{n}=8$; Saline + HET0016, $\mathrm{n}=8$; DEX + Vehicle, $\mathrm{n}=8$; DEX + HET0016, $\mathrm{n}=8$.

\subsubsection{Thymus weight}

There was a significant decrease in thymus weight associated with DEX treatment (saline + vehicle: $119 \pm 6$ vs DEX + vehicle: $41 \pm 6 \mathrm{mg} / \mathrm{g}$ body weight, $P^{\prime}<0.005$ ).

This decrease was not modified by HET0016 (DEX + vehicle: $41 \pm 6$ vs DEX + HET0016: $51 \pm 3 \mathrm{mg} / \mathrm{g}$ body weight, $n s$ ) (Figure 9.9 ). 


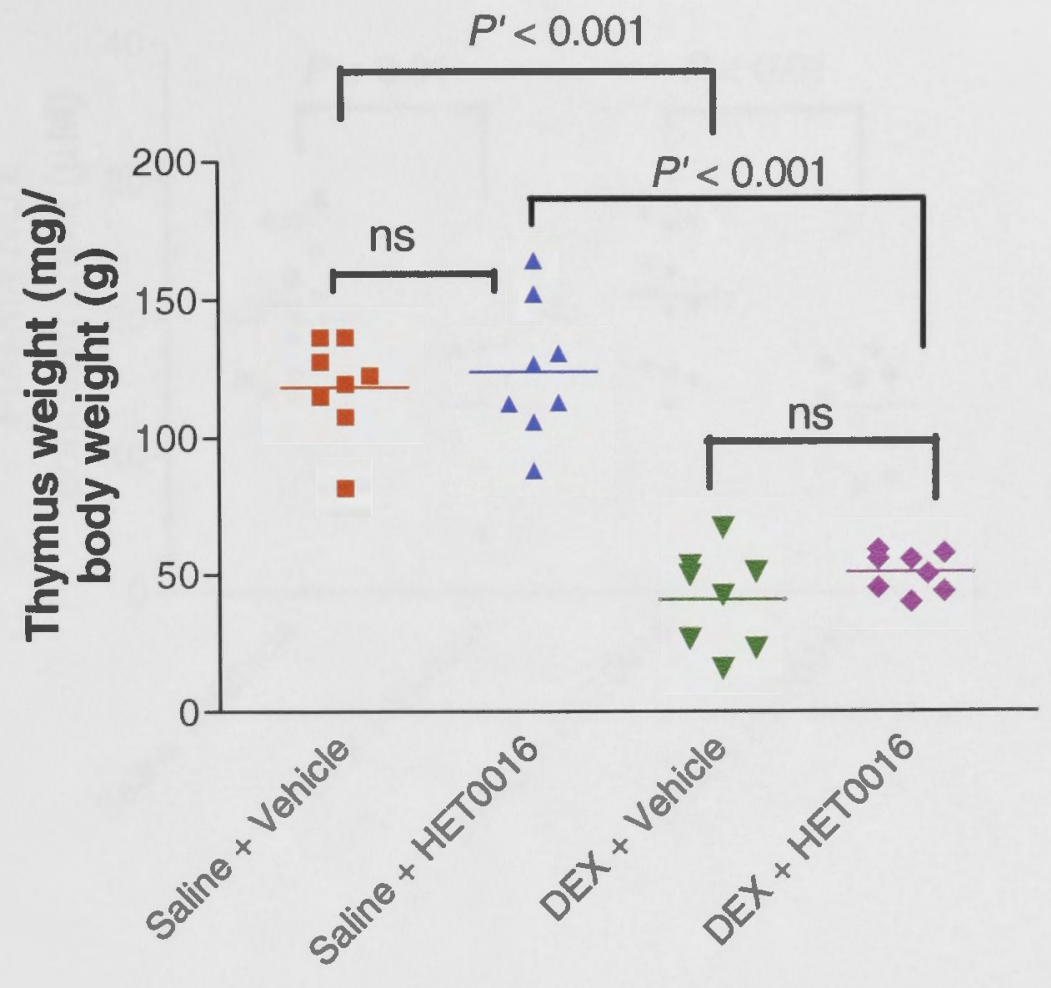

Figure 9.9: Thymus weight as marker of glucocorticoid activity. - Saline + Vehicle, $n$ $=8 ; \Delta$ Saline + HET0016, $\mathrm{n}=8 ; \boldsymbol{\nabla}$ DEX + Vehicle, $\mathrm{n}=8 ;$ DEX + HET0016, $\mathrm{n}=8$.

\subsubsection{Plasma nitrate and nitrite concentration}

DEX significantly decreased plasma NOx concentration from $20 \pm 1(n=16)$ to $14 \pm 2 \mu \mathrm{M}(\mathrm{n}=8, P<0.01)$ and this decrease was not reversed by HET0016 (saline + HET0016: $22 \pm 2, \mathrm{n}=13$ vs DEX + HET0016: $14 \pm 1 \mu \mathrm{M}, \mathrm{n}=8, P<0.01)$. HET0016 did not alter plasma NOx levels in either the saline or DEX-treated groups (Figure 9.10). 


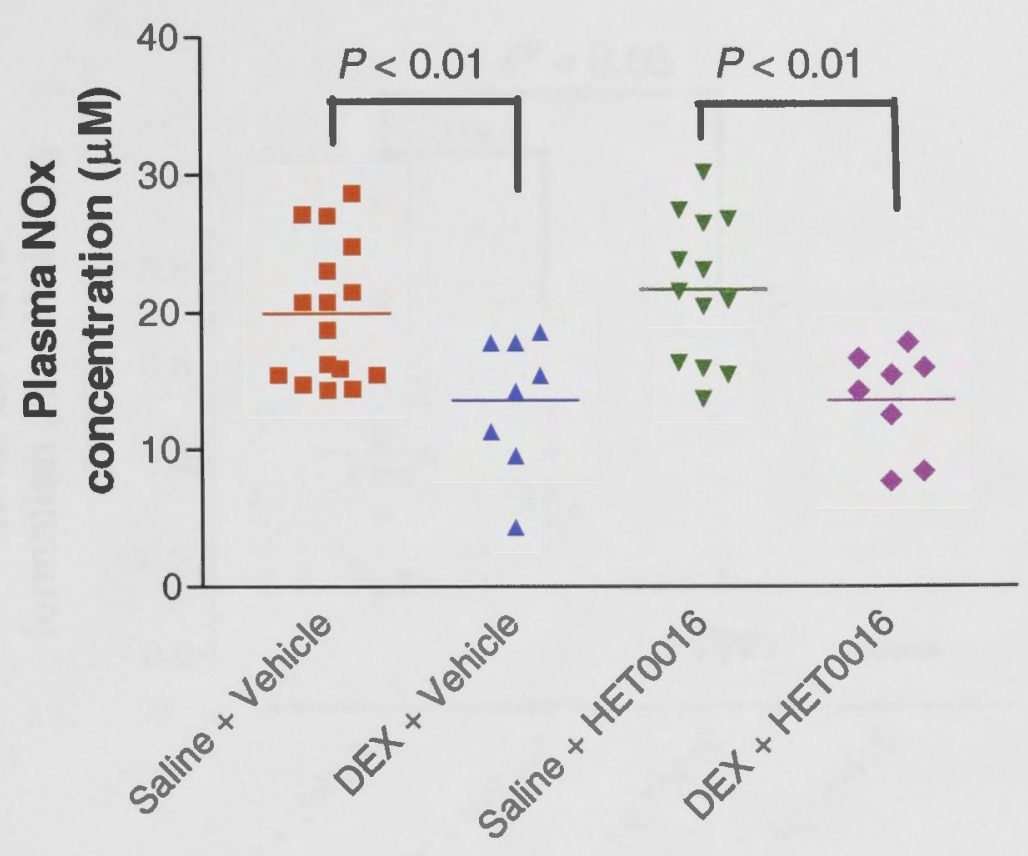

Figure 9.10: Plasma nitrate and nitrite concentrations. - Saline + Vehicle, $n=16 ; \boldsymbol{\nabla}$ DEX + Vehicle, $\mathrm{n}=8 ; \Delta$ Saline + HET0016, $\mathrm{n}=13 ; \mathrm{DEX}+$ HET0016, $\mathrm{n}=8$.

\subsubsection{Renal microsomal 20-HETE concentration}

DEX treatment did not alter renal microsomal 20-HETE concentration (saline + vehicle: $0.42 \pm 0.07, \mathrm{n}=12$ vs $\mathrm{DEX}+$ vehicle: $0.36 \pm 0.03 \mathrm{nmol} / \mathrm{min} / \mathrm{mg}$ protein, $\mathrm{n}=6$ ). Renal microsomal 20-HETE was significantly lower in saline + HET0016-treated rats $(0.03 \pm$ $0.02 \mathrm{nmol} / \mathrm{min} / \mathrm{mg}$ protein, $\mathrm{n}=8$ ) compared to saline + vehicle-treated rats $\left(0.42 \pm 0.07 \mathrm{nmol} / \mathrm{min} / \mathrm{mg}\right.$ protein, $\left.\mathrm{n}=12, P^{\prime}<0.05\right)$ and was undetectable in the DEX + HET1106 group (Figure 9.11). 


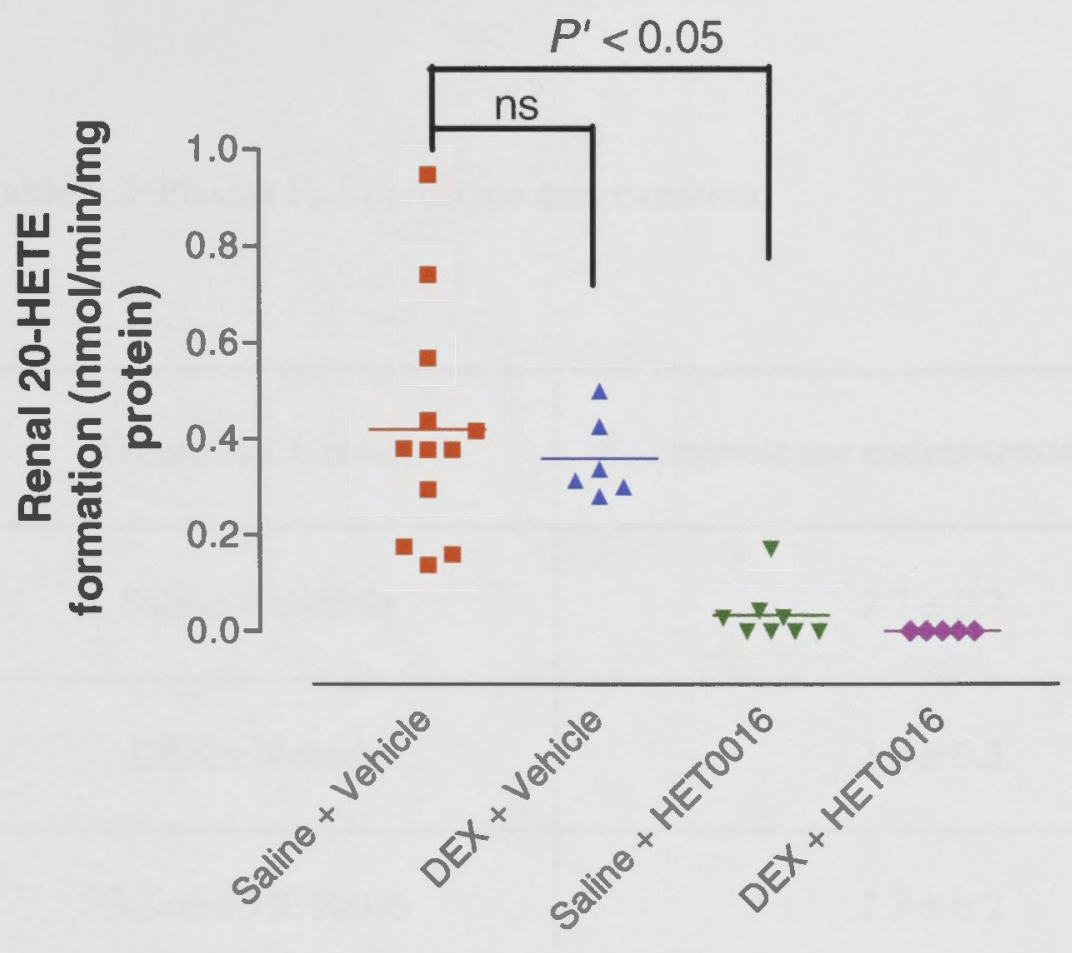

Figure 9.11: Renal microsomal 20-HETE concentration. - Saline + Vehicle, $n=12 ;$ DEX + Vehicle, $n=6 ; \Delta$ Saline + HET0016, $n=8 ; \bullet$ DEX + HET0016, $n=5$.

\subsubsection{Plasma $\mathrm{F}_{2}$-isoprostane concentration}

There was no significant difference in plasma $\mathrm{F}_{2}$-isoprostane concentration in any of the treatment groups. There was no significant difference in plasma $F_{2}$-isoprostane concentration between saline- and DEX-treated rats. HET0016 did not alter $F_{2}$ isoprostane concentration in DEX- or saline-treated rats (Table 9.2 and Figure 9.12). 
Table 9.2: Plasma $F_{2}$-isoprostane concentration

\begin{tabular}{|c|c|}
\hline Treatment Groups & F $_{2}$-isoprostane concentration (nmol/L) \\
\hline Saline + Vehicle & $2.7 \pm 0.2$ \\
\hline DEX + Vehicle & $3.3 \pm 0.3$ \\
\hline Saline + HET0016 & $2.7 \pm 0.2$ \\
\hline DEX + HET0016 & $2.8 \pm 0.3$ \\
\hline
\end{tabular}

Results were expressed as mean \pm SEM. 


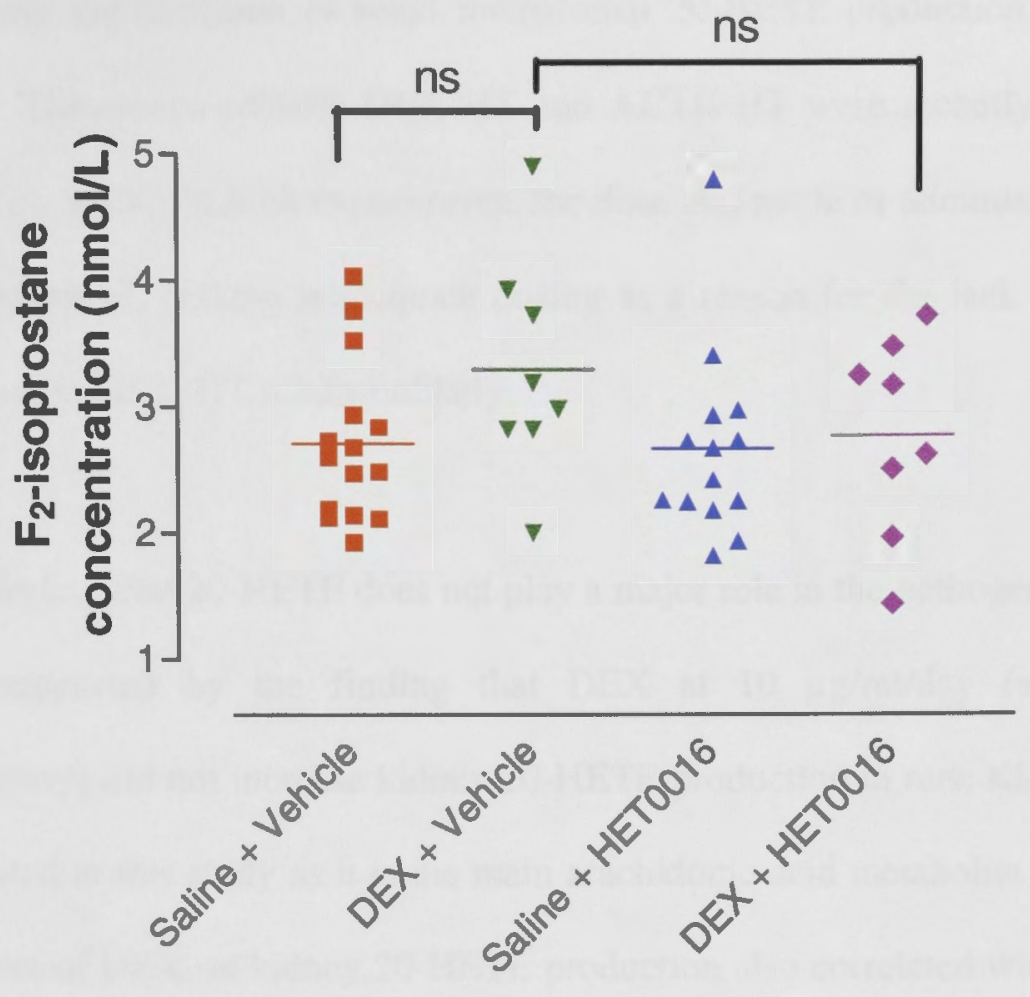

Figure 9.12: Plasma $F_{2}$-isoprostane concentration as marker of systemic oxidative stress. $=$ Saline + Vehicle, $\mathrm{n}=15 ; \boldsymbol{\nabla}$ DEX + Vehicle, $\mathrm{n}=8 ; \boldsymbol{\Delta}$ Saline + HET0016, $\mathrm{n}=$ 14; DEX + HET0016, $\mathrm{n}=8$.

\subsection{DISCUSSION}

The salient finding of this study was that the 20-HETE inhibitor HET0016 was unable to reverse established hypertension due to DEX despite completely inhibiting renal microsomal 20-HETE production. This was in contrast to the rat ACTH-HT model where HET0016 was effective in preventing and reversing hypertension as well as 
reversing the elevation of renal microsomal 20-HETE production (Zhang, Wu et al. 2009). The results of both DEX-HT and ACTH-HT were recently published (Zhang, Wu et al. 2009). In both experiments, the dose and mode of administration of HET0016 were identical, making inadequate dosing as a reason for the lack of antihypertensive response in DEX-HT seems unlikely.

This finding that 20-HETE does not play a major role in the pathogenesis of DEX-HT is also supported by the finding that DEX at $10 \mu \mathrm{g} / \mathrm{rat} /$ day (approximately 0.03 $\mathrm{mg} / \mathrm{kg} /$ day) did not increase kidney 20 -HETE production in rats. Kidney 20 -HETE was evaluated in this study as it is the main arachidonic acid metabolite in the rat. The lack of effect of DEX on kidney 20-HETE production also correlated with the evidence that urinary excretion of 20-HETE was not increased in DEX-hypertensive rats (Zhang, Hu et al. 2008). These findings, however, do not completely eliminate the possibility of a role of renal 20-HETE in DEX-HT as higher DEX doses in rats $(0.3-1 \mathrm{mg} / \mathrm{kg} / \mathrm{day})$ have been reported to result in increased renal 20-HETE production (Lin, Abraham et al. 1994; Sanchez-Mendoza, Lopez-Sanchez et al. 2000). Thus, DEX at higher doses could influence 20-HETE production and the likely effect on blood pressure regulation warrants further investigations in future studies.

As discussed in Section 1.7 of Chapter 1, there is increasing evidence implicating the role of oxidative stress and NO-redox imbalance in the pathogenesis of DEX-HT (Zhang, Croft et al. 2004; Hu, Zhang et al. 2006). The latter phenomenon is associated with NO deficiency. As in another study conducted in our laboratory (Krug, Zhang et al. 2008), this study once again demonstrated that DEX-hypertensive rats have lower 
plasma NOx. This finding is consistent with data shown by Wallerath et al. that DEX resulted in downregulation of eNOS in mice (Wallerath, Witte et al. 1999). In this study, inhibition of 20-HETE production with HET0016 failed to restore decreased plasma NOx availability induced by DEX. This suggests that NO does not have a regulatory effect on the renal 20-HETE production in the DEX-HT model. Furthermore, it is possible that the reduction in nitric oxide due to DEX may be independent of the 20-HETE pathway.

The association of 20-HETE and oxidative stress is recognised. 20-HETE has been shown to stimulate ROS production through eNOS uncoupling (Cheng, Ou et al. 2008) and activation of the NAD(P)H oxidase pathway (Medhora, Chen et al. 2008). In this study $\mathrm{F}_{2}$-isoprostane, a marker of systemic lipid peroxidation and oxidative stress, was not elevated by DEX treatment. This result is inconsistent with other studies (Chapters 6 and 8 ) in this project which showed significant a significant increase in plasma $\mathrm{F}_{2}$ isoprostane concentration due to DEX. This may represent a false negative result due to insufficient power of the relatively small sample size to detect the difference. Pooled results from the rats treated with only saline or DEX from the different studies in this project (Chapters 6, 8 and 9) showed that DEX significantly increased plasma $F_{2}$ isoprostane concentration (Figure 11.1, Chapter 11), consistent with our findings in Chapters 6 and 8. It is also unclear if the vehicle used in this study had caused this negative result. In the absence of a change in plasma $\mathrm{F}_{2}$-isoprostane in the DEX-only group, it is difficult to draw any conclusion about the role of HET0016 in reversing oxidative stress associated with DEX-HT. 
Whether HET0016 influences the metabolic effects induced by low dose DEX (20 $\mu \mathrm{g} / \mathrm{rat})$ such as growth stasis, acute transient natriuresis and diuresis, as will be described in Chapter 10, was assessed in this study. In this study, HET0016 did not reverse weight loss induced by DEX. Rather, the combination of HET0016 and DEX resulted in a slight but significant weight loss from day T8-T12. This phenomenon was not seen in DEX + vehicle treated rats. Whilst 20-HETE is known to have a diuretic effect, inhibition of 20-HETE with HET0016 failed to alter urine output. Furthermore, consistent with our findings in Chapter 10 , there is no significant difference in urine volume with DEX treatment on day T11. The lack of diuresis due to DEX further strengthens the notion that 20-HETE, a known diuretic, has no major influence in this hypertensive model.

There are several studies in the literature highlighting the differences between ACTHand DEX-HT (Chapter 1). The results demonstrated in this study, which were in contrast to those observed in ACTH-HT, further support the notion that ACTH- and DEX-HT involve different pathophysiological mechanisms. HET0016 successfully reversed ACTH-HT (Zhang, Wu et al. 2009) but not DEX-HT as shown in this study. DEX-HT was not associated with elevated renal microsomal 20-HETE level whilst an increase was observed in ACTH-HT (Zhang, Wu et al. 2009). This ACTH-induced increase was reversed by HET0016 (Zhang, Wu et al. 2009). Furthermore, urinary 20HETE excretion was increased in ACTH-HT but not DEX-HT (Zhang, Hu et al. 2008). 


\subsection{CONCLUSION}

DEX did not increase renal microsomal 20-HETE production and specific 20-HETE inhibition with HET0016 failed to reverse DEX-HT and DEX-induced reduction in plasma NOx. These results suggest that the arachidonic acid metabolite 20-HETE does not play a significant role in the pathogenesis of DEX-HT. 


\section{CHAPTER 10}

Metabolic Profiles of Dexamethasone-Induced

Hypertension in Rats 


\subsection{INTRODUCTION}

In rats, glucocorticoid excess is associated with negative sodium balance, and markedly reduced body weight secondary to skeletal muscle and fat catabolism (Tonolo, Fraser et al. 1988). The metabolic effects of excess glucocorticoid, which are dose-dependent, do not correlate with the hypertensive response it causes. Tonolo et al have demonstrated that a DEX dose as low as $1 \mu \mathrm{g} / \mathrm{rat} / \mathrm{day}$ could raise blood pressure without any change in body weight in rats (Tonolo, Fraser et al. 1988). Most of the studies on DEX-HT in the literature described DEX doses many times higher than the dose used by our laboratory or Tonolo et al. (Okuno, Suzuki et al. 1981; Handa, Kondo et al. 1984; Nakamoto, Suzuki et al. 1991), thus introducing potential significant confounding associated with major metabolic effects. The dose used in the haemodynamic studies (Chapters 3 and 4) was $20 \mu \mathrm{g} / \mathrm{rat} / \mathrm{day}$. Although this moderately low dose was much higher than that described by Tonolo et al. and slightly higher than the dose used in other DEX-related studies in this research project, it was still significantly lower that other studies in the literature (Okuno, Suzuki et al. 1981; Handa, Kondo et al. 1984; Nakamoto, Suzuki et al. 1991).

Even though this project aims to look at the mechanisms of DEX-HT, it is still important to note the metabolic changes associated with glucocorticoid administration as these changes not only act as surrogate markers of effective glucocorticoid administration, they may also influence blood pressure and other haemodynamic parameters. For example, changes in fluid balance can alter blood 
pressure regulation via the renin angiotensin system. DEX dose of $10 \mu \mathrm{g} / \mathrm{rat} / \mathrm{day}$ has been previously shown to raise blood pressure without altering body weight and water intake (Hu, Zhang et al. 2006). It, however, increased haematocrit (Hu, Zhang et al. 2006).

As the doses used in the haemodynamic studies (Chapters 3 and 4) were higher than the dose reported previously from our laboratory (Hu, Zhang et al. 2006), additional metabolic assessments were made on the rats used in the haemodynamic study (Chapter 3) to evaluate the metabolic consequences of DEX at $20 \mu \mathrm{g} / \mathrm{rat} / \mathrm{day}$ and to verify that the haemodynamic changes observed in Chapter 3 were not consequences of sodium retention and plasma volume expansion.

In this study, the following parameters were analysed:

- Food consumption

- Water consumption

- Urine volume

- Urine sodium and potassium excretion

- Urine sodium: potassium ratio

- Body weight

- Systolic blood pressure 


\subsection{METHODS}

\subsubsection{Experimental animals}

This study was approved by the Animal Experimentation Ethics Committee of the Australian National University (Protocol No. J.HB.22.06). The rats were housed and acclimatised as described in Sections 2.2 and 2.3 of Chapter 2.

As the rats were treated with either saline or DEX, the treatment protocol did not include pre-treatment days. Rather, there were 8 control days before treatment with either saline or DEX was commenced on day T0.

Fifteen rats received daily injections of saline $(0.2 \mathrm{~mL} / \mathrm{rat}, \mathrm{sc})$ and 10 rats received DEX $(20 \mu \mathrm{g} / \mathrm{rat}, \mathrm{sc})$ from T0-T10 using the method described in Section 2.3 in Chapter 2.

\subsubsection{Tail-cuff systolic blood pressure measurements}

Second-daily tail-cuff SBP measurements were performed on all rats using the method described in Section 2.5.1 in Chapter 2.

\subsubsection{Metabolic parameters}

As mentioned earlier, there were 8 control days before treatment with either saline or DEX was commenced. Metabolic data were recorded second-daily from C7 
onwards. Following the tail-cuff SBP and body weight measurements which were performed second-daily, the rats were then housed singly in metabolic cages that allow measurements of 24-hour urine volume, food and water consumption. These data were recorded second-daily from day C7 to T9 (Figure 10.1). Measurements of urine volume and water intake were expressed as $\mathrm{mL}$ per 24 hours. Food intake was adjusted for spillage and measurements were expressed in grams per 24 hours. 


\section{TAIL-CUFF SPHYGMOMANOMETRY AND BODY WEIGHT MEASUREMENTS}

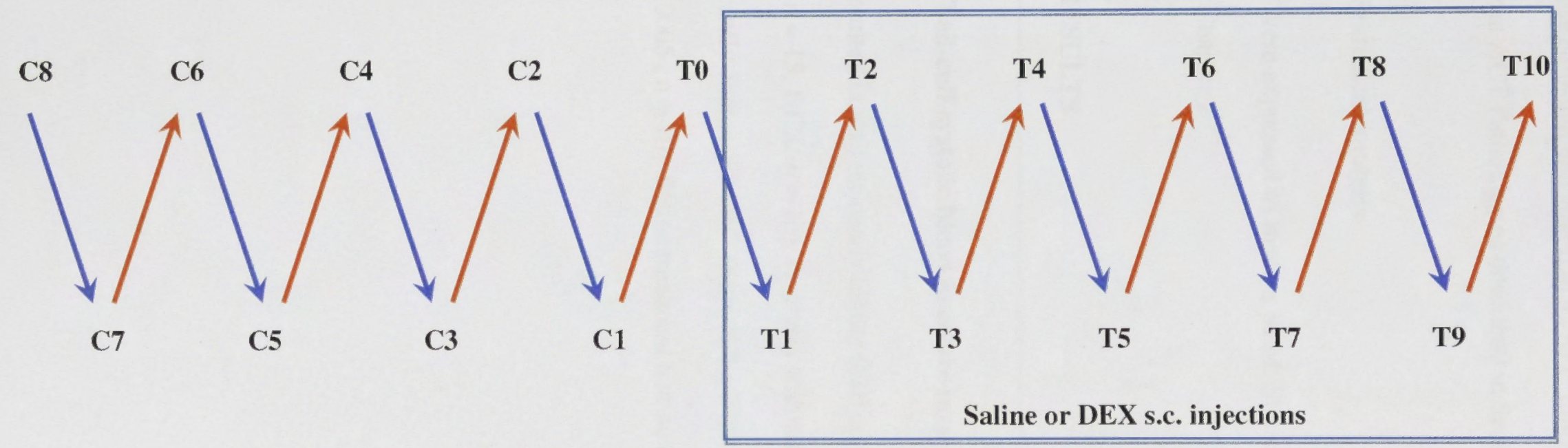

24-HOUR METABOLIC DATA RECORDINGS

Figure 10.1: Timeline of experimental protocol.

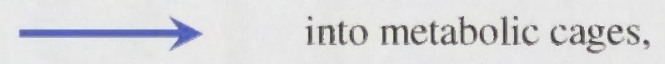

out of metabolic cages, $\mathbb{C}$ :

control days, T: treatment days. 
Urine was collected and stored in a $-70^{\circ} \mathrm{C}$ freezer until sodium, potassium and creatinine analysis at ACT Pathology as described in Sections 2.10.3 and 2.10.4.

\subsubsection{Statistical analysis}

Results were expressed as mean \pm SEM. Statistical analysis was as described in Section 2.12 of Chapter 2.

\subsection{RESULTS}

\subsubsection{Tail-cuff systolic blood pressure measurements}

DEX resulted in a significantly higher SBP compared with saline treatment $(P<0.0005$, saline: $n=15$, DEX: $n=10$ ). Analysis within the DEX-treated group showed that DEX significantly increased SBP from $120 \pm 2$ on day T0 to $149 \pm 6 \mathrm{mmHg}$ on day T10 $(P<0.0005, \mathrm{n}=10)$. Saline treatment had no effect on SBP $(n s, \mathrm{n}=15)$ (Figure 10.2). 


\section{Tail Cuff Systolic Blood \\ Pressure}

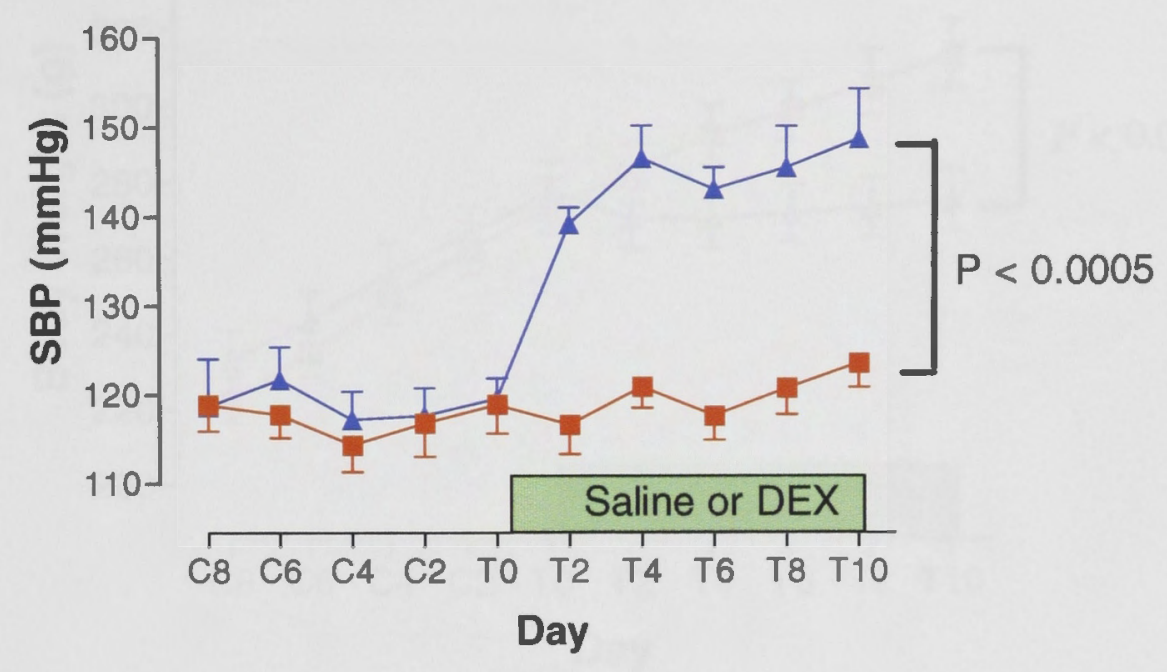

Figure 10.2: Tail-cuff systolic blood pressure. $\square$ Saline, $n=15 ; \Delta$ DEX, $n=10$.

\subsubsection{Body weight}

DEX treatment prevented body weight gain (T0: $278 \pm 8 \mathrm{~g}$ and T10: $275 \pm 8 \mathrm{~g}, n s$ ). Rats on saline treatment gained weight steadily (from T0: $274 \pm 7 \mathrm{~g}$ to $\mathrm{T} 10: 314 \pm 9 \mathrm{~g}$, $P<0.0005)$. Body weight was significantly lower in DEX-treated rats compared to controls $(P<0.0005)$ (Figure 10.3). 


\section{Body Weight}

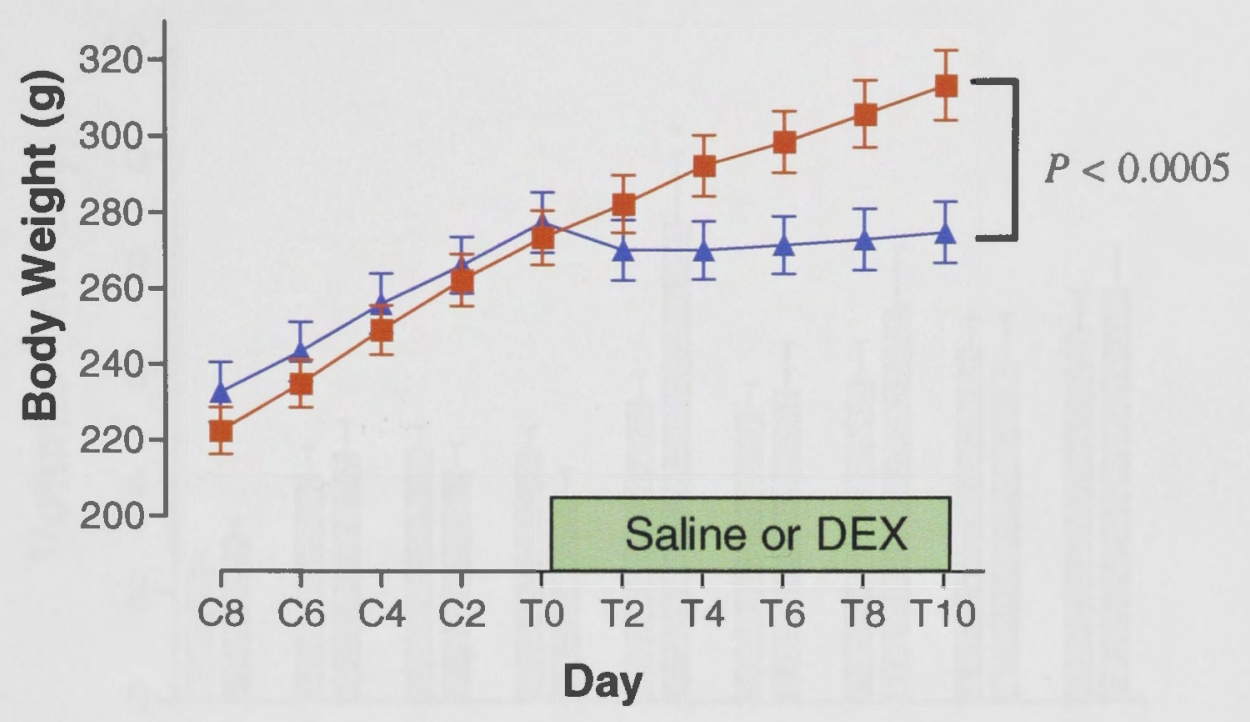

Figure 10.3: Body weight. $=$ Saline, $n=15 ; \Delta$ DEX, $n=10$.

\subsubsection{Urine volume}

Urine output was increased both in DEX-(from C1: $3.8 \pm 0.6 \mathrm{~mL}$ to T9: $7.6 \pm 0.9 \mathrm{~mL}$, $\mathrm{n}=10, P<0.005$ ) and saline-treated rats (from $\mathrm{C} 1: 4.6 \pm 0.1 \mathrm{~mL}$ to $\mathrm{T} 9: 6.9 \pm 0.7 \mathrm{~mL}$, $\mathrm{n}=15, P<0.01)($ Figure 10.4).

DEX resulted in a significant increase in urine volume in comparison with the saline treatment group ( $P<0.005$, saline: $\mathrm{n}=15, \mathrm{DEX}: \mathrm{n}=10$ ) (Figure 10.4). Day to day comparisons between the saline and DEX-treated groups showed a significant increase in urine volume by DEX-treated rats on day $\mathrm{T} 1$ (saline: $5.5 \pm 0.6 \mathrm{~mL}$ vs DEX: $8.8 \pm 1.3 \mathrm{~mL}, P<0.05$ ), which was a day following the commencement of DEX injections (Figure 10.4). 


\section{4-Hour Urine Volume}

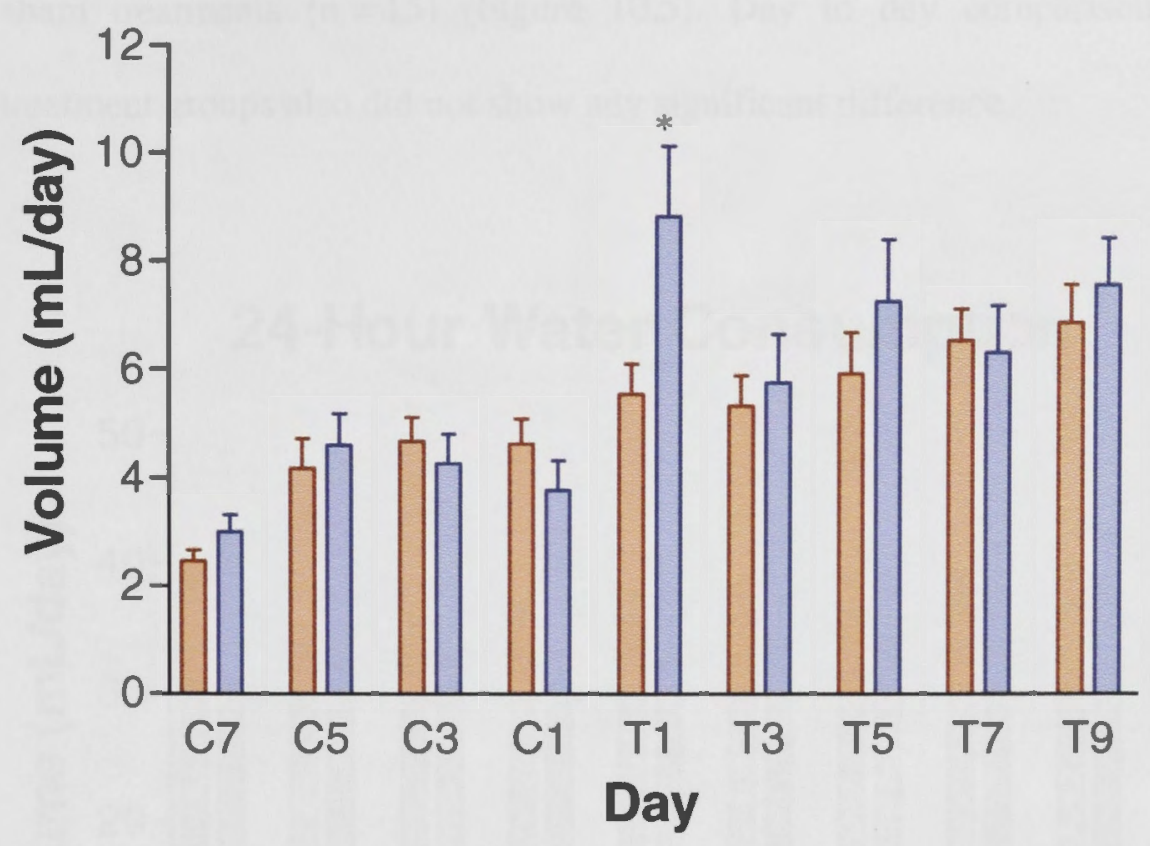

Figure 10.4: Urine volume over 24 hours. Saline, $\mathrm{n}=15$; $\mathrm{DEX}, \mathrm{n}=10 . * P<0.05$ compared to control on day T1.

\subsubsection{Water consumption}

Analysis within the saline $(n=15)$ and $\operatorname{DEX}(n=10)$ treatment groups did not show any difference in water consumption from day $\mathrm{C} 1$ (saline: $35 \pm 2 \mathrm{~mL}, \mathrm{DEX}: 31 \pm 3 \mathrm{~mL}$ ) to day T9 $\mathrm{Cl}$ (saline: $37 \pm 3 \mathrm{~mL}, \mathrm{DEX}: 38 \pm 4 \mathrm{~mL}$ ). In both groups, water intake per $100 \mathrm{~g}$ body weight was also not significantly different from day $\mathrm{C} 1$ (saline: $13 \pm 1 \mathrm{~mL}$, DEX: $11 \pm 1 \mathrm{~mL}$ ) to day T9 (saline: $12 \pm 1 \mathrm{~mL}, \mathrm{DEX}: 14 \pm 2 \mathrm{~mL}$ ). 
There was no significant difference in water intake between rats on DEX $(n=10)$ and sham treatments $(n=15)$ (Figure 10.5). Day to day comparisons between the 2 treatment groups also did not show any significant difference.

\section{4-Hour Water Consumption}

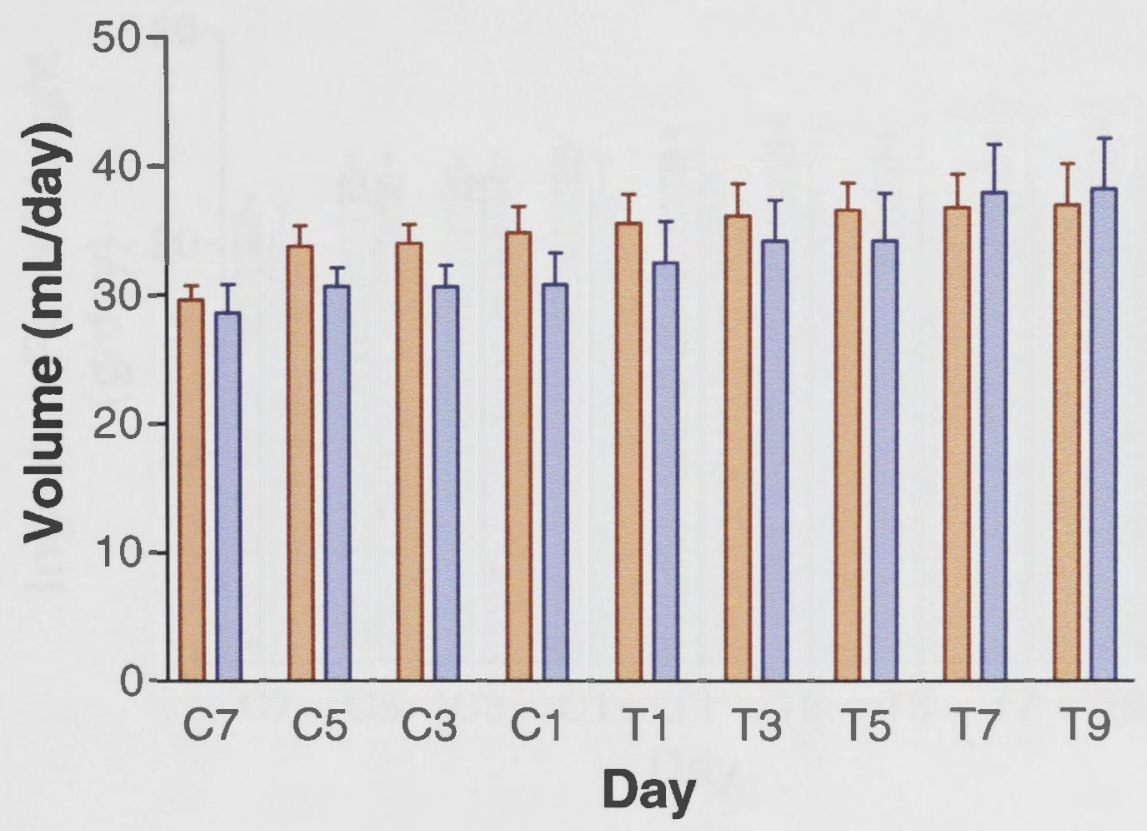

Figure 10.5: Water consumption over 24 hours. Saline, $n=15$; $\quad D E X, n=10$, not significant compared with saline.

\subsubsection{Food consumption}

There was no significant change in food consumption within the saline $(n=15)$ and DEX-treated groups $(n=10)$ from day Cl (saline: $25 \pm 1 \mathrm{~g}$; DEX: $22 \pm 2 \mathrm{~g}$ ) to T9 (saline: $24 \pm 1 \mathrm{~g}$; DEX: $23 \pm 1 \mathrm{~g}$ ) (Figure 10.6). 
There was no significant difference in overall food consumption between rats on DEX $(n=10)$ and sham treatments $(n=15)$. Day to day comparisons between the 2 treatment groups also did not show any significant difference (Figure 10.6).

\section{4-Hour Food Consumption}

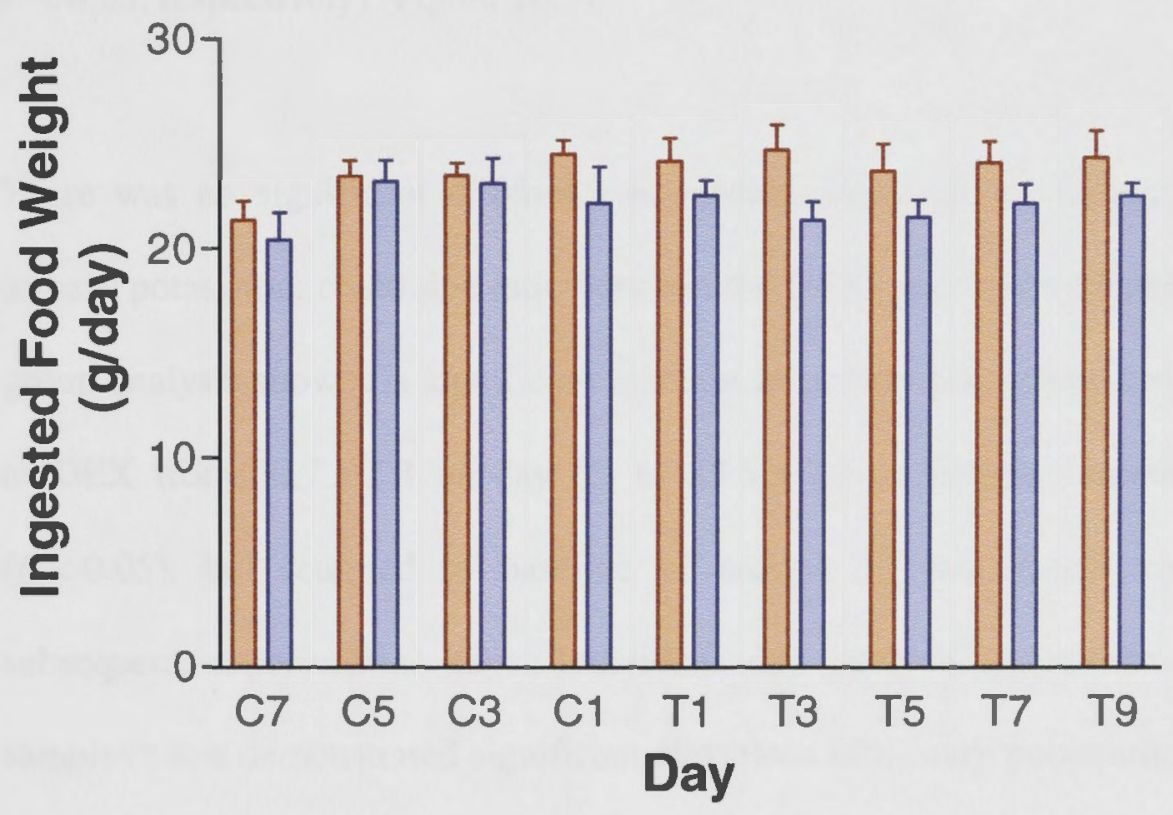

Figure 10.6: Food consumption over 24 hours. Saline, $n=15$; $D E X, n=10$, not significant compared with saline.

\subsubsection{Urinary electrolytes}

Urinary sodium excretion, as measured as urine sodium: creatinine ratio, was significantly higher in DEX-treated rats than saline-treated rats $(P<0.0005)$. Within the DEX-treated group, urine sodium: creatinine ratio was significantly increased from $23.7 \pm 0.9$ on day $\mathrm{C} 1$, a day before DEX administration; to $25.9 \pm 1.0$ on day $\mathrm{T} 9$, but 
most marked on day T1, 1 day after DEX commencement, with a ratio of $37.2 \pm 1.3$ $\mathrm{mmol} / \mu \mathrm{mol}$ creatinine $(\mathrm{n}=10, P<0.0005)$. When making comparisons between the saline- and DEX-treated groups on the individual experimental days using independent samples t-test, the sodium: creatinine ratios on days $\mathrm{T} 1$ and $\mathrm{T} 9$ were significantly elevated in the DEX-treated group compared to saline-treated group $(P<0.0005$ and $P<0.05$, respectively) (Figure 10.7 ).

There was no significant difference in urinary potassium excretion, as indicated by urinary potassium: creatinine ratio between the DEX-and saline-treated groups. Within group analysis showed a significant increase in urinary potassium: creatinine ratio due to DEX from $30.7 \pm 1.3$ on day $\mathrm{C} 1$ to $33.5 \pm 0.9 \mathrm{mmol} / \mu \mathrm{mol}$ creatinine on day $\mathrm{T} 1$ $(P<0.05)$, but returned to baseline of around $30 \mathrm{mmol} / \mu \mathrm{mol}$ creatinine on the subsequent experimental days. Individual day analysis by means of independent samples t-test demonstrated significant elevations of urinary potassium: creatinine ratio in DEX-treated rats compared with saline-treated rats on days T1 $(P<0.05)$ and T9 $(P<0.005)$ (Figure 10.8).

Urinary sodium: potassium ratio was significantly higher in the DEX-treated group in comparison with sham treatment group ( $\mathrm{n}=10$ each, $P<0.0005)$. DEX increased the sodium: potassium ratio from $0.78 \pm 0.04 \mathrm{mmol} / \mathrm{mmol}$ on day $\mathrm{Cl}$ to $0.85 \pm 0.03$ $\mathrm{mmol} / \mathrm{mmol}$ on day $\mathrm{T} 9$, but was most marked on day $\mathrm{T} 1(1.11 \pm 0.03 \mathrm{mmol} / \mathrm{mmol})$ $(P<0.0005)$. Day to day comparison using independent samples t-test showed that rats on DEX treatment on day $\mathrm{T} 1$ had significantly higher urine sodium: potassium ratio than those on saline treatment $(P<0.0005)$ (Figure 10.9). 


\section{Urine Sodium Excretion}

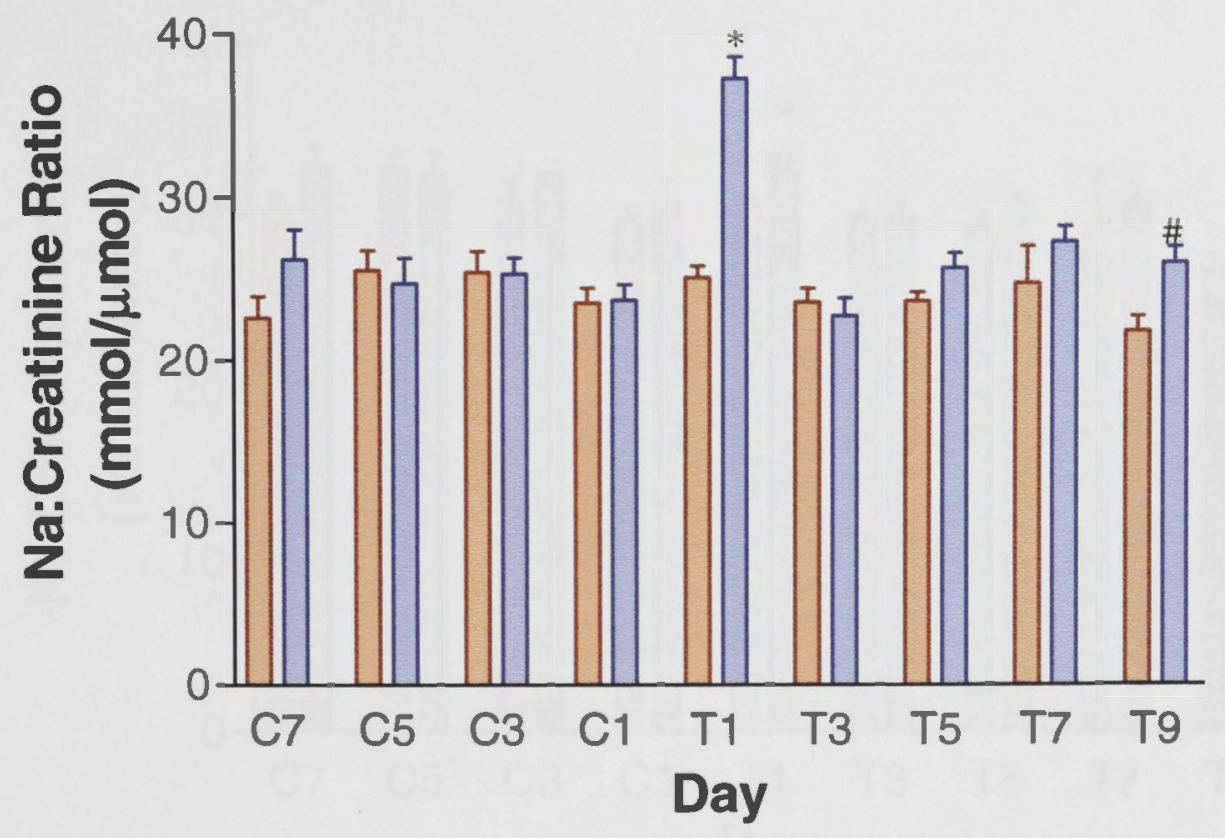

Figure 10.7: 24-hour urine sodium excretion. Saline, $n=10$; $D E X, n=10$. ${ }^{*} P<0.0005$ compared saline-treated rats on day $\mathrm{T} 1 ;{ }^{*} P<0.05$ compared to salinetreated rats on day $\mathrm{T} 9$. 


\section{Urine Potassium Excretion}

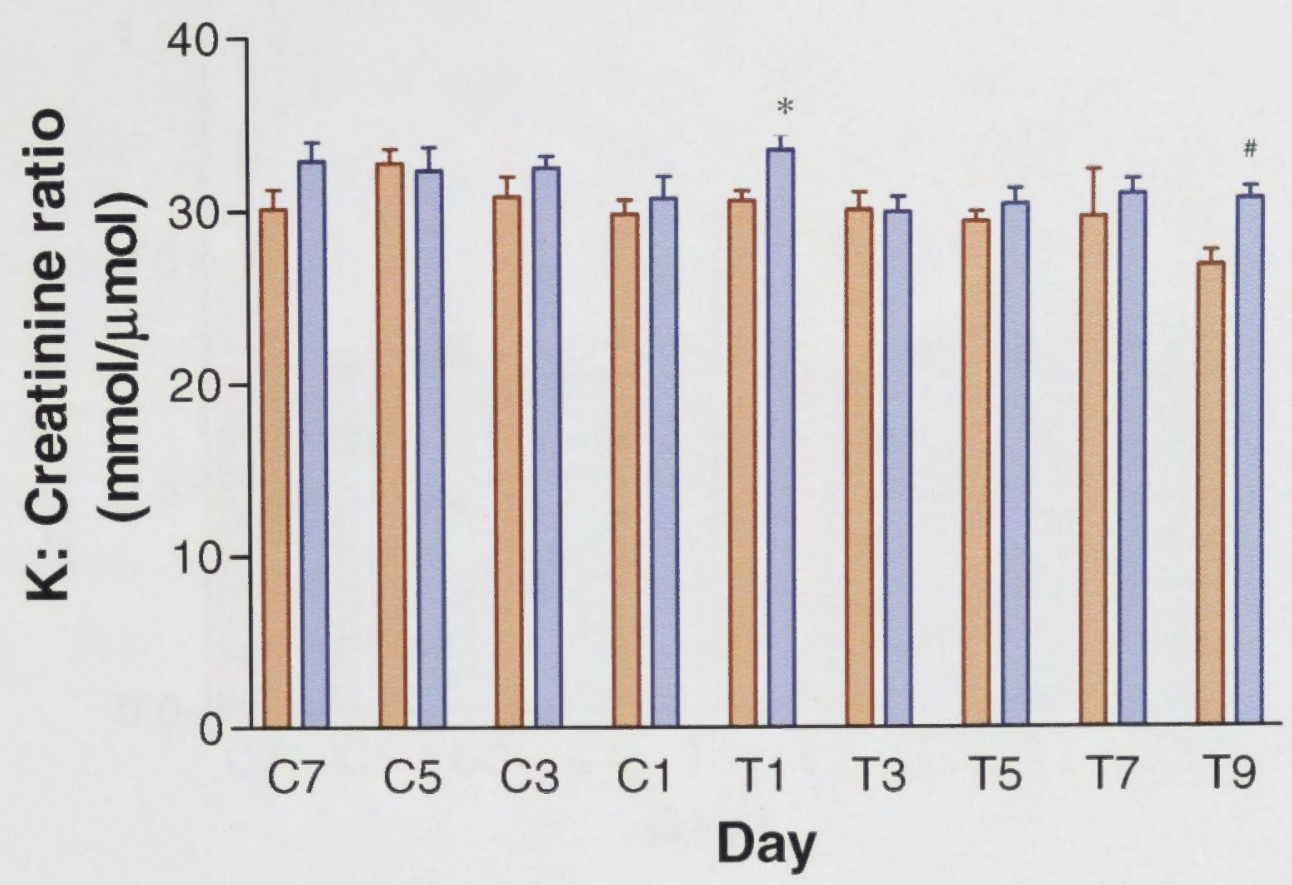

Figure 10.8: Urine potassium excretion. Saline, $\mathrm{n}=10$; $\mathrm{DEX}, \mathrm{n}=10 . * P<0.005$ compared to saline-treated rats on day $\mathrm{T} 1 ;{ }^{\#} P<0.05$ compared to saline-treated rats on day T9. 


\section{Mineralocorticoid Activity}

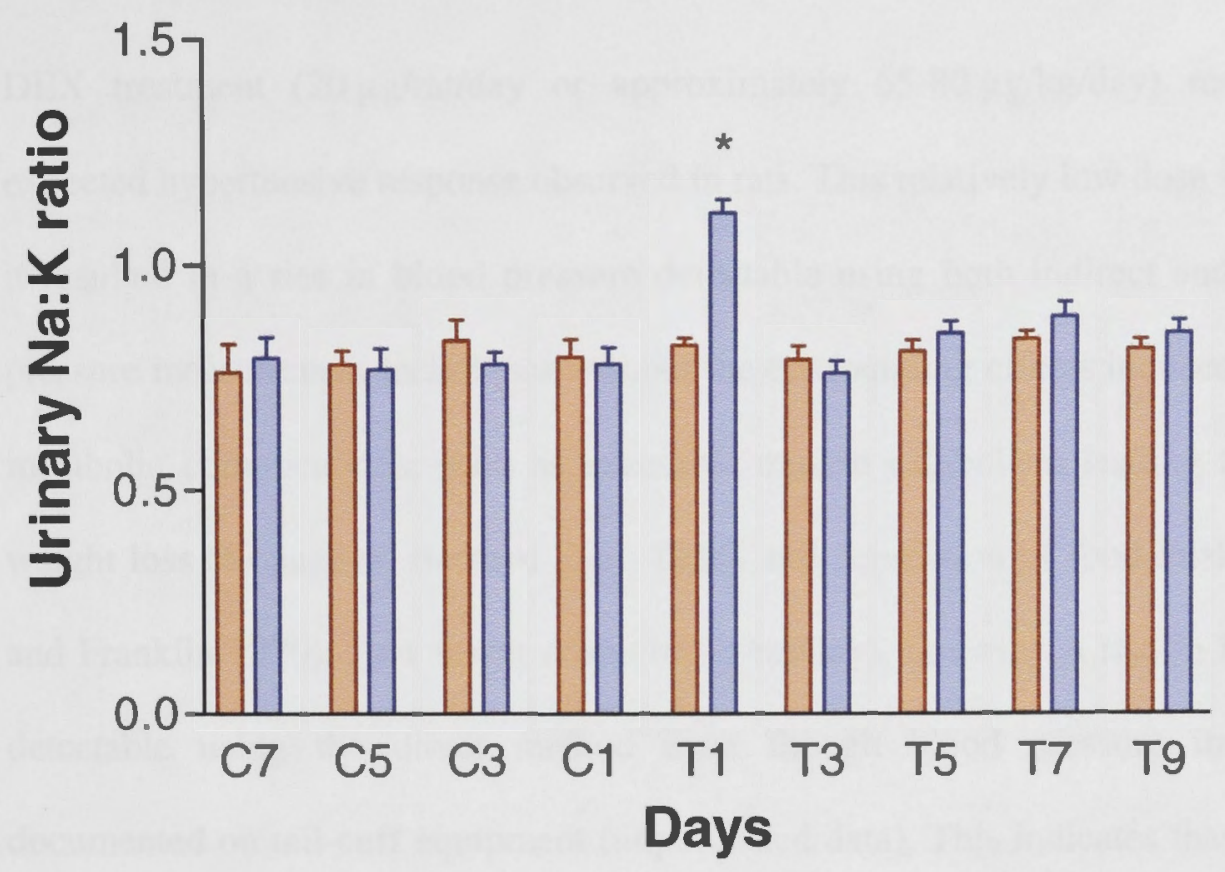

Figure 10.9: Urine sodium: potassium ratio as an index of mineralocorticoid activity. Saline, $\mathrm{n}=10$; DEX, $\mathrm{n}=10 . * P<0.0005$ compared to saline-treated rats on day $\mathrm{T} 1$. 


\subsection{DISCUSSION}

DEX treatment $(20 \mu \mathrm{g} / \mathrm{rat} /$ day or approximately $65-80 \mu \mathrm{g} / \mathrm{kg} /$ day $)$ resulted in the expected hypertensive response observed in rats. This relatively low dose was chosen as it resulted in a rise in blood pressure detectable using both indirect and direct blood pressure measurement techniques without the confounding effects induced by profound metabolic complications, such as excessive muscle catabolism leading to precipitous weight loss (Konagaya, Bernard et al. 1986) and depression of food intake (Stevenson and Franklin 1970). At a lower dose (10 $\mu \mathrm{g} / \mathrm{rat} / \mathrm{day})$, however, a rise in MAP was not detectable using the direct method even though blood pressure increases were documented on tail-cuff equipment (unpublished data). This indicates that a dose of 10 $\mu \mathrm{g} / \mathrm{rat} / \mathrm{day}$ is close to the threshold dose for a hypertensive response.

Rats on DEX treatment did not gain body weight; and had lower thymus and adrenal weights. These glucocorticoid effects in DEX-treated rats were indicative of effective administration and absorption of DEX. The observed growth stasis was not due to decreased oral food intake as there was no difference in food consumption between the DEX- and saline-treated groups. Rather, it was likely to be due to the effects of DEX on skeletal muscle mass (Konagaya, Bernard et al. 1986). The extent of these catabolic effects is proportional to the dose of DEX (Tonolo, Fraser et al. 1988; Ma, Mallidis et al. 2003). A significant loss of body weight due to high dose DEX administration in rats (approximately $100 \mu \mathrm{g} / \mathrm{rat} / \mathrm{day}$, in drinking water) has been reported (Handa, Kondo et al. 1984), but at lower doses (between 1-5 $\mu \mathrm{g} /$ day, continuous subcutaneous infusion), DEX resulted in growth stasis without inducing weight loss (Tonolo, Fraser et al. 1988). 
DEX-induced muscle atrophy is a consequence of decreased protein synthesis and increase protein degradation (Tomas, Knowles et al. 1992). Tomas et al demonstrated that subcutaneous DEX treatment at $20 \mu \mathrm{g} / \mathrm{rat} /$ day resulted in progressive weight loss, increased muscle protein breakdown as assessed by urinary excretion of 3-methyl histidine and decreased protein synthesis rate (Tomas, Knowles et al. 1992). A number of possible explanations have been put forward to explain the effect of glucocorticoid on skeletal muscles. DEX has been implicated in dose-dependent upregulation of myostatin mRNA and protein expression in vivo (Ma, Mallidis et al. 2003). In addition, inhibition of fetal rat insulin-like growth factor-1 (a hormone responsible for stimulating protein synthesis and inhibiting protein degradation) by maternal DEX treatment has been observed (Mosier, Spencer et al. 1987). Co-administration of insulin-like growth factor-1 and DEX in rats prevented the catabolic effects of DEX (Tomas, Knowles et al. 1992). Whilst there is evidence demonstrating the role of DEX in muscle catabolism, it remains unclear if stress from growth restriction plays a role in exacerbating the hypertensive response induced by DEX.

Despite the lower DEX dose used in this project to minimise the catabolic effects of DEX, we still observed growth restriction at this dosage. This effect is unlikely to be a significant confounder in this animal model of glucocorticoid-hypertension. Zhang et al have previously shown that $\mathrm{ACTH}$ administration resulted in hypertension and weight loss (Zhang, Jang et al. 2003). Treatment with tempol prevented and reversed hypertension due to ACTH even though it did not prevent or reversed the observed weight loss. In another study, the administration of $\mathrm{N}$-acetylcysteine to rats resulted in growth deceleration but did not alter blood pressure (Krug, Zhang et al. 2008). Rather, 
it prevented hypertension in DEX-treated rats (Krug, Zhang et al. 2008). Thus changes in body weight do not contribute significantly to glucocorticoid-induced hypertension.

In the ACTH-hypertensive model, differences have been observed across the different species that has been studied (human, rodent, sheep and dog) (Whitworth, Zhang et al. 2006). The rat and human are more similar in their response to ACTH although some differences exist. The discrepancy in the effect of glucocorticoid hormones on body weight in rats and humans is obvious but the blood pressure effects are similar.

Urine output in DEX-hypertensive rats was significantly higher than control rats. This diuretic response was more pronounced on day $\mathrm{Cl}$, which was day 1 post commencement of DEX treatment. A lesser increase was observed on subsequent experimental days. This increase in urine output was secondary to DEX-induced natriuresis which was also more prominent on day $\mathrm{Cl}$. Previous studies using DEX (2.5 x $10^{7}$ mol in drinking water) (Handa, Kondo et al. 1983), corticosterone (Haack, Mohring et al. 1977) and the glucocorticoid agonist RU 2698 (Grunfeld, Eloy et al. $1985)$ in rats, also produced diuretic and natriuretic responses. It is interesting to note that DEX treatment in the study by Handa et al. did not result in an acute transient increase in daily urinary sodium and water excretion observed in the present study. The different dosages used may be a contributory factor.

The mechanism of DEX-induced diuresis is unclear and is probably multifactorial. It is possible that the acute increase in urine output and sodium excretion is the renalpressure natriuresis response consequent on the DEX-induced acute rise in blood 
pressure (Hall, Mizelle et al. 1990; Rose and Post 2001). The observed diuresis and natriuresis can also be, in part, mediated through glucocorticoid-induced upregulation of atrial natriuretic peptide (ANP) gene expression and plasma ANP levels (Gardner, Hane et al. 1986). This finding was based on a higher DEX dose of $1 \mathrm{mg} /$ day, administered subcutaneously in rats. This is not a universal finding as, in another study, plasma ANP concentrations in rats were decreased by DEX (Tonolo, Richards et al. 1989). DEX dosages may be a reason for this difference. The effective DEX dosages $(1 \mu \mathrm{g} / \mathrm{day}$ to $10 \mu \mathrm{g} /$ day) used in the study by Tonolo et a.l (Tonolo, Fraser et al. 1988) were significantly lower than the dose used by Gardner et a.l (Gardner, Hane et al. 1986). It also possible that ANP stimulation by the lower DEX dose used by Tonolo et al was unable to overcome a larger ANP suppressive response induced by systemic volume contraction due to DEX.

As mentioned earlier, urine output and urinary sodium excretion were significantly raised transiently at the early stages of DEX treatment. These were partially corrected to some extent, probably by normal renal homeostatic mechanisms in response to DEXinduced decrease in effective circulating volume. These homeostatic mechanisms include the renin-angiotensin system (RAS) and antidiuretic hormone (ADH).

The $\mathrm{ADH}$ acts on the renal distal convoluted tubules and collecting tubules to allow water reabsorption and, therefore, excretion of a lower volume of concentrated urine. If this mechanism is being activated, it is unlikely to provide full correction as rats treated chronically with DEX have been shown to have elevated haematocrit (Hu, Zhang et al. 2006). Stimulation of the RAS by volume contraction in these DEX-hypertensive rats 
remains a possibility as Saruta has previously shown raised plasma renin activity in this model of hypertension (Saruta 1996).

Despite these changes, alteration to plasma volume, regulation of the RAS and ADH, these systems do not play a major role in the pathogenesis of DEX-HT as described in Sections 1.4.5.1 and 1.4.5.2 of Chapter 1.

DEX has been reported to have a very weak affinity for mineralocorticoid receptors (Grossmann, Scholz et al. 2004). In view of this, one would expect DEX excess to result in mineralocorticoid receptor activation. However, current evidence does not indicate the presence volume expansion in DEX-HT. In this study, serum sodium and potassium were not examined as the experimental conditions of the haemodynamic experiments on the last day (Chapter 3) would make serum electrolytes unreliable. Previous reports have shown that DEX (both at high and low doses) did not alter plasma sodium and potassium concentrations (Sinha, Rodriguez et al. 1981; Tonolo, Fraser et al. 1988). Furthermore, this study found that urine sodium: potassium ratio was significantly higher in DEX-hypertensive rats, suggesting that mineralocorticoid function was not activated in these DEX-hypertensive rats. These results further confirmed previous findings that DEX-HT is not associated with the mineralocorticoid effect of salt retention and volume expansion. 


\subsection{CONCLUSION}

DEX at $20 \mu \mathrm{g} / \mathrm{rat} /$ day, given subcutaneously, effectively increased blood pressure for the haemodynamic studies without the unwanted side effects of precipitous weight loss.

The increase in blood pressure induced by DEX was not due to increased mineralocorticoid activity and plasma volume expansion. This also explained why cardiac output and stroke volume (Chapter 3) were unchanged in DEX-HT. 


\section{CHAPTER 11}

Summary and Conclusion 
GC-HT was previously thought to be a consequence of mineralocorticoid receptor activation resulting in salt and water retention, and hence, plasma volume expansion, increase in cardiac output and hypertension. However, studies to date in humans and experimental animals have not supported this notion. GC-HT has been shown not to be associated with plasma expansion (Whitworth, Gordon et al. 1989; Montrella-Waybill, Clore et al. 1991; Williamson, Kelly et al. 1996; Li, Wen et al. 1999).

More recent evidence now revolves around the availability of NO and interactions between NO and ROS. Studies evaluating the mechanisms of GC-HT using the ACTH-HT model, a representation of hypertension due to naturally-occuring glucocorticoid, and the DEX-HT model, that of synthetic GC-HT, have revealed differences between the two.

This project examined the mechanism of DEX-HT in rats. The haemodynamics of DEX-HT along with the role of oxidative stress and its interactions with NO were tested in this project.

\subsection{HAEMODYNAMICS}

The study of the haemodynamics of DEX-HT in rats revealed that DEX at 20 $\mu \mathrm{g} / \mathrm{rat} / \mathrm{day}$ administered subcutaneously increased blood pressure rapidly within 2 days (Chapters 3 and 4). DEX-HT in rats was characterised by increased TPR, decreased TPC but no change in CO, SV, RVR, MVR and HVR. The increase in 
TPR and decrease in TPC were not due to individual changes in resistance and conductance in the mesenteric, renal and hindquarter vascular beds.

Pharmacological manipulation using the vasodilator minoxidil to test the role of TPR and TPC in the genesis of DEX-HT showed that these haemodynamic parameters are associated features of DEX-HT that are not essential for its development.

\subsection{OXIDATIVE STRESS}

There is a body of evidence highlighting the role of oxidative stress in DEX-HT. In this project, $\mathrm{DEX}-\mathrm{HT}$ was associated with an increase in plasma $\mathrm{F}_{2}$-isoprostane (Figure 11.1), a product of lipid peroxidation and marker of systemic oxidative stress. Treatment with the antioxidant alpha-lipoic acid prevented and partially reversed DEX-HT (Chapter 6). This finding was also consistent with previous studies using the antioxidants tempol (Zhang, Croft et al. 2004) and $\mathrm{N}$ acetylcysteine (Krug, Zhang et al. 2008). Increased production of superoxide from the NAD $(\mathrm{P}) \mathrm{H}$ pathway has been implicated in the pathogenesis of DEX-HT based on the prevention of DEX-HT in rats using apocynin (Hu, Zhang et al. 2006), a $\mathrm{NAD}(\mathrm{P}) \mathrm{H}$ oxidase inhibitor. Other sources of superoxide in the blood vessel wall have been evaluated in this project. Two main sources of superoxide, the mitochondria (Chapters 5 and 6) and the xanthine oxidase pathway (Chapter 7), were found in this project not to play significant roles in the pathogenesis of DEX-HT. Whilst there is an increase in lipid peroxidation in DEX-HT as 
indicated by an increase in plasma $\mathrm{F}_{2}$-isoprostane, inhibition of lipid peroxidation by propranolol (an antihypertensive drug known to have this effect) did not prevent DEX-HT (Chapter 8). This suggests that lipid peroxidation also does not play a major role in the development of DEX-HT.

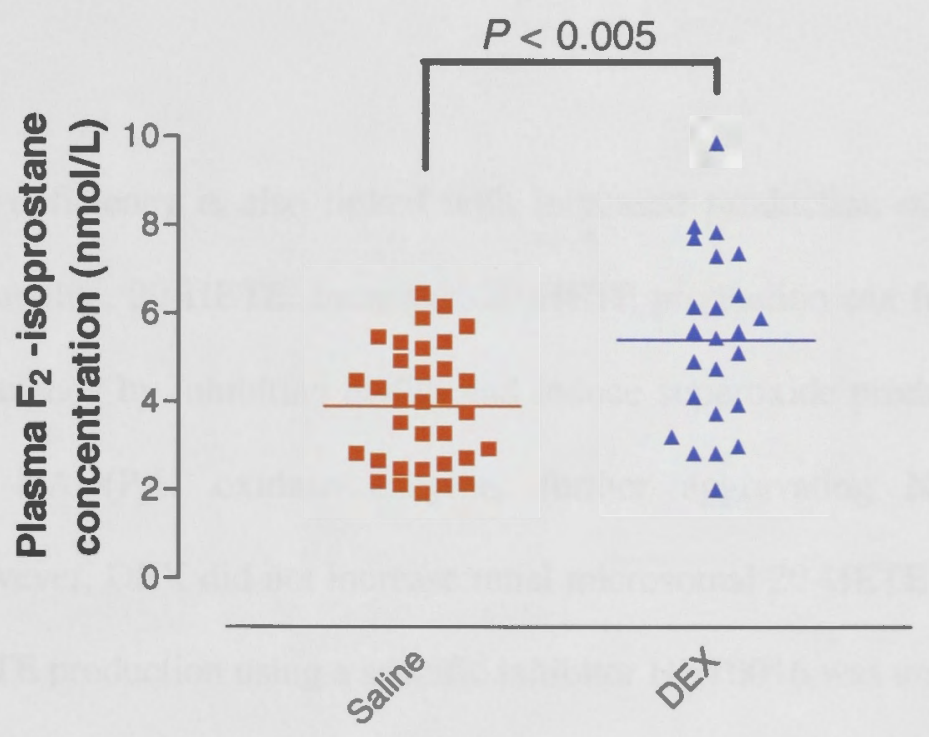

Figure 11.1: Pooled plasma $F_{2}$-isoprostane concentration results from all the relevant studies in this project. - Saline, $3.9 \pm 0.2 \mathrm{nmol} / \mathrm{L}, \mathrm{n}=33, \boldsymbol{\Delta}$ DEX, $5.4 \pm 0.4 \mathrm{nmol} / \mathrm{L}, \mathrm{n}=24$. 


\subsection{NITRIC OXIDE DEFICIENCY}

The pathways contributing to NO deficiency have been reviewed in Chapter 1 . The finding in this thesis (Chapters 7 and 9) that plasma NOx was reduced in DEX-HT is consistent with the notion that NO deficiency is implicated in DEXHT.

NO deficiency is also linked with increased production of an arachidonic acid metabolite, 20-HETE. Increased 20-HETE production can further exacerbate NO deficiency by inhibiting eNOS and induce superoxide production by stimulating the $\mathrm{NAD}(\mathrm{P}) \mathrm{H}$ oxidase enzyme, further aggravating NO-redox imbalance. However, DEX did not increase renal microsomal 20-HETE and inhibition of 20HETE production using a specific inhibitor HET0016 was unable to reverse DEXHT and DEX-induced reduction in plasma NOx (Chapter 9). These data suggest that 20 -HETE does not have a role in DEX-HT.

\subsection{OTHER MECHANISMS}

Propranolol is a common antihypertensive medication that acts via the inhibition of the $\beta$-adrenoreceptor. It failed to prevent DEX-HT despite effectively decreased HR. This study showed that $\beta$-adrenergic activation is not a main feature of DEX-HT and propranolol likely has a limited therapeutic role in DEXHT (Chapter 8). 


\subsection{OTHER OBSERVATIONS}

In all the studies described in this thesis, DEX at 10-20 $\mu \mathrm{g} / \mathrm{rat} /$ day increased blood pressure, prevented growth, decreased thymus weight, and decreased adrenal weight. Food and water consumption in these rats were not altered compared to controls (Chapter 10).

In the rat, DEX at $10 \mu \mathrm{g} / \mathrm{rat} /$ day given subcutaneously up to 11 days has no significant effect on blood glucose concentration (Chapter 6).

On the other hand, DEX treatment ( $20 \mu \mathrm{g} / \mathrm{rat} /$ day) resulted in natriuresis and diuresis, more pronounced in the first few days following the commencement of DEX (Chapter 10). Haematocrit was not altered after 11 days of DEX treatment (20 $\mu \mathrm{g} / \mathrm{rat} /$ day) (Chapters 3 and 4). These observations further substantiate the evidence that DEX-HT is not due to mineralocorticoid activation and plasma volume expansion.

\subsection{FURTHER DIFFERENCES BETWEEN ACTH- AND DEX- HYPERTENSION IDENTIFIED IN THIS PROJECT}

In addition to the differences highlighted in Chapter 1 (Section 1.9.2), further dissimilar features between ACTH- and DEX-HT have been demonstrated in this project. 


\subsubsection{Effects on body weight}

In this project, DEX resulted in growth stasis and maintenance in body weight whilst ACTH resulted in weight loss. This discrepancy was, in part, due to the dosages used. The dose of ACTH used in this study is unlikely to produce a level of endogenous glucocorticoid that is equivalent to the dose of DEX used. Whilst the low DEX dose used resulted in growth stasis but not weight loss, higher doses have been shown previously to result in significant weight loss (Handa, Kondo et al. 1984).

\subsubsection{Effects on cardiac output}

ACTH-HT, which is due to overproduction of cortisol in humans and corticosterone in rats, is associated with volume expansion due to stimulation of mineralocorticoid receptors. Consequently, cardiac output is increased in this model.(Wen, Fraser et al. 1998; Wen, Fraser et al. 1999) In contrast, this phenomenon of mineralocorticoid receptor activation, plasma volume expansion and increase in CO is absent in DEX-HT as DEX is a pure glucocorticoid with negligible mineralocorticoid effect (Table 1.1).

\subsubsection{Effects on plasma $F_{2}$-isoprostane concentration}

In this project, DEX treatment but not $\mathrm{ACTH}$ increased plasma $\mathrm{F}_{2}$-isoprostane concentration in rats. This, however, may not be a true difference as the response observed in the ACTH-treated rats was most likely influenced by the relatively 
small sample size. In an analysis using pooled data from our laboratory, $F_{2^{-}}$ isoprostane concentration was increased in ACTH-hypertensive rats (Ong, Zhang et al. 2008).

\subsubsection{The role of 20-HETE}

Increased production of urinary 20-HETE was previously observed in ACTHinduced hypertensive but not DEX-induced hypertensive rats.(Zhang, Hu et al. 2008) Futhermore, the use of the 20-HETE inhibitor HET0016 successfully prevented and reversed ACTH- (Zhang, Croft et al. 2008) but not DEX-induced hypertension as seen in Chapter 9.

\subsection{CONCLUSION AND FUTURE DIRECTIONS}

In conclusion, an increase in TPR and an imbalance between NO and ROS appear to be characteristics of DEX-HT in the rat. The exact mechanism by which DEX raises blood pressure remains unclear. Further studies exploring the differences in mechanisms between naturally-occuring and synthetic glucocorticoids, as well as the relevance of oxidative stress and NO-redox imbalance in human GC-HT will be important in advancing our understanding in this form of hypertension. Whilst the antioxidants tempol, apocynin and $\mathrm{N}$-acetylcysteine have been shown to be effective in preventing DEX-HT in the rat, the role of antioxidants in glucocorticoid- and DEX-HT in humans needs to be further evaluated. 
Application of the currently available evidence on GC-HT to research of clinical conditions associated with raised glucocorticoid levels should also be undertaken. One important area is the study of the role of defective $11 \beta$-hydroxysteroid dehydrogenase type 2 (leading to increased cortisol half life and cortisol: cortisone ratio) in the pathogenesis of hypertension in patients with chronic kidney disease. Although chronic kidney disease has previously been shown to be associated with defective cortisol inactivation (Homma, Tanaka et al. 2001; N'Gankam, Uehlinger et al. 2002), the causal relationship between glucocorticoid and hypertension in the CKD patients needs to be established. The relationships between 1) renal function and cortisol: cortisone ratio; and 2) cortisol: cortisone ratio, blood pressure and cardiovascular risks also need further evaluation. Further studies assessing the role of nitric oxide-redox imbalance, a mechanism that has been shown to implicate GC-HT, in the CKD-associated hypertension should also be pursued. 


\section{BIBLIOGRAPHY}

Adam-Vizi V. Production of reactive oxygen species in brain mitochondria: contribution by electron transport chain and non-electron transport chain sources. Antioxid Redox Signal 2005; 7(9-10): 1140-1149.

Addison T. On the constitution and local effects of disease of the supra-renal capsules. Medical Classics 1937; 2: 244-277.

Adrian TE, Allen JM, Bloom SR, Ghatei MA, Rossor MN, Roberts GW, Crow TJ, Tatemoto K, Polak JM. Neuropeptide Y distribution in human brain. Nature 1983; 306(5943): 584-586.

Allen YS, Adrian TE, Allen JM, Tatemoto K, Crow TJ, Bloom SR, Polak JM. Neuropeptide Y distribution in the rat brain. Science 1983; 221(4613): 877-879.

Alonso-Galicia M, Drummond HA, Reddy KK, Falck JR, Roman RJ. Inhibition of 20-HETE production contributes to the vascular responses to nitric oxide. Hypertension 1997; 29(1): 320-325.

Amudha G, Josephine A, Sudhahar V, Varalakshmi P. Protective effect of lipoic acid on oxidative and peroxidative damage in cyclosporine A-induced renal toxicity. Int Immunopharmacol 2007; 7(11): 1442-1449.

Antonaccio MJ, High J, DeForrest JM, Sybertz E. Antihypertensive effects of 12 beta adrenoceptor antagonists in conscious spontaneously hypertensive 
rats: relationship to changes in plasma renin activity, heart rate and sympathetic nerve function. J Pharmacol Exp Ther 1986; 238(1): 378-387.

Arimura K, Egashira K, Nakamura R, Ide T, Tsutsui H, Shimokawa H, Takeshita A. Increased inactivation of nitric oxide is involved in coronary endothelial dysfunction in heart failure. Am J Physiol Heart Circ Physiol 2001; 280(1): H68-75.

Armas-Padilla MC, Armas-Hernandez MJ, Sosa-Canache B, Cammarata R, Pacheco B, Guerrero J, Carvajal AR, Hernandez-Hernandez R, Israili ZH, Valasco M. Nitric oxide and malondialdehyde in human hypertension. Am J Ther 2007; 14(2): 172-176.

Aruoma OI, Smith C, Cecchini R, Evans PJ, Halliwell B. Free radical scavenging and inhibition of lipid peroxidation by $\beta$-blockers and by agents that interfere with calcium metabolism: A physiologically-significant process? Biochem Pharmacol 1991; 42(4): 735-743.

Azarov I, Huang KT, Basu S, Gladwin MT, Hogg N, Kim-Shapiro DB. Nitric oxide scavenging by red blood cells as a function of hematocrit and oxygenation. J Biol Chem 2005; 280(47): 39024-39032.

Ballinger SW. Mitochondrial dysfunction in cardiovascular disease. Free Radic Biol Med 2005; 38(10): 1278-1295.

Bauer J. The so-called Cushing's Syndrome, its history, terminology and differential diagnosis. Acta Med Scand 1950; 137(6): 411-416.

Bayir H. Reactive oxygen species. Crit Care Med 2005; 33(12 (Suppl)): S498501. 
Bengele HH, McNamara ER, Alexander EA. Natriuresis after adrenal enucleation: effect of spironolactone and dexamethasone. Am J Physiol Renal Physiol 1977; 233(1): F8-12.

Bengtsson SHM, Gulluyan LM, Dusting GJ, Drummond GR. Novel isoforms of NADPH oxidase in vascular physiology and pathophysiology. Clin Exp Pharmacol Physiol 2003; 30(11): 849-854.

Bennett T, Gardiner SM. Prevention and reversal of isolation-induced systolic arterial hypertension in rats by treatment with beta-adrenoceptor antagonists. Br J Pharmacol 1979; 65(2): 205-213.

Berry CE, Hare JM. Xanthine oxidoreductase and cardiovascular disease: molecular mechanisms and pathophysiological implications. J Physiol 2004; 555(3): 589-606.

Biewenga GP, Haenen GRMM, Bast A. The pharmacology of the antioxidant lipoic acid. Gen Pharmacol 1997; 29(3): 315-331.

Birmingham MK, Sar M, Stumpf WE. Dexamethasone target sites in the central nervous system and their potential relevance to mental illness. Cell Mol Neurobiol 1993; 13(4): 373-386.

Borcsok I, Schairer HU, Sommer U, Wakley GK, Schneider U, Geiger F, Niethard FU, Ziegler R, Kasperk $\mathrm{CH}$. Glucocorticoids regulate the expression of the human osteoblastic endothelin A receptor gene. $J$ Exp Med 1998; 188(9): 1563-1573. 
Bos AF, van Asselt WA, Okken A. Dexamethasone treatment and fluid balance in preterm infants at risk for chronic lung disease. Acta Paediatr 2000; 89(5): 562-565.

Bradford MM. A rapid and sensitive method for the quantitation of microgram quantities of protein utilizing the principle of protein-dye binding. Anal Biochem 1976; 72: 248-254.

Brookes PS, Morse K, Ray D, Tompkins A, Young SM, Hilchey S, Salim S, Konopleva M, Andreeff M, Phipps R, Bernstein SH. The triterpenoid 2cyano-3,12-dioxooleana-1,9-dien-28-oic acid and Its derivatives elicit human lymphoid cell apoptosis through a novel pathway involving the unregulated mitochondrial permeability transition pore. Cancer Res 2007; 67(4): 1793-1802.

Brown-Sequard ME. Recherches experimentales sur le physiologie et la pathologie des capsules surrenales. Academy of Science Paris 1856; 43: $422-425$.

Brown MR, Fisher LA. Glucocorticoid suppression of the sympathetic nervous system and adrenal medulla. Life Sci 1986; 39(11): 1003-1012.

Bunag RD. Propranolol in DOCA hypertensive rats: development of hypertension inhibited and pressor responsiveness enhanced. Eur J Pharmacol 1977; 43(4): 323-331.

Burrell LM, Phillips PA, Stephenson JM, Risvanis J, Rolls KA, Johnston CI. Blood pressure-lowering effect of an orally active vasopressin $\mathrm{V} 1$ receptor antagonist in mineralocorticoid hypertension in the rat. Hypertension 1994; 23(6 Pt 1): 737-743. 
Burris JF, Waeber B, Nussberger J, Brunner HR. Enhanced acute antihypertensive effect of propranolol in the absence of circulating epinephrine in the rat. $J$ Cardiovasc Pharmacol 1984; 6(4): 697-700.

Cai H, Harrison DG. Endothelial dysfunction in cardiovascular diseases: the role of oxidant stress. Circ Res 2000; 87(10): 840-844.

Çakatay U. Pro-oxidant actions of [alpha]-lipoic acid and dihydrolipoic acid. Med Hypotheses 2006; 66(1): 110-117.

Campo ML, Kinnally KW, Tedeschi H. The effect of antimycin A on mouse liver inner mitochondrial membrane channel activity. J Biol Chem 1992; 267(12): 8123-8127.

Capdevila JH, Falck JR, Dishman E, Karara A. Cytochrome P-450 arachidonate oxygenase. Methods Enzymol 1990; 187: 385-394.

Carletti M, Cantiello M, Giantin M, Nebbia C, Cannizzo FT, Bollo E, Dacasto M. Serum antioxidant enzyme activities and oxidative stress parameters as possible biomarkers of exposure in veal calves illegally treated with dexamethasone. Toxicol In Vitro 2007; 21(2): 277-283.

Chabria NL, Gaitonde BB. Effect of dexamethasone on urine output and electrolyte excretion in rats. Arch Int Pharmacodyn Ther 1966; 162(2): 364-370.

Chakrabarti A, Sharma PL. A study on withdrawal related haemodynamic response in chronic propranolol treated conscious rats. Indian J Physiol Pharmacol 1993; 37(3): 235-237. 
Cheng J, Ou J-S, Singh H, Falck JR, Narsimhaswamy D, Pritchard KA, Jr., Schwartzman ML. 20-Hydroxyeicosatetraenoic acid causes endothelial dysfunction via eNOS uncoupling. Am J Physiol Heart Circ Physiol 2008; 294(2): H1018-1026.

Codde JP, Beilin LJ. Dietary fish oil prevents dexamethasone induced hypertension in the rat. Clin Sci (Lond) 1985; 69(6): 691-699.

Connell JM, Whitworth JA, Davies DL, Lever AF, Richards AM, Fraser R. Effects of ACTH and cortisol administration on blood pressure, electrolyte metabolism, atrial natriuretic peptide and renal function in normal man. $J$ Hypertens 1987; 5(4): 425-433.

Connell JM, Whitworth JA, Davies DL, Richards AM, Fraser R. Haemodynamic, hormonal and renal effects of adrenocorticotrophic hormone in sodiumrestricted man. J Hypertens 1988; 6(1): 17-23.

Corder R, Pralong F, Turnill D, Saudan P, Muller AF, Gaillard RC. Dexamethasone treatment increases neuropeptide $\mathrm{Y}$ levels in rat hypothalamic neurones. Life Sci 1988; 43(23): 1879-1886.

Cordero M, De Miguel M, Moreno Fernandez A, Carmona Lopez I, Garrido Maraver J, Cotan D, Gomez Izquierdo L, Bonal P, Campa F, Bullon P, Navas P, Sanchez Alcazar J. Mitochondrial dysfunction and mitophagy activation in blood mononuclear cells of fibromyalgia patients: implications in the pathogenesis of the disease. Arthritis Res Ther 2010; 12(1): R17 [Epub ahead of print].

Cosentino F, Sill JC, Katusic ZS. Role of superoxide anions in the mediation of endothelium-dependent contractions. Hypertension 1994; 23(2): 229-235. 
Crawford DR, Davies KJ. Adaptive response and oxidative stress. Environ. Health Perspect. 1994; 102 Suppl 10: 25-28.

Cremer DR, Rabeler R, Roberts A, Lynch B. Long-term safety of $\alpha$-lipoic acid (ALA) consumption: A 2-year study. Regul Toxicol Pharmacol 2006; 46(3): 193-201.

Dalle-Donne I, Rossi R, Colombo R, Giustarini D, Milzani A. Biomarkers of oxidative damage in human disease. Clin. Chem. 2006; 52(4): 601-623.

Dawson TL, Gores GJ, Nieminen AL, Herman B, Lemasters JJ. Mitochondria as a source of reactive oxygen species during reductive stress in rat hepatocytes. Am J Physiol Cell Physiol 1993; 264(4): C961-967.

Degli Esposti M. Measuring mitochondrial reactive oxygen species. Methods 2002; 26(4): 335-340.

Dhalla NS, Temsah RM, Netticadan T. Role of oxidative stress in cardiovascular diseases. J Hypertens 2000; 18(6): 655-673.

Distelmaier F, Koopman WJH, Testa ER, de Jong AS, Swarts HG, Mayatepek E, Smeitink JAM, Willems PHGM. Life cell quantification of mitochondrial membrane potential at the single organelle level. Cytometry Part A 2008; 73A(2): 129-138.

Dobrian AD, Schriver SD, Prewitt RL. Role of angiotensin II and free radicals in blood pressure regulation in a rat model of renal hypertension. Hypertension 2001; 38(3): 361-366. 
Drost CJ. Vessel diameter-dependent volume flow measurements using ultrasound. Proc San Diego Biomed Symp 1978; 17: 463-485.

Du J, Daniels DH, Asbury C, Venkataraman S, Liu J, Spitz DR, Oberley LW, Cullen JJ. Mitochondrial production of reactive oxygen species mediate dicumarol-induced cytotoxicity in cancer cells. J Biol Chem 2006; 281(49): 37416-37426.

Duranteau J, Chandel NS, Kulisz A, Shao Z, Schumacker PT. Intracellular signaling by reactive oxygen species during hypoxia in cardiomyocytes. $J$ Biol Chem 1998; 273(19): 11619-11624.

Dvorak K, Payne CM, Chavarria M, Ramsey L, Dvorakova B, Bernstein H, Holubec H, Sampliner RE, Guy N, Condon A, Bernstein C, Green SB, Prasad A, Garewal HS. Bile acids in combination with low pH induce oxidative stress and oxidative DNA damage: relevance to the pathogenesis of Barrettâ $€^{\mathrm{TM}_{\mathrm{S}}}$ oesophagus. Gut 2007; 56(6): 763-771.

Ebii K, Fukunaga R, Taniguchi T, Fujiwara M, Nakayama S, Saitoh Y, Kimura Y. Effects of chronic administration of carteolol on beta-adrenoceptors in spontaneously hypertensive rat heart. Jpn J Pharmacol 1991; 56(4): 505512.

Echtenkamp SF, Dandridge PF. Renal actions of neuropeptide Y in the primate. Am J Physiol Renal Physiol 1989; 256(4): F524-531.

Edvinsson L, Ekblad E, Hakanson R, Wahlestedt C. Neuropeptide Y potentiates the effect of various vasoconstrictor agents on rabbit blood vessels. $\mathrm{Br} J$ Pharmacol 1984; 83(2): 519-525. 
Eggena P, Barrett JD. Renin substrate release in response to perturbations of renin-angiotensin system. Am J Physiol Endocrinol Metab 1988; 254(4): E389-393.

El Midaoui A, Elimadi A, Wu L, Haddad PS, de Champlain J. Lipoic acid prevents hypertension, hyperglycemia, and the increase in heart mitochondrial superoxide production. Am J Hypertens 2003; 16(3): 173179.

El Midaoui A, Wu R, de Champlain J. Prevention of hypertension, hyperglycemia and vascular oxidative stress by aspirin treatment in chronically glucosefed rats. J Hypertens 2002; 20(7): 1407-1412.

Eustachius B (1564). Opuscula Anatomica de Renum Structura, Efficio et Administratione. Venice, V. V. Lucchino.

Faggiano A, Pivonello R, Spiezia S, De Martino MC, Filippella M, Di Somma C, Lombardi G, Colao A. Cardiovascular risk factors and common carotid artery caliber and stiffness in patients with Cushing's disease during active disease and 1 year after disease remission. J Clin Endocrinol Metab 2003; 88(6): 2527-2533.

Falardeau P, Martineau A. Prostaglandin I2 and glucocorticoid-induced rise in arterial pressure in the rat. $J$ Hypertens 1989; 7(8): 625-632.

Ferrario CM, Chappell MC. Angiotensin formation and degradation. In: Izzo Jr J. L., Sica D. A.,Black H. R., eds. Hypertension Primer: the essentials of high blood pressure: basic science, population science and clinical management. 4th ed. Philadelphia: Lippincott Williams Wilkins; 2008:5258. 
Flower RJ, Blackwell GJ. Anti-inflammatory steroids induce biosynthesis of a phospholipase A2 inhibitor which prevents prostaglandin generation. Nature 1979; 278(5703): 456-459.

Fraillon D, Wynne KN, Funder JW. Further studies on neomycin and experimental hypertension. Clin Exp Pharmacol Physiol 1984; 11(4): 339341.

Fraser TB, Turner SW, Mangos GJ, Ludbrook J, Whitworth JA. Comparison of telemetric and tail-cuff blood pressure monitoring in adrenocorticotrophic hormone-treated rats. Clin Exp Pharmacol Physiol 2001; 28(10): 831-835.

Fraser TB, Turner SW, Wen C, Li M, Burrell LM, Whitworth JA. Vasopressin V1a receptor antagonism does not reverse adrenocorticotrophin-induced hypertension in the rat. Clin Exp Pharmacol Physiol 2000; 27(11): 866870.

Garcia R, Debinski W, Gutkowska J, Kuchel O, Thibault G, Genest J, Cantin M. Gluco- and mineralocorticoids may regulate the natriuretic effect and the synthesis and release of atrial natriuretic factor by the rat atria in vivo. Biochem Biophys Res Commun 1985; 131(2): 806-814.

Gardiner SM, Kemp PA, March JE, Bennett T. Effects of dexamethasone and SB 209670 on the regional haemodynamic responses to lipopolysaccharide in conscious rats. Br J Pharmacol 1996; 118(1): 141-149.

Gardner DG, Hane S, Trachewsky D, Schenk D, Baxter JD. Atrial natriuretic peptide mRNA is regulated by glucocorticoids in vivo. Biochem Biophys Res Commun 1986; 139(3): 1047-1054. 
Girouard H, Chulak C, Wu L, Lejossec M, de Champlain J. N-acetylcysteine improves nitric oxide and $\alpha$-adrenergic pathways in mesenteric beds of spontaneously hypertensive rats. Am J Hypertens 2003; 16(7): 577-584.

Glyn J. The discovery and early use of cortisone. J R Soc Med 1998; 91(10): 513517.

Gomes A, Costa D, Lima JLFC, Fernandes E. Antioxidant activity of [beta]blockers: An effect mediated by scavenging reactive oxygen and nitrogen species? Bioorganic \& Medicinal Chemistry 2006; 14(13): 4568-4577.

Graham WF, Allen KJ, Coghlan JP, Denton DA, Humphery TJ, Scoggins BA, Whitworth JA. Haemodynamic changes in ACTH-incuded hypertension in sheep. Clin Exp Pharmacol Physiol 1980; 7(5): 569-572.

Grassi G, Esler M. How to assess sympathetic activity in humans. $J$ Hypertens 1999; 17(6): 719-734.

Griendling KK, Minieri CA, Ollerenshaw JD, Alexander RW. Angiotensin II stimulates NADH and NADPH oxidase activity in cultured vascular smooth muscle cells. Circ Res 1994; 74(6): 1141-1148.

Groemping Y, Rittinger K. Activation and assembly of the NADPH oxidase: a structural perspective. Biochem J 2005; 386(Pt 3): 401-416.

Grosser N, Schroder H. Aspirin protects endothelial cells from oxidant Damage via the nitric oxide-cGMP pathway. Arterioscler Thromb Vasc Biol 2003; 23(8): 1345-1351. 
Grossmann C, Scholz T, Rochel M, Bumke-Vogt C, Oelkers W, Pfeiffer AF, Diederich S, Bahr V. Transactivation via the human glucocorticoid and mineralocorticoid receptor by therapeutically used steroids in CV-1 cells: a comparison of their glucocorticoid and mineralocorticoid properties. Eur $J$ Endocrinol 2004; 151(3): 397-406.

Grunfeld JP, Eloy L, Moura AM, Ganeval D, Ramos-Frendo B, Worcel M. Effects of antiglucocorticoids on glucocorticoid hypertension in the rat. Hypertension 1985; 7(2): 292-299.

Gu J, Polak JM, Adrian TE, Allen JM, Tatemoto K, Bloom SR. Neuropeptide tyrosine (NPY)--a major cardiac neuropeptide. Lancet 1983; 1(8332): 1008-1010.

Guyton AC, Hall JE. The adrenocortical hormones. In: Schmitt W., Gruliow R.,Norwitz A., eds. Textbook of medical physiology. 10 ed. Pennsylvania: W.B. Saunders Company; 2000:869-883.

Guyton AC, Hall JE. The autonomic nervous system; and the adrenal medulla. In: Schmitt W., Gruliow R.,Norwitz A., eds. Textbook of medical physiology. Pennsylvania: W. B. Saunders Company; 2000:697-715.

Haack D, Mohring J, Mohring B, Petri M, Hackenthal E. Comparative study on development of corticosterone and DOCA hypertension in rats. Am J Physiol Renal Physiol 1977; 233(5): F403-411.

Haas JA, Krier JD, Bolterman RJ, Juncos LA, Romero JC. Low-dose angiotensin II increases free isoprostane levels in plasma. Hypertension 1999; 34(4): 983-986. 
Haass M, Cheng B, Richardt G, Lang RE, Schomig A. Characterization and presynaptic modulation of stimulation-evoked exocytotic co-release of noradrenaline and neuropeptide $\mathrm{Y}$ in guinea pig heart. Naunyn Schmiedebergs Arch Pharmacol 1989; 339(1-2): 71-78.

Hagen TM, Ingersoll RT, Lykkesfeldt J, Liu J, Wehr CM, Vinarsky V, Bartholomew JC, Ames BN. (R)- $\alpha$-Lipoic acid-supplemented old rats have improved mitochondrial function, decreased oxidative damage, and increased metabolic rate. FASEB J. 1999; 13(2): 411-418.

Haigh RM, Jones CT. Effect of glucocorticoids on alpha-1 adrenergic receptorbinding in rat vascular smooth muscle. J Mol Endocrinol 1990; 5(1): 4148.

Hall JE, Mizelle HL, Hildebrandt DA, Brands MW. Abnormal pressure natriuresis. A cause or a consequence of hypertension? Hypertension 1990; 15(6): 547-559.

Han D, Antunes F, Canali R, Rettori D, Cadenas E. Voltage-dependent anion channels control the release of the superoxide anion from mitochondria to cytosol. J Biol Chem 2003; 278(8): 5557-5563.

Handa M, Kondo K, Suzuki H, Saruta T. Urinary prostaglandin E2 and kallikrein excretion in glucocorticoid hypertension in rats. Clin Sci (Lond) 1983; 65(1): 37-42.

Handa M, Kondo K, Suzuki H, Saruta T. Dexamethasone hypertension in rats: role of prostaglandins and pressor sensitivity to norepinephrine. Hypertension 1984; 6(2 Pt 1): 236-241. 
Harrison DG, Gongora MC, Guzik TJ, Widder J. Oxidative stress and hypertension. J Am Soc Hypertens 2007; 1(1): 30-44.

Hayamizu S, Kanda K, Ohmori S, Murata Y, Seo H. Glucocorticoids potentiate the action of atrial natriuretic polypeptide in adrenalectomized rats. Endocrinology 1994; 135(6): 2459-2464.

He Y. Angiotensin II receptor blockade in glucocorticoid-induced hypertension in rats. Canberra: The John Curtin School of Medical Research, The Australian National University; 2008.

Hein TW, Kuo L. LDLs impair vasomotor function of the coronary microcirculation : role of superoxide anions. Circ Res 1998; 83(4): 404414.

Heumuller S, Wind S, Barbosa-Sicard E, Schmidt HHHW, Busse R, Schroder K, Brandes RP. Apocynin is not an inhibitor of vascular NADPH oxidases but an antioxidant. Hypertension 2008; 51(2): 211-217.

Hirata F, Schiffmann E, Venkatasubramanian K, Salomon D, Axelrod J. A phospholipase A2 inhibitory protein in rabbit neutrophils induced by glucocorticoids. Proc Natl Acad Sci U S A 1980; 77(5): 2533-2536.

Homma M, Tanaka A, Hino K, Takamura H, Hirano T, Oka K, Kanazawa M, Miwa T, Notoya Y, Niitsuma T, Hayashi T. Assessing systemic 11 $\beta$ hydroxysteroid dehydrogenase with serum cortisone/cortisol ratios in healthy subjects and patients with diabetes mellitus and chronic renal failure. Metabolism 2001; 50(7): 801-804. 
Hu L, Zhang Y, Lim PS, Miao Y, Tan C, McKenzie KUS, Schyvens CG, Whitworth JA. Apocynin but not L-arginine prevents and reverses dexamethasone-induced hypertension in the rat. Am J Hypertens 2006; 19(4): 413-418.

Huang LQ, Whitworth JA, Chesterman CN. Effects of cyclosporin A and dexamethasone on haemostatic and vasoactive functions of vascular endothelial cells. Blood Coagul Fibrinolysis 1995; 6(5): 438-445.

Huang PL, Huang Z, Mashimo H, Bloch KD, Moskowitz MA, Bevan JA, Fishman MC. Hypertension in mice lacking the gene for endothelial nitric oxide synthase. Nature 1995; 377(6546): 239-242.

Humphrey TJ, Fan JS, Coghlan JP, Denton DA, Scoggins BA, Stewart KW, Whitworth JA. Inter-relationships between sodium and potassium intake and the blood pressure effects of ACTH in sheep. $J$ Hypertens $1983 ; 1(1)$ : 19-26.

Igarashi T, Nakajima Y, Ohtake S. Antihypertensive effect of combined treatment with alpha- and beta-adrenergic blockers in the spontaneously hypertensive rat. Jpn Circ J 1977; 41(8): 903-911.

Iijima F, Malik KU. Contribution of vasopressin in dexamethasone-induced hypertension in rats. Hypertension 1988; 11(2 Pt 2): I42-46.

Intengan HD, Schiffrin EL. Structure and mechanical properties of resistance arteries in hypertension : role of adhesion molecules and extracellular matrix determinants. Hypertension 2000; 36(3): 312-318. 
Ishihara T, Chin WP, Yoshida Y, Sun HT, Mitomi A, Ishibashi T, Tamura K, Imai S. Effects of chronic oral administration of a new beta-blocker, bopindolol, on serum lipoprotein concentrations and blood pressure of spontaneously hypertensive rats. Arch Int Pharmacodyn Ther 1989; 302: 145-157.

Iuchi T, Akaike M, Mitsui T, Ohshima Y, Shintani Y, Azuma H, Matsumoto T. Glucocorticoid excess induces superoxide production in vascular endothelial cells and elicits vascular endothelial dysfunction. Circ Res 2003; 92(1): 81-87.

Janssen BJA, De Celle T, Debets JJM, Brouns AE, Callahan MF, Smith TL. Effects of anesthetics on systemic hemodynamics in mice. Am J Physiol Heart Circ Physiol 2004; 287(4): H1618-1624.

Johns DG, Dorrance AM, Tramontini NL, Webb RC. Glucocorticoids inhibit tetrahydrobiopterin-dependent endothelial function. Exp Biol Med 2001; 226(1): 27-31.

Kalimi M. Role of antiglucocorticoid RU 486 on dexamethasone-induced hypertension in rats. Am J Physiol Endocrinol Metab 1989; 256(5): E682685.

Kanse SM, Takahashi K, Warren JB, Ghatei M, Bloom SR. Glucocorticoids induce endothelin release from vascular smooth muscle cells but not endothelial cells. Eur J Pharmacol 1991; 199(1): 99-101.

Kelly JJ, Tam SH, Williamson PM, Lawson J, Whitworth JA. The nitric oxide system and cortisol-induced hypertension in humans. Clin Exp Pharmacol Physiol 1998; 25(11): 945-946. 
Kennedy B, Elayan H, Ziegler MG. Glucocorticoid hypertension and nonadrenal phenylethanolamine N-methyltransferase. Hypertension 1993; 21(4): 415419.

Kennedy B, Ziegler MG. Cardiac epinephrine synthesis. Regulation by a glucocorticoid. Circulation 1991; 84(2): 891-895.

Kim H, Lee JM, Park JS, Jo SA, Kim Y-O, Kim C-W, Jo I. Dexamethasone coordinately regulates angiopoietin-1 and VEGF: A mechanism of glucocorticoid-induced stabilization of blood-brain barrier. Biochem Biophys Res Commun 2008; 372(1): 243-248.

Kim MS, Park JY, Namkoong C, Jang PG, Ryu JW, Song HS, Yun JY, Namgoong IS, Ha J, Park IS, Lee IK, Viollet B, Youn JH, Lee HK, Lee KU. Anti-obesity effects of alpha-lipoic acid mediated by suppression of hypothalamic AMP-activated protein kinase. Nat Med 2004; 10(7): 727733.

Kimura S, Zhang G-X, Nishiyama A, Shokoji T, Yao L, Fan Y-Y, Rahman M, Abe $Y$. Mitochondria-derived reactive oxygen species and vascular MAP kinases: comparison of angiotensin II and diazoxide. Hypertension 2005; 45(3): 438-444.

Kishi K, Kawashima K, Sokabe H, Saito K. Chronic effects of arotinolol (S-596) in spontaneously hypertensive rats. J Pharmacobiodyn 1985; 8(1): 50-55.

Knowles RG, Moncada S. Nitric oxide synthases in mammals. Biochem J 1994; 298 ( Pt 2): 249-258. 
Koeppen BM, Katz AI, Lindheimer MD. Effect of general anaesthesia on renal haemodynamics in the rat. Clin Sci (Lond) 1979; 57(5): 469-471.

Konagaya M, Bernard PA, Max SR. Blockade of glucocorticoid receptor binding and inhibition of dexamethasone-induced muscle atrophy in the rat by RU38486, a potent glucocorticoid antagonist. Endocrinology 1986; 119(1): $375-380$.

Kondo-Nakamura M, Shintani-Ishida K, Uemura K, Yoshida K-i. Brief exposure to carbon monoxide preconditions cardiomyogenic cells against apoptosis in ischemia-reperfusion. Biochem Biophys Res Commmun 2010; 393(3): 449-454.

Kontos HA, Wei EP, Ellis EF, Jenkins LW, Povlishock JT, Rowe GT, Hess ML. Appearance of superoxide anion radical in cerebral extracellular space during increased prostaglandin synthesis in cats. Circ Res 1985; 57(1): 142-151.

Koppers AJ, De Iuliis GN, Finnie JM, McLaughlin EA, Aitken RJ. Significance of mitochondrial reactive oxygen species in the generation of oxidative stress in spermatozoa. J Clin Endocrinol Metab 2008; 93(8): 3199-3207.

Krishna CM, Liebmann JE, Kaufman D, DeGraff W, Hahn SM, McMurry T, Mitchell JB, Russo A. The catecholic metal sequestering agent 1,2dihydroxybenzene-3,5-disulfonate confers protection against oxidative cell damage. Arch Biochem Biophys 1992; 294(1): 98-106.

Krug S, Zhang Y, Mori TA, Croft KD, Vickers JJ, Langton LK, Whitworth JA. NAcetylcysteine prevents but does not reverse dexamethasone-induced hypertension. Clin Exp Pharmacol Physiol 2008; 35(8): 979-981. 
Kubo T, Esumi K, Ennvu K. Effect of chronic treatment with propranolol on blood pressure and cardiovascular reactivity in spontaneously hypertensive rats. Arch Int Pharmacodyn Ther 1977; 227(1): 30-40.

Kumai T, Asoh K, Tateishi T, Tanaka M, Watanabe M, Shimizu H, Kobayashi S. Involvement of tyrosine hydroxylase up regulation in dexamethasoneinduced hypertension of rats. Life Sci 2000; 67(16): 1993-1999.

Kumar KV, Das UN. Are free radicals involved in the pathobiology of human essential hypertension? Free Radic Res Commun 1993; 19(1): 59-66.

Kurland GS, Freedberg AS. The potentiating effect of ACTH and of cortisone of pressor response to intravenous infusion of L-nor-epinephrine. Proc Soc Exp Biol Med 1951; 78(1): 28-31.

Kushiro T, Fujita H, Hisaki R, Asai T, Ichiyama I, Kitahara Y, Koike M, Sugiura H, Saito F, Otsuka Y, Kanmatsuse K. Oxidative stress in the Dahl saltsensitive hypertensive rat. Clin Exp Hypertens 2005; 27(1): 9 - 15.

Lachance D, Garcia R, Gutkowska J, Cantin M, Thibault G. Mechanisms of release of atrial natriuretic factor I. Effect of several agonists and steroids on its release by atrial minces. Biochem Biophys Res Commun 1986; 135(3): 1090-1098.

Lassegue B, Clempus RE. Vascular NAD(P)H oxidases: specific features, expression, and regulation. Am J Physiol Regul Integr Comp Physiol 2003; 285(2): R277-297.

Leenen FH, Prowse S. Time-course of changes in cardiac hypertrophy and pressor mechanisms in two-kidney, one clip hypertensive rats during treatment 
with minoxidil, enalapril or after uninephrectomy. $J$ Hypertens $1987 ; 5(1)$ : 73-83.

Lefer AM, Manwaring JL, Verrier RL. Effect of corticosteroids on the cardiovascular responses to angiotensin and norepinephrine. J Pharmacol Exp Ther 1966; 154(1): 83-91.

Lenaz G. Role of mitochondria in oxidative stress and ageing. Biochim Biophys Acta 1998; 1366(1-2): 53-67.

Lerman LO, Nath KA, Rodriguez-Porcel M, Krier JD, Schwartz RS, Napoli C, Romero JC. Increased oxidative stress in experimental renovascular hypertension. Hypertension 2001; 37(2): 541-546.

Lewis GD, Campbell WB, Johnson AR. Inhibition of prostaglandin synthesis by glucocorticoids in human endothelial cells. Endocrinology 1986; 119(1): $62-69$.

Li M, Fraser T, Wang J, Whitworth JA. Dexamethasone-induced hypertension in the rat: effects of L-arginine. Clin Exp Pharmacol Physiol 1997; 24(9-10): 730-732.

Li M, Wen C, Fraser T, Whitworth JA. Adrenocorticotrophin-induced hypertension: effects of mineralocorticoid and glucocorticoid receptor antagonism. J Hypertens 1999; 17(3): 419-426.

Li M, Wen C, Martin A, Whitworth JA. Dehydroepiandrosterone does not prevent adrenocorticotrophin-induced hypertension in conscious rats. Clin Exp Pharmacol Physiol 1996; 23(5): 435-437. 
Li M, Whitworth JA. Role of the sympathetic nervous system in the onset of hypertension in the rat: the effect of 6-OH-dopamine. Clin Exp Pharmacol Physiol 1991; 18(4): 197-204.

Lin F, Abraham NG, Schwartzman ML. Cytochrome P450 arachidonic acid omega-hydroxylation in the proximal tubule of the rat kidney. Ann $N Y$ Acad Sci 1994; 744(Cellular Generation, Transport, and Effects of Eicosanoids: Biological Roles and Pharmacological Intervention): 11-24.

Liu X, Zhao Y, Wang L, Yang X, Zheng Z, Zhang Y, Chen F, Liu H. Overexpression of cytochrome $\mathrm{P} 4504 \mathrm{~F} 2$ in mice increases 20hydroxyeicosatetraenoic acid production and arterial blood pressure. Kidney Int 2009; 75(12): 1288-1296.

Lou Y-k, Wen C, Li M, Adams DJ, Wang M-x, Yang F, Morris BJ, Whitworth JA. Decreased renal expression of nitric oxide synthase isoforms in adrenocorticotropin-induced and corticosterone-induced hypertension. Hypertension 2001; 37(4): 1164-1170.

Lund KC, Peterson LL, Wallace KB. Absence of a universal mechanism of Mitochondrial toxicity by nucleoside analogs. Antimicrob Agents Chemother 2007; 51(7): 2531-2539.

Lundberg JM, Terenius L, Hokfelt T, Goldstein M. High levels of neuropeptide Y in peripheral noradrenergic neurons in various mammals including man. Neurosci Lett 1983; 42(2): 167-172.

Ma K, Mallidis C, Bhasin S, Mahabadi V, Artaza J, Gonzalez-Cadavid N, Arias J, Salehian B. Glucocorticoid-induced skeletal muscle atrophy is associated with upregulation of myostatin gene expression. Am J Physiol Endocrinol Metab 2003; 285(2): E363-371. 
Macefield VG, Williamson PM, Wilson LR, Kelly JJ, Gandevia SC, Whitworth JA. Muscle sympathetic vasoconstrictor activity in hydrocortisone-induced hypertension in humans. Blood Press 1998; 7(4): 215-222.

MacKenzie A, Martin W. Loss of endothelium-derived nitric oxide in rabbit aorta by oxidant stress: restoration by superoxide dismutase mimetics. $\mathrm{Br} J$ Pharmacol 1998; 124(4): 719-728.

Madamanchi NR, Runge MS. Mitochondrial dysfunction in atherosclerosis. Circ Res 2007; 100(4): 460-473.

Magiakou MA, Smyrnaki P, Chrousos GP. Hypertension in Cushing's syndrome. Best Pract \& Res Clin Endocrinol Metab 2006; 20(3): 467-482.

Mak IT, Weglicki WB. Protection by beta-blocking agents against free radicalmediated sarcolemmal lipid peroxidation. Circ Res 1988; 63(1): 262-266.

Mak IT, Weglicki WB. Potent antioxidant properties of 4-hydroxyl-propranolol. J Pharmacol Exp Ther 2004; 308(1): 85-90.

Mangos G, Walker B, Williamson P, Whitworth J, Kelly J. Effect of Synthetic Corticosteroids on Vascular Reactivity in the Human Forearm. Clin Exp Hypertens 2006; 28(8): 707-718.

Mangos GJ, Turner SW, Fraser TB, Whitworth JA. The role of corticosterone in corticotrophin (ACTH)-induced hypertension in the rat. J Hypertens 2000; 18(12): 1849-1855. 
Marangon K, Devaraj S, Tirosh O, Packer L, Jialal I. Comparison of the effect of $\alpha$-lipoic acid and $\alpha$-tocopherol supplementation on measures of oxidative stress. Free Radic Biol Med 1999; 27(9-10): 1114-1121.

Marley R, Holt S, Fernando B, arry D, Anand R, Goodier D, Davies S, Moore K. Lipoic acid prevents development of the hyperdynamic circulation in anesthetized rats with biliary cirrhosis. Hepatology 1999; 29(5): 13581363.

Mason CS, Myers CS, Kendall EC. The chemistry of crystalline substances isolated from suprarenal gland. J Biol Chem 1936; 114: 613-631.

Mattiasson G. Analysis of mitochondrial generation and release of reactive oxygen species. Cytometry Part A 2004; 62A(2): 89-96.

Mattiasson G. Flow cytometric analysis of isolated liver mitochondria to detect changes relevant to cell death. Cytometry Part A 2004a; 60A(2): 145-154.

Meany DL, Poe BG, Navratil M, Moraes CT, Arriaga EA. Superoxide released into the mitochondrial matrix. Free Radic Biol Med 2006; 41(6): 950-959.

Medhora M, Chen Y, Gruenloh S, Harland D, Bodiga S, Zielonka J, Gebremedhin D, Gao Y, Falck JR, Anjaiah S, Jacobs ER. 20-HETE increases superoxide production and activates NAPDH oxidase in pulmonary artery endothelial cells. Am J Physiol Lung Cell Mol Physiol 2008; 294(5): L902-911.

Medvei VC. The history of Cushing's disease: a controversial tale. $J R$ Soc Med 1991; 84(6): 363-366. 
Mendlowitz M, Naftchi N, Weinreb HL, Gitlow SE. Effect of prednisone on digital vascular reactivity in normotensive and hypertensive subjects. $J$ Appl Physiol 1961; 16(1): 89-94.

Miao Y, Zhang Y, Lim PS, Kanjanapan Y, Mori TA, Croft KD, Earl J, Lee SY, McKenzie KU, Hu L, Whitworth JA. Folic Acid prevents and partially reverses glucocorticoid-induced hypertension in the rat. Am J Hypertens 2007; 20(3): 304-310.

Mills EH, Coghlan JP, Denton DA, Spence CD, Whitworth JA, Scoggins BA. The effect of sodium depletion and potassium loading on cortisol induced hypertension in sheep. Acta Endocrinol (Copenh) 1986; 113(2): 298-304.

MIMS_Online. (11 August 2008). "DBL Dexamethasone Sodium Phosphate Injection."

Mitchell BM, Dorrance AM, Webb RC. GTP cyclohydrolase 1 downregulation contributes to glucocorticoid hypertension in rats. Hypertension 2003; 41(3): 669-674.

Miyata N, Roman RJ. Role of 20-hydroxyeicosatetraenoic acid (20-HETE) in vascular system. J Smooth Muscle Res 2005; 41(4): 175-193.

Moini H, Packer L, Saris N-EL. Antioxidant and prooxidant activities of $\alpha$-lipoic acid and dihydrolipoic acid. Toxicol Appl Pharmacol 2002; 182(1): 84-90.

Mondo CK, Yang W-S, Su J-Z, Huang T-G. Atorvastatin prevented and reversed dexamethasone-induced hypertension in the rat. Clin Exp Hypertens 2006; 28(5): 499-509. 
Mondo CK, Yang W-S, Zhang N, Huang T-G. Anti-oxidant effects of atorvastatin in dexamethasone-induced hypertension in the rat. Clin Exp Pharmacol Physiol 2006a; 33(11): 1029-1034.

Mondo CK, Zhang Y, Possamai VdM, Miao Y, Schyvens CG, McKenzie KUS, $\mathrm{Hu}$ L, Guo Z, Whitworth JA. N-Acetylcysteine antagonizes the development but does not reverse ACTH-induced hypertension in the rat. Clin Exp Hypertens 2006; 28(2): 73 -84.

Montrella-Waybill M, Clore JN, Schoolwerth AC, Watlington CO. Evidence that high dose cortisol-induced $\mathrm{Na}+$ retention in man is not mediated by the mineralocorticoid receptor. J Clin Endocrinol Metab 1991; 72(5): 10601066.

Morgan ET, Ullrich V, Daiber A, Schmidt P, Takaya N, Shoun H, McGiff JC, Oyekan A, Hanke CJ, Campbell WB, Park C-S, Kang J-S, Yi H-G, Cha YN, Mansuy D, Boucher J-L. Cytochromes P450 and flavin monooxygenases-targets and sources of nitric oxide. Drug Metab Dispos 2001; 29(11): 1366-1376.

Mori TA, Croft KD, Puddey IB, Beilin LJ. An improved method for the measurement of urinary and plasma $F_{2}$-isoprostanes using gas chromatography-mass spectrometry. Anal Biochem 1999; 268(1): 117125.

Morrow JD, Hill KE, Burk RF, Nammour TM, Badr KF, Roberts LJ, 2nd. A series of prostaglandin $\mathrm{F}_{2}$-like compounds are produced in vivo in humans by a non-cyclooxygenase, free radical-catalyzed mechanism. Proc Natl Acad Sci U S A 1990; 87(23): 9383-9387. 
Morrow JD, Roberts LJ. The isoprostanes: Current knowledge and directions for future research. Biochem Pharmacol 1996; 51(1): 1-9.

Mosier HD, Jr., Spencer EM, Dearden LC, Jansons RA. The effect of glucocorticoids on plasma insulin-like growth factor I concentration in the rat fetus. Pediatr Res 1987; 22(1): 92-95.

Moura AM, Worcel M. Anti-hypertensive action of the effects of spironolactone on the permeability of smooth muscles to $\mathrm{Na}+$ and $\mathrm{Rb}+$. J Physiol (Paris) 1981; 77(8): A2-A3.

Mukhopadhyay P, Rajesh M, Hasko G, Hawkins BJ, Madesh M, Pacher P. Simultaneous detection of apoptosis and mitochondrial superoxide production in live cells by flow cytometry and confocal microscopy. Nat Protocols 2007; 2(9): 2295-2301.

Mukhopadhyay P, Rajesh M, Yoshihiro K, Hasko G, Pacher P. Simple quantitative detection of mitochondrial superoxide production in live cells. Biochem Biophys Res Commun 2007a; 358(1): 203-208.

Muller FL, Liu Y, Van Remmen H. Complex III releases superoxide to both sides of the inner mitochondrial membrane. J Biol Chem 2004; 279(47): 4906449073.

Munusamy S, MacMillan-Crow LA. Mitochondrial superoxide plays a crucial role in the development of mitochondrial dysfunction during high glucose exposure in rat renal proximal tubular cells. Free Radic Biol Med 2009; 46(8): 1149-1157. 
Murasawa S, Matsubara H, Kizima K, Maruyama K, Mori Y, Inada M. Glucocorticoids regulate $\mathrm{Vla}$ vasopressin receptor expression by increasing mRNA stability in vascular smooth muscle cells. Hypertension 1995; 26(4): 665-669.

Muthalif MM, Karzoun NA, Gaber L, Khandekar Z, Benter IF, Saeed AE, Parmentier J-H, Estes A, Malik KU. Angiotensin II-induced hypertension : contribution of Ras GTPase/mitogen-activated protein kinase and cytochrome P450 metabolites. Hypertension 2000; 36(4): 604-609.

Myrsen-Axcrona U, Karlsson S, Sundler F, Ahren B. Dexamethasone induces neuropeptide Y (NPY) expression and impairs insulin release in the insulin-producing cell line RINm5F. Release of NPY and insulin through different pathways. J Biol Chem 1997; 272(16): 10790-10796.

Myrsen U, Ahren B, Sundler F. Dexamethasone-induced neuropeptide Y expression in rat islet endocrine cells. Rapid reversibility and partial prevention by insulin. Diabetes 1996; 45(10): 1306-1316.

N'Gankam V, Uehlinger D, Dick B, Frey BM, Frey FJ. Increased cortisol metabolites and reduced activity of $11 \beta$-hydroxysteroid dehydrogenase in patients on hemodialysis. Kidney Int 2002; 61(5): 1859-1866.

Nabha L, Garbern JC, Buller CL, Charpie JR. Vascular oxidative stress precedes high blood pressure in spontaneously hypertensive rats. Clin. Exp. Hypertens. 2005; 27(1): $71-82$.

Nakamoto H, Suzuki H, Kageyama Y, Murakami M, Naitoh M, Saruta T. Central nervous system mediates an antihypertensive property in glucocorticoid hypertension in dogs. J Hypertens 1995; 13(10): 1169-1179. 
Nakamoto H, Suzuki H, Kageyama Y, Murakami M, Ohishi A, Naitoh M, Ichihara A, Saruta T. Depressor systems contribute to hypertension induced by glucocorticoid excess in dogs. $J$ Hypertens 1992; 10(6): 561569.

Nakamoto H, Suzuki H, Kageyama Y, Ohishi A, Murakami M, Naitoh M, Saruta T. Characterization of alterations of hemodynamics and neuroendocrine hormones in dexamethasone induced hypertension in dogs. Clin Exp Hypertens A 1991; 13(4): 587-606.

Nakayama K, Okamoto F, Harada Y. Antimycin A: isolation from a new Streptomyces and activity against rice plant blast fungi. J Antibiot (Tokyo) 1956; 9(2): 63-66.

Nakazono K, Watanabe N, Matsuno K, Sasaki J, Sato T, Inoue M. Does superoxide underlie the pathogenesis of hypertension? Proc Natl Acad Sci U S A 1991; 88(22): 10045-10048.

Nasjletti A, Erman A, Cagen LM, Baer PG. Plasma concentrations, renal excretion, and tissue release of prostaglandins in the rat with dexamethasone-induced hypertension. Endocrinology 1984; 114(3): 10331040.

Nieman LK, Biller BMK, Findling JW, Newell-Price J, Savage MO, Stewart PM, Montori VM. The diagnosis of Cushing's syndrome: An Endocrine Society clinical practice guideline. J Clin Endocrinol Metab 2008; 93(5): 15261540.

Nohl H, Gille L, Staniek K. Intracellular generation of reactive oxygen species by mitochondria. Biochem Pharmacol 2005; 69(5): 719-723. 
Okuno T, Suzuki H, Saruta T. Dexamethasone hypertension in rats. Clin Exp Hypertens 1981; 3(5): 1075-1086.

Ong SLH, Zhang Y, Whitworth JA. Reactive oxygen species and glucocorticoidinduced hypertension. Clin Exp Pharmacol Physiol 2008; 35(4): 477-482.

Owens GK. Influence of blood pressure on development of aortic medial smooth muscle hypertrophy in spontaneously hypertensive rats. Hypertension 1987; 9(2): 178-187.

Oyekan AO, Youseff T, Fulton D, Quilley J, McGiff JC. Renal cytochrome P450 omega-hydroxylase and epoxygenase activity are differentially modified by nitric oxide and sodium chloride. J Clin Invest 1999; 104(8): 11311137.

Pacher P, Beckman JS, Liaudet L. Nitric oxide and peroxynitrite in health and disease. Physiol Rev 2007; 87(1): 315-424.

Pagano PJ, Clark JK, Cifuentes-Pagano ME, Clark SM, Callis GM, Quinn MT. Localization of a constitutively active, phagocyte-like NADPH oxidase in rabbit aortic adventitia: Enhancement by angiotensin II. Proc Natl Acad Sci U S A 1997; 94(26): 14483-14488.

Pagano PJ, Ito Y, Tornheim K, Gallop PM, Tauber AI, Cohen RA. An NADPH oxidase superoxide-generating system in the rabbit aorta. Am J Physiol 1995; 268(6 Pt 2): H2274-2280.

Pallotti F, Lenaz G. Isolation and subfractionation of mitochondria from animal cells and tissue culture lines. Methods Cell Biol 2001; 65: 1-35. 
Pamnani MB, Clough DL, Haddy FJ. Altered activity of the sodium-potassium pump in arteries of rats with steroid hypertension. Clin Sci Mol Med Suppl 1978; 4: 41s-43s.

Paravicini TM, Gulluyan LM, Dusting GJ, Drummond GR. Increased NADPH oxidase activity, gp9lphox expression, and endothelium-dependent vasorelaxation during neointima formation in rabbits. Circ Res 2002; 91(1): 54-61.

Patrono C, FitzGerald GA. Isoprostanes: potential markers of oxidant stress in atherothrombotic disease. Arterioscler Thromb Vasc Biol 1997; 17(11): 2309-2315.

Payne CM, Weber C, Crowley-Skillicorn C, Dvorak K, Bernstein H, Bernstein C, Holubec H, Dvorakova B, Garewal H. Deoxycholate induces mitochondrial oxidative stress and activates NF-\{kappa\}B through multiple mechanisms in HCT-116 colon epithelial cells. Carcinogenesis 2007; 28(1): 215-222.

Pernow J, Lundberg JM. Modulation of noradrenaline and neuropeptide Y (NPY) release in the pig kidney in vivo: involvement of alpha 2, NPY and angiotensin II receptors. Naunyn Schmiedebergs Arch Pharmacol 1989; 340(4): 379-385.

Pham N-A, Robinson BH, Hedley DW. Simultaneous detection of mitochondrial respiratory chain activity and reactive oxygen in digitonin-permeabilized cells using flow cytometry. Cytometry 2000; 41(4): 245-251.

Pirpiris M, Sudhir K, Yeung S, Jennings G, Whitworth JA. Pressor responsiveness in corticosteroid-induced hypertension in humans. Hypertension 1992; 19(6): 567-574. 
Pirpiris M, Yeung S, Dewar E, Jennings GL, Whitworth JA. Hydrocortisoneinduced hypertension in men. The role of cardiac output. Am J Hypertens 1993; 6(4): 287-294.

Racotta R, Soto-Mora LM. Specificity of alpha- and beta-adrenergic inhibition of water and food intake. Physiol Behav 1993; 53(2): 361-365.

Rajagopalan S, Kurz S, Munzel T, Tarpey M, Freeman BA, Griendling KK, Harrison DG. Angiotensin II-mediated hypertension in the rat increases vascular superoxide production via membrane NADH/NADPH oxidase activation . Contribution to alterations of vasomotor tone. J Clin Invest 1996; 97(8): 1916-1923.

Rajashree S, Puvanakrishnan R. Dexamethasone induced alterations in the levels of proteases involved in blood pressure homeostasis and blood coagulation in rats. Mol Cell Biochem 1999; 197(1-2): 203-208.

Rajesh M, Mukhopadhyay P, Batkai S, Hasko G, Liaudet L, Drel VR, Obrosova IG, Pacher P. Cannabidiol attenuates high glucose-induced endothelial cell inflammatory response and barrier disruption. Am J Physiol Heart Circ Physiol 2007; 293(1): H610-619.

Ramachandran A, Levonen A-L, Brookes PS, Ceaser E, Shiva S, Barone MC, Darley-Usmar V. Mitochondria, nitric oxide, and cardiovascular dysfunction. Free Radic Biol Med 2002; 33(11): 1465-1474.

Rang HP, Dale MM, Ritter JM, Flower RJ. The pituitary and the adrenal cortex. Rang and Dale's Pharmacology. 6 ed. Philadelphia: Churchill Livingstone Elsevier; 2007:420-436. 
Rebuffat AG, Tam S, Nawrocki AR, Baker ME, Frey BM, Frey FJ, Odermatt A. The 11-ketosteroid 11-ketodexamethasone is a glucocorticoid receptor agonist. Mol Cell Endocrinol 2004; 214(1-2): 27-37.

Reckelhoff JF, Zhang H, Srivastava K, Roberts LJ, II, Morrow JD, Romero JC. Subpressor doses of angiotensin II increase plasma $F_{2}$-isoprostanes in rats. Hypertension 2000; 35(1): 476-479.

Rees DD, Palmer RMJ, Moncada S. Role of endothelium-derived nitric oxide in the regulation of blood pressure. Proc Natl Acad Sci U S A 1989; 86(9): 3375-3378.

Reichstein T (1962). Nobel Lectures (Physiology or Medicine). Amsterdam, Elsevier.

Richter C. Do mitochondrial DNA fragments promote cancer and aging? FEBS Letters 1988; 241(1-2): 1-5.

Robinson KM, Janes MS, Beckman JS. The selective detection of mitochondrial superoxide by live cell imaging. Nat. Protocols 2008; 3(6): 941-947.

Robinson KM, Janes MS, Pehar M, Monette JS, Ross MF, Hagen TM, Murphy MP, Beckman JS. Selective fluorescent imaging of superoxide in vivo using ethidium-based probes. Proc Natl Acad Sci U S A 2006: 0601945103.

Rodriguez-Iturbe B, Sepassi L, Quiroz Y, Ni Z, Vaziri ND. Association of mitochondrial SOD deficiency with salt-sensitive hypertension and accelerated renal senescence. $J$ Appl Physiol 2007; 102(1): 255-260. 
Roman RJ. P-450 metabolites of arachidonic acid in the control of cardiovascular function. Physiol Rev 2002; 82(1): 131-185.

Rose BD, Post TW. Regulation of the effective circulating volume. In: Wonsciewicz M., McCullough K.,Davis K., eds. Clinical physiology of acid base and electrolyte disorders. 5th ed. New York: McGraw-Hill; 2001:258-284.

Ross MF, Kelso GF, Blaikie FH, James AM, Cocheme HM, Filipovska A, Da Ros T, Hurd TR, Smith RA, Murphy MP. Lipophilic triphenylphosphonium cations as tools in mitochondrial bioenergetics and free radical biology. Biochemistry (Mosc) 2005; 70(2): 222-230.

Rothschild AJ, Langlais PJ, Schatzberg AF, Walsh FX, Cole JO, Bird ED. Dexamethasone increases plasma free dopamine in man. $J$ Psychiatr Res 1984; 18(3): 217-223.

Roubert P, Viossat I, Lonchampt MO, Chapelat M, Schulz J, Plas P, GillardRoubert V, Chabrier PE, Braquet P. Endothelin receptor regulation by endothelin synthesis in vascular smooth muscle cells: effects of dexamethasone and phosphoramidon. J Vasc Res 1993; 30(3): 139-144.

Roy SG, De P, Mukherjee D, Chander V, Konar A, Bandyopadhyay D, Bandyopadhyay A. Excess of glucocorticoid induces cardiac dysfunction via activating angiotensin II pathway. Cell Physiol Biochem 2009; 24(12): $1-10$.

Ruffmann R, Wendel A. GSH rescue by N-acetylcysteine. Klin Wochenschr 1991; 69(18): 857-862. 
Russo-Marie F, Duval D. Dexamethasone-induced inhibition of prostaglandin production dose not result from a direct action on phospholipase activities but is mediated through a steroid-inducible factor. Biochim Biophys Acta 1982; 712(1): 177-185.

Russo D, Fraser R, Kenyon CJ. Dexamethasone therapy selectively increases the sensitivity to noradrenaline of the rat mesenteric circulation. J Hypertens Suppl 1989; 7(6): S126-127.

Russo D, Fraser R, Kenyon CJ. Increased sensitivity to noradrenaline in glucocorticoid-treated rats: the effects of indomethacin and desipramine. $J$ Hypertens 1990; 8(9): 827-833.

Saha DC, Saha AC, Malik G, Astiz ME, Rackow EC. Comparison of cardiovascular effects of tiletamine-zolazepam, pentobarbital, and ketamine-xylazine in male rats. J Am Assoc Lab Anim Sci 2007; 46(2): 7480.

Sakaue M, Hoffman BB. Glucocorticoids induce transcription and expression of the alpha 1B adrenergic receptor gene in DTT1 MF-2 smooth muscle cells. J Clin Invest 1991; 88(2): 385-389.

Sambhi MP, Weil MH, Udhoji VN. Pressor responses to norepinephrine in humans before and after corticosteroids. Am J Physiol 1962; 203(5): 961963.

Sanchez-Mendoza A, Lopez-Sanchez P, Vazquez-Cruz B, Rios A, MartinezAyala S, Escalante B. Angiotensin II modulates ion transport in rat proximal tubules through CYP metabolites. Biochem Biophys Res Commun 2000; 272(2): 423-430. 
Sanz E, Lopez Novoa JM, Linares M, Digiuni E, Caramelo CA. Intravascular and interstitial fluid dynamics in rats treated with minoxidil. J Cardiovasc Pharmacol 1990; 15(3): 485-492.

Saruta T. Mechanism of glucocorticoid-induced hypertension. Hypertens Res 1996; 19(1): 1-8.

Sato A, Suzuki H, Iwaita Y, Nakazato Y, Kato H, Saruta T. Potentiation of inositol trisphosphate production by dexamethasone. Hypertension 1992; 19(1): 109-115.

Sato A, Suzuki H, Nakazato Y, Shibata H, Inagami T, Saruta T. Increased expression of vascular angiotensin II type $1 \mathrm{~A}$ receptor gene in glucocorticoid-induced hypertension. J Hypertens 1994; 12(5): 511-516.

Sayre LM, Smith MA, Perry G. Chemistry and biochemistry of oxidative stress in neurodegenerative disease. Curr Med Chem 2001; 8: 721-738.

Schafer SC, Wallerath T, Closs EI, Schmidt C, Schwarz PM, Forstermann U, Lehr HA. Dexamethasone suppresses eNOS and CAT-1 and induces oxidative stress in mouse resistance arterioles. Am J Physiol Heart Circ Physiol 2005; 288(1): H436-444.

Schimmer BP, Parker KL. Adrenocorticotrophic hormone; adrenocortical steroids and their synthetic analogs; inhibitors of the synthesis and actions of adrenocortical hormones. In: Hardman J. G.,Limbird L. E., eds. Goodman \& Gilman's the pharmacological basis of therapeutics. 10 ed. New York: McGraw-Hill; 2001:1649-1677. 
Schmidt K, Werner ER, Mayer B, Wachter H, Kukovetz WR. Tetrahydrobiopterin-dependent formation of endothelium-derived relaxing factor (nitric oxide) in aortic endothelial cells. Biochem J 1992; 281 ( Pt 2): $297-300$.

Scoggins BA, Allen KJ, Coghlan JP, Denton DA, Graham WF, Humphery TJ, Whitworth JA. Haemodynamics of ACTH-induced hypertension in sheep. Clin Sci (Lond) 1979; 57 Suppl 5: 333s-336s.

Sebaai N, Lesage J, Alaoui A, Dupouy JP, Deloof S. Effects of dehydration on endocrine regulation of the electrolyte and fluid balance and atrial natriuretic peptide-binding sites in perinatally malnourished adult male rats. Eur J Endocrinol 2002; 147(6): 835-848.

Sellden H, Sjovall H, Ricksten SE. Sympathetic nerve activity and central haemodynamics during mechanical ventilation with positive endexpiratory pressure in rats. Acta Physiol Scand 1986; 127(1): 51-60.

Shafagoj Y, Opoku J, Qureshi D, Regelson W, Kalimi M. Dehydroepiandrosterone prevents dexamethasone-induced hypertension in rats. Am J Physiol Endocrinol Metab 1992; 263(2): E210-213.

Shapiro HM. Membrane potential estimation by flow cytometry. Methods 2000; 21(3): 271-279.

Shapiro HM, Natale PJ, Kamentsky LA. Estimation of membrane potentials of individual lymphocytes by flow cytometry. Proc Natl Acad Sci U S A 1979; 76(11): 5728-5730. 
Siegal T, Soti F, Biegon A, Pop E, Brewster ME. Effect of a chemical delivery system for dexamethasone (Dex-CDS) on peritumoral edema in an experimental brain tumor model. Pharm Res 1997; 14(5): 672-675.

Silvan G, Martinez-Mateos MM, Blass A, Camacho L, Gonzalez-Gil A, GarciaPartida P, Illera JC. The effect of long-term exposure to combinations of growth promoters in Long Evans rats: Part 1: Endocrine adrenal function. Anal Chim Acta 2007; 586(1-2): 246-251.

Simmons WW, Ungureanu-Longrois D, Smith GK, Smith TW, Kelly RA. Glucocorticoids regulate inducible nitric oxide synthase by inhibiting tetrahydrobiopterin synthesis and L-arginine transport. J Biol Chem 1996; 271(39): 23928-23937.

Singh H, Cheng J, Deng H, Kemp R, Ishizuka T, Nasjletti A, Schwartzman ML. Vascular cytochrome P450 4A expression and 20-hydroxyeicosatetraenoic acid synthesis contribute to endothelial dysfunction in androgen-Induced hypertension. Hypertension 2007; 50(1): 123-129.

Sinha SK, Rodriguez HJ, Hogan WC, Klahr S. Mechanisms of activation of renal $(\mathrm{Na}++\mathrm{K}+)$-ATPase in the rat Effects of acute and chronic administration of dexamethasone. Biochim Biophys Acta 1981; 641(1): 20-35.

Sorescu D, Weiss D, Lassegue B, Clempus RE, Szocs K, Sorescu GP, Valppu L, Quinn MT, Lambeth JD, Vega JD, Taylor WR, Griendling KK. Superoxide production and expression of Nox family proteins in human atherosclerosis. Circulation 2002; 105(12): 1429-1435.

Spence CD, Mathai M, Mills EH, Coghlan JP, Whitworth JA, Scoggins BA. Centrally mediated increased sympathetic activity is not important in the 
genesis of ACTH-induced hypertension in sheep. Clin Exp Pharmacol Physiol 1989; 16(7): 607-619.

Starkov AA, Fiskum G, Chinopoulos C, Lorenzo BJ, Browne SE, Patel MS, Beal MF. Mitochondrial $\alpha$-ketoglutarate dehydrogenase complex generates reactive oxygen species. J Neurosci 2004; 24(36): 7779-7788.

Stern N, Palant C, Ozaki L, Tuck ML. Dexamethasone enhances active cation transport in cultured aortic smooth muscle cells. Am J Hypertens 1994; 7(2): 146-150.

Stevenson JA, Franklin C. Effects of ACTH and corticosteroids in the regulation of food and water intake. Prog Brain Res 1970; 32: 141-152.

Stewart P. The adrenal cortex. In: Kronenberg HM M. S., Polonsky KS, Larsen PR, ed. Williams Textbook of Endocrinology. 11 ed. Philadelphia: Saunders Elsevier; 2008:Chapter 14.

Stewart PM, Walker BR, Holder G, O'Halloran D, Shackleton CH. 11 betaHydroxysteroid dehydrogenase activity in Cushing's syndrome: explaining the mineralocorticoid excess state of the ectopic adrenocorticotropin syndrome. J Clin Endocrinol Metab 1995; 80(12): 3617-3620.

Stricker EM, Vagnucci AH, McDonald RH, Jr., Leenen FH. Renin and aldosterone secretions during hypovolemia in rats: relation to $\mathrm{NaCl}$ intake. Am J Physiol Regul Integr Comp Physiol 1979; 237(1): R45-51.

Sudhir K, Jennings GL, Esler MD, Korner PI, Blomberg PA, Lambert GW, Scoggins B, Whitworth JA. Hydrocortisone-induced hypertension in man. 
Pressor responsiveness and sympathetic function. Hypertension 1989; 13: 416.

Sun C-W, Alonso-Galicia M, Taheri MR, Falck JR, Harder DR, Roman RJ. Nitric oxide-20-hydroxyeicosatetraenoic acid interaction in the regulation of $\mathrm{K}+$ channel activity and vascular tone in renal arterioles. Circ Res 1998; 83(11): 1069-1079.

Sun XM, Dinsdale D, Snowden RT, Cohen GM, Skilleter DN. Characterization of apoptosis in thymocytes isolated from dexamethasone-treated rats. Biochemical Pharmacology 1992; 44(11): 2131-2137.

Sundler F, Bottcher G, Ekblad E, Hakanson R. PP, PYY and NPY. Occurrence and distrubution in the periphery. In: Colmers W.,Wahlestedt C., eds. The biology of neuropeptide $Y$ and related peptides. New Jersey: Humana Press; 1993:157-196.

Suzuki H, DeLano FA, Parks DA, Jamshidi N, Granger DN, Ishii H, Suematsu M, Zweifach BW, Schmid-Schonbein GW. Xanthine oxidase activity associated with arterial blood pressure in spontaneously hypertensive rats. Proc Natl Acad Sci U S A 1998; 95(8): 4754-4759.

Suzuki H, Handa M, Kondo K, Saruta T. Role of renin-angiotensin system in glucocorticoid hypertension in rats. Am J Physiol Endocrinol Metab 1982; 243(1): E48-51.

Suzuki H, Swei A, Zweifach BW, Schmid-Schonbein GW. In vivo evidence for microvascular oxidative stress in spontaneously hypertensive rats: hydroethidine microfluorography. Hypertension 1995; 25(5): 1083-1089. 
Swei A, Lacy F, Delano FA, Parks DA, Schmid-Schonbein GW. A mechanism of oxygen free radical production in the Dahl hypertensive rat. Microcirculation 1999; 6(3): 179 - 187.

Swei A, Lacy F, DeLano FA, Schmid-Schonbein GW. Oxidative stress in the Dahl hypertensive rat. Hypertension 1997; 30(6): 1628-1633.

Tabarin A, Minot AP, Dallochio M, Roger P, Ducassou D. Plasma concentration of neuropeptide Y in patients with adrenal hypertension. Regul Pept 1992; 42(1-2): 51-61.

Takahashi K, Suda K, Lam HC, Ghatei MA, Bloom SR. Endothelin-like immunoreactivity in rat models of diabetes mellitus. J Endocrinol 1991; 130(1): 123-127.

Takeda K, Bunag RD. Chronic propranolol treatment inhibits sympathetic nerve activity and keeps blood pressure from rising in spontaneously hypertensive rats. Hypertension 1980; 2(2): 228-235.

Tan C, Schyvens CG, P A, McKenzie KU, Zhang Y, Whitworth JA. The effect of adrenocorticotropic hormone treatment on nitrotyrosine levels in rat organs. Clin Exp Pharmacol Physiol 2005; 32(7): A26.

Tanaka MDE, Mori MDH, Chujo MM, Yamakawa MDA, Mohammed MDMU, Shinozaki Y, Tobita MDK, Sekka MDT, Ito MDK, Nakazawa MDH. Coronary vasoconstrictive effects of neuropeptide $\mathrm{Y}$ and their modulation by the ATP-sensitive potassium channel in anesthetized dogs. $J$ Am Coll Cardiol 1997; 29(6): 1380-1389. 
Tarpey MM, Fridovich I. Methods of detection of vascular reactive species: nitric oxide, superoxide, hydrogen peroxide, and peroxynitrite. Circ Res 2001; 89(3): 224-236.

Taubert D, Berkels R, Grosser N, Schroder H, Grundemann D, Schomig E. Aspirin induces nitric oxide release from vascular endothelium: a novel mechanism of action. Br J Pharmacol 2004; 143(1): 159-165.

Terzolo M, Allasino B, Bosio S, Brusa E, Daffara F, Ventura M, Aroasio E, Sacchetto G, Reimondo G, Angeli A, Camaschella C. Hyperhomocysteinemia in patients with Cushing's syndrome. J Clin Endocrinol Metab 2004; 89(8): 3745-3751.

Thida M, Earl J, Zhao Y, Wang H, Tse CS, Vickers JJ, Sutton M, Ong SLH, Mori TA, Croft KD, Whitworth JA, Zhang Y. Effects of sepiapterin supplementation and NOS inhibition on glucocorticoid-induced hypertension. Am J Hypertens 2010; 23(5): 569-574.

Tomas FM, Knowles SE, Owens PC, Chandler CS, Francis GL, Read LC, Ballard FJ. Insulin-like growth factor-I (IGF-I) and especially IGF-I variants are anabolic in dexamethasone-treated rats. Biochem J 1992; 282 ( Pt 1)(Pt 1): 91-97.

Tonolo G, Fraser R, Connell JM, Kenyon CJ. Chronic low-dose infusions of dexamethasone in rats: effects on blood pressure, body weight and plasma atrial natriuretic peptide. J Hypertens 1988; 6(1): 25-31.

Tonolo G, Richards AM, Manunta P, Troffa C, Pazzola A, Madeddu P, Towrie A, Fraser R, Glorioso N. Low-dose infusion of atrial natriuretic factor in mild essential hypertension. Circulation 1989; 80(4): 893-902. 
Tonolo G, Soro A, Madeddu P, Troffa C, Melis MG, Patteri G, Parpaglia PP, Sabino G, Maioli M, Glorioso N. Effect of chronic intracerebroventricular dexamethasone on blood pressure in normotensive rats. Am J Physiol Endocrinol Metab 1993; 264(6): E843-847.

Touyz RM, Schiffrin EL. Reactive oxygen species in vascular biology: implications in hypertension. Histochem Cell Biol 2004; 122(4): 339-352.

Tretter L, Adam-Vizi V. Alpha-ketoglutarate dehydrogenase: a target and generator of oxidative stress. Philos Trans R Soc Lond B Biol Sci 2005; 360(1464): 2335-2345.

Trivedi RC, Rebar L, Berta E, Stong L. New enzymatic method for serum uric acid at $500 \mathrm{~nm}$. Clin Chem 1978; 24(11): 1908-1911.

Tsoporis J, Fields N, Lee RM, Leenen FH. Arterial vasodilation and cardiovascular structural changes in normotensive rats. Am J Physiol Heart Circ Physiol 1991; 260(6): H1944-1952.

Tsunoda M, Takezawa K, Santa T, Ina Y, Nagashima K, Ohmori K, Kobayashi S, Imai K. New approach for measurement of sympathetic nervous abnormality in conscious, spontaneously hypertensive rats. Jpn $J$ Pharmacol 2000; 83(1): 39-45.

Turner SW, Mangos GJ, Whitworth JA. Nitric oxide synthase activity in adrenocorticotrophin-induced hypertension in the rat. Clin Exp Pharmacol Physiol 2001; 28(11): 881-883. 
Turner SW, Wen C, Li M, Fraser TB, Whitworth JA. Adrenocorticotrophin doseresponse relationships in the rat: haemodynamic, metabolic and hormonal effects. J Hypertens 1998; 16(5): 593-600.

Turner SW, Wen C, Li M, Whitworth JA. L-Arginine prevents corticotropininduced increases in blood pressure in the rat. Hypertension 1996; 27(2): 184-189.

Valko M, Leibfritz D, Moncol J, Cronin MTD, Mazur M, Telser J. Free radicals and antioxidants in normal physiological functions and human disease. Int J Biochem Cell Biol 2007; 39(1): 44-84.

van den Meiracker AH, Man in't Veld AJ, Boomsma F, Fischberg DJ, Molinoff PB, Schalekamp MA. Hemodynamic and beta-adrenergic receptor adaptations during long-term beta-adrenoceptor blockade. Studies with acebutolol, atenolol, pindolol, and propranolol in hypertensive patients. Circulation 1989; 80(4): 903-914.

Vanasco V, Cimolai MC, Evelson P, Alvarez S. The oxidative stress and the mitochondrial dysfunction caused by endotoxemia are prevented by $\alpha$ lipoic acid. Free Radic Res 2008; 42(9): 815 - 823.

Vanden Hoek TL, Shao Z, Li C, Schumacker PT, Becker LB. Mitochondrial electron transport can become a significant source of oxidative Injury in cardiomyocytes. J Mol Cell Cardiol 1997; 29(9): 2441-2450.

Vasquez-Vivar J, Kalyanaraman B, Martasek P, Hogg N, Masters BSS, Karoui H, Tordo P, Pritchard KA, Jr. Superoxide generation by endothelial nitric oxide synthase: The influence of cofactors. Proc Natl Acad Sci U S A 1998; 95(16): 9220-9225. 
Vasquez-Vivar J, Martasek P, Whitsett J, Joseph J, Kalyanaraman B. The ratio between tetrahydrobiopterin and oxidized tetrahydrobiopterin analogues controls superoxide release from endothelial nitric oxide synthase: an EPR spin trapping study. Biochem J 2002; 362(Pt 3): 733-739.

Vaziri ND, Wang XQ, Oveisi F, Rad B. Induction of oxidative stress by glutathione depletion causes severe hypertension in normal rats. Hypertension 2000; 36(1): 142-146.

Wakabayashi Y, Yamada E, Yoshida T, Takahashi H. Deficiency of endogenous arginine synthesis provokes hypertension by exhausting substrate arginine for nitric oxide synthesis. Biochem Biophys Res Commun 1994; 205(2): 1391-1398.

Walker BR, Best R, Shackleton CHL, Padfield PL, Edwards CRW. Increased vasoconstrictor sensitivity to glucocorticoids in essential hypertension. Hypertension 1996; 27(2): 190-196.

Wallerath T, Godecke A, Molojavyi A, Li H, Schrader J, Forstermann U. Dexamethasone lacks effect on blood pressure in mice with a disrupted endothelial NO synthase gene. Nitric Oxide 2004; 10(1): 36-41.

Wallerath T, Witte K, Schafer SC, Schwarz PM, Prellwitz W, Wohlfart P, Kleinert H, Lehr H-A, Lemmer B, Forstermann U. Down-regulation of the expression of endothelial NO synthase is likely to contribute to glucocorticoid-mediated hypertension. Proc Natl Acad Sci U S A 1999; 96(23): 13357-13362.

Wallwork CJ, Parks DA, Schmid-Schonbein GW. Xanthine oxidase activity in the dexamethasone-induced hypertensive rat. Microvasc. Res. 2003; 66(1): 30-37. 
Wang HD, Pagano PJ, Du Y, Cayatte AJ, Quinn MT, Brecher P, Cohen RA. Superoxide anion from the adventitia of the rat thoracic aorta inactivates nitric oxide. Circ Res 1998; 82(7): 810-818.

Wang J-S, Singh H, Zhang F, Ishizuka T, Deng H, Kemp R, Wolin MS, Hintze TH, Abraham NG, Nasjletti A, Laniado-Schwartzman M. Endothelial dysfunction and hypertension in rats transduced with CYP4A2 adenovirus. Circ Res 2006; 98(7): 962-969.

Wang L-L, Ou C-C, Chan JYH. Receptor-independent activation of GABAergic neurotransmission and receptor-dependent nontranscriptional activation of phosphatidylinositol 3-kinase/protein kinase Akt pathway in short-term cardiovascular actions of dexamethasone at the nucleus tractus solitarii of the rat. Mol Pharmacol 2005; 67(2): 489-498.

Wassmann S, Wassmann K, Nickenig G. Modulation of oxidant and antioxidant enzyme expression and function in vascular cells. Hypertension 2004; 44(4): 381-386.

Watanabe T, Noshiro T, Akama H, Kusakari T, Shibukawa S, Miura W, Abe K, Miura Y. Effect of dexamethasone on plasma free dopamine: dopaminergic modulation in hypertensive patients. Hypertens Res 1995; 18 (Suppl 1): S197-198.

Wen C, Fraser T, Li M, Turner SW, Whitworth JA. Haemodynamic mechanisms of corticotropin (ACTH)-induced hypertension in the rat. $J$ Hypertens 1999; 17(12 Pt 1): 1715-1723.

Wen C, Fraser T, Li M, Whitworth JA. Hemodynamic profile of corticotropininduced hypertension in the rat. J Hypertens 1998; 16(2): 187-194. 
Wen C, Li M, Fraser T, Wang J, Turner SW, Whitworth JA. L-Arginine partially reverses established adrenocorticotrophin-induced hypertension and nitric oxide deficiency in the rat. Blood Press 2000; 9(5): 298-304.

Wen C, Li M, Whitworth JA. Validation of transonic small animal flowmeter for measurement of cardiac output and regional blood flow in the rat. $J$ Cardiovasc Pharmacol 1996; 27(4): 482-486.

Wen C, Li M, Whitworth JA. Role of nitric oxide in adrenocorticotrophin-induced hypertension: L-arginine effects reversed by N-nitro-L-arginine. Clin Exp Pharmacol Physiol 2000a; 27(11): 887-890.

Whitworth JA. Adrenocorticotrophin and steroid-induced hypertension in humans. Kidney Int Suppl 1992; 37: S34-37.

Whitworth JA, Coghlan JP, Denton DA, Graham WF, Humphery TJ, Scoggins BA. Comparison of the effects of 'glucocorticoid' and 'mineralocortocoid' infusions on blood pressure in sheep. Clin Exp Hypertens 1979; 1(5): 649663.

Whitworth JA, Gordon D, Andrews J, Scoggins BA. The hypertensive effect of synthetic glucocorticoids in man: role of sodium and volume. $J$ Hypertens 1989; 7(7): 537-549.

Whitworth JA, Saines D, Scoggins BA. Blood pressure and metabolic effects of cortisol and deoxycorticosterone in man. Clin Exp Hypertens A 1984; 6(4): 795-809.

Whitworth JA, Saines D, Scoggins BA. Potentiation of ACTH hypertension in man with salt loading. Clin Exp Pharmacol Physiol 1985; 12(3): 239-243. 
Whitworth JA, Schyvens CG, Zhang Y, Mangos GJ, Kelly JJ. Glucocorticoidinduced hypertension: from mouse to man. Clin Exp Pharmacol Physiol 2001; 28(12): 993-996.

Whitworth JA, Zhang Y, Mangos G, Kelly JJ. Species variability in cardiovascular research: The example of adrenocorticotrophin-induced hypertension. Clin Exp Pharmacol Physiol 2006; 33(9): 887-891.

Wilding JP, Gilbey SG, Lambert PD, Ghatei MA, Bloom SR. Increases in neuropeptide $\mathrm{Y}$ content and gene expression in the hypothalamus of rats treated with dexamethasone are prevented by insulin. Neuroendocrinology 1993; 57(4): 581-587.

Williamson PM, Kelly JJ, Whitworth JA. Dose-response relationships and mineralocorticoid activity in cortisol-induced hypertension in humans. $J$ Hypertens 1996; 14 Suppl.(5): S37-41.

Wintour EM, Alcorn D, McFarlane A, Moritz K, Potocnik SJ, Tangalakis K. Effect of maternal glucocorticoid treatment on fetal fluids in sheep at 0.4 gestation. Am J Physiol Regul Integr Comp Physiol 1994; 266(4): R11741181.

Wong DL, Siddall B, Wang W. Hormonal control of rat adrenal phenylethanolamine N-methyltransferase. Enzyme activity, the final critical pathway. Neuropsychopharmacology 1995; 13(3): 223-234.

Wu R, Millette E, Wu L, de Champlain J. Enhanced superoxide anion formation in vascular tissues from spontaneously hypertensive and desoxycorticosterone acetate-salt hypertensive rats. J Hypertens 2001; 19(4): 741-748. 
Yoshimoto T, Naruse M, Irie K, Tanabe A, Seki T, Tanaka M, Imaki T, Naruse K, Muraki T, Matsuda Y, Demura H. Beta-adrenoceptor antagonist propranolol potentiates hypotensive action of natriuretic peptides. Eur J Pharmacol 1998; 351(1): 61-66.

Zavitsanou K, Nguyen V, Greguric I, Chapman J, Ballantyne P, Katsifis A. Detection of apoptotic cell death in the thymus of dexamethasone treated rats using [123I]Annexin $\mathrm{V}$ and in situ oligonucleotide ligation. $J \mathrm{Mol}$ Histol 2007; 38(4): 313-319.

Zhang L, Fujii S, Igarashi J, Kosaka H. Effects of thiol antioxidant on reduced nicotinamide adenine dinucleotide phosphate oxidase in hypertensive Dahl salt-sensitive rats. Free Radic Biol Med 2004; 37(11): 1813-1820.

Zhang Y, Chan MMK, Andrews MC, Mori TA, Croft KD, McKenzie KUS, Schyvens CG, Whitworth JA. Apocynin but not allopurinol prevents and reverses adrenocorticotropic hormone-induced hypertension in the rat. Am J Hypertens 2005; 18(7): 910-916.

Zhang Y, Croft KD, Mori TA, Schyvens CG, McKenzie KUS, Whitworth JA. The antioxidant tempol prevents and partially reverses dexamethasoneinduced hypertension in the rat. Am J Hypertens 2004; 17(3): 260-265.

Zhang Y, Croft KD, Veckers JJ, Ong SLH, Mori TA, Whitworth JA. HET0016, a 20-hydroxyeicosatetraenoic acid inhibitor, prevents and reverses adrenocorticotrophic hormone-induced hypertension. Journal of Hypertension 2008; 26: S76-S76.

Zhang Y, Hu L, Mori TA, Barden A, Croft KD, Whitworth JA. Arachidonic acid metabolism in glucocorticoid-induced hypertension. Clin Exp Pharmacol Physiol 2008; 35(5-6): 557-562. 
Zhang Y, Jang R, Mori TA, Croft KD, Schyvens CG, McKenzie KU, Whitworth JA. The anti-oxidant Tempol reverses and partially prevents adrenocorticotrophic hormone-induced hypertension in the rat. $J$ Hypertens 2003; 21(8): 1513-1518.

Zhang Y, Miao Y, Whitworth JA. Aspirin prevents and partially reverses adrenocorticotropic hormone-induced hypertension in the rat. Am J Hypertens 2007; 20(11): 1222-1228.

Zhang Y, Pang T, Earl J, Schyvens CG, McKenzie KU, Whitworth JA. Role of tetrahydrobiopterin in adrenocorticotropic hormone-induced hypertension in the rat. Clin Exp Hypertens 2004; 26(3): 231-241.

Zhang Y, Soboloff J, Zhu Z, Berger SA. Inhibition of Ca2+ influx is required for mitochondrial reactive oxygen species-induced endoplasmic reticulum Ca2+ depletion and cell death in leukemia cells. Mol Pharmacol 2006; 70(4): 1424-1434.

Zhang Y, Wu JHY, Vickers JJ, Ong SLH, Temple SEL, Mori TA, Croft KD, Whitworth JA. The role of 20-hydroxyeicosatetraenoic acid in glucocorticoid-induced hypertension. J Hypertens 2009; 27(8): 16091616. 